\title{
Report on Advanced Detector Development
}

J. Keith Jewell

Tony Hill

Fredrik Tovesson

Partha Chowdhury

Uwe Greife

Mike Slaughter

Eric Burgett

September 2012

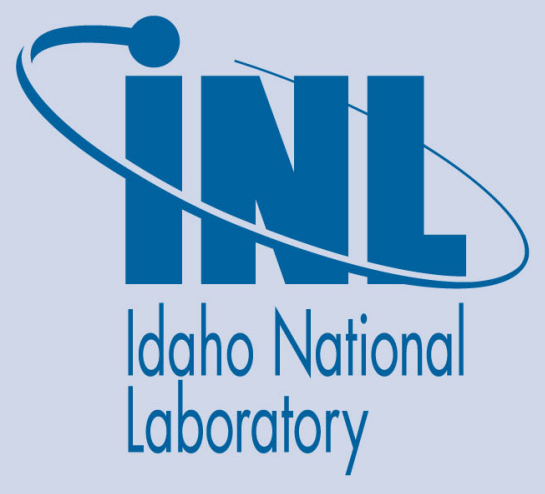

The INL is a U.S. Department of Energy National Laboratory operated by Battelle Energy Alliance 
INL/EXT-12-27268

FCRD-Fuel-2012-000357

\section{Report on Advanced Detector Development}

${ }^{1}$ LANL

${ }^{2} \mathrm{UML}$

${ }^{3} \mathrm{CSM}$

${ }^{4}$ WSU

${ }^{5}$ ISU
J. Keith Jewell

Tony Hill

Fredrik Tovesson ${ }^{1}$

Partha Chowdhury ${ }^{2}$

Uwe Greife ${ }^{3}$

Mike Slaughter ${ }^{4}$

Eric Burgett ${ }^{5}$

September 2012

Idaho National Laboratory
Idaho Falls, Idaho 83415

http://www.inl.gov
Prepared for the

U.S. Department of Energy

Office of Nuclear Energy

Under DOE Idaho Operations Office

Contract DE-AC07-05ID14517 


\section{Report on Advanced Detector Development Work Package Manager: J. Keith Jewell}

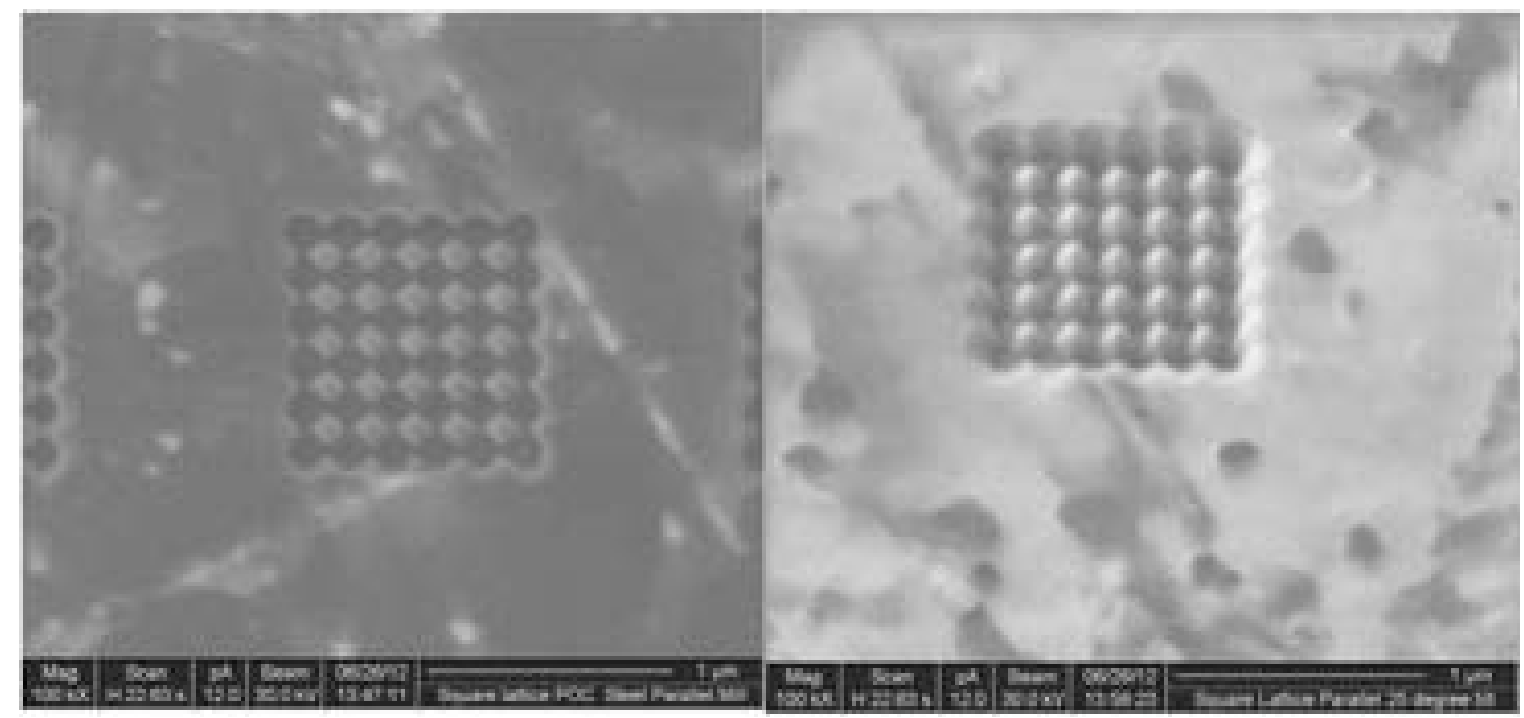

Focused lon Beam images of $\mathrm{ZnO}$ neutron scintillator development that incorporates a photonic crystal structure. A sapphire substrate was templated with a photonic crystal designed for the optical wavelength of $532 \mathrm{~nm}$. Zinc Oxide was grown on the template with a green band emission wavelength to match the photonic crystal structure. In future work, dopants other than carbon will be elicited to achieve a more desired emission spectrum. The structure was directly grown on the sapphire template through the modified ALD technique resulting in $\mathrm{ZnO}$ being grown conformally into the sample. Light output measurements were made of similar devices without the photonic crystal structure cut into them for a comparison baseline, and demonstrates that the light outcoupling has improved by a factor of two. 


\section{Blank Page}




\section{Table of Contents}

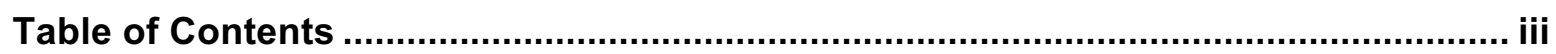

Major Contributors …....................................................................................... vi

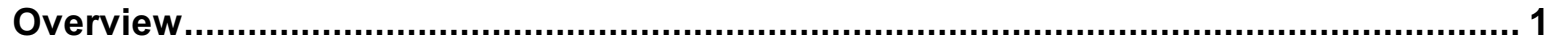

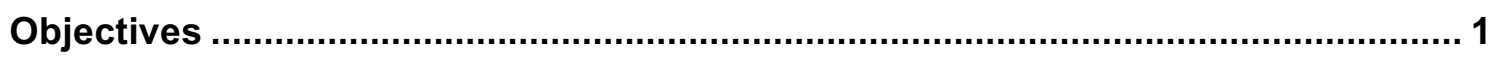

Nuclear Data and Advanced Measurements ............................................................... 2

Nuclear Data Measurement Program at LANL....................................................... 3

Overview

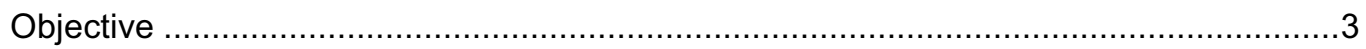

Assumptions and Prerequisites ……………………………………………….

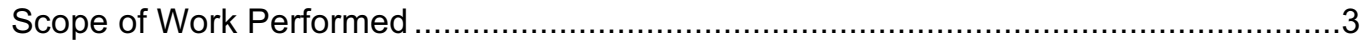

Los Alamos Neutron Science Center............................................................................... 4

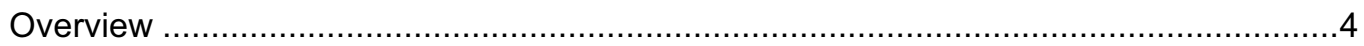

New building construction at LANSCE-WNR ……………………………….......

Nuclear Data Measurement Program …………………............................................ 6

Fission Measurement Program .............................................................................

Neutron Capture Measurement Program ………………………………………....30

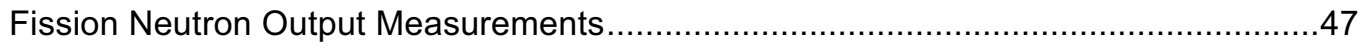

Time Projection Chamber ............................................................................... 60

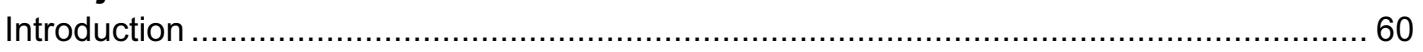

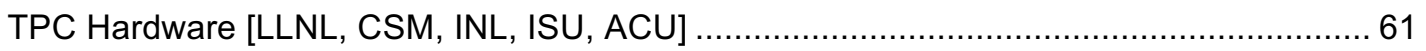

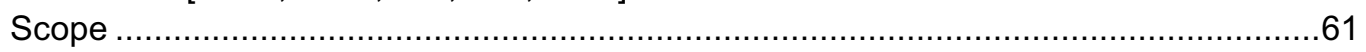

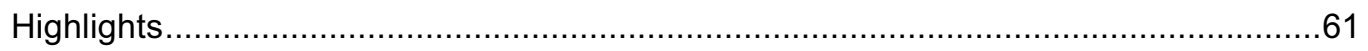

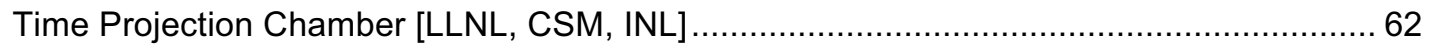

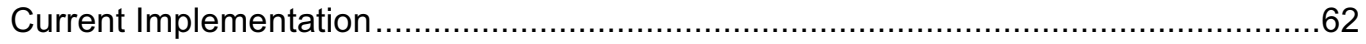

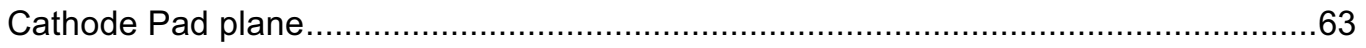

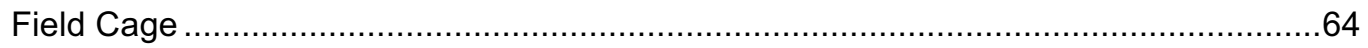

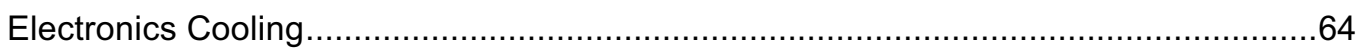

High Speed Cathode Readout (HiSCaR) ..............................................................65

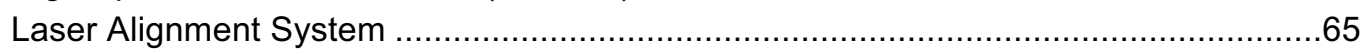

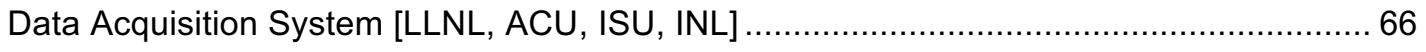

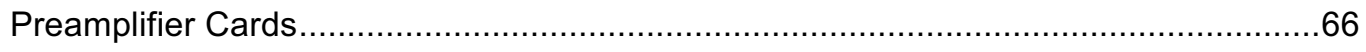

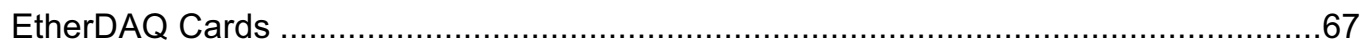

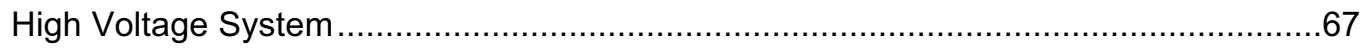

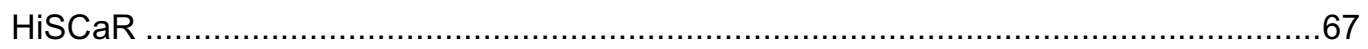

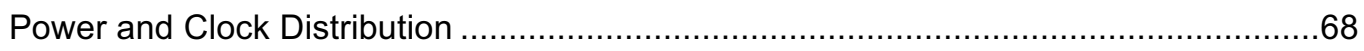

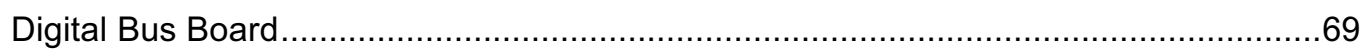

Gas Handling and Temperature Control Systems [CSM, LLNL] …………………….... 74

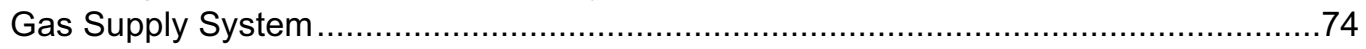

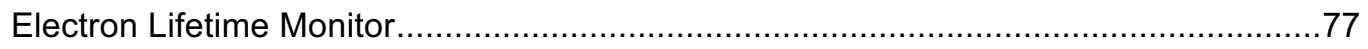

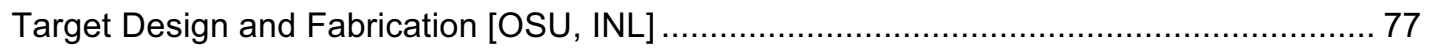




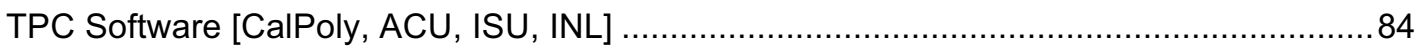

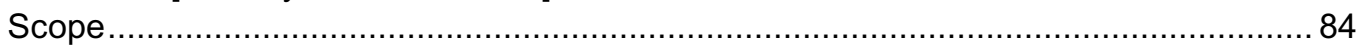

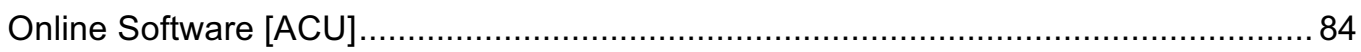

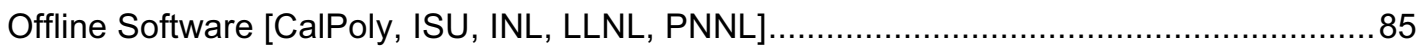

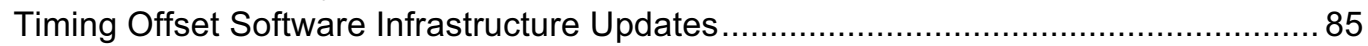

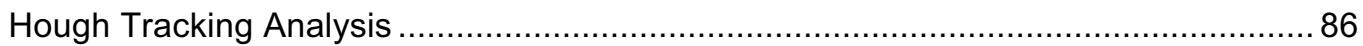

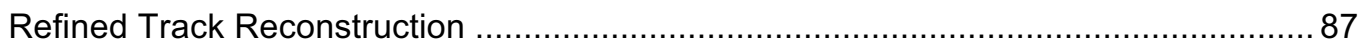

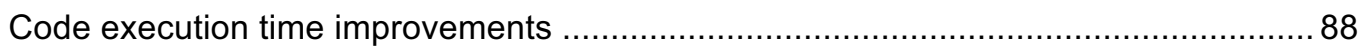

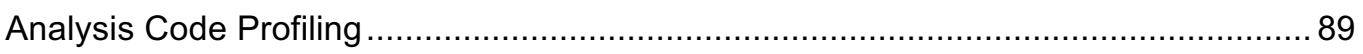

Track Reconstruction using the Combinatorial Hough method .................................... 90

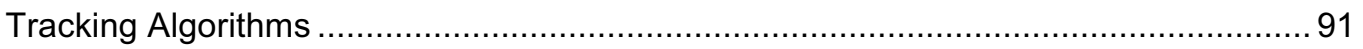

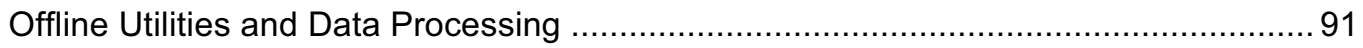

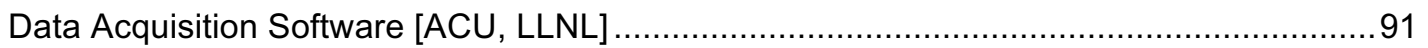

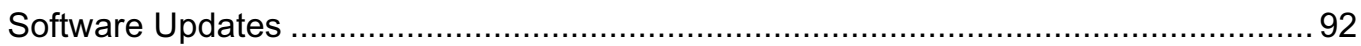

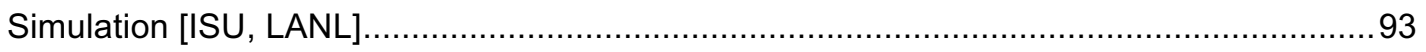

Scope

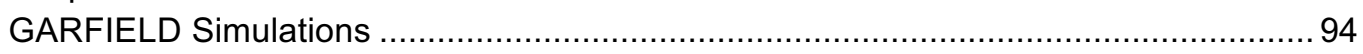

Improvements to Particle Transport and Event Generation inside Geant4 ..................96

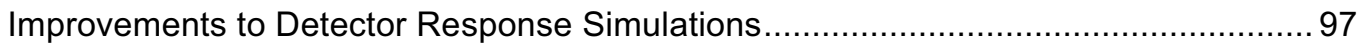

MCNP Simulation of the Fission Ionization Chamber ............................................... 97

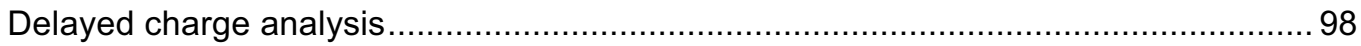

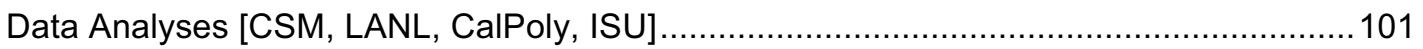

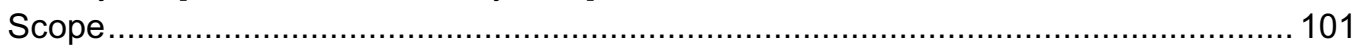

Cf-252 Alpha Decay to Spontaneous Fission Branching Ratio ................................ 101

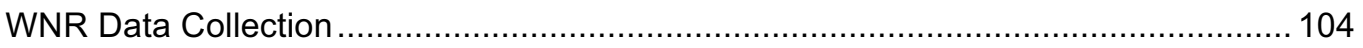

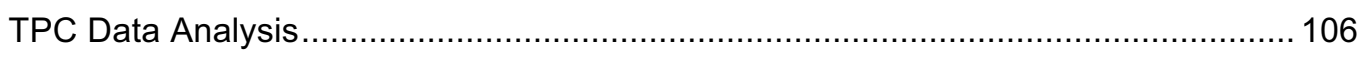

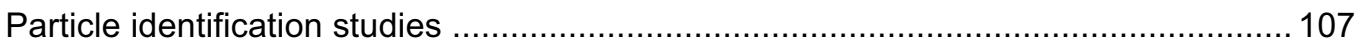

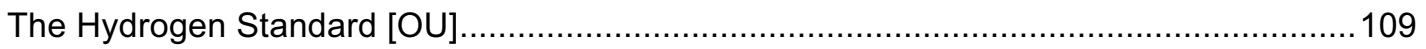

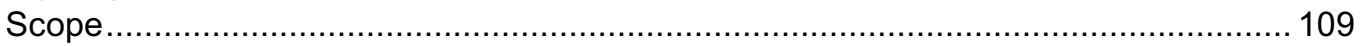

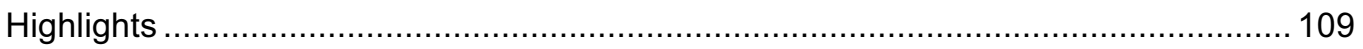

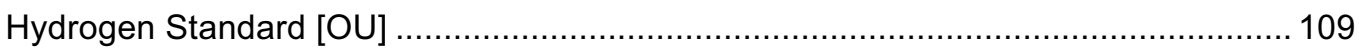

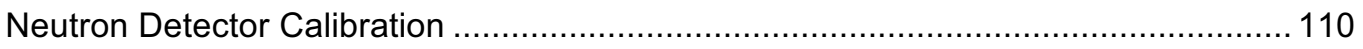

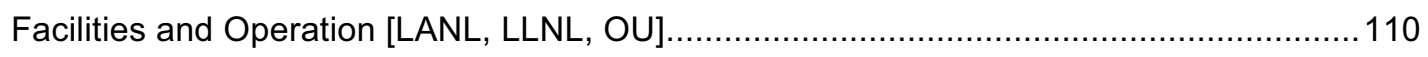

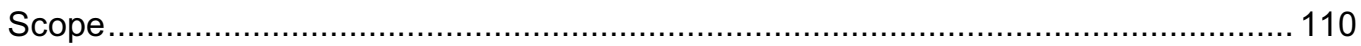

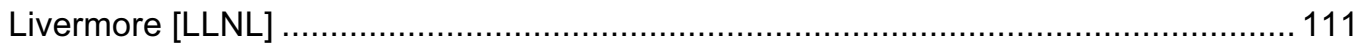

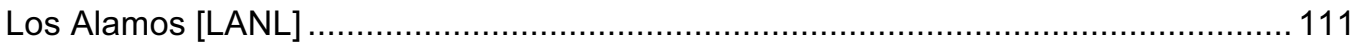

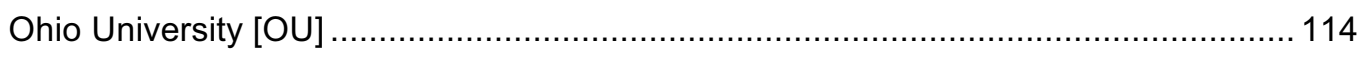

Nuclear Reaction Detection Capabilities ............................................................. 115

Advanced Detector Materials and Scintillators ................................................. 117

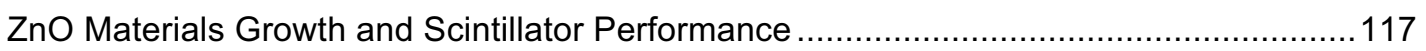

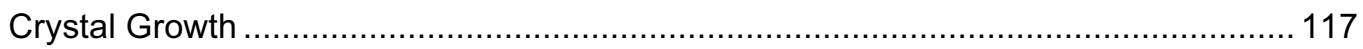

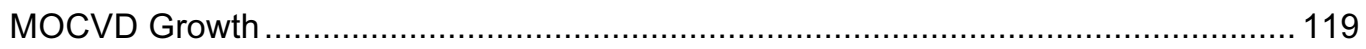

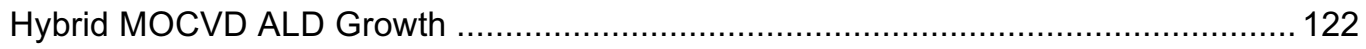

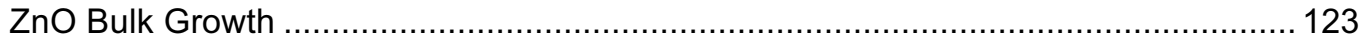

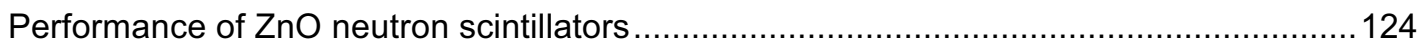

Impact of Growth Conditions on Scintillator Performance ...................................... 125

Impact of Dopants on ZnO Scintillator Performance ............................................. 128 


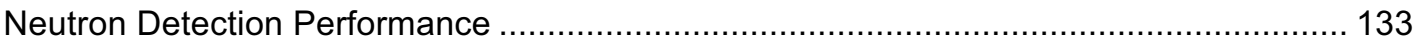

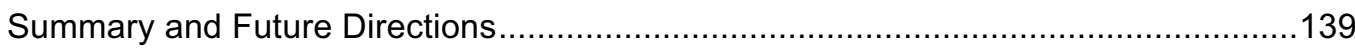

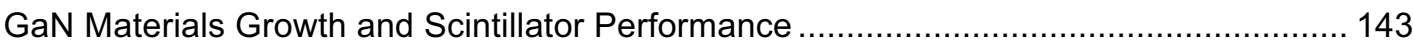

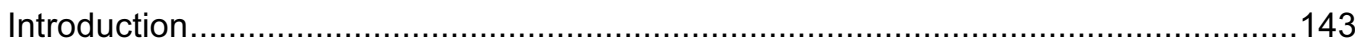

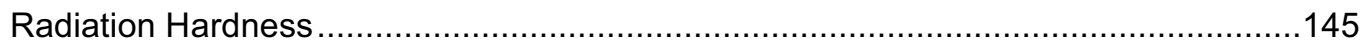

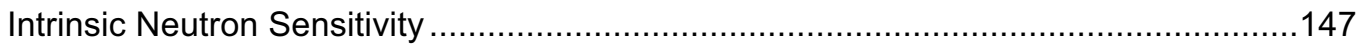

Challenges for Gallium Nitride Neutron Detectors ....................................................148

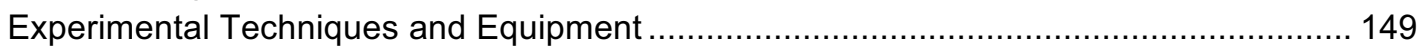

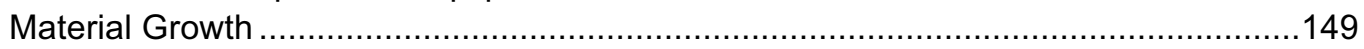

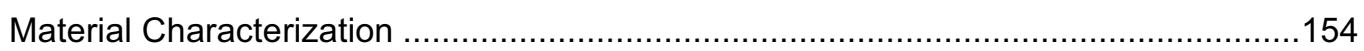

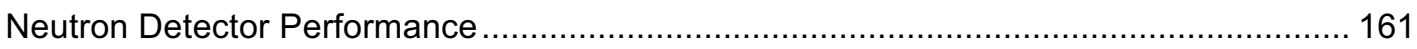

Scintillator Characterization and Performance ......................................................162

Plasmonically Cloaked and Metamaterial Neutron Scintillators..................................... 171

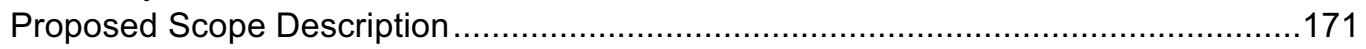

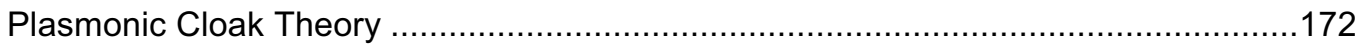

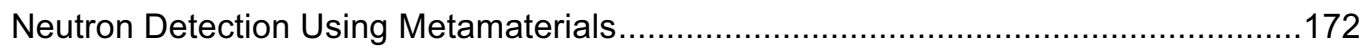

Fluidized Bed Reactor Design ................................................................... 177

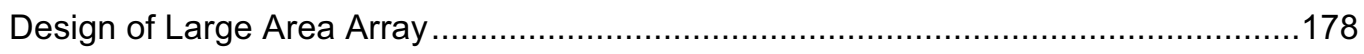

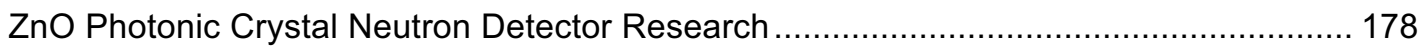

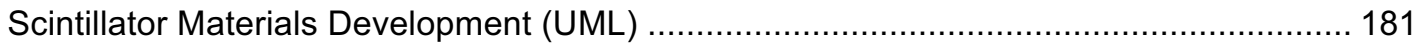

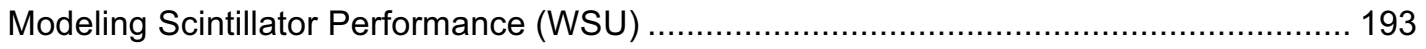

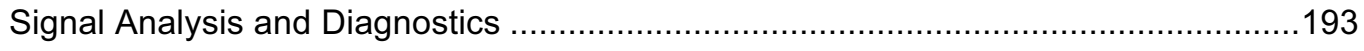

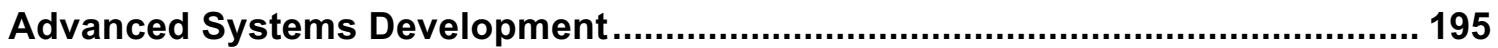

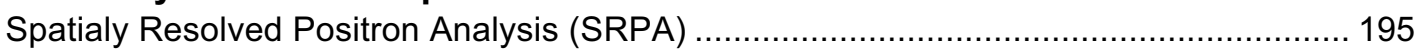

Pre-irradiation SRPA Materials Characterization ...................................................195

Irradiation and Post Irradiation SRPA Materials Characterization................................197

Integrated SEM/SRPA High Temperature System..................................................198

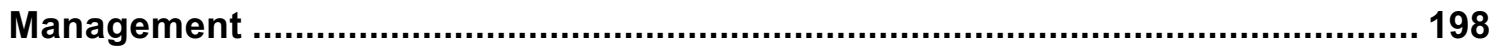

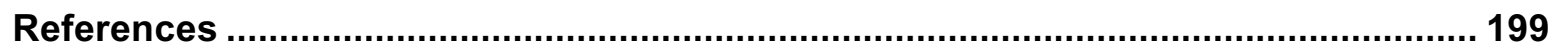




\section{Major Contributors}

INL

Tony Hill

Keith Jewell

LANL

Fredrik Tovesson

UML

Partha Chowdhury

CSM

Uwe Greife

WSU

Mike Slaughter

ISU

Eric Burgett 
Blank Page 



\section{Overview}

Neutron, gamma and charged particle detection improvements are key to supporting many of the foreseen measurements and systems envisioned in the R\&D programs and the future fuel cycle requirements, such as basic nuclear physics and data, modeling and simulation, reactor instrumentation, criticality safety, materials management and safeguards. This task will focus on the developmental needs of the FCR\&D experimental programs, such as elastic/inelastic scattering, total cross sections and fission neutron spectra measurements, and will leverage a number of existing neutron detector development efforts and programs, such as those at LANL, PNNL, INL, and IAC as well as those at many universities, some of whom are funded under NE grants and contracts. Novel materials and fabrication processes combined with state-of-the-art electronics and computing provide new opportunities for revolutionary detector systems that will be able to meet the high precision needs of the program. This work will be closely coordinated with the Nuclear Data Crosscut.

The Advanced Detector Development effort is a broadly-focused activity that supports the development of improved nuclear data measurements and improved detection of nuclear reactions and reactor conditions. This work supports the design and construction of large-scale, multiple component detectors to provide nuclear reaction data of unprecedented quality and precision. Examples include the Time Projection Chamber (TPC) and the DANCE detector at LANL. This work also supports the fabrication and end-user application of novel scintillator materials detection and monitoring.

\section{Objectives}

The objective of the Advanced Measurement Techniques effort is to provide fundamental, cross-cutting support for the NE R\&D Objectives $(2,3 \& 4)$ and the Fuel Cycle R\&D campaigns by identifying, prioritizing, developing and delivering the required nuclear and covariance data, at the needed precision, to the benefit of the operation, design and safety performance of the future nuclear systems and fuel cycles. Nuclear data plays a fundamental role in many calculations performed throughout the fuel cycle. Uncertainties in the nuclear data propagate into uncertainties in calculated integral quantities, driving margins and costs in advanced system design, operation and safeguards. This package contributes to the resolution of technical, cost, safety, security and proliferation concerns in a multi-pronged, systematic, science-based R\&D approach. The Nuclear Data effort identifies and develops small scale, phenomenon-specific experiments informed by theory and engineering to minimize the reliance and expense of large integral experiments. Also included in this effort is the identification and inclusion of high-quality data from previous large, expensive integral experiments, insuring past investments are realized and future data requirements are minimized. The Nuclear Data activities are leveraged by effective collaborations between experiment and theory, between DOE programs and offices, at national laboratories and universities, both domestic and international. 
Advanced detector R\&D continues to improve the NE nuclear data measurements by developing the detectors and systems capable of providing nuclear data at unprecedented precision. As the stringent requirements of the future fuel cycle are studied in detail, nuclear data uncertainties have been shown to be factors in cost and safety. In support of the broader advanced simulation and modeling engineering effort, this task provides support for evolutionary and revolutionary developments in existing and innovative measurement and monitoring tools, specifically, advanced neutron detectors and systems, room temperature gamma ray systems, capture measurement challenges, precision fission measurements, LSDS development and nuclear resonance fluorescence.

The Advanced Detector Development effort has two primary activities. One is to develop the measurement capabilities and methods to provide high-priority nuclear reaction data of unprecedented accuracy. Nuclear data sensitivity calculations have identified the isotopes and reactions that have the greatest impact on the integral parameters of reactor design and operation. The required data precisions present an experimental challenge that requires new techniques to ensure delivery of the highfidelity data for future generations of reactor development.

The second primary activity is the development of advanced detector materials for reactor applications. A science-based approach to reactor fuel design demands detectors that can provide real-time, in-situ monitoring within a reactor environment. Much of the data currently obtainable is limited to a post-irradiation analysis, and is inadequate to describe the time-evolution of reactor dynamics. A focus on novel materials and scintillators using new advances in nanotechnology will provide previously unobtainable data for in-core studies.

\section{Nuclear Data and Advanced Measurements}

The nuclear data measurement activities are driven by short-term and long-term nuclear data needs. Fast reactor sensitivity studies identify and quantify the nuclear data needs for the near term candidates of the fast reactor project. Uncertainties in the available nuclear data propagate to uncertainties in integral reactor parameters. Providing high precision nuclear data drives down the cost of construction and operation of a new plant. Nuclear theory development and future high fidelity reactor design calculations provide the long-term justification for an ongoing precision nuclear data measurement campaign. Nuclear physics is fundamental to reactor core simulations, as well safeguards and criticality safety. The design space for the future fleet of fast reactors and the future fuel cycle is severely limited by the uncertainties that currently exist in the nuclear data that have yet to be identified by specific designs. Truly innovative reactor designs and fuel cycles that will take full advantage of advanced computing techniques and platforms will be dependent on the fidelity of the underlying nuclear physics. The nuclear data measurement activities will help insure that nuclear physics will not be the limiting factor in future fuel cycle innovation. 


\section{Nuclear Data Measurement Program at LANL}

Included here is the 2012 annual report for Advanced Detector Development activities at Los Alamos National Laboratory. The activities included updates and advances to the fission measurement, capture-cross measurement, and fission neutron energy spectrum programs, as well as progress in detector development for the lead slowingdown spectrometer.

\section{Overview}

\section{Objective}

The objective of this work package is to provide fundamental, cross-cutting support for the NE R\&D Objectives (2, $3 \& 4)$ and the Fuel Cycle Technologies campaigns by identifying, prioritizing, developing and delivering the required nuclear and covariance data, at the needed precision, to the benefit of the operation, design and safety performance of the future nuclear systems and fuel cycles. Nuclear data plays a fundamental role in many calculations performed throughout the fuel cycle. Uncertainties in the nuclear data propagate into uncertainties in calculated integral quantities, driving margins and costs in advanced system design, operation, and safeguards. This package contributes to the resolution of technical, cost, safety, security, and proliferation concerns in a multi-pronged, systematic, science-based R\&D approach. The Nuclear Data effort identifies and develops small scale, phenomenon-specific experiments informed by theory and engineering to minimize the reliance and expense of large integral experiments. Also included in this effort is the identification and inclusion of high-quality data from previous large, expensive integral experiments, insuring past investments are realized and future data requirements are minimized. The Nuclear Data activities are leveraged by effective collaborations between experiment and theory, between DOE programs and offices, at national laboratories and universities, both domestic and international.

\section{Assumptions and Prerequisites}

Access to nuclear data measurement facilities and instruments at LANL and LLNL is continued.

\section{Scope of Work Performed}

Neutron and gamma detection improvements are key to supporting many of the foreseen measurements and systems envisioned in the R\&D programs and the future fuel cycle requirements, such as basic nuclear physics and data, modeling and simulation, reactor instrumentation, criticality safety, materials management and safeguards. This task will focus on the developmental needs of the FCR\&D experimental programs, such as elastic/inelastic scattering and fission neutron spectra measurements, and will leverage a number of existing neutron detector development efforts and programs, such as those at LANL, PNNL, INL, and IAC as well as those at many universities, some of whom are funded under NE grants and contracts. Novel materials and fabrication processes combined with state-of-the-art electronics and computing provide new opportunities for revolutionary detector 
systems that will be able to meet the high precision needs of the program. This work will be closely coordinated with the Nuclear Data Crosscut.

The developmental component to the Time Projection Chamber effort is included in this effort. This year, the prototype system with a full sextant will be used to collect fission ratio data from a specially designed target that contains both U-238 and U-235 deposits.

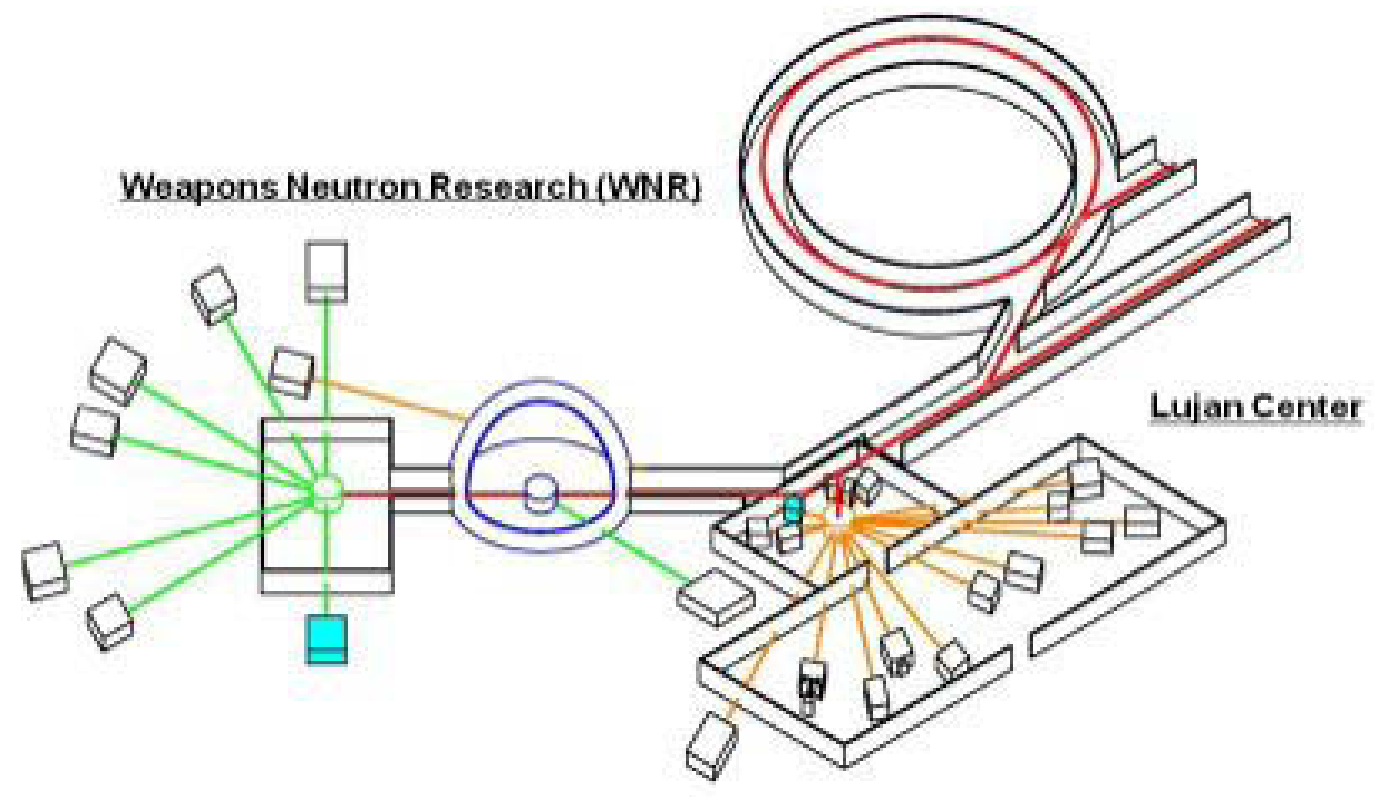

Figure 1: Schematic view of the Lujan Center and Weapons Neutron Research (WNR) facility at the Los Alamos Neutron Science Center (LANSCE).

\section{Los Alamos Neutron Science Center}

\section{Overview}

The work described here is carried out at the Los Alamos Neutron Science Center (LANSCE). There are two neutron facilities at LANSCE that are utilized: Lujan Center and Weapons Neutron Research (WNR) (Figure 1). The LANSCE accelerator delivers a pulsed $800 \mathrm{MeV}$ proton beam to tungsten spallation targets at both facilities producing white neutron beams. The neutron time-of-flight (TOF) method is used to determine the incident neutron energy. At the Lujan Center the spallation target is surrounded by various moderators, which results in a neutron spectrum with a strong thermal component and as well as a significant epithermal component. The statistically significant part of the neutron spectrum at this flight path ranges up to about $1 \mathrm{MeV}$. The WNR spallation target is bare, resulting in a fast neutron spectrum. The pulse structure at WNR allows the TOF method to be used for neutron energies from approximately $100 \mathrm{keV}$ to hundreds of MeV. 


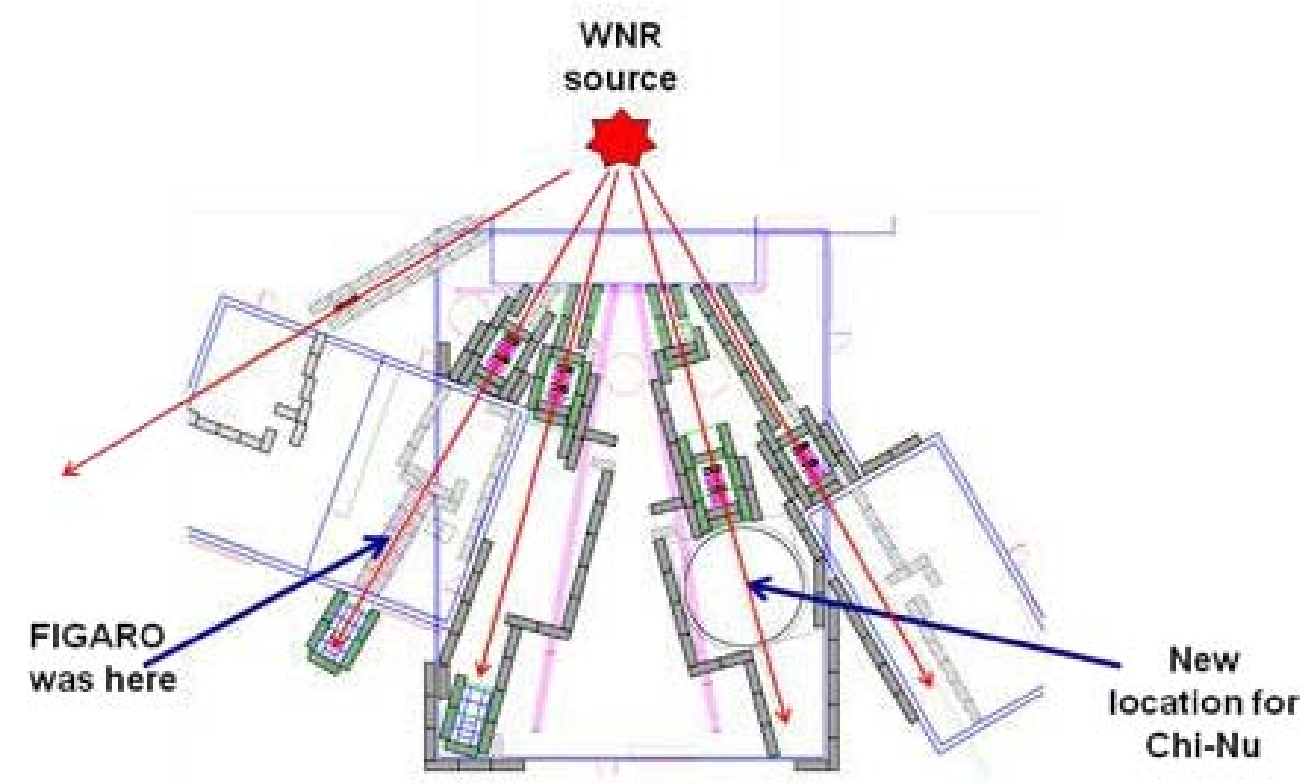

Figure 2: Layout of flight paths in the new LANSCE-WNR building.

\section{New building construction更t LANSCE-WNR}

A new building at the LANSCE-WNR facility was completed this year. A number of the flight paths at WNR are housed within this new building, and many experiments benefited from the construction. A second flight path opened is now available for electronics testing in neutron irradiation experiments, something that is in high demand from industrial users. The major benefit for the nuclear data program, however, came with the relocation of the Chi-Nu instrument that is being developed for fission neutron measurements (Figure 2). The new flight path that was configured for Chi-Nu has a 2 meter diameter and 2 meter deep pit with a false floor on which the detector system is located. The pit significantly reduces the room return of neutrons, which otherwise is a source of background. This is expected to reduce the systematic uncertainties associated with neutron output measurements.

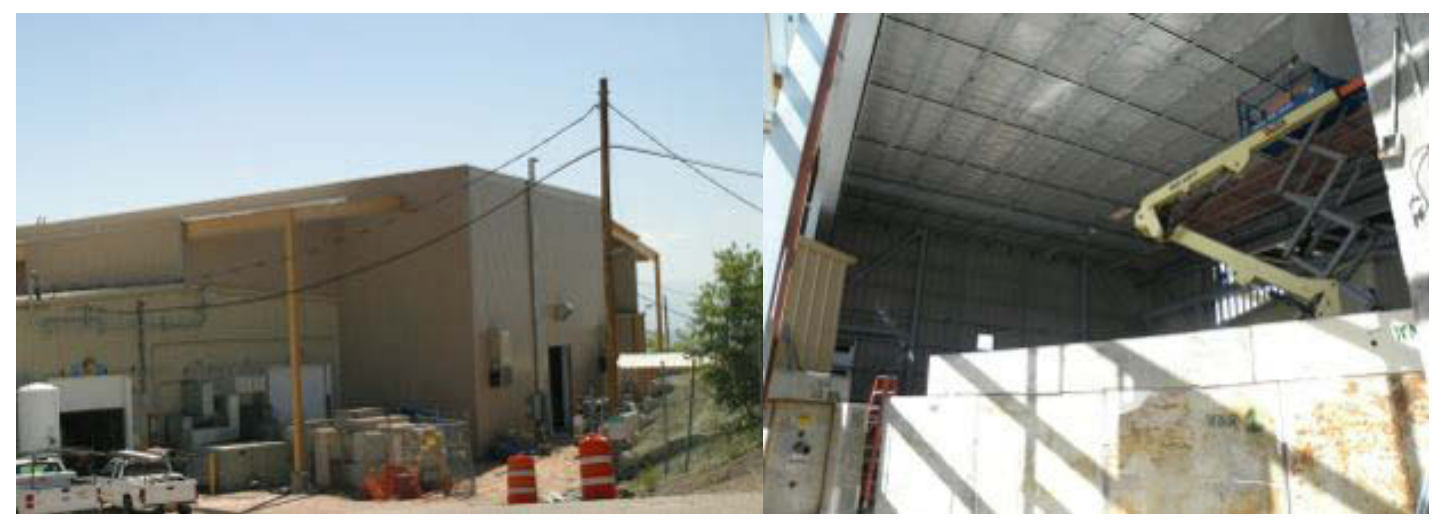

Figure 3: Left: Exterior view of the new building at LANSCE-WNR. Right: Interior view. 
Another benefit to the nuclear data program is the additional space that has been made available for the Time Projection Chamber (TPC) experiments. While the 4FP90L flight path where the TPC experiments are performed is not part of the new construction, it is adjacent to it. A work area and some office space has been setup for TPC in the new building, which made it possible to rearrange FP90L to make more space for the experiment itself. A schematic view of the flight path is shown in Figure 4.

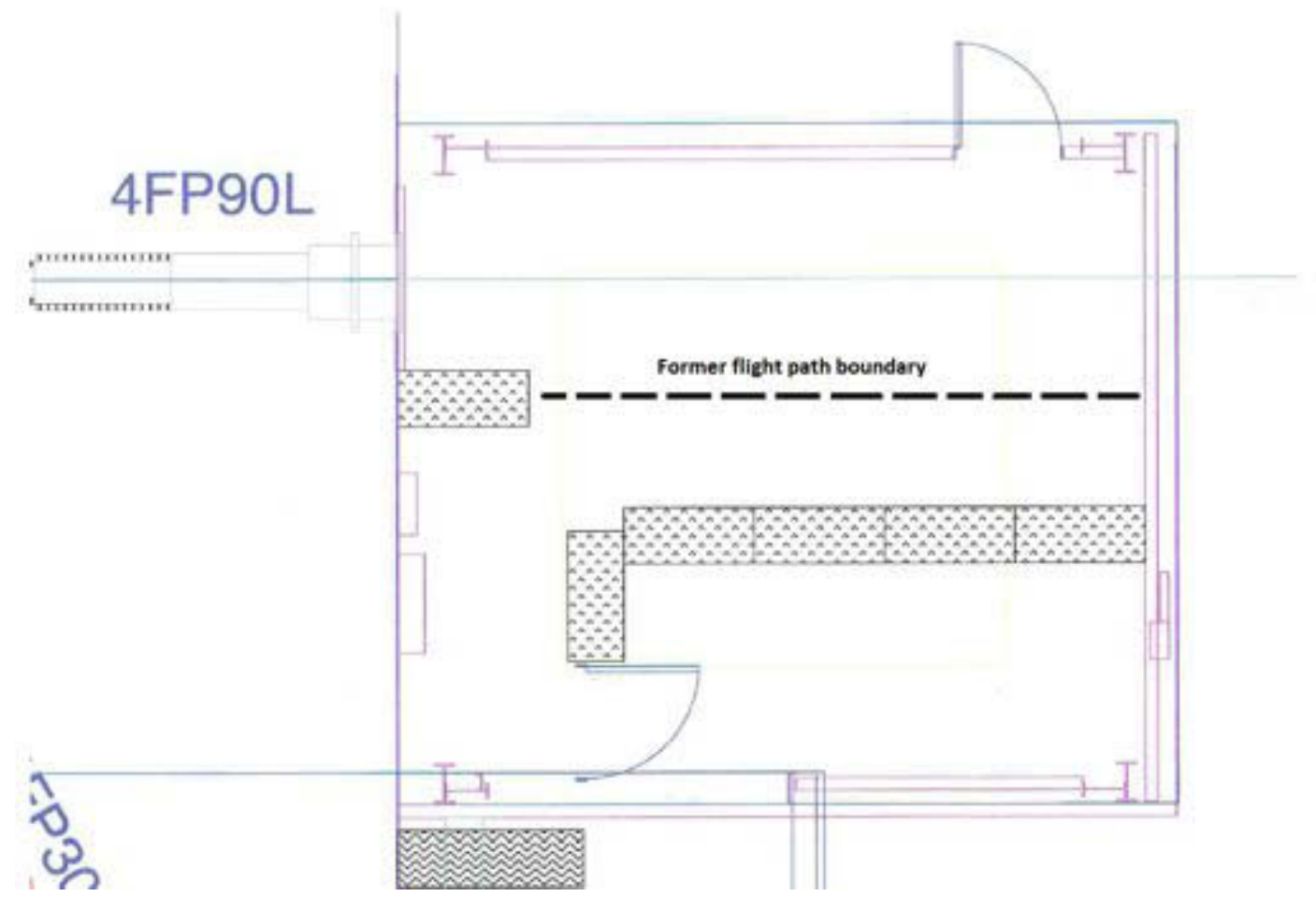

Figure 4: New layout of the 4FP90L flight path after the shielding blocks (dotted rectangles) were rearranged in May 2012. The dotted line shows the previous location.

\section{Nuclear Data Measurement Program}

The nuclear data measurement program is driven by short-term and long-term nuclear data needs. Fast reactor sensitivity studies identify and quantify the nuclear data needs for the near term candidates of the fast reactor project. Uncertainties in the available nuclear data propagate to uncertainties in integral reactor parameters. Providing high precision nuclear data drives down the cost of construction and operation of a new plant. Nuclear theory development and future high fidelity reactor design calculations provide the long-term justification for an ongoing precision nuclear data measurement campaign. Nuclear physics is fundamental to reactor core simulations, as well as safeguards and criticality safety. The design space for the future fleet of fast reactors and the future fuel cycle is severely limited by the uncertainties that currently exist in the nuclear data that have yet to be identified by specific designs. Truly innovative reactor designs and fuel cycles that will take full advantage of advanced computing techniques and platforms will be dependent on the fidelity of the underlying nuclear physics. The nuclear data measurement program 
will help insure that nuclear physics will not be the limiting factor in future fuel cycle innovation.

\section{Fission Measurement Program}

A program to measure fission cross sections in support of the AFCl program has been successful in delivering high impact results over the last four years. Some key fission measurements have been completed and more challenging experiments have been undertaken and considered. In order to further improve accuracies and perform more challenging measurements there are three main areas where developments are underway: improved precision by reducing systematic uncertainties, develop capabilities to measure shorter lived actinides and improve capabilities for measuring sub-threshold fission. The strategies to reach the goals in all these areas will be addressed in this report along with the specific accomplishments for this fiscal year.

\section{Introduction}

The fission measurement campaign for has been successful in delivering cross sections on such isotopes as ${ }^{237} \mathrm{~Np},{ }^{239-242} \mathrm{Pu},{ }^{243} \mathrm{Am}$ and ${ }^{233,236,238} \mathrm{U}$ using a parallel plate ionization chamber at LANSCE. There are different aspects of the isotopes measured to date that makes them challenging from an experimental point-of-view. The ${ }^{237} \mathrm{~Np}$ and ${ }^{240,242} \mathrm{Pu}$ isotopes are non-fissile and thus have very small fission cross sections below reaction threshold, which make even small levels of contamination of fissile isotopes in the samples difficult to handle. In addition, ${ }^{240,242} \mathrm{Pu}$ have relative high spontaneous fission rates that have to be separated from the neutron-induced fission events. ${ }^{241} \mathrm{Pu}$ has a 14.4 year half-life, and is thus highly radioactive. This makes the sample preparation and handling difficult, and limits the amount that can be handled safely. The smaller the samples are, the longer the irradiations and the higher neutron intensities need to be. Natural radioactive decay is also a source of background to the fission signals in the measurements and further complicates the experimental conditions. ${ }^{241} \mathrm{Pu}$ decays by beta emission, which produces less background in the detectors than alpha particles, meaning that an alpha-emitter with similar half-life would be even more challenging. Future measurements will include alpha-emitters with half-lives of less than a hundred years, some as short as a day, and will thus be even more challenging than the previously completed isotopes. The overall requirement is to increase the precision for these measurements, and in this report the strategy to meet these challenges will be outlined.

Before going in to the specific issues and strategies, an overview of the fission process and the current experimental program to measure fission cross sections at LANSCE will be given.

\section{General Properties of Nuclear Fission}

Nuclear fission is the process in which a nucleus splits into two lighter nuclei, which then in turn de-excites by neutron- and gamma-emission. This can be a natural decay process, called spontaneous fission, which occurs in a number of actinides such as ${ }^{240} \mathrm{Pu}$. Fission can also be induced by irradiation with, for example, neutrons or gammas. When a nucleus is excited in will deform, and when sufficiently deformed will eventually fission. The potential barrier along the deformation path will determine how much excitation energy is needed for it to undergo fission, and typical barrier heights for actinides are around 5-6 MeV (see an example of a fission barrier in 
Figure 5). Some isotopes such as ${ }^{235} \mathrm{U}$, when capturing a neutron, will have obtained enough excitation energy above the compound nucleus ground state that it will exceed the fission barrier, and will thus undergo fission regardless of the incident neutron energy. These actinides are typically referred to as being fissile. Other nuclei, in which fission can be induced, but have a threshold energy for neutron induced fission, are called fissionable.

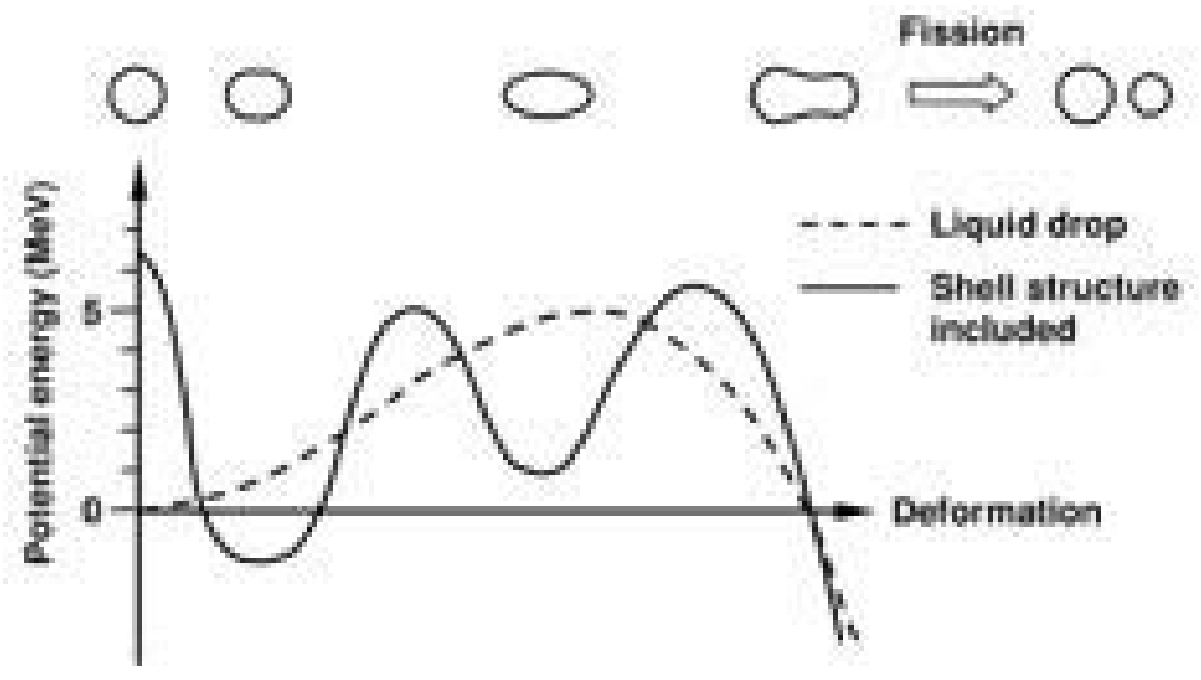

Figure 5: Example of an actinide potential energy fission barrier along the axis of deformation.

Approximately $200 \mathrm{MeV}$ of energy is released in a single fission event. About 180 $\mathrm{MeV}$ is in the form of kinetic energy of the two fission fragments and the remainder is energy carried away as neutrons and gamma-rays emitted by the fission fragments. Depending on the fissioning isotope and its excitation level, the mass yield distribution of the fission fragments will vary. In low-excitation fission of uranium and neighboring elements, asymmetric fission dominates. In asymmetric fission, the most probable mass split will be one light and one heavy fragment resulting in a doublehumped mass distribution of the fission fragments. At higher excitation energies and for certain elements, symmetric fission will be the dominating mode, meaning that the most probable mass split will be two fragments of equal masses.

The fission cross sections of different isotopes and energies have been measured using a variety of methods in the past. Fissile isotopes such as ${ }^{235} \mathrm{U}$ and ${ }^{239} \mathrm{Pu}$ have relatively large neutron-induced fission cross sections over the entire neutron energy range and are reasonably easy to measure. However even the non-fissile, fissionable isotopes have measurable fission cross sections below the reaction threshold. The reason is that even if the energy of the incident neutron is less than what is needed to bring the nucleus excitation above the fission barrier, there is some probability for transmission through the barrier due to quantum mechanical tunneling. The relatively small fission cross sections below the threshold energy are harder to measure but are important in applications such as fast reactors, where the neutron flux will be high in the fast region.

Fission can be observed using different techniques, by either detecting the energetic fission fragments, the prompt neutrons, the gamma-rays, or some combination of these. Historically, the most common method of measuring the energy-dependence of the fission cross section has been to directly measure the fission fragments. 


\section{Parallel plate ionization chambers}

The instrument used to detect fission is a parallel plate ionization chamber (PPIC) that was developed at LANL and which has been used extensively to monitor neutron flux at the WNR (the schematics of the PPIC is shown in Figure 6). Thin samples (typically $200 \mu \mathrm{g} / \mathrm{cm}^{2}$ ) of fissionable material on stainless steel backing are loaded into the PPIC, which can hold up to four samples at the time. The chamber is filled with an appropriate gas, in this case P-10 (a mixture of $90 \%$ argon and $10 \%$ methane), and kept at about two atmospheres. The chamber is introduced into the neutron beam in one of the two flight paths. When fission is induced in one of the samples the fission fragments are accelerated in opposite directions, which means the most of the time one of them will be stopped in the backing material and one will escape into the counting gas. The fragment will ionize the chamber gas along its trajectory. An electric field maintained between the cathode (the sample plate) and the anode will drift the ionization electrons, which induces a current on the anode, which in turn is read out using current sensitive preamplifiers. The efficiency to detect fission events is high, around 95\%, since the only events that are lost are those where the fragment trajectory is parallel to the cathode such that the fragment stops before entering the gas, or the fragment energy is low enough that it is indistinguishable from alpha particles or detector noise.

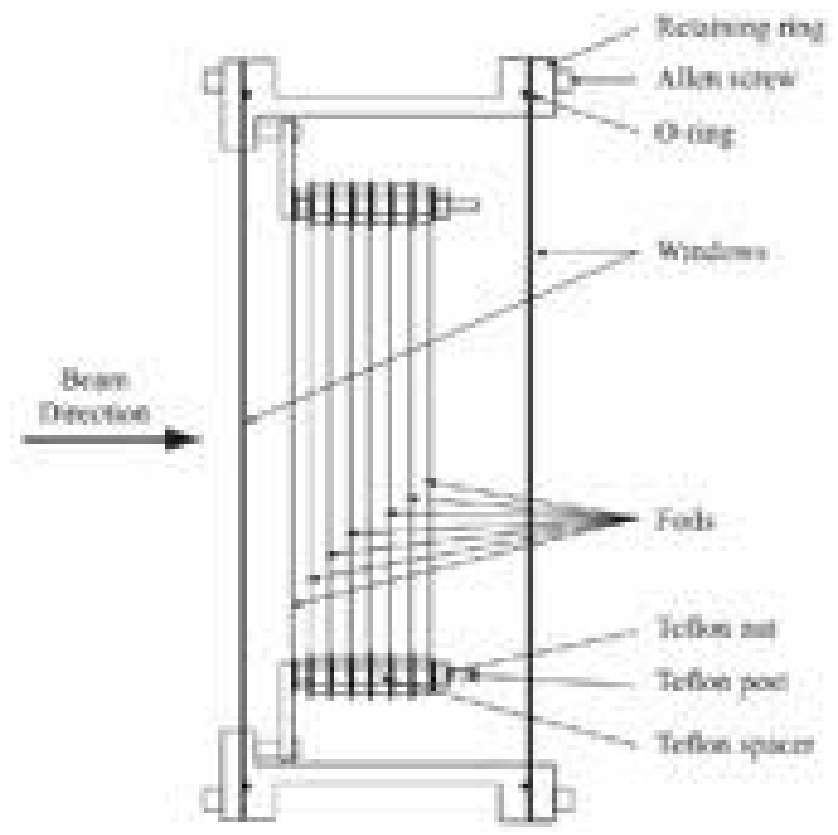

Figure 6: Drawing of the parallel plate ionization chamber.

\section{The Time Projection Chamber (TPC)}

The accuracy of cross section measurements performed with conventional techniques such as ionization chambers is limited to $3-5 \%$. In order to reach the level of accuracy that has been requested for certain isotopes (about 1\%) a new approach is needed.

The NIFFTE Collaboration, comprised of three national laboratories and six universities, is currently developing a Time Projection Chamber (TPC) capable of 
measuring fission cross sections to unprecedented accuracy. TPCs, first developed for high energy physics research in the late 1970s, are a well-established technology. This, however, marks the first use of a TPC for fission research.

There are many ways in which measuring fission cross sections with a TPC can help reduce systematic uncertainties. TPCs provide complete particle tracking by combining segmented readout (which generates 2-D projections of ionization tracks) with the drift time of the charge to obtain a full 3-D representation (Figure 7). This tracking information can help determine the sample uniformity, beam profile and other parameters that affect the accuracy of cross section measurements. Additionally, the fission TPC will provide much better identification of fission fragments than previously available with standard ionization chambers, and will facilitate the use of the $\mathrm{H}(n, n) \mathrm{H}$ standard for normalization of the neutron flux (a significant improvement over the less accurate $\mathrm{U}_{-}^{235}(\mathrm{n}, \mathrm{f})$ standard).

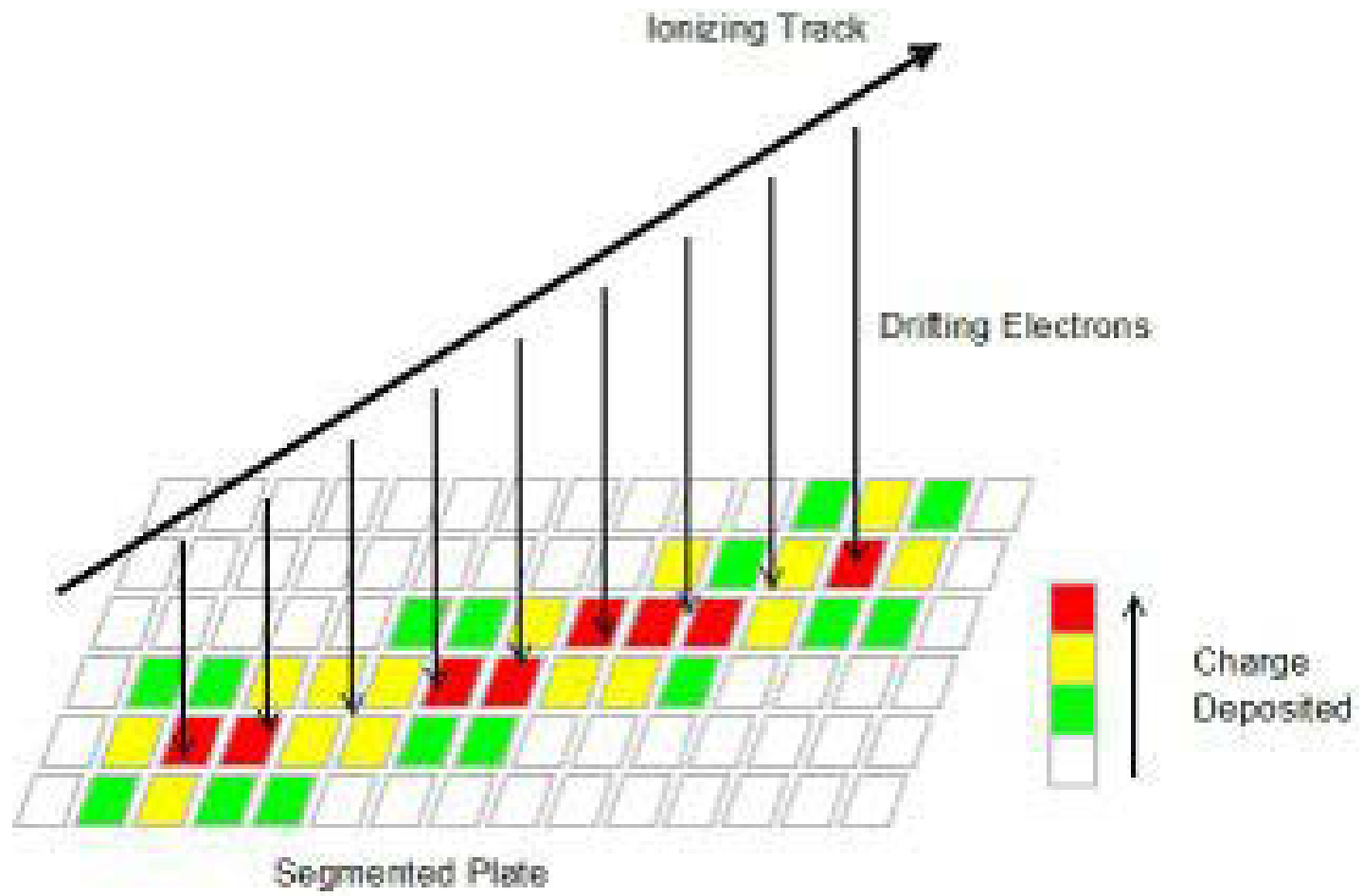

Figure 7: Cartoon describing how radiation is tracked in a Time Projection Chamber.

The first neutron beam experiments with the TPC were initiated in August 2010, and have continued with upgraded versions of the instrument up to the present.

A schematic of the fission TPC is shown in Figure 8 . The sample to be studied is located in the center of a gas-filled chamber, held in place by a central cathode plane. A field cage lines the interior of the chamber, supplying a uniform electric field to guide charged particles towards the end planes. At each end plane MicroMegas detectors, consisting of approximately 3000 segments each, amplify and read out the drifted charge. 


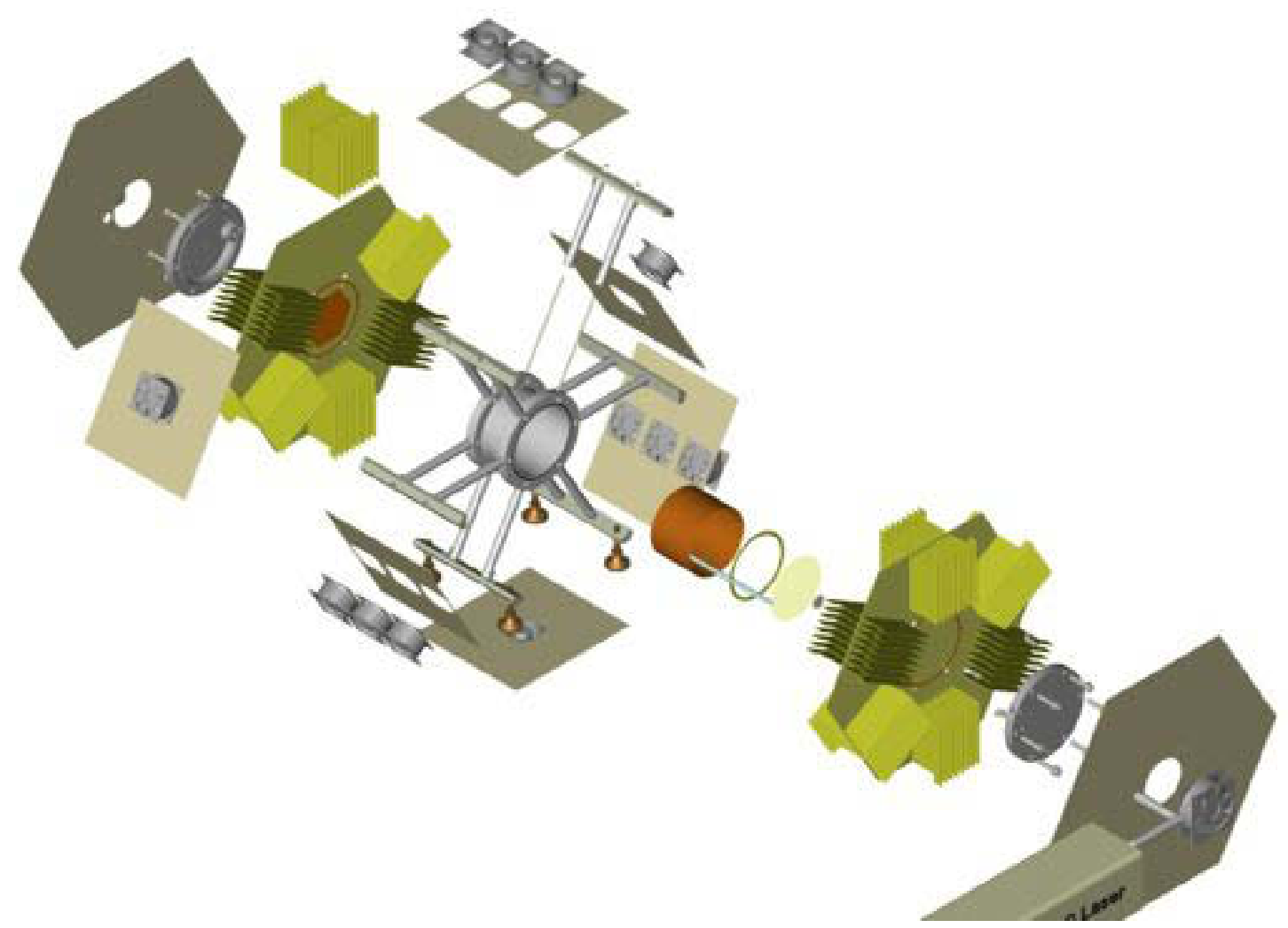

Figure 8: Exploded view of a CAD drawing of the fission TPC.

32-channel preamplifier cards process the signals from the individual segments. The preamplifiers are connected to digitizer cards equipped with on-board fieldprogrammable gate arrays (FPGAs), which digitize the signals at $50 \mathrm{MS} / \mathrm{s}$, and send the waveforms via Ethernet to the DAQ computer for further processing. Online software allows for real time viewing of the waveforms and reconstructed particle tracks. The samples used for fission cross section measurements needs to be sufficiently thin so that the fission fragments produced can escape from the sample with enough energy to produce a signal in the active volume of the detector. The intention with the TPC measurements is to detect both of the fragments emitted in binary fission, so a backing that is transparent to the fragments is needed.

The samples for the TPC are produced at Oregon State University, and consist of 30$120 \mathrm{\mu g} / \mathrm{cm}^{2}$ thick carbon backings with actinide material evaporated onto the surface. The deposits are typically $100-300 \mu \mathrm{g} / \mathrm{cm}^{2}$ thick, and have a radius of $1 \mathrm{~cm}$. Two samples were used for the experiments described in this report: an empty backing foil and an identical backing with a U-238 deposition. The backings were both $111 \mu \mathrm{g} / \mathrm{cm}^{2}$ thick, and the U-238 deposit had an areal density of $229.2 \mu \mathrm{g} / \mathrm{cm}^{2}$. Examples of samples are shown in Figure 9 and Figure 10. 


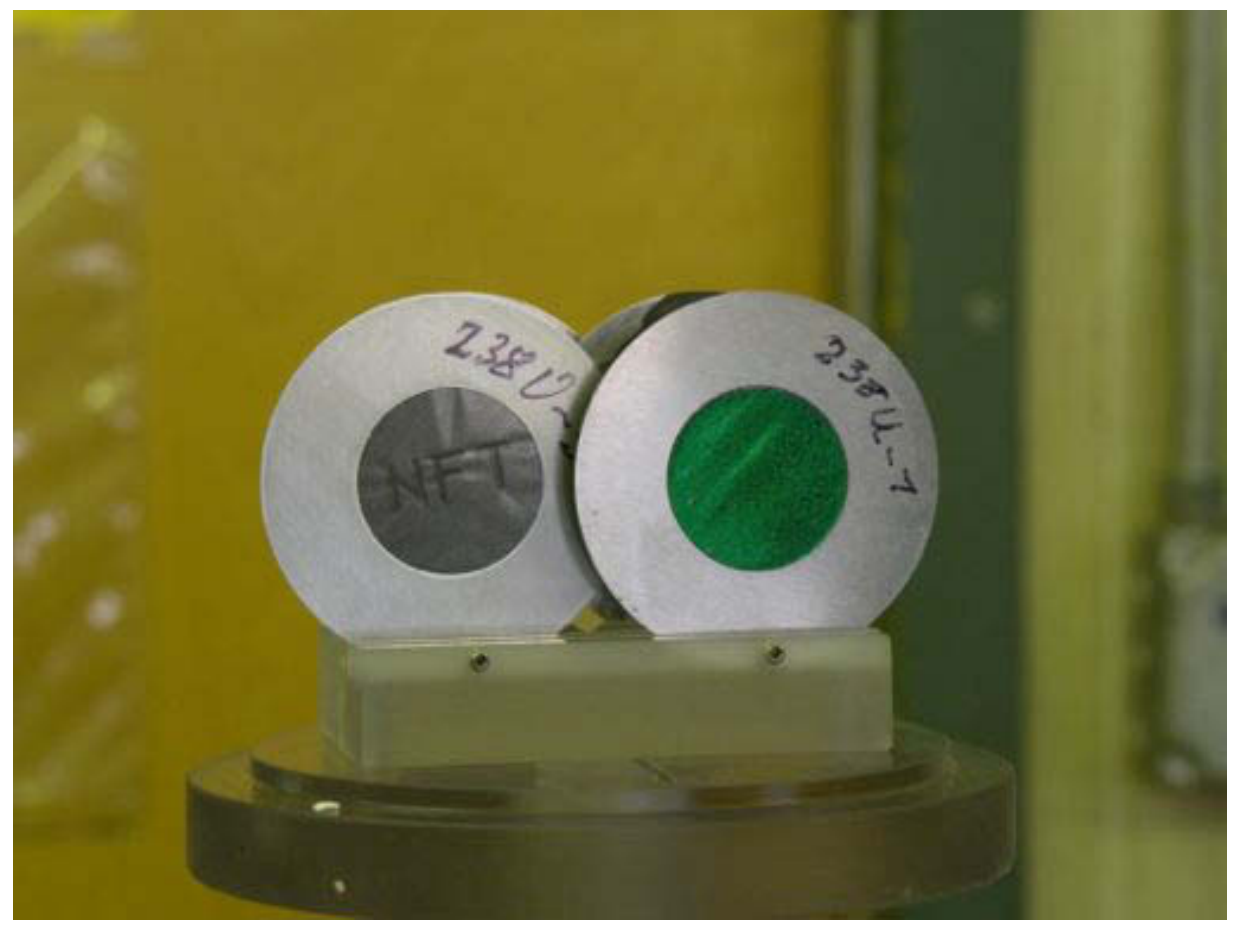

Figure 9: U-238 targets for the fission TPC. The left sample was deposited using a mask to generate a specific pattern. The right sample is similar to the one used in the 2010 LANSCE experiments.

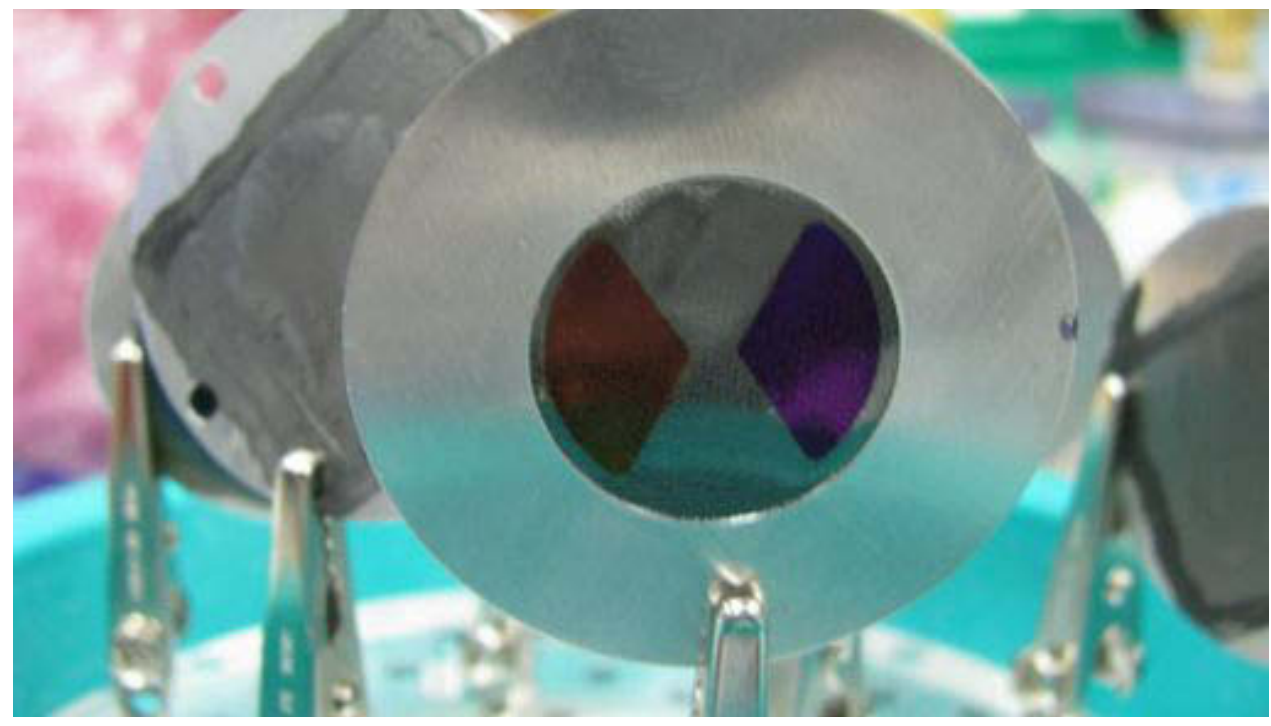

Figure 10: A mixed TPC sample with a U-238 deposit (left deposit), and Th-232 (right deposit).

This year, several new hardware and software components were integrated into the experimental setup at WNR. A new module was added to the Keithley 2701 multimeter: module 7706, which handles larger voltages and will complement the 7710 module already in place. In December 2011, a filter circuit was shipped from LLNL to LANL to allow remote control of all high voltage power supplies. A power distribution unit was also shipped that can shut down all low voltage power supplies remotely, placing the TPC into a safe idle state that can be maintained indefinitely. 
Software components added to the system included slow control monitoring of: beam current (Figure 11); gas pressure and flow rates (Figure 12); high voltage off and on controls; and low voltage off controls.
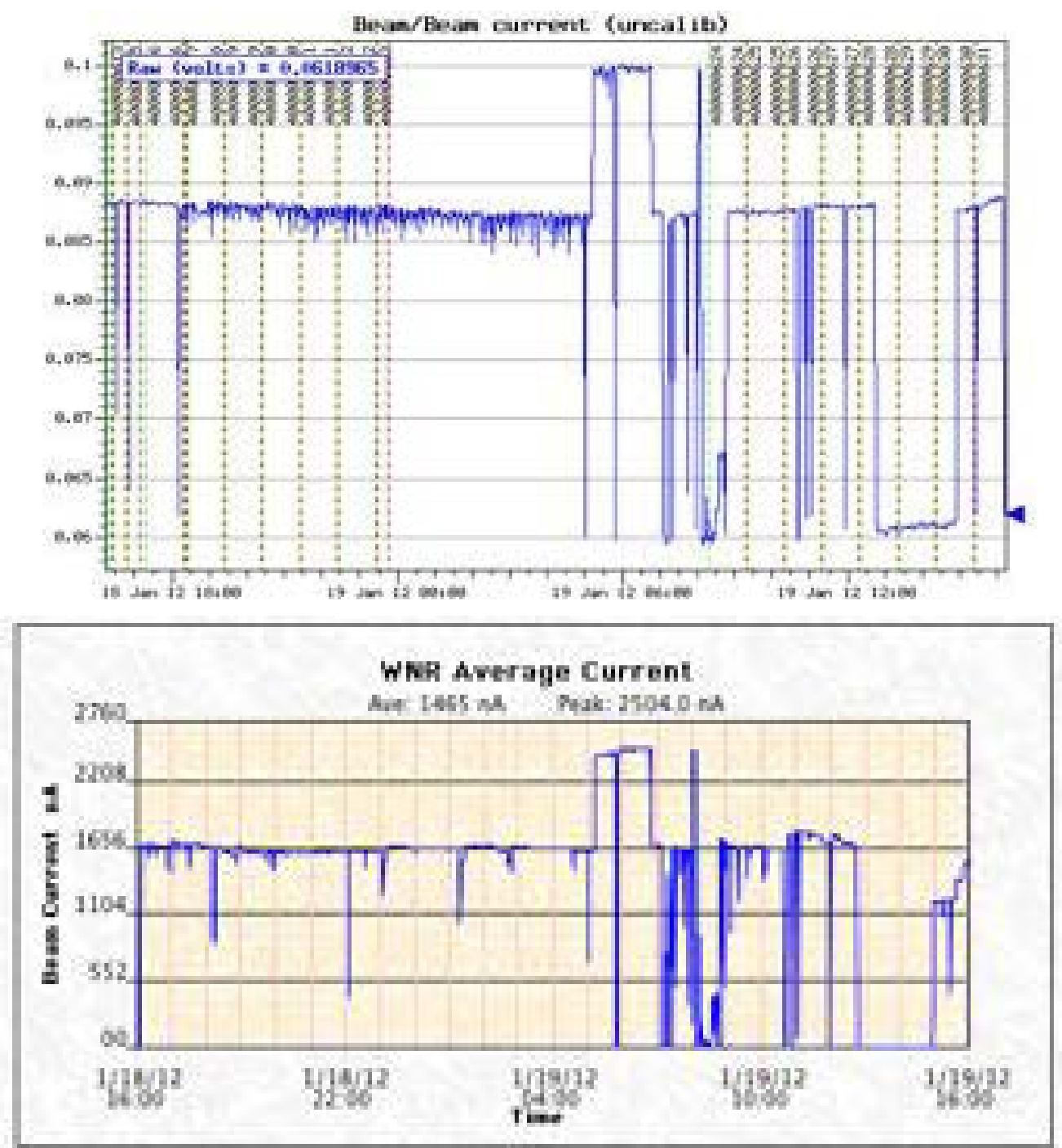

Figure 11: (Top) Beam current monitored by TPC slow controls; (Bottom) Beam current as reported by LANSCE Operations. 


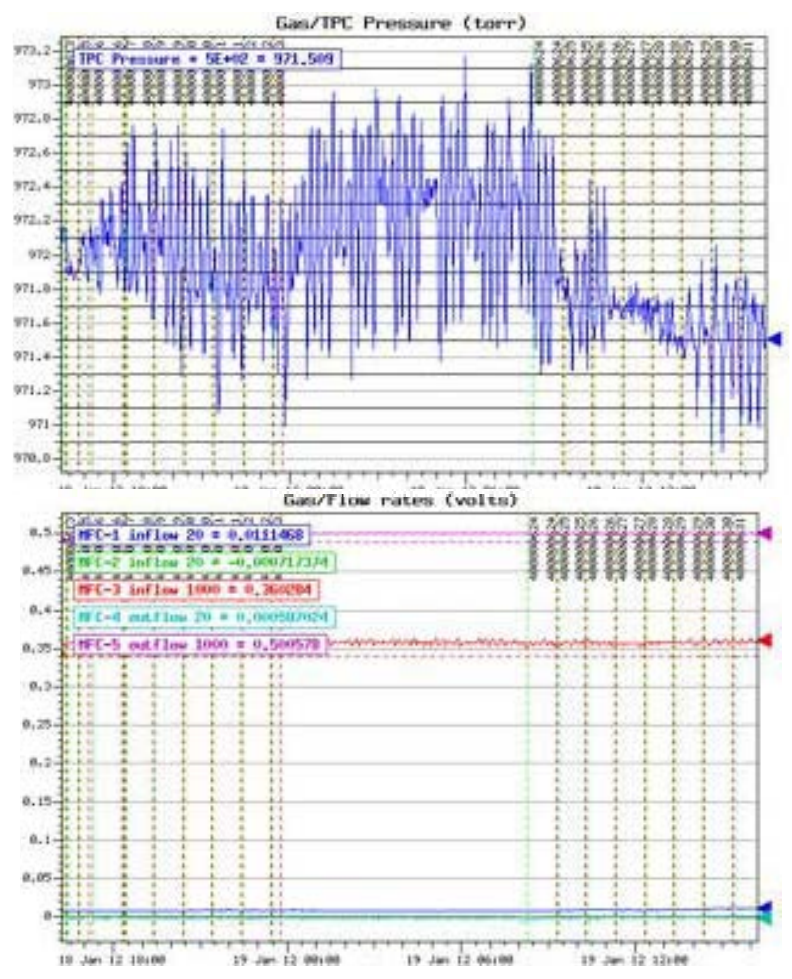

Figure 12: (Left) TPC pressure as measured with high-accuracy pressure gauge and read out through slow controls; (Right) TPC flow rates readout through slow controls.

New TPC hardware was implemented in the experimental setup at WNR in January 2012 to address issues identified in the previous quarter. A filter circuit, built to carefully control the high voltage settings of the MicroMegas, was integrated into the existing high-voltage system, allowing all high-voltage power supplies to be controlled remotely. The original design of this low-noise circuit ran on $9 \mathrm{~V}$ batteries, which needed to be changed approximately every two days. In February, a second design (running on LEDs and solar cells) was shipped to LANL, which ran smoothly and required little to no maintenance by on-site personnel. The second MicroMegas filter circuit is shown in Figure 13. 


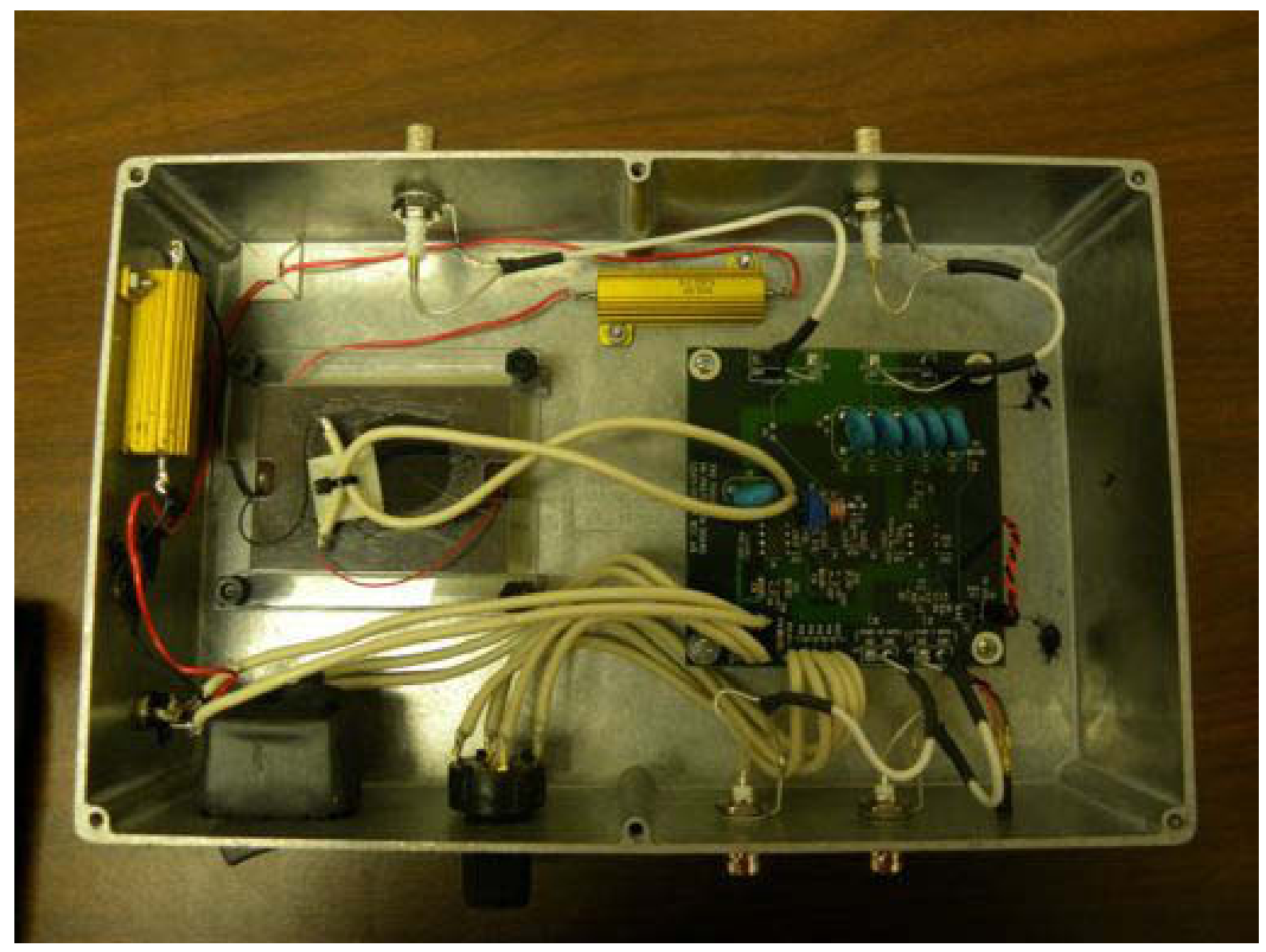

Figure 13: MicroMegas Filter Circuit, version 2.

The low voltage Power Distribution Unit (PDU, Figure 14) was also integrated into the TPC setup at WNR this quarter. The PDU controlled the clock, trigger, reset, and voltages for the digital bus boards and allowed remote users to shut down all low voltage power supplies, thereby placing the TPC into a safe, idle state that could be maintained indefinitely. New slow control monitors of the PDU state (on, off, tripped) were also implemented. Other slow controls monitors implemented in this quarter included: shutter status (open/closed); gas flow (on/off); beam intensity; and high voltage controls. 


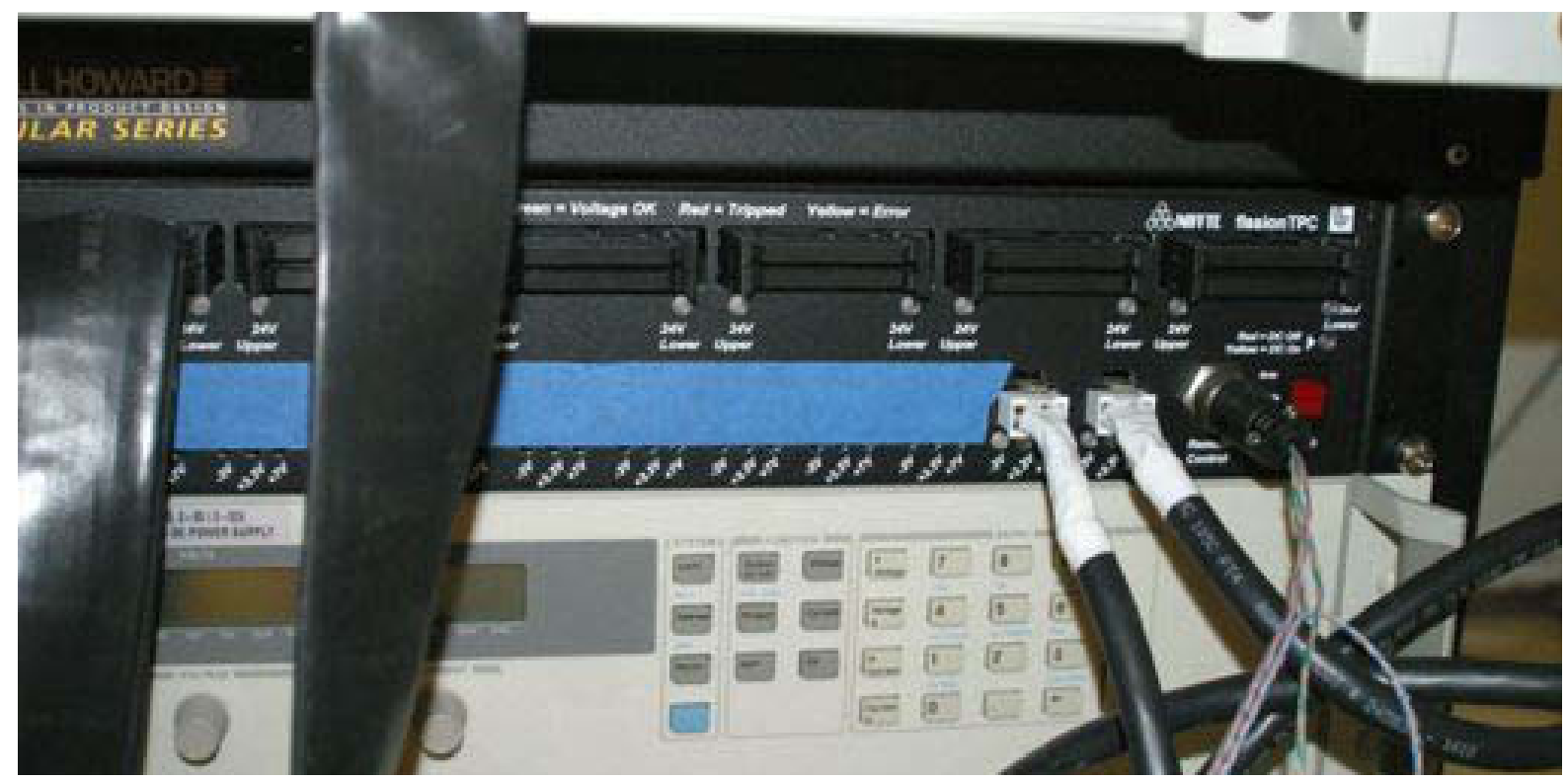

Figure 14: Low voltage Power Distribution Unit (PDU).

Final approvals for loading plutonium into the TPC and running in-beam were received in early February 2012 . The sample loading involves work in a customized radiological glove box (Figure 15) and the procedure was developed and practiced over several months using blank carbon samples. The first TPC Pu-239 sample loading was successfully completed in February. The highly enriched sample had a total mass of $0.35 \mathrm{mg}$, and was electroplated onto a solid aluminum backing. The deposit was $2 \mathrm{~cm}$ in diameter, corresponding to a thickness of $111 \mu \mathrm{g} / \mathrm{cm} 2$, and consistent with the samples that will be used for cross-section measurements. Figure 16 shows the Pu sample loaded into the TPC. 


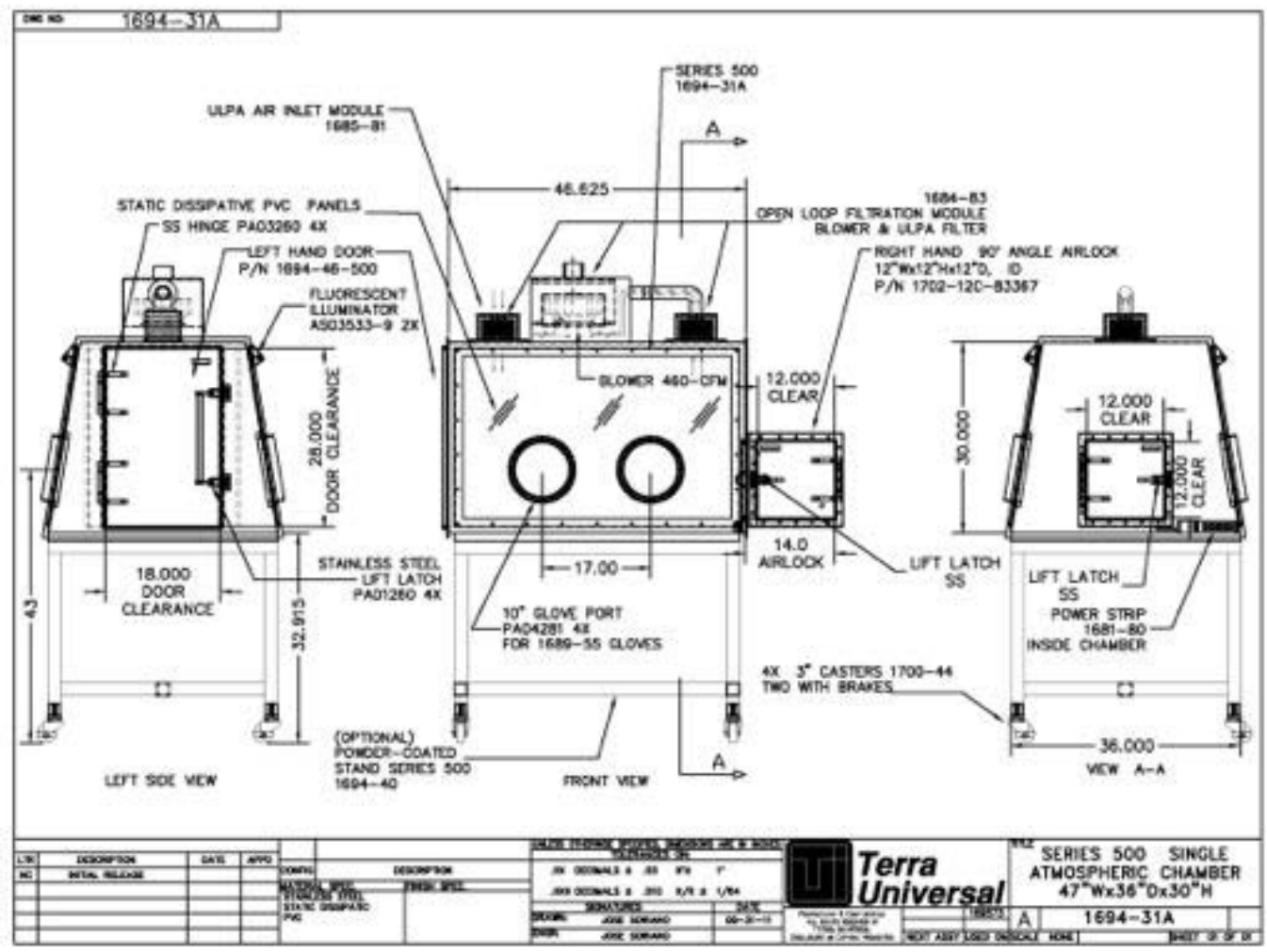

Figure 15: Glove box used for Pu loading at LANL.

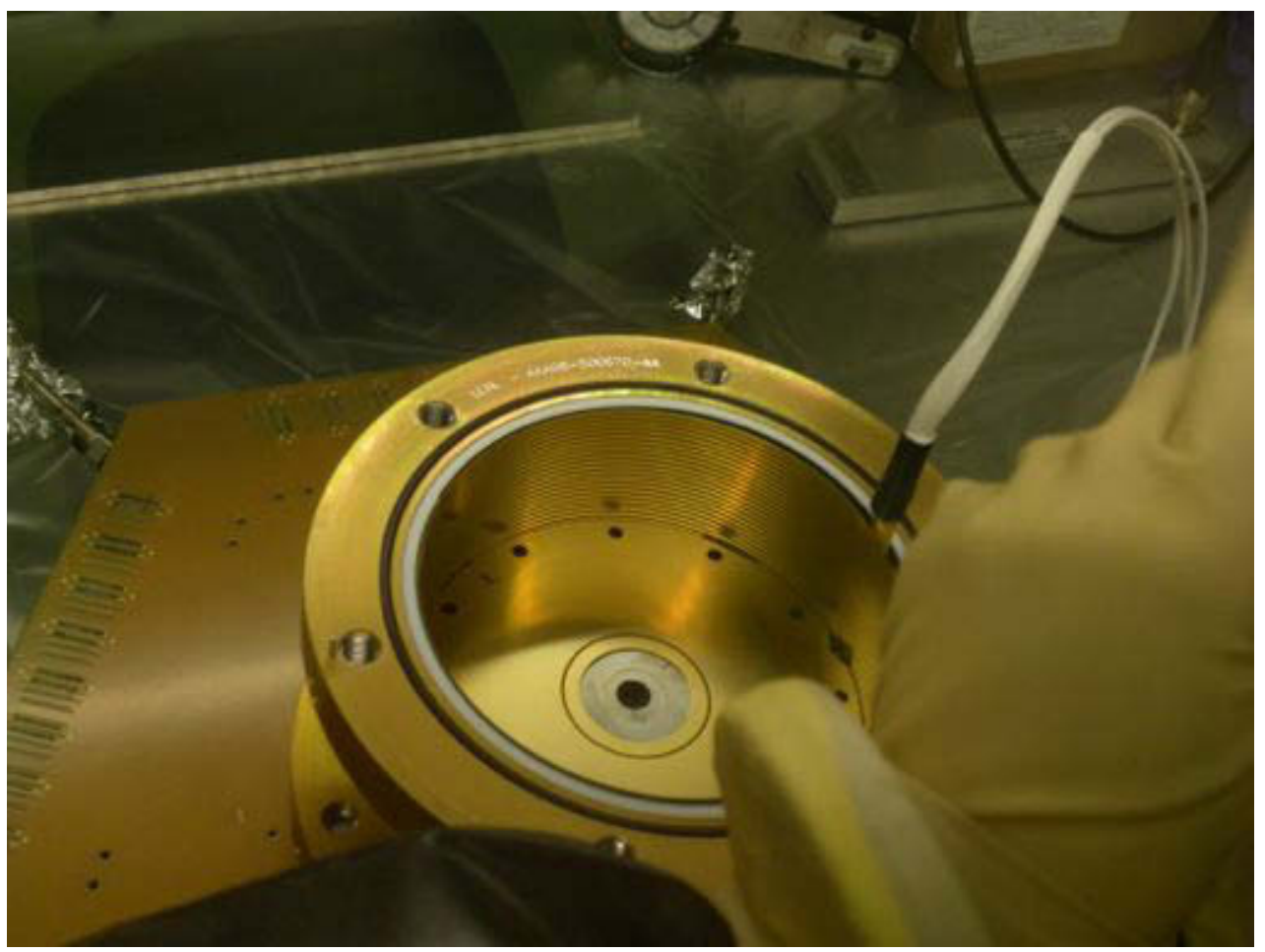

Figure 16: Pu-239 sample loaded into TPC. 
The second half of the 2011-2012 LANSCE run cycle began in January. The WNR construction project was paused to allow for 24 hour beam operations on the $90 \mathrm{~L}$ flight path. This flight path views the bare spallation target from a 90 degree angle relative to the proton beam axis, which is ideal for fission experiments since the flux is high in the 1-20 MeV range that is of particular interest for these experiments, and lower in the hundreds of MeV-range compared to the more forward flight paths at WNR, which helps reduce the experimental background levels. This is a relatively short flight path, with a nominal detector location of 10 meters from the neutron production target.

The flight path sits directly against the Target 4 bulk shielding. The beam pipe is evacuated up to the shutter at the end of the bulk shielding, and the beam is then transported in air to the detector. The neutron flux at the detector location is shown in Figure 17.

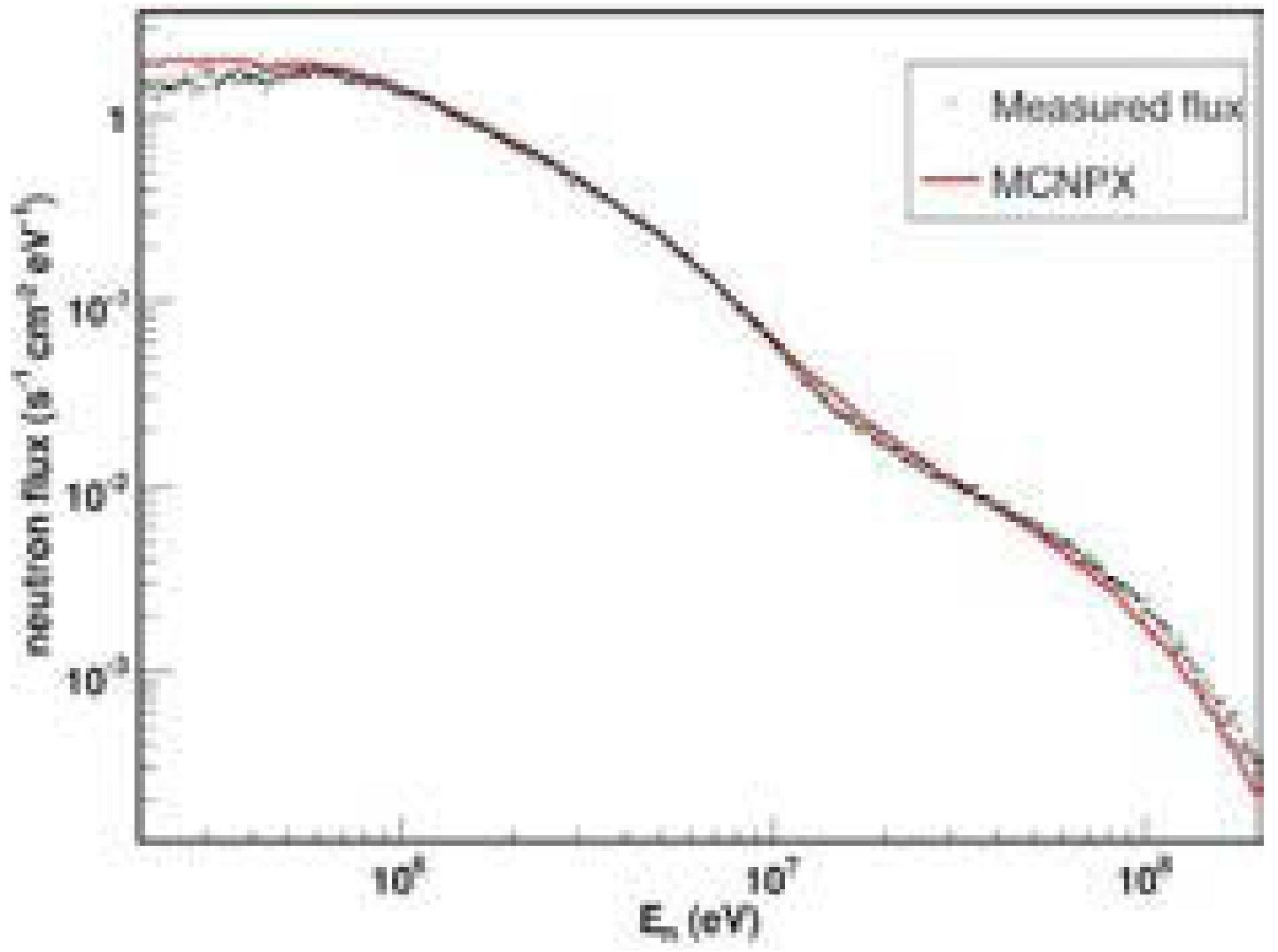

Figure 17: Measured (black) and calculated (red) neutron flux at 90L.

During the January maintenance period, a new U238/U235 mixed isotope target was loaded into the TPC (Figure 18). Beam experiments commenced January 12, running 24 hours per day until January 27. In early February the Pu-239 sample (see:

Plutonium Loading, above) was loaded into the TPC. The TPC performed well, despite the high alpha decay rate of the Pu-239 sample $(0.8 \mathrm{MBq})$. The high-rate $(1$ $\mathrm{Tb} /$ hour) data collected with low thresholds (to collect all alpha-particle tracks) will be very valuable when preparing for next run cycle's Pu-239 runs. 16 days of Pu-239 beam data was collected at LANSCE-WNR in February, before the facility entered the annual outage period February 28. 


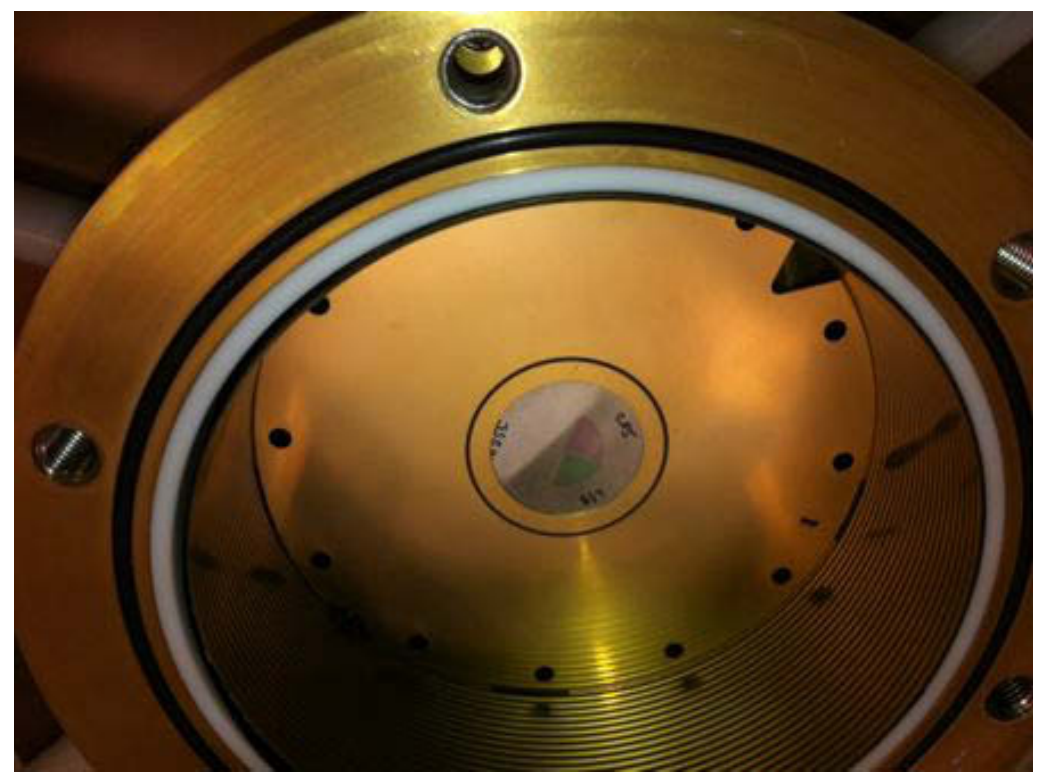

Figure 18: U235/U238 mixed isotope target, used in January 2012, loaded into TPC.

\section{Spectrometer for lon Detection in Fission Research (SPIDER)}

The SPIDER detector is a new instrument under development that will be used for high accuracy fission yield measurements. Fission yields are used as diagnostics of nuclear reactors, but the true accuracy of these yields has been under debate for some time, and new measurements were proposed to settle this controversy. The goal with the new measurements is not only to improve the accuracy for a give neutron energy spectrum, but also to better map out the energy dependence of the yields. This energy dependence is poorly understood, but will likely be more important as new advanced reactor designs are being explored.

The SPIDER instrument is being designed to measure fission yields by directly measuring the velocity, kinetic energy, and specific energy loss of the fragments emitted in binary fission. Using this information the mass and charge yields can be determined. The target mass accuracy is $1 \mathrm{amu}$, which will require sub-1\% accuracy when measuring the fragment velocity and energy. The focus of this year's development has been to demonstrate that performance using prototypes.

The velocity measurements are achieved using time pick-off detectors that measure fragment time-of-flight (TOF) over a flight path of approximately $50 \mathrm{~cm}$. The typical velocity of fission fragments is about $1 \mathrm{~ns} / \mathrm{cm}$, so timing resolution of 100-200ps is needed. The timing pick-offs consists of thin $\left(10-100 \mathrm{ug} / \mathrm{cm}^{2}\right)$ carbon foils, electrostatic mirrors, and Microchannel plate detectors (Figure 19). As fission fragments pass through the thin foils secondary electrons are emitted which get accelerated and bent out of the fission fragment path by the electrostatic mirror. Microchannel plate detectors register the secondary electron emission and provide the start/stop times for the time-of-flight. A prototype vacuum chamber for the TOF measurements was designed and built this year, and so were two electrostatic mirrors. The mirror consists of planes with 20 um diameter thick gold-plated tungsten wire with a $1 \mathrm{~mm}$ pitch. The wires are laid down on a plastic frame using a custom wire-winder, and attached by soldering to a copper strip on the frame. A strip of epoxy is also used further secure the wires to the frame (Figure 20). The planes are $98 \%$ transparent for 
fission fragments. The mirochannel plate (MCP) detectors and detector holders with position sensitive readouts were made by RoentDek Handels $\mathrm{GmbH}$, and have an active diameter of $75 \mathrm{~mm}$. The position resolution of the MCPs in combination with the electrostatic mirrors is about $2 \mathrm{~mm}$.

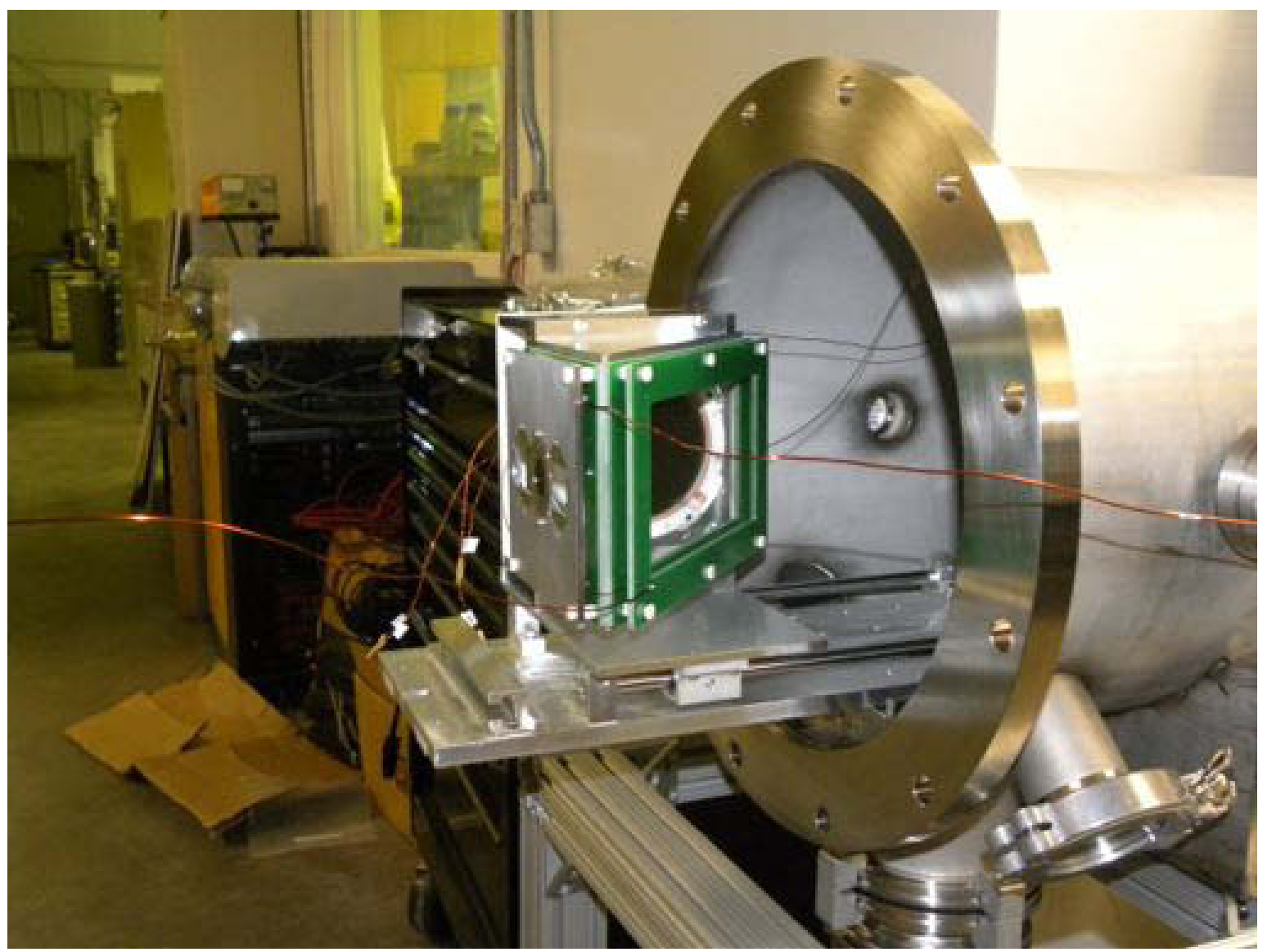

Figure 19: Timing detector mounted on a platform in the vacuum chamber used for fission fragment time-of-flight measurements. 


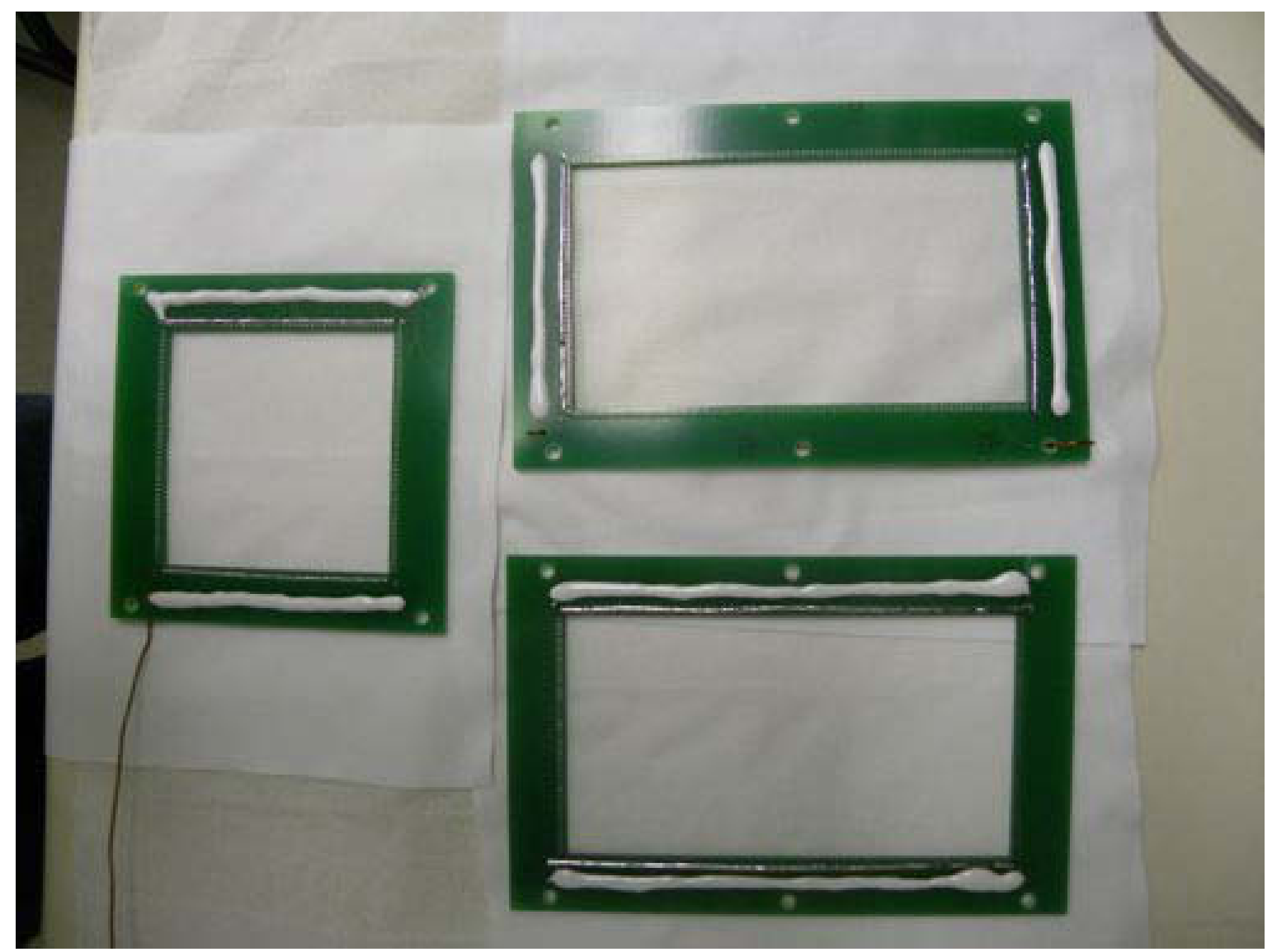

Figure 20: Frames with gold-plated tungsten wires for the electrostatic mirrors.

The vacuum chamber was used to study the timing response of the timing detector using radioactive sources. The first tests were performed with a $3 \mu \mathrm{Ci} \mathrm{Cf}-252$ source, which has both a spontaneous fission and alpha decay branch. The pulse height response from one of the timing detectors with the Cf-252 source in the chamber is shown in . A group of events with low pulse height, $<50$ channels, and another peak at larger pulse heights are evident in the histogram. The time-of-flight between two timing detectors with the same source is shown in Figure 22. The black line shows the TOF data for all particles from the source, i.e. fission fragments and alphaparticles. The red line is the data collected when requiring that the pulse height form the MCP is larger than 50 channels. The peak at $31 \mathrm{~ns}$ corresponds to the alpha particles, while the peaks at 41 and 55 ns correspond to the light and heavy fission fragment groups, respectively. When setting a 50 channel pulse height threshold the alpha-peak almost completely disappears, demonstrating that the two types of radiation can be distinguished both with time and pulse height cuts. 


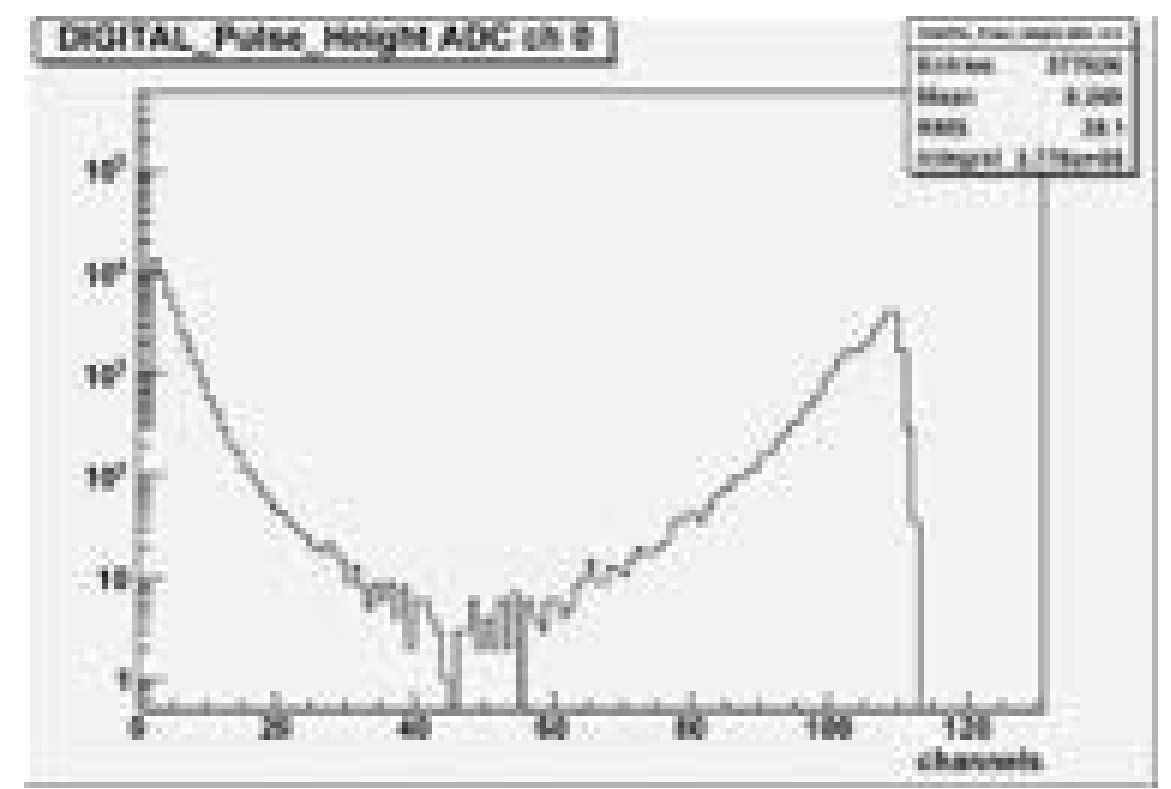

Figure 21: Pulse height response from the timing detector with a Cf-252 source.

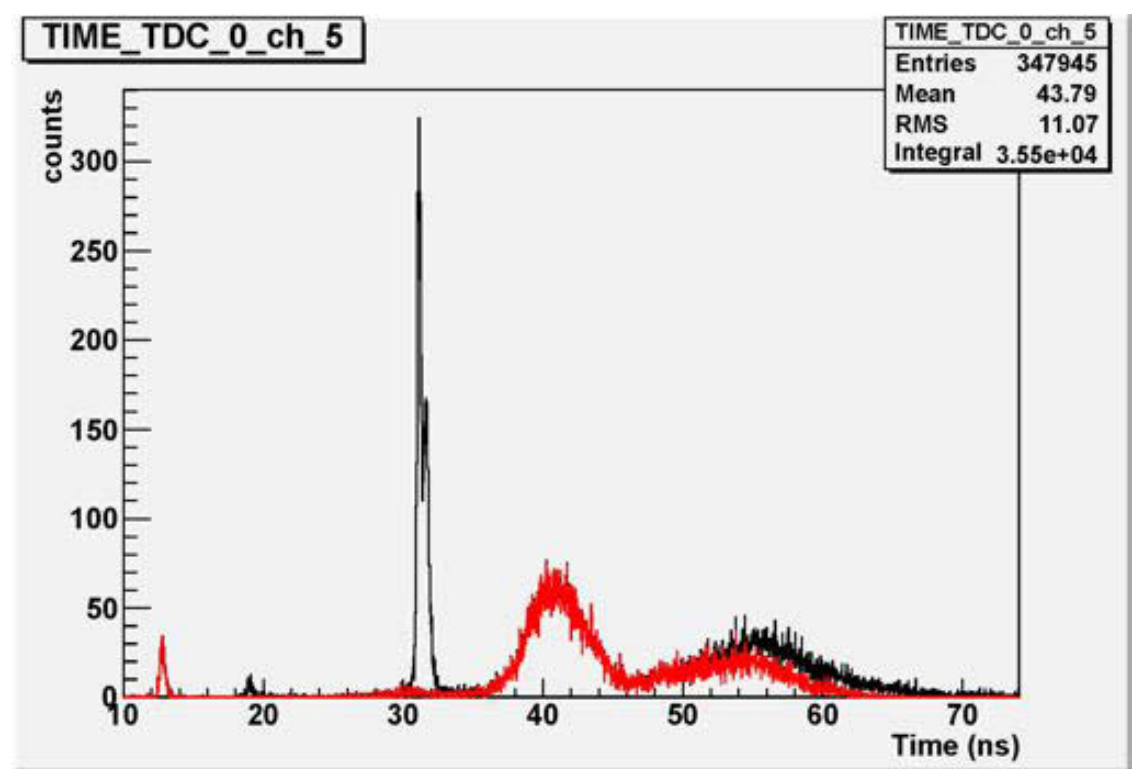

Figure 22. Time-of-flight for non-gated event (black line), and only events with pulse heights larger than 50 channels (red line).

Another set of timing tests were performed with a Th-229 alpha source. This source has no spontaneous fission branch, but multiple alpha lines with energies from about $5 \mathrm{MeV}$ up to $8 \mathrm{MeV}$ making it ideal for calibration purposes. The time-of-flight spectrum for that source is shown in Figure 23. By using the known energies in the decay and checking the TOF value using a linear fit the calibration demonstrated that the DAQ system was linear within the uncertainty of the fit.

In addition to the TOF calibration the Th-229 data was also used to determine the timing resolution of the system. The alpha TOF peaks were fitted assuming Gaussian 
shapes, which resulted in 190ps (FWHM). Since the alpha-source is very thin $\left(<50 \mathrm{ug} / \mathrm{cm}^{2}\right)$, the width of the peak is expected to be dominated by the time resolution of the system. This corresponds to 133 ps resolution per detector which is close to the $100 \mathrm{ps}$ target accuracy, and sufficient to reach 1 amu mass resolution for fission fragments.

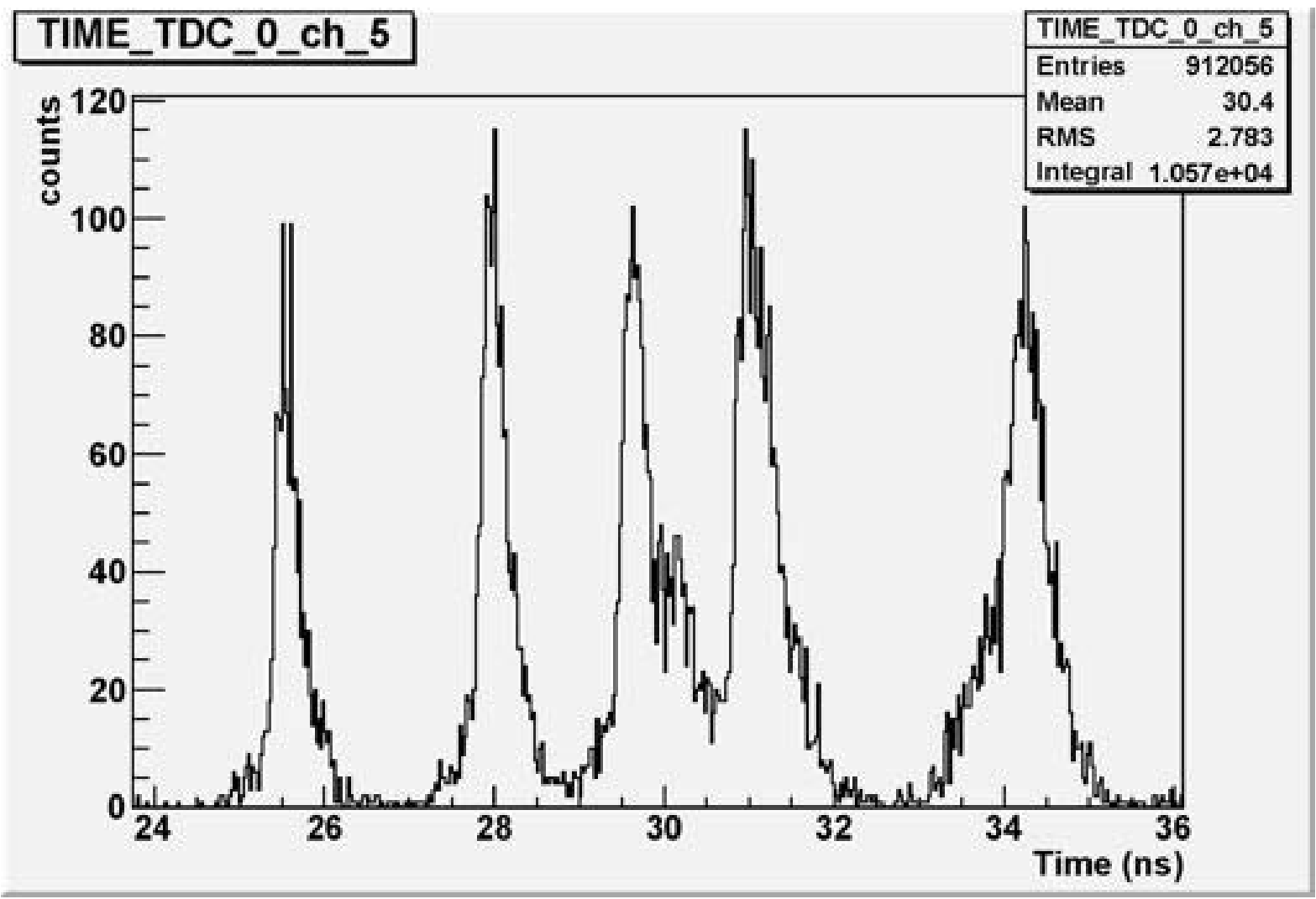

Figure 23: Alpha-particle time-of-flight spectrum collected with a Th-229 source.

The detection efficiency of the time pick-off detectors for alpha-particles was investigated by measuring the coincidence between these detectors and a silicon surface barrier detector. An alpha-source was placed in the vacuum chamber, and the emitted alphas passed first through a time pick-off, and then hit a silicon detector downstream from it. Silicon surface barrier detectors are known to have close to $100 \%$ detection efficiency for alpha particles. The measured efficiency is shown in Figure 24, as a function of acceleration potential used for the electrostatic mirrors. For very low potentials the electron collection on the MCP is reduced, resulting in low detection efficiency. Above about $300 \mathrm{~V}$ acceleration there is very little variation in the efficiency as the potential is increased. The efficiency is determined by the geometric transmission through the electrostatic mirror, which is about $94 \%$, and the probability to create enough ionization in the thin foils to trigger the MCP. The efficiency for fission fragments are expected to be higher since the specific ionization of these particles are higher, but a precise measurement of this efficiency still needs to be completed. 


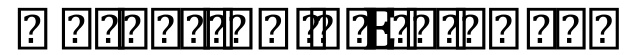

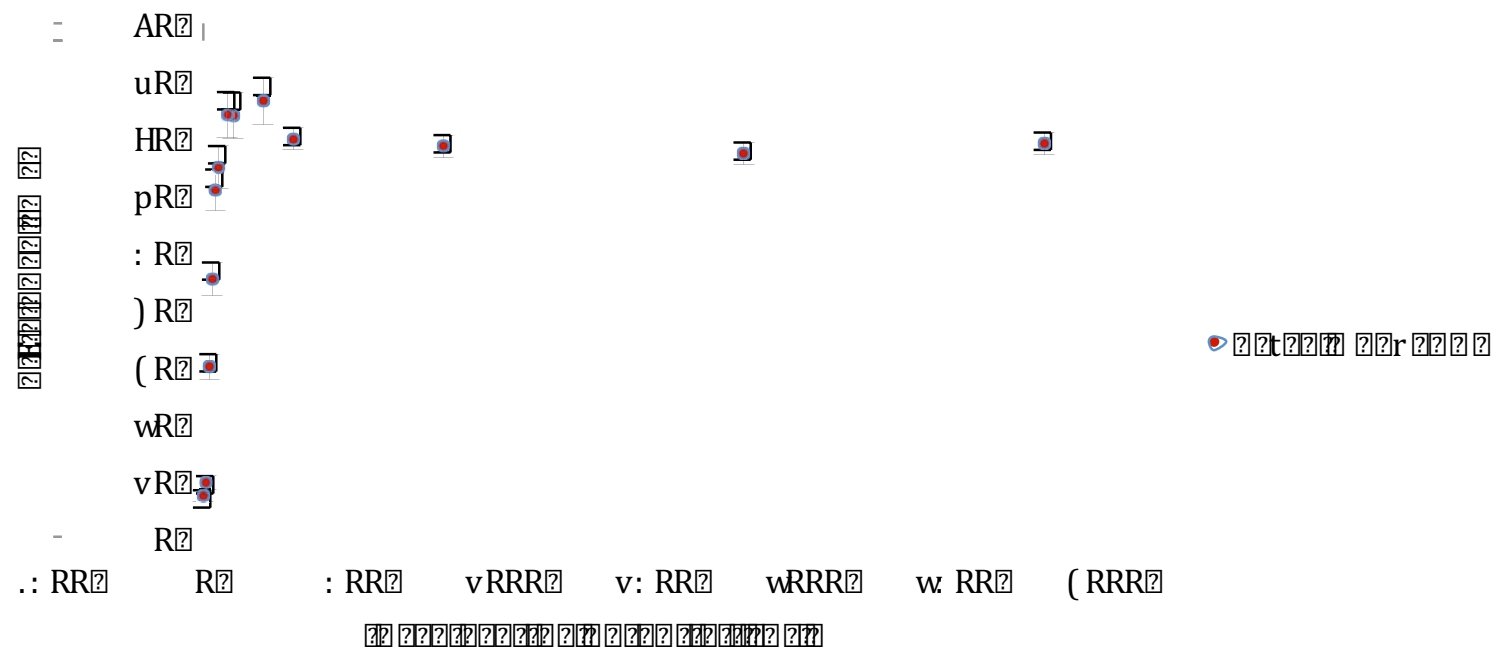

Figure 24: Alpha particle detection efficiency for the time pick-off detectors as a function of electron acceleration potential.

The kinetic energy measurements of fission fragments in SPIDER will be performed using ionization chambers. These chambers will also be used to perform Bragg spectroscopy to determine the nuclear charge of the fragments. The first prototype chamber was constructed and tested this year, and a second prototype is currently being assembled. An exterior view of the first prototype is shown in Figure 25. This prototype was designed and assembled at University of New Mexico, where the testing and evaluation was also performed. The chamber was operated with a P-10 gas mixture $(10 \%$ Argon $+90 \%$ Methane) at 1 atm pressure. The interior components of the chamber is shown in Figure 26, and consist of a anode that collects the electrons created when ionizing radiation passes through the gas volume, a Frisch grid that shields the anode from positive ions drifting to the cathode, and guard rings used to create a uniform electric field in the drift region. The first chamber prototype was used to check the energy resolution for alpha particles, and a spectrum for the $5.49 \mathrm{MeV}$ alpha decay of Am-241 obtained with the chamber is shown in Figure 27. The resolution that was obtained is $65 \mathrm{keV}(\mathrm{FWHM})$, or $1.2 \%$. This still needs to improved, as ionization chambers have been demonstrated to achieve about $35 \mathrm{keV}$ energy resolutions for alpha-particles. The relative resolution with ionization chambers for heavier ions with around $1 \mathrm{MeV}$ of kinetic energy per nucleon, such as fission fragments, has been shown to be approximately a factor of 2 better than for alpha-particles, or about $0.4 \%$. 


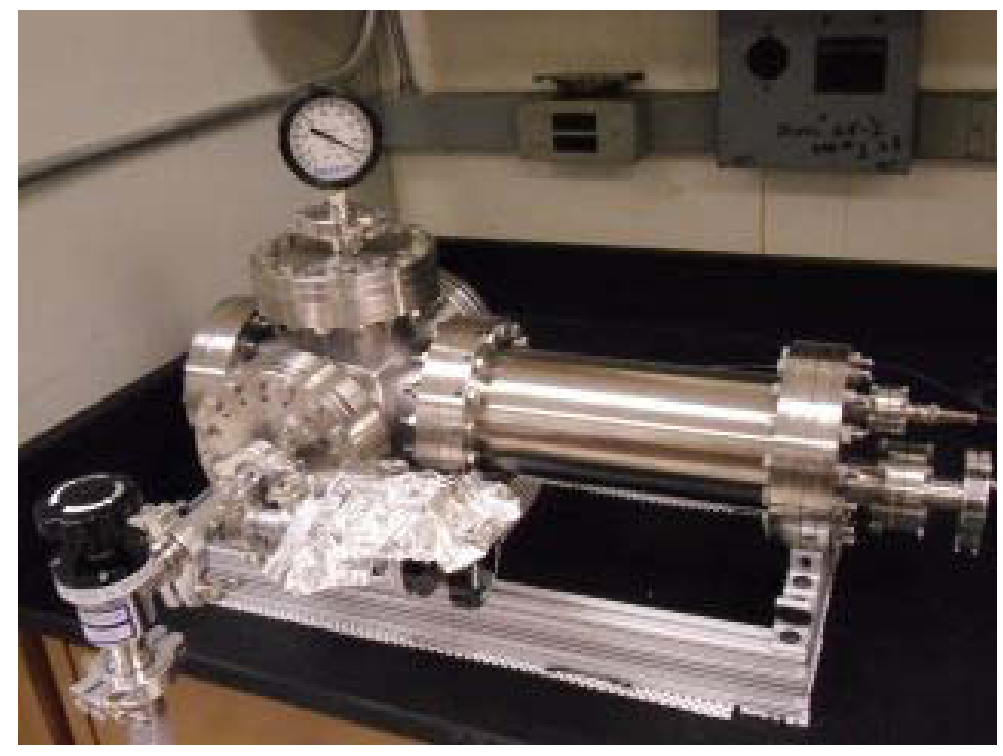

Figure 25: Ionization chamber used for testing at University of New Mexico (UNM).

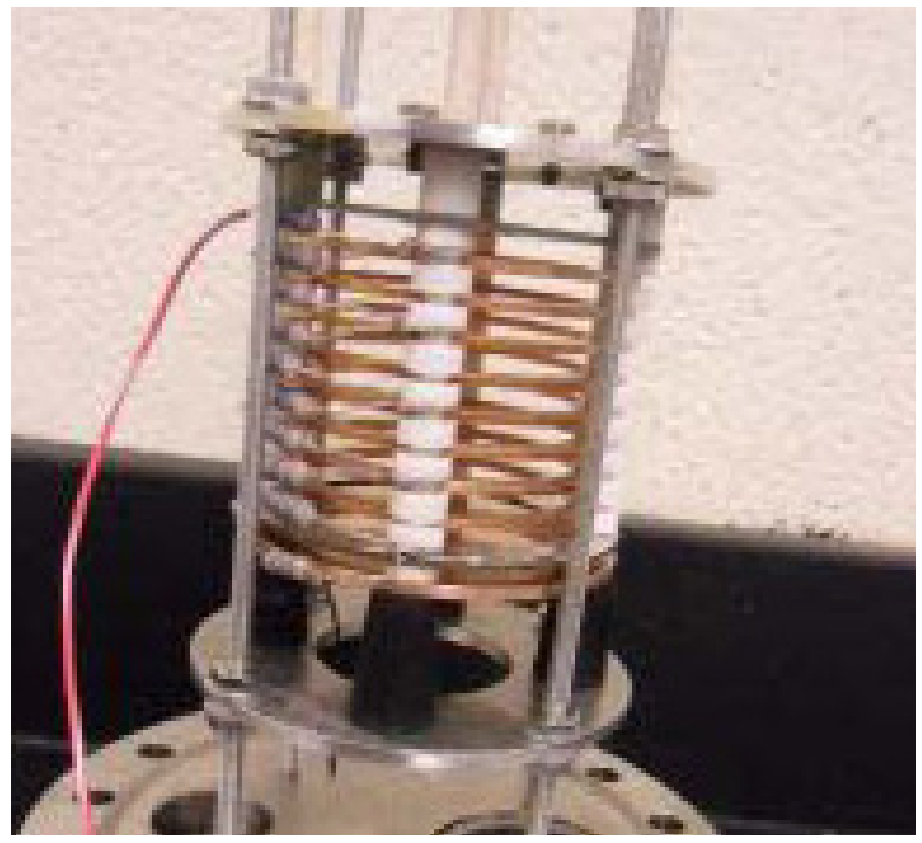

Figure 26: View of the internal components of the ionization chamber. 


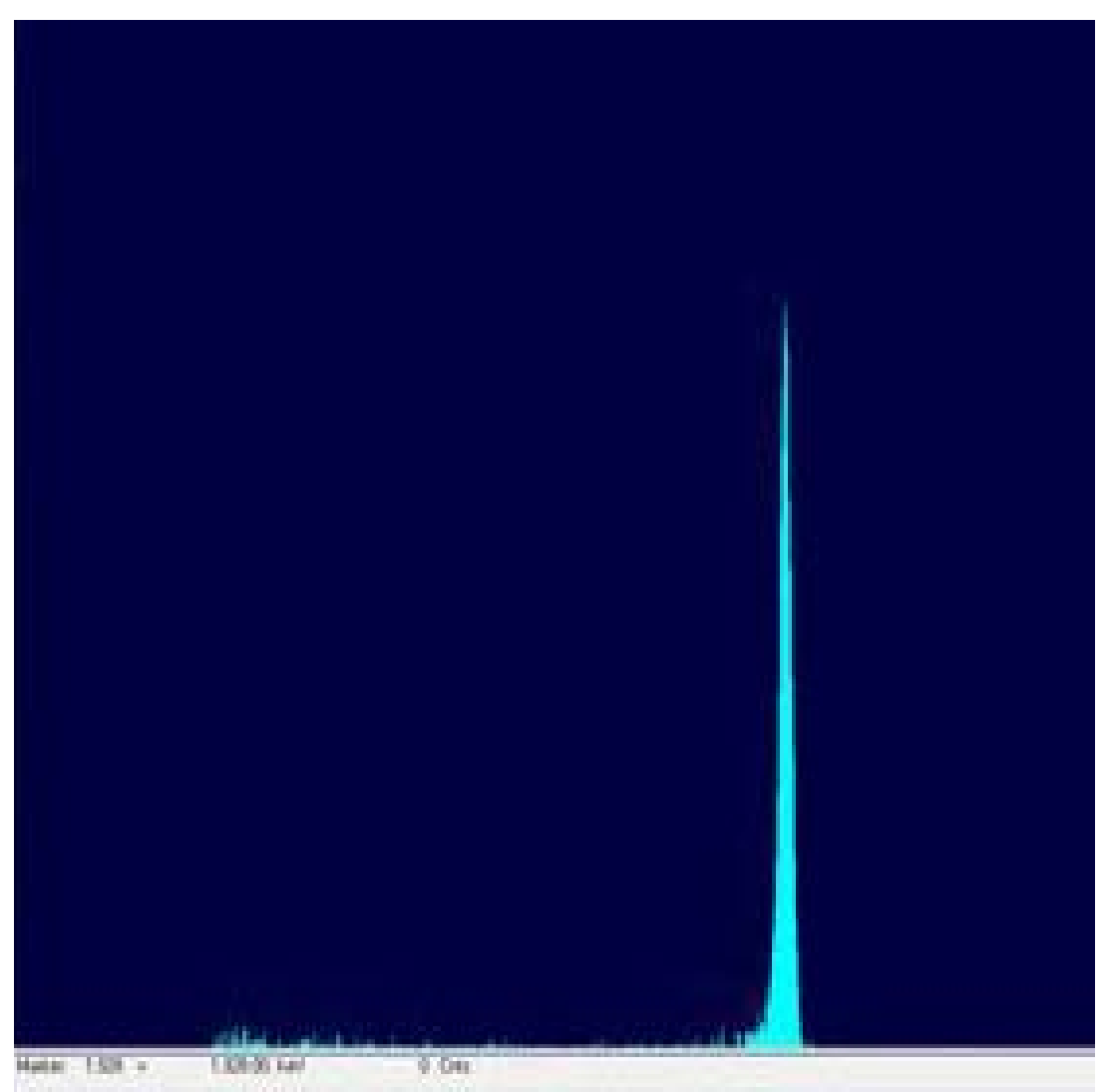

Figure 27: Energy spectrum for an Am-241 alpha-particle source collected with the ionization chamber prototype developed at UNM.

The second ionization chamber is currently being assembled, and will operate in the same mode as the production version. This chamber will be run with isobutane gas, which is favorable over P-10 for its higher energy resolution. It will also be run at about 150 torr, so that a thin window can be used between the TOF vacuum chamber and the gas volume. The prototype was designed with a vacuum section for testing different such thin windows. A drawing of the chamber is shown in Figure 28. The left section of the chamber will be under vacuum, while the right section will be filled with a counting gas at around 150 torr. The plane dividing the two sections will be designed such that different windows can be mounted and tested. 


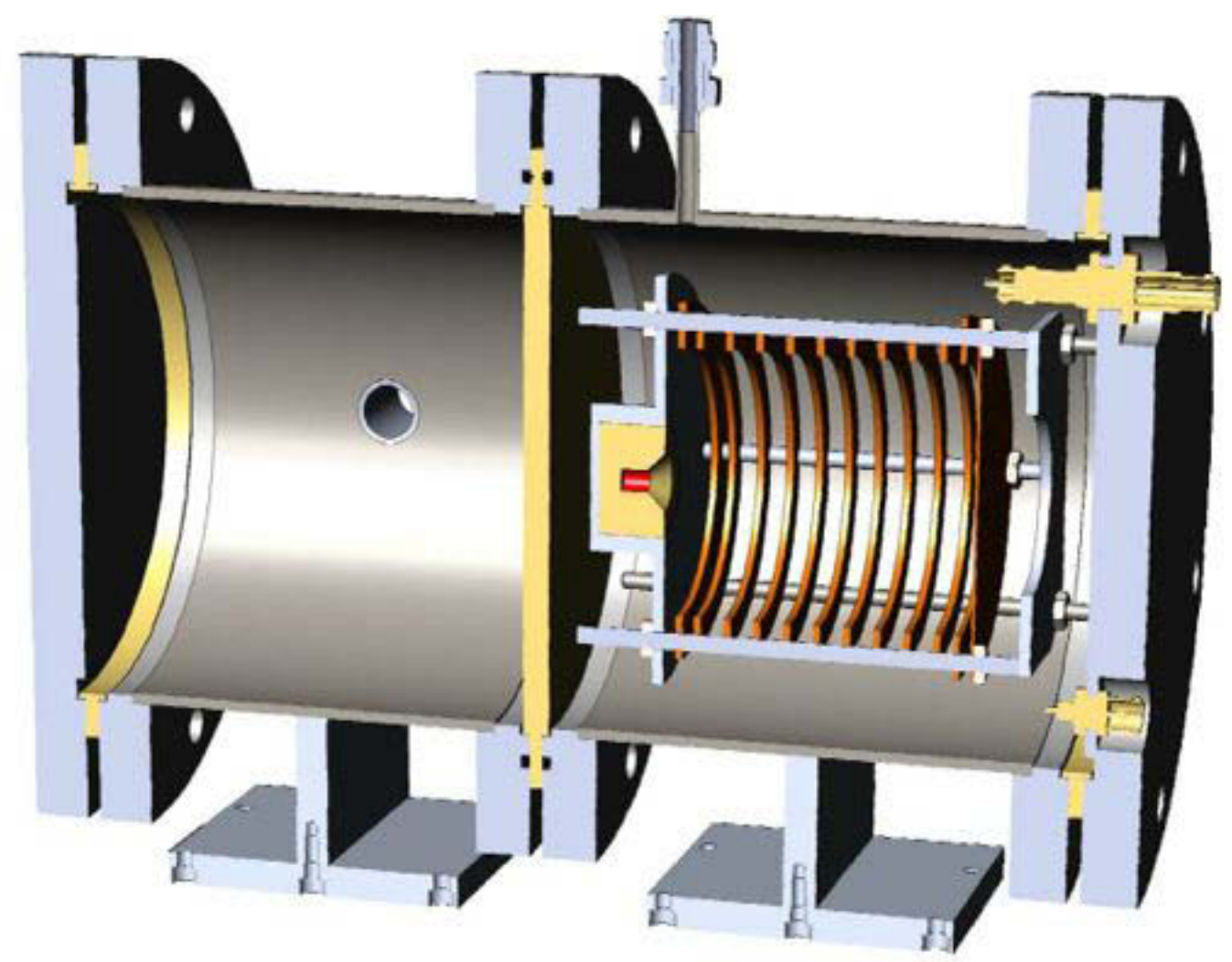

Figure 28: Cutaway CAD drawing of the second ionization chamber prototype.

\section{Detector Development for the Lead Slowing-Down Spectrometer}

The LSDS at LANSCE is a 20-ton cube of lead (Figure 29). Channels in the lead assembly allow the insertion of samples and detectors. Unused channels are filled with lead. The spectrometer is driven by the $800-\mathrm{MeV}$ pulsed proton beam incident on a tungsten target to produce a spallation neutron spectrum that extends from low energies to hundreds of MeV. The neutrons interact by reactions and inelastic scattering with the lead until the energies are less than the first excited state of ${ }^{207} \mathrm{~Pb}$, at $570 \mathrm{keV}$, which is the lowest first excited state of the stable lead isotopes. The neutrons then continue to lose energy, but they can only do so by elastic scattering, whereby the average energy lost per collision is only $1 \%$. The elastic scattering cross section is nearly constant below $60 \mathrm{keV}$ except for some isolated, narrow resonances. Since the faster neutrons in the distribution encounter more nuclei in a given time than do the slower ones, the fast neutrons slow down faster than the slower neutrons and there is therefore a focusing of the energy distribution as a function of time (Figure 30). Reactions can therefore be measured as a function of neutron energy by measuring the time when they occur as the neutron energy spectrum sweeps through this energy range. 


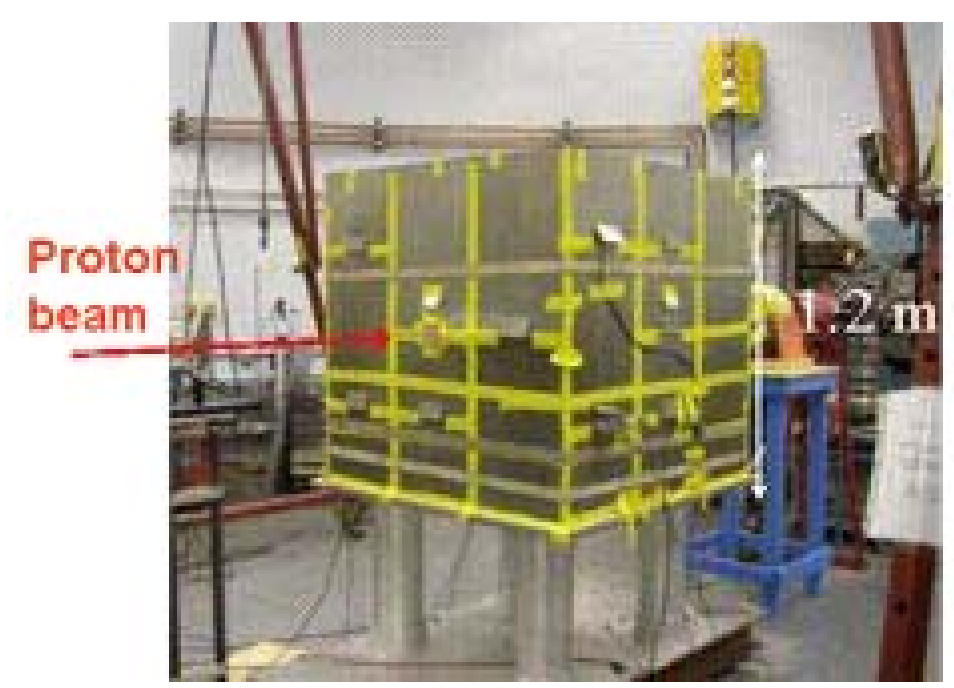

Figure 29: Lead slowing-down spectrometer installed at LANSCE. The pulsed 800-MeV proton beam from the accelerator enters a channel in the lead to strike a tungsten target. Samples are placed in channels in the spectrometer where they are subjected to a very high flux of neutrons. Thin sheets of cadmium cover the lead to reduce the number of neutrons returning to the spectrometer following scattering from the walls and floor of the room. The channels are just behind the flaps in the cadmium, visible in this photograph.

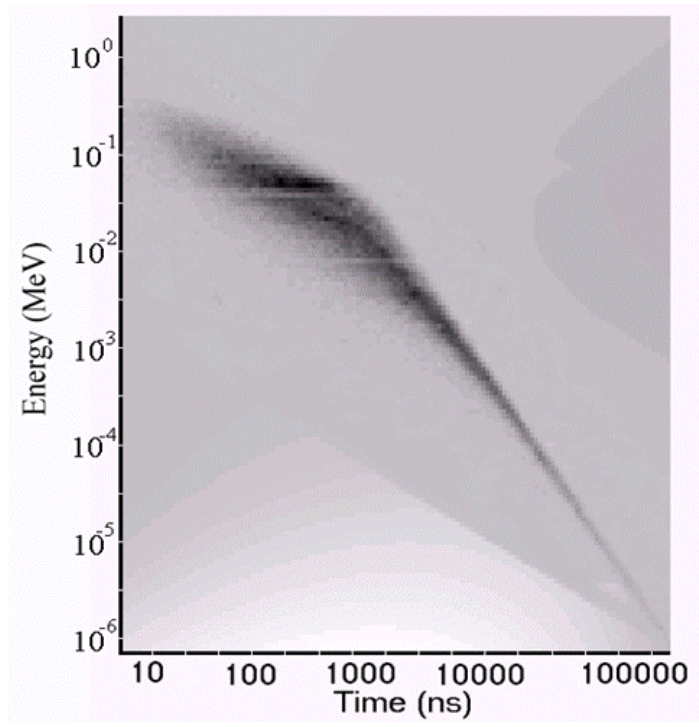

Figure 30: Simulation of the energy spectrum of neutrons within the spectrometer as a function of time showing the energy focusing of the distribution as time increases after 1 microsecond. The mean energy of the neutrons varies as $1 /\left(t+t_{0}\right) 2$, where the constant, $t_{0}$, has been determined previously [Rochman2005].

This LSDS is used for a variety of experiments where a very high neutron flux in the range $0.1 \mathrm{eV}$ to $100 \mathrm{keV}$ is required. Measurements of cross sections on very small samples or on isotopes that have a small cross section require very high fluxes. In past years, for example, we demonstrated that the fission cross section of ${ }^{239} \mathrm{Pu}$ could be measured with a sample of only $9.87 \mathrm{ng}$ (Figure 31). This capability has opened up the possibility of measuring other cross sections on radioactive samples where the intrinsic radioactivity limits the amount of material that can be handled safely. Last 
year, we began measurements of the fission cross section of ${ }^{237} U\left(t_{1 / 2}=6.7\right.$ day), and detector development was required for this work.

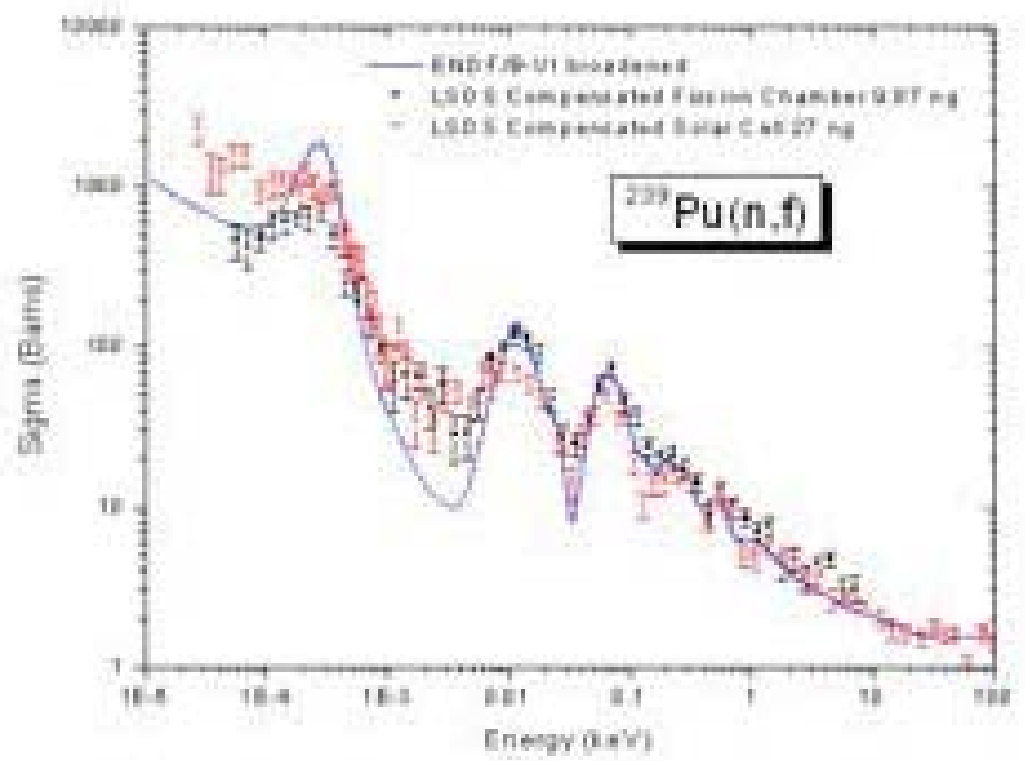

Figure 31: Fission cross section of ${ }^{239} \mathrm{Pu}$ as measured in the LSDS with sample sizes of 9.87 and 27ng. The energy resolution of the spectrometer, $d E / E$, is at best $30 \%$. The ENDF/B-VI evaluation was broadened with the measured energy resolution for this comparison with the LANSCE data.

Another application of the LSDS is in the assay of reactor fuel, both fresh and spent. The concept is to place a sample in the LSDS and then let the neutron spectrum sweep through the fission resonances characteristic of fissile isotopes. Fast (MeV) fission neutrons are emitted from the sample and the challenge is to detect these neutrons with a detector that is blind to the $\mathrm{eV}$ and $\mathrm{keV}$ neutrons inducing the fissions. We developed a fission chamber containing thorium, which has a fission threshold at about $600 \mathrm{keV}$ and therefore does not see lower energy neutrons.

This year we made the following progress: (1) a capillary containing a liquid scintillator and ${ }^{235} \mathrm{U}$ was tested to see if it is a suitable setup for measuring fission cross sections on very small samples; (2) gaseous proportional counters containing ${ }^{4} \mathrm{He}$ were used with samples of ${ }^{235} \mathrm{U}$ and ${ }^{239} \mathrm{Pu}$, each on the order of $25 \mathrm{grams}$, to see $\mathrm{n}-{ }^{4} \mathrm{He}$ scattering would be a better detector for actinide assay than the ${ }^{232} \mathrm{Th}$ and ultradepleted ${ }^{238} U$ fission chambers used last year, and (3) a paper was published on the use of silicon detectors called "PIPs" (Passivated implanted Planar Silicon) to study $(n, p)$ and $(n, a l p h a)$ reactions on very small samples [Ref. Thompson et al.]. The first two efforts are in their initial stages and results will be given in the annual report for next year. 


\section{Neutron Capture Measurement Program}

\section{Introduction}

The campaign for neutron capture measurements at Los Alamos is centered on the use of the Detector for Advanced Neutron Capture Experiments (DANCE). During FY12, DANCE ran experiments on ${ }^{235} \mathrm{U},{ }^{238} \mathrm{Pu}$, and decay studies of ${ }^{252} \mathrm{Cf}$ all of which are directly of interest for the Nuclear Energy program, even if all are not directly supported. In addition, experiments performed on ${ }^{173}$ Lu pushes the limits of highcount rate experiments on DANCE. Further, first measurements were performed with the new DANCE HPGe detectors in order to characterize their performance in DANCE. Finally, significant improvements were made to the data and support hardware infrastructure that have improved the reliability and stability of DANCE operations. In the remainder of this report, we will discuss the impacts of the following improvements

- Development of techniques for the study of fission gammas with DANCE

- Development of Ratio-to-fission Capture Cross Section Measurements

- Performance of DANCE PPAC

- Upgraded HV Mainframe

- Improved Gain Matching Algorithms

- $\quad$ Preparations for Major DAQ OS Upgrade

- $\quad$ First measurements with DANCE HPGe Detectors

- Gamma-ray Attenuation of the ${ }^{6} \mathrm{LiH}$ Sphere

\section{General Properties of Neutron Capture}

Neutron capture is a process that transmutes material to heavier isotopes of the same element. This is typically an exothermic process where 3-10 MeV of energy is released in the form of gamma rays. The product may or may not be stable once it has de-excited to its ground state. To walk through a specific, if simple example, we consider neutron capture on ${ }^{197} \mathrm{Au}$. This reaction is well studied. When a low energy $(<\sim 1 \mathrm{MeV})$ neutron is incident on ${ }^{197} \mathrm{Au}$, there are only two reaction channels open, elastic scattering and neutron capture. If neutron capture takes place, the resulting ${ }^{198^{\star}} \mathrm{Au}$ nucleus will have approximately $6.5 \mathrm{MeV}$ of excitation energy which is released in a gamma cascade of typically 3-4 gamma rays on a pico-second timescale. The residual ${ }^{198} \mathrm{Au}$ ground state nucleus is unstable and will decay to ${ }^{198} \mathrm{Hg}$ with a half-life of about 3 days. The incident neutron energy is not sufficient to significantly shift the energy of the gamma cascade. The gamma cascade is the primary signature of capture and the total energy of that capture is a unique nuclear property that can be used to identify the target nucleus. The capture cross section is sufficiently small that capture will not substantially transmute the composition of the sample in a typical experiment. If neutron scattering takes place, the neutron will be elastically scattered off of the ${ }^{197} \mathrm{Au}$ target into $4-\pi$. Due to the large mass difference between the neutron and $a{ }^{197}$ Au nucleus, the kinematic distortion is minimal. This neutron can now interact with detector material, structural supports, and the building where it will eventually thermalize and capture on one of the aforementioned materials. That capture will produce gamma rays which could be mis-identified as a capture in the ${ }^{197} \mathrm{Au}$ target. The two principal techniques for reducing this mis-identification are 1) 
use of calorimetric detectors to determine the total energy release and 2) reduction of surrounding material to minimize the probability of a nearby capture on structural materials causing energy release.

\section{Detector for Advanced Neutron Capture Experiments (DANCE)}

DANCE is a 4- $\pi, 160$ crystal, $\mathrm{BaF}_{2}$ detector array designed to determine the total gamma-ray cascade energy, consistent with the calorimetric principle mentioned above. A representation of the detector array is shown in Figure 32. The high granularity of the array reduces the instantaneous count rate in each crystal, reducing the probability of pile up and providing information about the event multiplicity. Barium fluoride scintillator was used as it has very high intrinsic efficiency for gamma rays, it does not activate in a neutron environment, it has moderate energy resolution, it is available in large volume $(750 \mathrm{ml})$ single crystals, and it exhibits relatively low neutron thermalization and capture properties. The total efficiency of DANCE for detecting a $1 \mathrm{MeV}$ photon emitted from the center of the ball is $86 \%$, while typical efficiencies for detecting a cascade are better than $95 \%$. These high efficiencies allow measurements on small samples. The total target size will depend upon the neutron flux available, but measurements have been completed at DANCE with as little as $160 \mu \mathrm{g}$ samples. Typical target masses are one to a few milligrams. While pure samples are always advantageous, it is possible to run samples with as low as $10 \%$ enrichment if the reaction $Q$-values of the contaminants are favorable. Isotopic contamination of material with $\Delta \mathrm{A}= \pm 1$ typically exhibit $\mathrm{Q}$-values with sufficient differences to allow measurements with DANCE. A ${ }^{6} \mathrm{LiH}$ shell is located between the sample and the detectors to attenuate scattered neutrons via the ${ }^{6} \mathrm{Li}(n, \alpha)$ t reaction, which does not produce gamma rays.

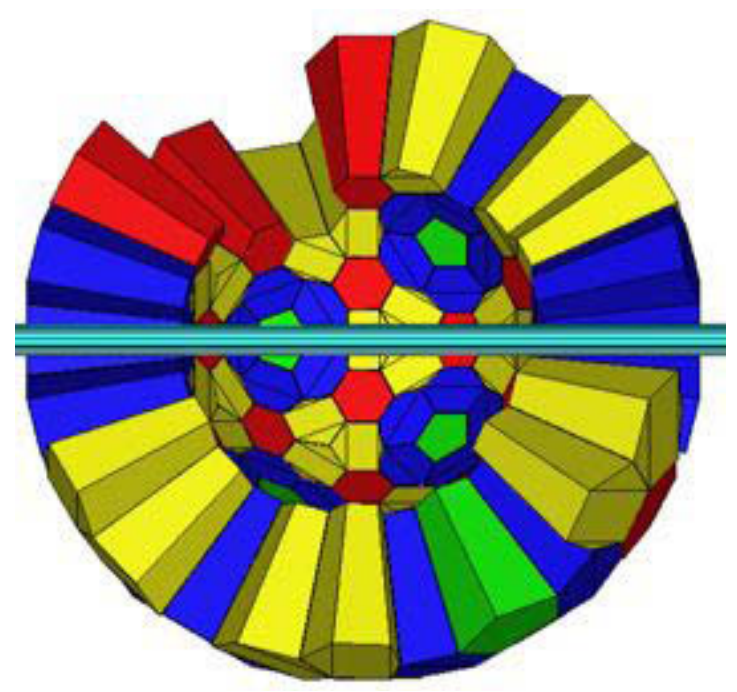

Figure 32: A representation of approximately half of the DANCE array is shown. Four distinct detector shapes cover the same solid angle. The different crystal shapes are shown in different colors. 


\section{Gamma-ray output from ${ }^{239} \mathrm{Pu}$ neutron-induced fission}

Information on the number of prompt gamma rays emitted following fission, as well as their individual energy spectrum and the total (summed) energy distribution is important for understanding the energy balance in fission and for modeling the fission process. These spectra are also important for applications including nuclear reactors and homeland security. There are relatively few measurements of the full spectrum of prompt gamma-rays from ${ }^{239} \mathrm{Pu}(\mathrm{n}, \mathrm{f})$, and both measurements were done at thermal energies using a single Nal detector. The average gamma-ray multiplicity and total energy were determined using mathematical unfolding techniques.

The 160-element calorimetric DANCE detector at LANSCE, which is a calorimetric 160 -crystal nearly $4 \pi \mathrm{BaF}_{2}$ array, is ideal for making measurements of the gammaray multiplicity, individual energy, and summed energy at neutron energies ranging from thermal to about $100 \mathrm{keV}$. These measurements made use of a fast fissiontagging parallel-plate avalanche counter (PPAC) designed to fit into the center of the DANCE array to identify fission events. Experiments were done on ${ }^{239} \mathrm{Pu},{ }^{242} \mathrm{Pu}$, ${ }^{238} \mathrm{Pu}$, and ${ }^{235} \mathrm{U}$ as well as ${ }^{252} \mathrm{Cf}$. The analysis is being done at several locations; the ${ }^{239} \mathrm{Pu}$ analysis is ready for publication, and the ${ }^{252} \mathrm{Cf}$ results have been published.

A drawing of the PPAC is shown in Figure 33. The PPAC is 1.75 inches diameter by 1.88 inches long, mounted on a thin aluminum extension tube. The detector gas was 4.5 Torr isobutane, flowing at roughly $4 \mathrm{ccm}$. The active region was an anodecathode-anode configuration, with the ${ }^{239} \mathrm{Pu}$ sample electroplated on both sides of the $3 \mu \mathrm{m} \mathrm{Ti}$ cathode foil. The anodes were operated at $+375 \mathrm{~V}$. A total of $937 \pm 9 \mu \mathrm{g}$ of Pu was electroplated over an area of $0.385 \mathrm{~cm}^{2}$, for a total target thickness of 2.43 $\mathrm{mg} / \mathrm{cm}^{2}$. The ${ }^{239} \mathrm{Pu}$ was enriched to $99.967 \%$, with the major contaminant being $0.03 \%$ of ${ }^{240} \mathrm{Pu}$.

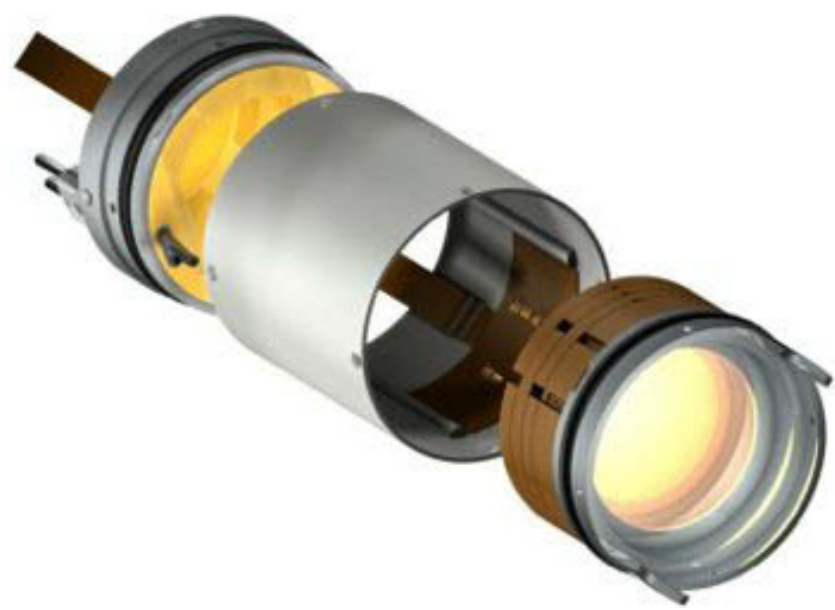

Figure 33: LLNL-LANL-MSI parallel-plate avalanche counter for fission tagging in DANCE.

The PPAC operated at about $70 \%$ efficiency for detecting fissions. The ${ }^{239} \mathrm{Pu}(\mathrm{n}, \mathrm{f})$ cross ection measured using the PPAC for neutrons from 10 to $140 \mathrm{eV}$ is shown Figure 34. Also shown is the $300 \mathrm{~K}$ Doppler-broadened evaluated cross section. 


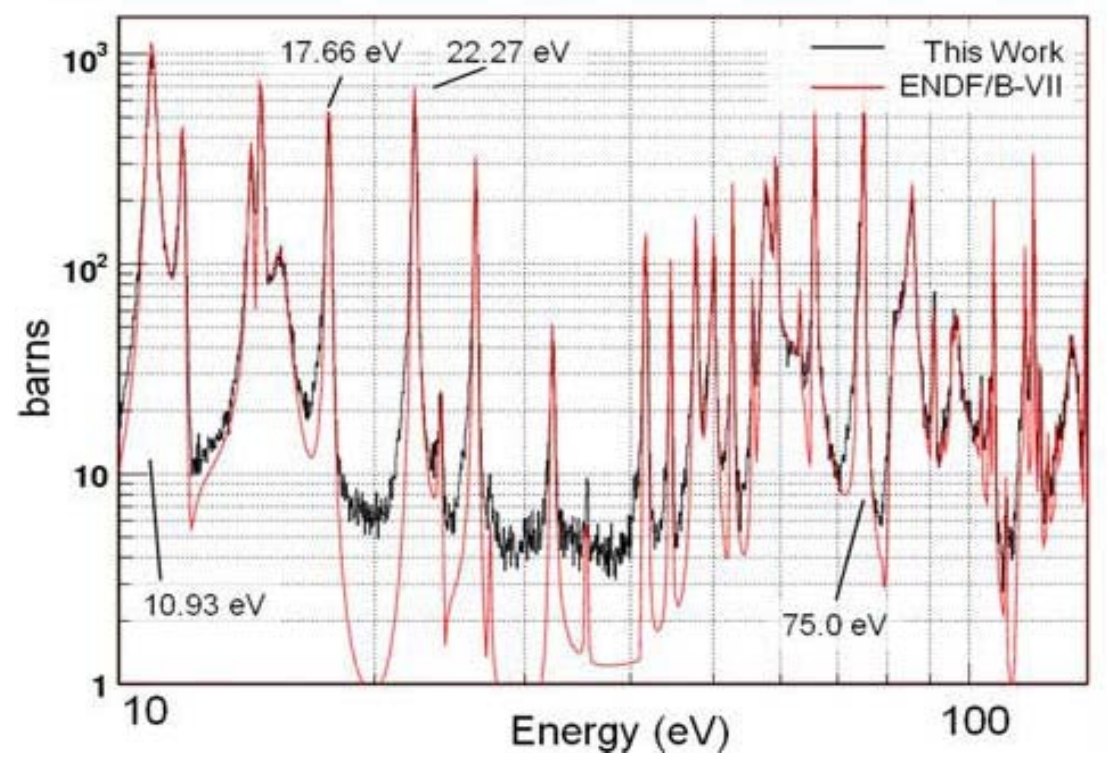

Figure $34:{ }^{239} \mathrm{Pu}(n, f)$ cross section measured for neutrons from 10 to $140 \mathrm{eV}$. Also shown is the Doppler-broadened evaluated cross section from ENDF/B-VII.

Even though DANCE covers about 3.5m, corrections must be made for detector response. The factors in the response include the geometric solid angle, Compton scattering and pair production in the crystals, scattering and attenuation in the ${ }^{6} \mathrm{LiH}$ shell, and detector threshold. This correction can be large for high-multiplicity events characteristic of fission since the probability for detecting all gammas goes as the efficiency to the power of multiplicity. There are two ways to make this correction. One way is to use a response function determined by measurement or Monte Carlo simulations to "unfold" the measured spectra. This method relies on accurately determining the response function, and it's inverse. Application of this method to the DANCE data is being pursued at LLNL.

In this work we have applied an alternate approach in which the physical emission spectra and multiplicity are described by analytic expressions. These expressions are used as probability distributions to generate by Monte Carlo a set of events that are then propagated through a well-tested GEANT model of the DANCE detector. The parameters of the expressions are then adjusted to minimize the $\mathrm{X} 2$. The gamma-ray multiplicity was characterized by two distribution functions:

where $\mathrm{M}_{\mathrm{i}}$ is the probability distribution:

$$
\begin{gathered}
M_{\gamma}=M_{1}+M_{2} \\
\left.M_{i}\right)=\left(2 M_{\dot{j}}+1\right) e^{-M_{i}\left(M_{i}+1\right) / B_{i}^{2}}
\end{gathered}
$$

The probability distribution of gamma rays emitted with energy $\varepsilon$ was taken to be:

$$
P_{1}(\varepsilon)=\varepsilon^{2} e^{-a_{1} \varepsilon} \quad P_{2}(\varepsilon)=\varepsilon^{3} e^{-\left(a_{2}+M_{\gamma} b_{2}\right) \varepsilon}
$$

The five parameters that were fitted are $\mathrm{B}_{1}^{2}, \mathrm{~B}_{2}, \mathrm{a}_{1}, \mathrm{a}_{2}$, and $\mathrm{b}_{2}$. The $\mathrm{x}^{2}$ minimization was based on comparing four Monte Carlo spectra with their measured values: total gamma-ray energy with crystal energy thresholds $150 \mathrm{keV}$ and $400 \mathrm{keV}$, crystal multiplicity with $150 \mathrm{keV}$ crystal threshold, and individual crystal energy with $150 \mathrm{keV}$ threshold. Note that in this analysis, we compared the quantities measured in individual crystals, while the parameterization is in terms of physical gamma rays. 
The measured data, of course, is in terms of individual crystals and the Monte Carlo simulations can easily determine individual crystal quantities. This is different from the usual DANCE analysis based on clusters since the clustering method starts to break down at the high gamma-ray multiplicities observed in fission.

The $x^{2}$ minimization used the iterative method of synthetic annealing based on the Metropolis algorithm. In this method, all five parameters were varied over a specified range at each iteration. The new parameters were accepted or rejected based on a probabilistic analysis and a new iteration started. Typically 500 to 1000 iterations were performed, and the parameters from the iteration with minimum $\mathrm{x}^{2}$ were used.

The analysis concentrated on data from the $\mathrm{J}=1$ resonance complex at $10.93+11.89$ $\mathrm{eV}$. Spectra were also obtained by gating on the $\mathrm{J}=1$ resonances at $7.82 \mathrm{eV}$ and $22.26 \mathrm{eV}$, the predominantly $\mathrm{J}=1$ complex at $75 \mathrm{eV}$, and the $\mathrm{J}=0$ resonance at 32.33 $\mathrm{eV}$. The spectra from all these regions were identical within statistics. Figure 35 and Figure 36 show spectra generated by Monte Carlo based on the parameterized probability distributions compared to preliminary Monte-Carlo Hauser Feshbach (MCHF) calculations. Figure 37 shows the parameterized spectrum, preliminary MCHF calculations, and evaluated (10) individual gamma-ray spectrum.

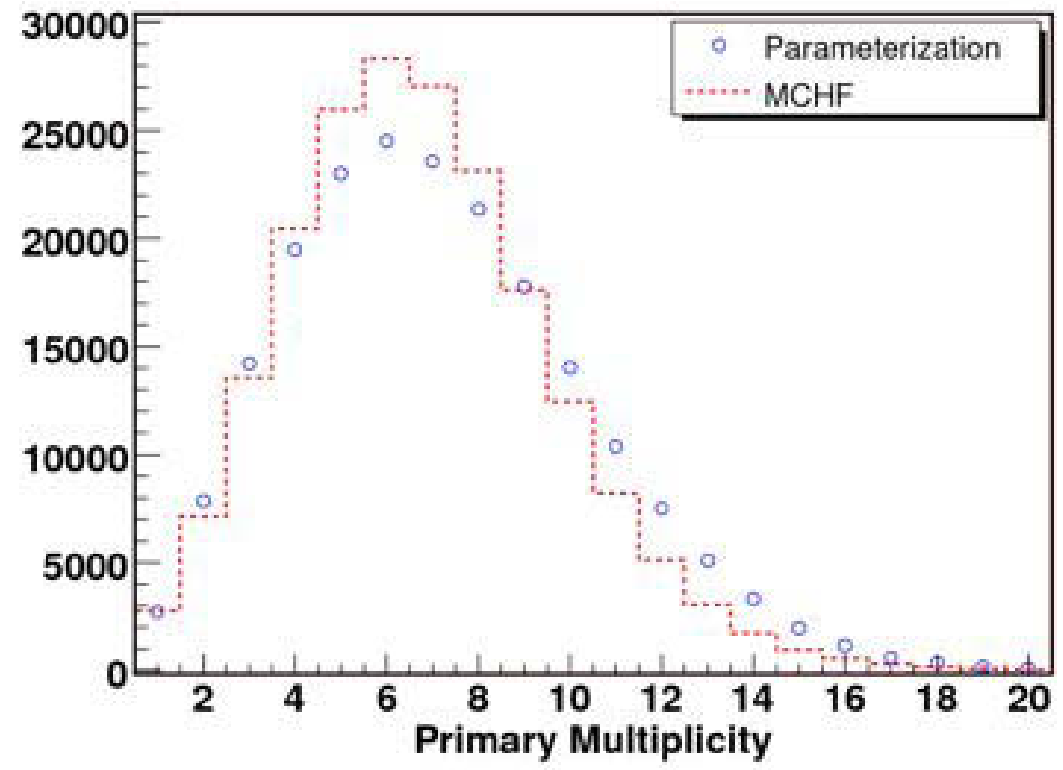

Figure 35: Gamma-ray multplicity from the best-fit parameterization compared to the preliminary MCHF calculations (9), with $150 \mathrm{keV}$ threshold. 


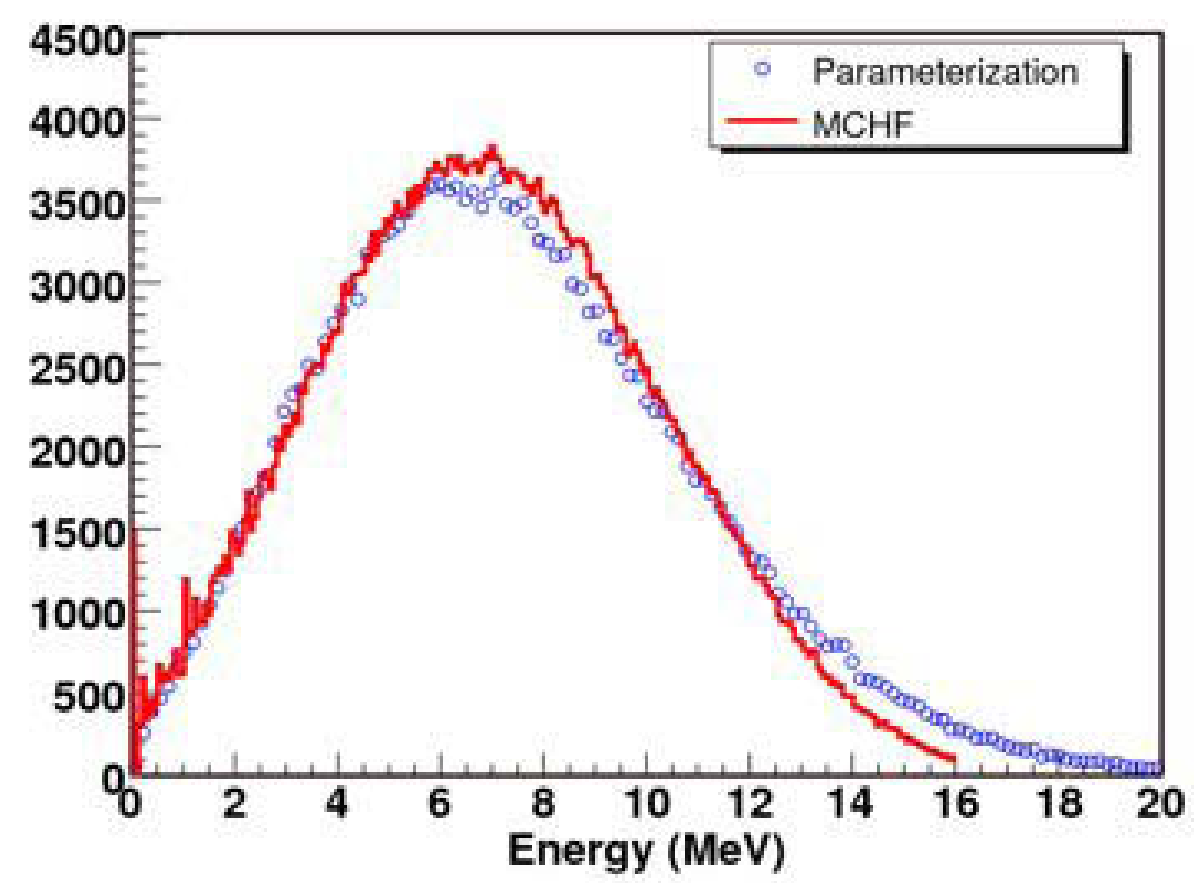

Figure 36: Total gamma-ray energy from the parameterization compared to preliminary MCHF calculations (9), with $150 \mathrm{keV}$ threshold.

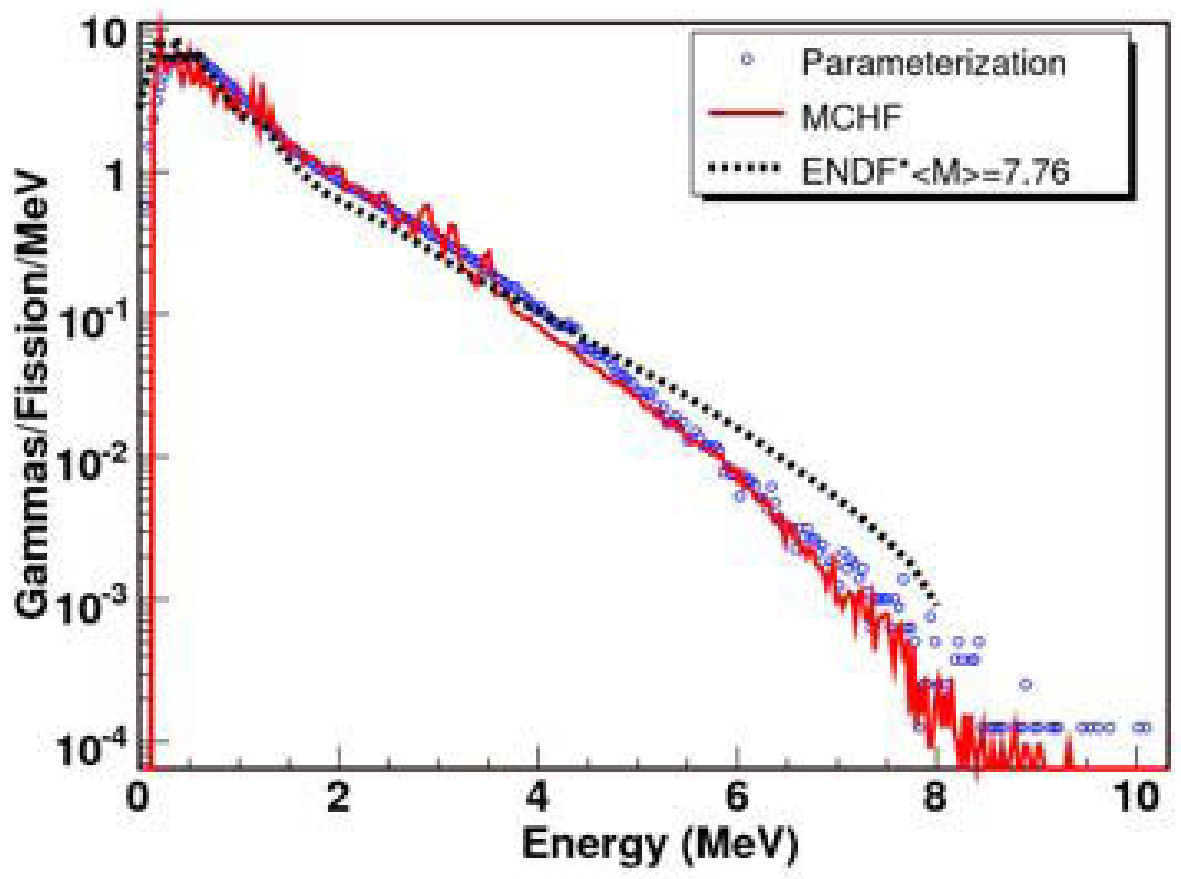

Figure 37: Individual gamma-ray energy from the best-fit parameters compared to the preliminary MCHF calculations (9) and the ENDF/B-VII evaluation (10). The evaluated spectrum was most likely based on ref (11).

The average total gamma-ray energy and multiplicity, compared to previous work, are shown in the table below. These values are very sensitive to the threshold in the 
experiment, as can be seen from Figure 37 This work and the calculations from (9) had a threshold of $0.150 \mathrm{keV}$, Verbinski (11) had a threshold of $0.140 \mathrm{keV}$, and Pleasonton (3) an apparent threshold of $0.085 \mathrm{keV}$.

Table 1: Average Multiplicity and Total Gamma Energy

\begin{tabular}{|c|c|c|}
\hline & $<\mathrm{M}>$ & $<\mathrm{E}_{\text {tot }}>$ \\
\hline This Work & $7.15 \pm 0.06$ & $7.37 \pm 0.06$ \\
Pleasonton (3) & $6.88 \pm 0.35$ & $6.73 \pm 0.35$ \\
Verbinski (11) & 7.23 & 6.81 \\
MCHF (Prelim) (9) & 6.76 & 7.03 \\
\hline
\end{tabular}

A publication on these results is nearing completion.

\section{Ratio-to-fission Capture Cross Section Measurements}

Independently measuring neutron capture cross sections at DANCE requires knowledge of the neutron flux at the target, the detailed detector response function, the sample mass distribution, and beam distribution on target, among other things. Understanding all of these quantities to the level needed to obtain high precision cross section measurements for the nuclear energy and defense programs is a challenging endeavor, so a novel method to measure the neutron capture cross section of fissile isotopes as a ratio to fission has been developed. Measuring capture and (known) fission cross sections simultaneously on the same target sample eliminates all of the above uncertainties and allows for reduced uncertainties in the measurement.

In the case of ${ }^{239} \mathrm{Pu}$, the experimental setup included a PPAC for fission tagging with an efficiency of $65 \%$. Neutron capture events were identified as qualitatively shown Figure 38, where fission and scattered neutron background must be subtracted off in order to obtain the neutron capture spectrum. This subtraction is performed as a function of energy to account for changes in both background rate and spectral shape. 


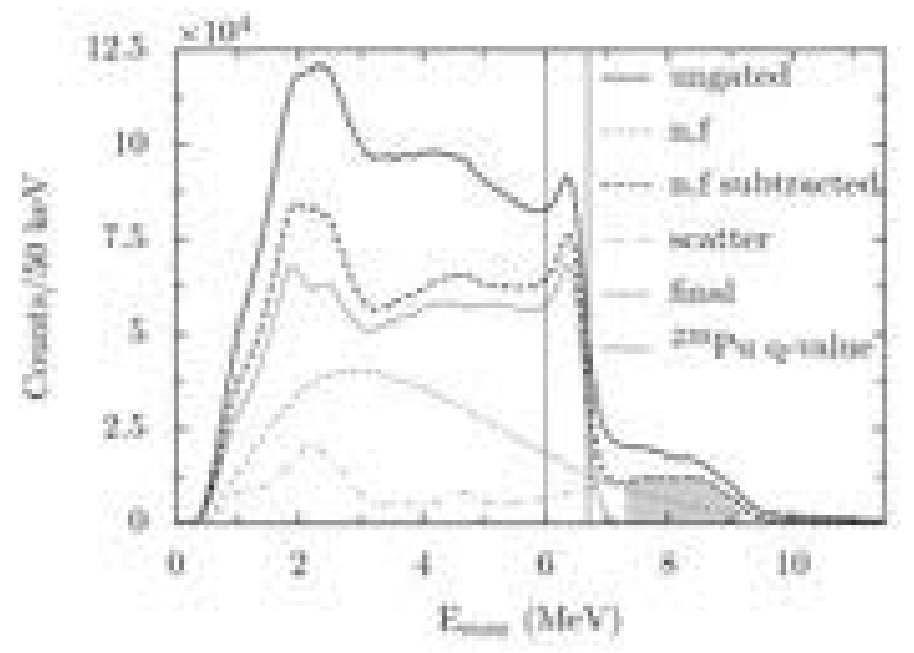

Figure 38: Total gamma-ray spectra demonstrating the background subtraction process. Fission (grey dashed line) is subtracted from the total spectrum (black solid). Then scattered neutron background (grey dot-dashed) is normalized to the resulting spectrum (black dashed) in the shaded region and subtracted to produce the final capture spectrum (grey solid). The 239Pu Qvalue is shown by the vertical lines.

The PPAC efficiency was determined as shown in Figure 39. In the plot of multiplicity vs. gamma energy in DANCE, only fission contributes to the boxed region. The ratio of the integrals of the boxed region for panels $(a)$ and $(b)$ can then be used to calculate the PPAC efficiency, which makes an accurate estimation of the fission background rate possible. 


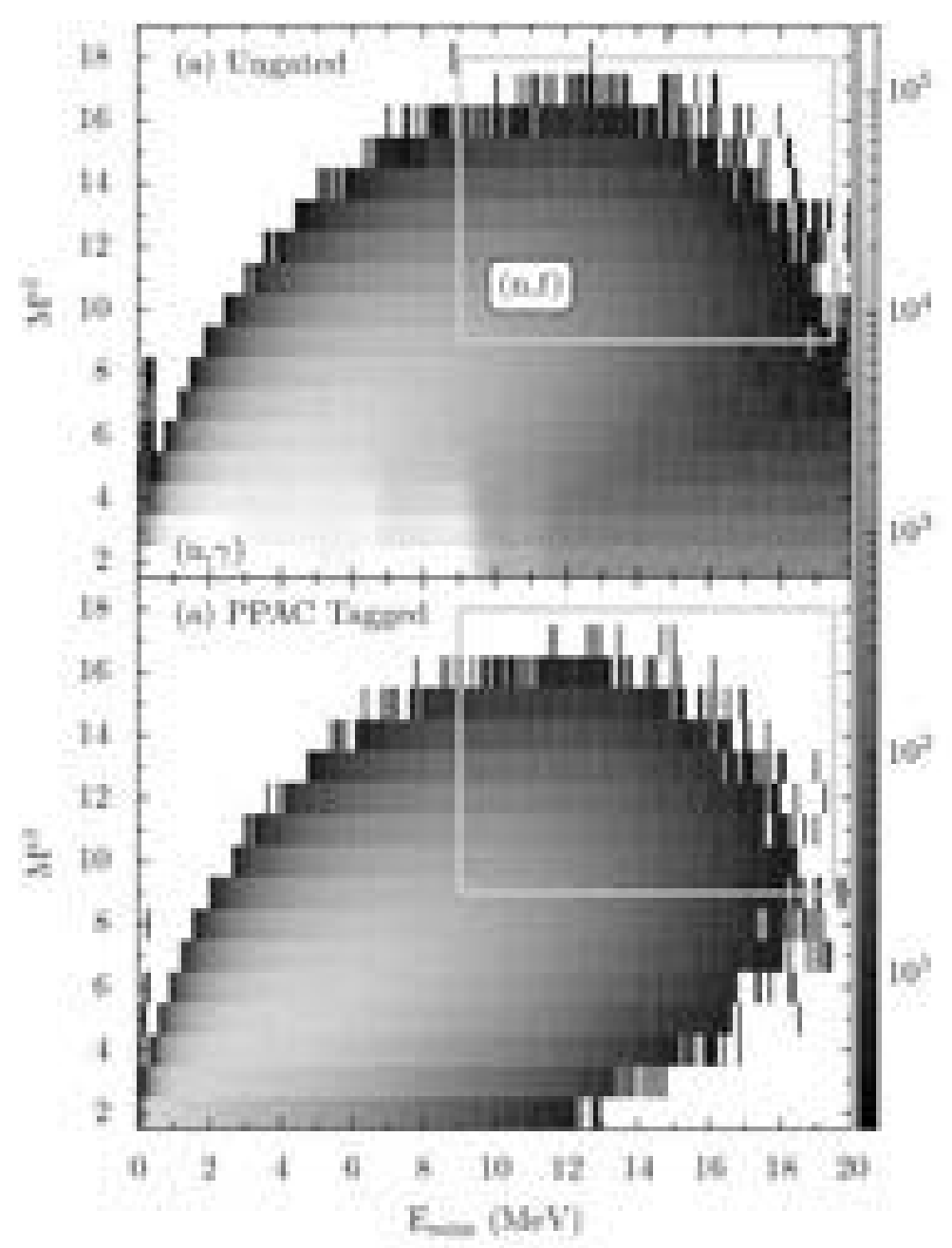

Figure 39: Multiplicity vs. Gamma energy for ungated (a) and PPAC coincidence (b). The box defines a region where only fission contributes to the spectrum, and is used to calculate the PPAC efficiency.

With the capture and fission yields both determined, the capture to fission ratio can be calculated by normalizing to a region where both the capture and fission cross sections are well known - in this case from 35 to $100 \mathrm{eV}$ in neutron kinetic energy. Because the fission cross section is well known across all accessible energies for this experiment, the measured cross section ratio can then be combined with the known fission cross section to calculate the neutron capture cross section.

\section{Performance of DANCE Parallel Plate Avalanche Counter (PPAC)}

One of the major improvements to the DANCE measurement capability has come from the drastically improved reliability delivered by the new DANCE PPAC design. Both of the previous sections discussing new techniques have taken advantage of this new capability. It was an integral part of the measurements for ${ }^{235} \mathrm{U},{ }^{238} \mathrm{Pu}$, and the fission decay properties for ${ }^{252} \mathrm{Cf}$ that were performed at DANCE this year. During FY12, the initial operating experience with the PPAC was studied in more detail and the results were published in Nuclear Instruments and Methods A. Rather than detailing the performance, we reference the journal article Wu et al. "A Compact gas- 
filled avalanche counter for DANCE", Nucl. Inst. And Meth. A 694 (2012) 78-81 and attach it in appendix $A$.

\section{Upgraded HV Mainframe}

Due to the 160 independent scintillator/PMT channels, DANCE requires a relatively large number of HV channels with independent control in order to drive the detector array. Further, as these are phototubes, at least $1 \mathrm{~mA}$ of available current is needed for each channel. For the last 10 year, DANCE has used a Universal Voltronics VISyN 1458 mainframe with 15 VISyN 1461 HV cards. Unfortunately, this system is based on legacy design from LeCroy and has not been significantly modernized by Universal Voltronics. As a result, while communication and control can be accomplished via telnet, it supports only one communication channel at a time, hampering diagnostics while running. Further, telnet communication must first be established via an RS-232 session as there is no front panel control and configuration of the system. While possible, establishing RS-232 communication has historically shown to be time consuming, making system debugging expensive in manpower. Finally, the mainframe stability had shown to be unreliable. Communication with the DAQ controller software would periodically drop out. Some of these connections would later be re-established, but others would not. After a small but appreciable percentage of these losses of communication, the system would turn off high voltage. Since there was no communication with the main frame at that point, the only signature was the loss of signals in the detectors as there was no bias on the PMTs.

As a result of these problems with the existing main frame, DANCE received funding from LANL defense programs to move to a CAEN HV mainframe. At the end of FY11, we procured at CAEN SY1527 Mainframe and 7 CAEN A1535S HV boards (24 channels each). Because the run cycle was already in progress, we did not immediately install the new HV mainframe. This new system satisfied several needs. First, it includes a front-panel control and configuration which drastically simplfies initial communication and debugging. Second, the system supports multiple communication protocols including TCP/IP. The important feature is that it supports multiple simultaneous TCP/IP connections at the same time, which allows it to communicate with the DAQ HV control system while experimenters can check on the status in an interactive session to ensure functionality. Next, the system is being actively developed and maintained by CAEN, which is one of the reasons that multisession TCP/IP was possible. Together with the mainframe, CAEN provided a C wrapper library in order to provide simple communication protocols for mainframe control. This was particularly important because in February of 2012, before the run cycle finished, the Universal Voltronics mainframe quit functioning completely. Within one week, a LANSCE-NS postdoc rewrote the HV control frontend to communicate with the CAEN mainframe, and DANCE was again taking data. This rapid turnaround was a direct result of the support delivered by CAEN for a high-level library for communication.

\section{Improved Gain Matching Algorithms}

The gain alignment procedure for DANCE has been automated for ease of use and consistency. The gains for all 160 crystals are aligned via light output from natural alpha decays within the crystals themselves. Figure 40 shows a representative spectrum resulting from 90 minutes of data (blue) along with the fit (red). A slope-like 
parameter controls the distance between alpha peaks in the fit, and voltages are adjusted to cause all crystals to have the same slope.

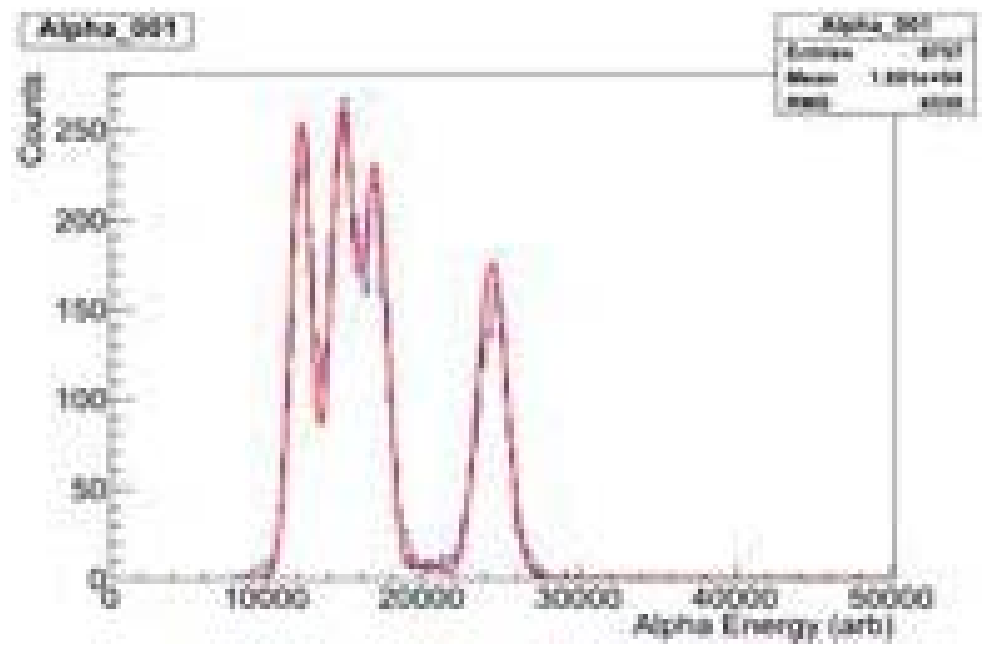

Figure 40: Raw energy spectrum (blue) for natural alpha decays within a representative DANCE crystal with fit (red) superimposed.

When running, the automation program takes and analyzes the data needed for the gain alignment, determines the old voltage settings, calculates new voltages, and creates several diagnostic output files. The only interaction needed is to start the program and confirm the usage of the calculated voltages. Figure 41 shows a representative set of crystals converging on the desired slope over 3-4 runs.

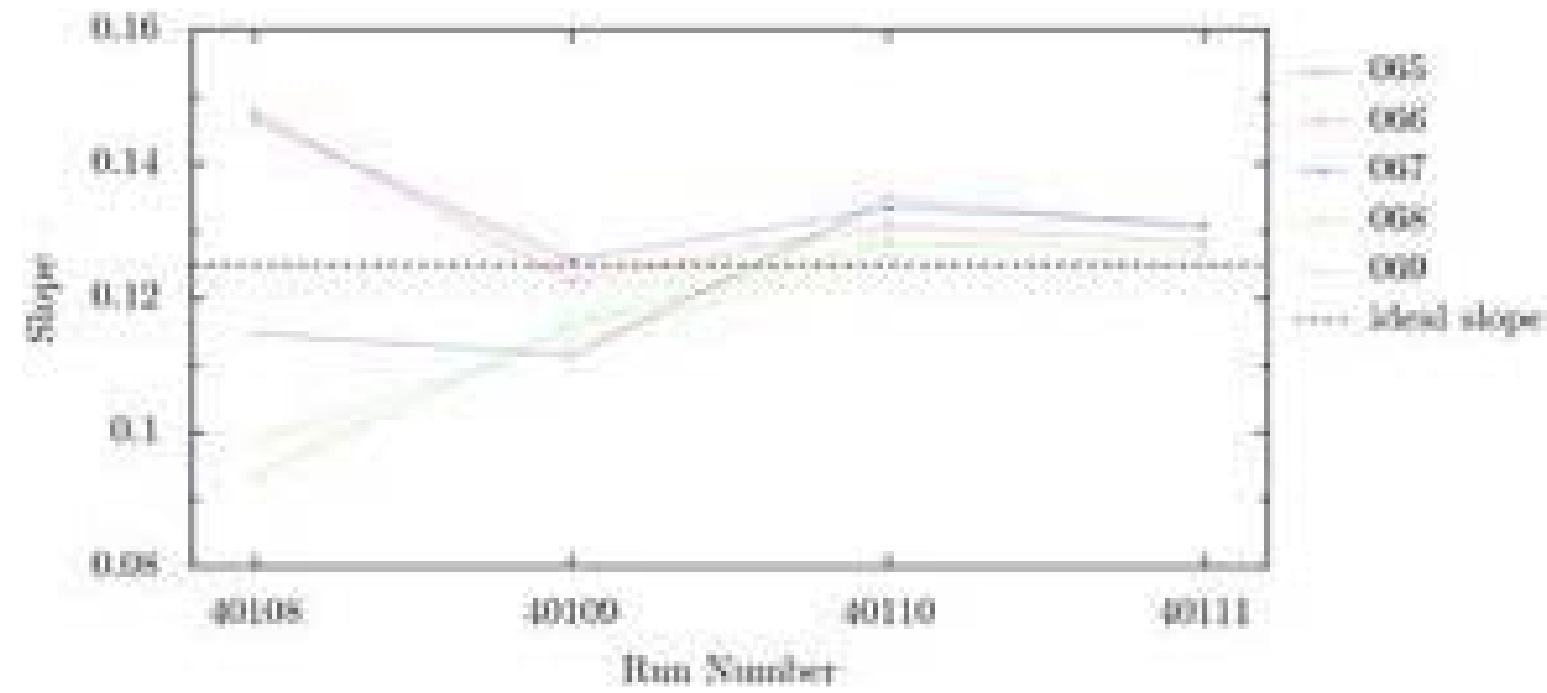

Figure 41: Slope vs. run number for five crystals (solid lines) converging on the desired slope (dashed line).

This program significantly reduces the time needed to gain match the detector, and increases the robustness of the entire procedure by consistently treating all crystals in a systematic fashion. 


\section{Preparations for Major DAQ upgrade}

In addition to the HV system which was rather outdated, the frontend computers and software for DANCE have been in place since 2005. DANCE uses $15 \mathrm{cPCl}$ singleboard computers to run the frontends the take data for the $\sim 330$ data channels. In addition, a rackmount server and a desktop complete the data acquisition system. The present operating system is Red Hat Enterprise Linux Workstation 3 (RHEL WS3), which is of a similar era as the hardware. The existing computers have started to show signs of hardware failure. These systems are no longer available for purchase. Repair is both expensive and time consuming. We have purchased a suite of replacement $\mathrm{cPCl}$ computers, but we cannot install RHEL WS3 on these machines as the hardware is too modern. During FY12, we started developing the upgrade path. In order to move forward, we need modern CPUs, a modern OS, drivers for the Acqiris DC265 digitizers, which form the backbone of the DANCE DAQ, drivers for Agilent U1066A digitizers, which are used for the HPGe upgrade, and a deadtime control solution. Deadtime control is presently managed via CAMAC with a Kinetic Systems 3922/2915 PCI-CAMAC interface. Kinetic Systems does not provide Linux drivers for this system. There is no other PCl-based CAMAC controller. When the DANCE OS was upgraded to RHEL WS3, custom drivers were written in-house. One possibility is to repeat that; another is to move to a VME based system which incurs hardware and frontend redevelopment costs.

To start on this challenge, we started with the ADLink cPCl-6510V controllers. This CPU offers several advantages of the existing DANCE controllers. First, it has 4 processors on board, increasing processing power. Second, it offers more memory. Third, it is still commercially available. Fourth, when we purchased them, we purchased 4 spares for immediate swap-out. That satisfies the modern CPU. These systems are recognized and build with both Red Hat Enterprise Linux Workstation 5 and 6 (RHEL WS5 and RHEL WS6). At that time, our standard DAQ was RHEL WS5. We installed new drivers from Agilent in order to communicate with the digitizers. While the Agilent drivers would install in RHEL WS5, they did not recognize the digitizer hardware. We then moved to an install of RHEL WS6. The Agilent drivers installed with RHEL WS6. They also recognized both the DC265 and the U1066A hardware. This was the first use of RHEL WS6 at LANSCE-NS, so it required customization of the environment to meet our needs. The most direct parts were a rebuild of MIDAS, the data acquisition framework, and ROOT, the analysis framework. At this point the system was ready to field test. All of the DANCE HPGe tests used this new hardware and OS framework. During this beamtime test for DANCE, we discovered a flaw in the Agilent driver software. After communication with Agilent, we have upgraded the drivers to a more stable version. At this point, most of the major pieces are in place for the DANCE OS and hardware upgrade which is planned for Q2-3 of FY13. There is still the remaining problem of the deadtime control. We have several solutions to this problem under investigation.

\section{First Measurements with DANCE High Purity Germanium (HPGe) Detectors}

The DANCE instrument is a very powerful tool for measuring neutron capture. The high efficiency for $Q$-value detection combined with the multiplicity information combine to allow measurement on small, impure samples. This capability is unmatched. However, the BaF2 scintillators have relatively poor resolution. This limits the selectivity for looking and individual gamma-transitions. There are several 
reasons which individual gamma resolution could be of interest. First, with a fissile sample, DANCE could be used to identify fission and the individual gamma energy could be used to tag the fission product if the low-lying energies are know. Second, there is interest in capture cross sections of certain materials with very large scatter:capture ratios. DANCE's ability to determine these cross sections is limited by the scattered neutron background. However, many of these isotopes with large scattering cross sections have realatively high-energy low-lying states, making them ideal candidates for additional tagging with HPGe. By combining the Q-value efficiency of DANCE with the resolution of HPGe detectors, the selectively can be increased by several orders of magnitude.

Two HPGe detectors were purchased in FY11 to test these methods. In Feb. of 2012, 10 days of DANCE beamtime were used for first tests with these detectors. There is still substantial work that needs to be done in order to fully integrate the HPGe signals with the DANCE DAQ, so the HPGe detectors were operated in standalone mode, replacing 2 of the DANCE BaF2 scintillators in order to determine the efficiency, timing resolution, and sensitivity to the gamma-flash of the HPGe detectors. This was also the time when the DAQ development discussed above was underway. It was during these measurements that the problems with the Agilent drivers were discovered. The solution was not found until after the experiment. As a result, only limited statistics were achieved.

The experiment was performed with a foil of ${ }^{117} \mathrm{Sn}$, populating excited states in ${ }^{118} \mathrm{Sn}$. This system was chosen as ${ }^{118} \mathrm{Sn}$ has a high-energy first excited state at roughly 1.2 $\mathrm{MeV}$, making it easy to identify. Further, the sample was available.

An HPGe detector in the specialized mount is shown in Figure 42. In Figure 43, the detectors are shown mounted in DANCE. Preliminary spectra are shown in Figure 44 and Figure 45.

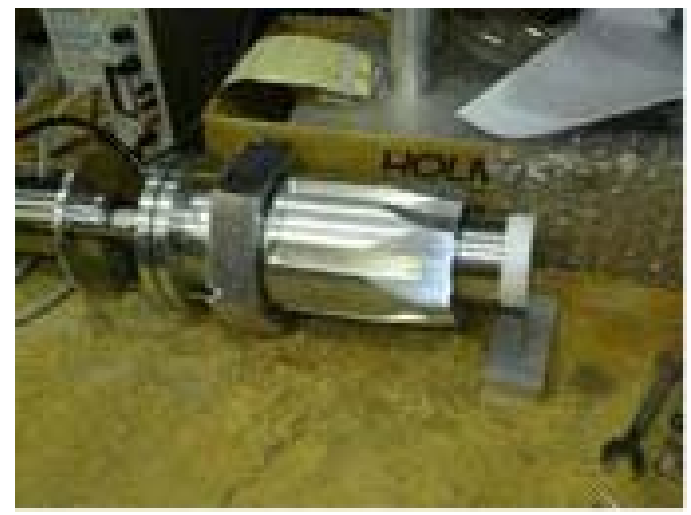

Figure 42: A DANCE HPGe Detector in the mounting bracket. 


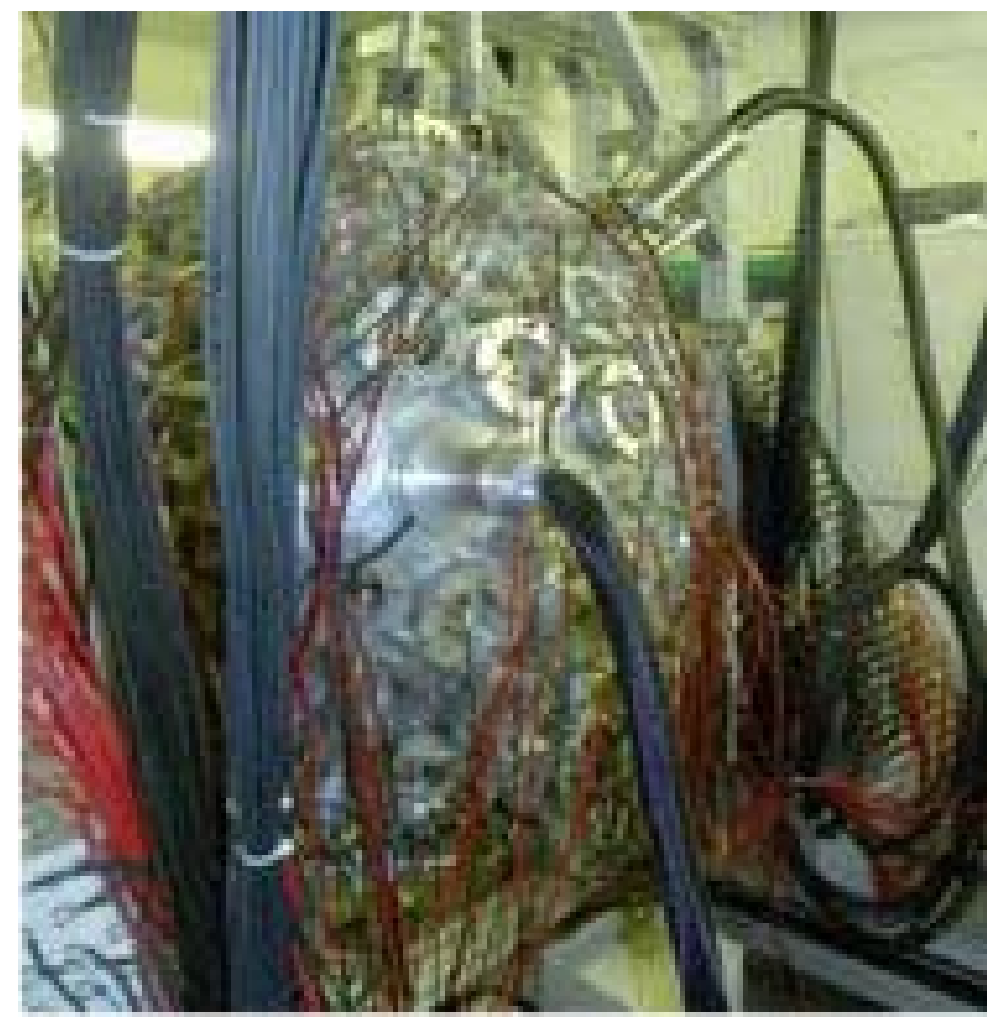

Figure 43: Shown above is the DANCE array with the two HPGe Detectors mounted. The black hoses coming off connect to ORTEC X-Cooler thermomechanical coolers.

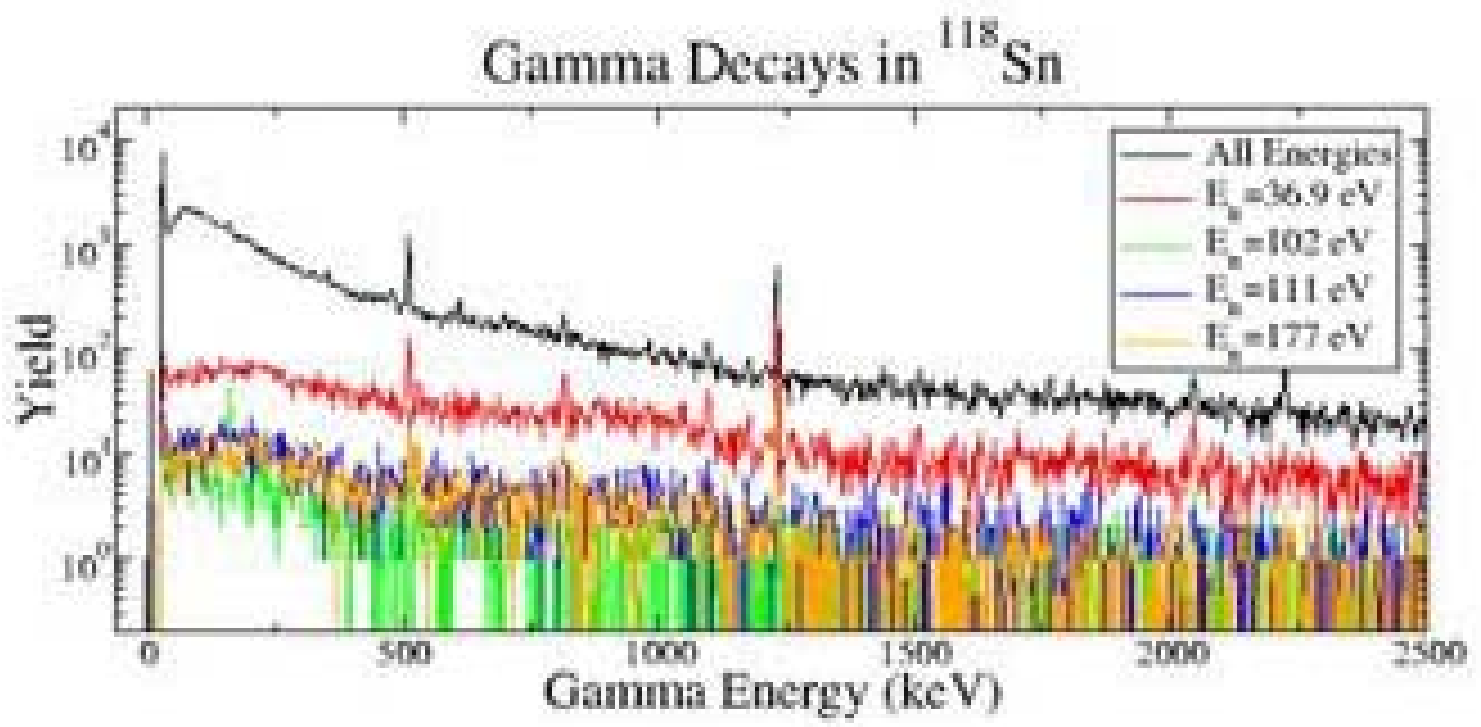

Figure 44: Shown above is the calibrated gamma energy spectra from the DANCE measurement with the HPGe detectors. In colors are shown the yield gated on neutron resonances. It is worth noting that the gamma at $\sim 200 \mathrm{keV}$ comes from a contaminant in the ${ }^{117} \mathrm{Sn}$ sample. 


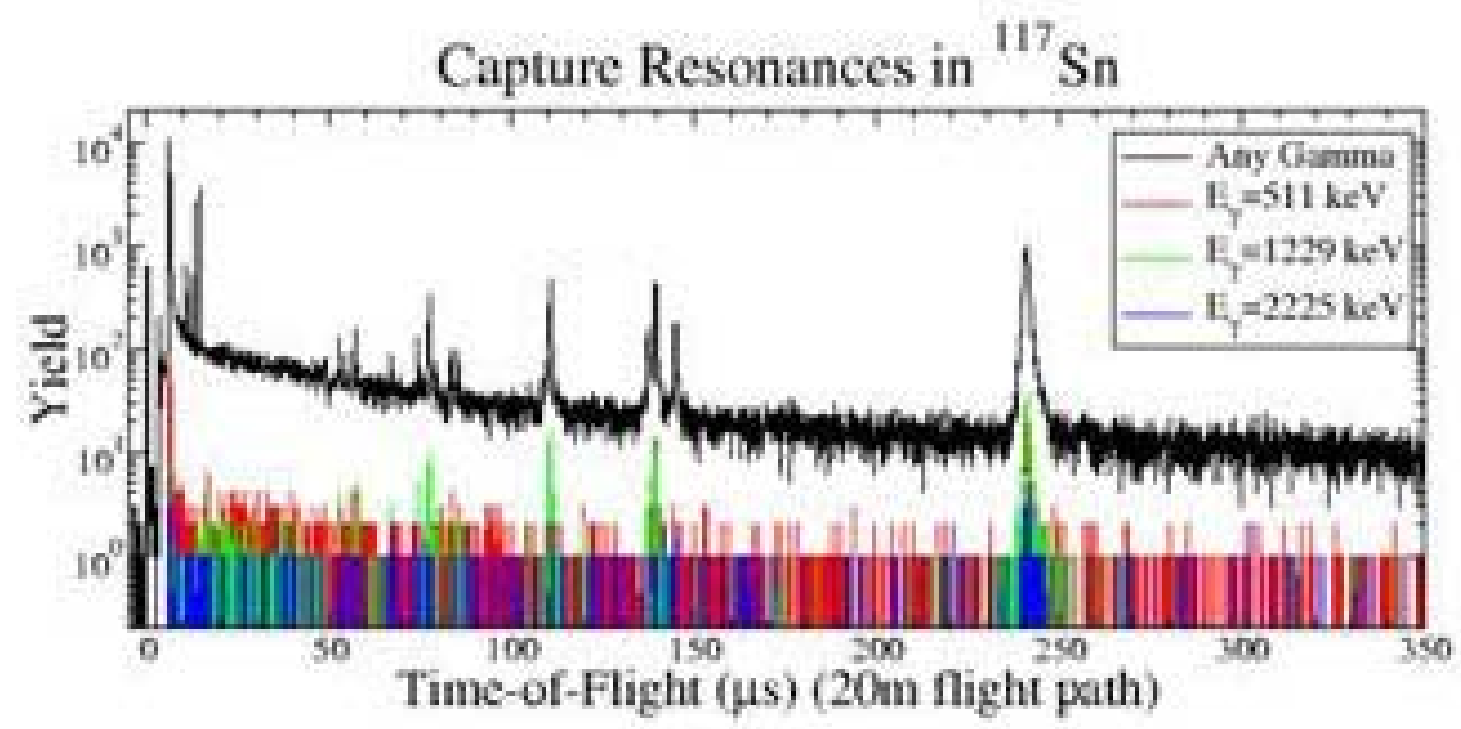

Figure 45: Shown above is the neutron time-of-flight spectra from the HPGe detectors. In black is the spectrum for the observation of any gamma in the HPGe. In colors are shown the spectra gating on a known transition. An approximate 2 order of magnitude improvement is seen from this selectivity. It is also worth noting that the detectors were sensitive down to quite short timeof-flights (neutron energies of several tens of $\mathrm{keV}$.)

\section{Gamma Ray Attenuation of the ${ }^{6} \mathrm{LiH}$ Sphere}

As mentioned above, one of the largest sources of background for DANCE comes from scattered neutrons. In order to mitigate this effect, a $6 \mathrm{~cm}$ thick ${ }^{6} \mathrm{LiH}$ sphere is used in DANCE as can be seen in Figure 46. This material was chosen as it is a relatively low- $\mathrm{Z}$ material, ${ }^{6} \mathrm{LiH}$ absorbs neutrons without gamma emission, and it is relatively effective at absorbing neutrons. However, it will still attenuate gamma rays from capture or fission. This effect becomes even more important when trying to determine the shape of the fission-gamma spectrum which has a large component of gamma-rays below $500 \mathrm{keV}$. 


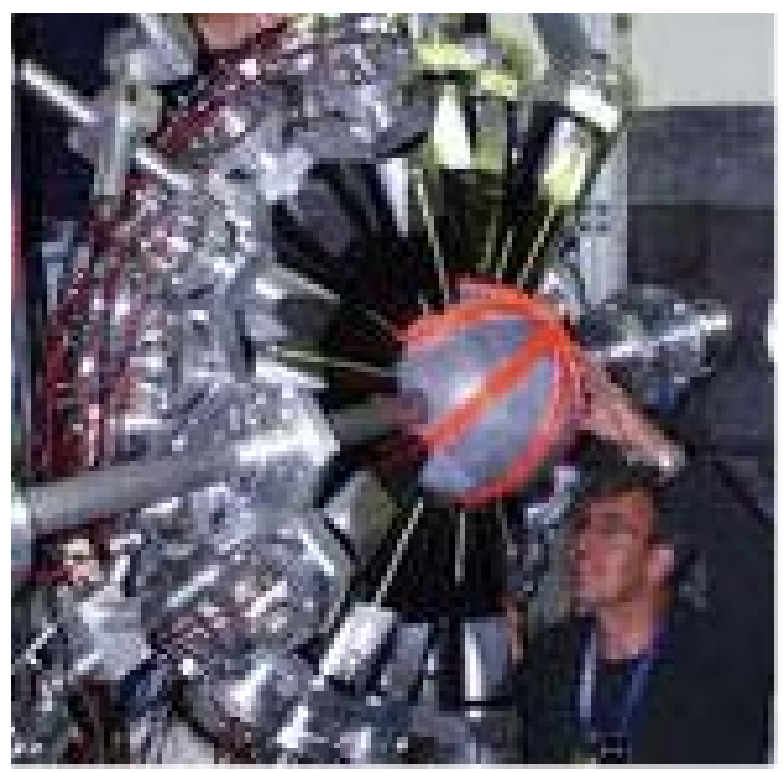

Figure 46: Shown is the interior of DANCE. The LiH sphere is held in place with the red velcro straps.

In the past, this attenuation has been modeled in GEANT4. In order to validate these models, a summer student measured the gamma-ray attenuation using the HPGe detectors for DANCE. The HPGe detectors offer the advantage that the resolution effects can be neglected and the ratio of photo-peak efficiencies with and without absorbers can be used to determine the absorption.

The simplest test would be to measure with and without the ${ }^{6} \mathrm{LiH}$ sphere on a tabletop. Unfortunately, the ${ }^{6} \mathrm{LiH}$ sphere cannot be simply removed from DANCE. Instead, the distance from the center of DANCE to the front face of the HPGe detector was determined geometrically. Gamma spectra were taken with sources at the center of DANCE and the HPGe in a crystal position. The HPGe detectors were then moved to a lab benchtop and the same sources were measured with the same detector-source distances. These two geometries were modeled in GEANT4 and the ratio of observed peaks with and without absorbers were compared as seen in Figure 47. The surprising observation was that GEANT4 did not reproduce the measured results. In fact, GEANT4 showed more absorption than the measurements. 


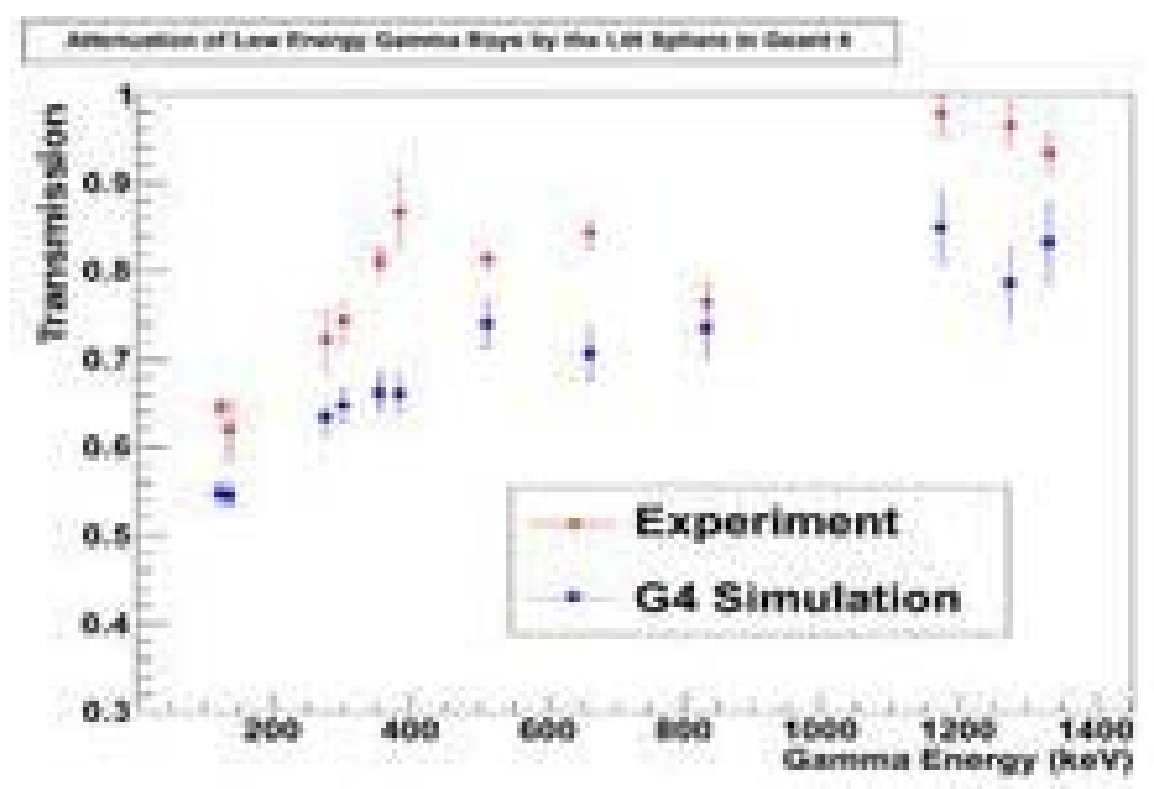

Figure 47: Shown is the transmission ratio from the measurement and the simulation. If the simulation reproduced the experiments, the red and blue points would lie on top of each other.

These initial results were quite surprising. The first thought was that there was a problem in the way that we had set up the GEANT4 simulation. In order to compare, we designed an experiment on the benchtop to model the absorption through Al blocks, as shown in Figure 48. As can be seen, in this case, the simulation and the measurement were in agreement. This presents a problem as the differences are not easily understood. These effects are still under study.

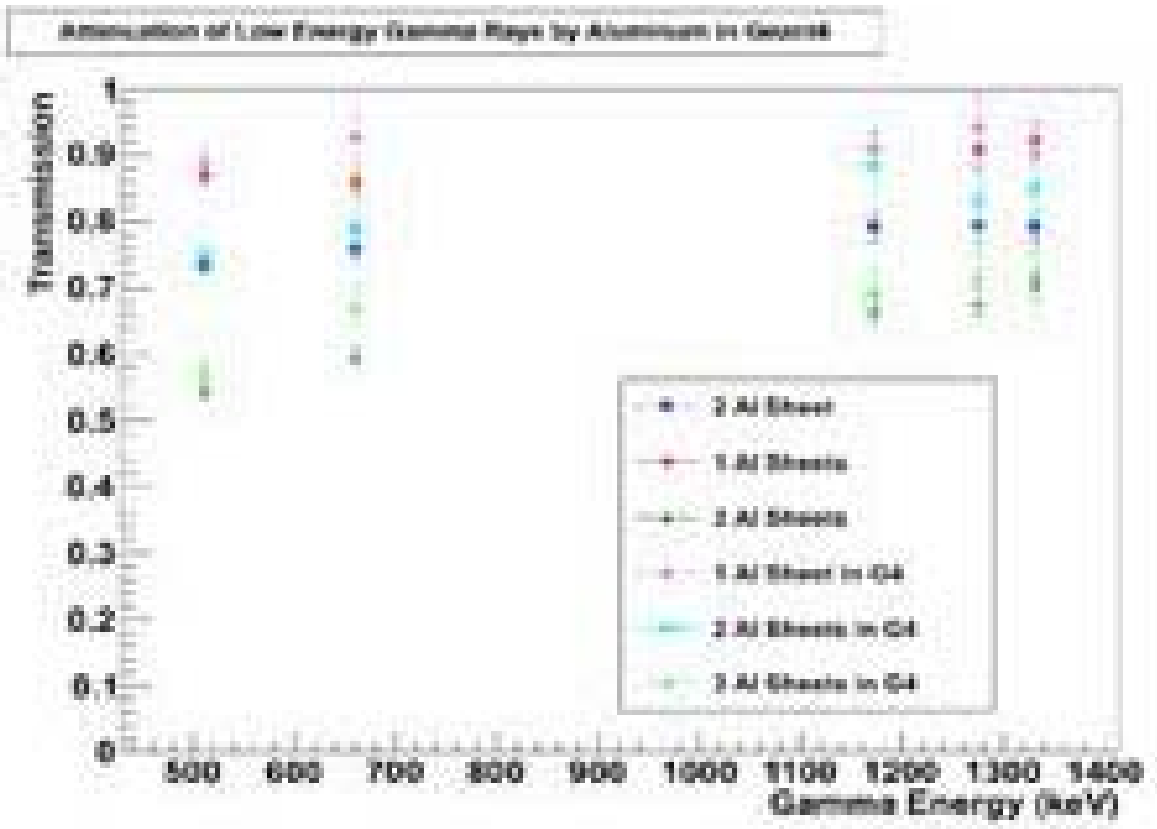

Figure 48: Shown above is the modeled and measured transmission through a series of Aluminum plates. They are generally in agreement. 


\section{Fission Neutron Output Measurements}

The source of neutrons in a reactor is from the neutron evaporation process of neutron-rich fission fragments. When actinides split, the fission products are neutron rich and can evaporate neutrons, most of which are emitted promptly, that is, within much less than one nanosecond. These neutrons are referred to as fission neutrons, and they are the vector responsible for creating fission chain reactions and criticality in nuclear reactors. The number and energy spectra for these neutrons as a function of incident neutron energy (responsible for fission) are the source terms in a reactor calculation. The need for better fission spectrum data has been uncovered in nuclear data sensitivity studies of fast reactor cores. Understanding the energy distribution of these neutrons is central to developing the required high fidelity reactor core calculations. Only sparse data are available for a handful of incident neutron energies and these data serve as the only tests of the theoretical "Los Alamos Model", which is used world-wide as the predictor of these quantities over a large number of isotopes and energies. Comprehensive measurements are essential to improving the available data and refining the Los Alamos Model. The goal of such a program is to improve, either by direct measurement or by better understanding of the physics, the population matrix of outgoing neutron energies versus incident neutron energies for a variety of isotopes. In the following sections we describe the approach, progress that has been made this year in detector and technique development, and simulations that indicate the accuracy that we expect to achieve.

\section{The Chi-Nu Array}

Our approach is to measure the spectrum of neutrons from neutron-induced fission by time-of-flight techniques at the Los Alamos Neutron Science Center (LANSCE). The experiment is a "double time-of-flight experiment" as described below.

Using the Weapons Neutron Research (WNR) pulsed spallation neutron source, we measure the energy of the incident neutron by its time of flight from the source to a fission chamber. The energies of fission neutrons are then determined by their time of flight to an array of neutron detectors. This measurement program has been in progress for nearly 10 years and has produced useful data on neutron-induced fission of ${ }^{235} \mathrm{U},{ }^{239} \mathrm{Pu}$ and several other actinides. To make significant improvements on the data required for applications, we are developing advanced techniques that will allow measurements of the full energy range of fission neutrons.

An example of data we have taken in previous years is shown in Figure 49. The data are quite good from 1 to $5 \mathrm{MeV}$. Our previous approach did not allow identification of neutrons below $1 \mathrm{MeV}$ and the statistical uncertainties above $5 \mathrm{MeV}$ are too large for improvement of the data base. So these are the areas of needed improvement in the experiments. Previous measurements show significant discrepancies in these regions and cannot differentiate between the predictions of various models (Figure 50). 


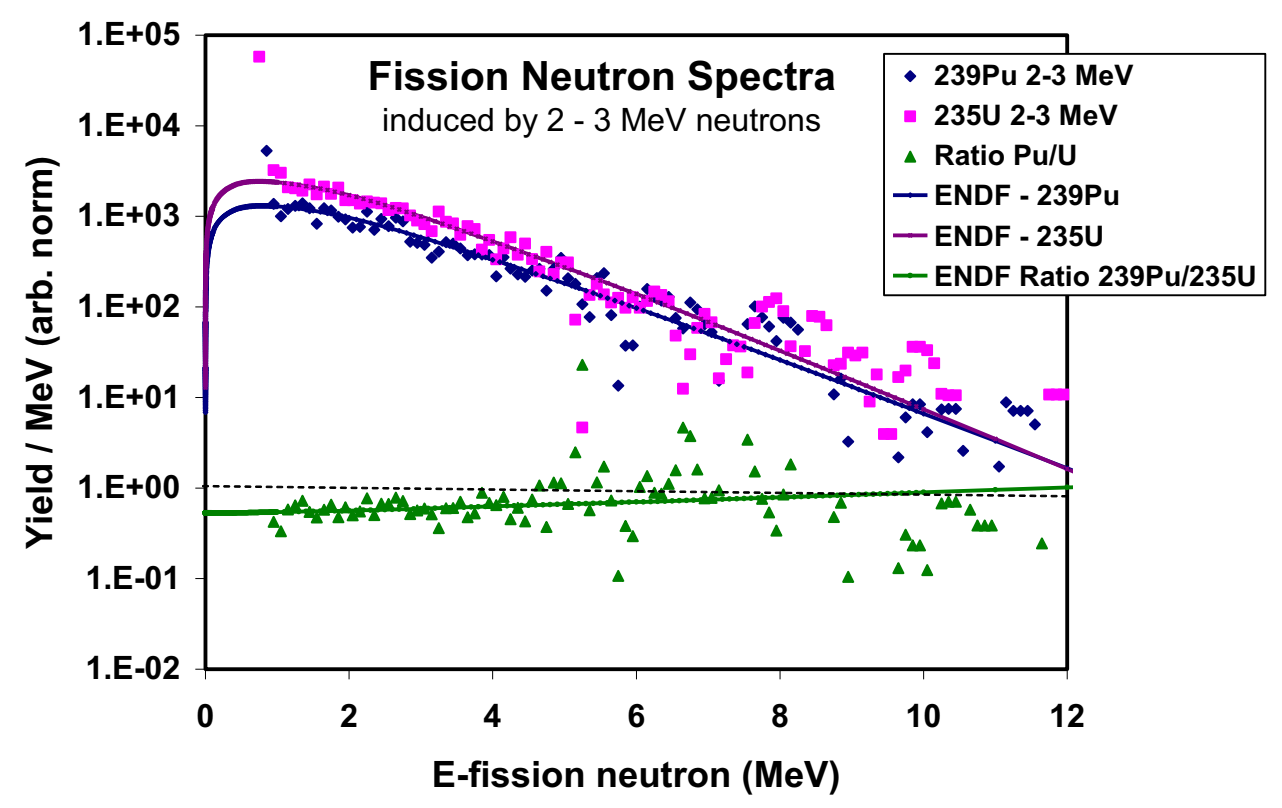

Figure 49: Fission neutron spectra for fission induced by neutrons from 2 to $3 \mathrm{MeV}$. Results for 239Pu are denoted by the black points and those for $235 \mathrm{U}$ by the purple points. The ratios are given by the green triangles. The data are compared with the ENDF/B-VII evaluations. Both the data and the evaluations have been arbitrarily normalized. The dashed horizontal line is a reference to show what a ratio of 1 would be in the fission neutron spectral shapes.

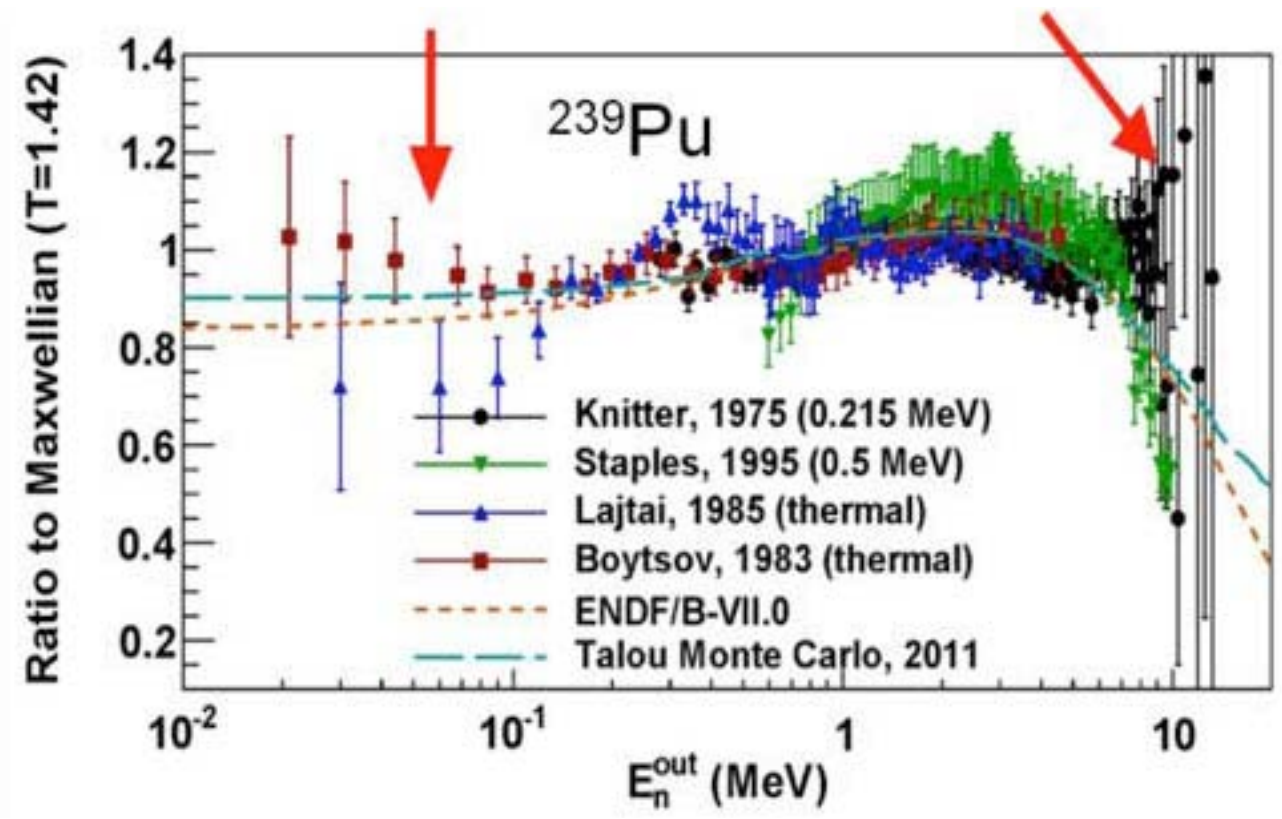

Figure 50: Literature data on prompt fission neutron spectra for neutron-induced fission of 239Pu. The areas of particular discrepancy are denoted by the red arrows. Ref: "Uncertainty

Quantification of Prompt Fission Neutron Spectrum for $n(0.5 \mathrm{MeV})+239 \mathrm{Pu}(n, f)$, , P. Talou, Nucl. Sci. Eng. 166, 254 (2010).

The challenges of this measurement are to detect and identify neutrons with energies below $1 \mathrm{MeV}$ and at high energies. Figure 51 displays the expected spectrum in three 
ways to exhibit (a) the general features of the spectrum, (b) the significant decrease at high neutron energies where, for example, the output at $10 \mathrm{MeV}$ is about $21 / 2$ orders of magnitude less than it is at the peak; and (c) the prediction that the spectrum extends down to energies of $1 \mathrm{keV}$.
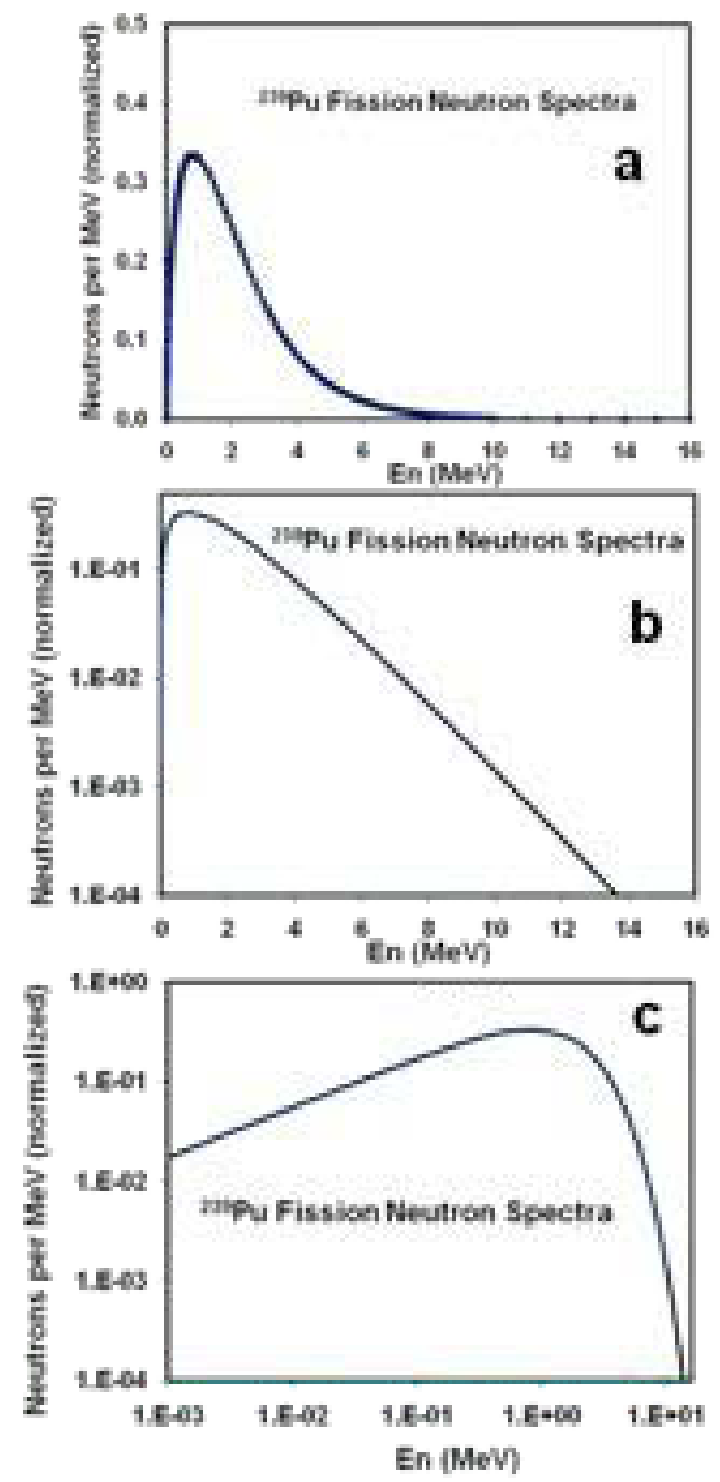

Figure 51: Fission neutron spectrum as evaluated in ENDF/B-VII.0 for 239Pu(n,f) with fission induced by $0.5 \mathrm{MeV}$ neutrons. We plot the data three ways to show (a) the spectrum on linear scales, (b) on a logarithmic $y$-axis to show that the spectrum decreases by 3 orders of magnitude from the peak at $0.75 \mathrm{MeV}$ to $12 \mathrm{MeV}$, and (c) on a log-log scale to show how the low energy part of the spectrum decreases by one order of magnitude from the peak to $1 \mathrm{keV}$ emitted neutron energy.

To make improvements in the experiment, the former FIGARO (Fast neutron-Induced Gamma Ray and Neutron Observer) array has been superseded by the Chi-Nu detector array. The name, "Chi-Nu," refers to the Chi-matrix which relates incident neutron energies to the distribution of fission neutron energies. Parts of this matrix 
are given in Figure 52 from the ENDF/B-VII.0 evaluation for ${ }^{239} \mathrm{Pu}$. For ease of display, we have divided the spectra by the values for fission induced by thermal neutrons. This figure shows that the fission neutron spectrum is expected to change with the energy of the neutron inducing the fission. Verifying and quantifying this sort of change is a principal goal of our experiments.

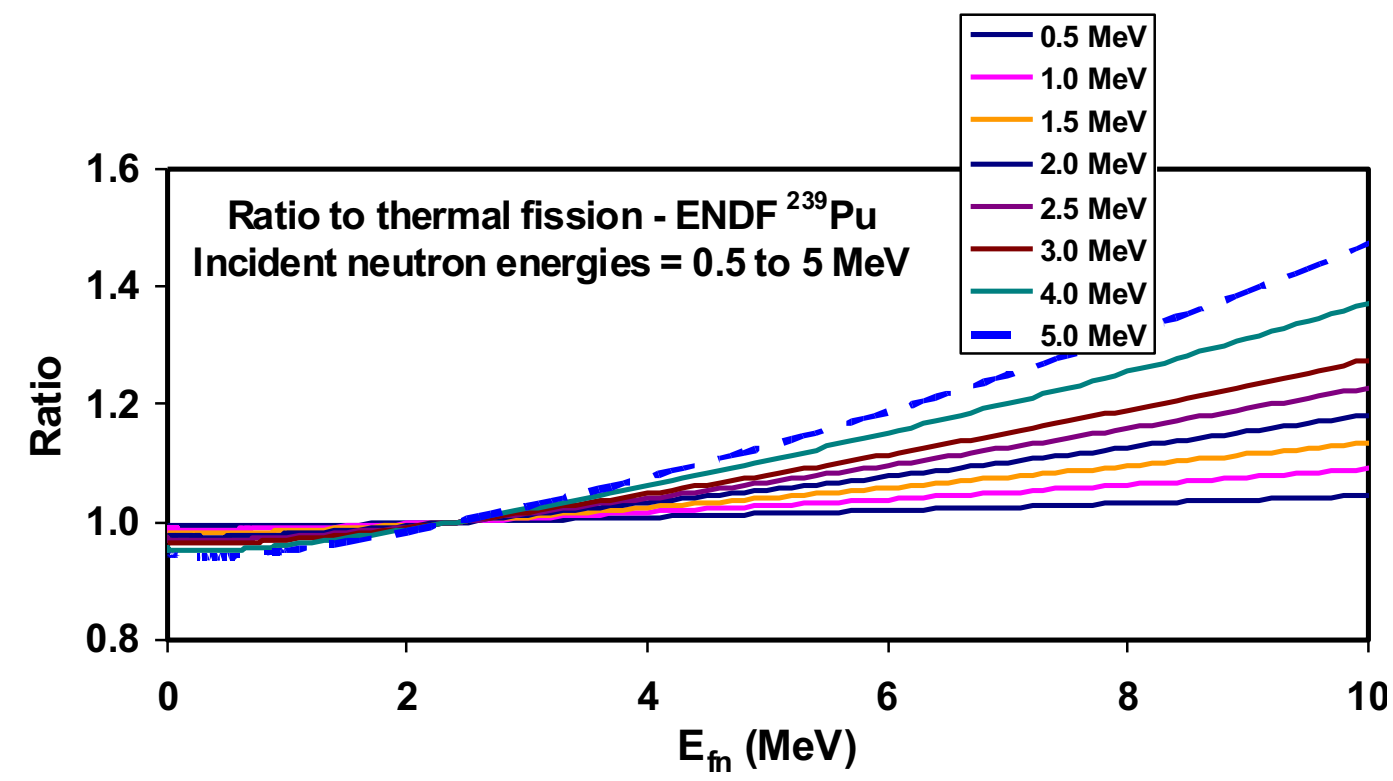

Figure 52: Fission neutron spectra for various incident neutron energies divided by the spectrum at thermal. The data are from the ENDF/B-VII.0 evaluation.

\section{A New Flight Path for Chi-Nu}

A new building (Figure 53) was completed in July 2012 to house a new flight path for fission neutron spectra measurements and also, in the future, other neutron emission measurements such as from elastic and inelastic neutron scattering. One feature of the building is a basement under the fission chamber to serve as a "get-lost" area for neutrons. The basement is 7 feet deep and 18 feet $x 18$ feet in area. It is covered with a $1 / 2$-inch aluminum plate, which we plan to replace in the future with an even lower mass plate or grid. The LANSCE accelerator began its new run cycle on August 6,2012 , and we have used the beam time to characterize the flight path. 


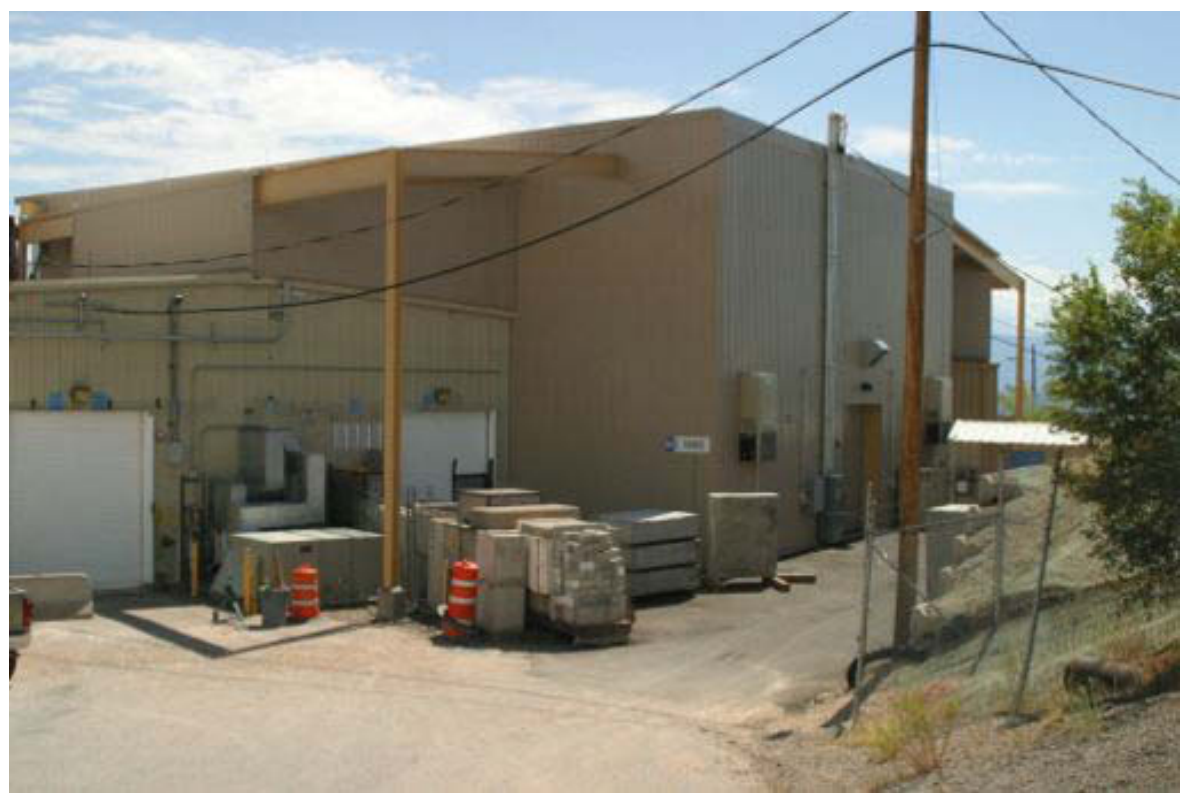

Figure 53: New experimental building (53-1302) where neutrons emitted in neutron-induced fission will be studied. Spallation neutrons from 0.1 to about $200 \mathrm{MeV}$ are produced by the pulsed $800 \mathrm{MeV}$ proton beam from the LANSCE accelerator and are collimated in flight paths for time-of-flight measurements.

Image plates were used to establish the beam centroid at several points on the flight path, to measure the uniformity of the beam, and to quantify any beam halos. See Figure 54 for layout of the beam line and detectors. After optimizing the shutter openings, the beam distribution and uniformity at the position of the actinide samples are indicated in Figure 55. Comparisons of the beam profile are shown for various shutter openings in Figure 56. Similar data were obtained at the position of the flux monitor (Figure 57).

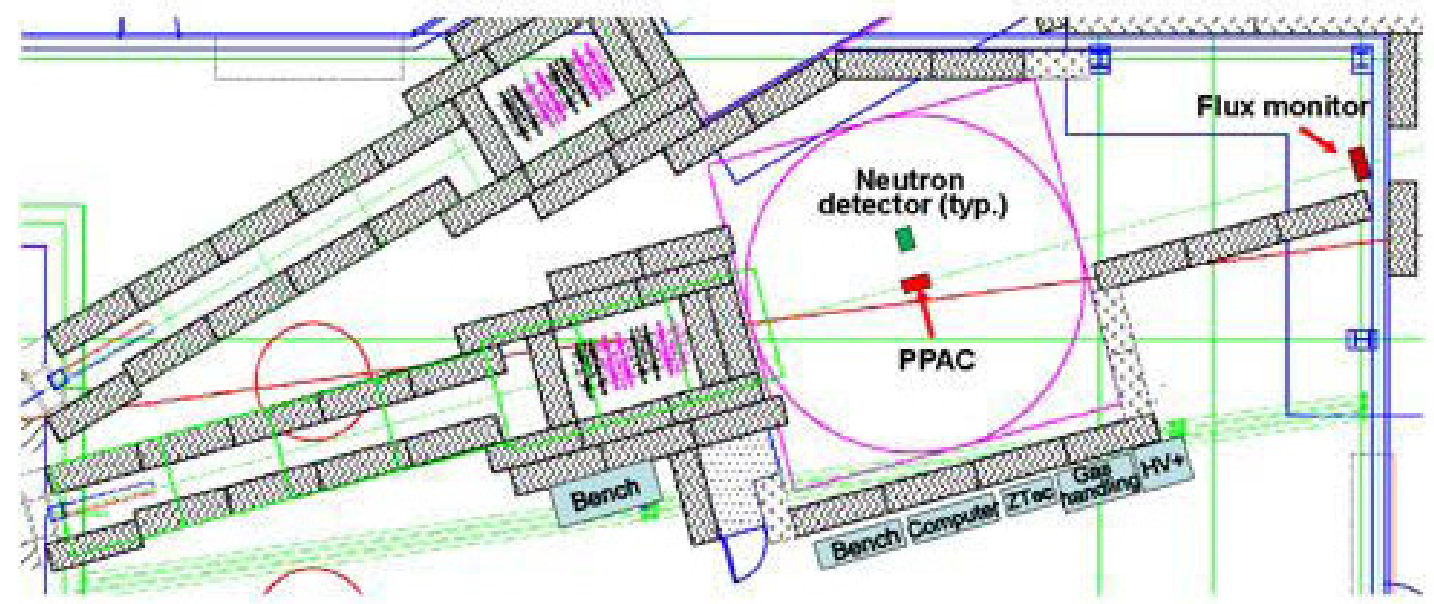

Figure 54: Layout of the Chi-Nu experiment on flight path 4FP15L at WNR/LANSCE. 


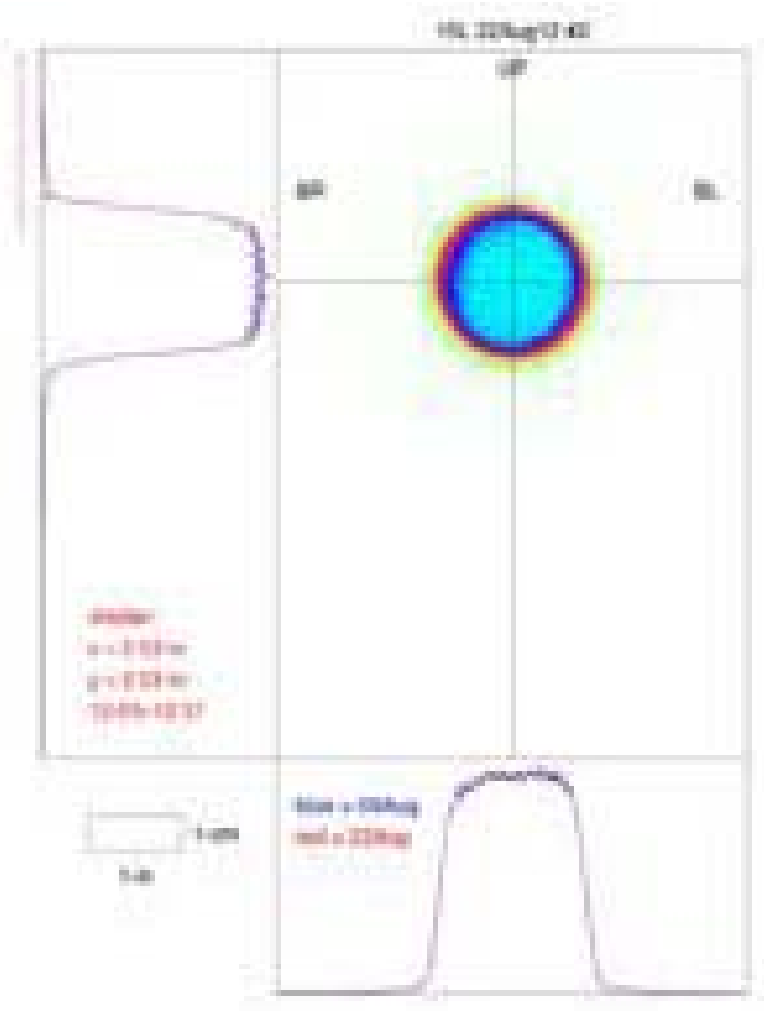

Figure 55: Image plate image of the neutron beam at the position of the fission counter (parallelplate avalanche detector). The blue and red curves were taken with different shutter openings: 4" $x$ 4" on 8/8/12 and 2.53" $(H)$ x 2.03" (V) on 8/23/12. This comparison shows that the smaller shutter opening gives the same beam profile, and it also gives smaller neutron backgrounds.

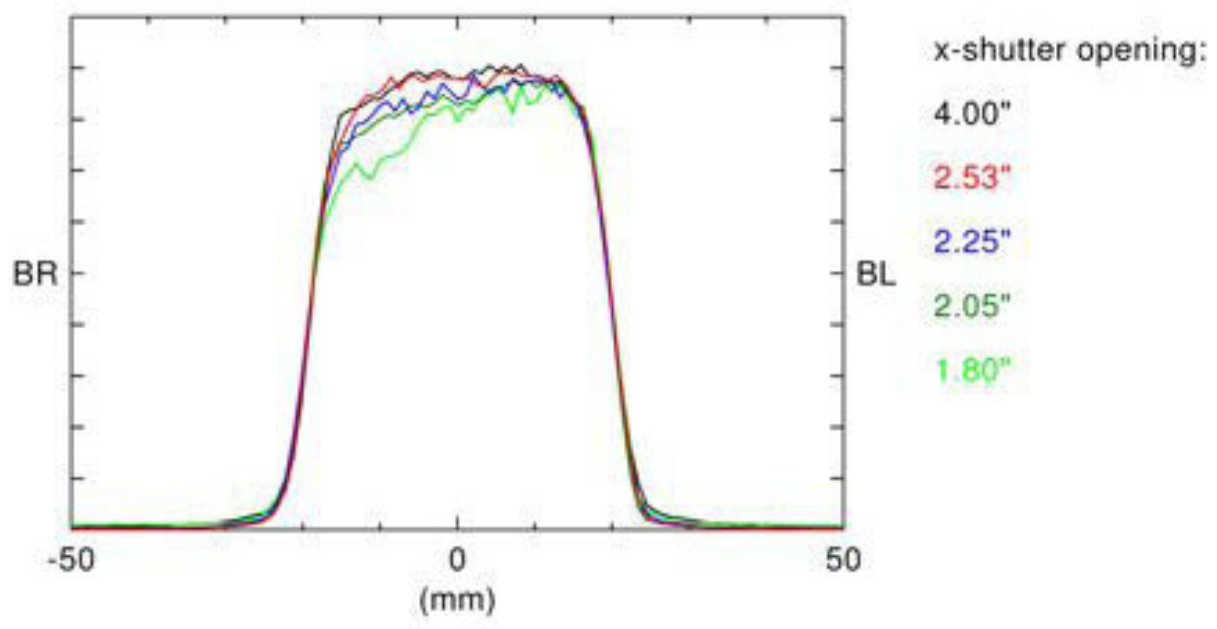

Figure 56: Horizontal lineouts in the center of the image of the beam, when the image plate is place at the fission counter position, for different horizontal shutter openings. This comparison shows that, for the best uniformity of the neutron beam, a shutter opening of 2.53" is preferred. 


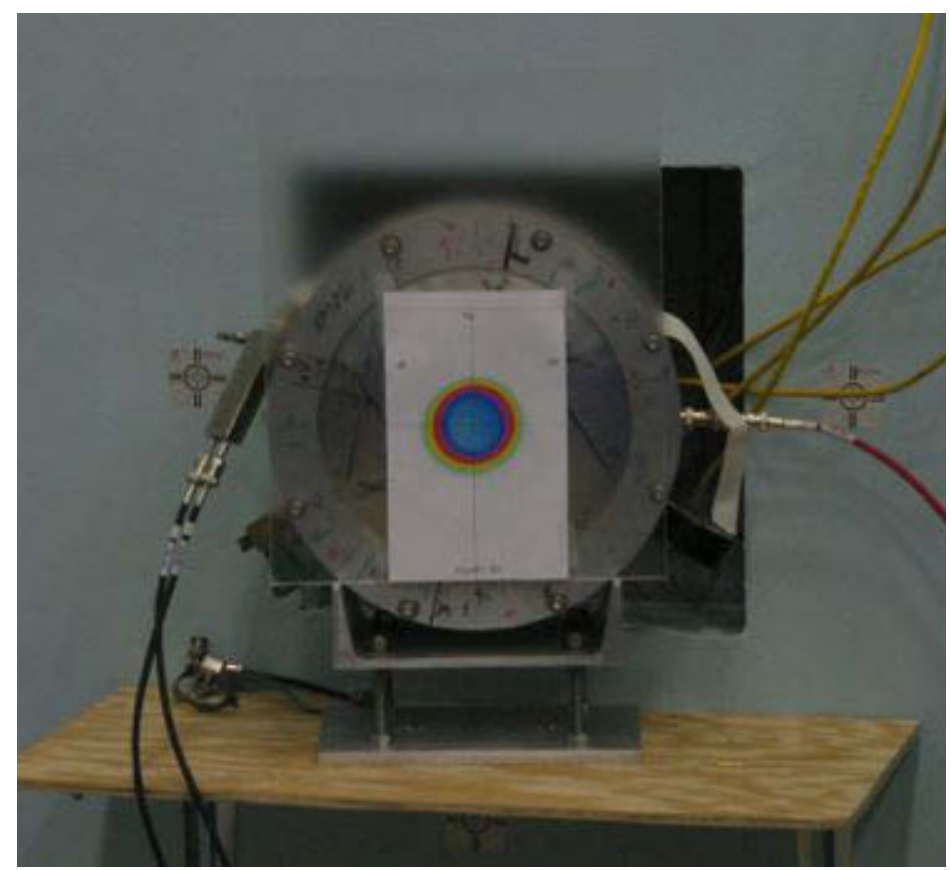

Figure 57: Image plate image of the beam registered to the flux monitor. The image is mounted on a plastic rectangular plate. The beam is well within the 5" diameter of the fission deposits inside the chamber.

Software development of the Universal Acquisition Code has enabled time-of-flight measurements with the ZTec 4441 digitizers. The following measurements were made with these digitizers and analysis of the digital waveform data.

The flight path lengths from the WNR neutron production source to the center of the fission chamber and to the flux monitor were measured with carbon resonances (see example in Figure 58). The flight path length to the center of the fission chamber is $21.49+/-0.01$ meters, which is close to that from surveying measurements of 21.47 meters. The flux monitor distance was also measured in this manner. 


\section{Incident Neutron Time of Flight}

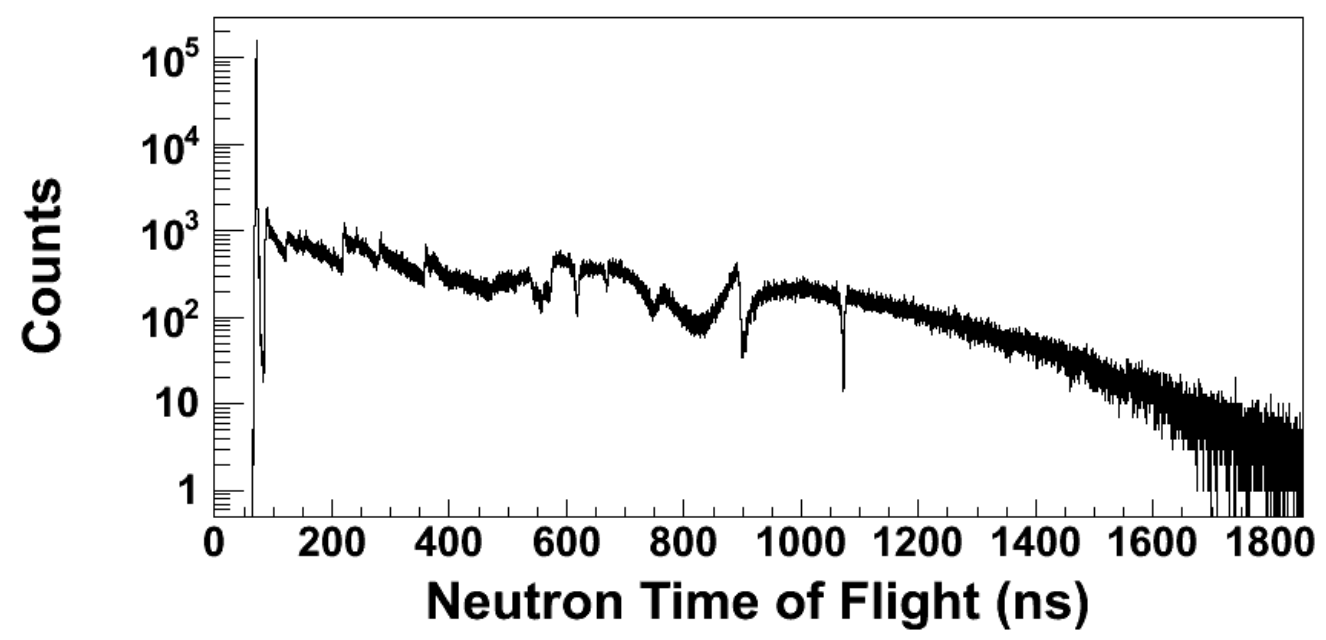

Figure 58: Time-of-flight spectrum from a thin, 1/16", scintillator placed at the position where the center of the fission chamber will be located. Resonances at 2.077 and $6.29 \mathrm{MeV}$ were used to deduce the flight path length of $21.49+/-0.01$ meters. More detailed analysis will be used to reduce the uncertainty.

Background measurements were made and some backgrounds were detected from neighboring flight paths and also from upstream areas in the Chi-Nu flight path.

These findings led to increasing the shielding on our flight path and to adding shield blocks between our flight path and the closest neighboring flight path, which was the source of most of the external background.

\section{A Station for Characterizing Neutron Detectors}

Spontaneous fission of ${ }^{252} \mathrm{Cf}$ emits neutrons with what is considered a standard spectrum. This year we established a test station (Figure 59) with a ${ }^{252} \mathrm{Cf}$ fission chamber produced at Oak Ridge National Laboratory. The source is 1.75 meters above the concrete floor to reduce room-scattered neutrons and, in the picture, a liquid scintillator neutron detector is installed 1 meter from the fission chamber. The fission chamber has good time resolution (Figure 60) so that the emitted neutrons can be used with TOF techniques to investigate the response of detectors to neutrons over a wide range of energies. A TOF spectrum is shown in Figure 61. 

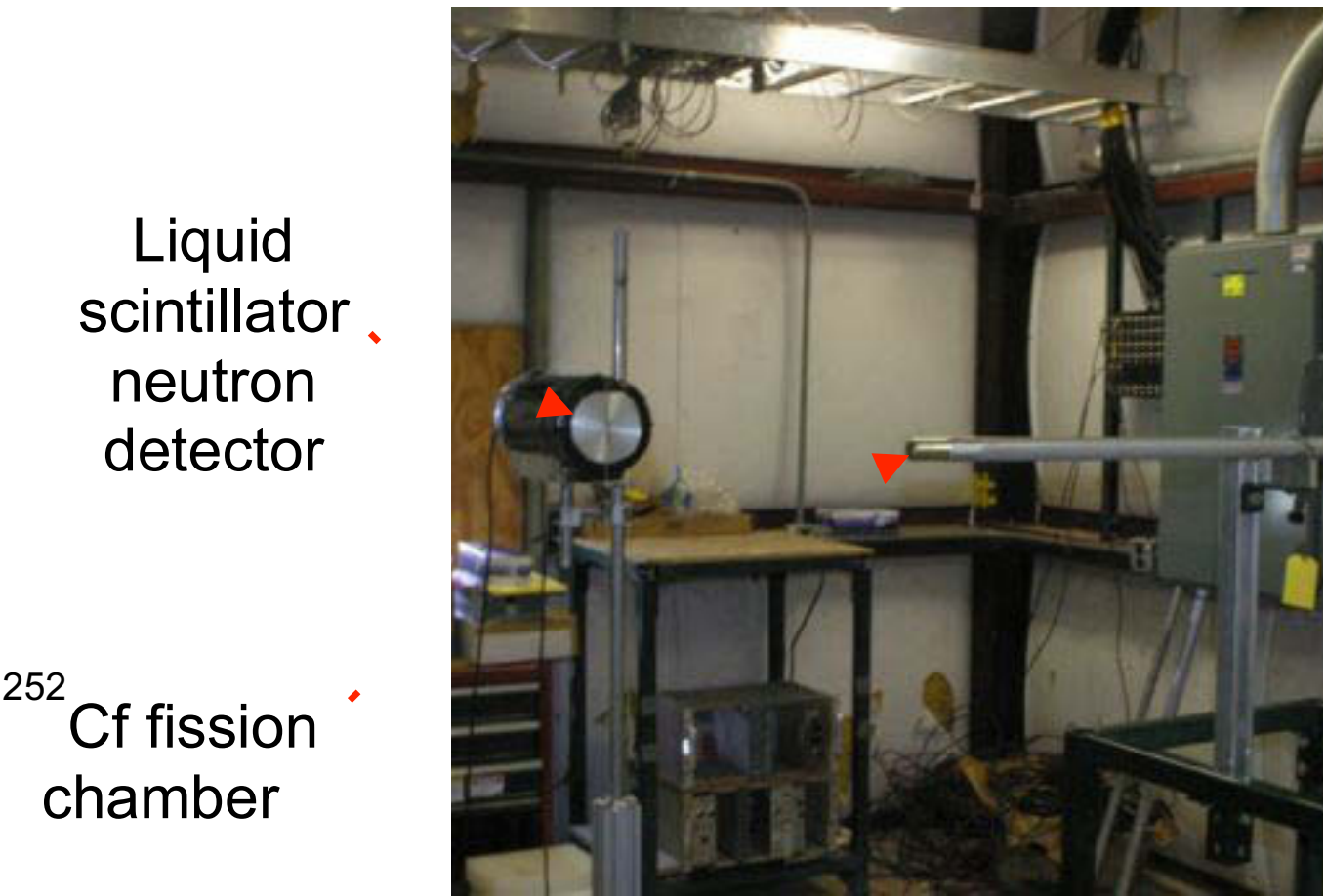

Figure 59: ${ }^{252} \mathrm{Cf}$ fission chamber and liquid scintillator neutron detector. With the two, we measure the time of flight of prompt fission neutrons over a 1meter flight path to deduce their energy spectrum. This system is being used to develop the data acquisition hardware and software for the full Chi-Nu project.

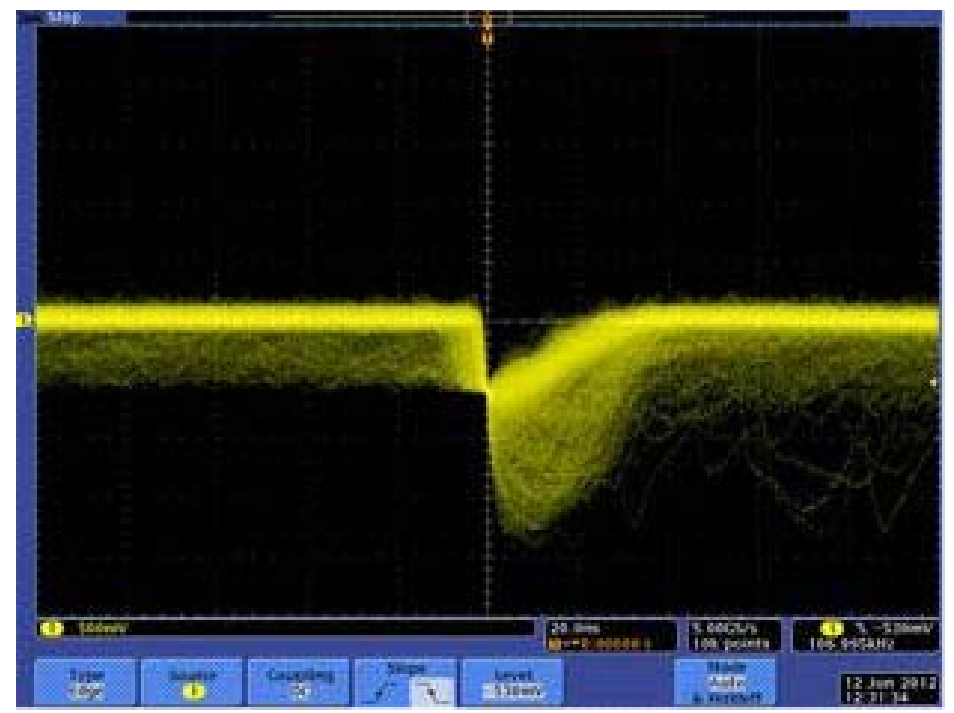

Figure 60: Pulses from the ${ }^{252} \mathrm{Cf}$ fission chamber showing the fast rise time necessary for good time-of-flight resolution. The many pulses at lower amplitude are from alpha particles that constitute about $97 \%$ of the spontaneous decay of this isotope. 


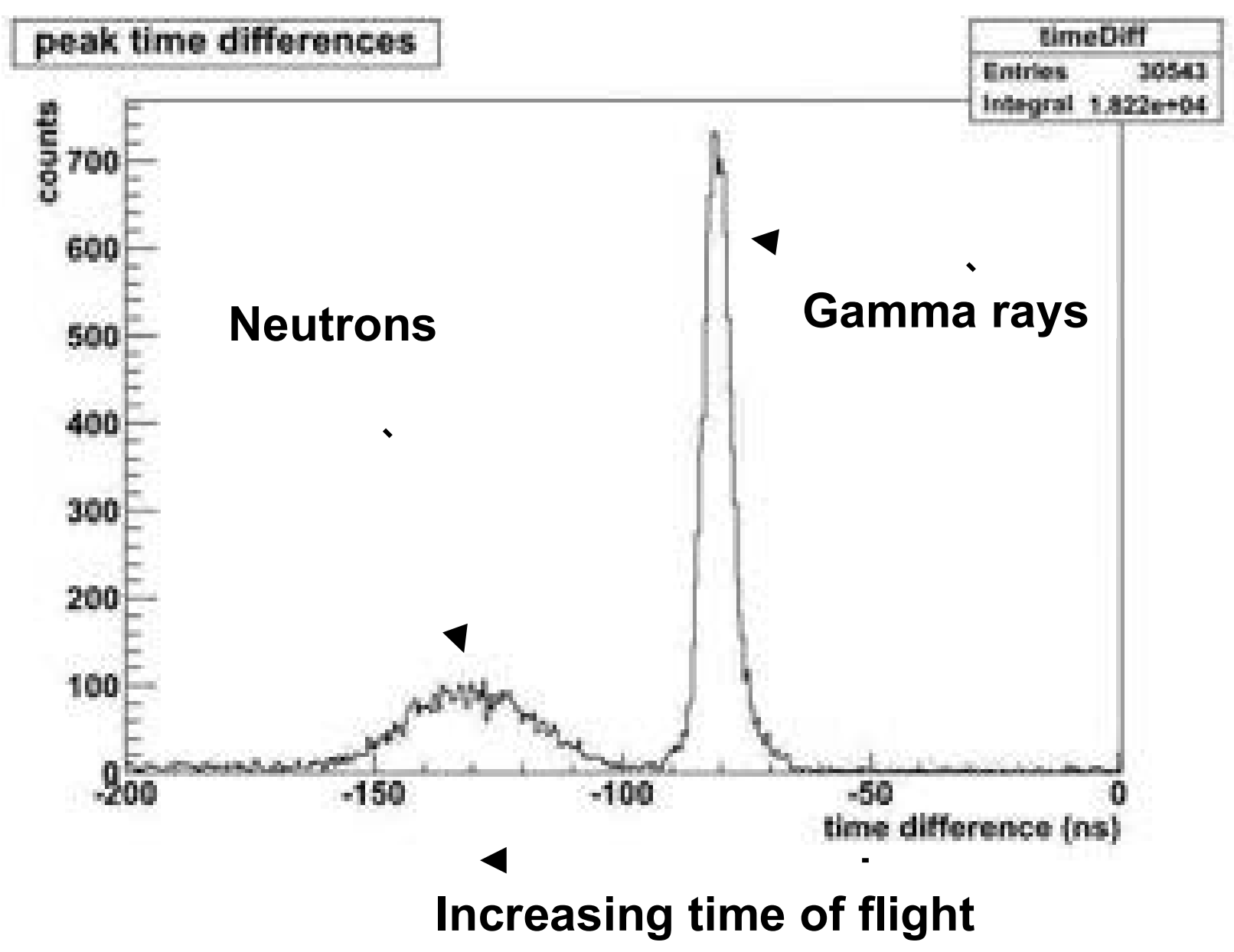

Figure 61: Time-of-flight spectrum for ${ }^{252} \mathrm{Cf}$ prompt fission neutrons and gamma rays taken with the Oak Ridge fission chamber and a liquid scintillator 1 meter from the source. Time in this figure goes from right to left and there is an offset of approximately -80 ns from the actual time of flight. These data were taken with the ZTec digitizers and the Universal Acquisition Code (UAC).

\section{Liquid scintillator development}

Organic liquid scintillators are the detectors of choice for measuring prompt fission neutron spectra from about $600 \mathrm{keV}$ up to the $12 \mathrm{MeV}$ or higher. The efficiency of the detector needs to be known, of course. To obtain accurate values for the efficiency, several approaches are used and include reference to the standard ${ }^{252} \mathrm{Cf}$ prompt fission neutrons, neutron tagging (we discussed in the previous annual report), monoenergetic neutron sources, and modeling the efficiency with a Monte-Carlo neutron transport code. To fully understand the detector and its environment, which can contain many scattering materials, the modeling approach is essential.

Neutron transport codes can give detailed information on the energy imparted in $n-p$ scattering and in reactions on carbon so that the energy distribution of the resultant protons, alpha particles and carbon recoils (and, at higher energies deuterons, tritons and other products) is quite well known. What is not so well known is how much light the energetic charged particles create in the scintillator. This is the so-called "light curve," which can be characterized through measurements and then modeling with MCNPX. This approach is independent of other methods to determine this important 
performance characteristic, which is central to understanding the efficiency of the neutron detector. An example of the fit to a measured ${ }^{252} \mathrm{Cf}$ spectrum is shown in Figure 62 together with two different parameterizations of the light curve.

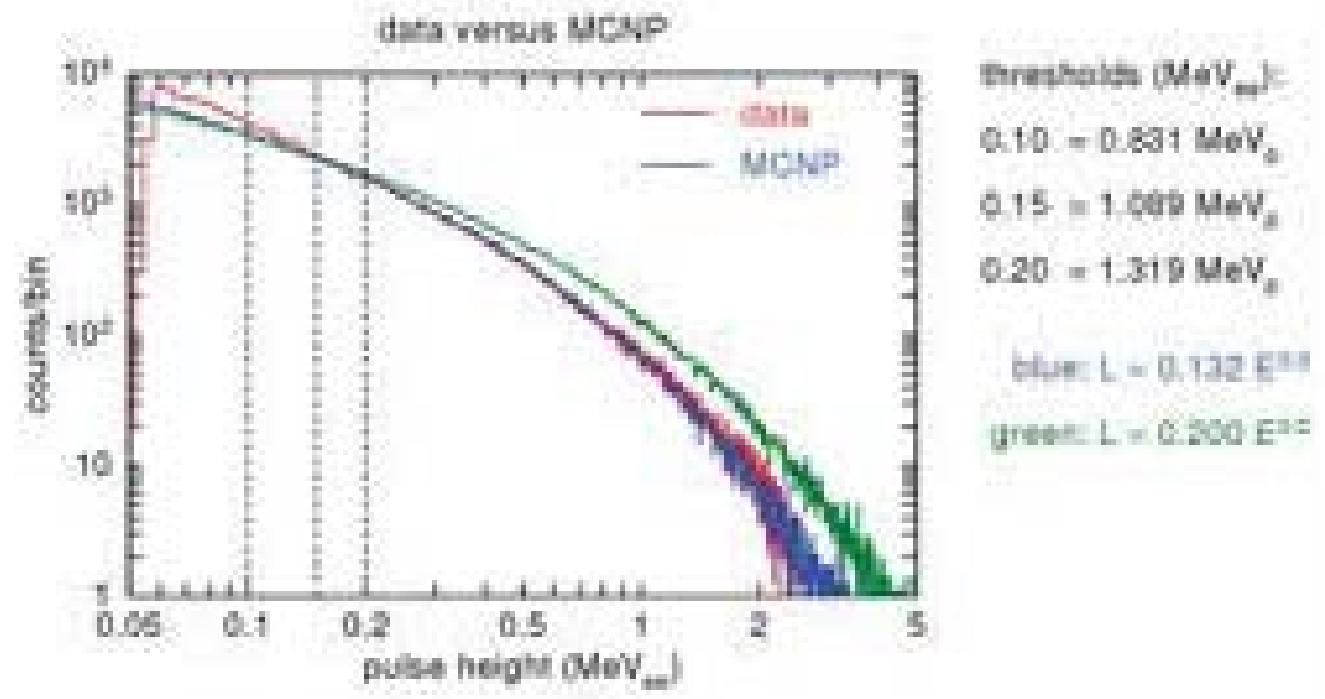

Figure 62: Pulse-height spectrum from a liquid scintillation detector looking at a ${ }^{252} \mathrm{Cf}$ source. Monte-Carlo calculations with two parameterizations of the light curve are shown.

\section{Installation of the ${ }^{235} \mathrm{U}$ Parallel Plate Avalanche Counter (PPAC)}

The LLNL fission chamber, a parallel-plate avalanche counter, with 10 foils containing ${ }^{235} \mathrm{U}$ was installed and made operational (pictured in Figure 63). This was the same chamber used in the experiments of late CY 2010. It was shown to work well. The performance can be illustrated by the pulse-height distribution (Figure 64) and a portion of the time-of-flight spectrum (Figure 65), both taken with the ZTec digitizers. 


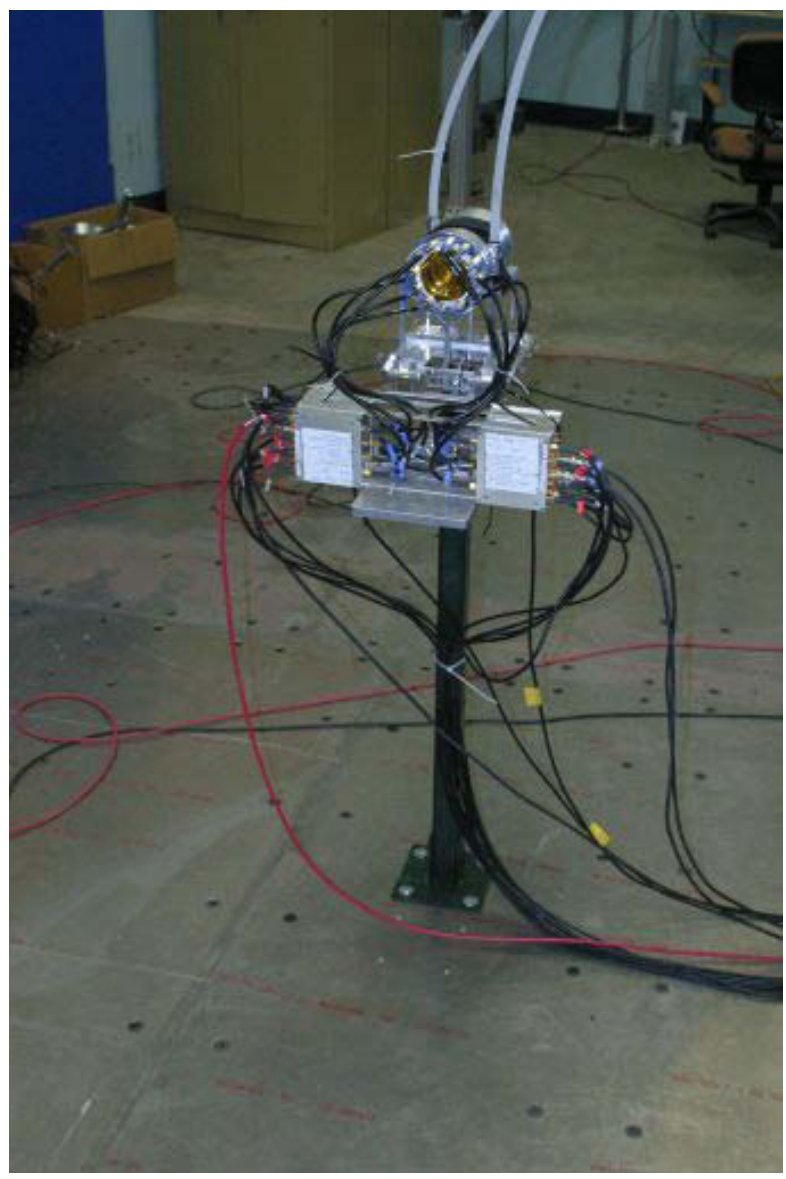

Figure 63: Parallel-plate avalanche counter (PPAC) made by LLNL in position in the Chi-Nu flight path. Below the PPAC are the preamplifiers. We are obtaining longer cables so that the preamplifiers can be moved farther below the PPAC and thereby reduce neutron scattering from them.

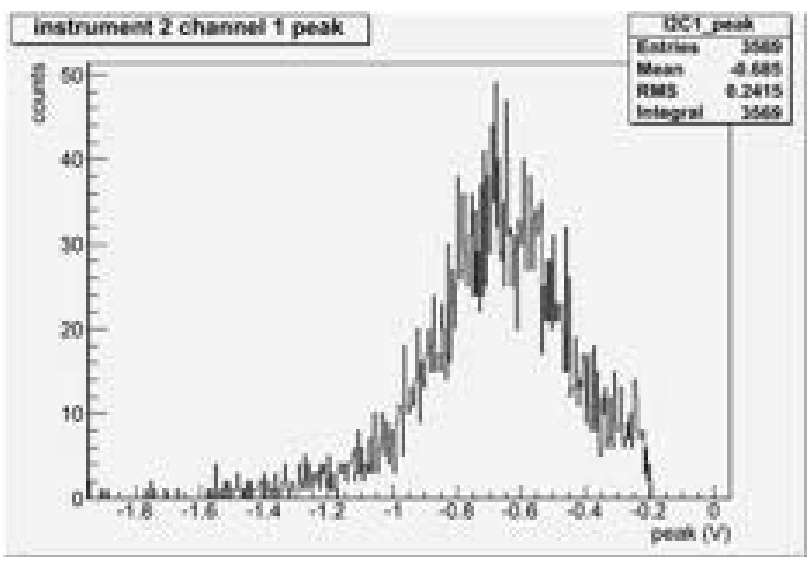

Figure 64: Pulse-height distribution from the PPAC showing the fission pulses, which are well resolved from alpha-particle signals, smaller than the threshold at -0.2 volts. The pulses are negative polarity, hence the scale on the abscissa that goes from -1.9 to zero volts. 


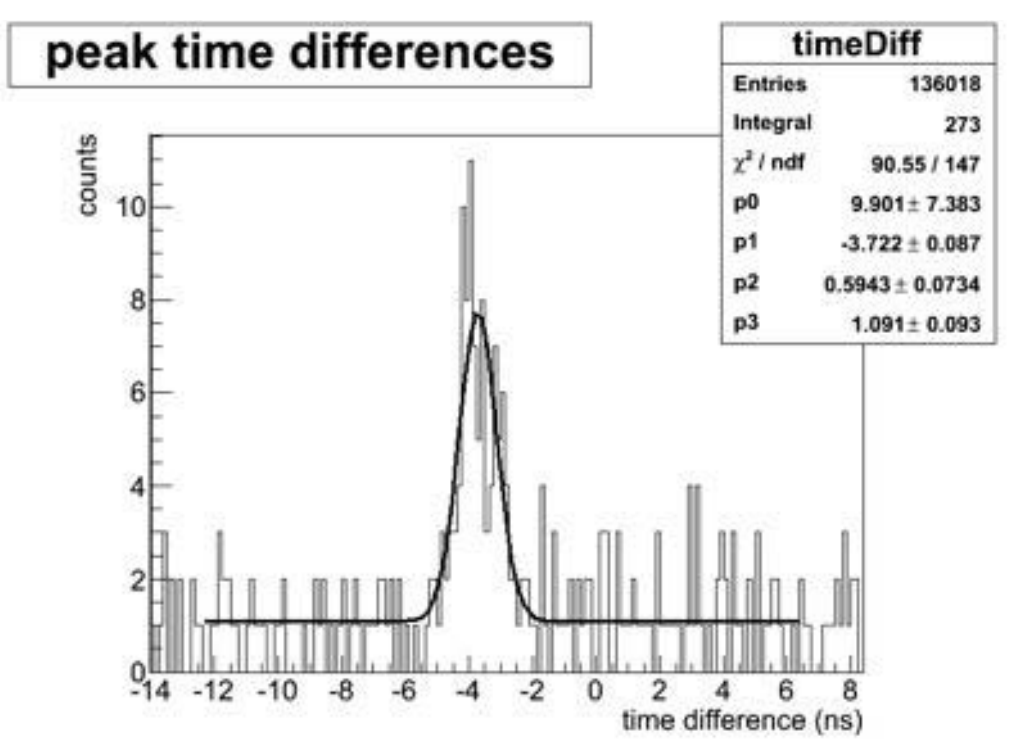

Figure 65: Photofission peak from the PPAC showing good resolution. We are working on making the resolution even better. The Gaussian fitting parameter $P 2$ is the sigma of the distribution, which gives a FWHM of 1.4 ns. We are working on making the resolution even better to a FWHM of $1.0 \mathrm{~ns}$ or better. 


\section{Time Projection Chamber}

The Time Projection Chamber is a collaborative effort to implement an innovative approach and deliver unprecedented fission measurements to DOE programs. This $4 \pi-$ detector system will provide unrivaled 3-D data about the fission process. Included here is the 2012 Report on Fission Time Projection Chamber that describes the project advances that crosscut the Advanced Detector Development and other research areas.

\section{Introduction}

Reactors, weapons and nucelosynthesis calculations are all dependent on nuclear physics for cross sections and particle kinematics. These applications are very sensitive to the nuclear physics in the fast neutron energy region and therefore have large overlaps in nuclear data needs. High performance computer codes interface the nuclear data through nuclear data libraries, which are a culmination of experimental results and nuclear theory and modeling. Uncertainties in the data contained in those libraries propagate into uncertainties in calculated performance parameters. The impact of nuclear data uncertainties has been studied in detail for reactor and weapon systems and sensitivity codes have subsequently been developed that provide nuclear data accuracy requirements based on adopted target accuracies on crucial design parameters. The sensitivity calculations have been performed for a number of candidate systems. These sensitivity studies provide specific requirements for uncertainties on many fission cross sections, many of which are beyond the reach of current experimental tools. The sensitivity codes are proving to be very useful for identifying the highest impact measurements for DOE programs and the TPC measurement program will help provide those data. The result of these new, high-accuracy precision measurements will be a refined understanding of performance results, thus reducing the liability nuclear data has on the overall uncertainties in calculated integral quantities. The new class of high-accuracy, highprecision fission measurements will not be easy. The proposed method is to employ a Time Projection Chamber and perform fission measurements relative to $\mathrm{H}(n, n) \mathrm{H}$ elastic scattering. The TPC technology has been in use in high-energy physics for over two decades - it is well developed and well understood. However, it will have to be optimized for this task that includes miniaturization, design for hydrogen gas, and large dynamic range electronics. The TPC is the perfect tool for minimizing most of the systematic errors associated with fission measurements. The idea is to engineer a TPC specifically for delivering fission cross section measurements with uncertainties below $1.0 \%$.

The long term goal is to fill theTPC with hydrogen gas and measure fission cross sections relative to $\mathrm{H}(\mathrm{n}, \mathrm{n}) \mathrm{H}$ elastic scattering, thus removing the uncertainties associated with using the U-235 fission cross section for normalization. In fact, we will provide the world's best differential measurement of the U-235 fission cross section and this will impact nearly all fission library data, since it has been used as a standard in much of the available fission experimental data. 
The immediate objective of this effort is to implement a fission cross section measurement program with the goal of providing the most needed measurements with unprecedented precision and accuracy using a time projection chamber. The first three years of this program will provide all the groundwork and infrastructure for a successful measurement campaign. Shortly following, we will provide precision fission ratio measurements for Pu-239/U-235 and U-238/U-235 along with a full design proposal to measure ${ }^{235} \mathrm{U} / \mathrm{n}(\mathrm{n}, \mathrm{p}) \mathrm{p}$. The ${ }^{235} \mathrm{U} / \mathrm{H}(\mathrm{n}, \mathrm{n}) \mathrm{H}$ measurement will provide the best single measurement of the U-235 fission cross section and will allow us to convert the initial, and any subsequent, ratio experiments to worlds best absolute measurements. After completion of the U-238 and $\mathrm{Pu}-239$ ratio measurements, the experimenters will move on to measurement of the minor actinide cross section, fission fragment distribution and neutron yield measurements. This information will play a crucial role in the long term DOE nuclear R\&D campaign.

The reporting for this project is broken down into four categories:

- TPC Hardware activities include design, testing and operation of the complete time projection chamber, including gas system and electronics.

- TPC Software activities will provide the project with the required programming for the online data acquisition system, data reduction and analysis as well as simulation.

- The Hydrogen Standard will be used to minimize total cross section errors. The ability to accurately and precisely determine fission cross sections hinges on the $\mathrm{H}(n, n) \mathrm{H}$ total cross section and angular distributions.

- Facilities and Operations will need to be identified and prepared for the construction, testing and operation of the TPC. This activity is spread amongst the collaborators, based on the work they are performing, such as target fabrication, computing, design, component testing, and operation.

- Management section describes the organizational work required for a project this size.

\section{TPC Hardware [LLNL, CSM, INL, ISU, ACU]}

\section{Scope}

The components that make up the TPC proper are included in this section. This includes the pressure vessel, field cage, pad-plane, gas amplifier, laser alignment system, targets, electronics and the engineering required to integrate all of the parts into a working system.

\section{Highlights}

- This quarter marks a major accomplishment in the aggressive schedule for the TPC project. For the first time, a full pad plane has been instrumented, tested and operated at the LLNL TPC laboratory.

- The TPC has been shipped to LANSCE for beam experiments at the dedicated $90 \mathrm{~L}$ flight path during the upcoming run cycle.

- The experiment can now be completely controlled from a remote internet location using the specially adapted MIDAS package. 
- All necessary targets have been prepared and shipped to LANL for the upcoming experiments.

\section{Time Projection Chamber [LLNL, CSM, INL]}

The TPC is the centerpiece of the experiment and consists of a number of parts and systems that are being designed and integrated into a working whole. All subsystems that support the operation of the chamber are either complete or nearing completion, where much of what is left are refinements and integral testing. Partially instrumented versions of the TPC have been used to successfully collect radioactive source data at LLNL and beam induced reactions at LANSCE that demonstrate the level of readiness for production running. This evolutionary approach has provided the design team with valuable performance information as feedback for final experimental design requirements. This section will describe the progress on each of those efforts.

\section{Current Implementation}

This quarter marks a major accomplishment in the aggressive schedule for the TPC project. For the first time, a full pad plane has been instrumented, tested and operated at the LLNL TPC laboratory (shown in Figure 66). This implementation allows for complete reconstruction of all charged particles in one half of the sensitive gas volume. This configuration is a complete test of the complete electronic infrastructure required for production running. This accomplishment required 92 EtherDAQ/preamplifier card pairs operating together ( 3000 channels) through several supporting architectures, evenly distributing power and precision clock signals in a coordinated way and debugging many of the issues expected with a complex, high-density, custom electrics system of this size. The other half of the TPC is simply a mirror image of the currently instrumented side where other than refinements, the further concerns that will need to be addressed in the months to come are total power consumption, thermal and data flow management. 


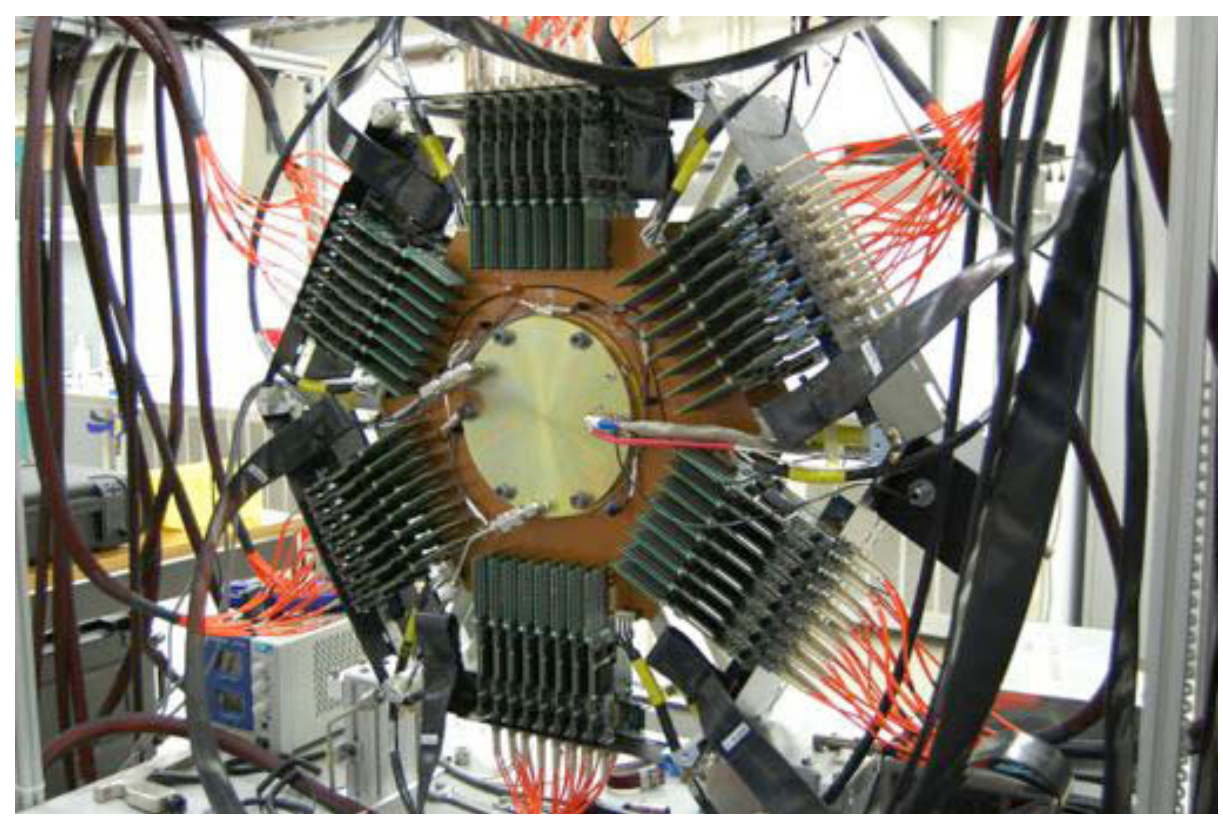

Figure 66: Here is the TPC at LLNL loaded with all 92 cards needed to operate one full pad plane, or one half of the detector. There are approximately 3000 channels instrumented in this photo.

The fully tested TPC has been packed and shipped to LANL for installation and subsequent operation during the next LANSCE run cycle, which begins in August.

\section{Cathode Pad plane}

Version 3 of the pad plane was designed and built last quarter. This version has improvements to the glue down region for the micromesh and more importantly is setup for robotic placement of the 96 connectors that go on each board. They were inspected for correct pillar height and some other preliminary inspections, and then sent to the loading shop for connector placement (see Figure 67). 


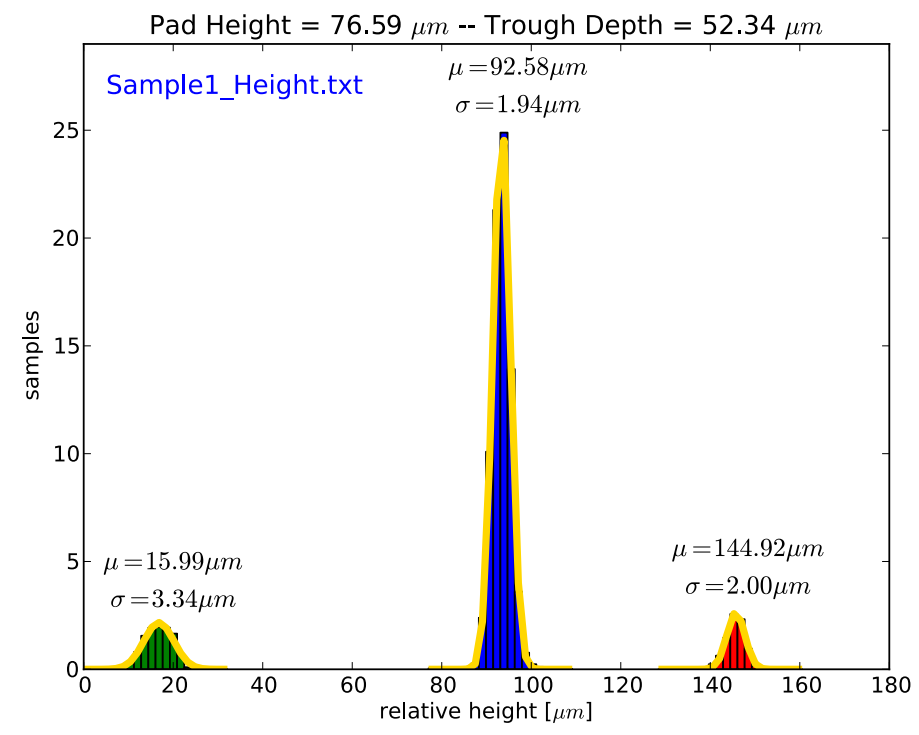

Figure 67: Shown here is the pad plane profilometry measurement summary revealing the consistency of construction.

The 12 new pad planes constructed the last quarter have all of the modification identified during the testing last year. In order to meet the goal of a fully instrumented pad plane we needed to load all 92 connectors attached to the pad plane, which is a large job given that each connector has 80 pins and they have a pitch of $0.4 \mathrm{~mm}$. The boards were sent to a component load shop for this work and they had problems that prevented us from getting a full set of working boards, but we did have one that worked well. There were some shorts on the connectors and we replaced them by hand. The result was a single fully functioning pad plane. We will revisit the auto loading of the connectors with a different company once we field the TPC at LANL. All of the pad plans that we used were carefully inspected both electrically and optically to look for defects.

\section{Field Cage}

Three Field cages were constructed and shipped to LANL for the upcoming runs at LANSCE. Each field cage holds one target and they can be swapped out as a single unit, minimizing the handling of fragile targets. We are planning on using a variety of targets at LANL during this run cycle and the field cage units will allow us minimize the time to swap them out and reduce the chances of breakage.

\section{Electronics Cooling}

The EtherDAQ/preamplifier card pairs consume enough power that cooling is essential for their proper operation. A convection system based on 36 standard electronic fan packs electronics has been designed to cool the 192 card pairs (see ). These fans were mounted on the TPC shield, which serves both as an EMF shield and air flow deflector. The power supply for the fan system was integrated into the run control for the experiment. 


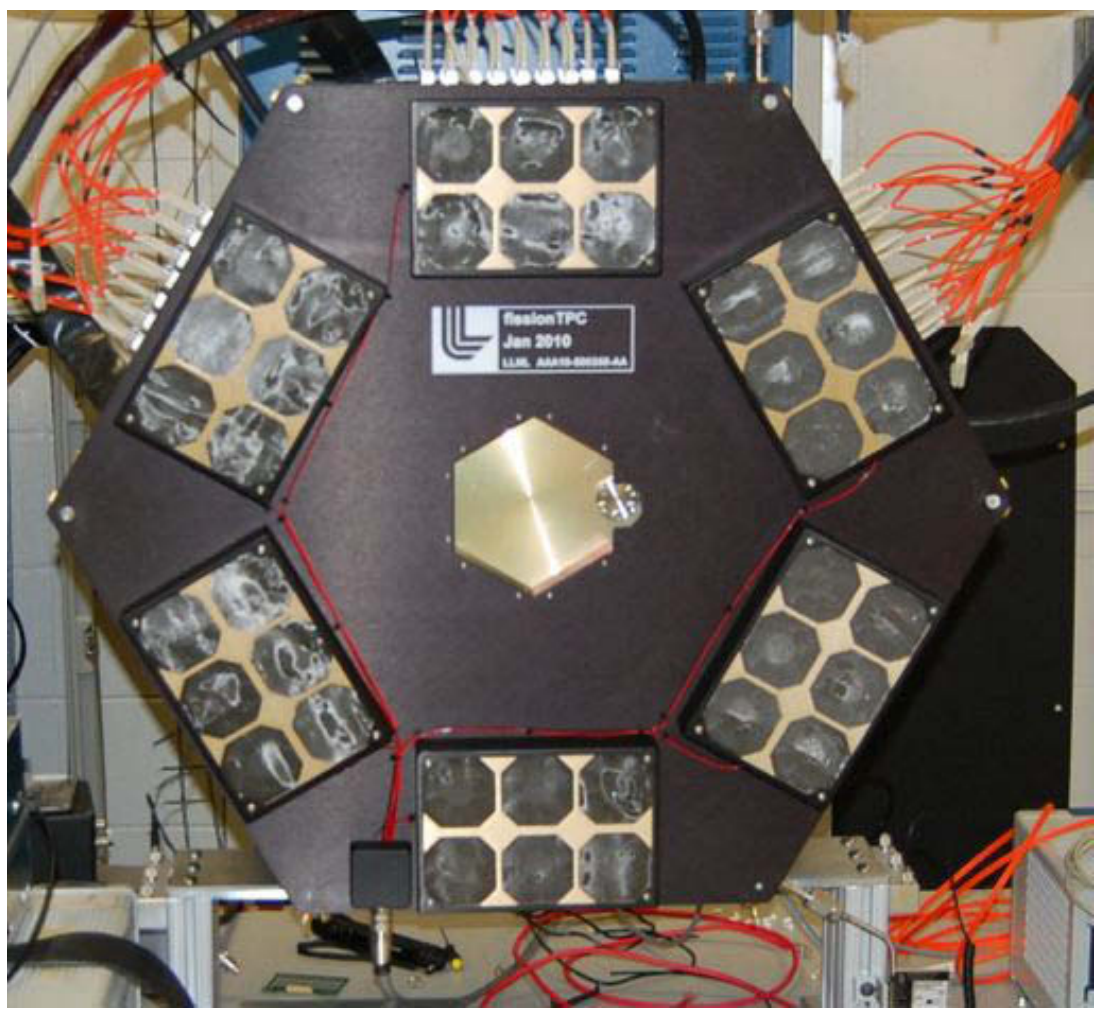

Figure 68: Shown here is the TPC shield that surrounds the gas chamber with the cooling fans attached. The single power connection is at the lower left and power is distributed across the face to each of the 6 packs of 6 fans that direct air over the EtherDAQ/preamplifier card pairs.

\section{High Speed Cathode Readout (HiSCaR)}

The High Speed Cathode Readout (HiSCaR) is the electronics that will allow the TPC experiment to determine the incident neutron energy that causes a charged particle reaction (fission, $(n, p)$ scattering, etc.). The precise HiSCaR timing signal is used in conjunction with the LANSCE accelerator timing signal to measure the neutron time of flight to derive the energy. This is a difficult measurement since in the end, we will need a fast signal with extemely low noise to pickup the proton signals. A shielding and grounding plan has been developed to minimize the noise and pickup that is difficult to remove. For example the $25 \mathrm{MHz}$ clock to the TPC does make it into the signal at some level. We have a plan to improve this and this version is just a quick start so we have a signal for the first running of the full pad plane. The boxes that accomplish this measurement have been built, tested and shipped to LANL for installation

\section{Laser Alignment System}

The laser calibration system for the TPC is important to measure field distortions caused by positive ions in the drift gas. The first design was to use a fiber to transport the laser beam from the laser head to the pressure vessel and down optical rods with prisms at the ends. After a number of attempts to use a fiber we found that it simply can not handle the power. We have decided to change the design to match 
closer to a proven design used in the STAR TPC experiment. A conceptual design of the TPC laser system has been completed, and work has started on the details of constructing that design. The first difficult problem is mounting and carefully aligning the very small $(1 \mathrm{~mm})$ and fragile glass rods. It was determined that a small flat ground into the glass could be used with a fixture to align the rods and then epoxy them in place. The alignment fixture has been designed, and the next step is to test this procedure.

\section{Data Acquisition System [LLNL, ACU, ISU, INL]}

The TPC will have over 6000 pads, each of which need to be instrumented with a preamplifier, ADC and digital readout. The challenge of a large number of densely packed high-speed channels has been met in the past with custom ASIC chips. The technology of both ADC and FPGA has improved considerably over the last decade and it is now possible to use off-the-shelf components to accomplish the same task for considerably less development cost, less time to working prototypes, and considerably more flexibility in the final design.

\section{Preamplifier Cards}

It was observed at LANSCE that the micromegas was not as stable as the operations at LLNL. The discharges happen every few days or so, and each time the discharge hits a preamp it kills the preamp fet ( $\$ 1$ part) that is difficult to replace in the field. We think the discharges are the result of dust entering the TPC and the environment at LLNL is substantially cleaner than at LANSCE. We are working to clean up the work area at LANSCE and also working to make the preamps more robust against sparking. A prototype for the protection circuit was designed and tested. It was tested both for the ability to withstand sparks, and that is does not increase the noise of the system. The PCB was modified and is ready for production. We don't currently have the funds to make this run so we are holding off for now. We will use the older unprotected preamps for the next run cycle.

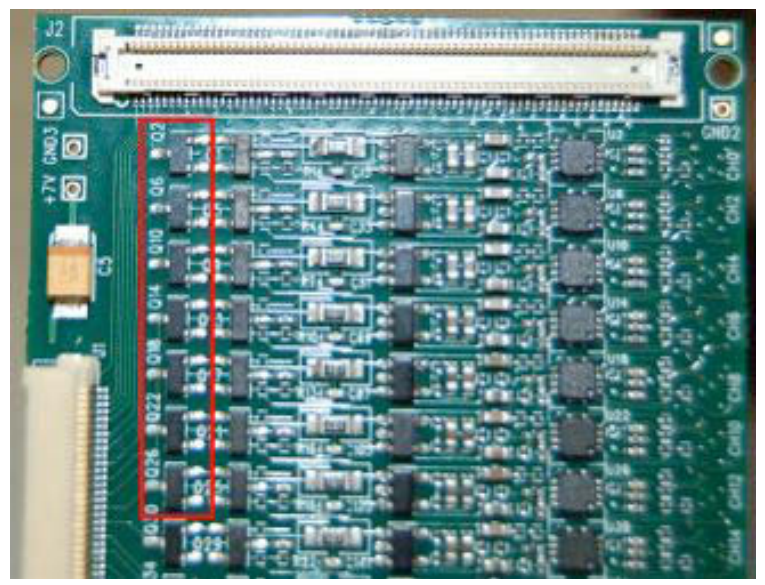

Figure 69: The FETs that are damaged by sparking in the TPC. 


\section{EtherDAQ Cards}

Over half of the production electronics were built this quarter. There was a significant problem found with the soldering of the production EtherDAQ cards. It looks like from the $\mathrm{x}$-ray that the pad on the bottom of some chips was not soldered. We visited the assembly shop to diagnose this problem. A similar problem with soldering may also effect about 6 or the 30 digital bus board cards. In this case, it is a smaller problem since there are fewer needed cards, they are easier to repair, and they cost less. The problem was resolved at the pick-and-place shop by having them rework all of the boards. The end result was that we all but a few to pass which is an excellent yield. A production run of 80 EtherDAq board was also completed last quarter. After receiving the cards they were inspected, tested and programmed. In this quarter, we tested and programmed over 100 EtherDAQ cards and were placed into service.

When operating a large number of EtherDAQ cards we noticed that sometimes the cards would not obtain a necessary Ethernet link. This issue was debugged and solved with a modification to the FPGA firmware.

A separate problem with the electronics is that they would sometimes fall into a mode where they stopped working. We found that this was correlated with the quality of the clock that was distributed to all cards to keep them in sync. The power and clock distribution unit (PCDU) is a custom box was modified to change the clock from a simple TTL signal to a differential LVDS format. This improved the clock and the failure mode of the electronics has not reappeared. We will continue to investigate in the firmware why this was happening, but this fix will allow the experiment to continue. The modified PCDU was shipped to LANL for in-beam runs.

\section{High Voltage System}

The gain stage of the TPC requires careful filtering of the high voltage power and also needs a very sensitive trip circuit to prevent damage to the TPC in the event of a discharge. This is accomplished with the custom filter box that we designed. This has operated very well and a second one was constructed for operation at LANL and has been shipped.

\section{HiSCaR}

The cathode of the TPC can be instrumented to determine the time-of-flight of neutrons arriving at the TPC. This is used to determine the energy of the neutrons to make the cross section measurement. This system consists of two parts. The first is an analog amplifier that takes the small signal from the cathode, amplifies it and conditions it for recording by a digital system. This amplifier has to be fast and low noise which is difficult to accomplish simultaneously. A first version of this amplifier has been built and we are working to refine it. The second part is the digital readout. This is complicated by the fact that we need the timing with respect to the master TPC clock. We have solved this by using a TPC card and making a special interface card that takes the signal from the preamp and delays it 20 times each for just $1 \mathrm{~ns}$. The delayed signals are fed into 20 channels and from this we can get very good timing. This part has been tested with a pulser and works well. This quarter we have integrated the two parts and we now have a higher speed readout of fission at the 
cathode. This was done at LLNL with a $252 \mathrm{Cf}$ source. The rise time is limited by the cable capacitance at the front end and is currently 300-400ns. This is too long, but will be used to get started in the next run cycle. Working with a pulser, it looks like 10-20ns rise is possible once the amplifier is placed inside the TPC; something we plan to have by at least the run cycle in CY13.

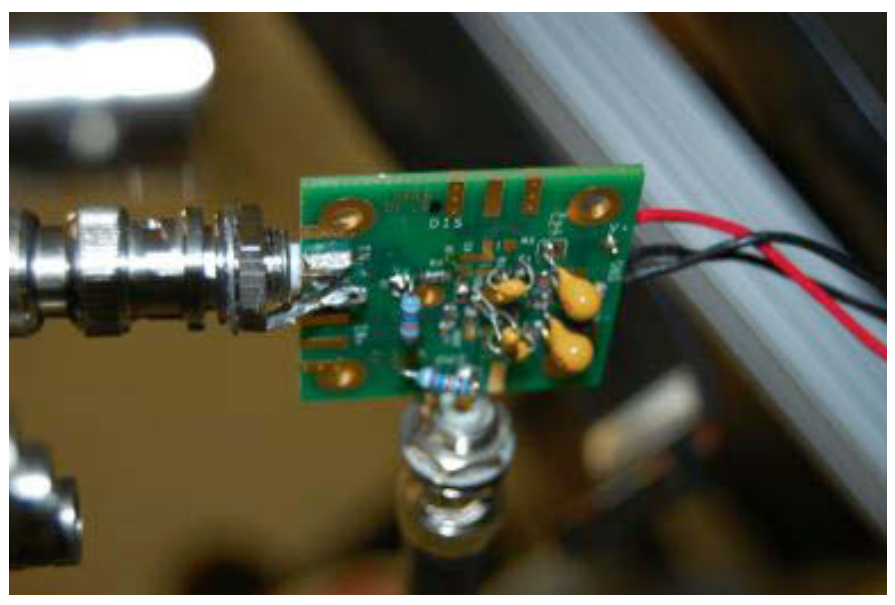

Figure 70: Prototype preamp used on the TPC cathode.

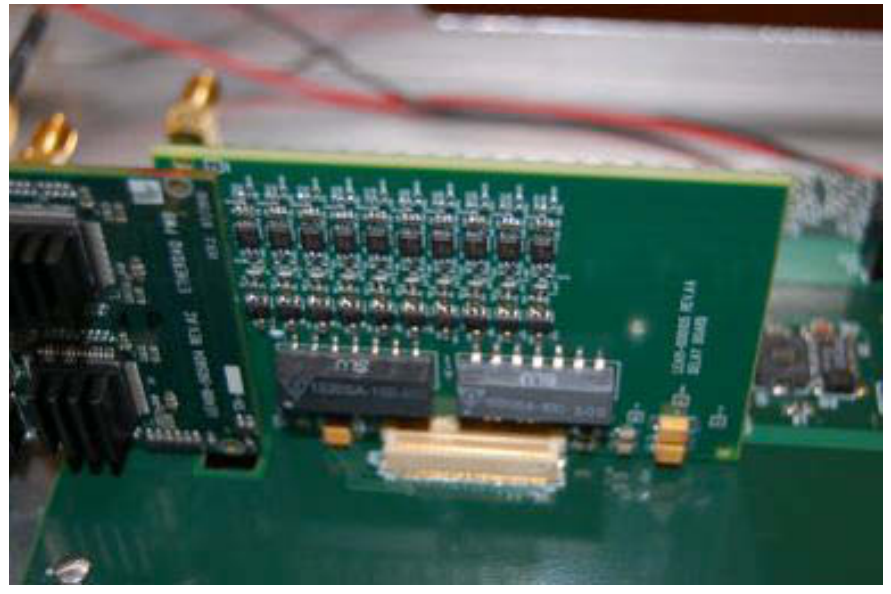

Figure 71: Delay board used to get high speed timing with the TPC electronics need to get the signal readout on the TPC timebase.

\section{Power and Clock Distribution}

The power and clock distribution system (PCDU) is shown in Figure 72 was completed for 6 channels and tested. This allows for operation of 96 front end cards, and is a demonstration of what is needed to power the whole TPC. It also has feature such as a remote shutoff and status for remote operation. 


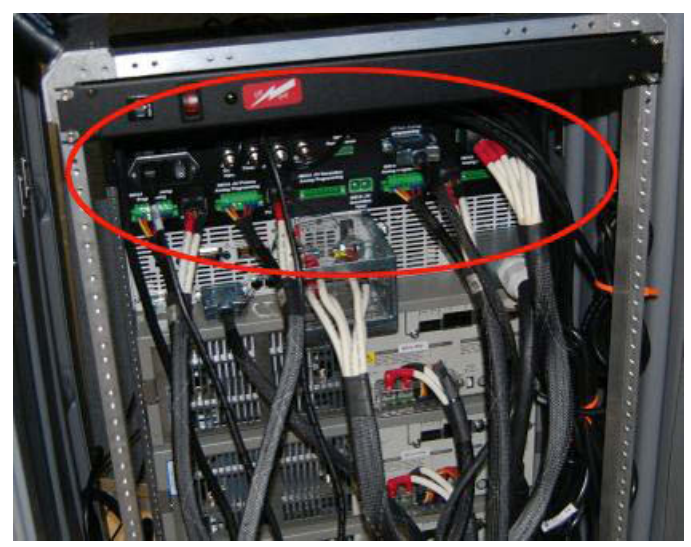

Figure 72: The power and clock distribution system.

\section{Digital Bus Board}

A production run of 30 digital bus boards was completed this quarter, and a full testing procedure has been developed. The boards will be tested this summer for the next run cycle.

\section{Preamp Test Stand}

The preamp card testing capabilities of the EtherDAQ GUI (GUI) have been greatly expanded. Previously, the GUI was used primarily as a method for controlling the pulse generator, as well as collecting the raw ADC data and dumping the data to a text file. The raw data was then externally analyzed using the ROOT framework. All the external analysis functions have now been incorporated into the GUI, thus providing a "one-stop shop" to provide all the tools necessary to analyze and characterize an EtherDAQ/preamp card pair. There are currently three tests: 1 ) burnin, 2) linearity, and 3) cross-talk. 


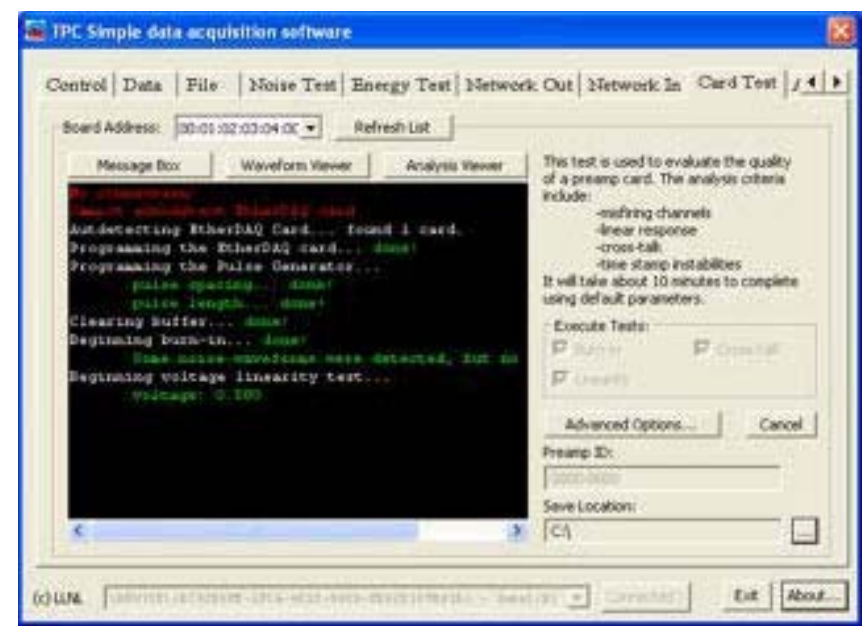

Figure 73. The EtherDAQ GUI in action with the burn-in test completed and the linearity test just beginning.

The burn-in test is used to pre-screen the EtherDAQ/preamp pair. All 32 preamp channels are enabled but the pulse generator is silent. All triggered data is collected by the GUI and analyzed. If a channel is determined to be noisy, i.e. generating false triggers, then that channel is internally masked by the software and the user is notified. If the number of noisy channels exceeds a predetermined limit then the test is halted and the user is informed that the EtherDAQ/preamp card pair is faulty. The card can then be shipped to LLNL for inspection.

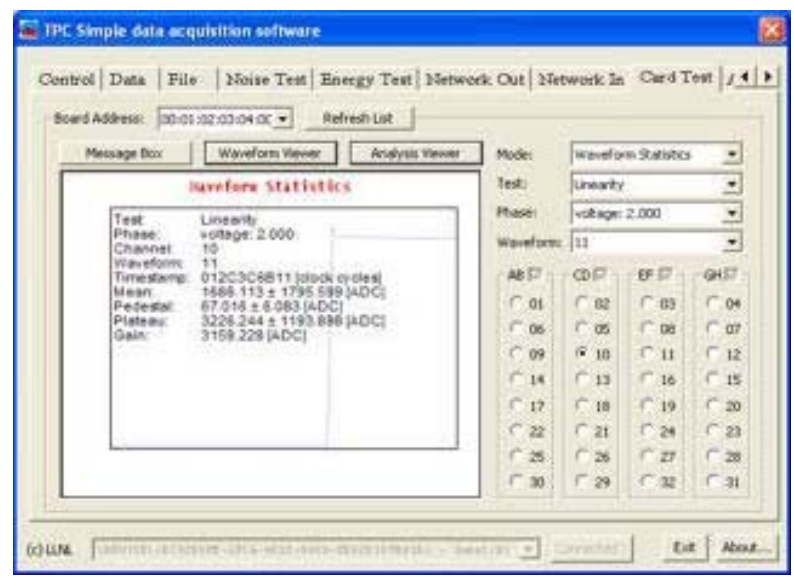

Figure 74. The statistics for each waveform can be inspected on an individual basis.

The linearity test routine analyzes the responses from each of the 32 channels.

Primarily, the linearity analysis tests the response for each channel to ensure that the converted ADC values are proportional to the applied voltage. This is accomplished by sending pulses that cover a range of voltages. A linear regression is then fit to the gain as a function of the input voltage. The standard deviations from the gain 
calculations and the R2 value from the linear regression fit can be used as a metric of the quality of the response.

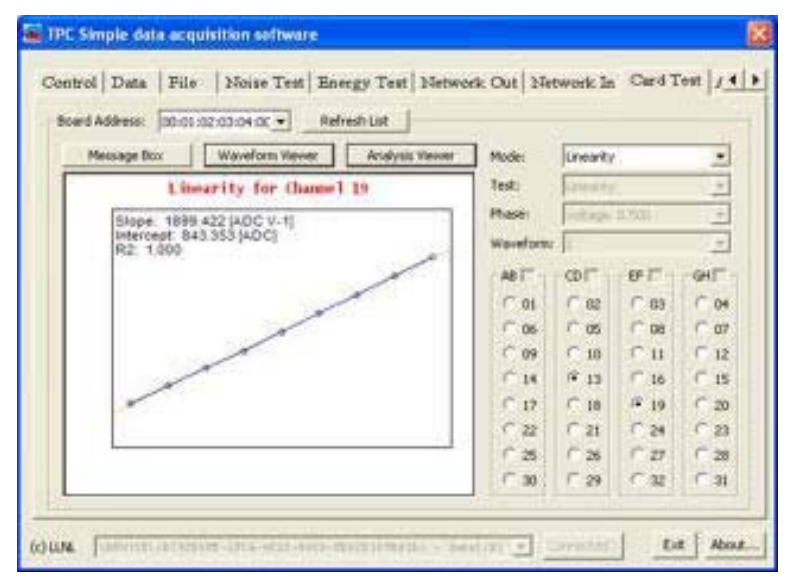

Figure 75. Results of the linearity calculations with corresponding linear fit.

Additionally, minor variances between the analog components introduce slight variations in the response amplitude between channels for the same input voltage. This is the case even though there are very tight tolerances for the components on the preamp card. The linearity portion of the test provides for a channel-by-channel calibration. The slope and intercept values from the linear regression fit can be stored in a database and later dynamically accessed to provide energy calibration for the actual TPC data analysis. This is especially important to enabling the $<1 \%$ measurement error goal for the collaboration.

The final test is the cross-talk routine, which is still in development. Cross-talk cannot be neglected for the NIFFTE project because of the density of the electronics and proximity of the channels to each other. Essentially, cross talk occurs when a charge build-up in one channel causes a resulting charge to accumulate in neighboring channels. It is important to understand the magnitude of this charge sharing so that the effects can be accounted for and calibrated.

To analyze cross-talk contributions, an external trigger was created from the (t0) pulse of the pulse generator to enable a global readout of all channels coinciding in time with the leading edge of the first non-zero event pulse. By creating several sequential inputs, each with a leading pulse that corresponds to a different channel, the cross talk can be analyzed for all non-event channels. After discovering some timestamp anomalies, a new Etherdaq Card was obtained from LLNL that shows the expected timestamp even behavior. A copper Faraday Cage was constructed in order to reduce the observed noise (see Figure 76) when implementing the external trigger. 


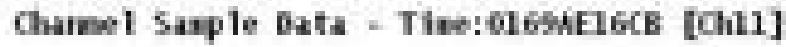

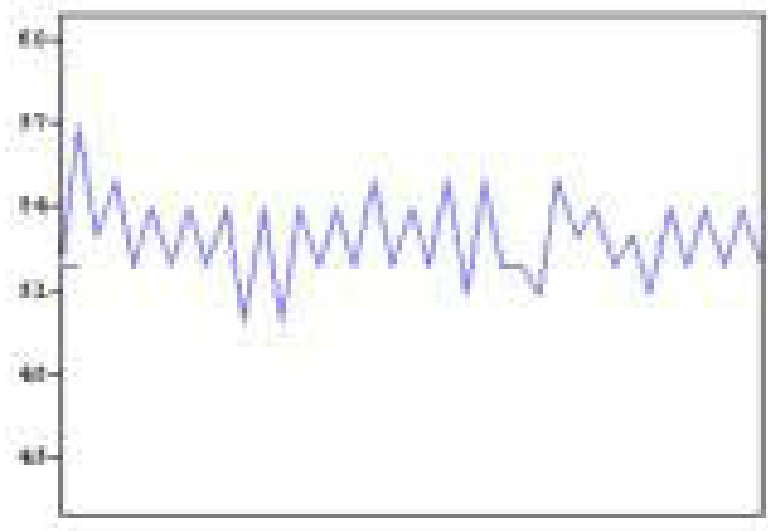

Figure 76. Noise in the pedestal of channel 11.

The GUI has been programmed with functionalities to:

- Visually display multiple waveforms simultaneously

- Zoom in on regions of interest

- Analyze timestamp consistency

- Analysis of pedestal values

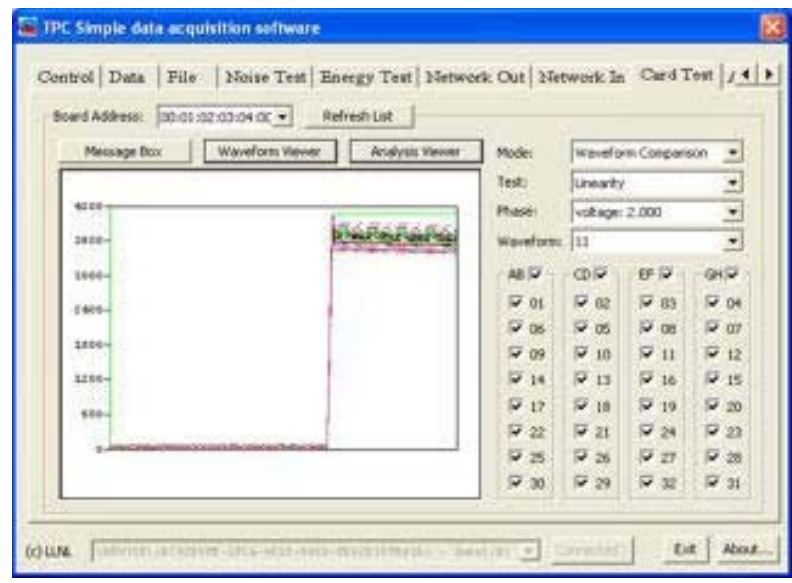

Figure 77. Waveforms from all 32 channels displayed simultaneously. 


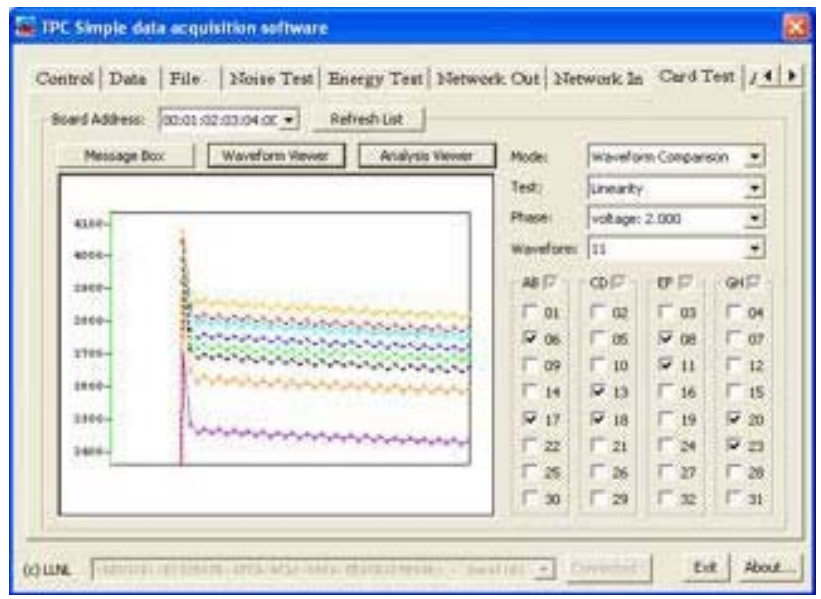

Figure 78. Zoomed-in view of a few randomly selected waveforms.

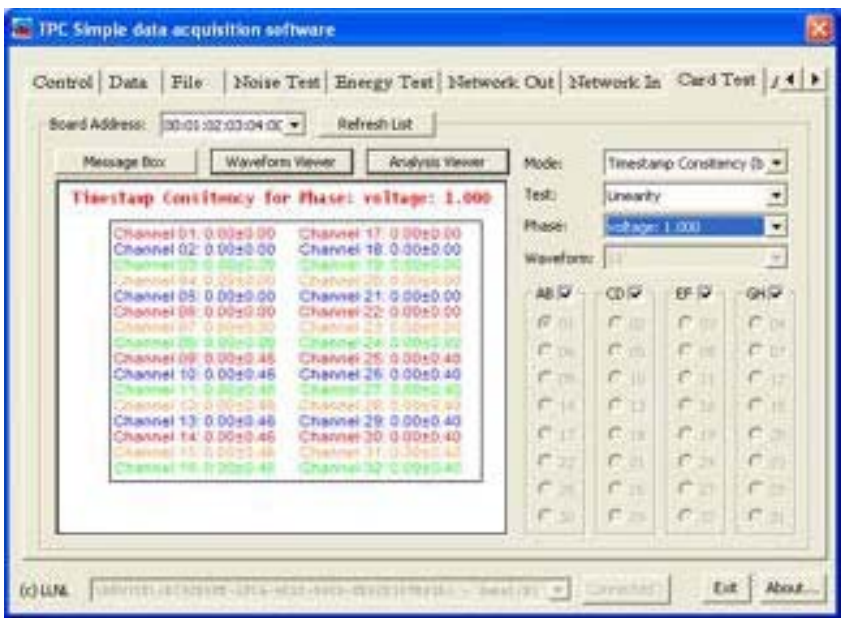

Figure 79. Timestamp analysis functionality. 


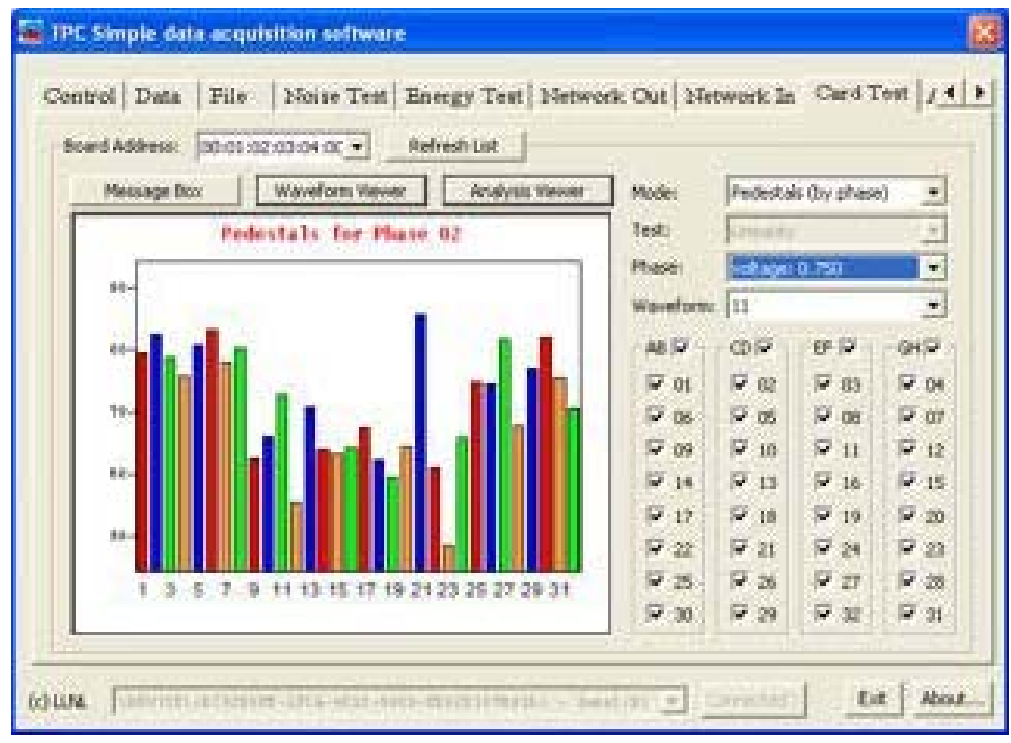

Figure 80. Pedestal analysis functionality.

\section{Gas Handling and Temperature Control Systems [CSM, LLNL]}

One dominant source of error in the absolute measurement of fission cross sections is the normalization of fission data to the U-235 standard. Any campaign that wants to improve on existing data will have to include a new absolute U-235 measurement. The U-235 reference is used to determine the total neutron beam flux, however this method incurs an error of no less than $1 \%$ due to the cross section error. The most promising alternative is the reaction ${ }^{1} \mathrm{H}(n, n)^{1} \mathrm{H}$, which is known to $0.2 \%$. This would though require the use of either pure Hydrogen or a very well known admixture as both target and detection gas in the TPC. In order to be not the limiting factor for the precision of the experiment, the hydrogen density will need to be known and kept constant within $0.1 \%$. For calibration purposes, a gas admixture of $\mathrm{Kr}-83$ will be used and in a later stage, one experimental possibility would be the use of a gaseous actinide target. We are planning for a system that allows for the use of three gases being mixed and supplied to the TPC.

\section{Gas Supply System}

The Colorado School of Mines group's main task in the NIFFTE collaboration is the maintenance and operation of the gas supply and control system. After the previous run cycle, the collaboration had requested that the general control of the system be migrated from the Windows to the Linux computer environment. This would enable the integration of the system into the same computing environment as the rest of the NIFFTE DAQ and slow controls. Additionally, it would provide the collaboration with remote control access to the system.

The migration to Linux required much of the proprietary hardware present in the old system to be replaced by relay boards. Much of the wiring from the previous gas system needed to be replaced. Taking advantage of the rebuilding opportunity, we also replaced the hardware enclosure to provide easier access to both the electronics and hardware elements. 


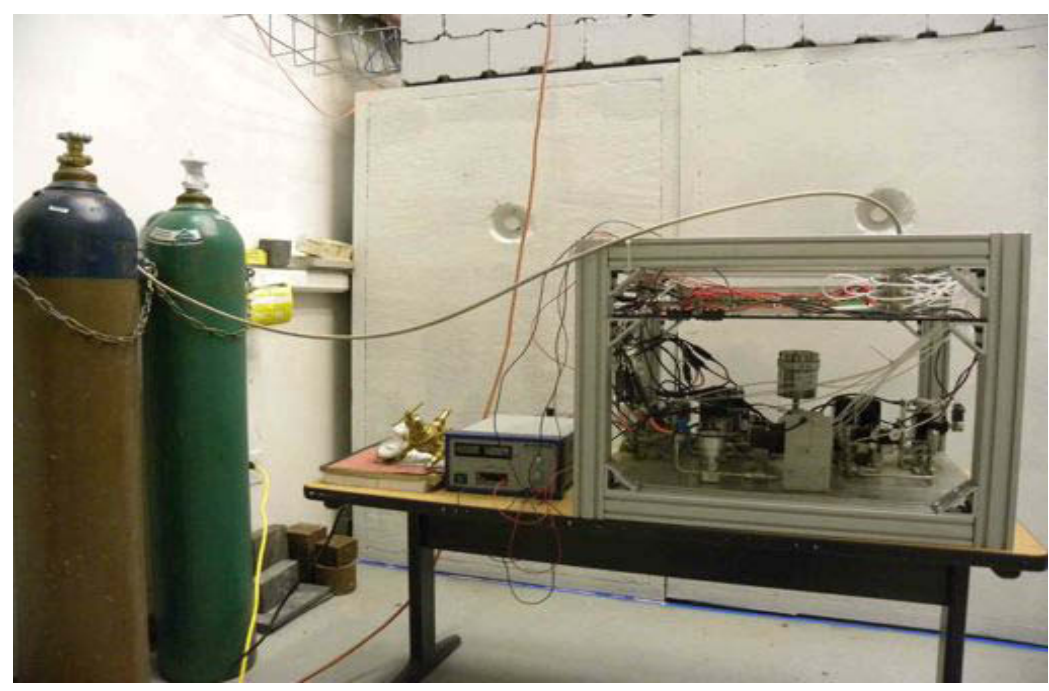

Figure 81: The complete gas supply system hardware with relay board and power supply in the flight path.

The top of the enclosure contains the electronics control that consists of two relay boards. The Arduino Uno SMD is used to read out the status of the individual valves and transmits the information via USB to the NIFFTE gas system computer. The relay board, a ProXR RS-232 DPDT relay controller from National Control Devices, distributes the power to open the individual valves.

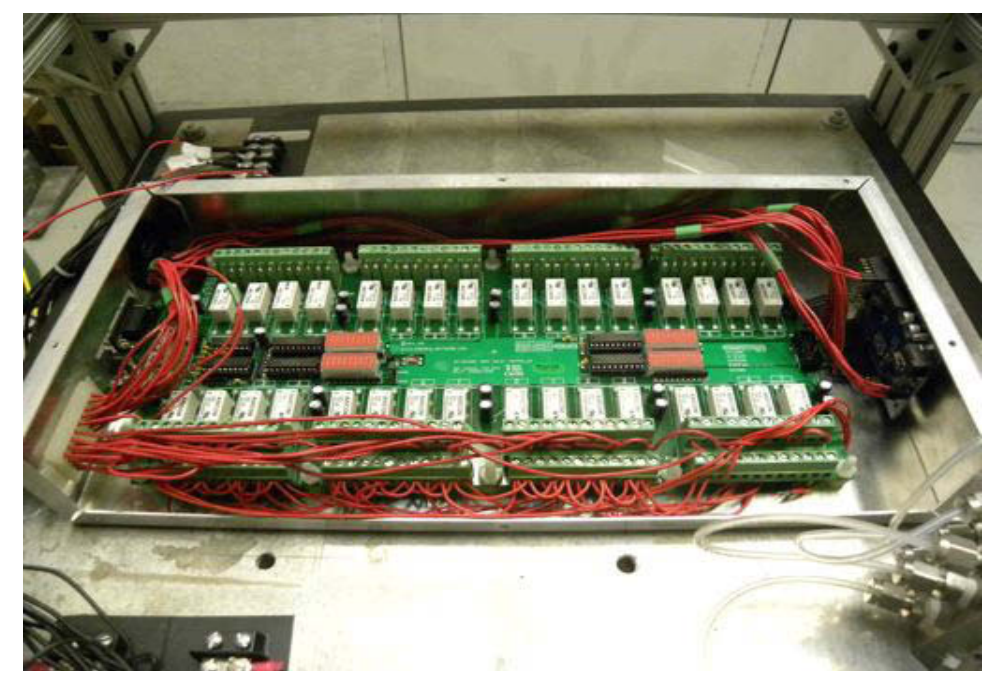

Figure 82: Relay board for solenoid valve control.

The relay boards are mounted directly above the pressure control hardware in order to keep the electronics wires as short as possible. This way, the complete setup only needs to be supplied with one ground connection, $5 \mathrm{~V}$ and $24 \mathrm{~V}$ from a common 
power supply, pressurized air at $80 \mathrm{psi}$, one USB and one RS-232 computer connection.

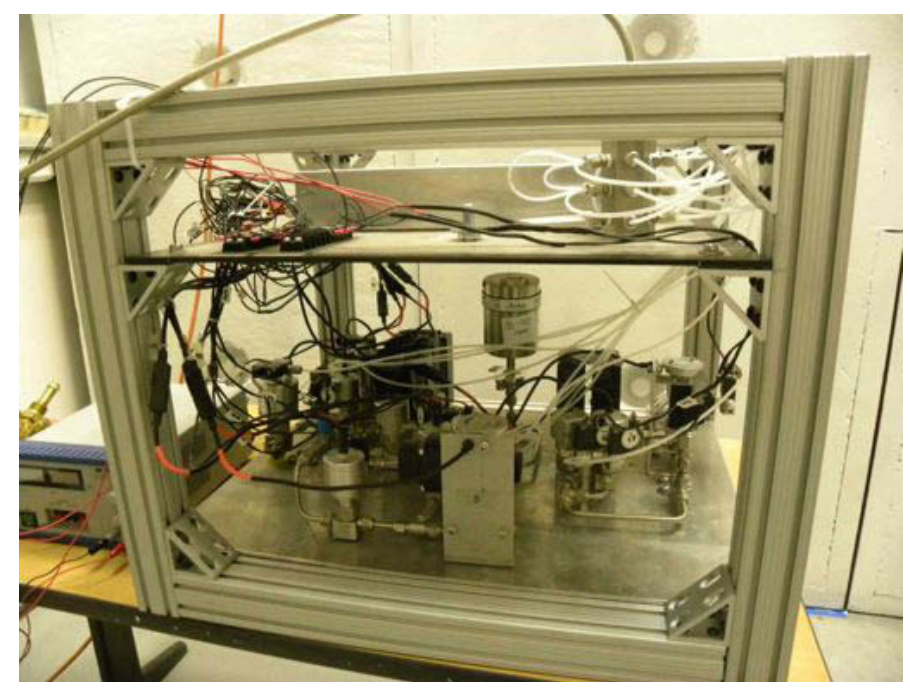

Figure 83: New gas system hardware enclosure for improved access.

As it appears that the measurement using radioactive target gasses is still a few years away, we decided to simplify the gas system hardware to the amount necessary for current measurements. This allowed more accessible placement of the elements in the same footprint as the previous system.

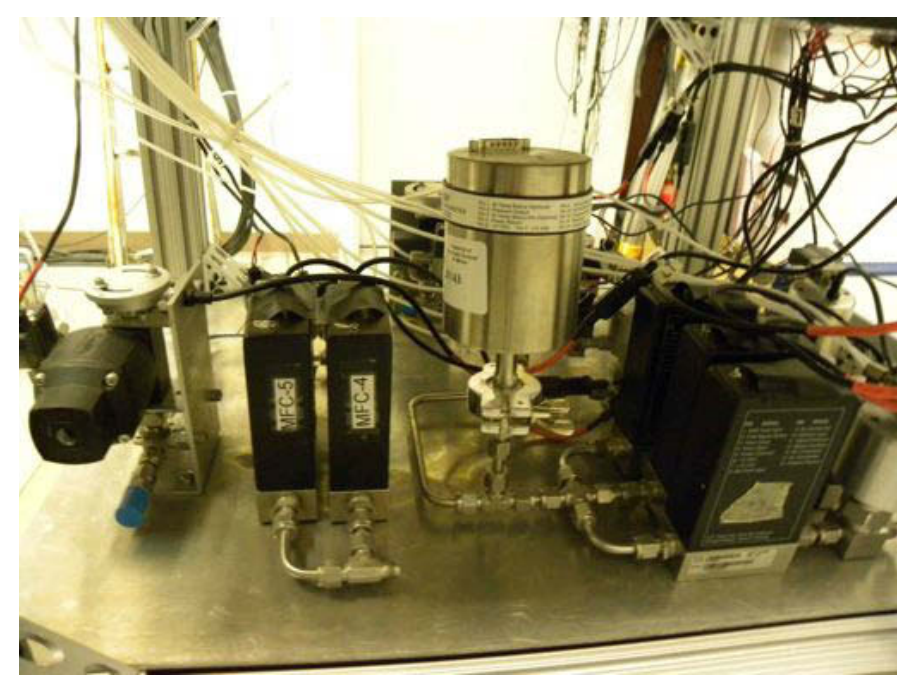

Figure 84: Close up view of the hardware components of the gas supply system as mounted in the new enclosure.

The valve control software is a $\mathrm{C}++$ program and is completely operational. The gas flow and pressure monitoring is performed by MKS Instruments PR $4000 \mathrm{~F}$ and 647C 
controllers. An open source program called Minicom is responsible for communication and control of these devices. In the coming weeks, we will integrate the system with the TPC and provide a pressure readout line to be included in the MIDAS experimental control system.

\section{Electron Lifetime Monitor}

The electron lifetime monitor is an important gas monitor to determine both the drift velocity of the electrons in the TPC as well as how much the gas impurities attach to the drifting electrons. In this quarter it was tested with P10 gas. Different algorithms were evaluated for the extraction of the relevant parameters (charge, lifetime, drift time, parallel diffusion constant). The cathode total photo-emitted charge was observed to drift over time. The reason for this behavior is being investigated. A PCcontrolled oscilloscope was purchased and tested for use with the electron lifetime monitor. A custom $C$ code was written to configure and control the scope. Operation on a Linux machine was also successfully verified. The analysis of the waveforms from the electron lifetime monitor was also improved. The use of Pockels cells and polarizers to reduce the pulse width of the Xe flash lamp was considered but then discarded due to the very poor transmittance of Pockels cells crystals in the VUV regime.

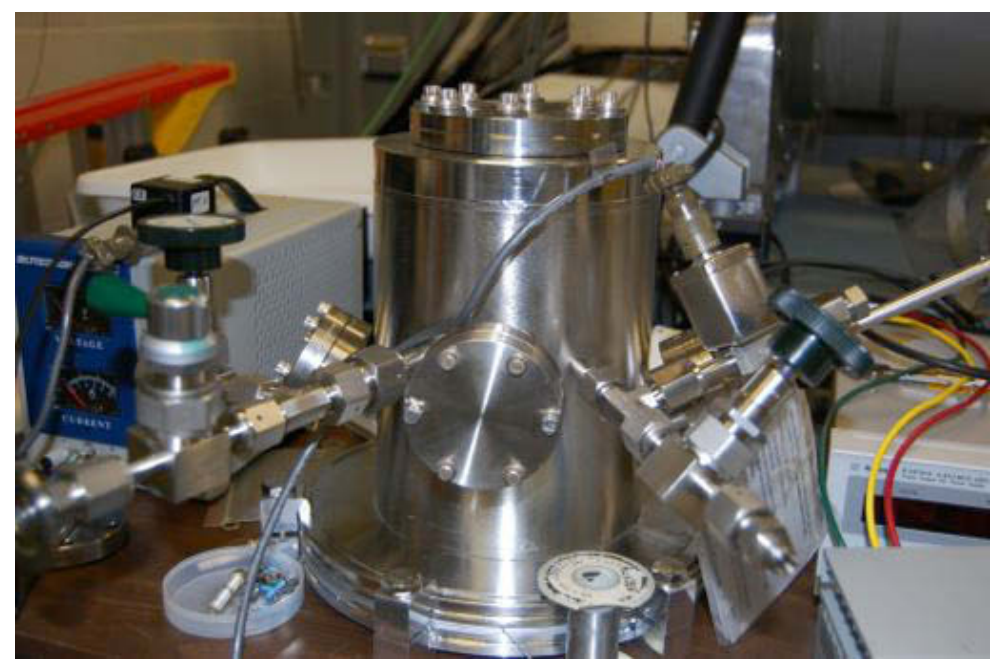

Figure 85: Shown here is the electron lifetime monitor vessel on the test bench at the LLNL TPC laboratory.

\section{Target Design and Fabrication [OSU, INL]}

A well-prepared set of targets is very important for high quality measurements of fission cross sections. Uncertainties in fission cross section measurements with fission chambers can be attributed, in part, to uncertainties in the target mass, non uniformities in the target, surface defects in the targets and surface contaminants in the targets, as well as impure target materials. While the proposed TPC for fission studies will allow detailed corrections for many of these problems, it is of great benefit to start with the highest quality actinide targets. 


\section{Target Design}

We have established a proven ability to furnish targets of ${ }^{238} \mathrm{U},{ }^{235} \mathrm{U},{ }^{232} \mathrm{Th}$ and ${ }^{239} \mathrm{Pu}$ on both thick $\left(540 \mu \mathrm{g} / \mathrm{cm}^{2}\right.$ or $\left.81400 \mu \mathrm{g} / \mathrm{cm}^{2} \mathrm{Al}\right)$ and thin $\left(80-100 \mu \mathrm{g} / \mathrm{cm}^{2} \mathrm{C}\right)$. We have shipped $\sim 50$ targets to the TPC collaboration. The targets we have furnished have been used to perform the initial testing of the TPC.

Now we are entering into a second phase of target development for the upcoming run cycle (CY12) in which the TPC collaboration will perform a number of critical measurements of the TPC. To make these tests, we will need a set of special targets designed to allow definitive testing of the TPC performance. These new targets, requiring unusual shapes of actinide deposits, variable thicknesses on a single target, the use of thin solid hydrogen containing targets and special deposit geometries to test the track reconstruction pointing accuracy were defined in an on-going dialog between us and the other members of the collaboration.

Some of the design issues that arose and their solution are as follows:

(a) Design of the solid hydrogen target. Several materials were evaluated as solid hydrogen targets with polypropylene being chosen. This choice was made because of the relatively well-known composition of this material, the ability to get this material in appropriate thicknesses, and the lack of neutron absorbing materials (other than carbon and hydrogen) in the target. To prepare deposits of actinides on this material, we are using molecular plating on a polypropylene surface that is coated with about $2.4 \mathrm{ug} / \mathrm{cm}^{2} \mathrm{Al}$.

(b) Design of a ${ }^{252} \mathrm{Cf}$ "spot" target to test the track reconstruction pointing accuracy. This design effort is a work in progress with plans to produce these targets in two iterations, a simple "low resolution" spot target containing deposits of ${ }^{252} \mathrm{Cf}$ on $81400 \mathrm{ug} / \mathrm{cm}^{2} \mathrm{Al}$ prepared by evaporation, and a final "high resolution" version of the target prepared by molecular plating of ${ }^{252} \mathrm{Cf}$ on the same Al backing. Among the challenges that had to be solved were to obtain the ${ }^{252} \mathrm{Cf}$ material, to construct a special set of masks (see below) to define the high resolution spot patterns, to develop precise positioning devices for spotting the Al surface using automatic pipettors, the development of spreading agents to insure uniform evaporated deposits, the development of an appropriate surrogate for ${ }^{252} \mathrm{Cf}$ to test procedures, and to fabricate new plating cells for ${ }^{252} \mathrm{Cf}$. Spreading agents being evaluated are a $5 \%$ solution of insulin in water and 2-(heptadecafluorodecylsulfanyl)-ethylphosphonic acid. The latter compound will be synthesized using a radical chain reaction. [M. Essahi, et al., Journal of Fluorine Chemistry 127 (2006) 854.] As a surrogate for the ${ }^{252} \mathrm{Cf}$ in testing the procedures, we are using ${ }^{153} \mathrm{Gd}$ tracer $\left(\mathrm{t}_{1 / 2}=239.5 \mathrm{~d}\right)$ prepared by activation of gadolinium nitrate in our TRIGA reactor. The masks for the "high resolution" molecular plating were machined from Delrin by Katon Precision Machining and involved hole patterns of 100 holes in a $9 \times 9 \mathrm{~mm}$ square with hole sizes of $0.1 \mathrm{~mm}$ diameter. The "low resolution" targets are being spotted with $2 \mathrm{~mm}$ spacing between the spots on a grid of $10 \times 10 \mathrm{~mm}$ with spot sizes of about $1 \mathrm{~mm}$ diameter.

(c) Design of thin carbon targets with multiple coatings/thicknesses of ${ }^{239} \mathrm{Pu}$ on them in the form of wedges (see figures below). We have had a low success rate at putting four separate wedges of ${ }^{239} \mathrm{Pu}$ (by molecular plating) on $100 \mathrm{ug} / \mathrm{cm}^{2} \mathrm{C}$. Even when we are successful in preparing the targets, we have observed them to dis-integrate spontaneously upon standing in air. 
The "final" requested target designs are shown in the figures below.

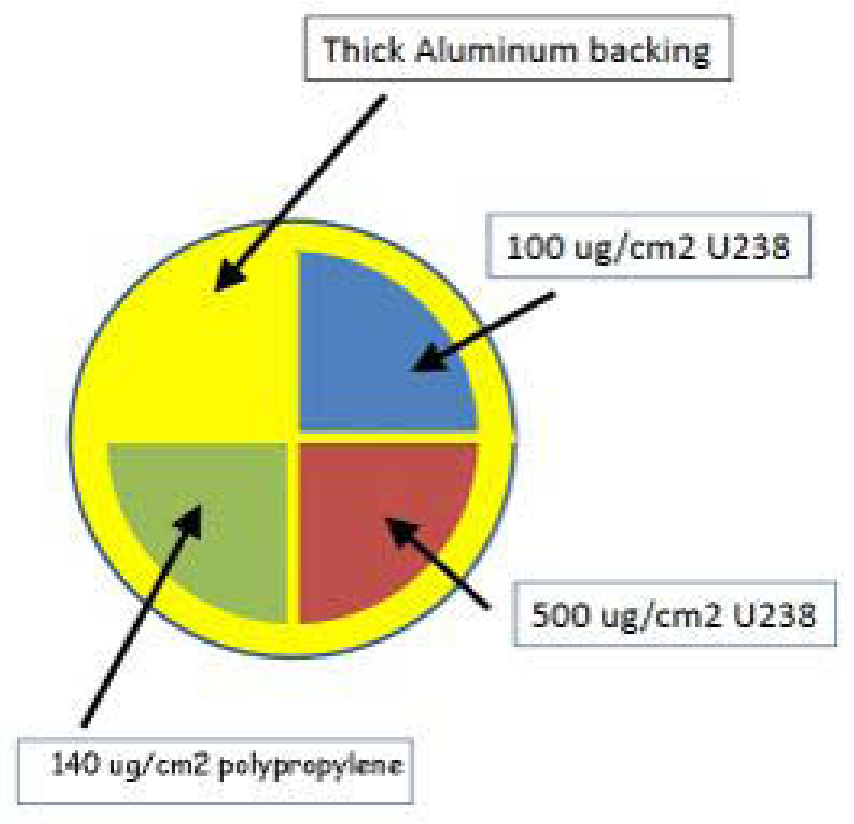

Figure 86: Front and back views of target CY12R0TO, The back side of the target is a uniform Pu239 coating at $40 \mu \mathrm{g} / \mathrm{cm}^{2}$.

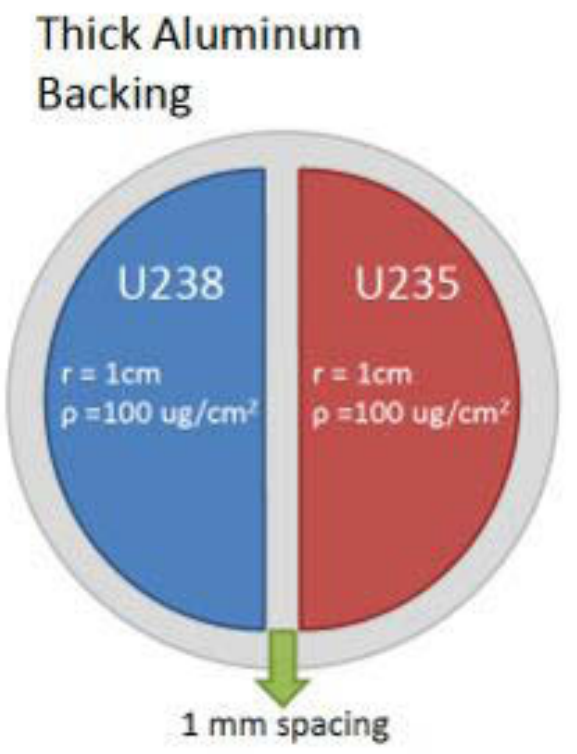

Figure 87: Design of target CY12R1T0. 


\section{Thick Aluminum Backing}

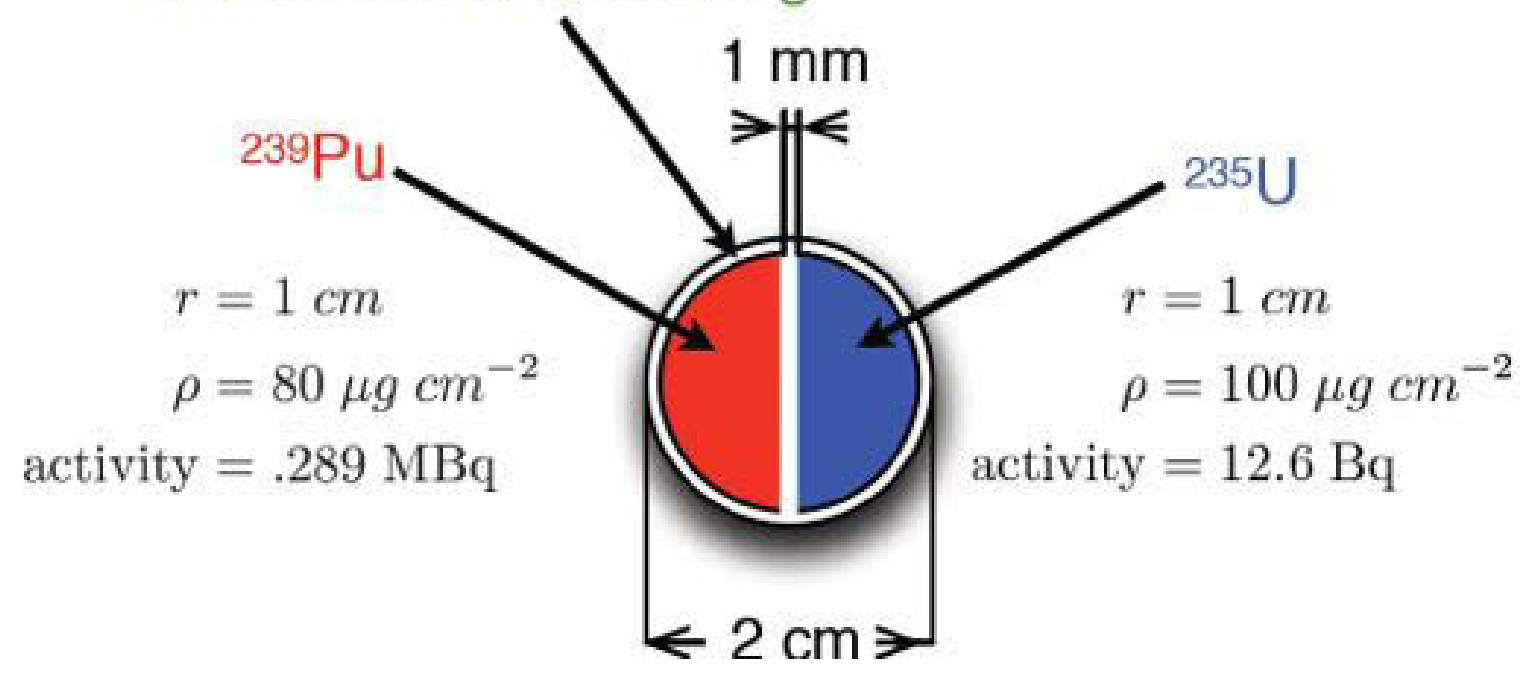

Figure 88: Design of target CY12R2TO.

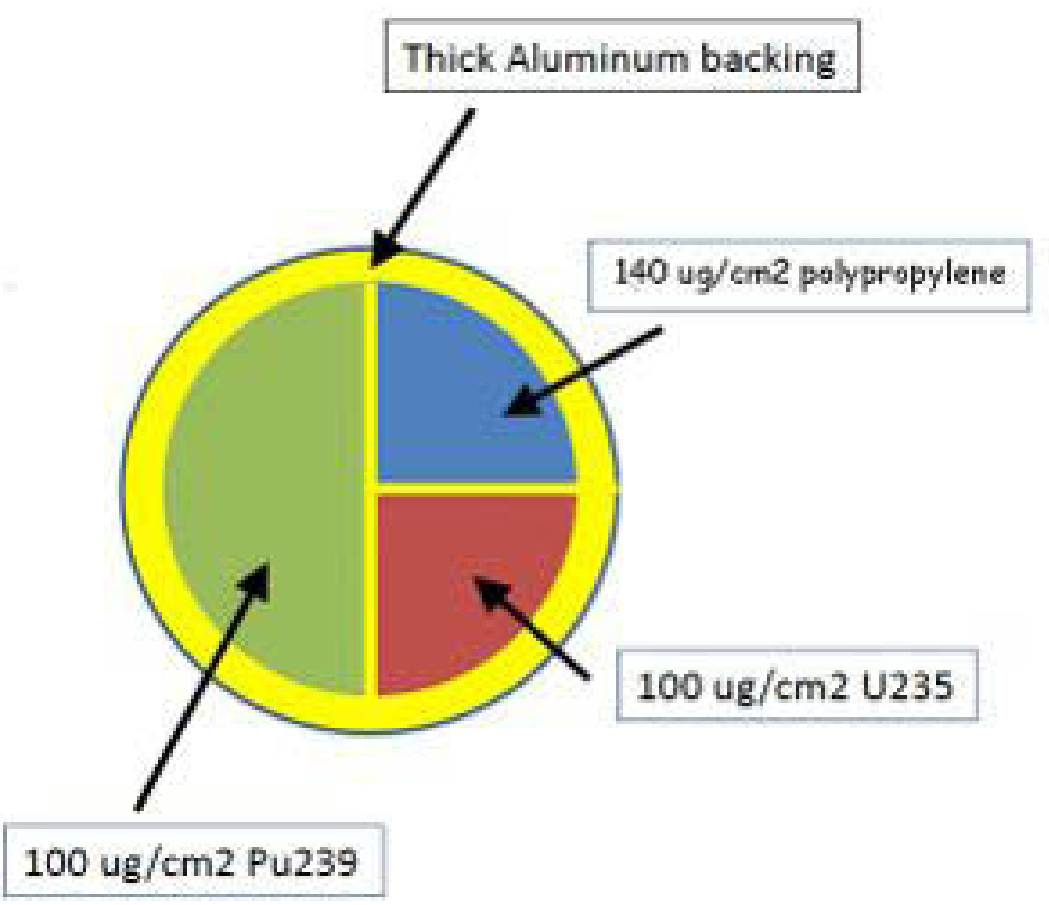

Figure 89: Design of target CY12R3T1. 


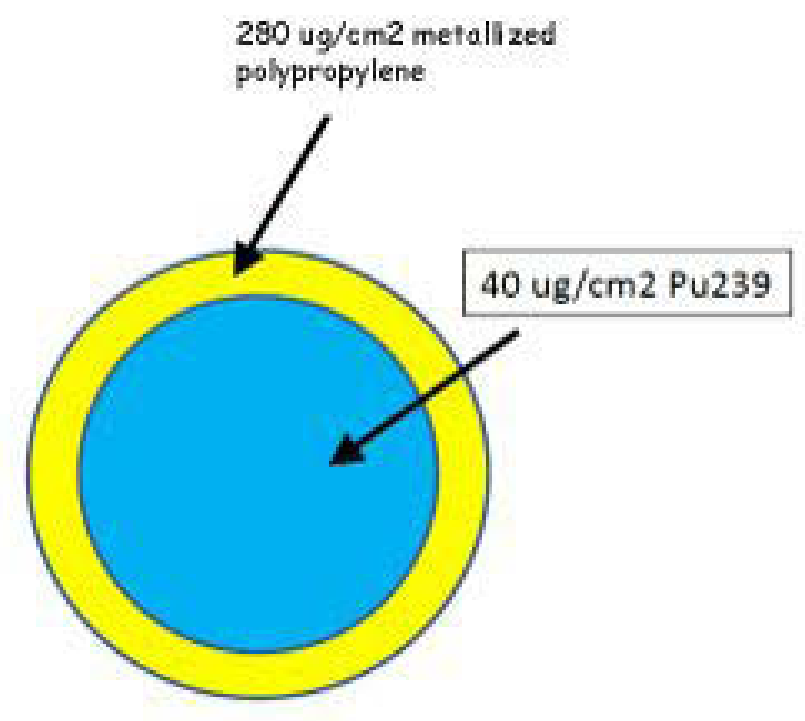

Figure 90: Design of target CY12R3T0.

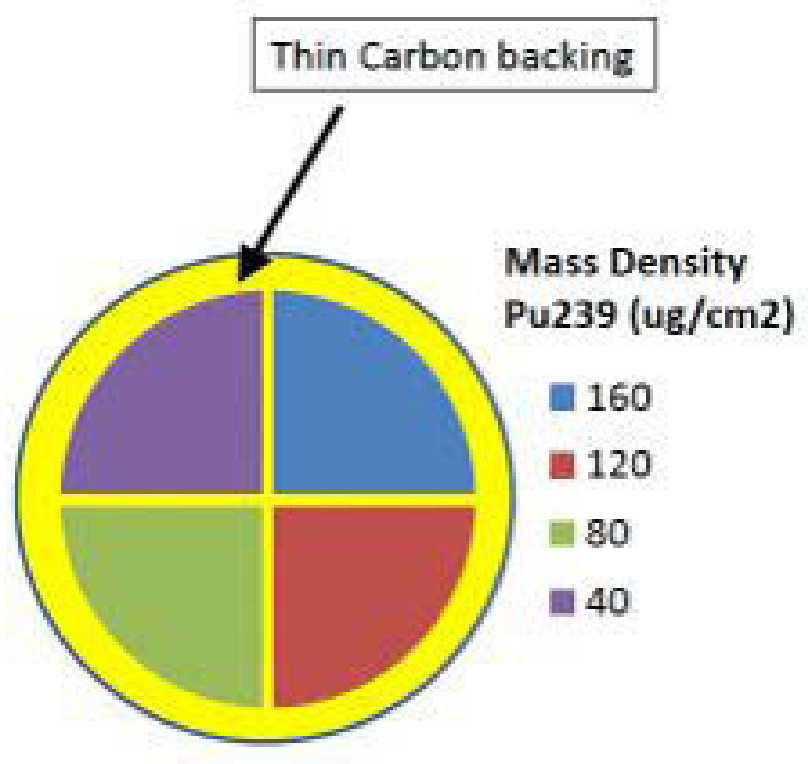

Figure 91: Initial design of target CY12R4T0. This design has not proven to be viable and modifications are being made-see text. 


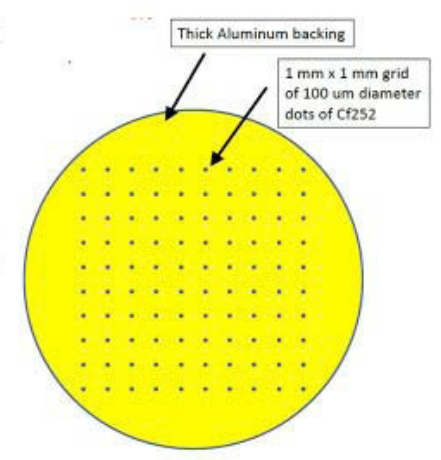

Figure 92: Front view of 252Cf spot target design.

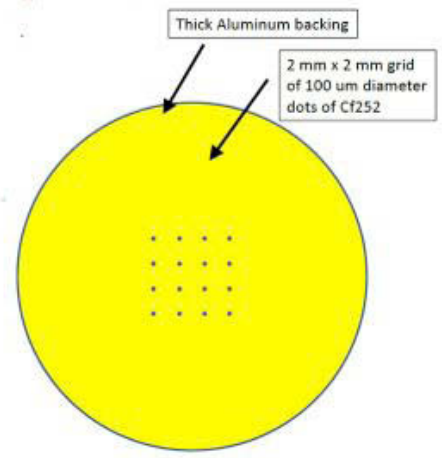

Figure 93: Back viewsof 252Cf spot target design.

\section{Target Fabrication}

The following "target fabrication" activities were undertaken:

(a) We hosted a group of three scientists from PNNL who are seeking to set up their own actinide target making facility. We provided demonstrations of preparation of ${ }^{239} \mathrm{Pu}$ targets by molecular plating and preparation of ${ }^{232} \mathrm{Th}$ targets by vacuum volatilization. We discussed several general issues in actinide target preparation. We prepared and shipped a test target to PNNL. The target designator was LY 5. The target consisted of half-circles of ${ }^{238} U$ and ${ }^{235} U$ of thickness 443 and 472 $\mathrm{ug} / \mathrm{cm}^{2}$.

(b) We loaned a nominal $100 \mathrm{nCi}{ }^{252} \mathrm{Cf}$ source to LLNL for TPC testing.

(c) We shipped a previously prepared ${ }^{6} \mathrm{Li}$ coated scintillator to ISU.

(d) We worked on the re-design and alternate fabrication methods for targets CY12R4T0 and the 252Cf spot target (see above).

(e) In the process of assaying the various multi-isotope targets, we have noticed that the ${ }^{239} \mathrm{Pu}$ deposits "creep", i.e., the activity moves outside the boundary of the deposit area. The transfer of material is very small ( $<<0.01$ atom percent) and will not affect the use of the targets to measure cross sections. What this may affect is the use of the TPC to radiograph the targets because one will have to 
distinguish the alpha decay of the low specific activity nuclides $\left({ }^{235} U,{ }^{238} U\right)$ from the high specific activity ${ }^{239} \mathrm{Pu}$.

\section{Target preparation}

The following targets were prepared and shipped to LANL:

\begin{tabular}{|c|c|c|c|}
\hline $\begin{array}{l}\text { TPC Target } \\
\text { Designator }\end{array}$ & OSU Designator & Backing & Deposits \\
\hline \multirow[t]{6}{*}{ CY12R1T0 } & LY7 & $81400 \mathrm{ug} / \mathrm{cm}^{2} \mathrm{Al}$ & $302 \mathrm{ug} / \mathrm{cm}^{2}{ }^{238} \mathrm{U}$ \\
\hline & & & $239 \mathrm{ug} / \mathrm{cm}^{2}{ }^{235} \mathrm{U}$ \\
\hline & LY 9 & $81400 \mathrm{ug} / \mathrm{cm}^{2} \mathrm{Al}$ & $122.5 \mathrm{ug} / \mathrm{cm}^{2}{ }^{238} \mathrm{U}$ \\
\hline & & & $173 \mathrm{ug} / \mathrm{cm}^{2235} \mathrm{U}$ \\
\hline & LY 11 & $81400 \mathrm{ug} / \mathrm{cm}^{2} \mathrm{Al}$ & $115.3 \mathrm{ug} / \mathrm{cm}^{2}{ }^{238} \mathrm{U}$ \\
\hline & & & $106.6 \mathrm{ug} / \mathrm{cm}^{2235} \mathrm{U}$ \\
\hline \multirow[t]{4}{*}{ CY12R2T0 } & LY10 & $81400 \mathrm{ug} / \mathrm{cm}^{2} \mathrm{Al}$ & $105.1 \mathrm{ug} / \mathrm{cm}^{2}{ }^{239} \mathrm{Pu}$ \\
\hline & & & $70.4 \mathrm{ug} / \mathrm{cm}^{2}{ }^{235} \mathrm{U}$ \\
\hline & LY12 & $81400 \mathrm{ug} / \mathrm{cm}^{2} \mathrm{Al}$ & $74.3 \mathrm{ug} / \mathrm{cm}^{2}{ }^{239} \mathrm{Pu}$ \\
\hline & & & $63.9 \mathrm{ug} / \mathrm{cm}^{2}{ }^{235} \mathrm{U}$ \\
\hline \multirow[t]{8}{*}{ CY12R0T0 } & LY6 & $81400 \mathrm{ug} / \mathrm{cm}^{2} \mathrm{Al}$ & $29.7 \mathrm{ug} / \mathrm{cm}^{2}{ }^{239} \mathrm{Pu}$ \\
\hline & & & $456.9 \mathrm{ug} / \mathrm{cm}^{2}{ }^{238} \mathrm{U}$ \\
\hline & & & $133.8 \mathrm{ug} / \mathrm{cm}^{2}{ }^{238} \mathrm{U}$ \\
\hline & & & $\begin{array}{l}140 \mathrm{ug} / \mathrm{cm}^{2} \\
\text { polypropylene }\end{array}$ \\
\hline & LY14 & $81400 \mathrm{ug} / \mathrm{cm}^{2} \mathrm{Al}$ & $29.1 \mathrm{ug} / \mathrm{cm}^{2}{ }^{239} \mathrm{Pu}$ \\
\hline & & & $954 \mathrm{ug} / \mathrm{cm}^{2}{ }^{238} \mathrm{U}$ \\
\hline & & & $105.7 \mathrm{ug} / \mathrm{cm}^{2}{ }^{238} \mathrm{U}$ \\
\hline & & & $\begin{array}{l}140 \mathrm{ug} / \mathrm{cm}^{2} \\
\text { polypropylene }\end{array}$ \\
\hline \multirow[t]{3}{*}{ CY12R3T1 } & LY13 & $81400 \mathrm{ug} / \mathrm{cm}^{2} \mathrm{Al}$ & $112.4 \mathrm{ug} / \mathrm{cm}^{2}{ }^{235} \mathrm{U}$ \\
\hline & & & $110.7 \mathrm{ug} / \mathrm{cm}^{2}{ }^{239} \mathrm{Pu}$ \\
\hline & & & $\begin{array}{l}140 \mathrm{ug} / \mathrm{cm}^{2} \\
\text { polypropylene }\end{array}$ \\
\hline CY12R3T0 & LY15 & $\begin{array}{l}280 \mathrm{ug} / \mathrm{cm}^{2} \\
\text { polypropylene } \\
\text { metalized with } 2.4 \\
\mathrm{ug} / \mathrm{cm}^{2} \mathrm{Al}\end{array}$ & $25.3 \mathrm{ug} / \mathrm{cm}^{2}{ }^{239} \mathrm{Pu}$ \\
\hline
\end{tabular}




\section{TPC Software [CalPoly, ACU, ISU, INL]}

\section{Scope}

The TPC Software effort will include online and offline coding, FPGA programming for the data acquisition system, and simulation. In addition to reconstruction and analysis of data acquired by the NIFFTE detector, the offline simulation and reconstruction software will be used to generate substantial "Mock Data Challenge" datasets of simulated fission events, alpha decays and background events that will be processed through the full simulation and reconstruction chain. In addition to testing the readiness and functionality of the offline software, these MDC efforts are intended to improve modeling and design of the actual NIFFTE detector.

\section{Online Software [ACU]}

The portion of this experiment's software library that is used during the data taking is called the online software. It overlaps in many ways with the data acquisition software, since it must take the data output from the readout cards after it has been processed with the FPGAs. The features that fall within the online category include: data receiver, event builder, data cataloging and storage, run control, on-the-fly data inspection, data base management, electronic log book, and remote experiment monitoring and control. Some of this software is available and only needs to installed and maintained on the online computers, other software will have to be written and maintained in the collaboration subversion library. All written software will be written following a modular design for reusability in $\mathrm{C}++$. In addition, the online software team will take on the role of administer for the online computers.

\section{Support for Operations at LANL}

The online software has seen continuous development during the past quarter. Data collection at LANL was supported by installing, debugging, and maintaining the DAQ, slow controls, and online data quality assurance software. An important issue with the online demux performance was addressed. After analyzing some initial data with the TPC in the neutron beam, it was determined per-channel TPC trigger thresholds should be lowered to record significantly more recoil proton tracks from beam neutron interactions within the TPC.

\section{Online Monitoring Maintenance and Development}

The packet receiver and channel demux software were updated to handle waveforms from the HiSCaR. The event loop for the online channel demux software was modified to improve its data throughput capability.

The online event builder was modified and implemented to save a user controlled fraction of its output. For example, the program can save $100 \%$ of waveform events in when data rates are low or only $1 \%$ when rates are very high. This feature allows fine control over the amount of data passed to the CPU intensive online analysis and histogramming program. 
MIDAS frontend program to store slow control measurements in the persistent SQL database has been updated to include recently added and modified channels. All the recorded variables are now saved with UNIX timestamp and the dates/minutes/seconds with time zone. This program was also modified to significantly reduce its CPU utilization.

\section{Offline Software [CalPoly, ISU, INL, LLNL, PNNL]}

The thrust of this task is to transform the data from their raw form to final calibrated results, which requires a complete data analysis chain. The offline software required to perform this analysis must be designed, organized, written and documented. In order to achieve maximum flexibility, the design should focus on providing simple interfaces within a modular framework. For ease of use by collaborating experimenters, the software should also be well documented and maintained in a central repository available to the entire collaboration.

\section{Timing Offset Software Infrastructure Updates}

Before the implementation of the High Speed Cathode Readout (HiSCaR) system in the TPC hardware, the $z$ coordinate of an event was not well determined - all $z$ coordinates were relative and calculated from the signal time (bucket number) recorded with the TPC waveforms. With the introduction of the HiSCaR precision timing signal, an absolute time reference $(t=0)$ is established, so the absolute $z$ coordinates can be determined. While implementing the changes required for the bucket number to $z$ coordinate mapping, it became clear that it would be desirable to redesign the stored data objects so that the bucket number is stored and the $z$ coordinate is always calculated. This allows for the possibility that post-processing can improve the HiSCaR calibration and modify the $t=0$ determination. The actual mapping between bucket number and $z$ coordinate depends on a timing offset that must be determined for each event (and separately for each TPC volume as the triggers for each volume are independent).

The two plots in Figure 94 below demonstrate the implementation of the timing offset infrastructure. In the first plot, the $z$ coordinates of the start of all reconstructed tracks are shown before any calculation of timing offsets. The distribution of start positions is due to diffusion near the start of the track. In the second plot, an artificial timing offset correction is applied which forces the vertex of each track to be at $z=0$. Although the HiSCaR timing offset algorithm is still under development, these plots demonstrate that the infrastructure is in place to manage the offsets once the algorithm is ready. 


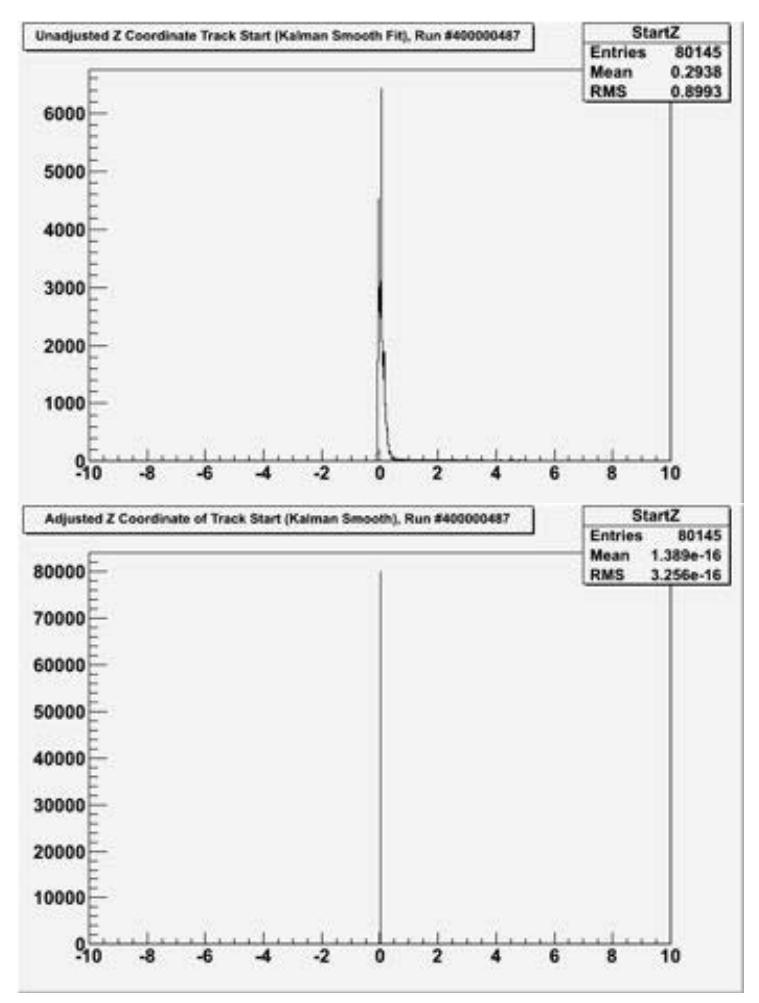

Figure 94: The $z$ coordinates of the start of all tracks before and after the application of an artificial timing offset correction.

\section{Hough Tracking Analysis}

An analysis of the Hough track finder was undertaken by a visiting student at PNNL. The existing Hough track finder relies on several input parameters, as listed in Table 1 , and works reasonably well on data taken so far with the default values listed. However, a systematic study of the effects of varying these parameters has not yet been done, and the student who wrote the Hough tracking code is no longer available, so this is a worthwhile investigation. A PNNL report on the results of this study will be released next quarter.

Table 1. Hough track finder input parameters.

\begin{tabular}{|l|c|l|}
\hline Parameter & Default Value & Description \\
\hline WeighByADC & On & $\begin{array}{l}\text { On = weight the digits by their ADC values } \\
\text { Off = all digits weighted equally }\end{array}$ \\
\hline DigitSharing & Off & $\begin{array}{l}\text { Off = Each digit can only be assign to one track } \\
\text { On = Allow digits to be shared between tracks }\end{array}$ \\
\hline DigitThreshold & 1 & $\begin{array}{l}\text { Digit ADC value must be above this to be considered for } \\
\text { inclusion in a track }\end{array}$ \\
\hline
\end{tabular}




\begin{tabular}{|l|c|l|}
\hline TrackThreshold & 10 & Minimum track ADC sum to be accepted as a track \\
\hline HoughRadius & 1.0 & Expected radius of a track in the XY plane \\
\hline HoughDiffusion & 0.90 & Expected radius of diffusion of a track \\
\hline
\end{tabular}

\section{Refined Track Reconstruction}

Work has begun to develop a refined tracking package with superior vertexing and particle identification that will be capable of extracting information for fission yields and charge configurations in the fission process. The proposed tracking package will include a fully informed global fitter that deconvolutes physical and detector-induced distortions on the ADC signals, like electron diffusion and charge movements in the detector and the electronics.

The paradigm for the new fitter is to start the reconstruction process using tracklet hypotheses in the hexagonal voxels imposed by the geometric structure of the TPC anode plane. A fitter will refine and connect these tracklets to form a track instead of using points with an assumed a charge deposition occurring in the center of an anode pad.

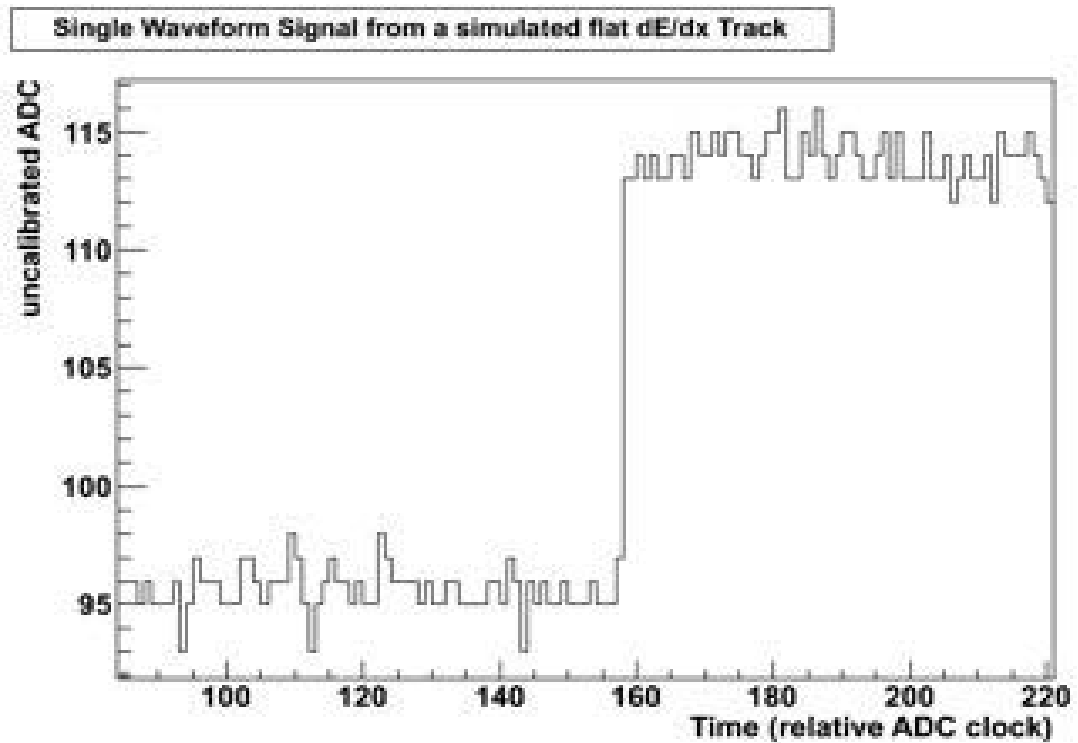

Figure 95: A single pad waveform signal in the shape of a step function from a simulated flat ionization loss track.

In order to learn more about the structure of the single waveform and to serve as probe for any future tracking developments, the simulation package has been used to produce 'perfect' unperturbed waveform signals. For the particular task of testing and exploring waveform signals, the simulation was modified to produce signals from tracks that have a flat $\mathrm{dE} / \mathrm{dx}$ profile (as opposed to ionization loss according to a 
Bragg-curve) and do not include any distorting effects like electron diffusion or charge distortion in the electronics. The obtained waveform is an almost perfect step function with a slight decay on the top of the rise due to the RC-constant in the electronics. Figure 95 shows an unperturbed signal from a track with flat ionization loss. This unperturbed waveform can be fit using a piecewise polynomial, while all three parts are a straight line with different slopes. The onset and edge of the rise can be found using a simple 7-point derivative filter. Due to the simplicity of the fit, it is actually possible to use an analytic least-squares fit using matrices, which increases the processing speed compared to a numerical fit.

When a more realistic ionization loss following a Bragg-curve is introduced into the simulation, the waveforms deviate from the idealized perfect step-functions. The edges of the rise become more curved and less pronounced, and the rise does not necessarily follow a straight line. A Fermi function coupled with an exponential RCdecay has proven to best fit those realistic signals.

The fit parameters of the signal waveforms are used to determine the impact of different distortions to the waveform by including them individually in the simulation. Examples for distortions investigated are non-flat ionization loss, changing the RC decay constant and Preamp gain, diffusion in 3-dimensional space and a variety of charge distortions - charge sharing between neighboring pads or crosstalk between electronics. The goal of this effort is to deconvolute the distorting effects on the signal to be able to use unperturbed signals in the track reconstruction.

The radial information of the reconstructed track is pulled from the $(x, y)$ coordinates of the anode pad for which the signal arrived. A well-informed tracking algorithm has to know the geometry of each hexagon, as the tracklets are made up by intersection points of the track with the hexagon structure rather than just the center of a hexagon. The $(x, y)$ coordinates in the active volume of the TPC have been mapped to (row, column) coordinates uniquely identifying each pad on the anode. This enables the tracking software to extract an ADC signal from the corresponding pad from a certain $(x, y)$ position. Recent efforts have led to a 3-dimensional mapping of the TPC by including a time bucket number in the mapping to produce 3-dimensional hexagonal prisms that make up the detector volume.

The initial $(x, y)$ direction hypothesis of individual tracklets will be refined by forcing them to connect, and by tuning their length within a voxel based on polar angle and specific ionization.

\section{Code execution time improvements}

Potential improvements in code execution were identified in the analysis code profiling last quarter. Several of these were implemented this quarter in the Hough track finder and Kalman track fitter modules, resulting in minor speed improvements. In addition, updates to the persistent data objects, utilizing the fast I/O streamers built into the ROOT framework, were introduced. These changes yielded $70 \%$ improvement in the time to write reconstructed events to disk and a $30 \%$ improvement in the overall per event processing time. Several more significant code improvements are planned for next quarter. 


\section{Analysis Code Profiling}

During the analysis of raw data from the 2011-12 LANSCE run cycle, it was quickly identified that the analysis was taking a remarkably long time to complete, particularly for the Pu239 data. It was not immediately obvious which part of the analysis chain was causing this, or even if it was due to a particular module in the chain, so a code profiling effort was undertaken to better identify any critical bottlenecks or inefficiencies.

Valgrind (www.valgrind.org) is an open-source programming tool suite capable of identifying memory leaks, memory cache use, and call tree profiling, among other things. It accomplishes this by first converting the original binary executable into a processor-neutral intermediate form, which is then run on a virtual machine. The resulting execution speed is $20-100$ times slower than the original, but the executable otherwise performs identically. Complete call tree and memory usage statistics are collected and reported during the execution.

The profiling was performed for the full production analysis chain for 1000 events from the $U$ and Pu run. Some results are shown in Table 2 and Table 3.

\begin{tabular}{|l|c|}
\multicolumn{1}{l}{ Function } & $\begin{array}{c}\text { Percent of total } \\
\text { execution time }\end{array}$ \\
\hline NiffteRootlO::WriteEvent & $40.8 \%$ \\
\hline TPCHoughD::ProcessEvent & $27.5 \%$ \\
\hline TPCKalman::ProcessEvent & $25.1 \%$ \\
\hline
\end{tabular}

Table 2. Percent of total execution time for selected functions in the NIFFTE production analysis chain, as reported by Valgrind.

The obvious conclusion from Table $X$ is that writing the events to disk is taking a significant portion of the total analysis time. CalPoly collaborations quickly identified that NIFFTE data is stored in C standard template library arrays, but could instead be stored in ROOT TArrays which would reduce the amount of data reformatting required when writing events to disk. Preliminary estimates of switching from STL arrays to TArrays in the NIFFTE analysis code implied a potential speedup of 2.8x; see the corresponding subsection for more details on that analysis.

\begin{tabular}{|l|c|}
\multicolumn{1}{l}{ Function } & \multicolumn{1}{c}{$\begin{array}{c}\text { Percent of Hough } \\
\text { execution time }\end{array}$} \\
\hline ceil & $5.6 \%$ \\
\hline $\cos$ & $31.2 \%$ \\
\hline $\sin$ & $30.2 \%$ \\
\hline ResetHistogram & $10.8 \%$ \\
\hline
\end{tabular}

Table 3. Percent of total execution time for selected functions in the NIFFTE production analysis chain, as reported by Valgrind.

Once the data storage format has been changed, the file I/O should drop to roughly $20 \%$ of the total execution time, and the optimization of the analysis code will become more important. Table $\mathrm{Y}$ demonstrates areas of potential improvement for the Hough 
track finder. In particular, standard library calls for sine and cosine trigonometric functions take over half of the processing time. Provided that the required resolution is achievable, this could be significantly sped up by replacing the function calls with a lookup table. As another example, the ResetHistogram() function simply clears the contents of a histogram to zeroes, but the existing code was using an inefficient nested loop to do so.

Several easy optimizations are also present in the Kalman filter module. Improved code for the issues described above are being submitted for the next release of the analysis code, and Valgrind will be used to continue optimization of the NIFFTE production chain.

\section{Track Reconstruction using the Combinatorial Hough method}

The software profiling work identified the Hough subroutine as a place for some performance improvement. The Hough subroutine identifies hits in the TPC that fall on the same line in $2 \mathrm{D}$ space. The Hough transform maps points in $(X, Y)$ space to a polar coordinates space $(\rho, \theta)$ where lines are represented by angle and offset from the origin. The idea is that points that fall on the same line in $(X, Y)$ space will map to the same point in the $(\rho, \theta)$ space. Searching the $(\rho, \theta)$ space for the most intense regions will give the points (digits in the TPC analysis language) that are all on the same line. Some minor inefficiencies in the search and initialization routines were identified and fixed. Those resulted in only minor performance improvements. The major inefficiency in the code is fundamental to the routine and involves filling the $(\rho, \theta)$ space with the family of lines that all go through the $(X, Y)$ point. The number of lines allowed to pass through that point should be large for precision results but small for the routine to complete quickly. By definition, the vast majority of the looping will be for lines that are not representative of the data.

A potentially faster method is to use the Combinatorial Hough transform. Instead of cycling through hundreds of lines for each point, each pair of points in the collection of digits for a particular event is cycled through and the $(\rho, \theta)$ points representing the line between those points are recorded. Again, the most intense region of $(\rho, \theta)$ space then represents the line that encompasses the most points. This method will contain fewer iterations as long as the number of digits remains less than half of the number of lines tested in the original algorithm. For events with small numbers of digits, the Combinatorial Hough routine should be substantially faster.

A study was performed to test the performance improvement. The results for both track reconstruction routines were compared using the first 1000 events of a typical uranium target run (400000487) and the first 1000 events of a typical plutonium target run (400000838). The results of the study are shown in Table 4. The Combinatorial Hough method is certainly faster and the improvement is even more for the Pu data. Due to the large intrinsic activity of the plutonium, a higher detector threshold was used resulting in shorter stored tracks. These shorter tracks have fewer digits and so the percent improvement is larger for the Combinatorial Hough as would be expected given the two routines different dependencies on the number of digits mentioned above.

Table 4. Study of the time to convert from a raw file and perform track reconstruction for the first 1000 events using the original Hough and the Combinatorial Hough routines for the two different targets. 


\begin{tabular}{|c|c|c|c|}
\hline Target & $\begin{array}{c}\text { Time to execute } \\
\text { with } \\
\text { Original Hough (s) }\end{array}$ & $\begin{array}{c}\text { Time to execute with } \\
\text { Combinatorial Hough } \\
(\mathrm{s})\end{array}$ & $\begin{array}{c}\text { Percent } \\
\text { improvement }\end{array}$ \\
\hline Uranium & 9.17 & 7.65 & $17 \%$ \\
\hline Plutonium & 6.72 & 4.22 & $37 \%$ \\
\hline
\end{tabular}

An initial assessment of the performance of the Combinatorial Hough was completed. In some regions of the phase space there was good agreement between the original and Combinatorial routine while in other regions, there was less agreement. However, an examination of the tracking results for both algorithms reveals that neither is optimal yet. There are adjustments and optimizations that need to be made to both algorithms to allow for more reliable track reconstruction. Examples of the necessary adjustments are the weighting method used for each event, the width of the tracks, and the coarseness of the $(\rho, \theta)$ space. Further study has begun using simulated data to compare the strengths and weaknesses of the two routines and hopefully lead to improvements in the algorithms. The initial particle conditions are better controlled in the simulation which allows the different algorithm behaviors to be examined in more detail.

\section{Tracking Algorithms}

A new 3D Hough transform track finding algorithm was implemented in the offline framework and is currently being debugged and tuned. Initial comparison between the new and old algorithm vs. Monte Carlo simulations indicate that we can expect some improvement in tracking accuracy once the new algorithm is optimized.

\section{Offline Utilities and Data Processing}

A web-based software to manage shifts for the LANSCE run was installed and configured, and an extenisve shift page was setup for shifters. The shifts have been going well and we are getting good participation from folks to run the shifts around the clock.

New bug tracking system (TRAC) is installed and in use to track and prioritize both software and hardware related issues.

Automatic data transfers form LANL to LLNL are now in production mode. Run data is archived on tape within 2 hours of being taken.

\section{Data Acquisition Software [ACU, LLNL]}

This effort will develop all the software required to control the TPC experiment and log the data. An experiment control interface will be developed to allow collaborators to run and monitor the experiment from remote sites, including a slow control system with appropriate interfaces. The front-end cards for the TPC will be quite powerful and flexible because of the Field Programmable Gate Arrays (FPGA). The FPGAs do require programming which we will organize in a framework of modules (each module representing one task) for easy reconfiguration of the device. The modules that would be written for the FPGA would include (1) an ADC receiver that interfaces with the 
ADC chip, sending and receiving clock signals, receiving the serial data and presenting the data in a pipeline for the next module, (2) preprocessing modules would work with the data before zero suppression, and would include functions such as, ballistic deficit correction, fast proton timing, rebinning, and digital shaping.

\section{Software Updates}

The online software was used extensively during the WNR operation period by remote shifters and local operators. Monitoring for EtherDAQ status, TPC data rate, and Ethernet traffic helped to identify several cases of degraded communication with multiple frontend DAQ cards. The hardware configuration change that coincided with these communication issues was reverted.

The overall data rate from the U238 and U235/U238 wedge combo targets is 200 $\mathrm{kB} / \mathrm{s}$ with properly defined trigger threshold. The beam trigger mask was not completely deployed for this run cycle so the threshold was set very high to reject most of the alpha background in most of the Pu data runs. In this case, it turned out the data rate was at $700 \mathrm{kB} / \mathrm{s}$ as seen from Figure 96. Effort was taken to record data with low trigger thresholds for the Pu target as well to test the performance of the whole system chain. Thresholds are set up as 4up, 3 down for most of the channels while few noisy channels were configured with slightly higher thresholds. Figure 97 shows the reconstructed track length vs ADC from a part of a low threshold run. As expected, it appears that the most events are from alpha decay.

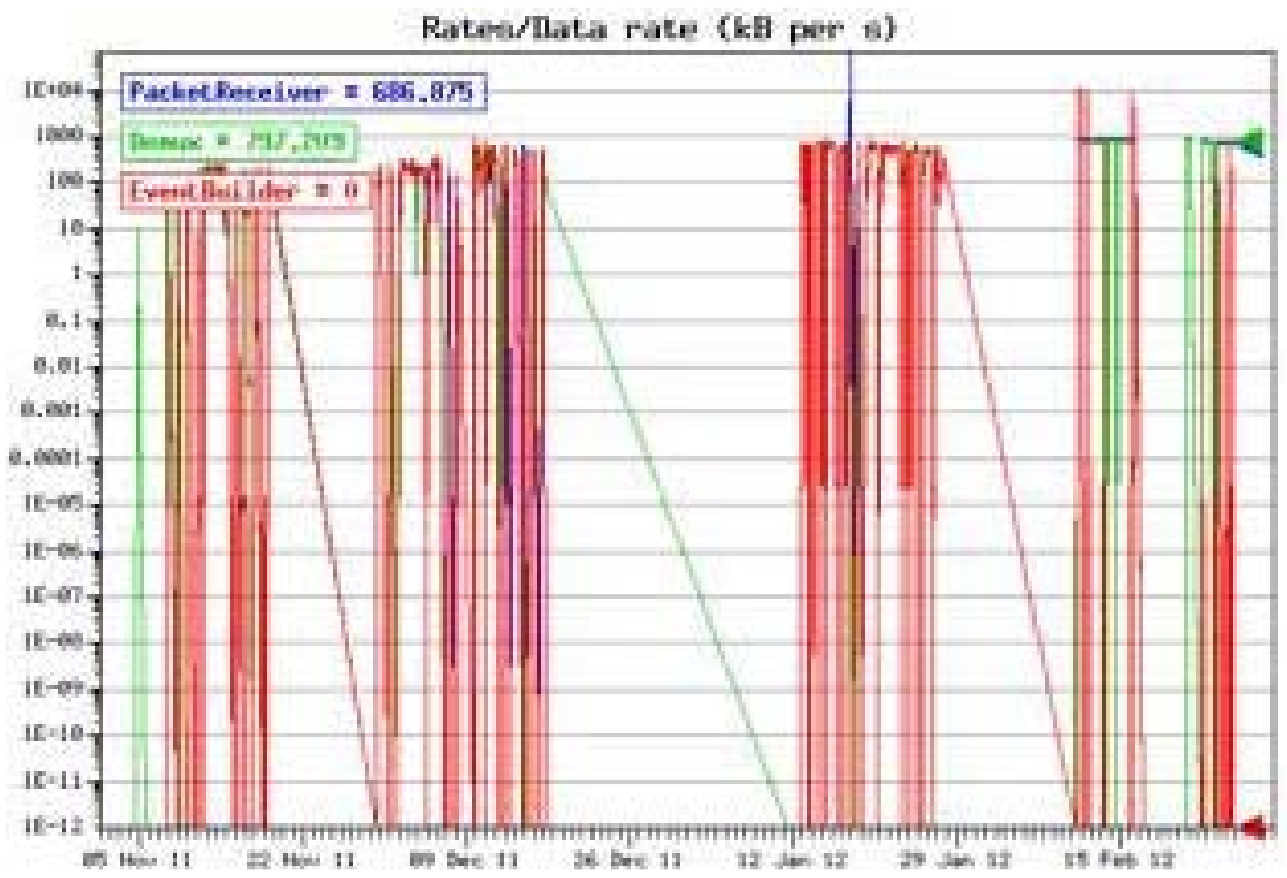

Figure 96: Shown here are the data rate monitoring for the run period. 


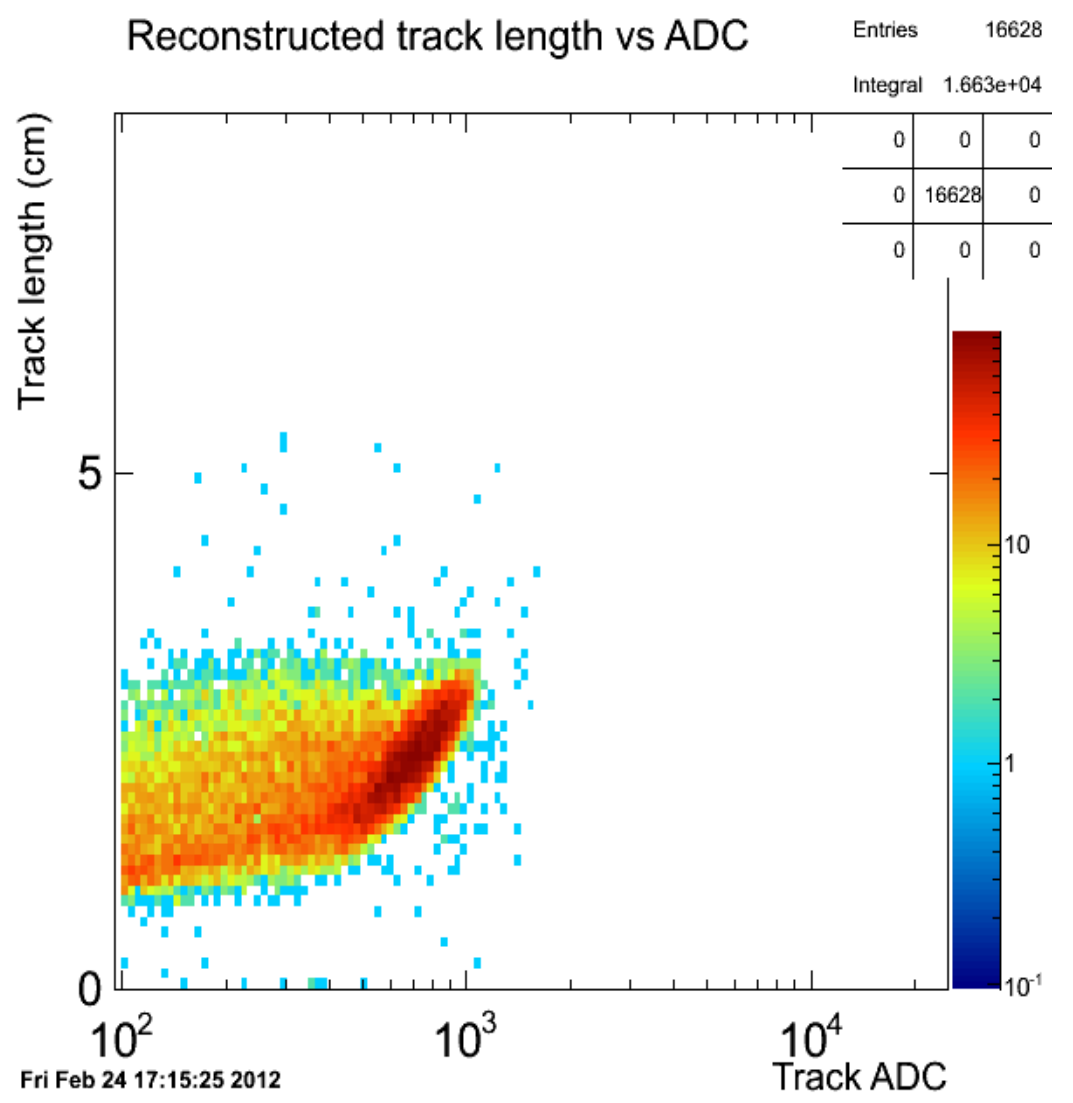

Figure 97: Shown here is the reconstructed track length with the ADC distribution from a portion of a low threshold Pu run from online monitoring. The run conditions were: pressure 972 torr, FCT/FCB/MMG -1200/-339/-340 V, average beam current 1730 nA, Lookback=65.

\section{Simulation [ISU, LANL]}

\section{Scope}

In order completely understand how the TPC will respond to various neutron environments and to accurately determine the fission parameters of uranium and the minor actinides, a complex simulation effort will be undertaken. The environments that the TPC will be used in will require accurate modeling of the detector systems used as well as the neutron production. MCNPX will be used to model the experimental setup at both the LANSCE, the quasi-monoenergetic neutron source at LLNL and Ohio University mono-energetic experimental facilities. These fully detailed four-dimensional models (3D space and time) will be used to create the source term for the GEANT4 modeling of the detector itself. Since MCNPX does not have the ability to transport heavy fission fragments, GEANT has been selected for this task. GEANT only has data for uranium fission events in the G4NDL library and the data for the remaining fissionable isotopes is based on a low precision neutron yield model. GEANT will need to be modified to use the Los Alamos model, also known as the Madland-Nix model, in which fission data will be added for U-238 and Pu-239 and the minor actinides. The modified GEANT module will allow the user to select the Los 
Alamos model or a fission distribution file supplied by the user. The fission fragmentation model will also be added to this module. To allow for a full model of the detector, another GEANT modification will be the addition of a static electric field modeling capability. This module will be used to accurately model the gas electron amplification inside the detector system. This will allow GEANT to completely model the detection system from birth (through MCNPX) to charge collection in the TPC pads. Using the high fidelity models of the experimental setup facilities, a series of databases will be created for various isotopes. This will allow for rapid comparison with experimental data.

\section{GARFIELD Simulations}

As mentioned in the previous quarterly report, a particular type of track defect was identified for fission fragments, where a much slower data collection rate occurs for a subset of the charge. The hypothesis presented at that time was that the much larger number of electron-ion pairs being generated by fission fragments was resulting in a significant ion cloud in the region of the track, which was affecting the drifting electrons. An example from actual data is shown in Figure 98.

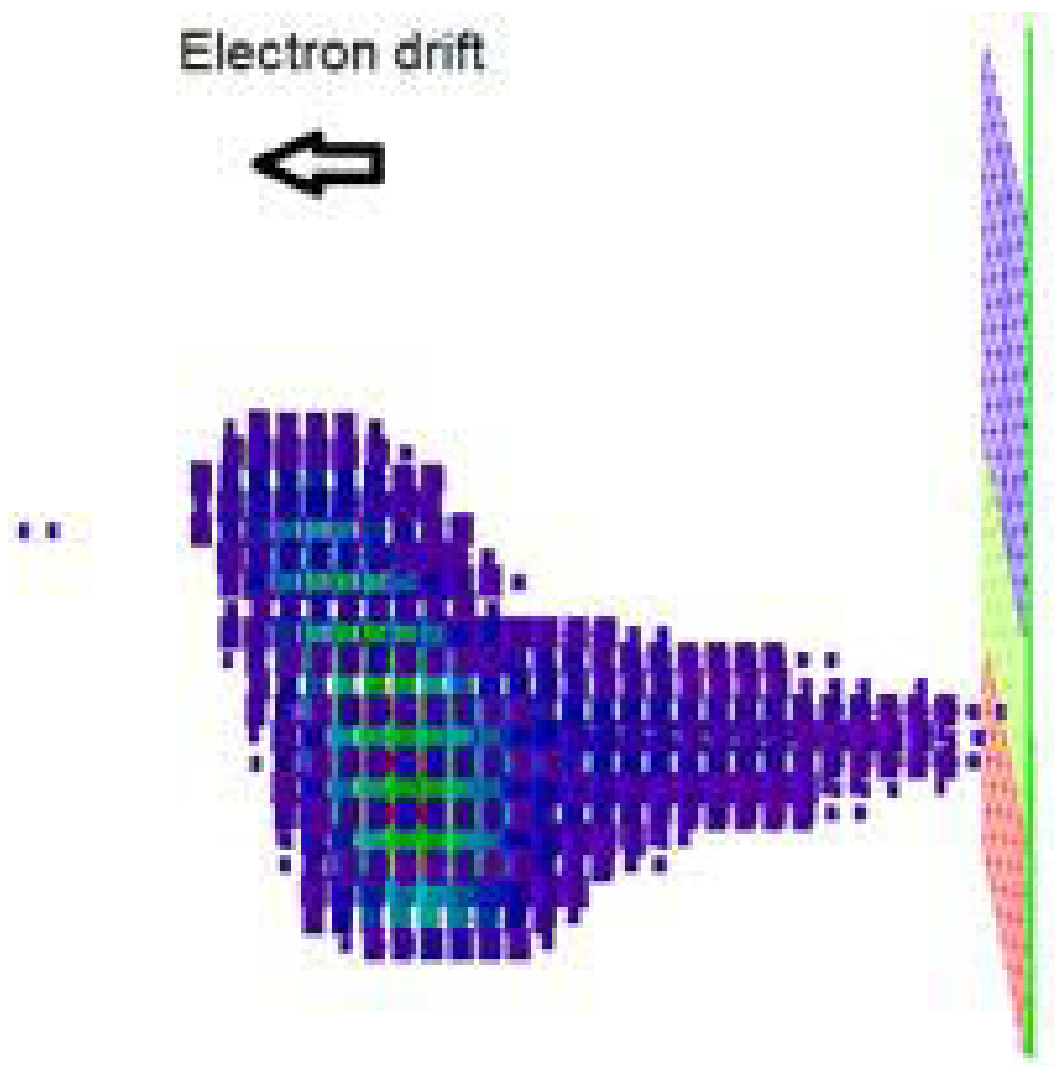

Figure 98. Example of the delayed drift time effect in actual data (electron drift and collection occurs to the left). The "tail" of charge to the right of the track actually represents electrons arriving later than expected. The track has incorrectly identified it as a separate track. 
To better study this, a simple GARFIELD simulation was run, with the simple geometry of two $8 \mathrm{~cm} \times 8 \mathrm{~cm}$ plates with voltages of OV and $-840 \mathrm{~V}$ placed $5.5 \mathrm{~cm}$ apart representing the TPC. Provided that the drifting electrons stayed near the center of the plates, then the lack of field cage had only a minor effect on the simulation. To get around limitations of GARFIELD, which assumes that all defined shapes are solids and thus block electron drift, an electric field was calculated with an external tool and imported into GARFIELD. The finite element analysis tool Maxwell was available and used for this effort.

With Maxwell, cylindrical charge clouds of radii from 10um out to 100um were generated horizontally, and electrons drifted from a line below and perpendicular to the cloud drifted past (and through) it. If the hypothesis was correct, then electrons that drift through the cloud should show longer drift times than those that drift some distance from it. Figure 99 shows results for radii of 10um, 25um, and 100um.

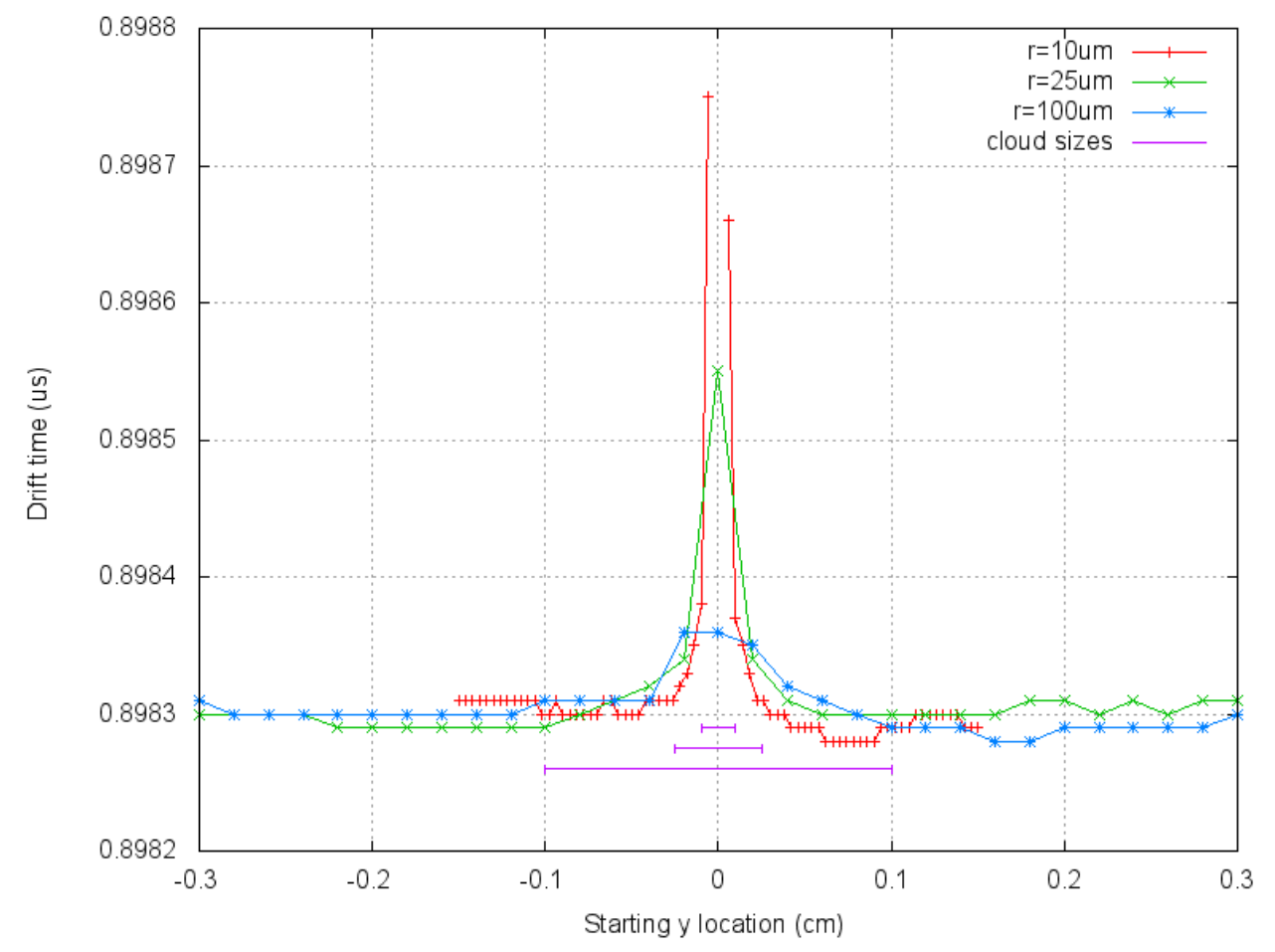

Figure 99. Calculated drift times for electrons drifting

While it can be seen that electrons that pass through the charge clouds have longer drift times, and the drift times increase as the charge cloud radii decreases, the effect is only about $1 / 1000$ as large as has been identified in the actual data.

Investigation of the parameters used in these simulations is underway to identify any potential errors in the analysis. In addition, analytical estimates of the effect are now being undertaken to confirm the magnitude of the effect as seen in simulation. 


\section{Improvements to Particle Transport and Event Generation inside Geant4}

Several improvements have been made to the GEANT4 geometry of the TPC in order to be able to simulate the different target designs that were proposed for the CY2012 run cycle. These improvements include the ability to insert different sections of the source to simulate the wedges or separate circles (see Figure 100). Other improvements include how the position of the event is chosen.

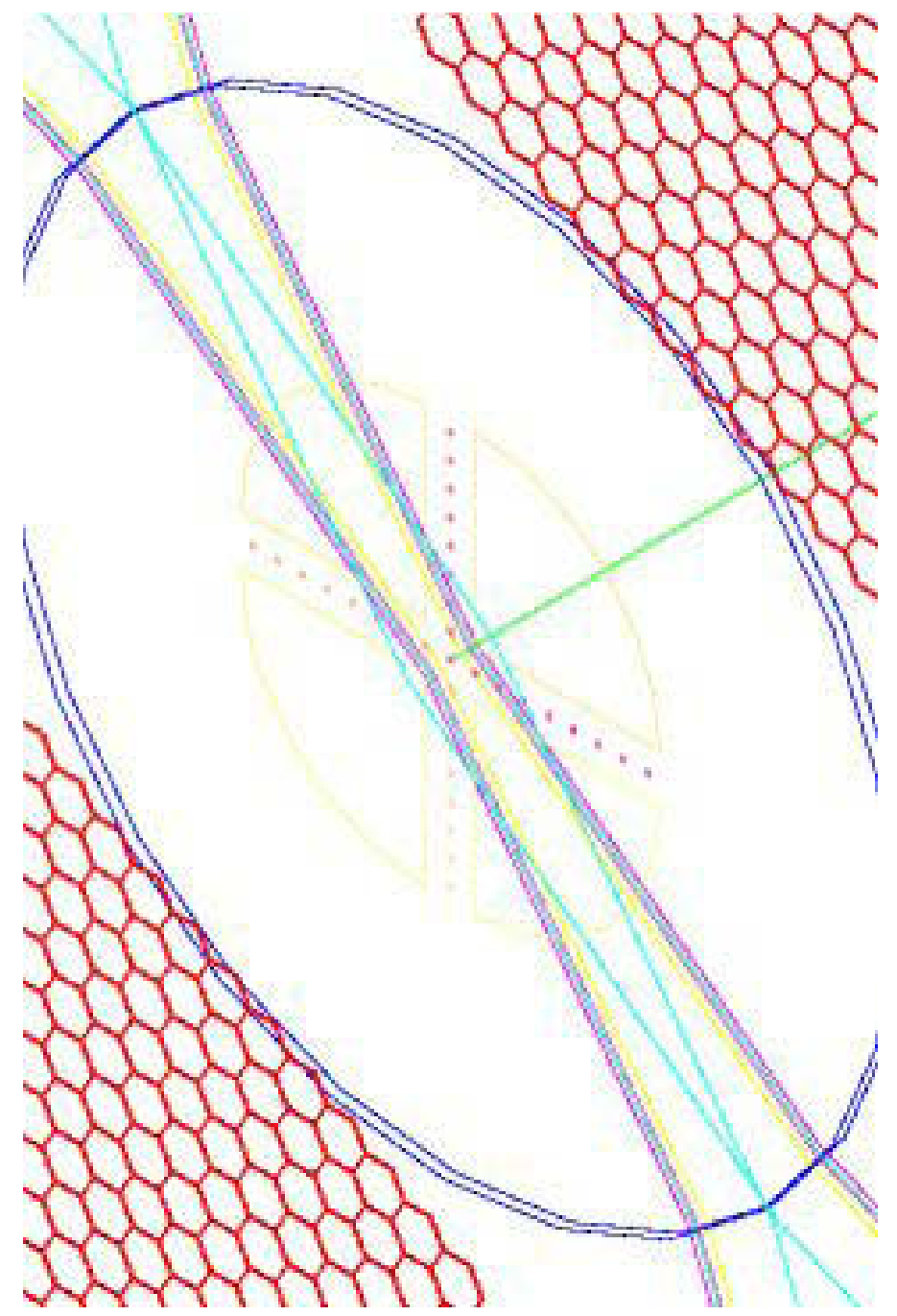

Figure 100. Source material shown in Geant4 as a cross and four wedges.

The initial positioning part of the event generation is now running inside of Geant 4 as an independent module, and the user has the ability when running Geant 4 to choose which method should dictate the starting location of the event. The methods include "fixed", "uniform", "uniform based off source intensity", and "histogram". Uniform based off source intensity chooses the source insert according to the intensity and then uniformly chooses the start position inside that insert.

Similar improvements have been made to the G4FissionFragmentGenerator where only a fixed neutron energy was available. The user now has the ability to choose between a fixed, uniform between given range, absolute Gaussian, user defined 
histogram, and published spectrum for LANCE-WNR 90L beam. The details of the incident neutron are now also saved in the output data file as an MCParticle.

In addition, the user can now turn off multiple scattering while running Geant4. This was added to test how the reconstruction changes with and without multiple scattering.

Kapton dielectric, electric field, and the pressure vessel were added to the default geometry, to better simulate what happens when a particle interacts with the edge of the active volume.

\section{Improvements to Detector Response Simulations}

In order to better understand the results coming from the TPC, two cross talk modules have been created for the detector response simulations. One module is to simulate the cross talk between traces on the pad plane and the other to simulate the cross talk between traces on the preamplifier card. Each module at the moment assumes a uniform percentage of charge shared to all nearest neighbors for every trace.

To be able to test out a new track fitter, a module to normalize the $\mathrm{dEdx}$ for one track events was added to the detector response simulations. The module will only work for one track events using a hexagonal geometry. Full tests of the module are currently ongoing.

\section{MCNP Simulation of the Fission Ionization Chamber}

In an effort to better characterize the neutron source and neutron interactions in the TPC volume, including the scattering and room return effects, a new MCNP model of the 90L station and TPC volume is being developed (see Figure 101). The basic MCNP geometric model of the area is based on recent measurements and photographs, and will be benchmarked against published data from a standard fission chamber (Figure 102) used in that location. A realistic TPC volume will then be added to the MCNP model.

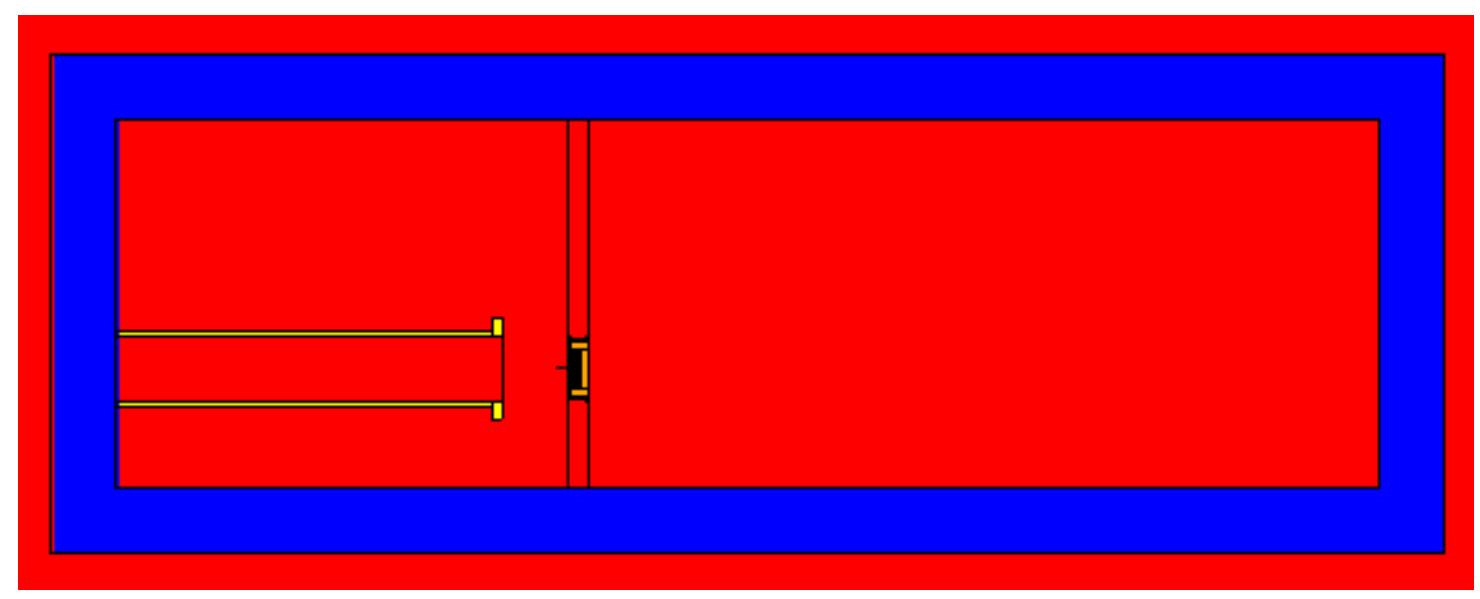

Figure 101. A side view of the MCNP model of the 90L station. Each individual color represents a particular material. 


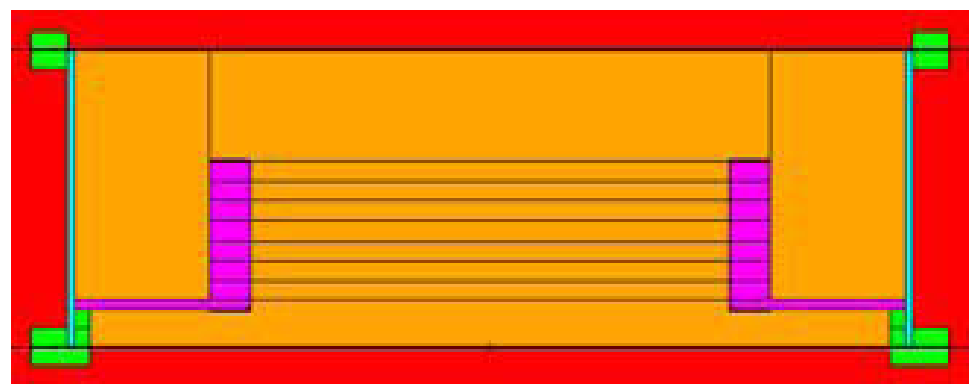

Figure 102. An updated cross-sectional view of the representative fission ionization chamber.

\section{Delayed charge analysis}

Last quarter, GARFIELD simulations were run to identify whether the ion cloud left by a fission fragment traversing the TPC was potentially the cause of the "delayed charge" effect seen on fission fragment tracks, where a small portion of the charge was collected much later than the rest of the track (see Figure 103). The hypothesis was that electrons that had to drift through the ion cloud were being delayed by the effects of the ion cloud on the local electric field. The preliminary simulation results indicated that a delaying effect was occurring and that the effect was greater for narrower ion clouds, but that the magnitude was about three orders of magnitude too small to match what was seen in the TPC data. The results were rechecked for accuracy and confirmed.

Secondary electron emission from the cathode plane (or target itself) was investigated as a possible source of the delayed charge as well. As a highly-charged fission fragment traverses a metal, it generates secondary electrons due to both Coulomb interactions with atomic electrons as well as knock-on electron generation. At depths in the metal below about $10^{-7} \mathrm{~cm}$, the electrons tend to recombine in the metal, but near the surface these electrons can escape (Sternglass, 1957).

Measurements of secondary electron emission from $\mathrm{UO}_{2}$ metal have been shown to produce hundreds of electrons per fission fragment, depending on the metal thickness (Anno, May 1962), so the number of electrons is sufficient to potentially provide a signal in the TPC.

Whether the geometry of secondary electron emission matches the signal is currently under investigation; the interplay between the depth of the interaction in the metal and the $\mathrm{dE} / \mathrm{dx}$ deposited per unit length makes it unclear whether the observed delayed charge matches the expected distribution. 


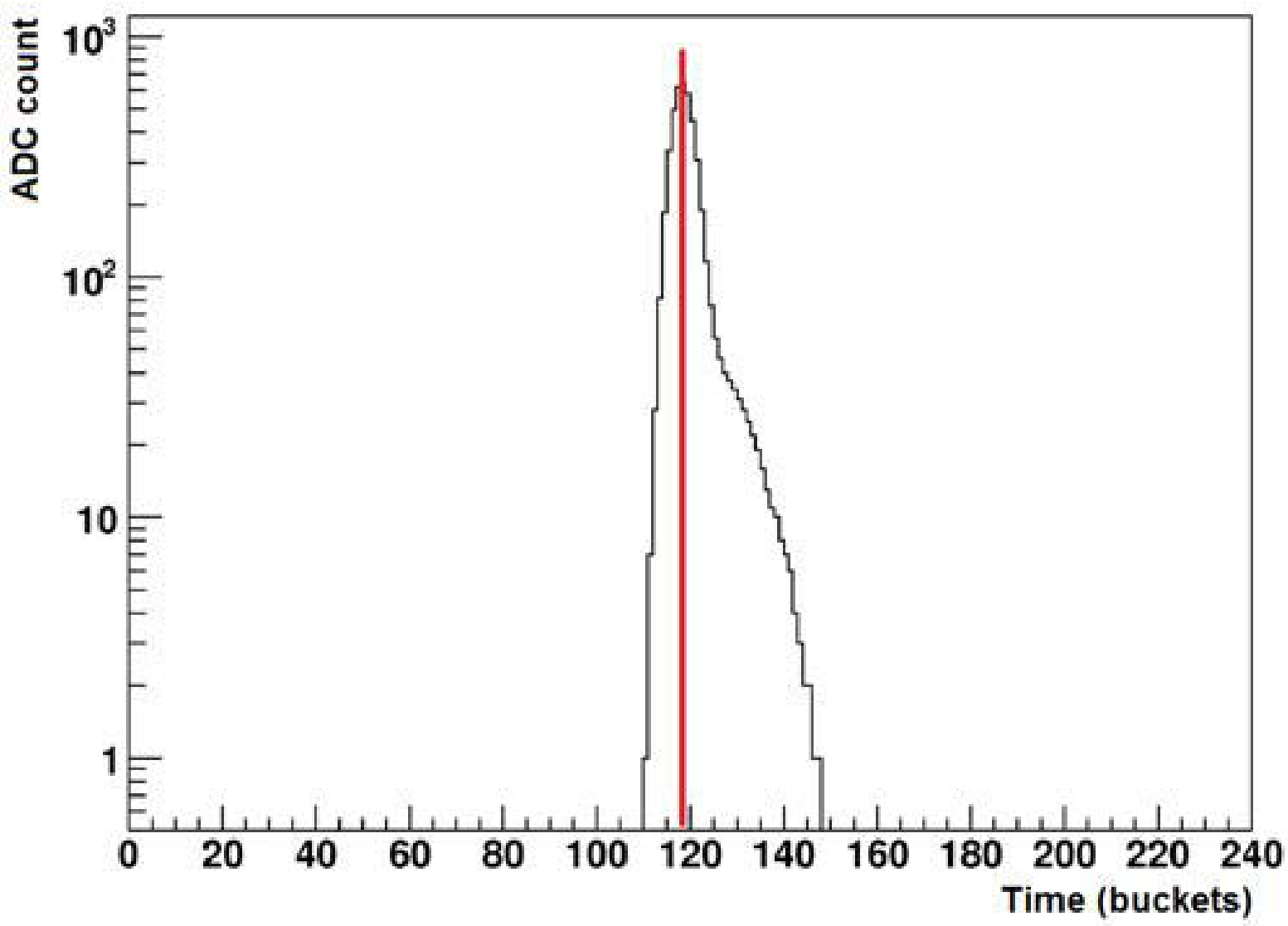

Figure 103. $A D C$ count vs. time for a single channel. The red line represents the mean value of a gaussian centered on the charge from the primary track. The delayed charge cloud is evident in the additional counts to the right, signifying a later arrival time.

It was identified that the ability to automatically select events with the delayed charge was necessary, and so the track geometry of those events was investigated. The presence of two tracks in the event was required as a simple method of selecting events of interest, since the tracker frequently fit the delayed charge tail with a track; plotting the total ADC against the number of tracks per event (see Figure 104) demonstrates that 2-track events are more likely to have higher ADC values and hence contain a fission fragment. On top of that selection criteria, an azimuthal angle cut on the primary track of greater than either $45^{\circ}$ or $80^{\circ}$ was added to identify any dependence on primary track angle.

It should be noted that the track's azimuthal angle has several obvious effects on the identification of the delayed charge cloud as a secondary track. First, if we assume that the delayed charge cloud always points back to the cathode plane (which is what is observed), then the tracks will tend to combine primary tracks with low azimuthal angles with any potential delayed charge cloud, since they essentially lay on top of each other. Second, primary tracks with azimuthal angles of $30-45^{\circ}$ tend to "steal" charge from any delayed charge cloud, essentially bending the charge cloud's track identification in the same direction as the primary track. Only at larger angles such as 
$80^{\circ}$, where the delayed charge is at nearly right angles to the primary track, is this effect minimized.

Preliminary results from this track geometry analysis show that it is possible to select delayed charge events with these simple cuts, but only if the tracker identifies the delayed charge as a separate track. The study will continue in the next quarter to better assess event selection to include events where the delayed charge is not so obvious.

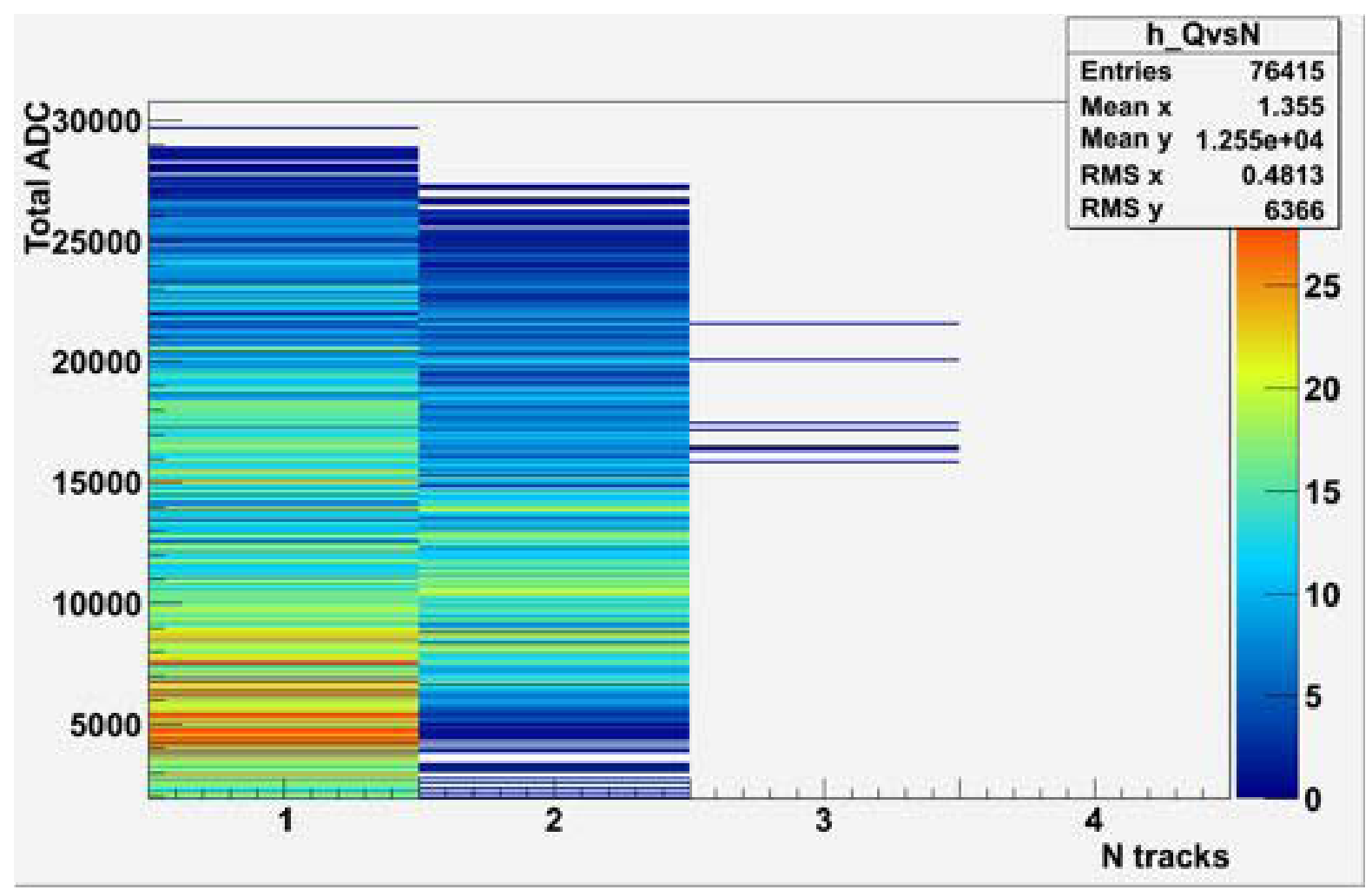

Figure 104. Total $A D C$ vs. number of tracks per event. There is a clear indication that 2-track events have greater $A D C$ values, implying that they are more likely to contain a fission fragment.

In an attempt to better understand the cause of the delayed charge effect, the channel ADC data (such as in Figure 104) was examined in more detail for events exhibiting the effect. The signals for each channel were fitted with a Gaussian shape, and the ADC count outside that peak due to the delayed charge was plotted versus the area of the fitted signal pulse (i.e. total ADC counts). Preliminary results appear to indicate that events with larger pulse heights have a higher likelihood of having a delayed charge tail, but some events with large amounts of charge have no charge tail. Also, the fraction of the charge in the tail to the total does not remain constant, but leads to a range of pulse shapes. The location and direction of the tracks is being examined for correlations with events with delayed charge. 


\section{Data Analyses [CSM, LANL, CalPoly, ISU]}

\section{Scope}

The TPC data is analyzed early in the program to validate operation and design choices. The early analyses include data collected using partially instrumented instruments, using sources and neutron beams, including the proto-type TPC as well as the production versions. These analyses are used to test the complete system and to determine the overall quality of the data through the complete hardware and software chain.

\section{Cf-252 Alpha Decay to Spontaneous Fission Branching Ratio}

Working toward the goal of developing robust particle tracking and identification capabilities, the fission TPC is being used to measure the alpha/SF branching ratio in Cf-252. An initial analysis of the Cf-252 data is complete and the branching ratio was measured to within $1 \%$ of the accepted value. The measurement of alpha/SF was completed with 1 sextant of the TPC instrumented, or $1 / 12^{\text {th }}$ of the total detector, and a $100 \mathrm{nCi} \mathrm{Cf-252} \mathrm{source} \mathrm{mounted} \mathrm{on} \mathrm{a} \mathrm{platinum} \mathrm{backing.} \mathrm{This} \mathrm{is} \mathrm{a} \mathrm{challenging}$ exercise as the limited fiducial coverage leads to effects that constitute the major contributors to the overall systematic errors in this particular study. A fully instrumented TPC will not suffer from these limitations.

The source composition was determined by measuring the alpha decay spectrum of the source with a silicon diode detector. The alpha and fission spectrum of the Cf252 button source as measured by the silicon diode detector can be seen in Figure 105. The source activity was determined to be approximately $97.3 \%$ Cf- 252 at the time of the measurement. The alpha/SF branching ratio was also extracted from this data and was found to be within $1 \%$ of the accepted value. 

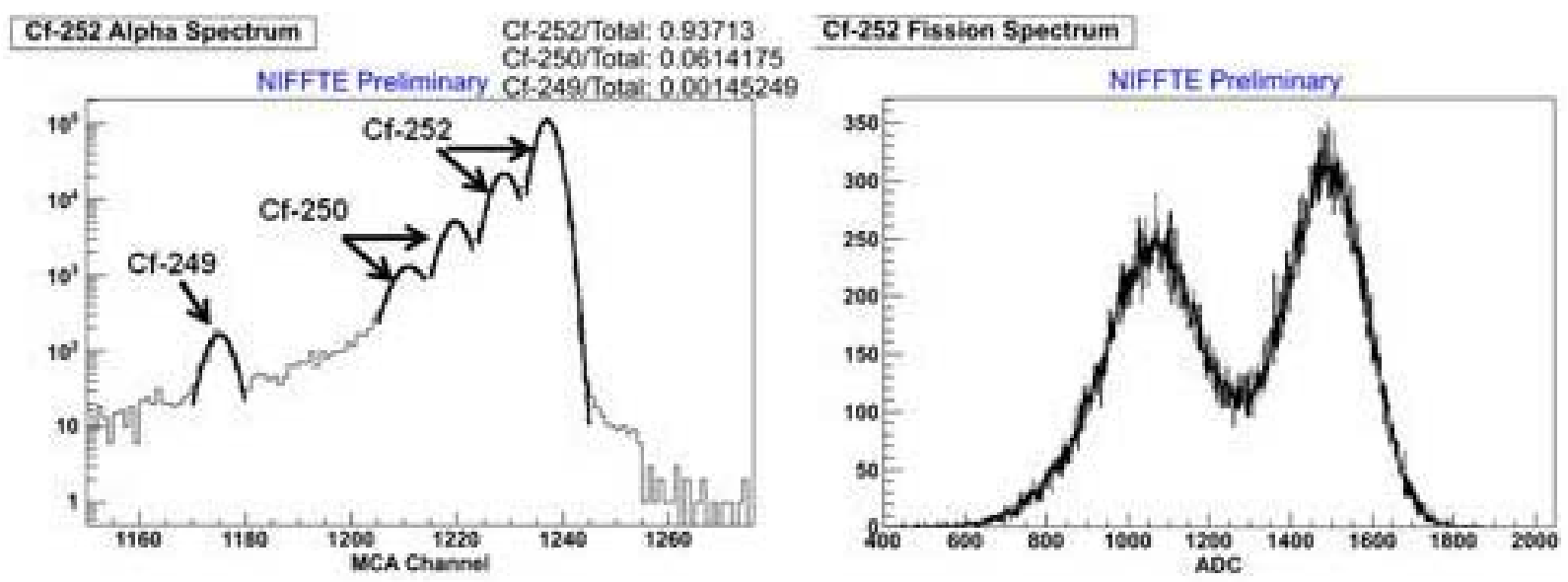

Figure 105: The alpha spectrum of the Cf-252 button source as measured with a silicon diode detector. The plot on the left shows the alpha spectrum, the $y$-axis is in logarithmic scale. The plot on the right is the fission spectrum. The expected major contaminates were Cf-249 with an alpha energy of $5.813 \mathrm{MeV}$ (82.21\%) and Cf-250 with alpha energies of $6.030 \mathrm{MeV}$ and $5.989 \mathrm{MeV}$ (84.61\% and $15.11 \%$ respectively). The expected alpha energies from Cf-252 are 6.118 MeV and 6.075 MeV (84.21\% and $15.71 \%$ respectively). These five peaks are fit with Gaussian functions. The ratios shown on the top right the alpha spectrum plot is the area under the associated peaks divided by the total of all the peak areas.

SRIM simulations and published data were used to account for the effects of the scattering of alpha particles and fission fragments in the source. The Cf-252 source was mounted on a thick platinum backing and covered by approximately $100 \mu \mathrm{g} / \mathrm{cm}^{2}$ of gold. In principle the available solid angle for emission from the sources is $2 \pi$, however it is not expected that the detection efficiency will be $50 \%$ due to the effects of scattering in the source backing and cover. Alpha particles (and in the case of the Cf-252 source, fission fragments) incident upon a metal backing will occasionally scatter through a large angle due to an interaction with a nucleus (Rutherford scattering), and be emitted from the surface of the source and into the active area of the detector. Even more often than Rutherford scattering, an ion will undergo multiple scattering at small angles with the atomic electrons, the cumulative effects of which can result in backscattering, particularly if the initial trajectory of the ion was at a grazing angle with the surface of the metal backing. In this context then, backscattered refers to an ion with and initial velocity vector towards a surface which then scatters and is left with a velocity vector away from the surface, not necessarily an ion that is scattered through an angle greater than 90 degrees. Figure 106 shows the polar angle distribution of alpha particles from a TRIM simulation of a $4 \pi$ alpha point source mounted on a thick platinum backing and covered by a thin gold foil, also shown is the data from the TPC. The active volume of the TPC was defined to be between a polar angle of 90-180 degrees, where the polar angle is the angle with the axis that is perpendicular to the plane of the source. An ideal point source would then be expected to have a flat distribution of tracks as a function of the cosine of the polar angle. The simulation and the data both show a predominance of tracks near a cosine of zero, which is near parallel with the surface of the source. This is a result of alphas that have backscattered in the platinum backing and then been transmitted through the active, gold covered side of the source. 

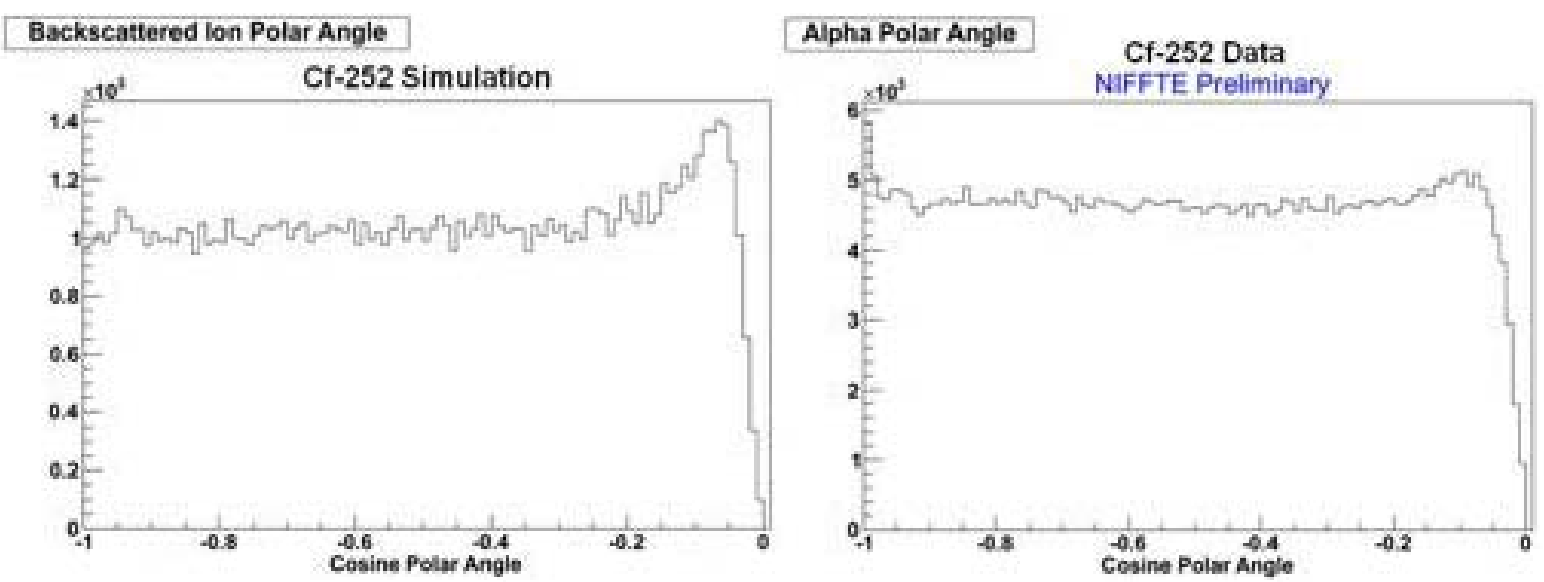

Figure 106: The left plot is a simulation of the Polar angle distribution of the alpha particles that have either been transmitted directly through a $100 \mu \mathrm{g} / \mathrm{cm}^{2}$ gold covering or backscattered off a thick platinum backing and then transmitted through the thin gold covering. The simulation was based on a $4 \pi$ point source of $6.118 \mathrm{MeV}$ alpha particles positioned between the gold covering and platinum backing. Note the peak in the count rate near cosine equal to zero, which is a result of excess particle tracks from backscattering. The Plot on the right is the polar angle distribution of alpha tracks in the TPC. A similar peak in the count rate near cosine equal to zero to the one in the simulation is exhibited. The polar angle is defined as the angle with the axis that is perpendicular to the surface of the source. Cosine equal to zero then is parallel to the surface of the source.

Figure 107 shows the length vs. ADC of fission fragment and alpha particles in the TPC. Graphical cuts show the number of alpha particles and fission fragments and the ratio of the two numbers. The branching ratio shown in the graph must be multiplied by a factor 2 to account for the fact that with a $2 \pi$ source there are at least two chances to detect every fission event. Doing so will give a ratio of approximately 33.36 which is higher than the expected value of 31.34. The ratio has not been corrected for backscattering however. Due to the high nuclear charge of fission fragments, they are generally more susceptible to backscattering than alpha particles resulting in the ratio being higher than expected. The details of a backscattering correction will not be discussed here, but after applying a preliminary correction a ratio of approximately $31.08-31.19$ is calculated (the exact value depends on the details of the application of the backscattering correction). While this value is preliminary, it is within $1 \%$ of the accepted value of the alpha/SF branching ratio of Cf-252. Considering the limitations imposed by having a partial detector and the use of a non-optimum source (i.e. the thick platinum backing which causes backscattering), the results are quite good and go far in confirming the abilities of the fission TPC to track, identify and distinguish alpha particles from fission fragments. 


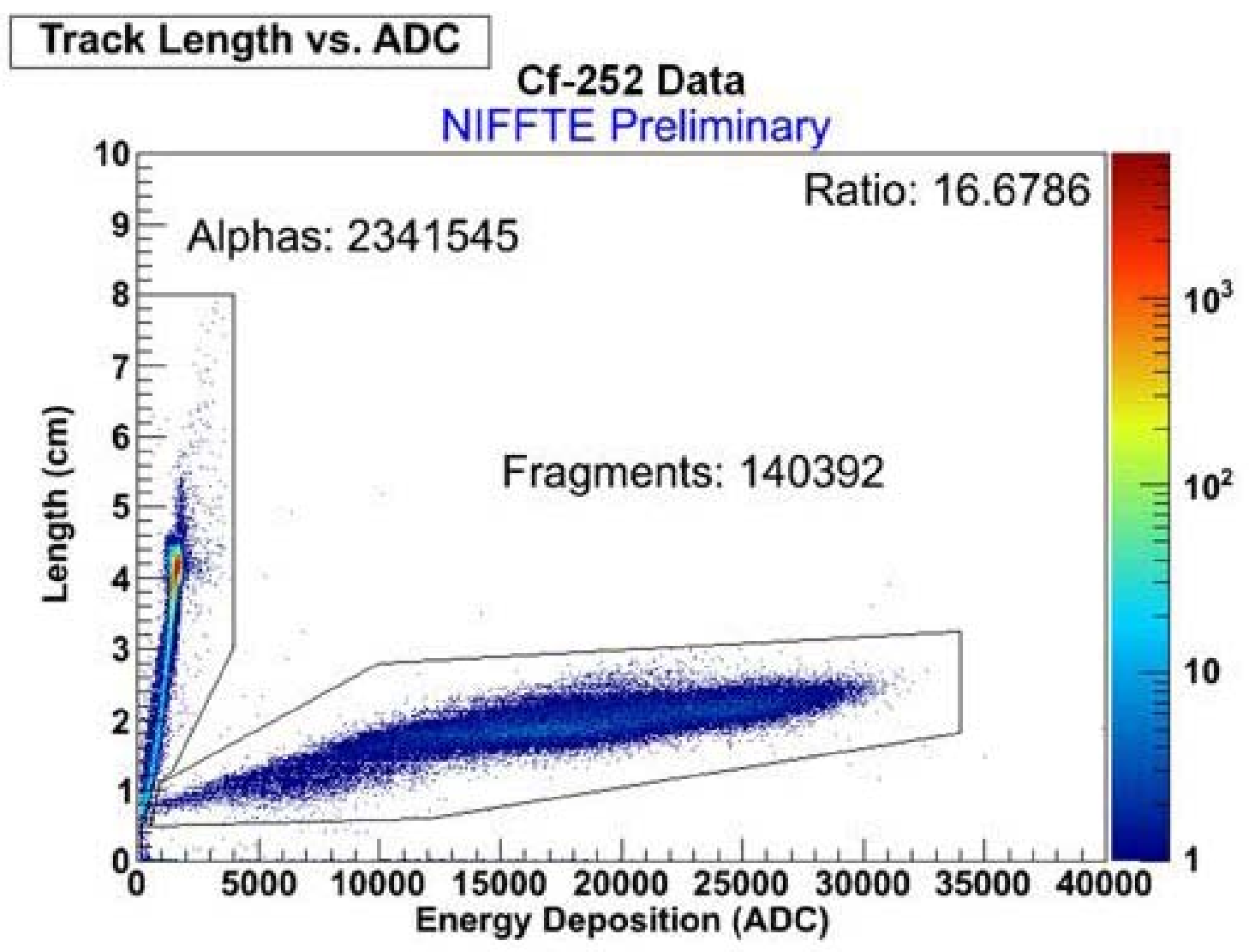

Figure 107: The track length vs. the ADC of particle tracks in the TPC. The color scale is logarithmic. The band on the left at low energy with long track lengths are alpha particles, while the high energy short tracks are fission fragments. The number of fission fragments and alpha particles are based on the graphical cuts drawn. The tracks were between $35-85$ degrees azimuth and 90 to 180 degrees polar.

Luke Snyder from the Colorado School of Mines defended his PhD thesis successfully in March based on the first data from the one segment (1/12) operation of the NIFFTE TPC. His thesis describes an analysis of the alpha to spontaneous decay channel ratio in the nucleus 252-Cf. His results are compatible within error (experiment achieved competitive precision) with previous measurements. Additionally, due to the tracking capabilities of the TPC it was possible for the first time to directly measure the fraction of back-scattered particles originating from the platinum source backing. Given the complex geometry of the partial detector the analysis was very successful and provided an excellent start to the physics program of NIFFTE.

\section{WNR Data Collection}

There are total 950 runs (846 physics runs) of data collected with raw pcap data file size of 2.18TB $(2,183,313,959,341$ Bytes). Figure 108 shows the run number versus the raw data file size. At the end of the run cycle, effort was taken to collect the $\mathrm{Pu}$ beam data with low threshold. It turned out the data rate went up to $\sim 100 \mathrm{MB} / \mathrm{s}$. Few short runs with low threshold were successfully taken with 25GB in about 5 minutes each. The average beam current is in about between 1500 and $1700 \mathrm{nA}$ and is stable 
during operation period (Shown in Figure 109). The performance of TPC electronics shows very stable and calm, the warmest card temperature recorded was never above 38 degree (Shown in Figure 110).

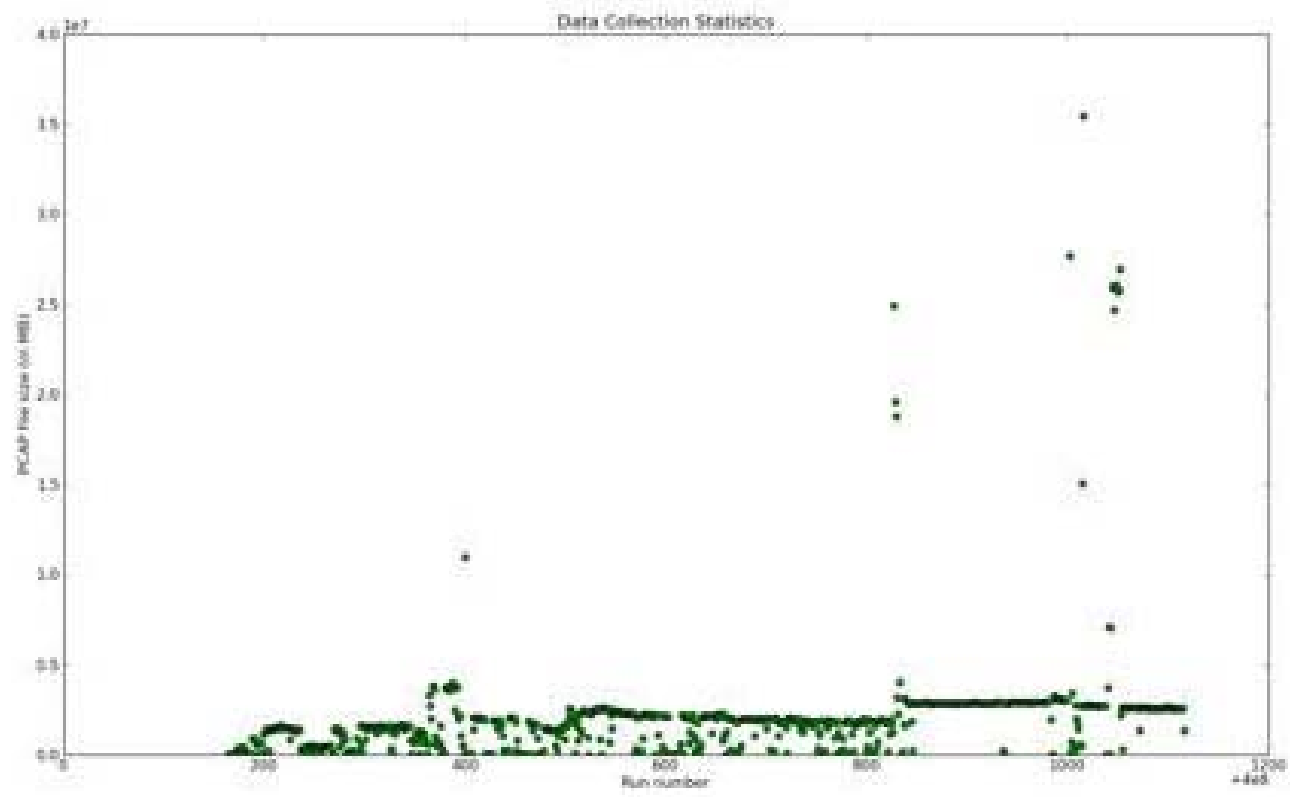

Figure 108: Shown here are the data statistics versus run number.

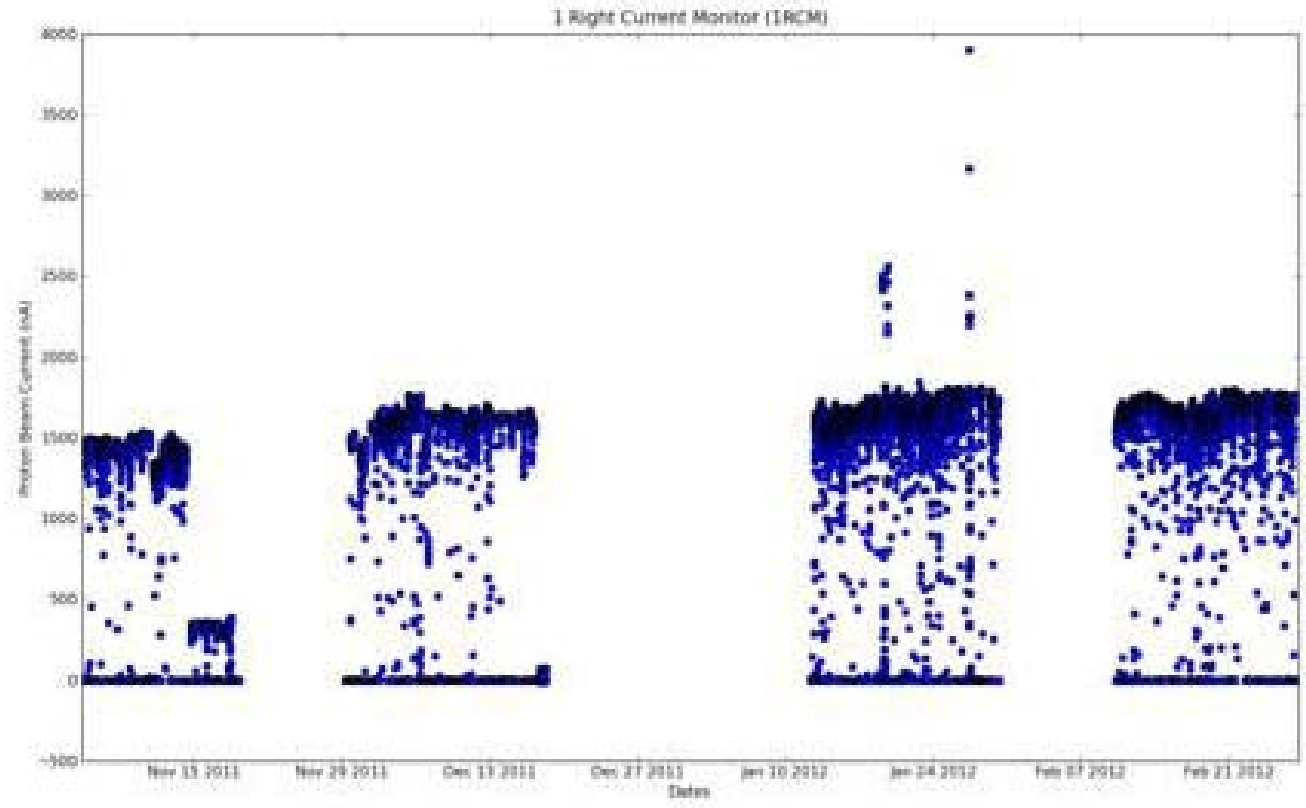

Figure 109: Shown here is the beam current monitoring at the 1 Right path.. 


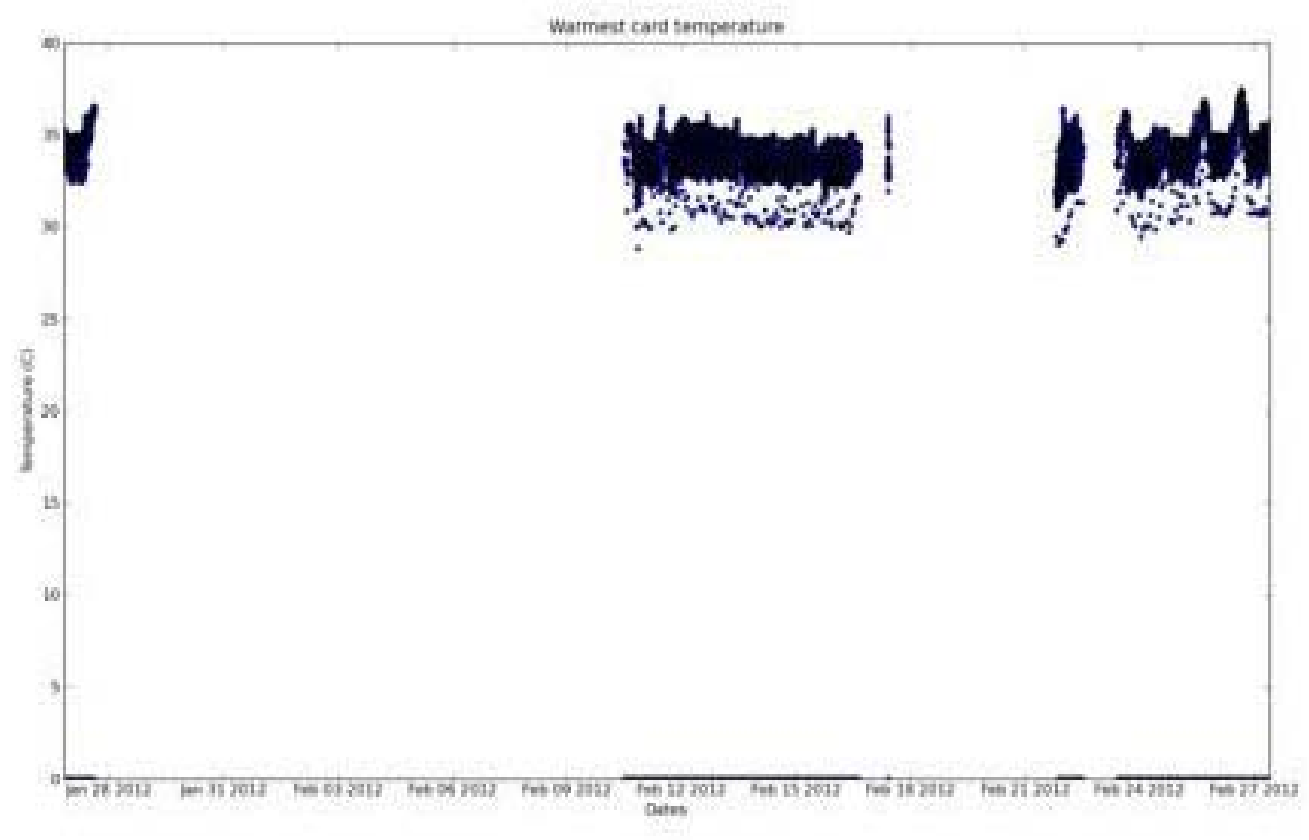

Figure 110: Shown here is the warmest EtherDAQ card temperature variation during data taking.

\section{TPC Data Analysis}

The particle ionization characteristic from both $\mathrm{Pu}$ and Uranium data has been studied to better understand the detector performance, such as the space charge effect from the track density inside the TPC gas volume. Figure 111 shows the specific ionization of alpha tracks from both data set. The overall shape of the ionization along the track looks similar while difference shows at the beginning part of the track. The $\mathrm{dE} / \mathrm{dx}$ shape is lower near the start part of the alpha track from Pu data compared to the alpha track from Uranium data. It could due to some kind of attenuation of drift electrons by gas ions created from high density tracks per unit time for the Pu compared to Uranium. The effect seems to be most significant at the beginning of the track where the drift electrons travel the longest distance to pad plane. Similar effects may be present in recoil proton and fission fragment events but is not distinct for the much smaller deposit energy of proton and the more complicated space charge effect of the fission fragment. 
Track specific ionization

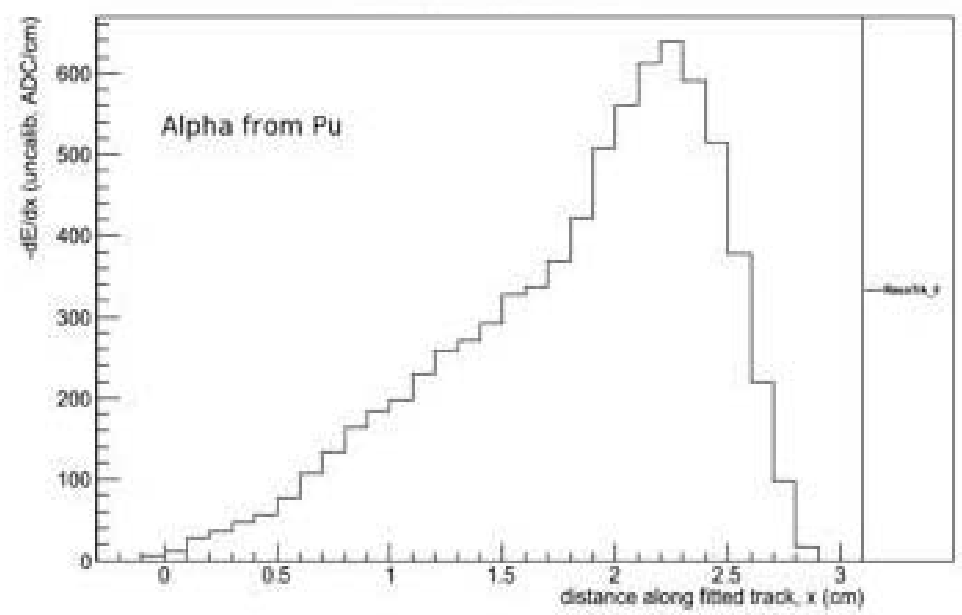

Track specific ionization

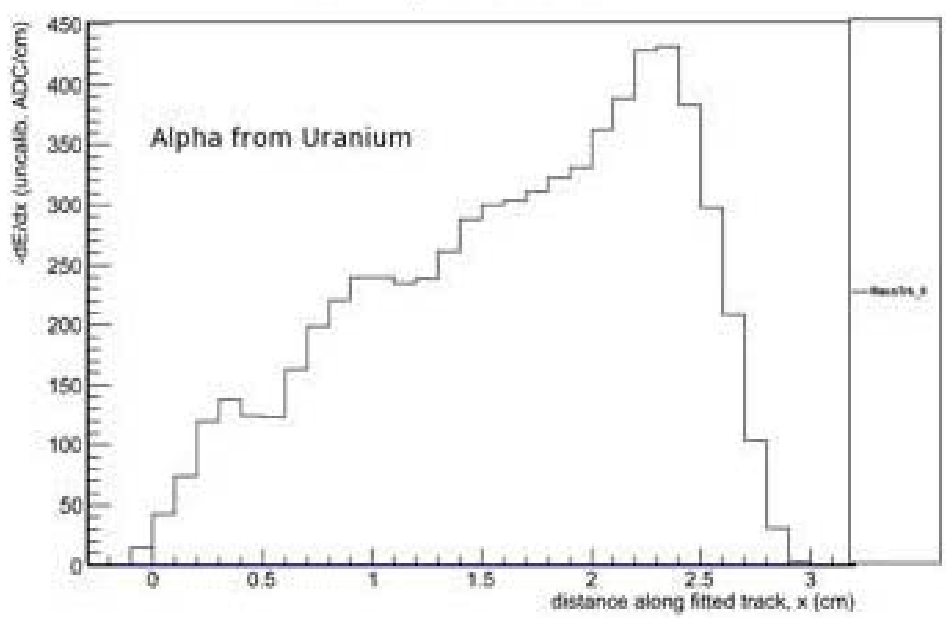

Figure 111: Shown here is the deposited energy per unit length along the reconstructed track of the alpha from the Pu and Uranium data.

\section{Particle identification studies}

The Uranium data set has been analyzed to check and compare the track ionization for the different types of produced particles. Before applying any calibration, the relative ADC comparison is used to identify and separate the types of particles. An identified proton track is shown in Figure 112. The $\mathrm{dE} / \mathrm{dx}$ shows the peak ionization energy is around $100 \mathrm{ADC} / \mathrm{cm}$. The discontinuity of shape of the specific ionization along the track is due to dead channels in the TPC. An alpha track is identified in Figure 113. The track ionization peak is about $500 \mathrm{ADC} / \mathrm{cm}$. In Figure 114, a fission fragment track is identified by the peak ionization energy at the several thousand ADC/cm level. The large charge tail appears at the start position of the real fission track in this image. 


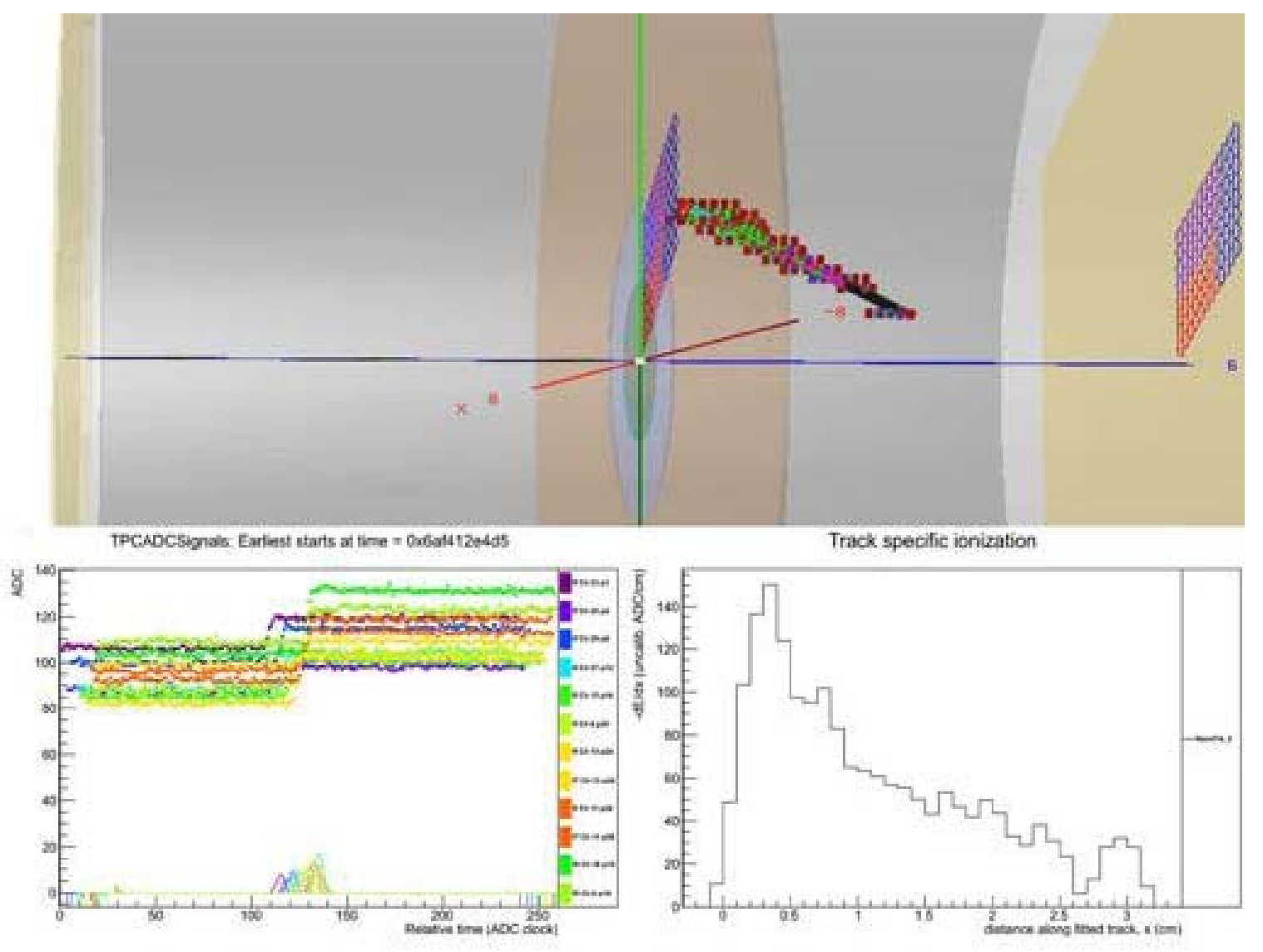

Figure 112: $d E / d x$ of an identified proton track showing peak ionization.
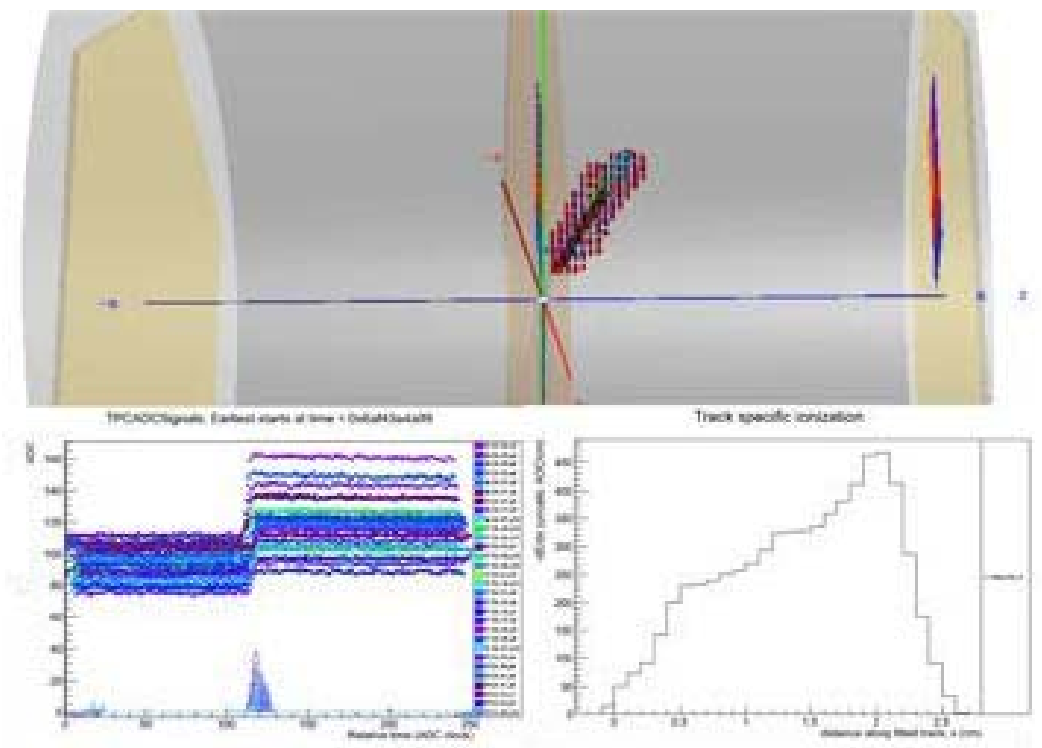

Figure 113: $d E / d x$ of an identified alpha track showing peak ionization. 


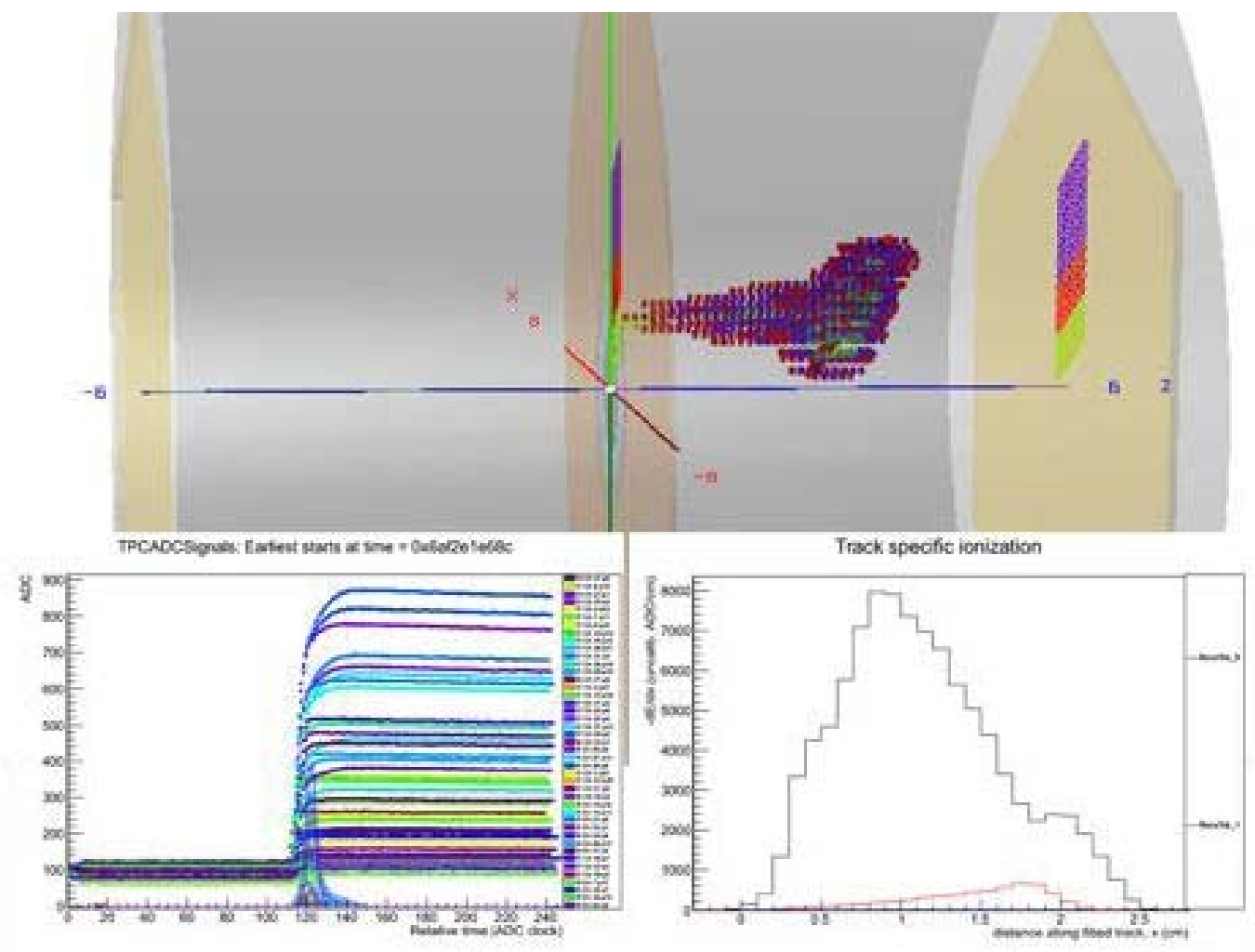

Figure 114: $d E / d x$ of an identified fission fragment showing peak ionization.

\section{The Hydrogen Standard [OU]}

\section{Scope}

The project to accurately and precisely determine fission cross sections hinges on the $\mathrm{H}(\mathrm{n}, \mathrm{n}) \mathrm{H}$ total cross section and angular distributions. The $\mathrm{H}(\mathrm{n}, \mathrm{n}) \mathrm{H}$ total cross section is well determined with errors less than $0.5 \%$. In the planned TPC measurements, hydrogen will to be used as the working gas for a new standards measurement of $U$ 235. The $\mathrm{H}(\mathrm{n}, \mathrm{n}) \mathrm{H}$ angular distribution must be known to connect the total cross section to the measured elastic recoils in the TPC.

\section{Highlights}

- High accuracy neutron detector calibrations are underway.

\section{Hydrogen Standard [OU]}

The TPC offers a major advance in technology for measuring the $\mathrm{H}(\mathrm{n}, \mathrm{n}) \mathrm{H}$ angular distribution. The solid angle for this detector is a factor of 100 larger than that used in our current measurements at $14.9 \mathrm{MeV}$. The target thickness would be comparable. This would result in a counting rate increase by a nearly a factor of 100 . This may allow high accuracy measurements of the angular distributions. A further factor is that the beam need not be as strongly collimated which would give as much as another 
factor of 10 in statistical improvement. This may reduce the experimental running time for a $1 \%$ measurement from 3 months to days. The fitted angular distribution can then be compared to calculations based on potential models such as Bonn or on phase shifts such as Arndt. This may eventually provide a test of the QCD-based model of nuclear forces. We are proposing to collaborate on the modeling of hydrogen scattering in the TPC chamber. We will look at the minimum energy detected. We will also investigate the angular resolution for $\mathrm{H}(\mathrm{n}, \mathrm{n}) \mathrm{H}$ scattering in the chamber. A pure hydrogen atmosphere will be investigated along with addition of quenching and/or scintillating gases. Further work will also be done on determining the gas composition and density to better than $0.2 \%$. The systematic errors in a standard measurement must be fully explored in order to reach the desired goal. The modeling work will be focused on these problems. Consideration will also be given to possible inter-comparison of the neutron standards such as ${ }^{6} \mathrm{Li}(n, \alpha){ }^{3} \mathrm{H},{ }^{10} \mathrm{~B}(\mathrm{n}, \alpha){ }^{7} \mathrm{Li}$ and ${ }^{235} U(n, f)$.

\section{Neutron Detector Calibration}

We are working on calibration of a neutron detector to $1-2 \%$ error in the efficiency using a Cf-238 fission chamber. A measurement of a Cf-238 in a fission chamber has been in progress for over 2 months with good stability. We are investigating some changes that occurred when a pulsed deuteron beam was delivered to another beam leg. This detector will be used in the $\mathrm{H}(n, n) \mathrm{H}$ scattering experiments and in symmetric nuclear reactions to extend the efficiency range from $0.020 \mathrm{MeV}$ to 20 $\mathrm{MeV}$ with similar accuracy.

\section{Facilities and Operation [LANL, LLNL, OU]}

\section{Scope}

Due to the necessity to have a finely tuned neutron beam, with as little contamination as possible, the experimental area needs to be groomed for TPC installation and running. This will mean additional collimation will be needed to adjust the $90 \mathrm{~L}$ flight path to work with the TPC. MCNPX simulations will be made of the $90 \mathrm{~L}$ flight path.

The TPC mount will need to be fabricated. The TPC mount will consist of a 3-axis positioner that the TPC will mount to that will allow for precise positioning of the TPC in the neutron beam. The design specifications will come from the TPC design team, as well as 3 axis movement specifications for fine-tuning in the LANSCE beam. The experimental infrastructure will be partially provided by facilities currently at the LANSCE facility. A good working rapport has been established with the facilities personnel at LANSCE through this collaborative effort.

The TPC experiment will be maintained and monitored while located at LANSCE. The Nuclear Science group employs a number of qualified technicians who will perform the required upkeep and maintenance of the TPC and related systems. The facilities will be maintained to that the instrument will function properly and beams can be supplied to the area. The TPC detector and associated electronics will be maintained as necessary. The gas system will be monitored and maintained, including gas bottle replacements and any required periodic testing. The data acquisition system will be maintained by experimenters and a LANSCE supplied computer technician. 
In addition to running at LANSCE, the TPC will also run at other facilities to cross check systematic errors. This will be critical to achieve the small systematic errors that are the goal of this experiment. One possibility is the ALEXIS facility under construction at LLNL. This mono energetic neutron source is notable for the low cost $\left(\$ 150 / \mathrm{hr}\right.$ to have the whole facility) and high luminosity $\left(10^{8} \mathrm{n} / \mathrm{s}\right.$ at $\left.10 \mathrm{~cm}\right)$ neutron beam that will complement the LANSCE facility.

Another notable resource is the accelerator at Ohio University, which will be used to study the hydrogen standard for this project and develop the data required to extend the small uncertainties in the $\mathrm{H}(n, n) \mathrm{H}$ total cross section to the actinide measurements.

\section{Livermore [LLNL]}

There are numerous facilities at LLNL that are of interest to this project. The construction of ALEXIS, Accelerator at Livermore for EXperiments in Isotope Sciences, is still being considered. This facility will generate pseudo-monoenergetic neutrons up to $108 \mathrm{n} / \mathrm{s} / \mathrm{cm}^{2}$ at energies from $100 \mathrm{keV}$ up to $14 \mathrm{MeV}$ at low operating cost. The LC computing system has large CPU clusters and storage systems that have been successfully utilized by similar computing projects such as Phenix at RHIC, MIPP at FNAL, and is currently working on setting up ALICE at CERN.

\section{TPC Laboratory Improvements}

Expanded the TPC lab space at LLNL by $50 \%$. Work on the lab infrastructure includes new A/C units, which slowed lab work on the TPC this quarter.

\section{Los Alamos [LANL]}

The Nuclear Science group at Los Alamos Neutron Science Center operates and maintains the Weapons Neutron Research facility that provides spallation neutrons to five flight paths. The group also maintains and operates two moderated neutron flight paths in the Lujan Center. The group operates and maintains the Blue Room facility, with access to an $800 \mathrm{MeV}$ proton beam and a Lead Slowing Down Spectrometer. The Nuclear Science team will provide the floor space and neutron beam access to the TPC project primarily on the 90Left flight path at the WNR and flight path 5 of the Lujan Center. The $90 \mathrm{~L}$ flight path experimental area is inside a new construction that contains an overhead crane, light lab space, a vented hood, source safes, computers and easy access to the neutron beam line. Flight path 5 experimental area includes an overhead crane, light lab space, source safes, computers and easy access to the neutron beam line. Recently refurnished light lab space will also be available for TPC work. Monitored stacks are in the vicinity of the two flight paths for TPC gas system and hood exhausts. Radiological shipments and handling facilities are also available. The LANSCE facility provides outside users with all necessary training, a cafeteria and meeting rooms.

\section{WNR Construction and 90L Flight Path Reconfiguration}

The construction of a new building that will cover a large part of the WNR south yard is nearly complete (Figure 115, Figure 116). The area is no longer considered a construction site, and only minor tasks (additional emergency lighting, signage, etc.) 
remain. WNR scientists are expected to obtain full occupancy of the building in midJune.

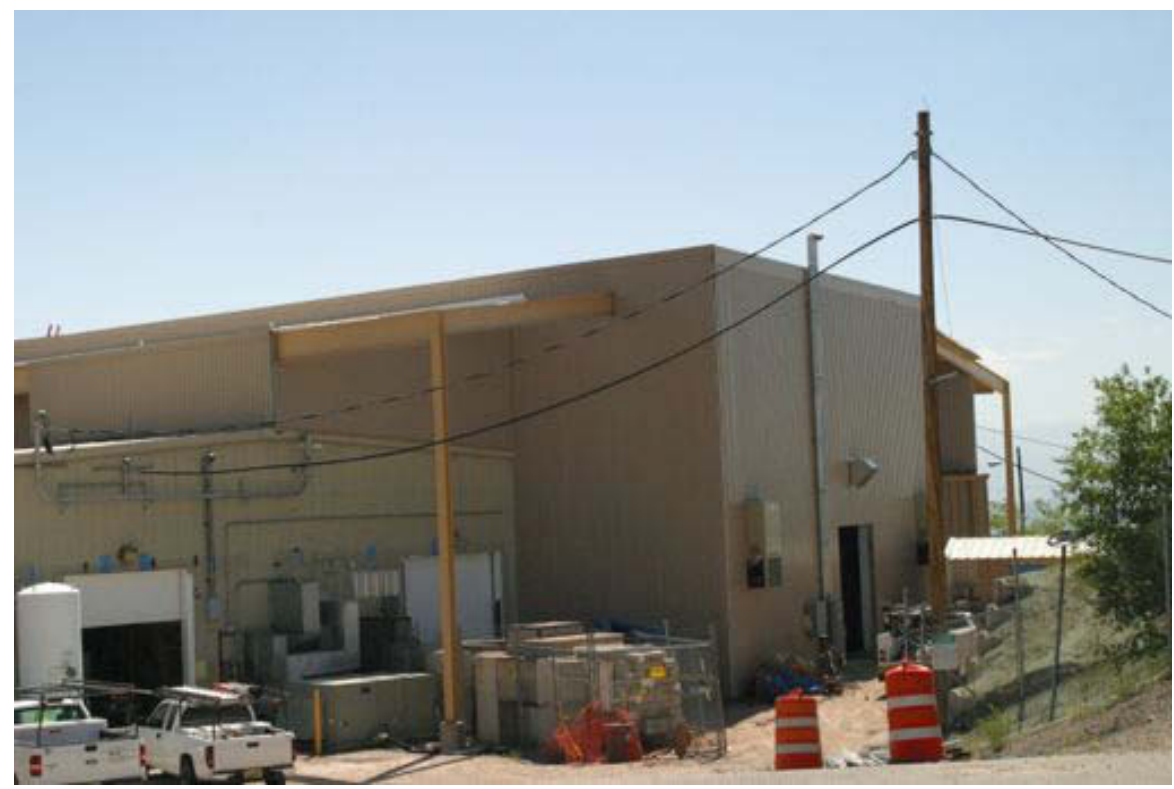

Figure 115: View of new building from WNR south yard.

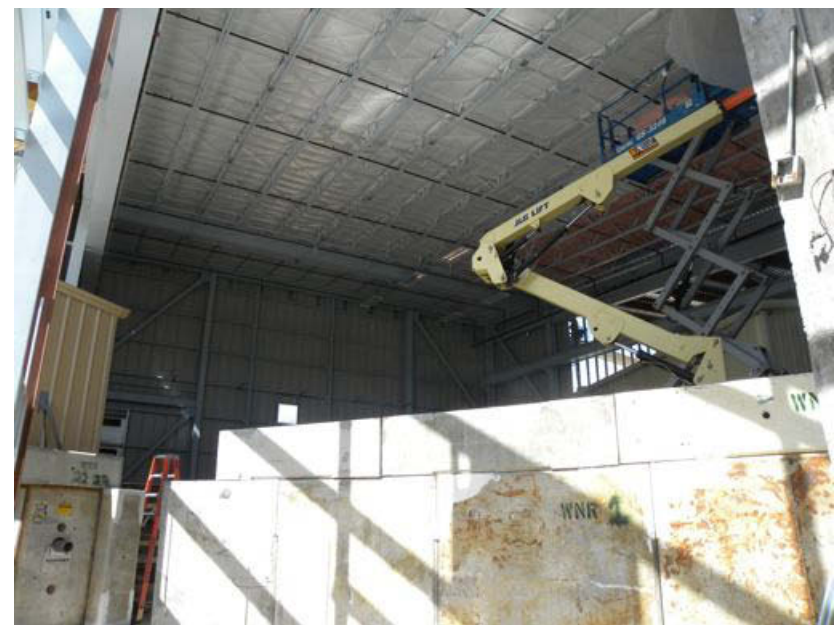

Figure 116: Interior view of the new WNR building.

The $90 \mathrm{~L}$ flight path was reconfigured to reduce room return and allow experimenters to more easily access equipment. The new flight path, widened from 6 feet to 9.5 feet, is shown in Figure 117. Since the TPC will now be placed closer to the shutter position, all in-room collimation has been removed. The flight path is completely accessible to experimenters and has been cleaned and organized for the 2012 run cycle. 


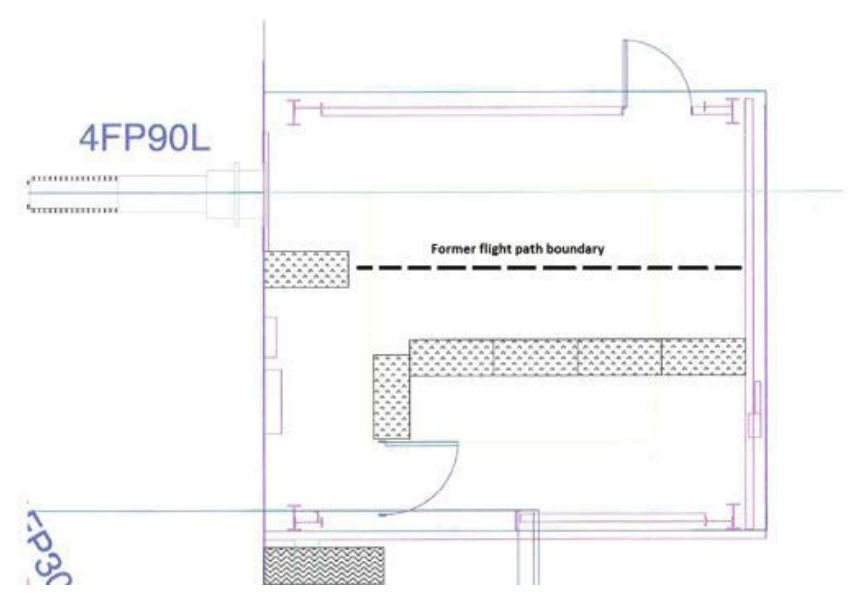

Figure 117: Schematic of reconfigured flight path. Dashed line indicates where shielding blocks were prior to the reconfiguration. Dotted rectangles indicate current location of shielding blocks.

\section{LANSCE Run Cycle}

The LANSCE Program Advisory Committee met in April to consider beam time allocation in the 2012 LANSCE run cycle (August - December 2012). The TPC collaboration submitted four proposals. One development proposal, to test the integration of new hardware and software capabilities, and three proposals to investigate the U238/U235, Pu239/U235, and $\mathrm{Pu} 239 / \mathrm{H}(\mathrm{n}, \mathrm{n})$ cross-section ratios. All proposals were approved and the $90 \mathrm{~L}$ flight path at WNR has been dedicated to TPC operations over the full 2012 run cycle.

Several purchases were made to facilitate the 2012 run cycle operations, including a complete set of cooling fans, 100 new fiber-optic cables, and an Uninterruptable Power Source (UPS) system which will reduce electronic noise by isolating the TPC system from the LANSCE power grid. The UPS was funded by the LANL Laboratory Directed Research and Development (LDRD) Office.

\section{LANL Summer Student Contributions}

Four summer students have been stationed at LANL to help with the TPC project. Lucas Montoya (NMSU) and Paul Greife (CSU), both undergraduate engineering students, have been assisting with infrastructure developments including: upgrades to the TPC positioning table, cable relief systems to manage fiber optic cables, storage containers for field cages that have been loaded with Pu samples, and installing cooling fans onto TPC shielding. William (Spenser) Lynn (ACU, undergraduate), is testing and developing a plan to turn the glove box purchased in 2010 into a Class-1 clean room environment, to facilitate loading of high-activity samples into the TPC. Dana Duke (CSM, graduate) is returning to LANL for the third summer to upgrade the TPC gas handling system from Windows to Linux operating systems and from Labview to $\mathrm{C}++$ programming. 


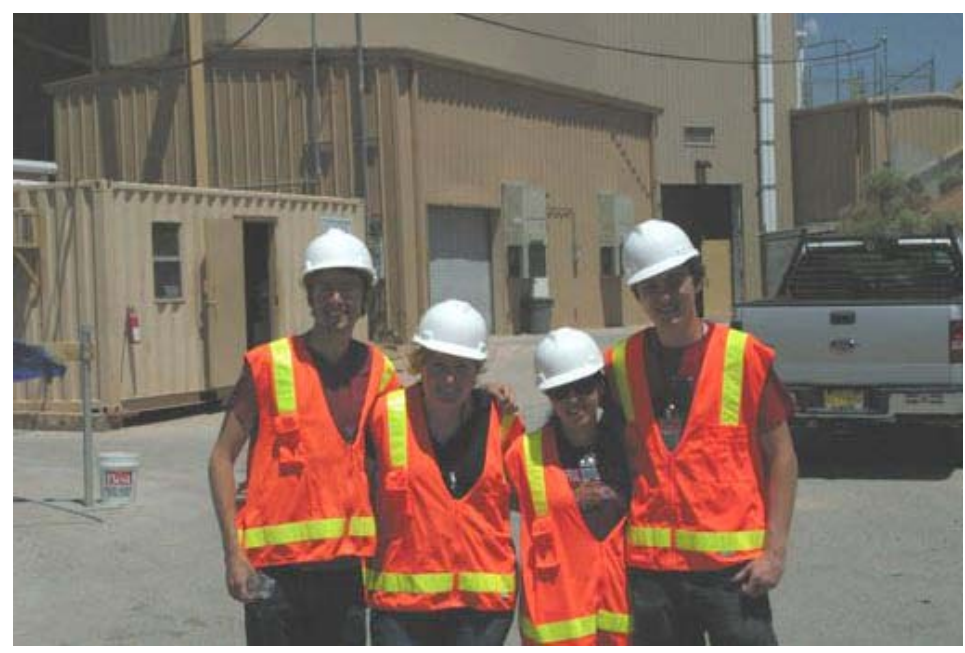

Figure 118: (From left) LANL summer students Spenser Lynn, Dana Duke, (postdoc) Rhiannon Meharchand, and Paul Greife.

\section{Ohio University [OU]}

The work proposed will be undertaken in the Edwards Accelerator Laboratory of the Department of Physics and Astronomy at Ohio University. The laboratory includes a vault for the accelerator, two target rooms, a control room, a thin film preparation and chemistry room with a fume hood, an electronics shop, a teaching laboratory for small non-accelerator based nuclear experiments, and offices for students, staff, and faculty. The Laboratory building supplies approximately 10,000 square feet of lab space and 5,000 square feet of office space. In the Clippinger Research Laboratories, the Department of Physics and Astronomy has a 3000 square foot mechanical shop, staffed by two machinists, that supports all the experimental work of the department. The machinists have numerically controlled machines that they use in the fabrication of apparatus used in experiments, they are accomplished at making parts from exotic materials such as refractory metals, and can perform heli-arc welding and other sophisticated joining techniques.

The heart of the Edwards Accelerator Laboratory is the 4.5-MV tandem Van de Graaff accelerator and six beam lines. This machine is equipped with a sputter ion source and a duoplasmatron charge-exchange ion source for the production of proton, deuteron, 3,4 He, and heavy ion beams. DC beams of up to $30 \mu \mathrm{A}$ are routinely available for protons, deuterons and many other species from the sputter ion source. Pulsing and bunching equipment are capable of achieving $1 \mathrm{~ns}$ bursts for proton and deuteron beams, $2.5 \mathrm{~ns}$ bursts for $3,4 \mathrm{He}$ beams, and $3 \mathrm{~ns}$ bursts for $7 \mathrm{Li}$. The accelerator belt was replaced most recently in March 2004; the accelerator has performed very well since that time with good stability for terminal voltages up to $4.0 \mathrm{MV}$. The SF6 compressor and gas-handling system were refurbished in April 2005. The Laboratory is very well equipped for neutron time-of-flight experiments. The building is very well shielded thus allowing the production of neutrons from reactions such as $d(d, n)$. A beam swinger magnet and time-of-flight tunnel allow flight paths ranging from 4 to $30 \mathrm{~m}$. The tunnel is well shielded, and the swinger-magnet assembly allows angular distributions to be measured with a single flight path.

\section{Tandem Operations}


We have completed the conversion of our tandem into a pelletron. We anticipate on months testing before experiments resume.We have reached a terminal voltage of 4.0 MV with 75 PSI SF6 in the tandem. We will increase the SF6 pressure to 95 PSI to allow stable operation to higher voltages. We have 15 operators taking refresher training to comply with the new Ohio Department of Health rules for industrial accelerators. We have recently lost the facility chiller due to freezing the heat exchanger, repairs are expected to take at least 3 weeks.

\section{$\underline{H(n, n) H \text { Scattering Experiment }}$}

We produced a $6 \mathrm{LiCl}$ target on a platinum backing and a covering of gold. This will be used for efficiency determination using the symmetric reaction $6 \mathrm{Li}+6 \mathrm{Li}$. We have begun the setup of the Hydrogen scattering experiment. The 252Cf calibration work to achieve $\sim 2 \%$ errors in the efficiency between 1 and $8 \mathrm{MeV}$ is currently underway. We are continuing the preparation work to perform the measurement of $\mathrm{H}(n, n) \mathrm{H}$ scattering at small neutron scattering angles by measurement of the scattered neutron.

\section{Nuclear Reaction Detection Capabilities}

The INL has special capabilities in neutron, charged particle, and gamma ray coincidence detection, at the heart of which is an array of Compton-suppressed, highpurity germanium detectors (CSHPGe) that have been used in the past for neutron reaction studies on actinide targets at ANL-IPNS. This array has been used to measure cross sections and reaction yields from actinide targets of interest to NE and NNSA. The array stand is designed to hold 12 germanium detectors and the active BGO shields.

Compton suppressed detector arrays and their associated electronics and data systems are considered one of the best ways to instrument reaction experiments of all types. The Compton suppression shields reduce the scattered background in each germanium detector, thus lowering the photo peak intensity error. The coincidence requirement of multiple detectors further suppresses random background by requiring two or more detectors to "see" simultaneous radiation from one nuclear event before the signals from the detector are digitized. The array uses a "list mode" data system that stores the data in packets on an event-by-event basis as a continuous stream of data. This allows analysis programs to select or sort the data based on a particular event condition in the data stream. This can be an energy selection, a type of radiation selection, or most commonly a time selection, i.e., multiple radiations must occur within a selected time of one another.

All of these factors: Compton suppression, an array of multiple detectors, fast coincidence electronics, and an event-by-event, list-mode data system, result in highly accurate separation of reaction channels, low statistical error, and reduction of systematic error with simultaneous measurement of different reaction channels 
In addition to the more standard reaction measurements, the array can be used to measure prompt fission yields of either induced or spontaneous fission. This measurement method does not require chemical separation, and thus the generation of radioactive waste. With the array the errors are again low and this is a market improvement over previous measurements and statistical model predictions.

The coupling of this array of CSHPGe detectors with additional charged particle detectors, neutron detectors and TOF capabilities allows the measurement of neutron reaction channels that have been previously overlooked. These unmeasured charged particle channels are often of higher probability than fission, inelastic scattering, or neutron capture. Not only does measurement of these other reaction channels like $(n, \alpha)$ and $(n, p)$ provide the missing data for nuclear energy research but also provides missing factors that are important in the development of nuclear models that better predict reaction results. This is the result of using better tools like the CSHPGe array INL has.

Figure 119 shows the array in use at ANL. The array operates in coincidence mode and allows the measurement of yields and neutron reaction cross sections with a very low background and thus small uncertainty. It has been used to measure simultaneously the fission and capture gamma-ray spectra from $235 \mathrm{U}$ and $239 \mathrm{Pu}$ thus eliminating the need to correlate two independent measurements to obtain the reaction channel branching ratios. The initial plan has been to measure the spontaneous fission yields of 240Pu as INL has a unique source that was obtained for this purpose. In addition, with the new pelletron and TOF beam line neutron reaction cross sections can be measured as they were done at ANL.

All support electronics exists and a new data acquisition system was installed in the past year to allow better use of the list mode data format. The detector array, electronics, data system and support stand represent approximately $\$ 2,000,000$ of a special capability at the INL.

This array represent part of the effort to use new and better techniques that have been developed in the nuclear physics community to improve and provide better nuclear data that is needed in the nuclear energy work. This represents the "science based" approach to nuclear research at its most fundamental level.

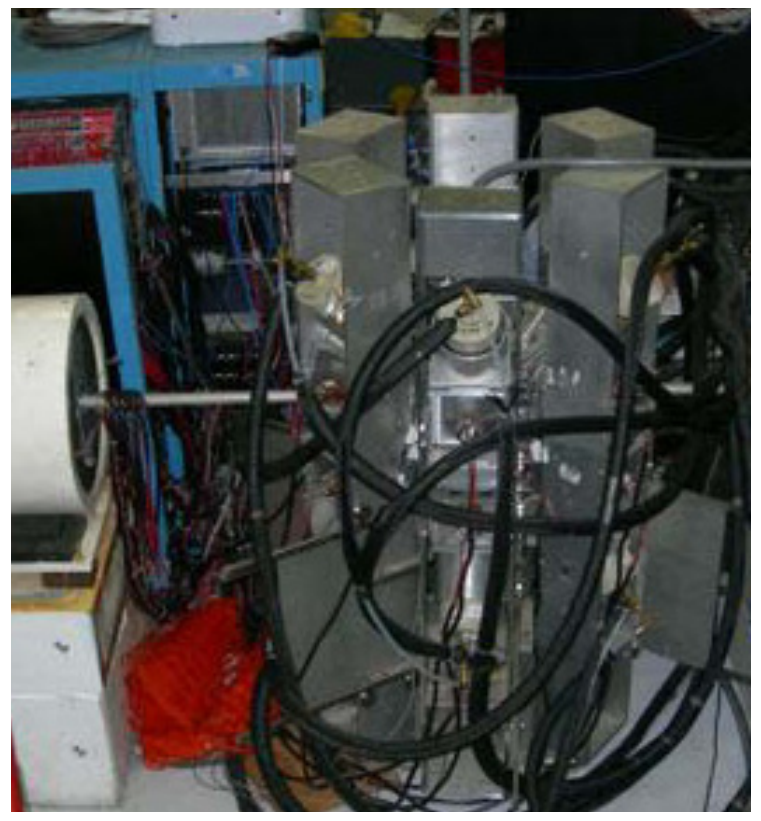

Figure 119. Compton Suppressed HPGE array in use at ANL/IPNS. 


\section{Advanced Detector Materials and Scintillators}

\section{ZnO Materials Growth and Scintillator Performance}

$\mathrm{ZnO}$ is a promising inorganic crystal for application as a scintillator for thermal neutron detection. The following section details progress made in fabricating the $\mathrm{ZnO}$ material and its scintillator application.

\section{Crystal Growth}

There are a number of different crystal growth methods for $\mathrm{ZnO}$ scintillators. They are broken into two distinct groups, bulk growth and thin film/nanoparticle growth. Bulk growth can be divided into melt growth, hydrothermal growth, Seeded Chemical Vapor Transport (SCVT), Chemical Vapor Transport (CVT), and Chemical Vapor Deposition (CVD) growth. Each growth method was pioneered by a primary commercial company.

SCVT growth is primarily performed by Eagle Pitcher, which has reported growth rates of $40 \mu \mathrm{m}$ per hour in a horizontal tube furnace at $1000-1200^{\circ} \mathrm{C}$. This company uses a zinc source and hydrogen as a carrier gas with an overpressure of water vapor to maintain stoichiometry. In this process, the crystal is seeded by an initial $\mathrm{ZnO}$ crystal, and grows on a $5.08 \mathrm{~cm}$ (2 in) diameter [2]. The growth rate for this method, however, is constrained and asymptotically approaches a steady state value lower than the $40 \mu \mathrm{m}$ per hour. Doping, which requires a suitable source capable of sublimation to the gas phase, is difficult with this method.

Chemical Vapor Transport is similar to SCVT, but there is no seed crystal to nucleate growth. This method usually has a lower crystalline quality due to the random nature of the initial growth. However, it is a low-cost option to produce bulk materials. Using a standard horizontal tube furnace, the growth is conducted by sublimation of zinc under a hydrogen carrier gas. Oxygen or water is used as the other reagent, while monitoring the partial pressure of carrier gas and reactant gases [3].

CERMET, Inc., is the leader in bulk melt grown materials. CERMET utilizes a patented crystal growth technology, cold wall bulk melt growth at high pressure, to produce doped and undoped $\mathrm{ZnO}$ single crystals. An RF heat source induces fields in the starter $\mathrm{ZnO}$ material in the water-cooled cold wall chamber. These fields produce eddy currents, which promote Joulean heating in the material until it melts. The highly refractory melt is contained in a cold wall crucible by a solid thermal barrier that forms between it and the molten material. The cooled material prevents the molten material from directly contacting the crucible cooling surface. This entire melting process is carried out in a controlled gas atmosphere ranging from $1 \mathrm{~atm}$ to over $100 \mathrm{~atm}$. This prevents the loss of volatile components, as well as the decomposition of compounds into atomic components [4]. CERMET has melted $\mathrm{ZnO}$ in 6 inch diameter crucibles, producing kilogram-sized boules from which inch-sized single crystals were cut. A schematic of the $\mathrm{ZnO}$ crystal growth furnace is shown in Figure 120. High dopant levels can be achieved using this method. 


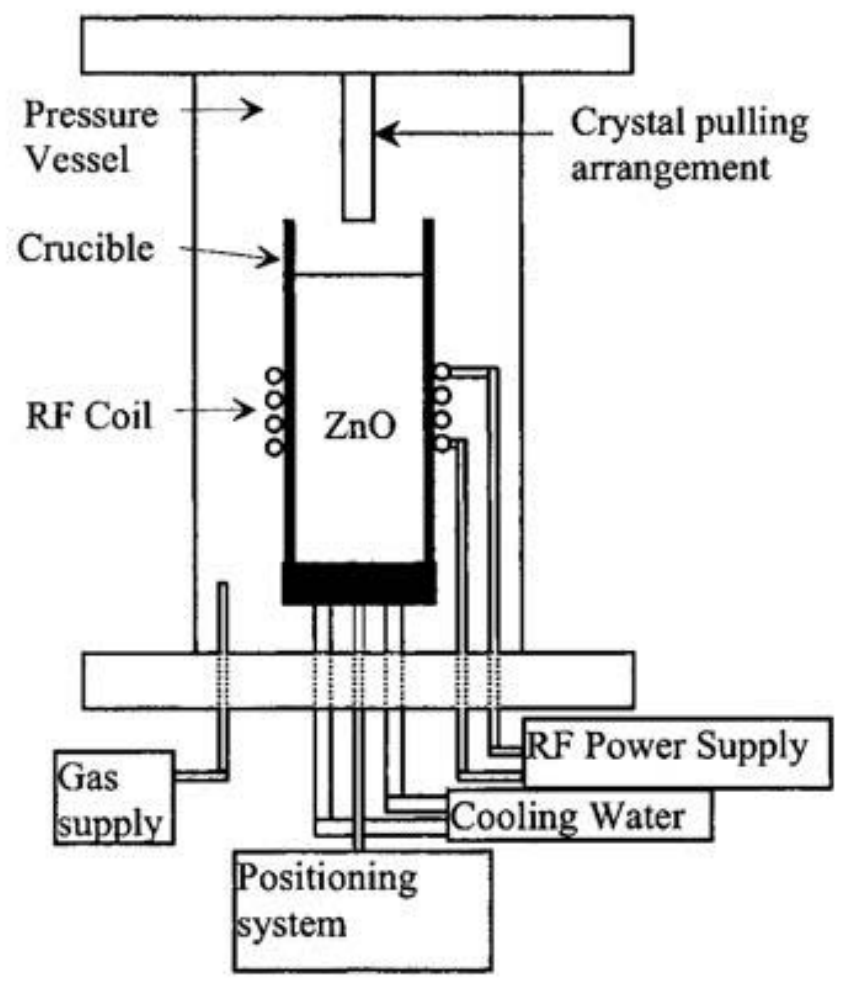

Figure 120. Schematic of the Cermet high pressure ZnO growth furnace [6]. The ZnO powder is heated up to $\sim 1900^{\circ} \mathrm{C}$ via pressurized induction melting apparatus, and the melt is contained in a cooled crucible.

Hydrothermal growth of $\mathrm{ZnO}$ consists of a solution using $\mathrm{LiOH}, \mathrm{NaOH}$ or $\mathrm{KOH}$ as a solvent at very high pressures $(150 \mathrm{ATM}+)$ and a temperature less than $400^{\circ} \mathrm{C}$. Zinc oxide is dissolved under extreme conditions and then allowed to precipitate slowly out of solution as the pressure/temperature is reduced [7]. This growth rate is exceptionally slow ( $\mathrm{mm}$ per day), and the $\mathrm{ZnO}$ contains high concentrations of contaminant nuclei from the solvent [8]. However, there has been a report of exceptional crystalline quality with a full width half maximum (FWHM) of 8 arc seconds in the XRD plot [9].

Each method described above has been investigated for nearly twenty years. While hydrothermal growth has produced the best reported crystalline quality, it incorporates contaminants undesirable for scintillation applications. Melt growth provides the highest speed and largest boules of usable scintillation material. Melt growth also shows the most promise for doping with neutron target materials and allows for in situ doping of almost any practical dopant with relative ease. While it is possible to grow at high growth rates by CVT and SCVT, the cost of fabrication of materials by these methods tend to be prohibitive. Inability to dope the materials makes these methods unsuitable for the fabrication of scintillation detectors. 


\section{MOCVD Growth}

One promising growth mechanism for $\mathrm{ZnO}$ is by Metalorganic Chemical Vapor Deposition. MOCVD is an epitaxial growth method of chemical vapor deposition in which the alkyl precursors are attached to an organic molecule. Metalorganics are usually volatile pyrophoric liquid materials, which aid in the growth process. Special growth reactors are required for MOCVD crystal growth and can grow various materials including $\mathrm{GaN}, \mathrm{GaAs}, \mathrm{InP}$, and $\mathrm{ZnO}$ [10]. Subtle changes to the internal components of these reactors enable application of different precursors. The change required to accommodate different growth materials is costly and time consuming, as the tool is disassembled and rebuilt. MOCVD permits very fine control of the structure and dopant levels, and can produce a very uniform epitaxial layer. A schematic of the reactor chamber specially designed for $\mathrm{ZnO}$ growth is illustrated in Figure 121.

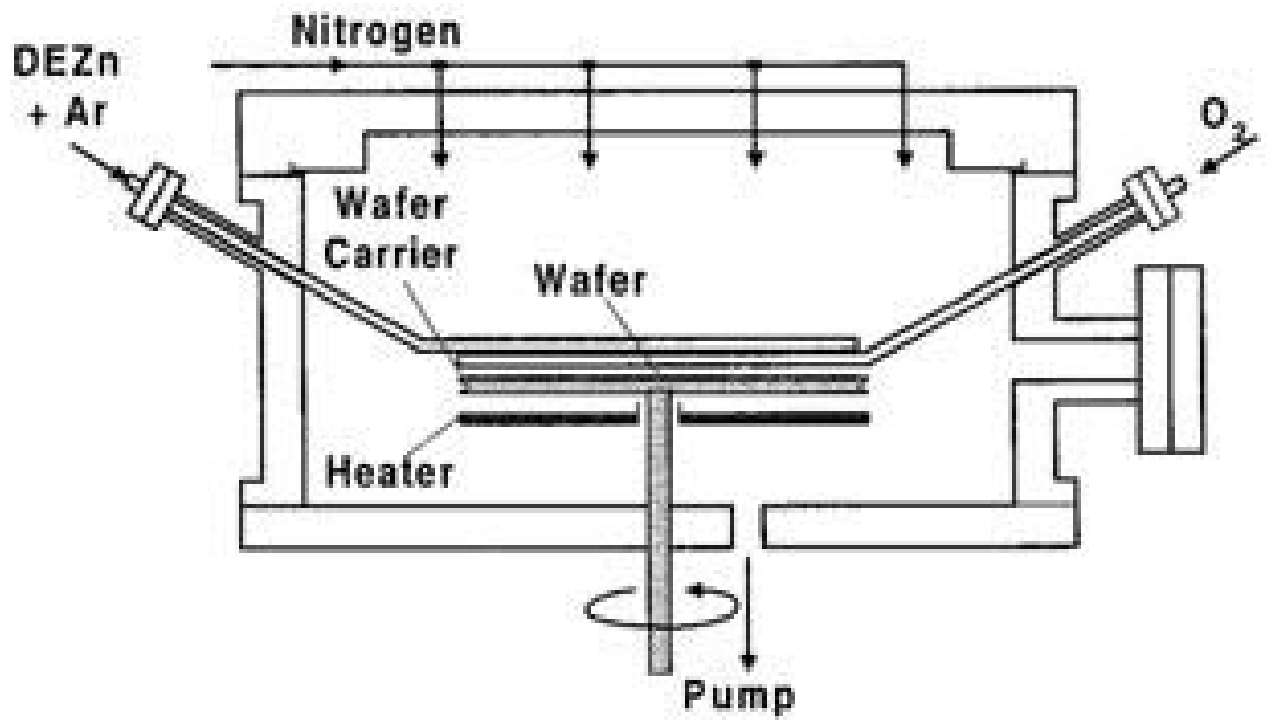

Figure 121. Schematic of a standard MOCVD growth chamber [5]. The wafer is located on a heated, rotating wafer carrier in an evacuated reactor chamber. Precursors flow into the chamber through their individual inlets.

The heater is capable of rapid temperature changes and can operate in vacuum as well as extreme environments, such as oxidizing or reducing atmospheres. Different designs of reactors have been proposed over the years. However, the most common one in mass production is the rotating disk model [11]. The rotating disk spins under a shower head, from which the metalorganic precursor is introduced [12]. The growth chamber's pressure may vary between 0.1 and 100 Torr [13],[14] and for ZnO growth low pressure growth is preferable. The design relies on a stable reactor condition in which laminar flow is maintained over the growth platter [15]. Significant effort is put into designing the vessel head to achieve laminar flow [20]. As the metalorganic precursor is heated, it is cracked, breaking the hydrogen and carbon nuclei and leaving the free metal ion in the gas phase flow. A reactant gas is then flowed simultaneously or in pulsed mode over the growth platter. The reactant gas completes the other half of the compound semiconductor. 
In multicomponent semiconductors, several alkyl sources and reactant gases can be pulsed or flowed into the chamber. For example, in $\mathrm{ZnO}$ growth, diethylzinc is the metalorganic of choice, and either oxygen (O2), nitrous oxide, or nitrogen monoxide can be used as the reactant gas [16]. Ideally, oxygen or nitrous oxide are typically the best choices. These two gas molecules crack to form free oxygen. Nitrous oxide also produces N2, which is stable and is not incorporated into the matrix. N2 is the common carrier gas that is flowed into the growth chamber to maintain laminar flow over the substrate for this very reason. Nitrogen monoxide, however, when cracked leaves free nitrogen. This free nitrogen is absorbed readily into the matrix resulting in nitrogen doped samples [17]. The reaction for $\mathrm{ZnO}$ MOCVD growth using oxygen as the gas can be seen in the following equation. MOCVD growth of $\mathrm{ZnO}$ is one of the few completely green reactions producing safe, non-toxic gases as byproducts.

$$
\left(\mathrm{C}_{2} \mathrm{H}_{5}\right)_{2} \mathrm{Zn}+7 \mathrm{O}_{2} \rightarrow \mathrm{ZnO}+5 \mathrm{H}_{2} \mathrm{O}+4 \mathrm{CO}_{2}
$$

Dopant gases are ideally metalorganic liquids similar to diethylzinc. Trimethylgallium (TMGa), trimethylindium (TMIn), and diethylboron are frequently used for gallium, indium, and boron doping, respectively. These metalorganics are sometimes too efficient at doping in comparison to the zinc oxide growth. Metalorganic sources are highly volatile, as many have boiling points below $10^{\circ} \mathrm{C}$. In ZnO MOCVD, nitrogen (carrier gas) is flowed through the liquid source. The flow rate of a metalorganic through the reactor is determined by partial pressures of the carrier gas and the source through a bubbler. The composition and doping of a material is precisely controlled by varying the source temperatures, the carrier gas flow, and the partial pressures. Bypass lines are often installed to minimize the pressure differential in the bubbler. This allows rapid switching at both constant pressure and constant flow rate between the metalorganic source and carrier gas to minimize spiking in the doping profile.

A series of mass flow controllers, solenoid valves, and pressure controllers/transducers regulates the gas flow of the carrier gas, reactant gas, and the metalorganic sources. Vacuum is maintained by a high volume, high vacuum pump, which usually includes a two stage vacuum/blower configuration. The internal chamber pressure is regulated on the exhaust side through a digitally controlled exhaust butterfly valve. Temperature and rotation measurements are performed with a resistive thermal detector (RTD) and a pyrometer (for operation above $700^{\circ} \mathrm{C}$ ) and an optical RPM meter. Computer control of MOCVD components ensures precise determination of the growth conditions.

Characterization tools can be integrated into the MOCVD chamber: For example, surface reflectance and ellipsometry [18]. Surface reflectance is measured using a fiber optic wide band light source to direct light into the growth chamber and measure the reflected light from the surface of the rotating wafer.

The measurements are gated on the rotational speed of the wafer so as to only measure the reflectance of the wafer and not the spinning platter [19]. For $\mathrm{ZnO}$, one full oscillation at 730 $\mathrm{nm}$ light corresponds to $\sim 250 \mathrm{~nm}$ of growth, and a sample surface reflectance measurement for growth of $\mathrm{ZnO}$ can be seen in Figure 122. 


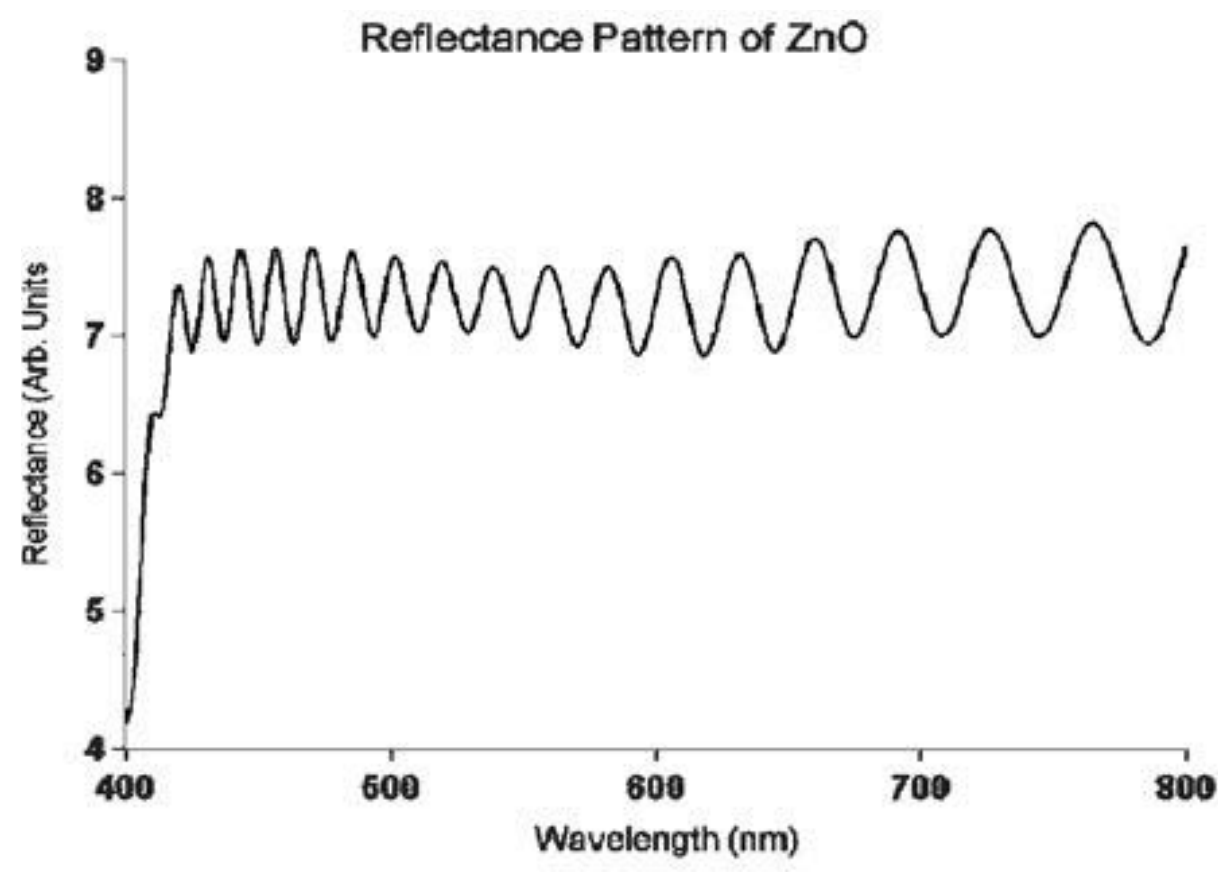

Figure 122. Reflectance pattern from a thin film ZnO scintillator.

This in-situ characterization technique is crucial for optimizing the growth parameters such as chamber pressure effects on growth, growth rates, dopant levels and optical quality. The status of the crystal growth process can be easily monitored without the need to stop the reaction, cool the chamber, and bring the chamber back to atmospheric conditions.

Two dimensional (2D) thin film growth generates highly uniform films with very few defects at high growth rates and requires a specific range of growth conditions. In contrast, 3D film growth gives rise to nanorod-like structures and polycrystalline materials, which are impractical for application in scintillators. The crystalline defects and grain boundaries produce trapping locations, longer scintillation decay times, and scintillation outside the band gap. ZnO growth in the MOCVD process has a very narrow window in which 2D growth can occur and is especially sensitive to the reactor pressure (between 10 and 50 Torr). Outside this region, the metalorganic preferentially adheres to the surface and does not diffuse into a smooth 2D growth. $2 \mathrm{D}$ growth requires a reactor temperature between $475^{\circ} \mathrm{C}$ and $550{ }^{\circ} \mathrm{C}$. Above 550 ${ }^{\circ} \mathrm{C}$, the growth rate is reduced below $100 \mathrm{~nm} / \mathrm{h}$. Below $475^{\circ} \mathrm{C}$, a sufficient quantity of oxygen cannot be cracked. By switching oxygen precursor gases may improve this requirement. Hybrid 2D and 3D $\mathrm{ZnO}$ has also been grown [21]. 
The crystal quality of the MOCVD grown $\mathrm{ZnO}$ samples is heavily dependent on the substrate, although $\mathrm{ZnO}$ offers significant tolerance to strain the first few layers. Ideally, the lattice mismatch between the substrate and the $\mathrm{ZnO}$ structure should be minimized. Sapphire is the preferred growth substrate with a lattice mismatch parameter of approximately $30 \%$.

However, despite the large lattice mismatch, $\mathrm{ZnO}$ can compensate for it within the first $100 \AA$, promoting high quality $2 \mathrm{D}$ growth [21]. ZnO has been grown on other substrates, including silicon, silicon oxide, and glass. High quality films can be grown on almost any material including non-wurzite structures and amorphous materials by utilizing techniques described in the following section.

\section{Hybrid MOCVD ALD Growth}

Atomic Layer Deposition (ALD) permits very precise thin film growth by introducing (or pulsing) one precursor at a time [22]. Following the pulse of the first precursor, a purge pulse of carrier gas clears the chamber. Subsequently, a gas is introduced to "crack" the dangling molecule. Then, a purge gas pulse clears this cracking gas. These four steps are repeated for the second precursor. In contrast to MOCVD, only one gas is in the reactor chamber at a time. To further ensure that uniform thin films are formed, the growth conditions are selflimiting. Leaving a dangling chain exposed on one surface limits the reaction to nucleate atoms one atom thick across the surface. The diethylzinc source is still used, but the oxygen source is replaced with water vapor. Water vapor reacts with the surface and forms a hydroxide molecule. The stoichiometric balance equations are shown in the following equations:

$$
\begin{aligned}
& \mathrm{ZnOH}^{*}+\mathrm{Zn}\left(\mathrm{CH}_{2} \mathrm{CH}_{3}\right)_{2}{ }_{2}^{*} \rightarrow \mathrm{ZnOZn}\left(\mathrm{CH}_{2} \mathrm{CH}_{3}\right)^{*}+\left(\mathrm{CH}_{3} \mathrm{CH}_{3}\right) \\
& \mathrm{Zn}\left(\mathrm{CH}_{2} \mathrm{CH}_{3}\right)^{*}+\mathrm{H}_{2} \mathrm{O} \rightarrow \mathrm{ZnOH}{ }^{*}+\left(\mathrm{CH}_{3} \mathrm{CH}_{3}\right)
\end{aligned}
$$

The pulsed growth cycle is alternated between diethylzinc and water vapor. Growth rates were found to be approximately $2 \AA$ per pulse cycle. This was determined both by in situ reflectance measurements as well as post growth filmetrics reflectance measurements. An image of the ALD growth process for $\mathrm{ZnO}$ can be seen in Figure 123. One atomic layer is deposited per pulse.

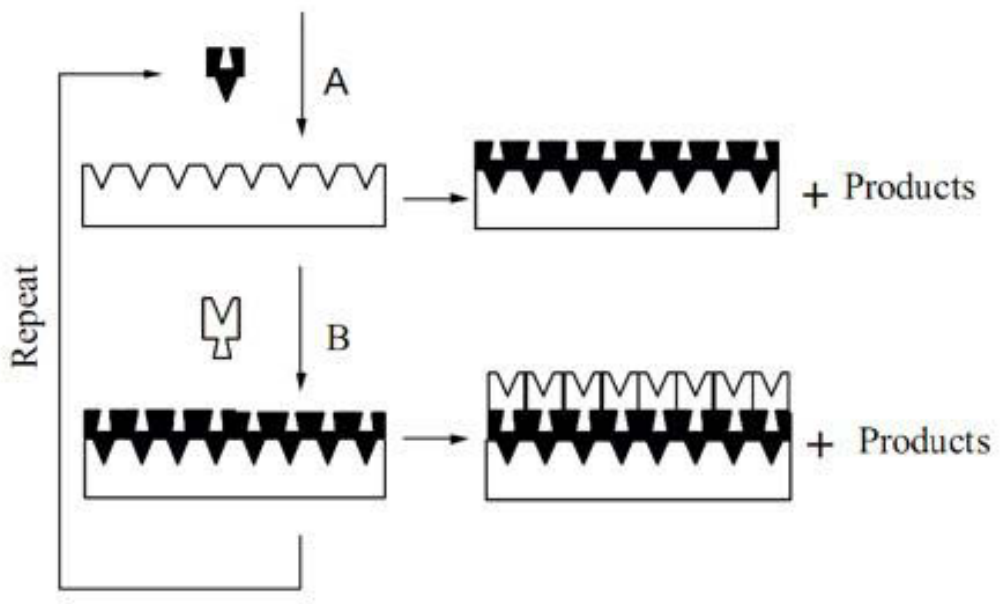

Figure 123. ALD of $\mathrm{ZnO}$ using water molecules and diethylzinc [23]. 
An advantage of ALD growth for initial growth over a substrate or patterned structure is the generation of very high conformal coatings with extreme aspect ratios of $10,000: 1$. This is only possible due to the surface limiting reaction. In contrast MOCVD's rapid growth would cover up the small features of patterned structures and generate a smooth layer on top. ALD by its very nature is a slow growth process and due to the self-limiting reaction usually produces amorphous material, especially, with a large lattice mismatch between the desired material and the substrate.

\section{ZnO Bulk Growth}

Bulk and MOCVD grown ZnO samples exist in various shapes, including cubes, cylindrical boules, and thin wafers. $10 \mu \mathrm{m}$ thick layered scintillators are also shown. Figure 124 shows various undoped and doped $\mathrm{ZnO}$ boules and wafers. All of these wafers were grown at Cermet, Inc.

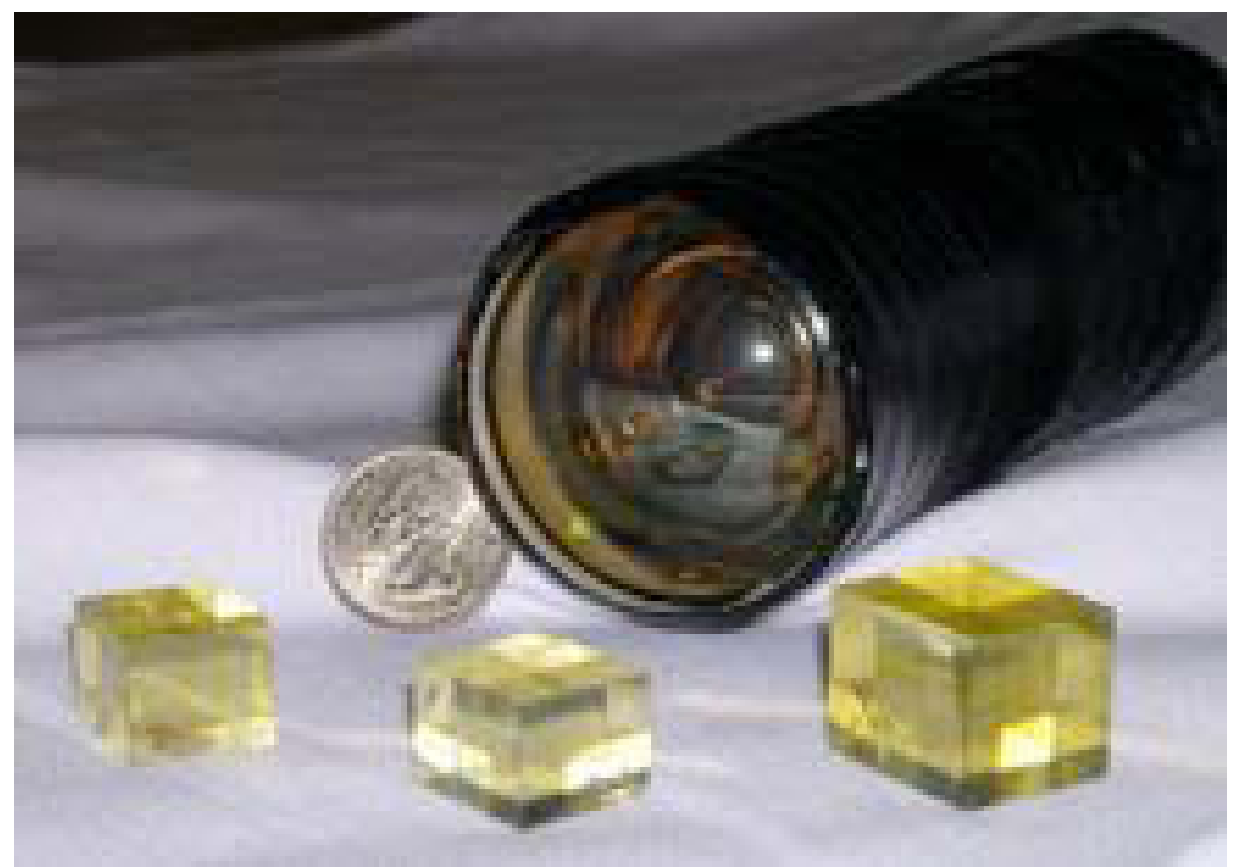

Figure 124. Large cubes of polished $\mathrm{ZnO}$. The left and right cubes are undoped $\mathrm{ZnO}$ while the center cube is lithium doped $\mathrm{ZnO}$. 


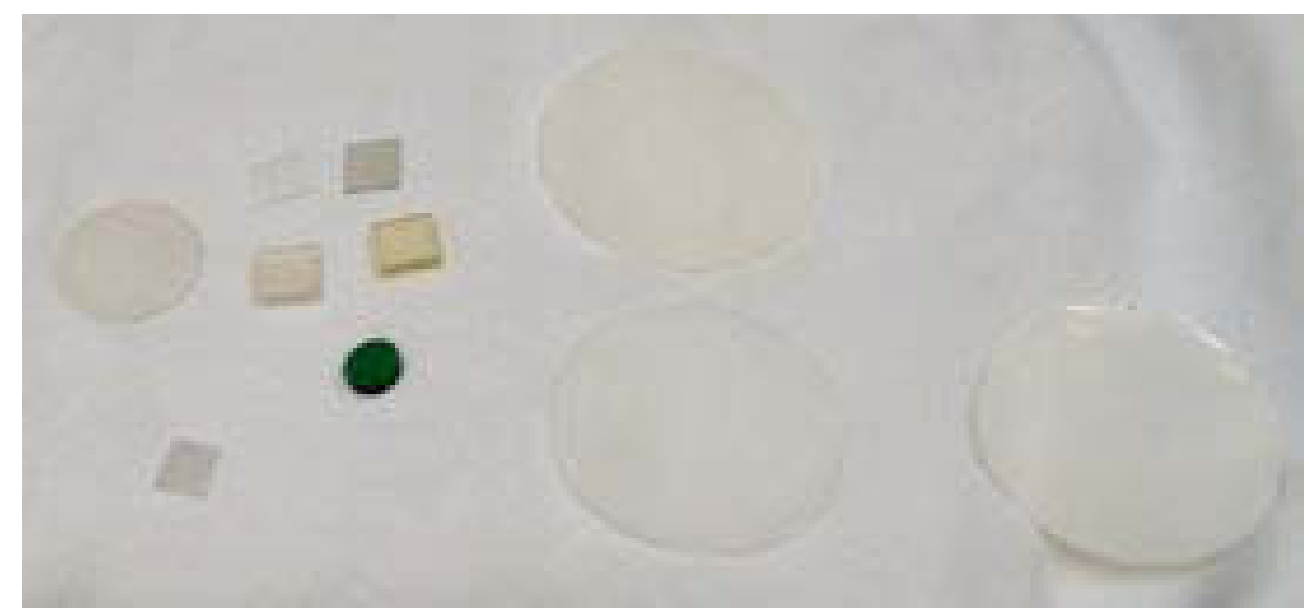

Figure 125. Shown on the left, small bulk grown ZnO samples including indium, lithium, cobalt and undoped $\mathrm{ZnO}$. On the right, the two inch wafers showing MOCVD grown $\mathrm{ZnO}$.

Figure 125 compares the bulk grown $\mathrm{ZnO}$ samples to MOCVD grown $\mathrm{ZnO}$ samples. The samples are of high quality, optically clear, and will prove to be good, high efficiency neutron detectors.

\section{Performance of $\mathrm{ZnO}$ neutron scintillators}

In this section we will review the impact of growth methods/conditions and of various dopants on scintillation detectors created from zinc oxide. Two particular types of $\mathrm{ZnO}$ scintillators were tested, bulk melt grown $\mathrm{ZnO}$, and MOCVD grown $\mathrm{ZnO}$. Three classes of dopants were investigated, (1) neutron detector dopant materials, (2) dopants to improve the scintillation wavelength providing acceptor and donor bands, and (3) dopants to improve crystalline clarity and scintillator transparency. These dopants are not mutually exclusive as often the incorporation of dopants impacts two or more of these parameters. Six dopants were studied in particular: lithium, boron, gadolinium, aluminum, gallium and nitrogen. A preliminary investigation of bulk grown samples of cobalt, indium and magnesium doped scintillators eliminated these materials from further consideration. Lithium and gadolinium were studied in both the bulk growth and MOCVD samples. Aluminum, boron, gallium, and nitrogen were only studied in MOCVD samples. Undoped samples were studied to assess the impact of growth conditions on the samples grown with MOCVD.

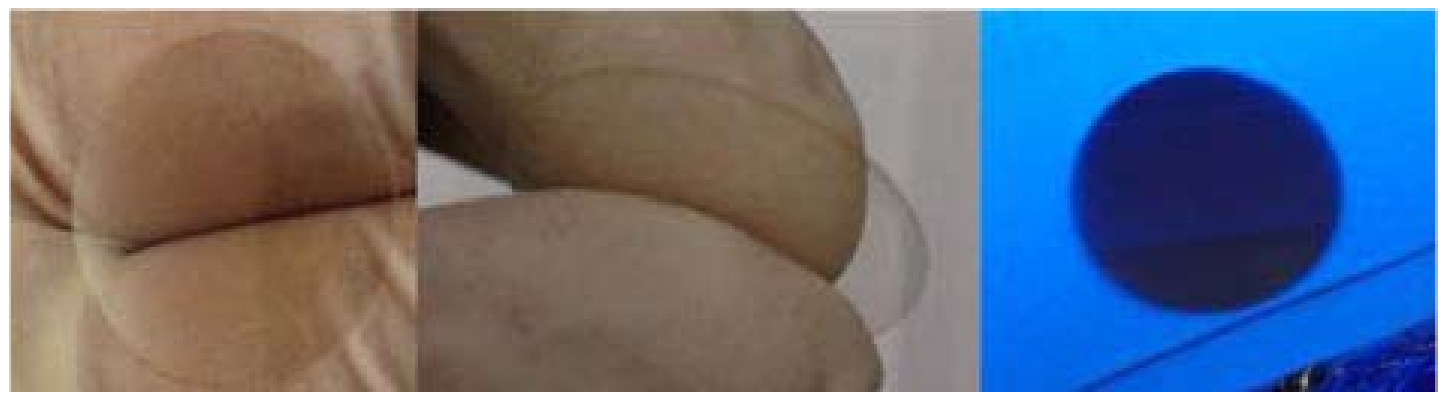

Figure 126 New grown compound wafer scintillators being prepared. Figure on the right is under UV illumination on a white background. 


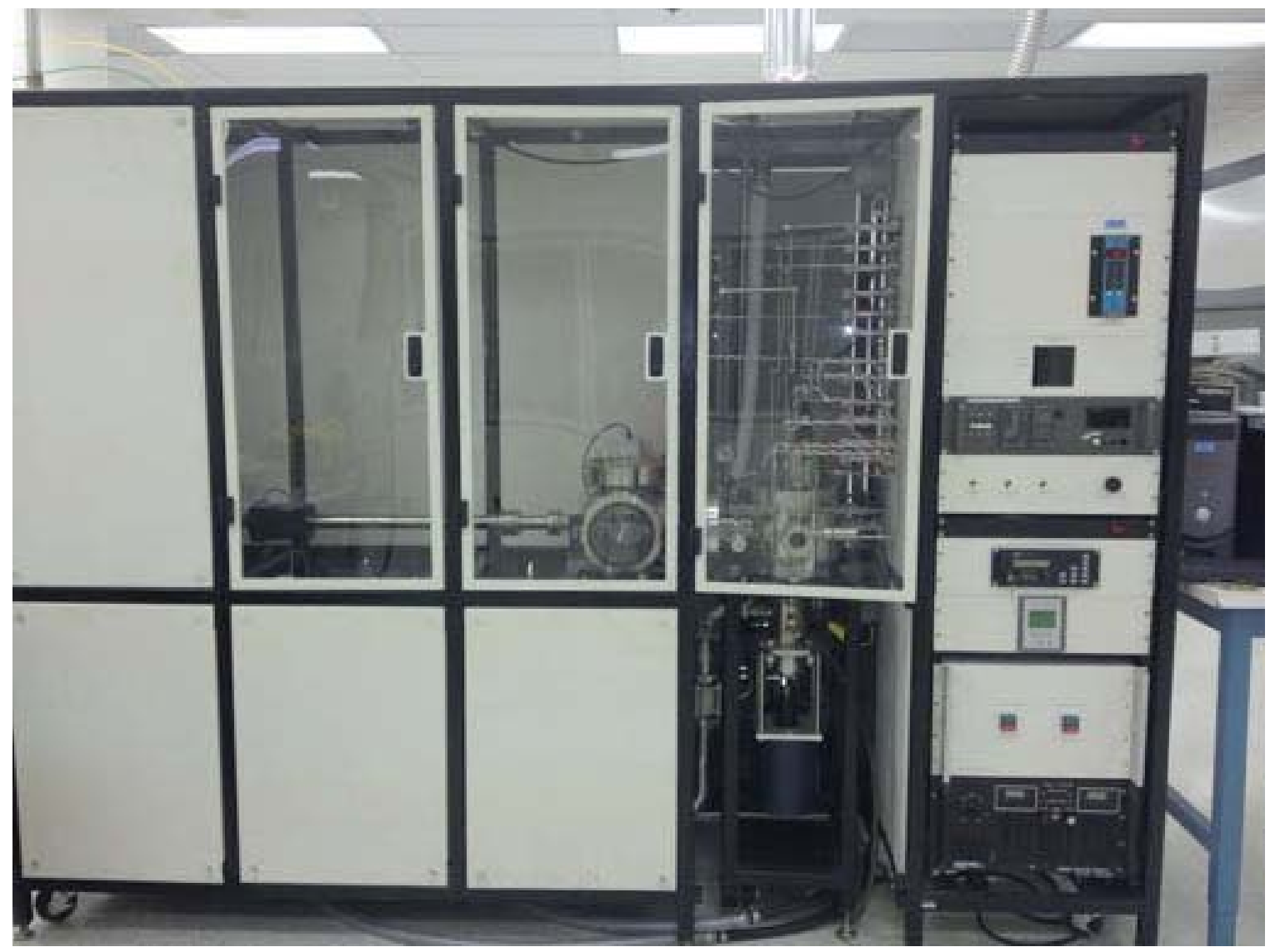

Figure 127 New MOCVD tool installed at the ISU RISE Complex for growing high performance ZnO scintillators.

\section{Impact of Growth Conditions on Scintillator Performance}

Three main parameters were investigated to ascertain the optimal growth conditions for MOCVD growth of ZnO scintillators, (1) the II-VI ratio as determined by oxygen and zinc flow rates, (2) temperature, and (3) pressure.

\section{Effect of II-VI Ratio on ZnO Samples}

The effect of the II-VI ratio on growth is dramatic. Increasing the zinc to oxygen ratio improves the photoluminescence (PL). Moreover, the samples became visibly opaque from excess zinc. A slight excess of zinc appeared to be optimal for PL measurements and resulted in a nearly transparent thin film. When excess zinc is present, there is usually excess carbon trapping due to the inability to form carbon dioxide during growth. The carbon trapping can be seen as defect luminescence in the Blue Band (BL shift). Carbon trapping slows the response by introducing a longer tail in the decay pulse heights in addition to reducing the transparency of the scintillator. Excess oxygen samples became white and did not scintillate nearly as efficiently as those which were slightly oxygen depleted. It should be noted that in this work we are optimizing the $\mathrm{ZnO}$ for scintillation applications rather than for its electronic properties.

Plotted below are the relative PL spectra after varying the ratio between diethylzinc (DEZ) flow and oxygen flow. Oxygen flow rate was held constant to maintain identical flow patterns in the chamber. Pressure was held at a constant 3 torr during 
growth. The impact of the ratio between zinc and oxygen is shown below in Figure 128.a). A flow rate ratio between 150 and 350 to 900 was determined to be optimal.

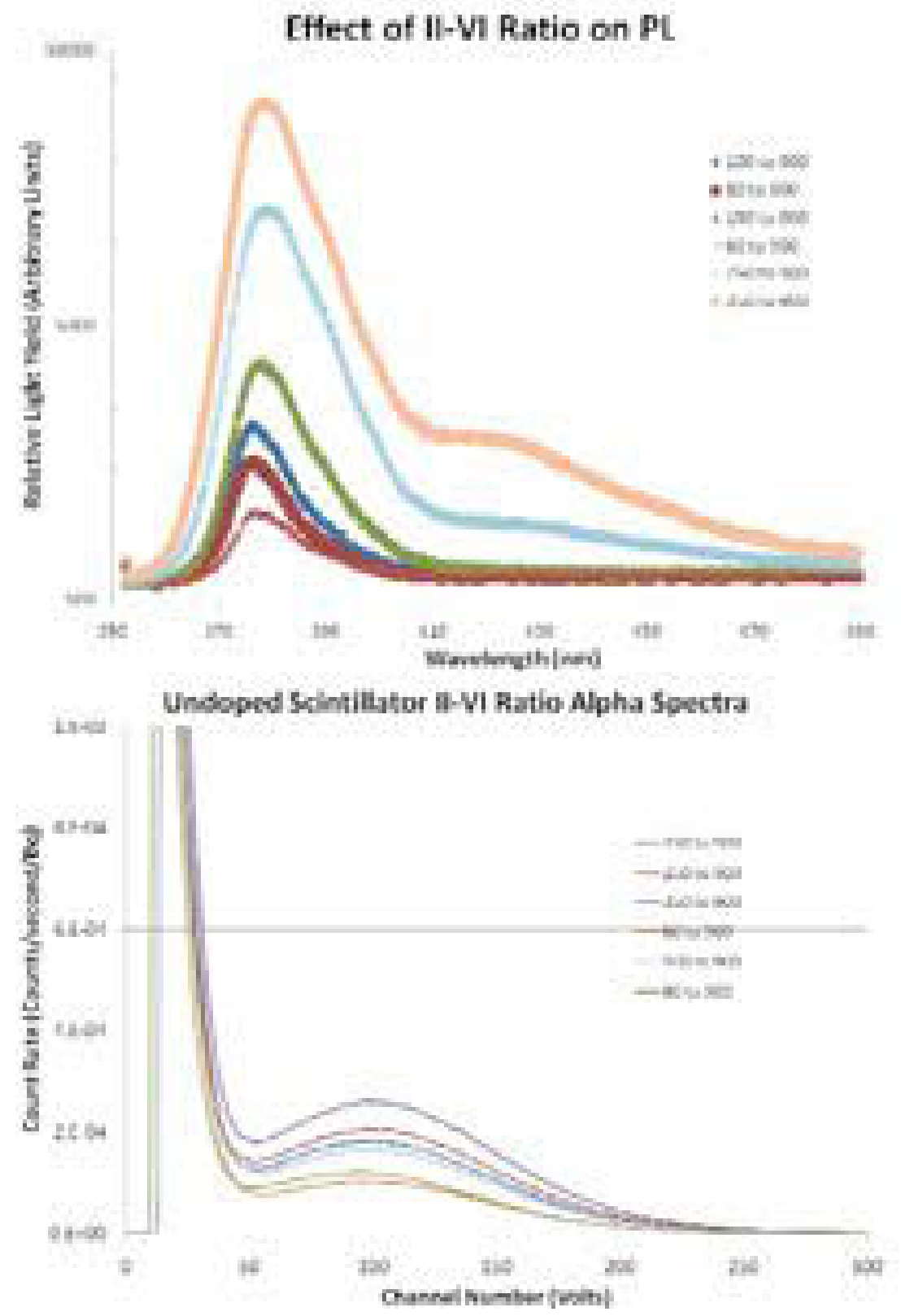

Figure 128. .a) Effect of growth ratio between zinc flow rates and oxygen flow rates in the MOCVD growth chamber, and b) 230Th alpha spectra comparison for undoped ZnO.

The alpha spectra in Figure 128.b) reveal a larger alpha peak for the 350 to 900 ratio, indicating higher detection efficiency. However, this sample has a very low transparency. In larger samples, these higher zinc to oxygen ratios do not yield practical devices. At thicknesses greater than $4 \mu \mathrm{m}$, these structures would be unusable. A balance between efficiency and optical clarity was reached in the 150 to 900 ratio. 


\section{Effect of Growth Temperature on ZnO Samples}

Temperatures in the range of 400 to $600{ }^{\circ} \mathrm{C}$ were studied to determine their effect on growth. Growth rates were found to increase up to $550^{\circ} \mathrm{C}$ and then noticeably decrease above that temperature. The impact of the temperature on the PL spectra of the scintillators is shown in Figure 129. When the temperature is not high enough, not enough oxygen is cracked, and the scintillators come out dark with carbon trapped interstitially. Carbon trapping can again be seen in the blue luminescence peak that begins to form around $425 \mathrm{~nm}$. Crystalline quality is also noticeably lower with 3D growth dominating. Good quality samples at reasonable growth rates were maintained at $550^{\circ} \mathrm{C}$. Above $575^{\circ} \mathrm{C}$, part of the PL band gap peak begins to shift and broaden at lower wavelengths. At $600^{\circ} \mathrm{C}$ the growth rate slows considerably and the material quality is poor.
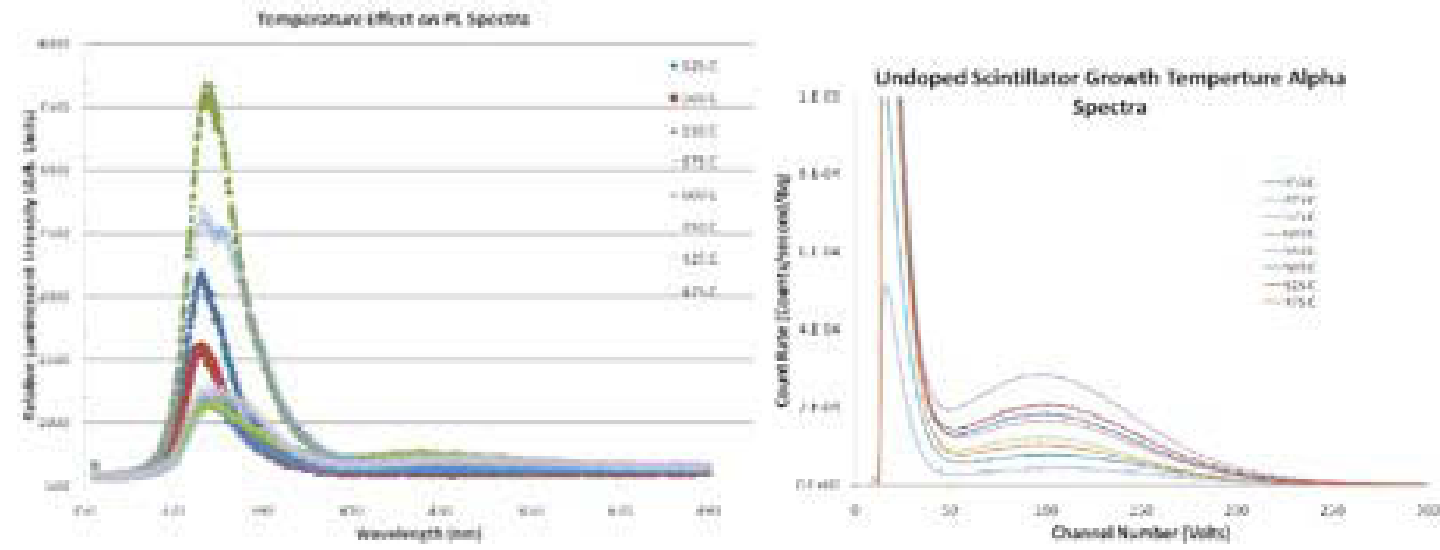

Figure 129. .a) Effect of temperature on PL data, and (b) 230Th alpha spectra comparison for different MOCVD growth temperatures.

\section{Effect of Growth Pressure on ZnO Samples}

Growth pressure does not have a significant impact on the PL as temperature or the II-VI ratio. It does, however, impact the maximum growth rate and crystalline quality, and this can be seen in high resolution X-ray measurements. Figure 130 shows XRD results for $10 \mu \mathrm{m}$ thick scintillators in the range of 2 to 10 Torr. 


\section{XRD Resulis for Pressure Varled Zno Grawth}

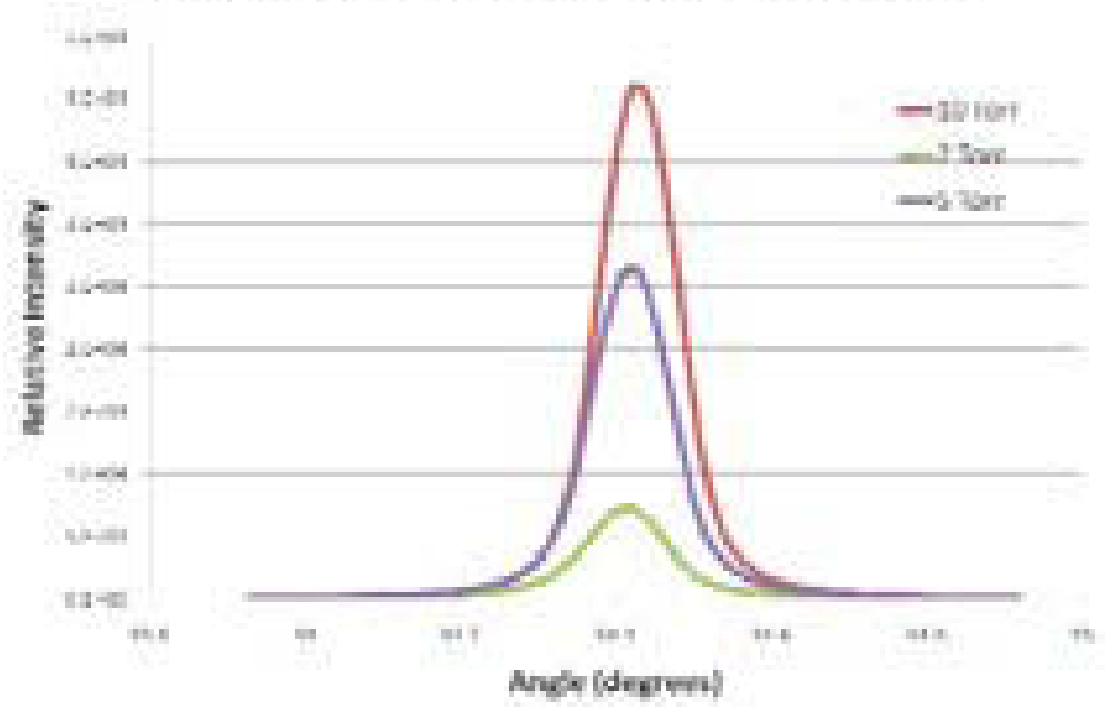

Figure 130. Effect of pressure in the growth chamber on XRD crystalline quality.

\section{Impact of Dopants on ZnO Scintillator Performance}

\section{Impact of Nitrogen Doping on Scintillator Performance}

Nitrogen doping was performed during MOCVD of $\mathrm{ZnO}$ with ammonia, NH3.

Ammonia, when cracked, provides one nitrogen atom and three hydrogen atoms.

When performing nitrogen doping with ammonia, the oxygen flow rate must be increased to compensate for the excess hydrogen present. Nitrogen doping was found to improve the growth of $\mathrm{ZnO}$ to $>4 \mu \mathrm{m} / \mathrm{h}$. Crystalline transparency also improved, and samples thicker than $15 \mu \mathrm{m}$ were still clear. The ammonia was found to act as a surfactant and appears to clean the surface of excess carbon during growth. The structures usually exhibited 3D growth with nanorod-like structures present at a larger degree than undoped scintillators.

There was one large drawback with just nitrogen doping. When using ammonia, the three excess hydrogen atoms had a tendency to become trapped in the crystalline structure. This is the first time free hydrogen atoms were released into the chamber without being attached to carbon nuclei. Hydrogen functions as a deep acceptor in the scintillator and passivates the PL centers by trapping the excited electrons, and no luminescence is observed.

A rapid thermal anneal of the scintillator under an oxygen overpressure was found to restore, to a limited extent, the original PL spectra. Oxygen overpressure and higher temperatures liberate the trapped hydrogen atoms, and oxygen combines with hydrogen to form water vapor. Rapid thermal annealing was completed at $800{ }^{\circ} \mathrm{C}$ with various time durations and oxygen flow rates of $30 \mathrm{sccm}$ to $80 \mathrm{sccm}$ to remove the hydrogen. The samples were allowed to cool in an oxygen atmosphere. A two wafer growth run was conducted under the "optimal" pulsed ammonia growth conditions to prepare samples for the ammonia doped scintillator tests.

The annealed samples gradually regained the peak in the PL spectra (Figure 131.a)). The wavelength of scintillation was blue-shifted by approximately $10 \mathrm{~nm}$. The peak 
continued to increase but never reached the undoped level as the sample annealing time was increased. A 5 minute anneal at $800^{\circ} \mathrm{C}$ of the nitrogen doped samples yielded the highest intensity peak in the PL spectrum among the doped samples but never reached the peak of the undoped sample. The samples became oxygen saturated and cloudy with longer anneal times.
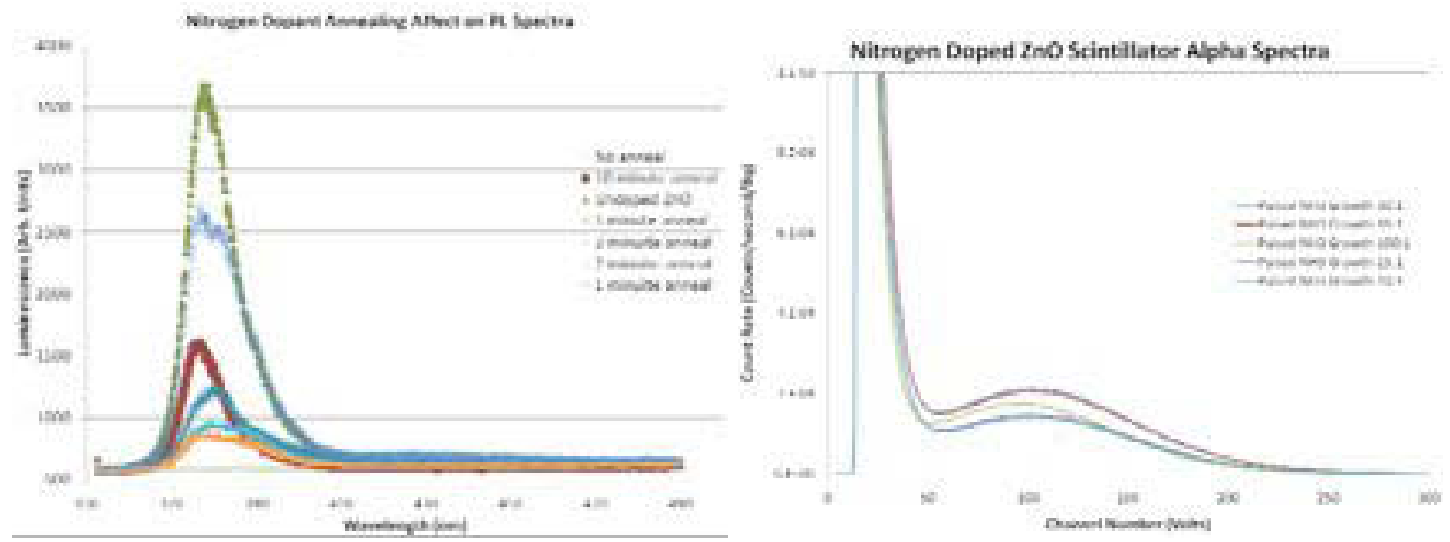

Figure 131. a) Nitrogen doped samples after annealing under oxygen, and b) 230Th alpha spectra comparison for nitrogen doped $\mathrm{ZnO}$ after 5 minute anneal under oxygen at $800^{\circ} \mathrm{C}$.

The 5 minute anneal also resulted in the return of the alpha peak (Figure 131.b)). The peak position shifted to the right indicating a slight increase in light collection.

\section{Impact of Gallium Doping on Scintillator Performance}

Gallium also was investigated in an attempt to shift the scintillator spectrum's peak by introducing additional transition levels. Trimethylgallium (TMG) was used as the dopant precursor, and gallium is a far more reactive metalorganic source than zinc. Continuous flow rates often caused the samples to blacken due to excess gallium or the formation of gallium oxide, and so PL was only performed on transparent samples. $10 \mu \mathrm{m}$ thick samples were grown which were not optically transparent, and the PL spectra show an average wavelength shift of $7 \mathrm{~nm}$ (Figure 132.a)). The peaks with gallium are skewed, indicative of the gallium alloying in the $\mathrm{ZnO}$ matrix. Only pulsed introduction to the reactor could incorporate gallium into the matrix.
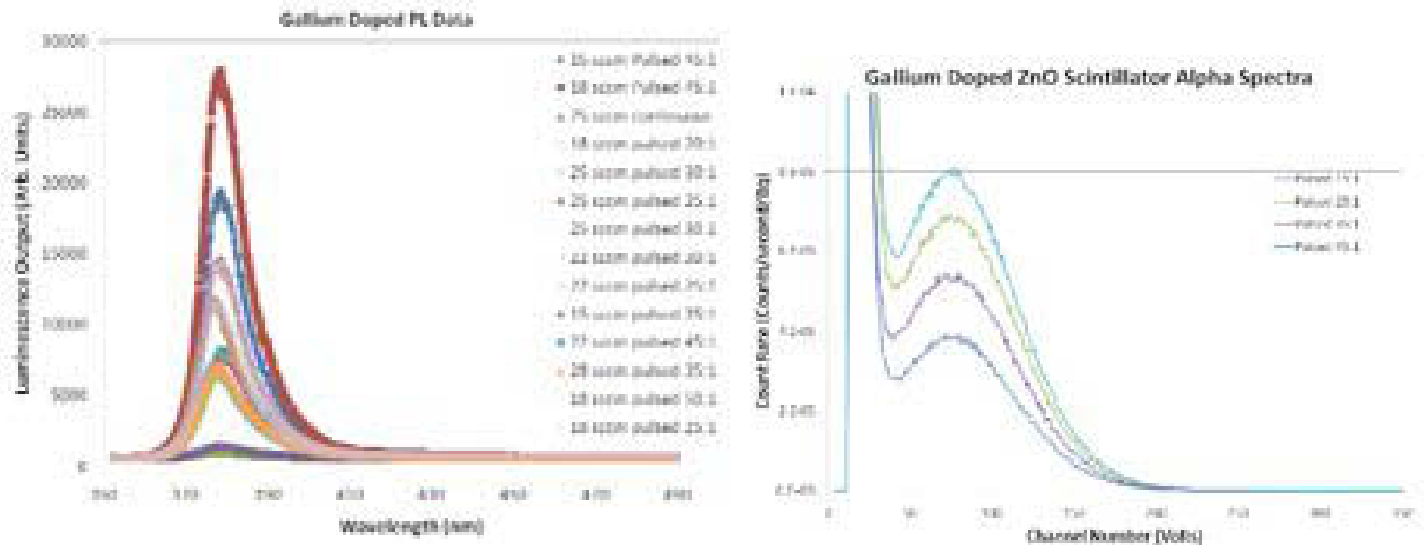

Figure 132. a) Gallium doped PL data, and (b) 230Th alpha spectra comparison for gallium doped $\mathrm{ZnO}$. 


\section{Impact of Aluminum Doping on Scintillator Performance}

Aluminum doped $\mathrm{ZnO}(\mathrm{AZO})$ is highly transparent, crystalline, and highly conductive. It is commonly used as an ohmic contact for solar cell work. AZO grows very well on sapphire (AI2O3) since both exhibit wurzite structures. AZO can be grown by MOCVD to over $20 \mu \mathrm{m}$ thick with high crystalline quality with dopant levels of up to $20 \%$. During initial growths continuous Al precursor flow rates completely passivated the samples, even at low flow rates and high pressures. The passivated samples also consisted of $50 \%-80 \%$ aluminum oxide and a small fraction of zinc interstitials. In contrast, pulsed Al precursor yielded high quality AZO samples. Aluminum doping of $\mathrm{ZnO}$, however, tends to increase the scintillation wavelength to the UV region instead of decreasing it (Blue region). This effect must be balanced with other dopants, as the increase in scintillation bandgap beyond that of $\mathrm{ZnO}$ reduces the luminosity. Otherwise, AZO scintillates below its band gap edge and preferentially reabsorbs its scintillation light. The $\mathrm{ZnO}$ peak is still dominant (Figure 133.a)), but it is skewed to the left slightly with a peak wavelength of $376.3 \mathrm{~nm}$. This results from the truncation of the scintillation spectrum by the band edge of the material.
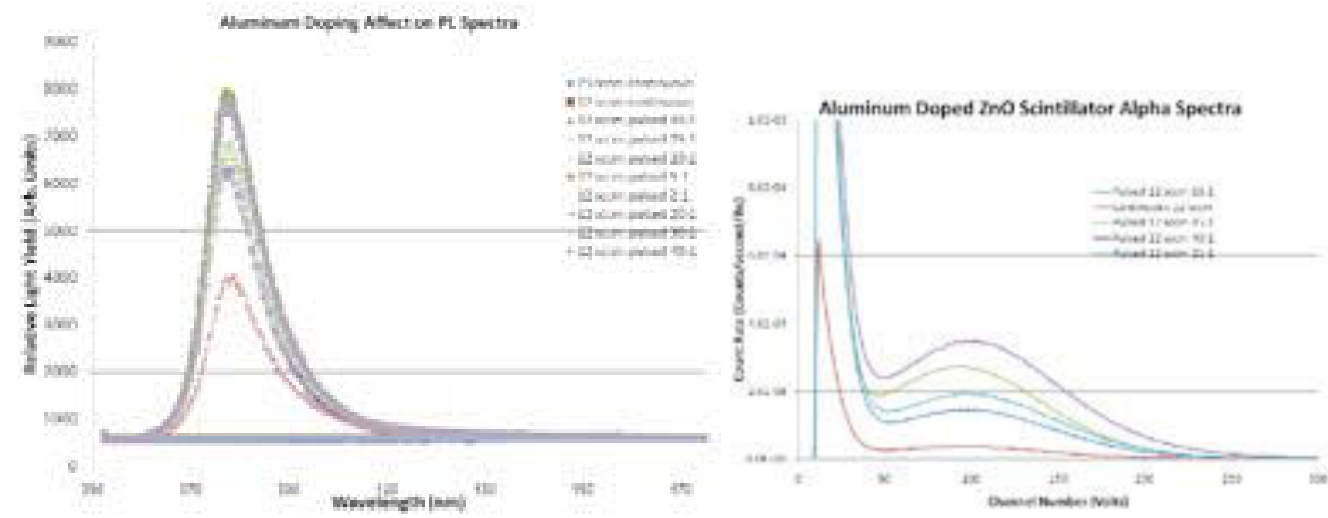

Figure 133. a) Aluminum doping impact on PL spectra, and (b) 230Th alpha spectra comparison for aluminum doped $\mathrm{ZnO}$.

\section{Impact of Boron on Scintillator performance}

Boron, a neutron target material can be directly doped into the scintillator. Although boron has a tendency to form boron oxide, boron doped $\mathrm{ZnO}$ demonstrated good PL intensity (Figure 134). Boron added several acceptor activation levels, which increased the scintillation wavelength by approximately $10 \mathrm{~nm}$. Extensive studies on boron were conducted because it is a dual-purpose dopant, adding neutron detection functionality and improving the light yield. Diethylboron was used (DEB) as the boron precursor. This precursor must be kept at extremely low temperatures, at $-20^{\circ} \mathrm{C}$ at a pressure of 800 Torr, due to its low vapor pressure. 

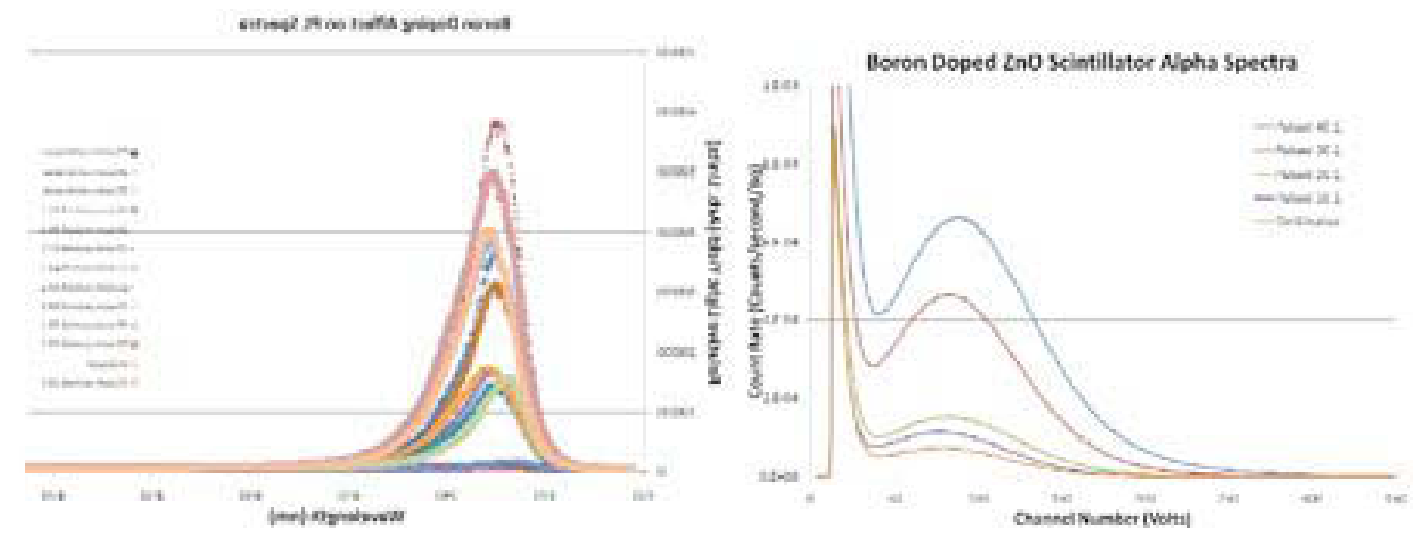

Figure 134. a) Impact of Boron doping on ZnO PL spectra, and (b) ${ }^{230} \mathrm{Th}$ alpha spectra comparison for boron doped ZnO. Flow across the boron bubbler was $20 \mathrm{sccm}$ at 800 Torr.

\section{Impact of Lithium Doping on ZnO Scintillator Performance}

Lithium was also thoroughly investigated as a potential dopant. Lithium samples are difficult to grow due to the lack of a suitable lithium precursor. There are no diethyl lithium or dimethyl lithium sources. Similar sources are lithium ß-diketyonates and lithium TMHD. However, either liquid injection or heated precursor lines are needed. In this work lithium cyclopentadienide, lithium TMHD, t-butyllithium, and lithium acetylacetonate were all investigated as potential lithium sources. Lithium cyclopentadienide was eliminated quickly as the very low vapor pressure resulted in sample sublimation and decomposition of the source in a vacuum.

Lithium TMHD was more promising, but required the lines to be heated, as it condensates on cold walls, clogging the injection line. T-butyllithium dissolved in pentane worked well with continuous agitation. Otherwise, the t-butyllithium precipitated at the bottom of the bubbler. A separate liquid injection line was installed for this source but, due to the large flow volume, it was considerably more difficult to control and to produce a transparent crystal. Finally, the lithium acetylacetonate provided a good solution in a heated vapor phase. After a few days, it typically solidified from its original powder form. Subsequently, this same sample was dissolved in acetone and used as a liquid injection source. The samples grown by MOCVD were comparable to the bulk growth samples prepared by Cermet Inc. But it was difficult to obtain clear lithium doped samples by MOCVD. The resulting PL spectra are shown in Figure 135.
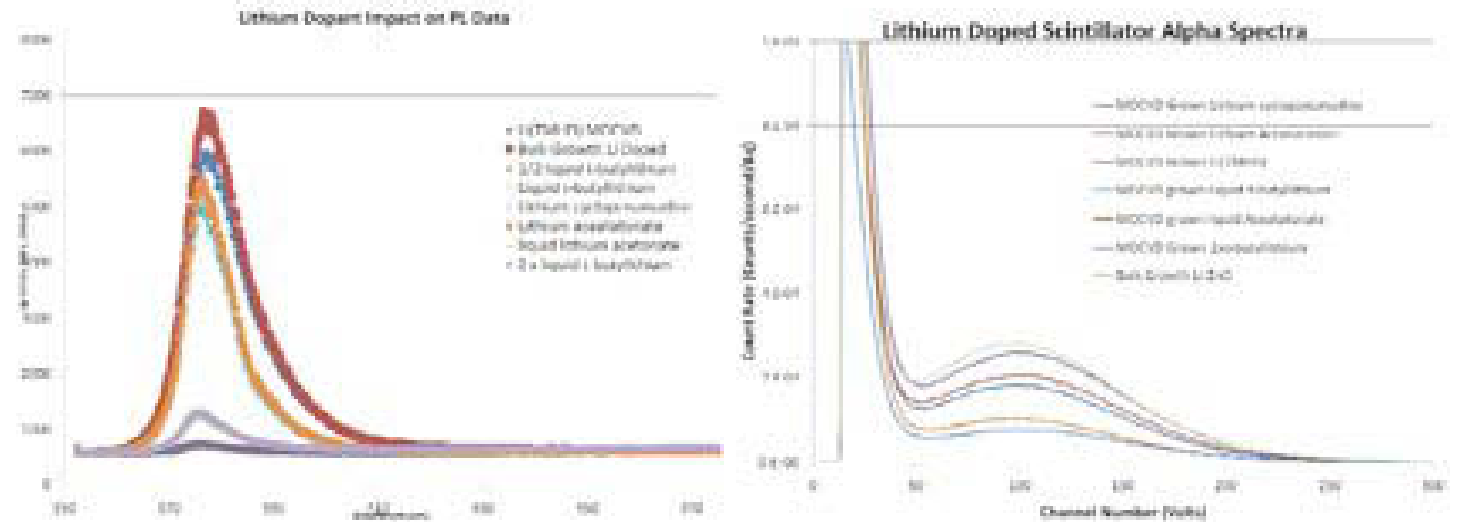

Figure 135. a) Lithium dopant impact on PL light intensity, and (b) ${ }^{230}$ Th alpha spectra comparison for lithium doped $\mathrm{ZnO}$. 


\section{Impact of Gadolinium on ZnO Scintillator Performance}

Gadolinium was the last major dopant to be investigated in both bulk and MOCVD samples. A Gd(TMHD) source was used as the MOCVD precursor. The bulk growth samples were comparable to the MOCVD. Gadolinium, with its extensive electron structure, provides extra acceptor energy states for excited electrons, and it dramatically raises the effective $Z$ number of the material. The $3+$ state and $\mathrm{Gd}$ bond were studied. The availability of only one MOCVD Gd source limited the study to flow rate variation over the sample. PL demonstrated high intensities from these samples. But the use of gadolinium doping was not conclusive at this time.

Pulse rise and decay time (Figure 136.a)) distributions were analyzed for all of the scintillators (Figure 136.b)). The pulses were digitized using a $1 \mathrm{GHz}$ sampling oscilloscope. Rise and decay times were measured for all of the samples discussed above.
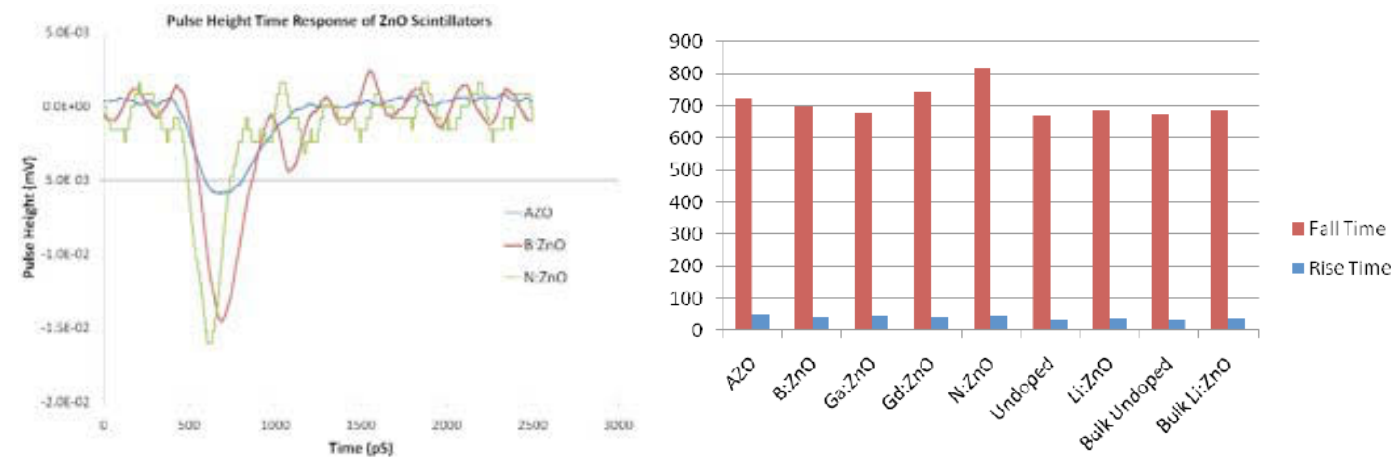

Figure 136. . a) The pulse height rise and fall times for three scintillators, and (b) the rise and fall times of the best production quality scintillators.

The undoped $\mathrm{ZnO}$ scintillators show the fast fall times, but doping $\mathrm{ZnO}$ dramatically increases luminescence. Nitrogen doped $\mathrm{ZnO}$ has both the longest fall time and very low intensity PL peak. Boron doped and gadolinium doped $\mathrm{ZnO}$ have the highest PL intensities and average rise and fall times.

An additional study was conducted using a microchannel plate photodetector as opposed to a photomultiplier tube. The microchannel plate does not suffer from the same time resolution problems the PMT does. The $\mathrm{ZnO}$ scintillator was tested in comparison to a fast plastic scintillator, $\mathrm{BC}-422 \mathrm{Q}$ quenched fast plastic scintillator. With a rise time of $35 \mathrm{pS}$, and a decay time of $0.65 \mathrm{nS}$, the $\mathrm{ZnO}$ is a factor of two faster. This can be seen below in Figure 137. 

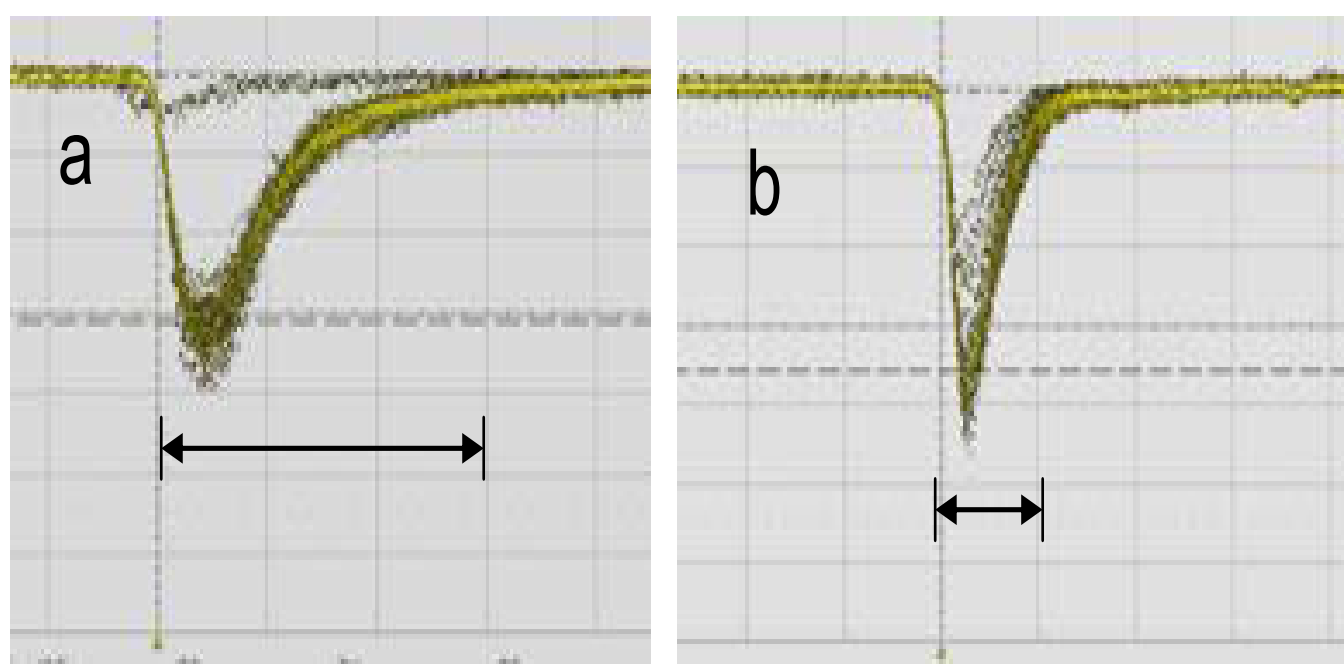

Figure 137 a) shows a BC-422Q, the fastest plastic scintillator available. B) shows the ZnO scintillator. This is roughly a factor of two faster.

\section{Neutron Detection Performance}

The $\mathrm{ZnO}$ neutron scintillators were tested by (1) measuring their response to highenergy neutrons such as those directly from source $\mathrm{PuBe}$ neutron source, i.e. the ${ }^{9} \mathrm{Be}(\alpha, n){ }^{12} \mathrm{C}$ reaction neutrons where the alpha particles are from ${ }^{239} \mathrm{Pu}$ decay for plutonium intimately mixed with the beryllium ) and (2) performing several Monte Carlo simulations to test various scintillator configurations. Results from the $\mathrm{ZnO}$ scintillator's response in free space recorded by a multichannel MCA demonstrate that undoped $\mathrm{ZnO}$ could possibly discriminate between gamma rays and neutrons (Figure 138).

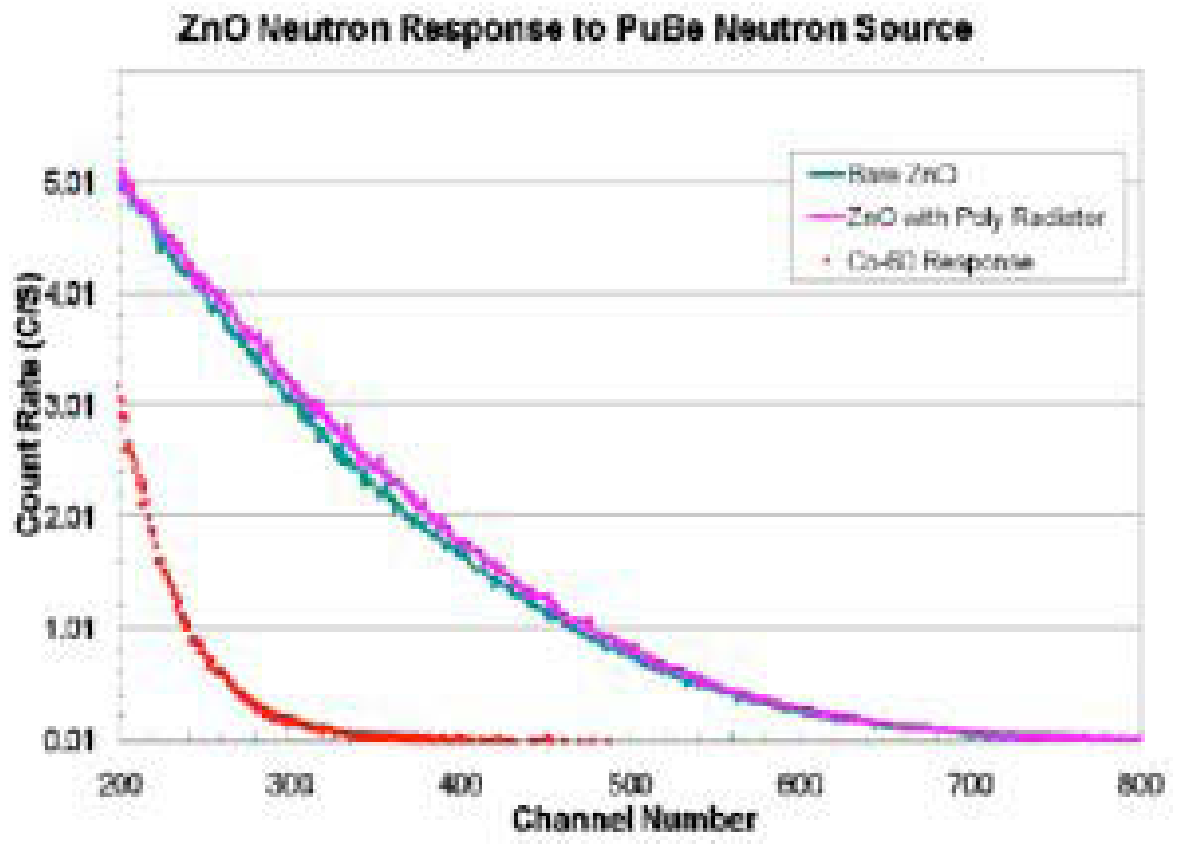

Figure 138. ZnO neutron detector response to fast neutrons. 
MCNPX v2.7d was used to simulate the response of various scintillator configurations to both neutron and gamma ray fields. The energy deposition tally was used to correctly calculate the energy imparted to the scintillator, as it takes the effects of secondary particles, such as gamma particles, tritons, and protons, into account. The neutron capture isotope algorithm (NCIA) was used to model the neutron capture reaction. These were then summed and plotted with a 3D contour mapping package in GNUPlot [24].

Configurations of only uniformly doped scintillators, stacks of one-sided, and stacks of two-sided scintillators for a variety of thicknesses were run. Simulation results for the $1 \mathrm{~mm}$ samples are shown below in Figure 139. Samples thicker than approximately $15 \mu \mathrm{m}$ demonstrated compromised neutron versus gamma discrimination. At 0.5 $\mathrm{mm}$, the signal from the gamma-ray field emitted from a standard fission gamma spectrum is comparable to the signal from the $(n, \alpha)$ reactions in the scintillator. Above $1 \mathrm{~mm}$ the detector is saturated by the gamma ray signal.
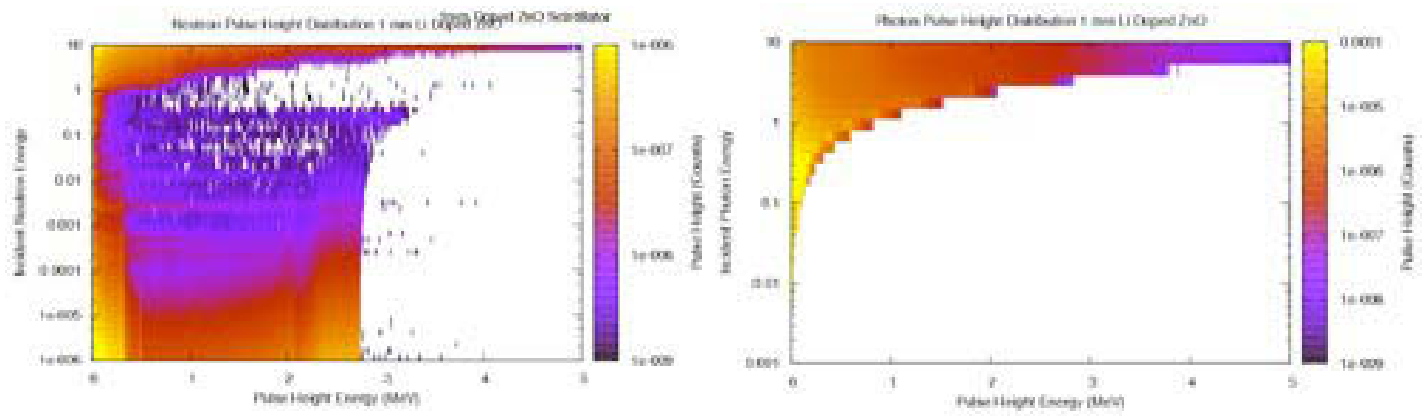

Figure 139. a) Pulse height distribution simulation resulting from neutrons incident on a $1 \mathrm{~mm}$ lithium doped $\mathrm{ZnO}$ with a $5 \mu \mathrm{m}$ 6LiF conformal coating, and (b) the pulse height distribution resulting from various photon energies in a $1 \mathrm{~mm}$ thick $\mathrm{ZnO}$ doped scintillator.

To adequately test the neutron detectors, four commercial reference detectors were used (

Table 5).

Table 5. Commercial reference detectors.

\begin{tabular}{|l|c|c|}
\hline \multicolumn{1}{|c|}{ Detector type } & Diameter & Length \\
\hline${ }^{3} \mathrm{He}$ tube (LND Inc.) & $0.635 \mathrm{~cm}$ & $10.16 \mathrm{~cm}$ \\
\hline${ }^{10} \mathrm{~B}$ lined gas filled & $2.54 \mathrm{~cm}$ & $30.48 \mathrm{~cm}$ \\
\hline${ }^{10} \mathrm{BF} 3$ & $2.54 \mathrm{~cm}$ & $30.48 \mathrm{~cm}$ \\
\hline $\begin{array}{l}\text { Lithiated glass } \\
\text { scintillator }\end{array}$ & $5.08 \mathrm{~cm}$ & $1.27 \mathrm{~cm}$ \\
\hline
\end{tabular}

The neutron sources were a $241 \mathrm{AmBe}$ driven steady state graphite pile which yields a fairly thermalized spectrum at the measurement point and a Graphite Slowing Down Spectrometer (GSDS). While the steady state graphite pile provides a simple test 
platform for thermal neutron detectors, the GSDS provides a means to select energy responses.

The lithiated glass scintillator has a characteristic $(n, \alpha)$ peak centered.$;$ Integrating the peak and subtracting detector background yieldss the neutron count.

The 10B lined gas filled tube does not exhibit a discernible $(n, \alpha)$ peak. Unless the incident neutron has very high energy, one of the particles is generally emitted into the detector's wall and, therefore, does not produce a useful ionization event.

The 10BF3 tube has three peaks due to the "wall effect" at approximately channels 250 (recoiling7Li nucleus), 350 (recoiling), and 600 counts (recoiling a particle and energetic $7 \mathrm{Li}$ nucleus). The last peak at channel 900 corresponds to the recoiling $7 \mathrm{Li}$ at ground state.

The $3 \mathrm{He}$ tube pulse height distribution for thermal neutrons also exhibits the "wall effect". The continuum of charged reaction products lies between channels 300 and 900. It has less of a stair-step because the reaction generates particles of similar charge. The total energy peak is centered around channel 1250. The sharp drop in the count rate of the gamma distribution region around channel 100 facilitates neutron versus gamma discrimination.

The neutron and gamma-ray responses of six novel neutron detectors were measured. Two of the $\mathrm{ZnO}$ detectors contained neutron target nuclei ( $\mathrm{Li}$ and $\mathrm{B}$ ). The structures of these detectors are summarized in Table 6.

Table 6. Dimensions of novel neutron scintillators.

\begin{tabular}{|c|c|c|}
\hline Detector type & Thickness & Coating \\
\hline B doped $\mathrm{ZnO}$ & $6 \mu \mathrm{m}$ & $10 \mu \mathrm{m}{ }^{6} \mathrm{LiF}$ conformal coating \\
\hline Li doped $\mathrm{ZnO}$ & $6.2 \mu \mathrm{m}$ & $10 \mu \mathrm{m}{ }^{6} \mathrm{LiF}$ conformal coating \\
\hline Al doped $\mathrm{ZnO}$ & $10 \mu \mathrm{m}$ & $10 \mu \mathrm{m}{ }^{6} \mathrm{LiF}$ conformal coating \\
\hline Ga doped $\mathrm{ZnO}$ & $8.1 \mu \mathrm{m}$ & $10 \mu \mathrm{m}^{6} \mathrm{LiF}$ conformal coating \\
\hline $\mathrm{N}$ doped $\mathrm{ZnO}$ & $9.3 \mu \mathrm{m}$ & $10 \mu \mathrm{m}{ }^{6} \mathrm{LiF}$ conformal coating \\
\hline Gd doped $\mathrm{ZnO}$ & $5.9 \mu \mathrm{m}$ & $10 \mu \mathrm{m}{ }^{6} \mathrm{LiF}$ conformal coating \\
\hline Undoped $\mathrm{ZnO}$ & $10 \mu \mathrm{m}$ & $10 \mu \mathrm{m}^{6} \mathrm{LiF}$ conformal coating \\
\hline
\end{tabular}

The first novel neutron detector to be compared is MOCVD grown boron doped $\mathrm{ZnO}$. The $(n, \alpha)$ peak results from a combination of $B$ and $L i$ based reactions and is separate from the gamma radiation region (Figure 140.b) and d)). The detector has good neutron versus gamma discrimination due to its thin geometry. 

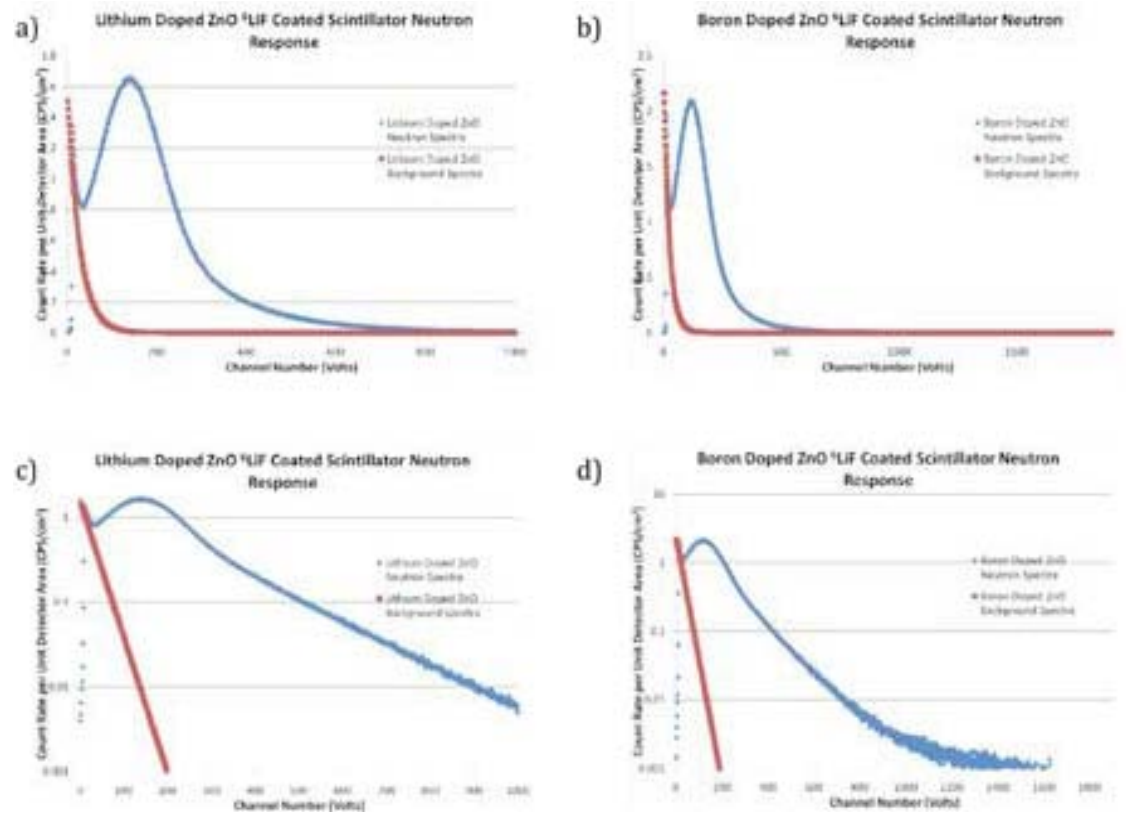

Figure 140. $(n, \alpha)$ spectra from 6 LiF coated $B$ doped $\mathrm{ZnO}(a)(c)$. ( $n, \alpha)$ spectra from 6 LiF coated $B$ doped $\mathrm{ZnO}$ (b)(d). (a) and (b) have linear vertical axes, whereas (c) and (d) have logarithmic vertical axes. All graphs show the $(n, \alpha)$ peaks clearly.

The lithium doped $\mathrm{ZnO}$ scintillator exhibits the characteristic $(n, \alpha)$ peak also visible in the boron and lithiated glass responses (Figure 140.a) and c)). The (n, $\alpha)$ peak results from the $6 \mathrm{Li}$ based reaction and can be distinguished from the gamma radiation response. The detector has good neutron versus gamma discrimination due to its thin geometry.

Bulk and a $\sim 6 \mu \mathrm{m}$ thick MOCVD grown Li doped $\mathrm{ZnO}$ scintillator were tested. The count rate is significantly higher in the bulk sample, since the thicker sample can absorb higher energy and generate a higher number of reactions.

The neutron responses of lithium doped and boron doped $\mathrm{ZnO}$ detectors were compared (Figure 141). Since both Li and B are neutron target nuclei, most of the energy of both particles is collected. The lower intensity of lithium doped $\mathrm{ZnO}$ sample's response corresponds to its lower number of dopants compared to the boron doped $\mathrm{ZnO}$ sample. 


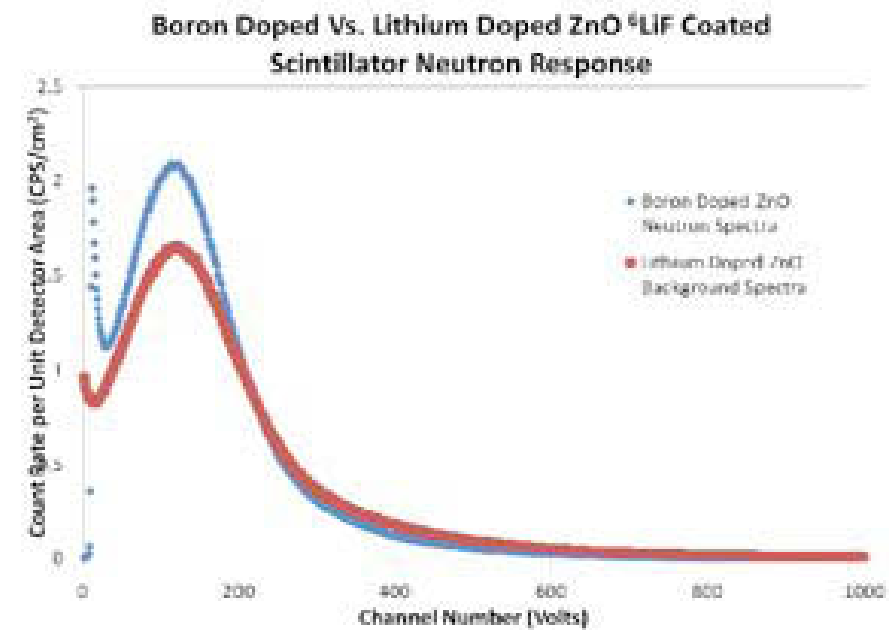

Figure 141. $(n, \alpha)$ spectra from $6 \mathrm{LiF}$ coated Li doped $\mathrm{ZnO}$ in comparison to B doped $\mathrm{ZnO}$. The $n, \alpha$ peak is clearly visible.

The remaining $\mathrm{ZnO}$ samples still detect neutrons well, even though they are not doped with neutron target nuclei. These samples had a $10 \mu \mathrm{m} 6 \mathrm{LiF}$ conformal coating (an evaporative coating process). As a result they could only collect one of the charged particles produced in the $6 \mathrm{Li}(\mathrm{n}, \alpha)$ reaction due to the isotropic nature of this reaction in the center of mass. Consequently, their responses resemble those of the $10 \mathrm{~B}$ lined tube pulse height distribution.

The AZO exhibits a lower pulse height distribution because of its lower scintillation light emission (Figure 142.a)). Neutron versus gamma discrimination is still possible, but the gamma region of the spectrum is almost a continuum similar to the $10 \mathrm{~B}$ lined tube.

Gallium doped $\mathrm{ZnO}$ performed well with acceptable neutron versus gamma discrimination. The sample used was $8.1 \mu \mathrm{m}$ thick with a $10 \mu \mathrm{m} 6 \mathrm{LiF}$ conformal coating. The background $100 \mathrm{mR} / \mathrm{h}$ gamma ray field is easily distinguished from the neutron component of the spectrum (Figure 142.b)).

Nitrogen doped $\mathrm{ZnO}$ performed with acceptable neutron versus gamma discrimination (Figure 142.c)). The sample was $9.3 \mu \mathrm{m}$ thick with a $10 \mu \mathrm{m}$ conformal coating of $6 \mathrm{LiF}$. Although the sample was annealed for 5 minutes in oxygen, some of the trapped hydrogen remained and reduced the light emission. The background 100 $\mathrm{mR} / \mathrm{hr}$ gamma ray field is easily distinguished from the neutron region of the spectrum. The total energy peak edge is located at a lower energy due to the significantly lower light emission. While the sample was optically transparent, the lower light yield is not desirable in scintillator performance.

Undoped $\mathrm{ZnO}$ performed well with decent neutron versus gamma discrimination (Figure 142.d)). The sample was $6.4 \mu \mathrm{m}$ thick with a $10 \mu \mathrm{m}$ conformal coating of $6 \mathrm{LiF}$. The background $100 \mathrm{mR} / \mathrm{hr}$ gamma ray field is easily discerned from the neutron component of the spectra.

Gadolinium doped ZnO was also tested (Figure 142.e)). The gadolinium samples performed significantly worse than the other lithium doped samples, and exhibited poor neutron versus gamma discrimination. The background $100 \mathrm{mR} / \mathrm{hr}$ gamma ray field is no longer easily distinguished from the neutron region of the spectrum, since the spectra overlap from channel 100 to 300 . 
a)

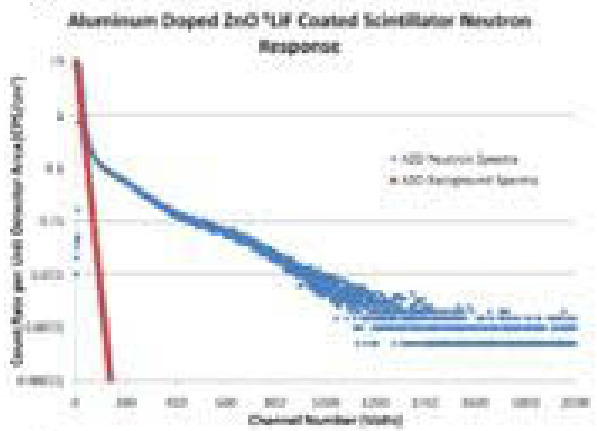

c)

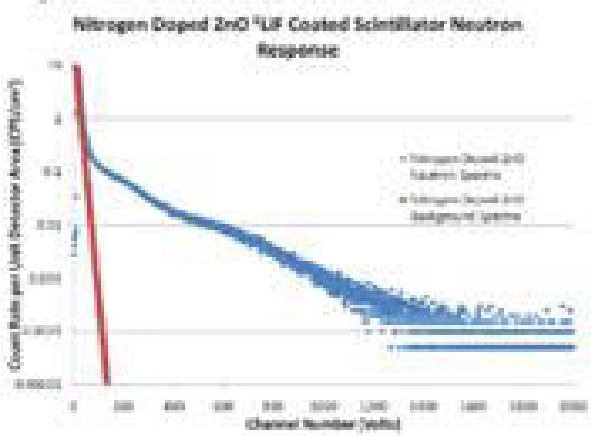

b)

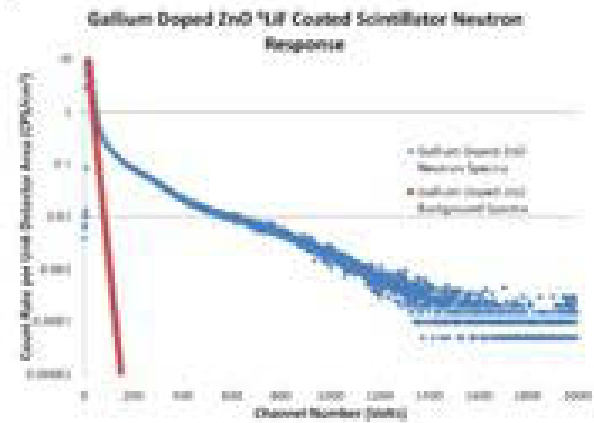

d)

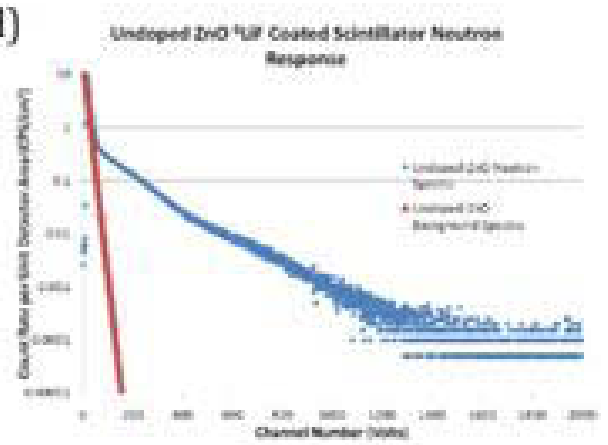

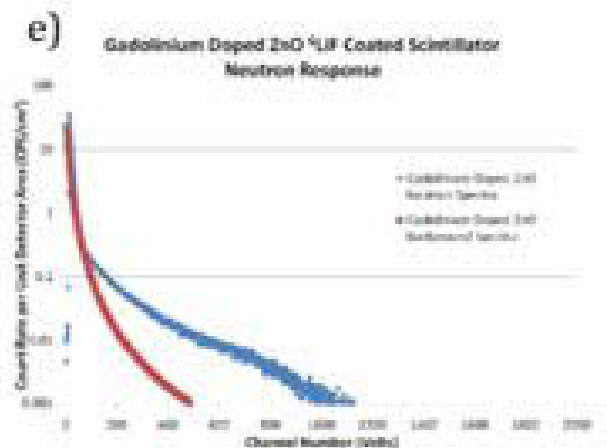

Figure 142. $(n, \alpha)$ spectra for simple coated 6 LiF structured AZO and the corresponding 100 $\mathrm{mR} / \mathrm{hr}$ gamma ray field (a). ( $n, \alpha)$ spectra for simple coated $6 \mathrm{LiF}$ structured gallium doped $\mathrm{ZnO}$ and the corresponding $100 \mathrm{mR} / \mathrm{hr}$ gamma ray field (b). (n, a) spectra for simple coated 6LiF structured nitrogen doped $\mathrm{ZnO}$ corresponding $100 \mathrm{mR} / \mathrm{hr}$ gamma ray field (c). (n, a) spectra for simple coated $6 \mathrm{LiF}$ structured undoped $\mathrm{ZnO}$ and the corresponding $100 \mathrm{mR} / \mathrm{hr}$ gamma ray field (d). $n, y$-> conversion electron spectra for uniformly doped gadolinium $\mathrm{ZnO}$ and the corresponding $100 \mathrm{mR} / \mathrm{hr}$ y ray field (e). Neutron versus gamma discrimination is acceptable for all spectra except for the $\mathrm{Gd}$ doped $\mathrm{ZnO}$ spectrum. The a spectrum has turned into a continuum which resembles a $10 B$ lined tube for all of the plots except for the $G d$ doped $\mathrm{ZnO}$ spectrum. In the $\mathrm{Gd}$ doped $\mathrm{ZnO}$ spectrum, the electron conversion is a continuum resembling a $\mathrm{y}$-ray response with a slight peak around channel 800.

To quantitatively compare the results of the neutron detection system, the detectors response to the steady state thermal neutron flux, the detector count rates as a function of detector area were computed. The neutron detection efficiency per unit area of each of the scintillators is compared in Figure 143.a). Figure 143.b) shows the comparison of neutron detection efficiency per unit volume. 


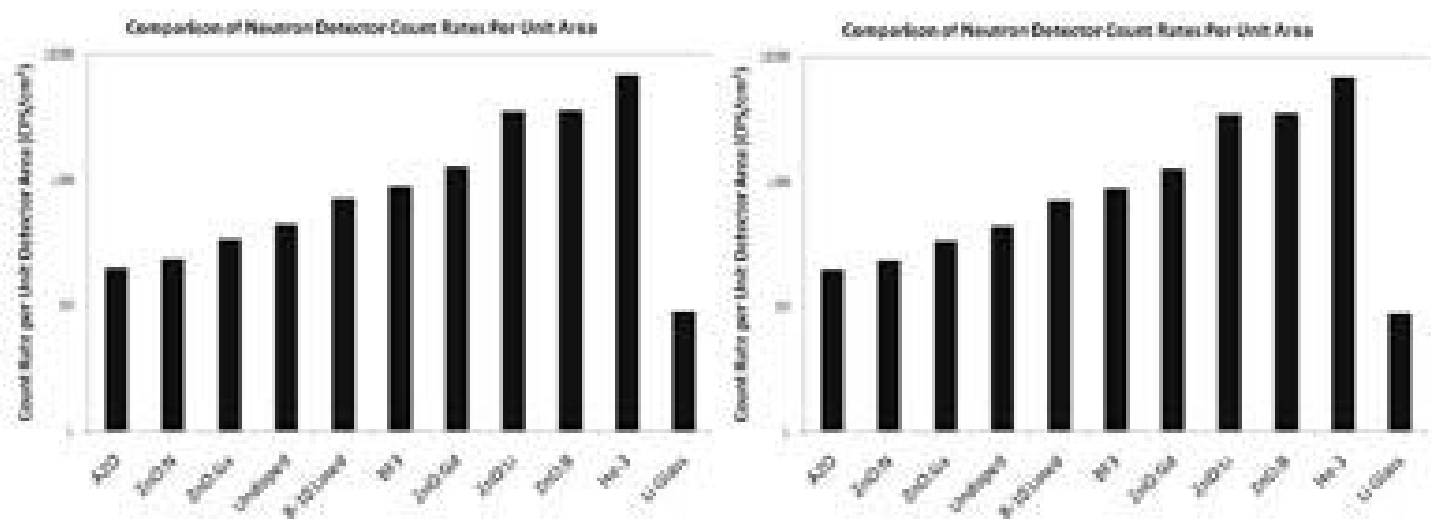

Figure 143. a) Comparison of count rates per unit area for neutron detectors tested. The count rate was summed above the zero value gamma ray cutoff. (b) Comparison of count rates per unit volume for neutron detectors tested. The count rate was summed above the zero value gamma ray cutoff.

\section{Summary and Future Directions}

The zinc oxide scintillator is a good neutron radiation detector. Zinc oxide can be grown in a number of different ways, including bulk melt growth and MOCVD growth. Thin films can be grown which exhibit equivalent or superior neutron versus gamma discrimination to conventional neutron detectors. High thermal neutron cross sections were observed which produce highly efficient neutron detectors. It is not necessary to observe a $(n, \alpha)$ peak to create a neutron detector with good neutron versus gamma discrimination, although this feature should be present in an ideal neutron detector so that the $Q$ value can be used for neutron vs. gamma discrimination. The $(n, \alpha)$ peak/region cannot overlap the gamma ray region.

Novel ZnO neutron detectors have been simulated by Monte Carlo methods. Using these detectors, growth studies were undertaken successfully to optimize the scintillator structures for both MOCVD and bulk melt growth. The samples which do not possess lithium or boron interstitially mixed into the matrix currently resemble the response of a boron lined gas filled tube. This is due to the inability to collect both particles because of the coating layer thickness. Two sided scintillators can be grown over conformal $6 \mathrm{LiF}$ coatings. The full energy peak for both the triton and alpha particle in the $6 \mathrm{Li}(n, \alpha)$ reaction can be collected in doped scintillators. This $Q$ value could be used for neutron spectroscopy.

It has been shown that doped $\mathrm{ZnO}$ scintillators exhibit superior characteristics compared to undoped $\mathrm{ZnO}$ in optical clarity, light emission, and neutron detection. However, when doping the scintillator, there is an optimal point of dopant concentration. Past this optimal value the material becomes either conducting or insulating, opaque, and decreases in performance. Determining the peak value for doping was the key to this work. Increasing the dopant concentration beyond this point, even though in theory it should have raised detection efficiencies, in fact impedes performance.

The thermal neutron reaction was dominated by surface reactions. Comparing count rates versus surface area, the detectors at one particular layer thickness perform well in comparison to conventional neutron detectors. When comparing the count rate per unit volume of the detector, the thin film $\mathrm{ZnO}$ scintillators show superior neutron detection compared to other conventional neutron detectors. The conformal coating 
thickness was initially selected to be $10 \mu \mathrm{m}$ because of ease of growth. However, this thickness was neither optimal for neutron detection nor for fabrication. A switch from thermal evaporation to electron beam evaporation should generate thinner, higher efficiency coatings. $1050 \mathrm{~nm}$ layers of $6 \mathrm{LiF}$ deposited multiple times over layers of doped $\mathrm{ZnO}$ would yield higher efficiencies and be easier to fabricate. With the $10 \mu \mathrm{m}$ thick coatings, the probability that both particles will enter the scintillator, even in a 2 sided device, is low. By minimizing this thickness to $10-50 \mathrm{~nm}$ and increasing the number of layers, it is possible to improve scintillator efficiency.

The Monte Carlo simulations of the neutron detectors show that the optimal neutron versus gamma discrimination capabilities of the $\mathrm{ZnO}$ scintillator are realized when the scintillator thickness is kept as thin as possible. Making stacks of one or two sided scintillators offset with sapphire substrates (or other non-scintillating / non-Cherenkov radiation emitting materials) is beneficial for increasing the neutron detection efficiency, but effectively separates the responses of each scintillator, minimizing the gamma response. Neutron versus gamma discrimination can be achieved at larger thicknesses, but at a noticeable reduction in efficiency if the zero crossing value of the photon response for the low energy cutoff is used.

When examining the models produced in the Monte Carlo simulations, and extrapolating from current data, stacks of 3 or 4 of these scintillators can have efficiencies equal to or greater than $3 \mathrm{He}$ tubes. The detectors are already equivalent in neutron versus gamma discrimination. Comparing the speed of the $3 \mathrm{He}$ tube to the $\mathrm{ZnO}$ scintillator, the $\mathrm{ZnO}$ scintillator is a superior choice. The $\mathrm{ZnO}$ scintillator can be grown in large surface area devices producing very efficient neutron detectors, with efficiencies superior to $3 \mathrm{He}$ tubes. These novel devices are superior to lithiated glass, 10BF3 tubes and 10B-lined tubes in overall efficiencies and speed. The fabrication cost of these scintillators is relatively low in comparison to that of $3 \mathrm{He}$ or other structures. In addition, there exists a direct road to commercialization of these devices through companies such as Cermet Inc.

This doped $\mathrm{ZnO}$ structure shows promise in fulfilling the need for a near term $3 \mathrm{He}$ tube replacement for homeland security applications. Either bulk melt grown materials or MOCVD grown materials are suitable for this endeavor. Bulk melt grown materials have the promise of large throughput and very thick samples. However, bulk melt growth would not be a good use of limited enriched $6 \mathrm{Li}$ compounds, but this still needs to be further investigated. Application of conformal coatings of uniform samples is the most economic path to commercialization with bulk melt growth materials. MOCVD grown materials grown with a larger, scaled up version of the reactor do show promise. Here, rapid layer changes and enriched lithium can be used to their advantage to make thin multi-layered stacks of materials. Materials grown by this method could approach the necessary $100 \%$ intrinsic efficiency. The single stacks achieved $43 \%$ intrinsic efficiencies for thermal neutrons.

Overall, this work has shown that regardless of the growth method, doped $\mathrm{ZnO}$ and $6 \mathrm{LiF}$ coated $\mathrm{ZnO}$ with or without dopants can produce a highly efficient neutron scintillator. Dopant studies were conducted for three types of dopants, (1) those for wavelength shifting, (2) crystal transparency improvement, and (3) neutron detector nuclei. In the crystal transparency regime, aluminum improves the optical clarity of the sample, but shifts the scintillation spectrum toward the UV. Of the wavelength shifting dopants, gallium and boron, exhibit a good blue wavelength shift. However, they produce optically clouded materials because the oxide formation of each of these materials is a white, opaque material. Lithium and boron can be doped as 
neutron detector nuclei. Lithium produces a more optically clear material. Lithium does blue shift the scintillation spectrum slightly but is difficult to grow by MOCVD due to lack of a good precursor. Lithium acetatonate shows the most promise for the growth of MOCVD lithium doped $\mathrm{ZnO}$. Dopant materials and scintillation luminosity are inversely proportional. While the dopant materials add beneficial characteristics to the scintillator, high dopant levels degrade the scintillation. A fine balance must be struck between dopant characteristics and scintillation luminescence.

To further improve the detector efficiency of the $\mathrm{ZnO}$ scintillator, a new heterostructure has been developed. This involves alternating layers of $\mathrm{ZnO}{ }^{6} \mathrm{LiF}$, and sapphire substrates. A schematic is shown below followed by an SEM image of the grown wafers.

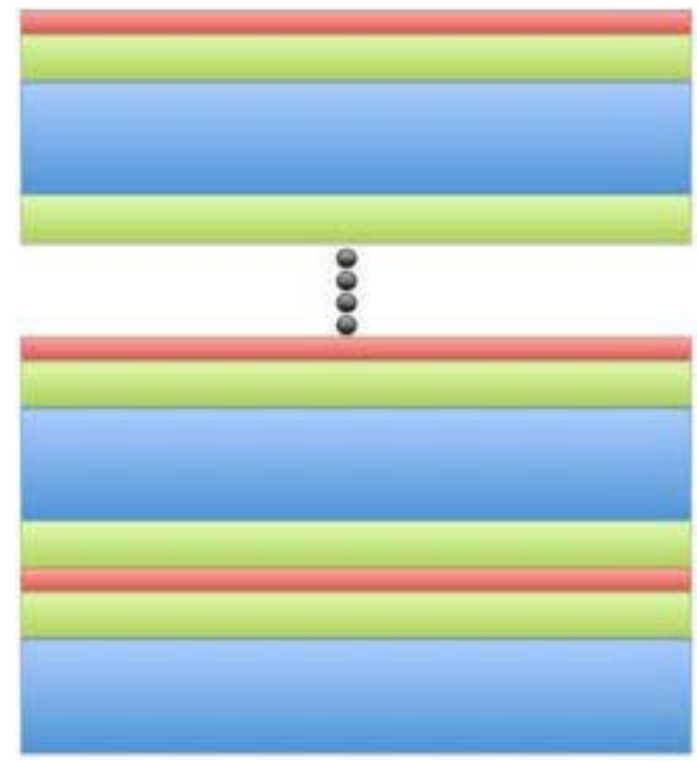

Figure 144 a heterostack of $\mathrm{ZnO}$ scintillators, sapphire substrates and LiF layers. The blue represents sapphire, green is $\mathrm{ZnO}$ and red is LiF. The top unit comprises of one base unit which is stacked to produce a high resolution device. 


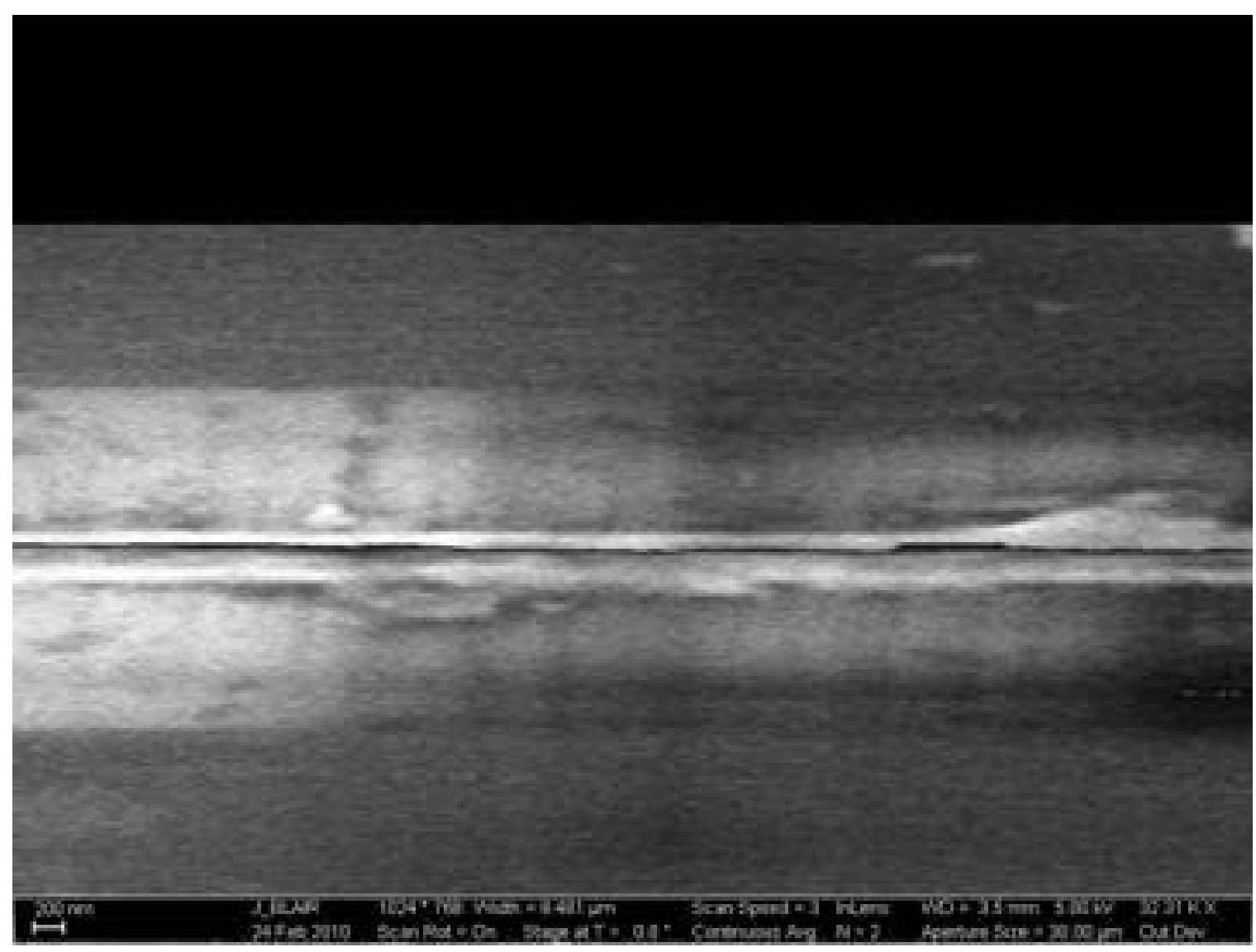

Figure $145 \mathrm{ZnO}$ heterostructure grown by MOCVD. The $\mathrm{ZnO}$ scintillator is the light grey color. 


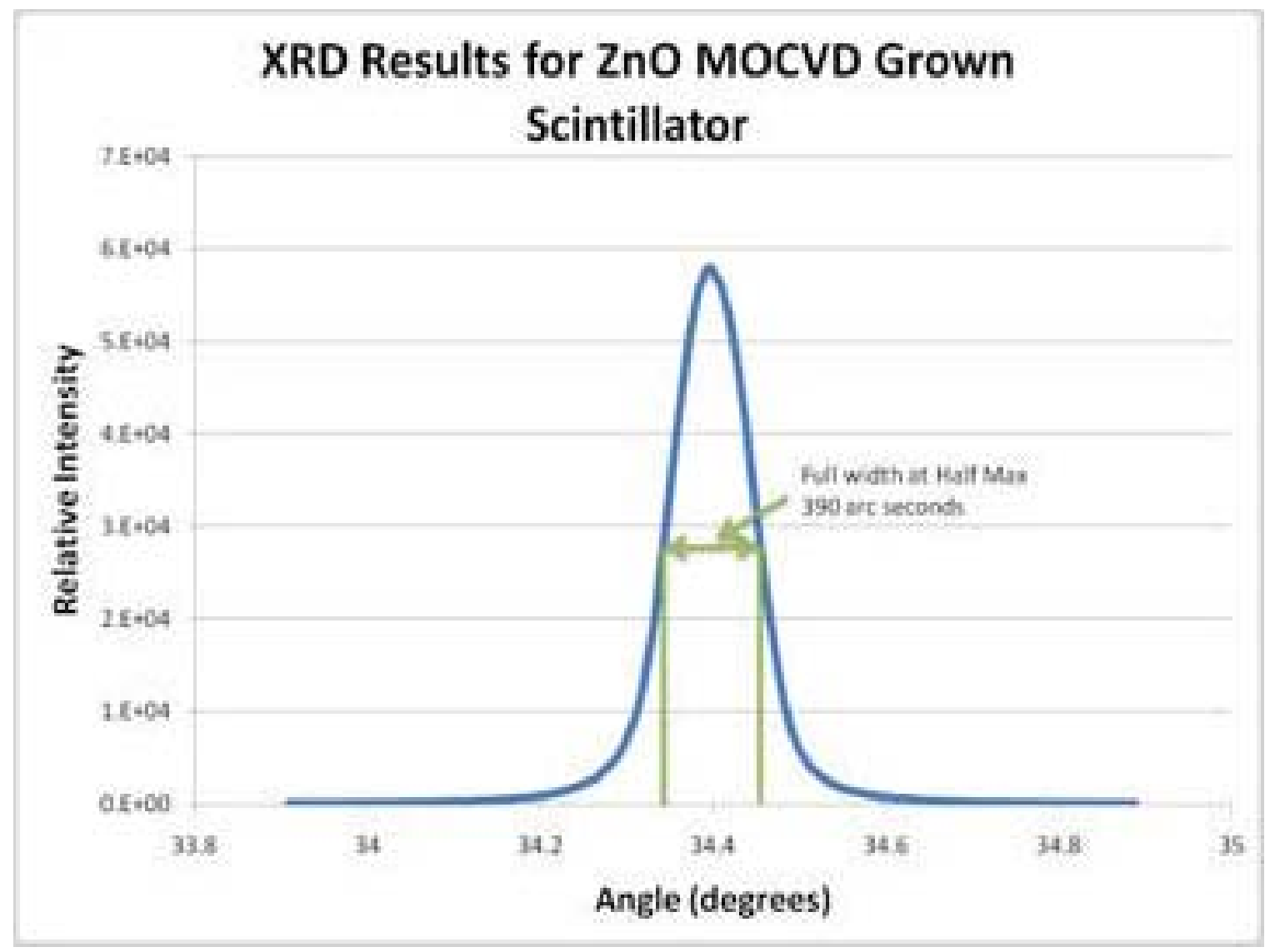

Figure 146 Crystalline quality shown by XRD rocking curve analysis. Very high quality samples are being grown through this method.

\section{GaN Materials Growth and Scintillator Performance}

$\mathrm{GaN}$ is a promising candidate as a neutron detection material compared to other semiconductor materials due to its superior radiation hardness. Additionally, GaN can be doped and/or alloyed with neutron- sensitive elements such as boron and gadolinium. There are challenges, however, to creating GaN-based neutron detectors. These include the difficulty of achieving efficient neutron detection with thin films and the difficulty of achieving gamma-discrimination when using Gadolinium (Gd).

\section{Introduction}

$\mathrm{GaN}$ is the most important of the three binary compounds that form the basis of the IIINitride semiconductor material system, the others being Aluminum Nitride (AIN) and Indium Nitride $(\operatorname{InN})$. These materials have a wurtzite structure (shown schematically in Figure 147 ) and direct bandgaps. Since Shuji Nakamura's material and device breakthroughs in the mid-1990's [37], the III-Nitrides have seen extensive use in optoelectronic devices such as 


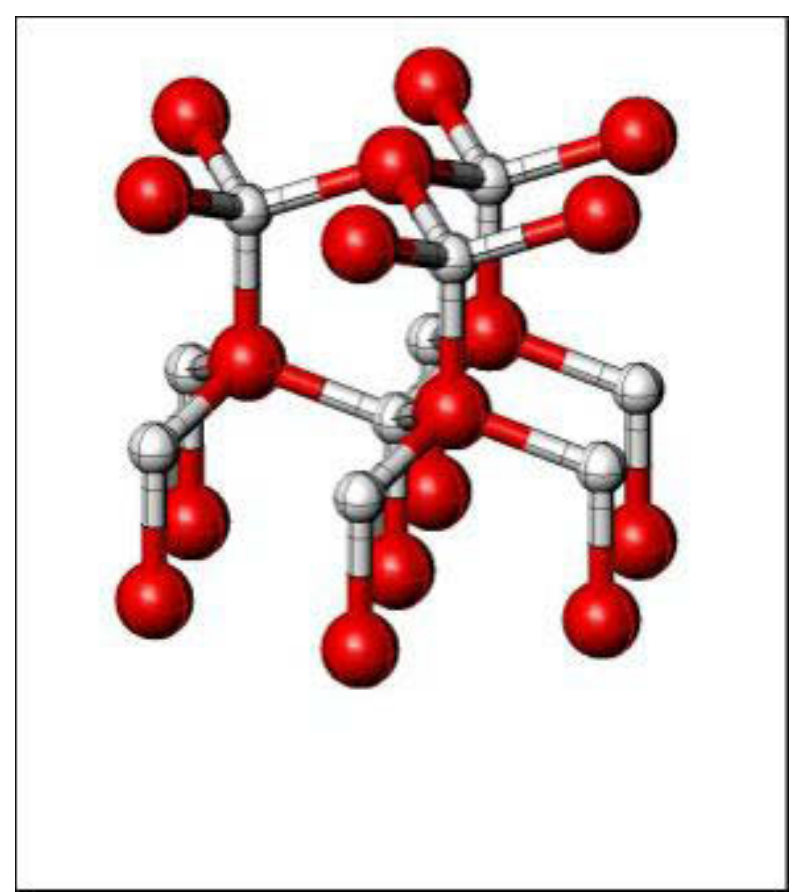

Figure 147. The wurtzite structure of III-Nitrides.

light-emitting diodes (LED), laser diodes (LD), and photodiodes. Commercial technologies such as solid-state lighting (SSL) and Blu-rayTM optical storage are based on such III-Nitride devices. High electron mobility transistors (HEMT) [38] and heterojunction bipolar transistors (HBT) [39] have also been produced using these materials, as they have several desirable characteristics including high carrier saturation velocity and high breakdown electric field. In addition, GaN has a wide bandgap of $3.4 \mathrm{eV}$ which can be tuned to higher values through alloying with AIN (EG = 6.2 eV) and to lower values through alloying with InN (EG = $0.7 \mathrm{eV})$. The lattice constant to bandgap relationship is shown in Figure 148 with bandgap bowing parameters taken from Liou et al. [40]. The wide bandgap of GaN offers the potential for producing very low noise devices, which is a common problem for Si-based technologies. Table 7 Properties of Gallium Nitride and other semiconductors [41] gives a comparison of the semiconductor properties of $\mathrm{GaN}$ and other common semiconductor materials [41]. 


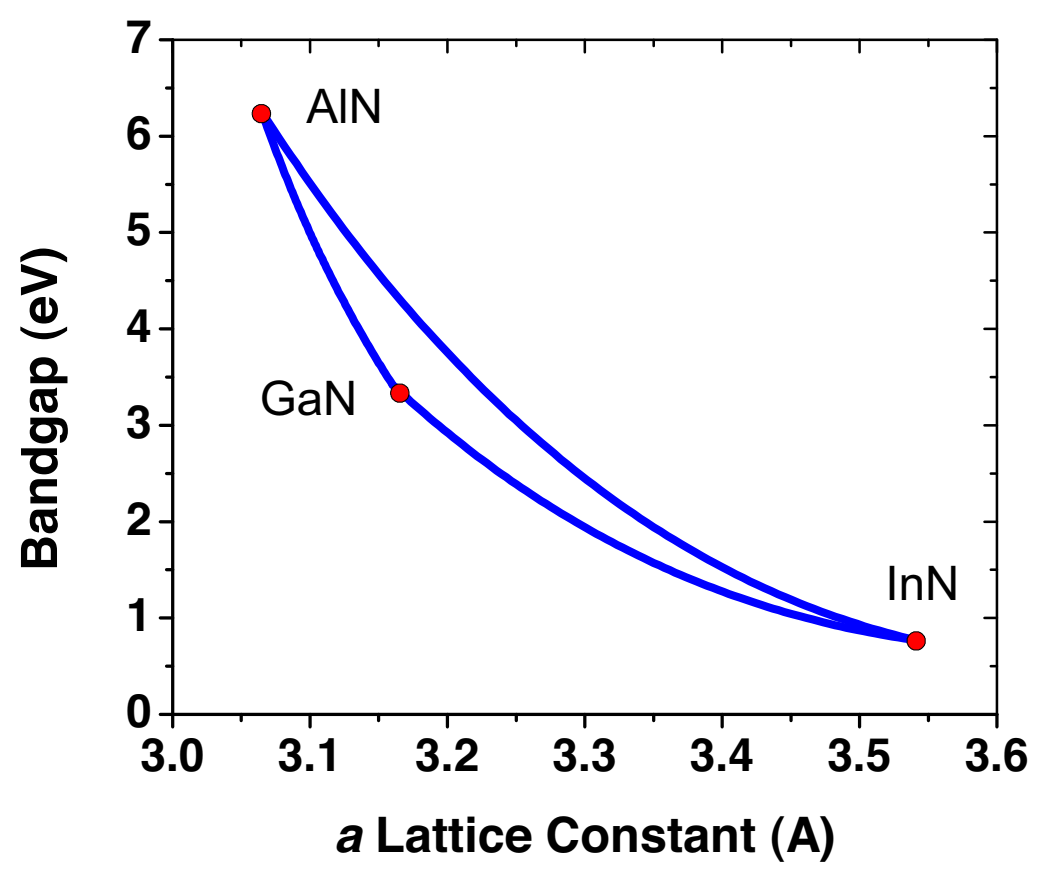

Figure 148 Lattice constant versus bandgap values for III-Nitrides.

Table 7 Properties of Gallium Nitride and other semiconductors [41]

[1]

\begin{tabular}{|l|c|c|c|c|}
\hline & Silicon & $\begin{array}{c}\text { Gallium } \\
\text { Arsenide }\end{array}$ & $\begin{array}{c}\text { Silicon } \\
\text { Carbide }\end{array}$ & $\begin{array}{c}\text { Gallium } \\
\text { Nitride }\end{array}$ \\
\hline $\begin{array}{l}\text { Bandgap } \\
\text { (eV) }\end{array}$ & 1.1 & 1.42 & 3.26 & 3.4 \\
\hline $\begin{array}{l}\text { Electron mobility } \\
\text { (cm } \mathbf{2} / \mathbf{s})\end{array}$ & 1500 & 8500 & 700 & $1000-2000$ \\
\hline $\begin{array}{l}\text { Saturated electron velocity } \\
\text { (cm/s) }\end{array}$ & 1.0 & 1.3 & 2.0 & 1.3 \\
\hline $\begin{array}{l}\text { Critical breakdown field } \\
\text { (MV/cm) }\end{array}$ & 0.3 & 0.4 & 3.0 & 3.0 \\
\hline $\begin{array}{l}\text { Thermal Conductivity } \\
\text { (W/cm·K) }\end{array}$ & 1.5 & 0.5 & 4.5 & $>1.5$ \\
\hline Relative dielectric constant & 11.8 & 12.8 & 10.0 & 9.0 \\
\hline
\end{tabular}

\section{Radiation Hardness}

The aforementioned superior semiconductor characteristics make GaN worthy of consideration for use in most solid-state technologies. In most applications requiring neutron detection it is desirable to use a detector with a long lifetime. For any material to achieve this it must be either resistant to or tolerant of radiation-induced damage from bombardment with gamma rays and heavy particles. GaN has been studied by several groups for potential use in high radiation environments and continues to be a promising candidate for such applications. 
The atomic threshold displacement energy (Ed) is a useful parameter for predicting damage accumulation caused by electron, neutron, and light ion bombardment [42]. Ed can be thought of as the minimum kinetic energy that must be transferred to an atom to break it from its lattice site and become an interstitial. Look et al. irradiated epitaxial GaN films on sapphire with high energy electrons (0.7-1 MeV) and calculated displacement energies for the $\mathrm{Ga}$ and $\mathrm{N}$ atoms of $20.5 \mathrm{eV}$ and $10.8 \mathrm{eV}$, respectively [43]. Ionascut-Nedelcescu et al. [44] calculated a similar $\mathrm{Ga}$ atom displacement energy of $\sim 20 \mathrm{eV}$ based on high energy electron bombardment of bare GaN/InGaN blue LED die [44]. This is quite high compared to other common semiconductor materials, such as $\mathrm{Si}, \mathrm{GaAs}$, and Silicon Carbide ( $\mathrm{SiC}$ ). No N displacement was observed in this experiment, which was interpreted as an indication that the nitrogen sub-lattice is self-repairing. A comparison of atomic displacement energy for common semiconductors is shown in Figure 149. More recent simulations have shown that $\mathrm{Ed}$ is highly dependent on direction for both $\mathrm{Ga}$ and $\mathrm{N}$. Nord et al. applied molecular dynamics simulation to $\mathrm{GaN}$ - using atomic potentials derived from empirical fitting - and found minimum displacement energies of $22 \mathrm{eV}$ for $\mathrm{Ga}$ and $25 \mathrm{eV}$ for $\mathrm{N}$ [45]. They also simulated bombardment from 1000 random directions and found average Ed values of $45 \mathrm{eV}$ for $\mathrm{Ga}$ and $109 \mathrm{eV}$ for $\mathrm{N}$. While the minimum displacement energy for $\mathrm{Ga}$ agrees well with the reported experimental values, it is surprising that the $\mathrm{N}$ displacement energy was predicted to be higher than that for $\mathrm{Ga}$. Xiao et al. [42] also used molecular dynamics simulation to analyze.

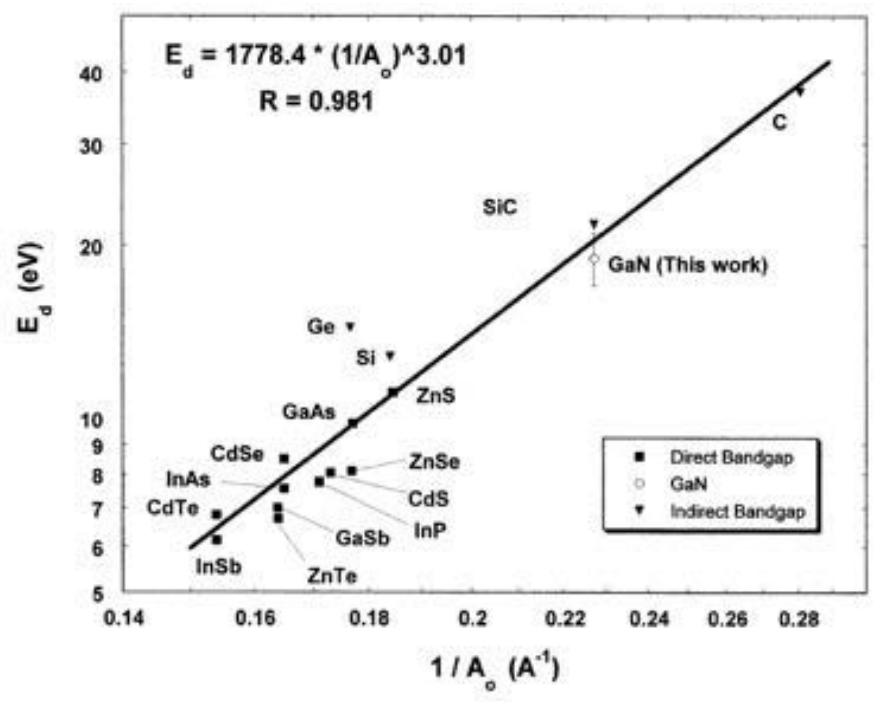

Figure 149. Lattice constant versus atomic displacement energies for common semiconductors. [44] 
Investigation of the material robustness of $\mathrm{GaN}$ under fast and thermal neutron bombardment has been performed by several research groups. In 2004 Park et al. experimented with thermal neutron-induced transmutation doping (TMD) of Si-doped GaN [46]. They observed successful conversion from n-type to p-type, which was attributed to conversion of Si to Phosphorous $(\mathrm{P})$, however poor hole mobility and deterioration of the photoluminescence $(P L)$ spectra indicated that the films became quite defective. Kuriyama et al. irradiated $\mathrm{GaN}$ with fast neutrons and found that $\mathrm{GaN}$ had half the number of $\mathrm{Ga}$ displacements per primary knock-on (PKO) than Gallium Phosphide ( $\mathrm{GaP})$, confirming that the chemical bonding of $\mathrm{GaN}$ is stronger than that of $\mathrm{GaP}$ [47]. Marques et al. found that both thermal and fast neutrons contributed to damage in $\mathrm{GaN}$ after respective fluencies of $\sim 1019 \mathrm{~cm}-2$ each [48]. In particular, significant expansion of the lattice in the c-direction was observed, with no significant changes in the a-parameter. This indicates that atoms were displaced preferentially along the c-direction. Thermal neutrons have far lower energy than the quoted displacement energies of either $\mathrm{Ga}$ or $\mathrm{N}$, however it was speculated that they could still contribute to displacement by undergoing radiative capture reactions $(n, y)$ which produce high energy gamma rays. These gamma rays cause recoil of the emitting atom which can be enough to displace it. Grant et al. found that epitaxial semi-insulating $\mathrm{GaN}$ films retained $>80 \%$ charge collection efficiency for fast neutron fluences up to $1015 \mathrm{~cm}$ 2 [49]. Polyakov et al. have performed several studies on neutron effects in GaN [5052]. Among their findings: fast neutrons introduce electron traps with $0.75 \mathrm{eV}$ activation energy and produce an electron removal rate of $\sim 5 \mathrm{~cm}-1$ in undoped $\mathrm{GaN}$; high fluence $(>1016 \mathrm{~cm}-2)$ of fast neutrons produces compensation of p-type GaN, eventually converting it to highly resistive n-type after $\sim 1018 \mathrm{~cm}-2$.

The general consensus to be taken from the literature is that the appearance of device degrading damage in $\mathrm{GaN}$ due to neutron irradiation does not occur until fluences $\geq$ $1015 \mathrm{~cm}-2$ of fast neutrons. This confirmed radiation hardness coupled with the excellent semiconductor properties of GaN make it a very good candidate for use in novel solidstate neutron detectors.

\section{Intrinsic Neutron Sensitivity}

Nitrogen makes up $50 \%$ of the GaN crystal structure, with a density of $4.4 \times 1022 \mathrm{~cm}-3$. Thus $\mathrm{GaN}$ is intrinsically neutron sensitive through the $14 \mathrm{~N}(\mathrm{n}, \mathrm{p})$ reaction, as mentioned earlier. The two isotopes of gallium, $69 \mathrm{Ga}(60 \%)$ and $71 \mathrm{Ga}(40 \%)$, both have comparable neutron cross-sections as $14 \mathrm{~N}$, however they primarily interact via elastic scattering, which does not result in ionization or ionizing secondary products.

Another advantage of $\mathrm{GaN}$ over most other semiconductors is the possibility of doping or alloying with neutron-sensitive elements, such as B and $\mathrm{Gd}$. Boron alloys with $\mathrm{GaN}$, replacing $\mathrm{Ga}$ in the crystal lattice and resulting in a higher bandgap. Crystalline Ga1$x B x N$ thin films have been grown by metalorganic chemical vapor deposition (MOCVD) with $\mathrm{x}$ as high as $2 \%$ [53]. Gadolinium-doping of $\mathrm{GaN}$ is another way to introduce neutron-sensitive nuclei. This has been achieved during epitaxial growth of thin films $[54,55]$, during bulk growth [56], and through ion-implatation [57]. Ga1-xGdxN films with $x$ as high as $12.5 \%$ have been reported [55]. These films can be incorporated into GaN-based neutron detectors, such as scintillators and diode detectors. 


\section{Challenges for Gallium Nitride Neutron Detectors}

\section{Thin Film}

GAN has many characteristics that make it a promising candidate for solid-state neutron detection, however there are a few challenges that must be overcome to realize this potential. The first of these challenges is the low thickness of epitaxial films. It is very difficult and expensive to produce bulk GaN, due to the intense pressure and temperature requirements to keep it from breaking down into Ga and N2 [58]. Recently, bulk GaN substrates produced by hydride vapor phase epitaxy (HVPE) and ammonothermal growth have become available [5961]. However, these substrates are prohibitively expensive for most devices, and thus the majority of GaN devices are produced by heteroepitaxy on sapphire substrates using molecular beam epitaxy (MBE) or MOCVD. These techniques are generally limited to growth rates of a few microns per hour, making them only practical for thin film growth. Due to the relatively long mean free path of high energy particles (and neutrons in particular) in matter, it is generally desirable to maximize the interaction volume in radiation detectors. Thus, the limited thickness of epitaxial GaN films limits the achievable neutron detection efficiency. However, the efficiency can continue to be improved as bulk GaN substrates become more available.

\section{Large Area Devices}

In low flux applications it is desirable to maximize the exposed area of the neutron detector - in addition to maximizing the detector volume - in order to maximize the number of impinging neutrons. This is challenging for GaN-based devices due to the relatively poor lateral conduction in $\mathrm{p}-\mathrm{GaN}$ and nonuniformities in the $\mathrm{p}$-i-n layers. The chances for a device killing defect also increase with increased device area. These issues can be largely overcome by utilizing arrays of small devices rather than large-area single devices. Such an array would also enable source location functionality.

\section{Dark Current}

Another challenge to using $\mathrm{GaN}$ diodes for radiation detection is the relatively high reverse-bias dark current found in these devices. This issue is primarily caused by the high threading dislocation density found in heteroepitaxial GaN films. These dislocations provide shunt current paths, thus reducing the achievable signal-to-noise ratio. Noise from dark current in GaN-based detectors can be reduced by reducing threading dislocations, by passivating surface defects caused by plasma etching, and by operating at low to no bias.

\section{Gamma Discrimination}

Gamma discrimination is an important characteristic of neutron detectors because most neutron sources also produce gamma photons. Gamma discrimination will be a challenge for neutron detectors using $\mathrm{Gd}$, because the products of the $157 \mathrm{Gd}(\mathrm{n}, \mathrm{y})$ reaction are gamma photons and electrons with energies similar to gamma photons. Thus, the ionization resulting from neutron conversion will be indistinguishable from ionization resulting from incident gamma photons through only pulse height discrimination. Device designs similar to Li-sandwich detectors could allow for isolation of neutron-induced signals through co-incident signal isolation. 


\section{Experimental Techniques and Equipment}

In this research, GaN-based neutron detectors consist of structures grown epitaxially by MOCVD. In addition to standard GaN growth, Gd-doped GaN was also grown. Several standard material and device characterization techniques have been used to aid in the development of these neutron detectors. Material characterizations include structural, optical, electrical, and surface techniques. X-ray diffraction (XRD) was the primary structural characterization technique. The primary optical characterization was PL. Electrical characterization was achieved using Hall effect measurements and contactless resistivity mapping. Devices were fabricated using standard photolithography, plasma etching, and metallization techniques for III-Nitrides. Diode devices were tested for current-voltage (I-V) behavior, dark current, and photoresponse as well as capacitancevoltage behavior. Neutron testing of devices was performed using an Am-Be neutron source and an AGN-201 thermal reactor.

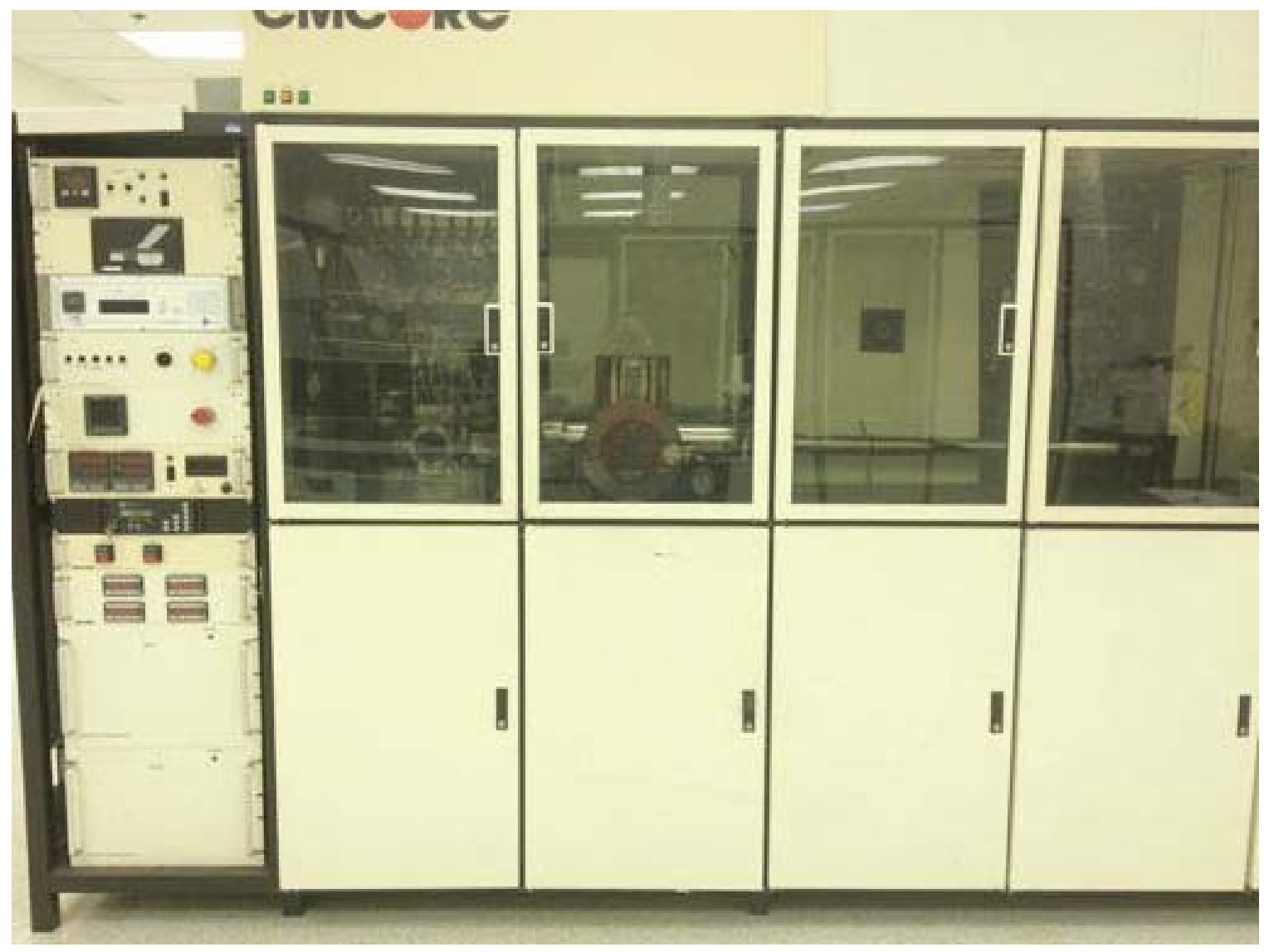

Figure 150 New MOCVD GaN tool installed at the ISU RISE Complex.

\section{Material Growth}

\section{MOCVD Growth of GaN}

MOCVD is an epitaxial crystal growth technique used to produce high quality semiconductor thin films. The most common application of MOCVD is in the production of compound semiconductor devices structures such as solar cells, LEDs, 
and LDs. MOCVD is the most widely used epitaxy technique in commercial production of optoelectronic devices due to its excellent scalability and high throughput. Deposition is achieved through the reaction of organometallic and hydride precursor gases in the presence of a suitable substrate. In the case of $\mathrm{GaN}$, the precursors are usually trimethylgallium (TMG) and ammonia (NH3). The quality of the resulting films is dependent on precise control of growth conditions, including temperature, pressure, gas flow rates, and precursor ratios. The choice of substrate also has a large impact on film quality, as in all forms of epitaxy.

The first applications of MOCVD were producing III-Arsenide and III-Phosphide materials, for which native bulk substrates are available. In the case of III-Nitrides, however, traditional melt-type bulk production techniques are not possible, due to the extremely high temperature and pressure required to prevent decomposition [58]. GaN substrates produced by ammonothermal or hydride vapor phase epitaxy (HVPE) have recently become available (at very high cost) [59-61], however most commercial and research III-Nitride growth continues to be on non-native substrates. Heteroepitaxy is problematic, however, because lattice size and thermal expansion mismatch between the substrate and the deposited film cause dislocations to form. For III-Nitride growth, sapphire (16\% lattice mismatch) is the most common substrate, followed by $6-\mathrm{H} \mathrm{SiC} \mathrm{(3.6 \%} \mathrm{lattice} \mathrm{mismatch).}$

A buffer layer is necessary in order to achieve high quality GaN thin films on sapphire. Historically, AIN buffer layers were the first to enable high quality GaN growth [62]. Later, GaN buffer layers were developed and are more commonly used today [63]. These buffer layers are grown at low temperature and are initially amorphous. They are then brought to high temperature, which crystallizes the material and causes it to form islands. These islands eventually coalesce, forming a two-dimensional film. The steps involved in growing GaN on sapphire are illustrated in Figure 151.

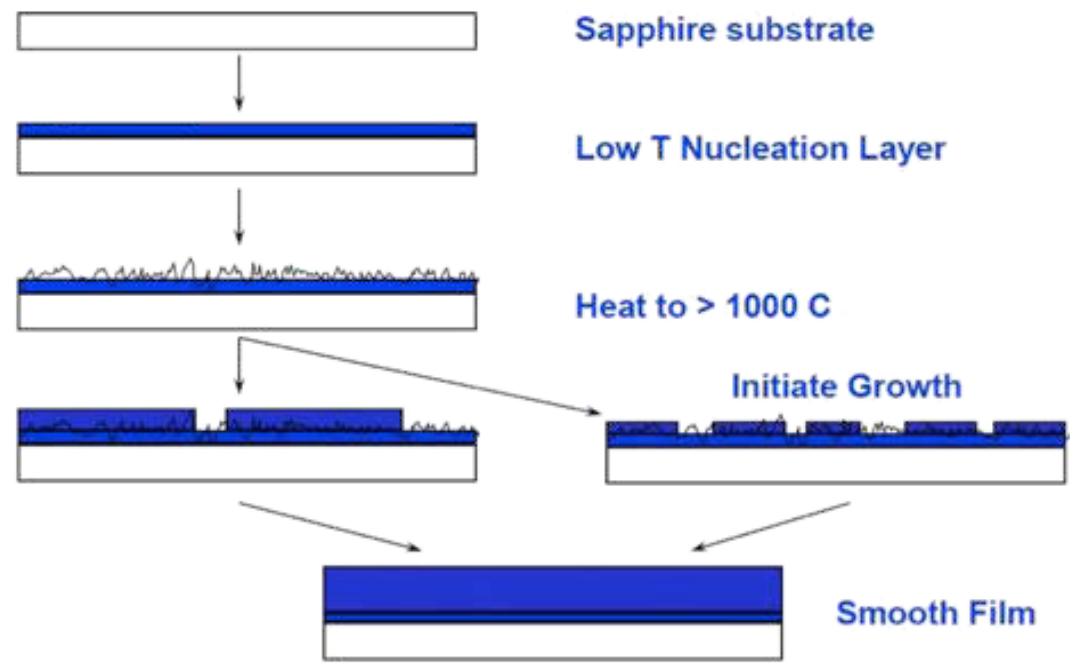

Figure 151. Typical growth process for GaN on sapphire, using a low-temperature nucleation layer.

Heteroepitaxial GaN has much higher threading dislocation density $(108-109 \mathrm{~cm}-2)$ than typical III-Arsenide and III-Phosphide thin films, even with the use of lowtemperature buffer layers [64]. GaAs-based LED performance is greatly limited by 
dislocation densities greater than $104 \mathrm{~cm}-2$, while GaN-based LEDs have shown remarkable insensitivity to dislocation densities 4-5 orders of magnitude higher [65]. In addition to high dislocation density, GaN epilayers generally have a high unintentional free electron concentration of $\sim 1016 \mathrm{~cm}-3$ contributed by crystal defects related to heteroepitaxy. Shallow donor impurities such as carbon and oxygen contributed by precursor gases used in MOCVD also contribute to the unintentional doping.

One of the foremost challenges of III-Nitride materials is the difficulty of achieving highly conductive p-type material. The high unintentional $n$-type doping in $\mathrm{GaN}$ is one of the causes of the causes of this. The other factor contributing to poor quality $\mathrm{p}$ $\mathrm{GaN}$ is the relatively deep acceptor level ( 200 meV) of Magnesium (Mg) in GaN, which results in only $\sim 1 \%$ ionization at room temperature [66]. Despite being so inefficient, Mg remains the best known acceptor dopant for III-Nitrides. However, the low ionization efficiency necessitates high $\mathrm{Mg}$ doping levels to achieve reasonable hole concentrations. This further degrades crystal quality and lowers hole mobility, resulting in relatively low conductivity $p-G a N$. Room temperature hole concentrations are generally limited to $<1018 \mathrm{~cm}-3$. These problems have proved persistent and have hindered progress in III-Nitride device performance. While modulated growth techniques such as delta-doping have yielded hole concentrations $>1018 \mathrm{~cm}-3$, these films have generally showed low hole mobility and have not been widely reproducible $[67,68]$. Quaternary AIGalnN alloys have been proposed as a way to achieve materials with both high bandgap and low acceptor ionization energy [69].

In contrast, high quality n-type GaN is quite easy to achieve using Si doping. Silicon is a very shallow donor in $\mathrm{GaN}$, and room temperature electron concentrations of up to $1019 \mathrm{~cm}-3$ can be achieved while still maintaining high crystal quality [70] [Lee 1998]. The doping asymmetry in the III-Nitrides is typical of other wide bandgap semiconductor materials, which generally have either a low valence band minimum or a high conduction band maximum relative to the vacuum level [71]. Examples of other "unipolar" materials are diamond (difficult to achieve n-type) and Zinc Oxide ( $\mathrm{ZnO}$, difficult to achieve p-type). In the case of III-Nitrides, increasing the bandgap (increasing $\mathrm{Al}$ concentration) further decreases the $\mathrm{Mg}$ ionization efficiency, while decreasing the bandgap (increasing In concentration) increases the Mg ionization efficiency [72].

Table 8

\begin{tabular}{|l|l|} 
Element & Precursor \\
\hline Gallium $(\mathrm{Ga})$ & Trimethylgallium $(\mathrm{TMG})$ \\
\hline Nitrogen $(\mathrm{N})$ & Ammonia $(\mathrm{NH} 3)$ \\
\hline Silicon $(\mathrm{Si})$ & Silane $(\mathrm{SiH} 4)$ \\
\hline Magnesium $(\mathrm{Mg})$ & Bis(cyclopentadienyl)magnesium $(\mathrm{Cp} 2 \mathrm{Mg})$ \\
\hline
\end{tabular}

The modified EMCORE D125 MOCVD growth tool used in this research is shown in Figure 152. This tool is a vertical-injection commercial system with a rotating disk and short jar configuration. It has been modified by the addition of a second injector block to prevent cross-contamination between processes and to allow for the use of a large 
number of unique metalorganic precursors. The gas panel of the tool has also been modified to allow for the use of either hydrogen or nitrogen as a carrier gas. The electrical power system of the tool allows for growth temperatures of up to $1200{ }^{\circ} \mathrm{C}$. The tool is equipped with a load lock system for control of the reactor environment to ensure that no impurities enter the reactor from the atmosphere thus preventing contamination of the growth chamber.

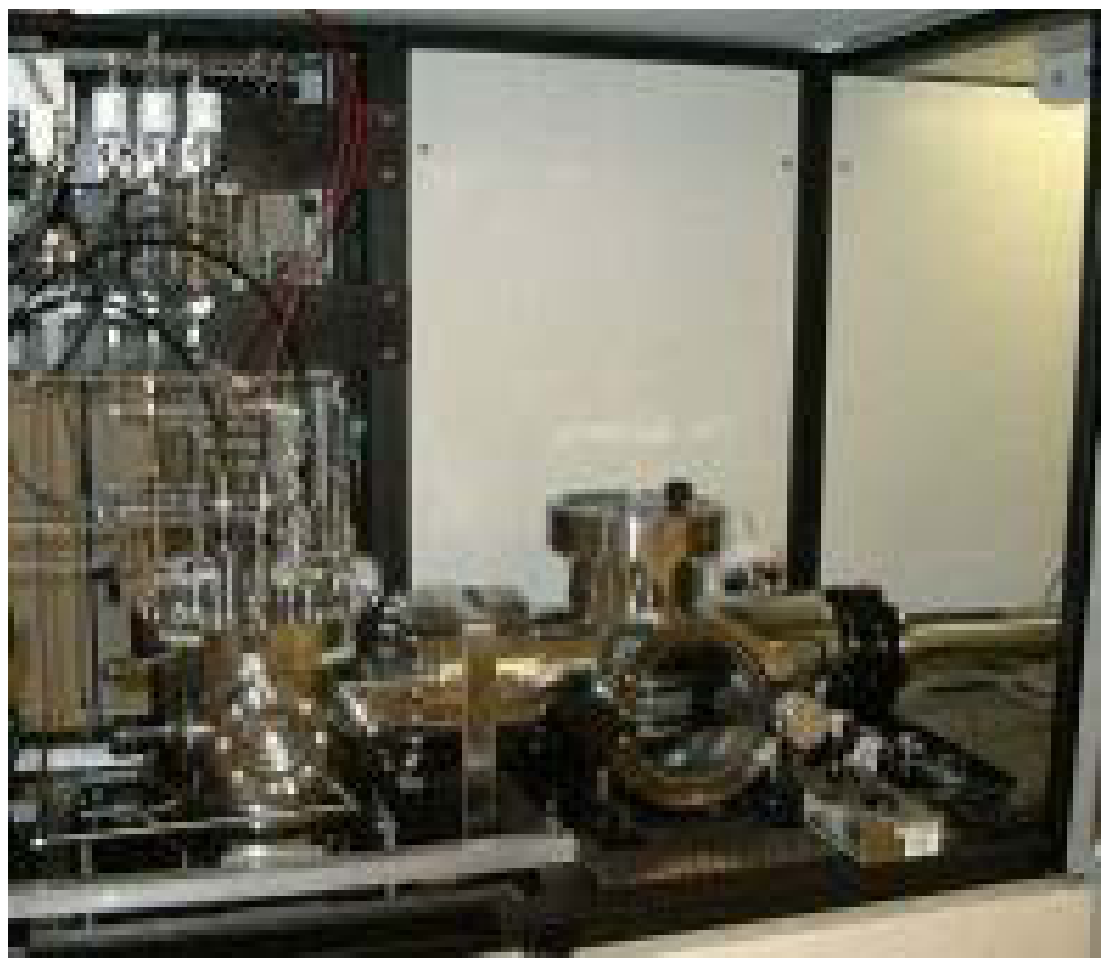

Figure 152: MOCVD tool used in this research.

In situ monitoring of film growth was achieved using optical reflectometry. The reflectance of a thin film on top of a substrate is given by

$$
R=A+B \cdot \cos (4 \cdot \pi \cdot n \cdot d / \lambda)
$$

where $A$ and $B$ are constants, $n$ is the refractive index of the film, $d$ is the thickness of the film, and $\lambda$ is the wavelength of light. This relationship is used to monitor the film thickness and growth rate. During growth, the film thickness is given by

$$
d=g \cdot t
$$

where $g$ is the growth rate and $t$ is time. The reflectance of the film for a given wavelength of light will vary sinusoidally with time. After measuring the period of oscillation, $\mathrm{T}$, the growth rate is given by: 


$$
g=\lambda /(2 \cdot n \cdot T)
$$

The film thickness, $d$, can be calculated by multiplying the growth time by the growth rate. This same technique can be used to measure film thickness ex situ. For a fixed film thickness, the reflectance will vary sinusoidally with inverse of the wavelength, $\lambda$. The film thickness, $d$, can be found by finding the best numerical fit to the measured oscillations.

\section{Growth of GaN:Gd}

Gadolinium has a very high neutron cross-section, making it potentially useful in neutron detectors. Therefore $\mathrm{Gd}$-doped $\mathrm{GaN}$ was investigated in this work. There are many reports of $\mathrm{Gd}$-doped $\mathrm{GaN}$ in literature by $\mathrm{MBE}[73,74]$ and ion implantation $[57,75]$. These reports primarily focused on the magnetic properties of the resulting films. Gd incorporates into the GaN crystal lattice as a 3+ ion, replacing $\mathrm{Ga}$. In this research, Gd-doped GaN films were produced by MOCVD. Two different metalorganic precursors for $\mathrm{Gd}$ were used in this work: tris(cyclopentadienyl)gadolinium (Cp3Gd) and tris(2,2,6,6-tetramethyl-3,5heptanedionato)gadolinium (Gd(TMHD)3). Both $\mathrm{Cp} 3 \mathrm{Gd}$ and $\mathrm{Gd}(\mathrm{TMHD}) 3$ have very low vapor pressure, unlike other commonly used metalorganic precursors. The properties of these two precursors are compared to those of TMG and Cp2Mg in Table 9. The standard III-Nitride precursors, TMG and Cp2Mg, have higher vapor pressures at much lower temperatures than the two Gd precursors. The ClausiusClapeyron plot showing the vapor pressure versus temperature for $\operatorname{Gd}(T M H D) 3$ and Cp3Gd is shown in Figure 153 [76, 77].

Table 9-Comparison of $\mathrm{Gd}$ precursors with typical MOCVD precursors

\begin{tabular}{|c|c|c|}
\hline Precursor & Melting Point & $\begin{array}{l}\text { Vapor Pressure } \\
\text { (Temperature) }\end{array}$ \\
\hline Gd(TMHD)3 & $180^{\circ} \mathrm{C}$ & 0.047 Torr $\left(150^{\circ} \mathrm{C}\right)$ \\
\hline Cp3Gd & $295^{\circ} \mathrm{C}$ & ${ }_{7}^{0.01} \operatorname{Torr}\left(150^{\circ} \mathrm{C}\right)$ \\
\hline Cp2Mg & $176{ }^{\circ} \mathrm{C}$ & ${ }_{7}^{0.02}$ Torr $\left(20^{\circ} \mathrm{C}\right)$ \\
\hline TMG & $-15.8^{\circ} \mathrm{C}$ & $\begin{array}{ll}87.9 & \\
2 & \operatorname{Torr}\left(5^{\circ} \mathrm{C}\right)\end{array}$ \\
\hline
\end{tabular}




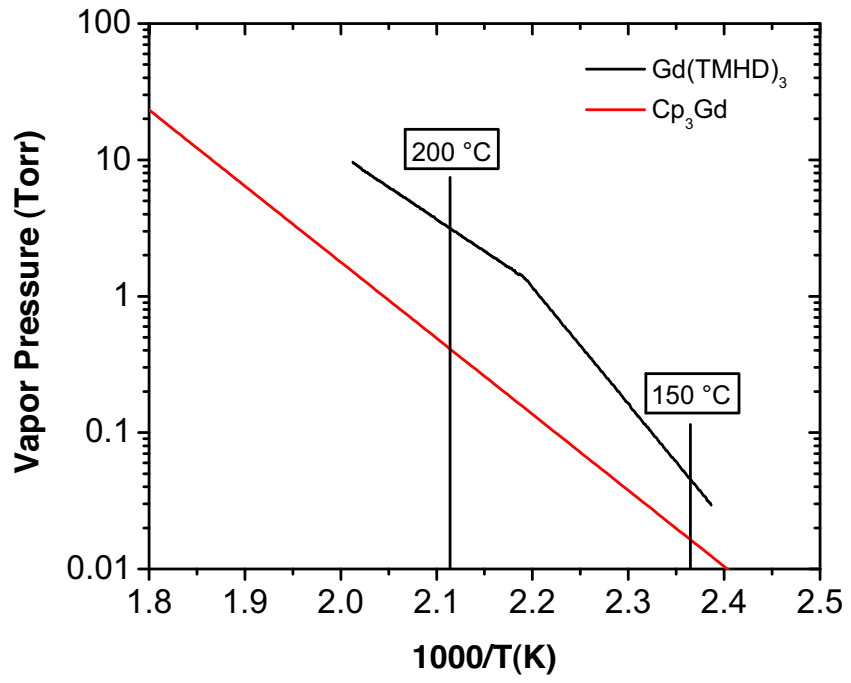

Figure 153 Vapor pressures of metalorganic Gd precursors.

Typically, metalorganic precursors are kept at a regulated temperature using water baths. These baths are limited to $140{ }^{\circ} \mathrm{C}$, which is not high enough to produce a reasonable vapor pressure for the Gd precursors. Therefore, in this work an electrical heater jacket and specialized heating wraps were used to heat the sources and gas lines. These components are capable of heating up to $350{ }^{\circ} \mathrm{C}$ and are shown in Figure 154. In addition, a dedicated gas line was installed to prevent contamination of existing gas lines with Gd precursors.
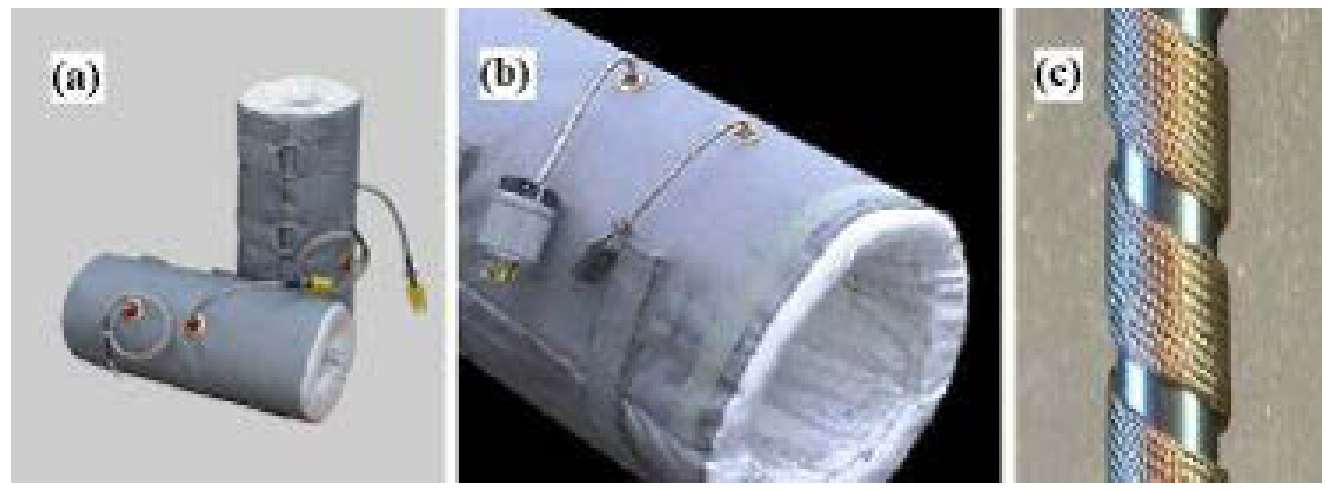

Figure 154. Heater jacket system used for metalorganic Gd precursors. (a) Heater jacket, (b) thermocouple feedback, (c) line heaters.

\section{Material Characterization}

\section{Optical}

Photoluminescence is a non-destructive characterization technique used to study the band structure and impurity levels in semiconductor materials and structures. In this technique electrons in the semiconductor are excited into an unstable energy state by illuminating the sample with a photon source, usually a laser. This excitation source is generally chosen to have photon energy greater than the band gap of the 
semiconductor under test. After excitation, electrons then relax to the conduction band edge by releasing phonons and subsequently recombine with valence band holes through several possible mechanisms. In PL, light produced by radiative recombination is collected and analyzed using a spectrometer. Possible radiative recombination paths include band to band (B-B), donor to valence band (D-V), conduction band to acceptor (C-A), and donor to acceptor (D-A). It is also possible for recombination to occur via deep-level mid-gap states caused by defects or through Auger recombination. These recombination pathways are illustrated in Figure 155. The presence and relative intensities of spectral peaks corresponding to these recombination paths can yield detailed information about the composition and quality of the material or structure under test.

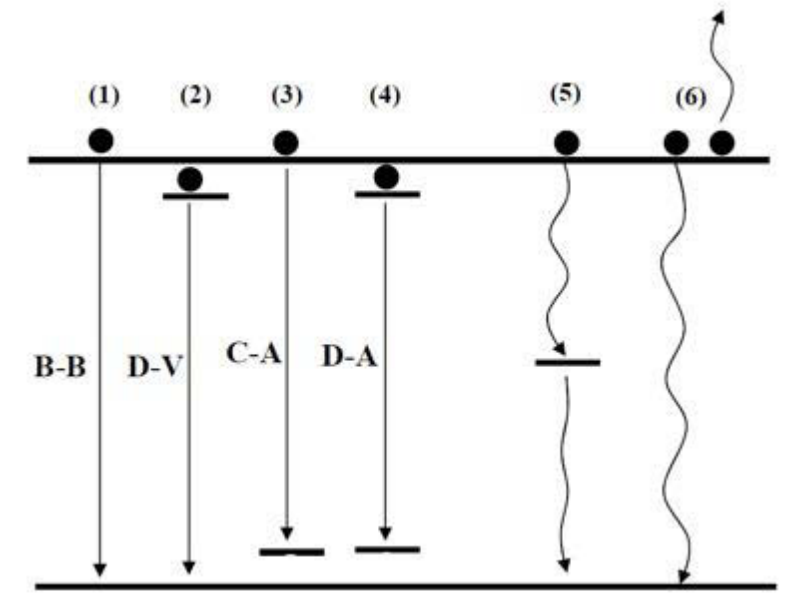

Figure 155. Various electron-hole recombination paths.

Because PL relies on radiative recombination, it has been most commonly applied to III-V and other direct bandgap semiconductors, which have much higher radiative efficiencies than indirect bandgap materials such as silicon. Recently, however, PL has been used with increasing frequency to check doping levels in silicon, despite the intrinsic radiative inefficiency [78].

In this work PL spectra were measured using a Melles-Griot Helium-Cadmium (HeCd) laser (325 nm emission) as the excitation source with an Acton Spectra Pro 2300i monochromator and PIXIS 100BR CCD camera used for spectrometry. Two orientations were used: front collection and back collection. The excitation source is incident on the film surface in both orientations; the difference is that in front collection the film surface faces the spectrometer, while in back collection the substrate faces the spectrometer (and luminescence is collected after traveling through the substrate). Diagrams of these orientations are shown in Figure 156. 


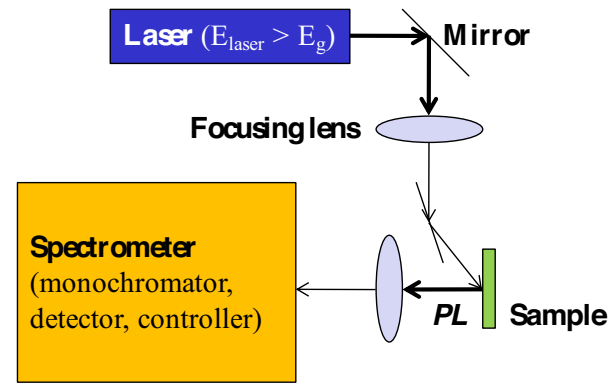

(a)

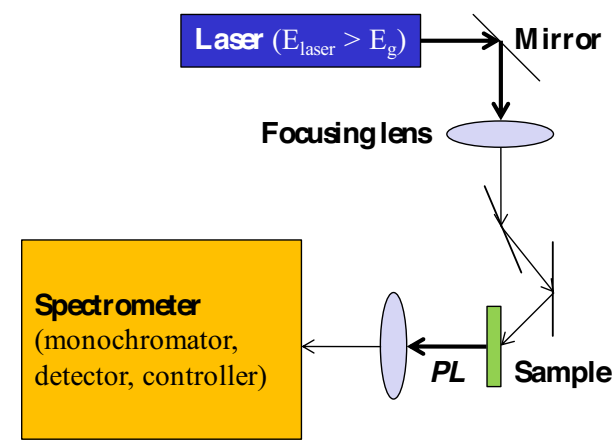

(b)

Figure 156. Schematics of (a) front collected PL and (b) back collected PL.

\section{Structural}

III-Nitrides films are often assumed to have a "mosaic" structure which consists of hexagonal columns separated by threading dislocations [79]. In this model the columns are formed during growth when individual islands coalesce into a single twodimensional film. Tilt and twist of the columns, as well as lateral coherence length, represent imperfections in the crystal structure of epitaxial III-Nitrides. The mosaic structure is illustrated in Figure 157. Illustration of the mosaic structure of III-Nitrides.. This model for threading dislocation generation has been questioned recently [64] but remains the standard view.

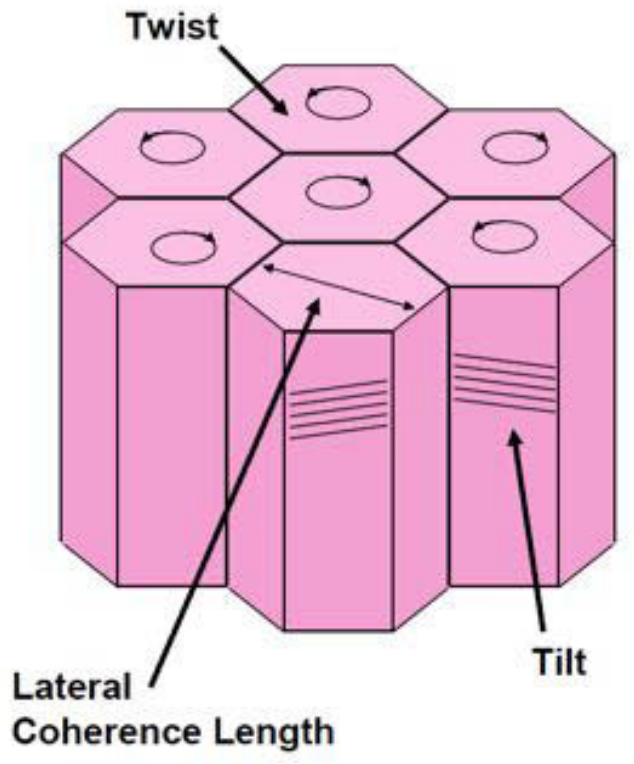

Figure 157. Illustration of the mosaic structure of III-Nitrides.

Structural characterization was achieved primarily through the use of XRD measurements. XRD is a non-destructive technique which uses the diffraction of $X-$ rays by crystal planes to determine the lattice parameters of the material under test. Bragg's law is given by

$$
\mathrm{n} \lambda=2 \mathrm{dhkl} \cdot \sin \theta
$$


where $\mathrm{n}$ is an integer, $\lambda$ is the $\mathrm{X}$-ray wavelength, dhkl is the spacing between crystal planes, and $\theta$ is the Bragg angle. The basic concept of XRD is illustrated in Figure 158.

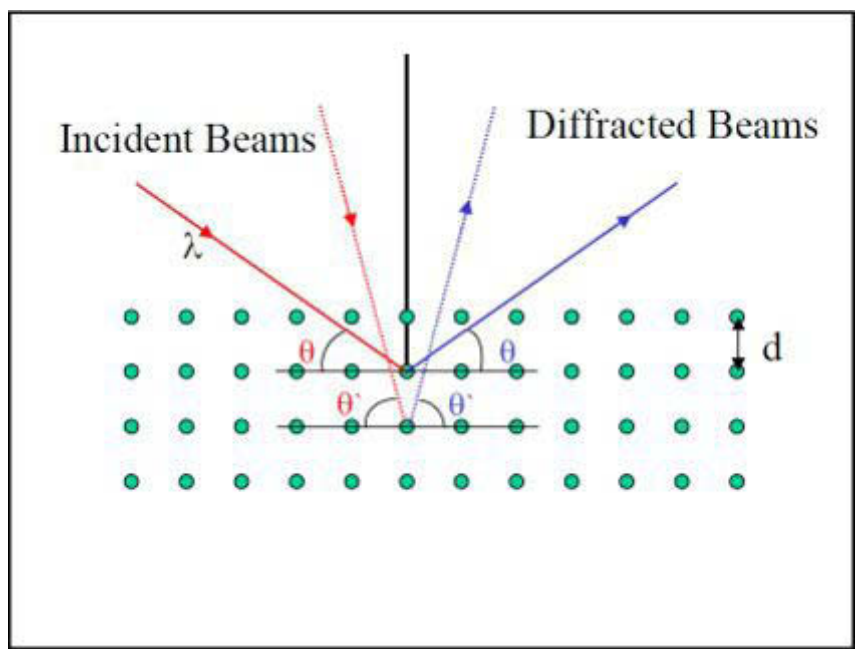

Figure 158. Illustration of $X$-ray diffraction.

Exact lattice size and crystal structure are unique to a given material, so XRD is often used for material identification or to quantify alloy composition. Crystal quality can be assessed using XRD by analyzing the full-width at half maximum (FWHM) of diffraction peaks. Defect density and strain in a crystalline material can also be determined through XRD [80].

In III-Nitrides, the most basic indicator of crystal quality is generally taken to be the FWHM of the (002) rocking curve diffraction peak. Broadening of the (002) rocking curve peak is primarily caused by columnar tilt and screw dislocations, which impact the lateral coherence length. The diffraction angle of the (002) peak allows calculation of the c lattice parameter, and the [002] direction is the direction of growth for most IIINitrides epitaxy. The relationship between planar orientation and planar spacing is given by

$$
\frac{1}{\mathrm{~d}_{\mathrm{hkl}}^{2}}=\frac{4}{3} \cdot \frac{\mathrm{h}^{2}+\mathrm{k}^{2}+\mathrm{hk}}{\mathrm{a}^{2}}+\frac{\mathrm{l}^{2}}{\mathrm{c}^{2}}
$$

where dhkl is the spacing between the (hkl) planes and a and c are the hexagonal lattice parameters.

In this work, XRD was performed using a Phillips XPert Pro MRD diffractometer with a CuKa source, four bounce Ge (220) monochromator, and a two reflection Ge (220) analyzer.

\section{Electrical}

Electrical characterization of materials in this work was achieved through two techniques: Hall effect measurement and contactless resistivity mapping. The Hall effect is a phenomenon based on the Lorentz force wherein a static magnetic field applied perpendicular to electric current flow in a conductor will induce an electric field that is perpendicular to both the applied magnetic field and the current flow. This is illustrated in Figure 159. Illustration of the Hall effect.. It is possible to calculate the 
resistivity and majority carrier type, concentration, and mobility of a film by measuring the Hall voltages induced in the film while in certain geometries.

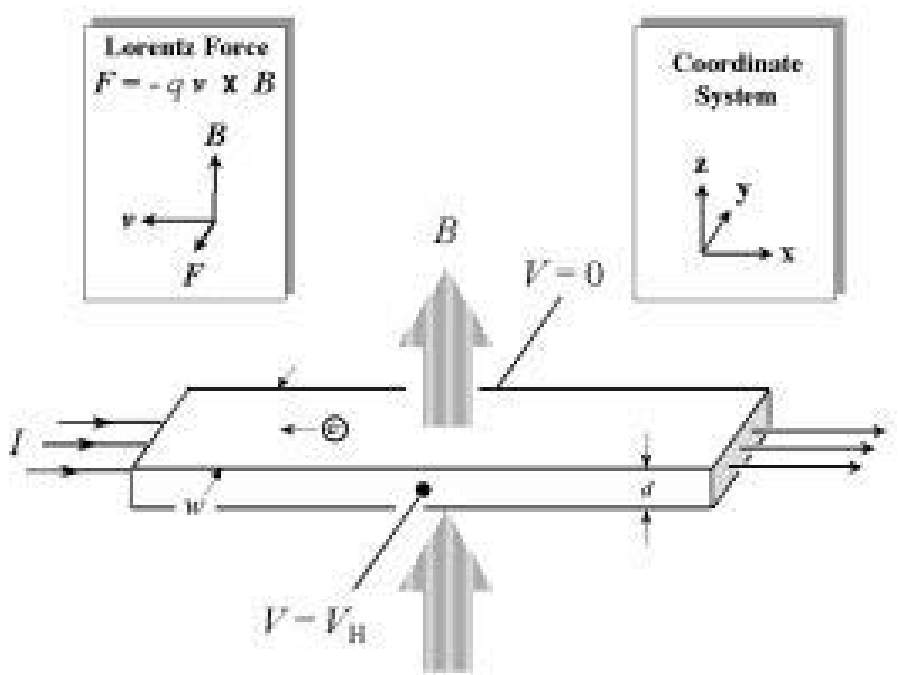

Figure 159. Illustration of the Hall effect.

The van der Pauw technique is commonly used to characterize flat samples of arbitrary shape [81]. The technique requires that the sample under test be uniformly thick and that four electrical contacts be made to the sample and that they be located at the perimeter of the sample. The electrical contacts should be sufficiently small that the ratio of the contact diameter to the distance between contacts is approximately zero, as this is the order of errors caused by non-infinitely small contacts [82]. Eight Hall voltages must be measured at the same electric current level in order to determine the carrier concentration and type: V24, V42, V13, and V31, each at positive and negative applied magnetic field. The carrier concentration (in $\mathrm{cm}-3$ ) is then given by:

$$
\text { concentration }=8 \times 10^{-8} \cdot \frac{\mathrm{I} \cdot \mathrm{B}}{\mathrm{d} \cdot \mathrm{q} \cdot\left(\mathrm{V}_{\mathrm{P}-} \mathrm{V}_{\mathrm{N}}\right)}
$$

where I is the applied current (in amps), B is the magnitude of the applied magnetic field (in Gauss), d is the sample thickness (in $\mathrm{cm}$ ), $\mathrm{q}$ is the elementary charge (positive), VP is the sum of the Hall voltages with positive magnetic field, and VN is the sum of the Hall voltages with negative applied magnetic field. If the calculated concentration is negative, electrons are the majority carrier; if negative, holes are the majority carrier. An Ecopia HMS-3000 Hall effect measurement system with a 5.1 kGauss permanent magnet was used in this work.

Contactless resistivity mapping was also used to electrically characterize epitaxial films. In this technique, the sample under test is placed between two coupled RF coils to which a sinusoidal voltage is applied. The alternating magnetic field of the coil induces eddy currents in the conductive sample, and the power absorbed is inversely proportional to the sheet resistance of the sample. The sheet resistance of the sample can be calculated by keeping the coil drive voltage constant and measuring 
the drive current [78]. A Lehighton Electronics Inc. model 1310 contactless resistivity mapper was used in this work.

\section{Device Processing}

Prior to device fabrication it is necessary to activate the p-type GaN by annealing at $800{ }^{\circ} \mathrm{C}$ for four minutes in order to drive off passivating hydrogen atoms [83]. Several fabrication steps must be performed in order for device characterization and testing to be possible. The required processes are identical to the processes used to make IIINitride LEDs and include the following steps: deposition of current spreading layer, etching to isolate device mesas, oxidation of current spreading layer, deposition of $p$ contact pad, deposition of $n$-contact pad. These steps require the use of standard photolithography processes and the use of two tools: an inductively coupled plasma (ICP) etcher and an electron beam (e-beam) evaporator.

The current spreading layer consists of a $\mathrm{Ni}(5 \mathrm{~nm}) / \mathrm{Au}(5 \mathrm{~nm})$ layer which is oxidized to form a NiOx tunneling contact to the p-type GaN [84]. The oxidation is accomplished through annealing at $550{ }^{\circ} \mathrm{C}$ with ambient air for 5 minutes. The p-contact pad consists of $\mathrm{Ni}(50 \mathrm{~nm}) / \mathrm{Au}(200 \mathrm{~nm})$, and the n-contact pad consists of $\mathrm{Ti}(10 \mathrm{~nm}) / \mathrm{Al}(30$ $\mathrm{nm}) / \mathrm{Ti}(10 \mathrm{~nm}) / \mathrm{Au}(200 \mathrm{~nm})$. These electrical contacts were deposited by e-beam evaporation. Gd conversion layers were also deposited by e-beam evaporation, while the $6 \mathrm{LiF}$ alpha radiator was desposited by thermal evaporation. Prior to device characterization and testing, the fabricated structures were diced and mounted into DIP packages with the contacts wirebonded to the package pins.

\section{Diode Characterization}

Device characterization included dark and illuminated I-V testing and C-V testing. I-V testing was performed using a computer-controlled Keithley 2420 source-meter. Optical sensitivity was tested using a broadband Xenon (Xe) lamp illumation source with significant UV spectral content. The illuminated I-V testing was used to check the functioning of the $p-i-n$ structures as photodiodes. C-V testing allowed analysis of the depletion width of the devices.

\section{Alpha Particle Testing}

Enriched conversion layers such as $6 \mathrm{LiF}$ are a common way to achieve enhanced neutron sensitivity in semiconductors, however these materials are expensive. Additionally, Li-containing compounds require dedicated deposition tools, due to the potential for contamination in subsequent films. The response of a neutron detector that would utilize the $6 \mathrm{Li}(\mathrm{n}, \alpha)$ reaction can be simulated using a spontaneous alpha particle emitter instead. This testing was performed using an Americium-241 (241Am) alpha emitter, which emits alphas with energy of approximately $5.48 \mathrm{MeV}$.

\section{Neutron Detection Testing}

Neutron sensitivity was characterized using two neutron sources: an Americium241/Beryllium (Am-Be) spontaneous fission source at Georgia Tech and an AGN-201 reactor at Idaho State. IThe $5.48 \mathrm{MeV}$ alpha particles produced by the $241 \mathrm{Am}$ are converted to neutrons by the $9 \mathrm{Be}(\alpha, \mathrm{n})$ reaction. The neutron energy spectrum produced by an Am-Be source is shown in Figure 3.11 [85]. The Am-Be source used in this work was spherical in shape with a diameter of $\sim 2.5 \mathrm{~cm}$. The source was 
placed in a graphite pile in order to produce thermal neutrons. A shielding box made of a neutron absorbing material was used to produce a collimated thermal beam for testing detectors [86]. The thermal neutron flux at the detector in this configuration was approximately $103 \mathrm{~cm}-2 \cdot \mathrm{s}-1$.

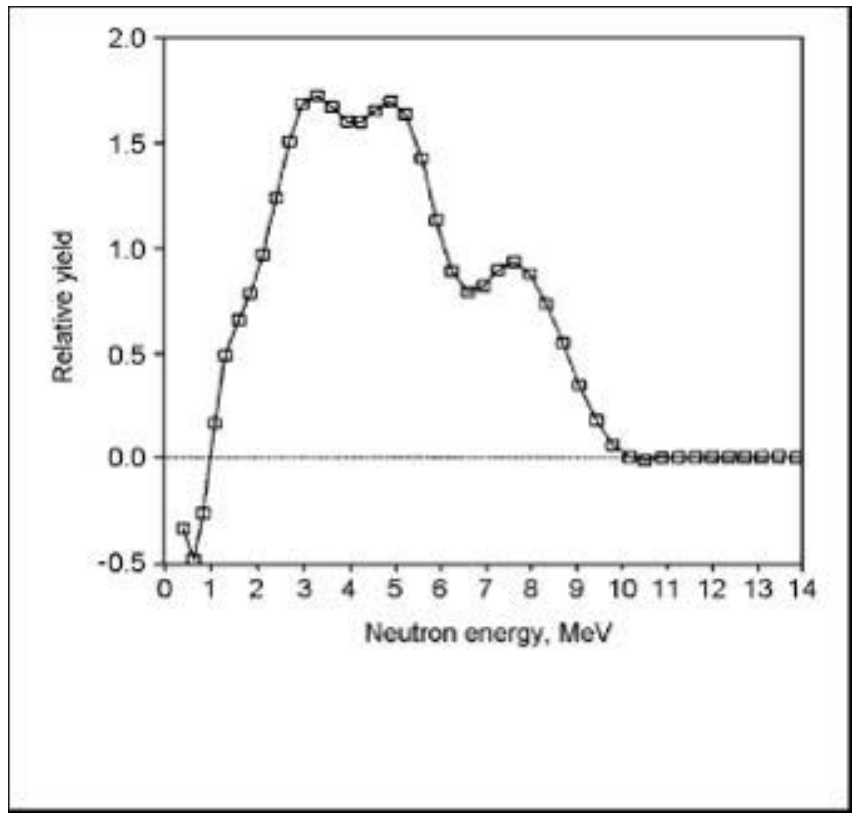

Figure 160 Neutron spectrum from an un-moderated Am-Be source.

The other neutron source used in this work was an Aerojet General Nucleonics AGN201 homogeneous thermal reactor. A schematic of this reactor is shown in Figure 161. This reactor uses $672.93 \mathrm{~g}$ of $20 \%$ enriched Uranium Oxide (UO2) powder encased in polyethylene. It has a maximum operating power of $5 \mathrm{~W}$. The reactor core is shielded by $55 \mathrm{~cm}$ of water and $10 \mathrm{~cm}$ of lead. On top of the reactor core is a thermal neutron column, which is where detector testing was performed. The thermal neutron flux on top of the thermal column was approximately $5.3 \times 106 \mathrm{~cm}-2 \cdot \mathrm{s}-1 \cdot \mathrm{W}-1$. 


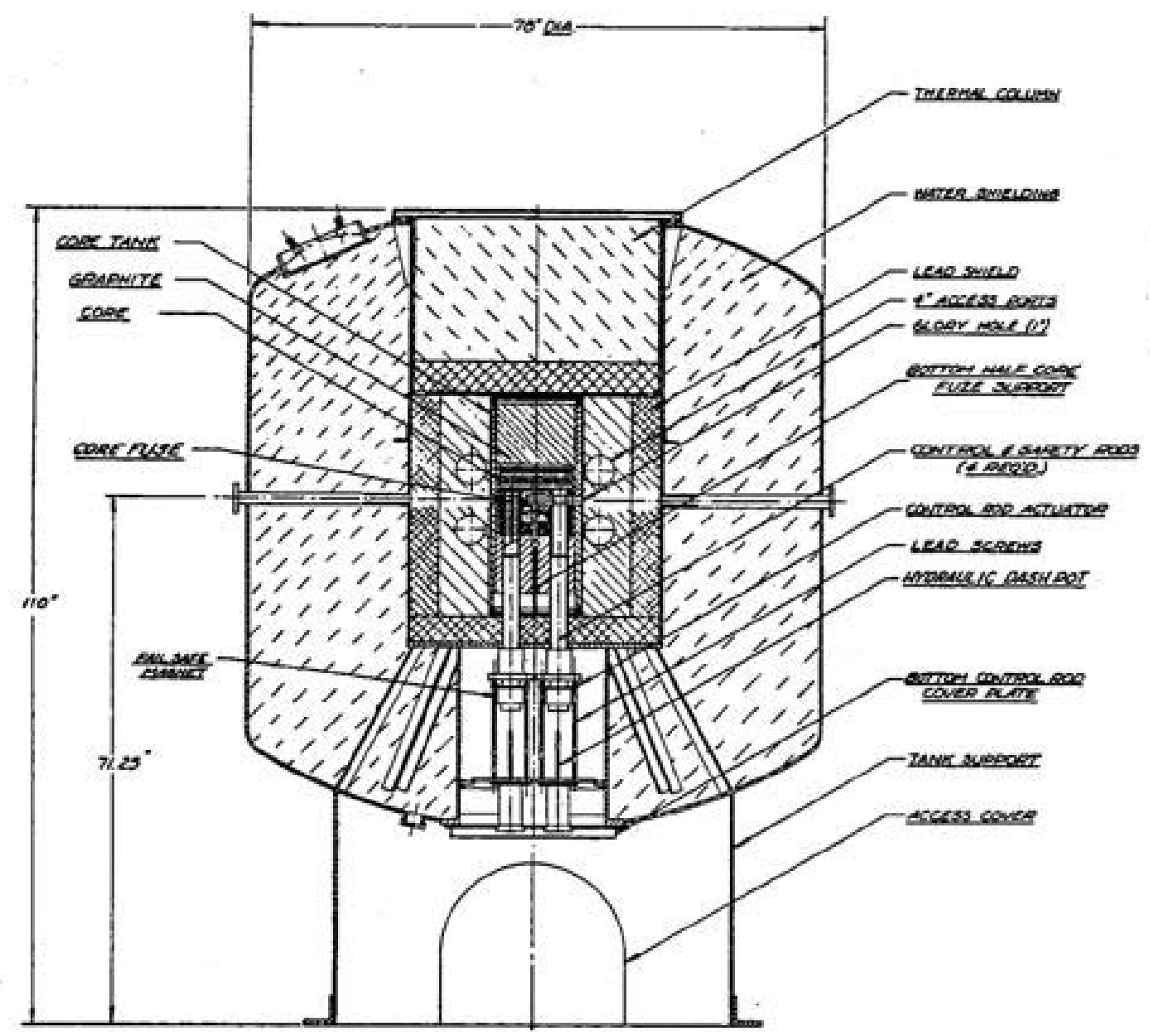

Figure 161. The ISU AGN-201 reactor.

GaN-based neutron detecting structures were grown by MOCVD. Material was characterized optically by PL, structurally by XRD, and electrically by Hall effect and contactless resistivity mapping. Devices were fabricated using standard III-Nitride processing techniques and tested by I-V and $\mathrm{C}-\mathrm{V}$. The detectors were tested for neutron sensitivity using two neutron sources: an Am-Be source and a reactor. Thermal neutrons were used in both cases: the Am-Be source was placed in a graphite pile, and the thermal neutron column on top of the reactor was used.

\section{Neutron Detector Performance}

The GaN-based scintillators and diode neutron detectors were tested using neutron sources at Georgia Tech and Idaho State University. The devices showed intrinsic neutron sensitivity which was enhanced by the use of a $6 \mathrm{LiF}$ radiator. Excellent gamma-discrimination was observed. The detectors were also shown to scale linearly with reactor power, confirming their usefulness in reactor monitoring applications. Structural and optical properties of the scintillators were examined, as well as the electrical performance of the diodes. 


\section{Scintillator Characterization and Performance}

\section{Material Characterization}

Figure 162 shows the in situ reflectometry signal from the growth of undoped, Sidoped, and Gd-doped GaN scintillators. The growth rates for all of these films were approximately $2 \mu \mathrm{m} /$ hour. It can be seen that the average reflectance of the undoped and Gd-doped GaN stabilized, while the reflectance of the Si-doped GaN eventually began to degrade. This is an indication that the surface of the film is getting rougher which is not unusual, as $\mathrm{Si}$ is known to act as an anti-surfactant during MOCVD growth of GaN [99-101]. Gd-doing of GaN is commonly observed to have only subtle effects on the observable structural properties of the material so it is not surprising that the reflectance signal is similar to that of undoped GaN [55, 103]. XRD omega rocking curves of the (002) reflection for undoped, Si-doped, and Gd-doped GaN are shown in Figure 163.
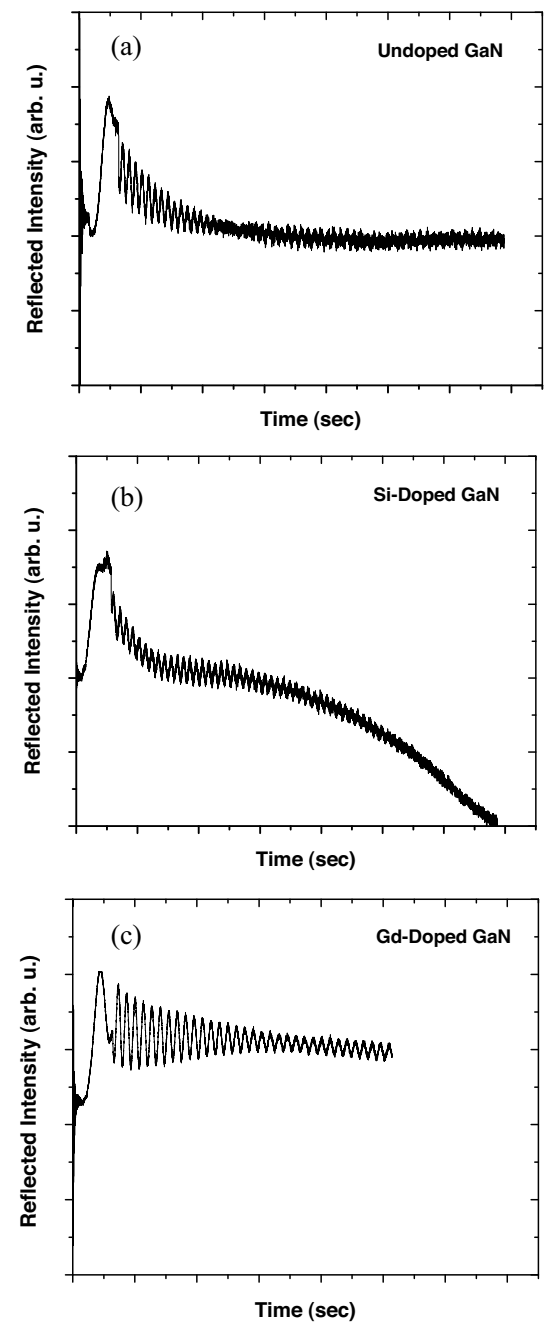

Figure 162. In situ reflectometry signals for (a) undoped, (b) Si-doped, and (c) Gd-doped GaN. 


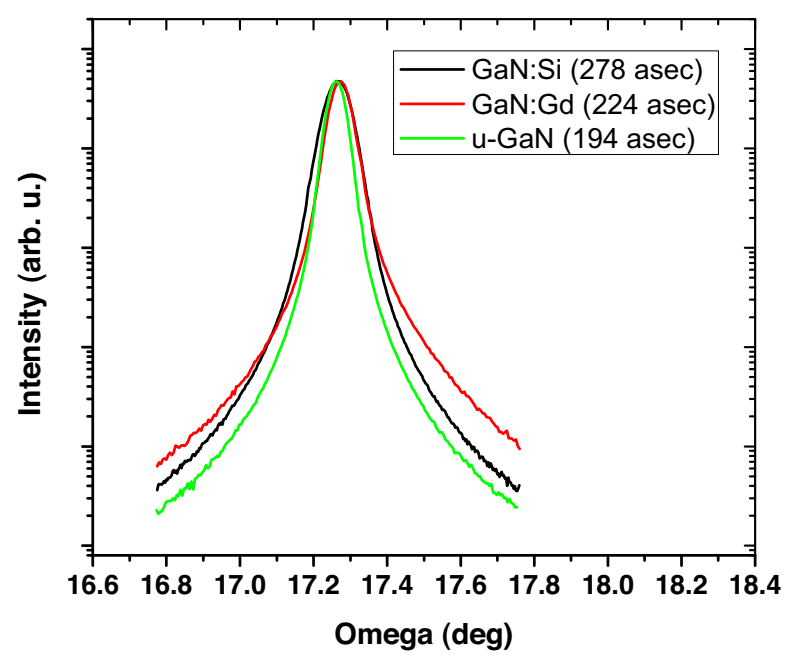

Figure 163. Omega rocking curves of GaN scintillators.

Undoped GaN had the lowest FWHM, however all three were under 300 arcseconds, indicating good crystal quality.

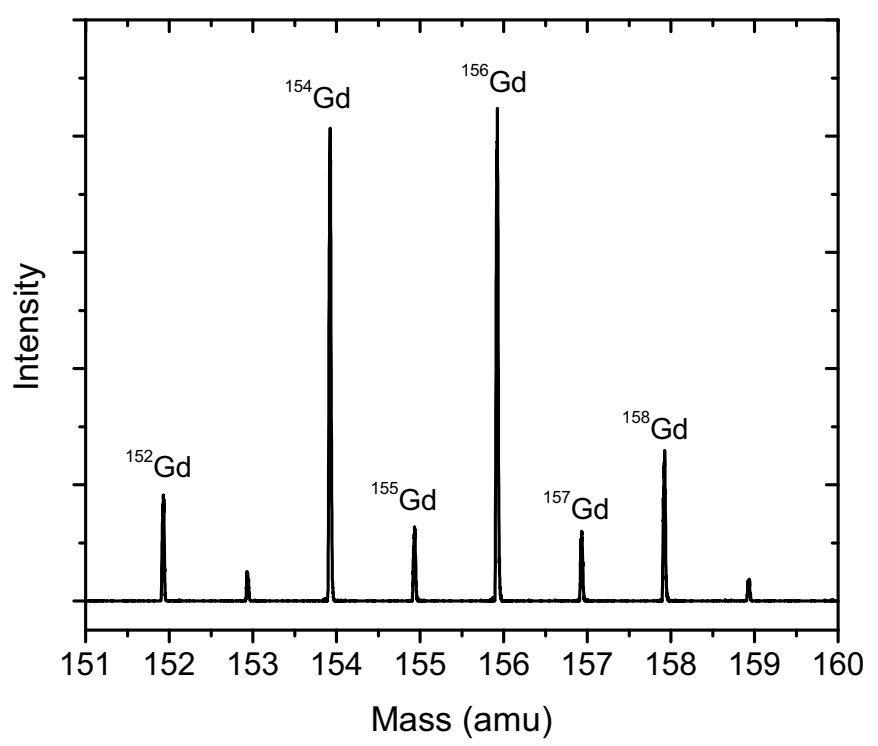

Figure 164. Mass spec for Gd sample.

Atomic identification techniques are necessary to confirm the presence of $\mathrm{Gd}$ in epitaxially grown $\mathrm{GaN}$ films, due to the subtle impact it has on the material properties. Such techniques include electron dispersive Figure 164 shows peaks from a TOF secondary ion mass spectrometry (TOF-SIMS) measurement of the Gd-doped GaN scintillator. Peaks corresponding to $152 \mathrm{Gd}, 154 \mathrm{Gd}, 155 \mathrm{Gd}, 156 \mathrm{Gd}, 157 \mathrm{Gd}$, and $158 \mathrm{Gd}$ were observed. The small peak corresponding to atomic mass of 153 could be due to Europium-153 (153Eu), and the small peak at 159 could be due to Terbium159 (159Tb). The presence isotopes in the film is possible due to the presence of low 
levels of rare earth impurities in the $\mathrm{Gd}$ precursor. Quantification of the atomic percentages for each of these isotopes was not possible due to the lack of a GaN:Gd standard sample.

Resistivity maps of the three scintillators are shown in Figure 165. The mean sheet resistances for the samples were 1104, 2.713, and 1763 Ohms/square for the undoped, Si-doped, and Gd-doped scintillators. Assuming that all these films are ntype, these measurements allow estimation of the electron concentration using the following approximation:

$$
\mathrm{n} \approx \frac{1}{\mathrm{q} \mu_{\mathrm{n}} \mathrm{R}_{\mathrm{s}} \mathrm{t}}
$$

where $\mu n$ is the electron mobility, RS is the sheet resistance, and $t$ is the film thickness. Each of the films was $10 \mu \mathrm{m}$ thick and a typical value for $\mu \mathrm{n}$ in n-type $\mathrm{GaN}$ is $\sim 500 \mathrm{~cm} 2 / \mathrm{Vs}$. This gives electron concentration values of $\sim 1 \times 1016 \mathrm{~cm}-3, \sim 5 \times 1018$ $\mathrm{cm}-3$, and $\sim 7 \times 1015 \mathrm{~cm}-3$ for the undoped, Si-doped, and Gd-doped films, respectively. These values conform to expectation, as $\mathrm{Si}$ is a shallow donor in $\mathrm{GaN}$ and $\mathrm{Gd}$ is isovalent with $\mathrm{Ga}$ and thus does not directly contribute any additional freeelectrons. 

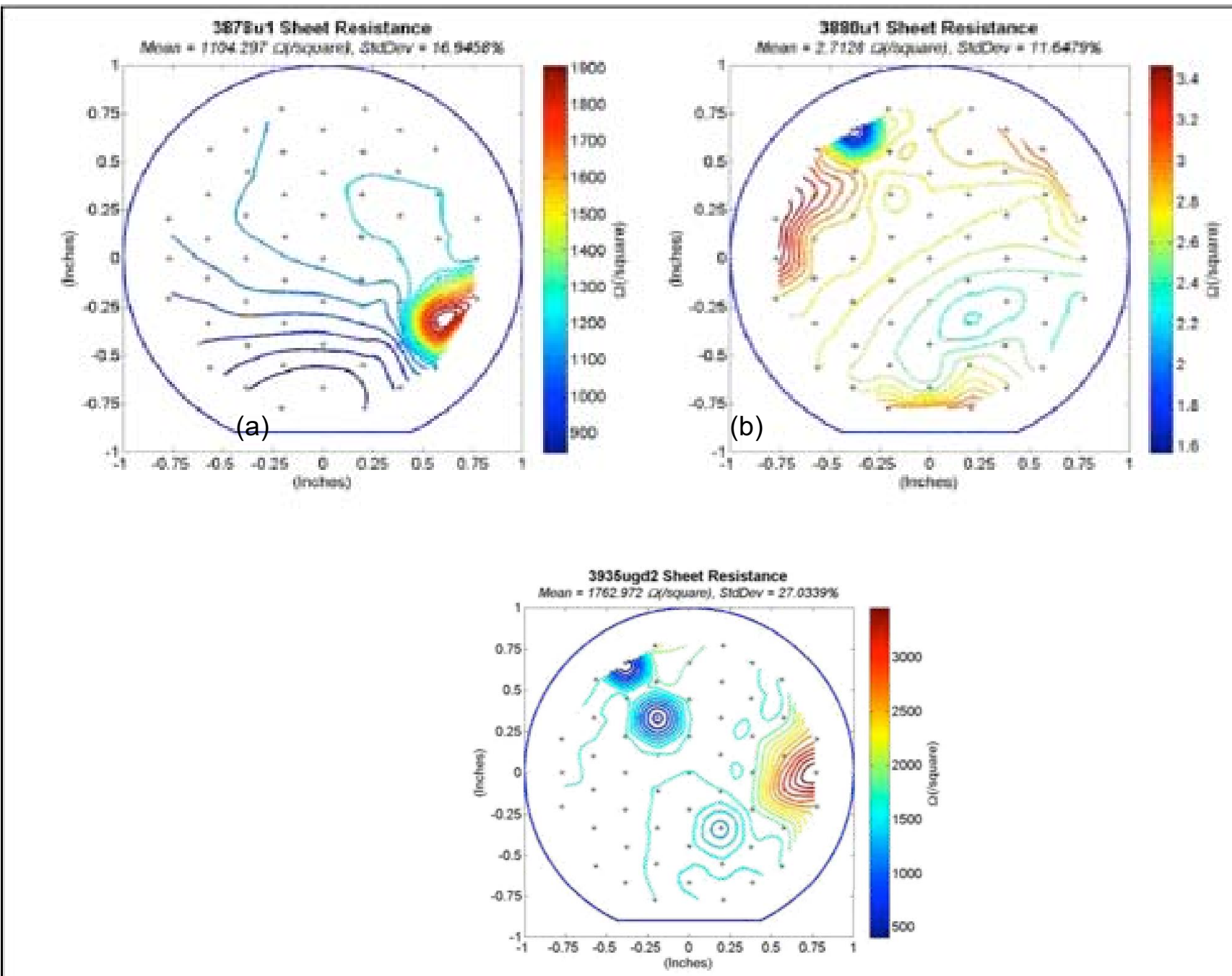

Figure 165. Resistivity maps for a) undoped, b) Si-doped, and c) Gd-doped GaN. 
The front collected PL spectra for undoped and Si-doped GaN are shown in Figure 166. PL spectra of Si-doped and undoped GaN scintillators.. As expected, Si-doping resulted in a more than order of magnitude increase in the near-bande-edge emission intensity caused by the increase in free electron concentration. The near-band-edge emission peak in the Si-doped GaN is also red-shifted (from $3.433 \mathrm{eV}$ to $3.421 \mathrm{eV}$ ) and broadened (FWHM from $0.038 \mathrm{eV}$ to $0.092 \mathrm{eV}$ ) with respect to the undoped GaN. Both films also exhibited the broad yellow luminescence band centered at $\sim 2.2 \mathrm{eV}$, which is typical of GaN films.

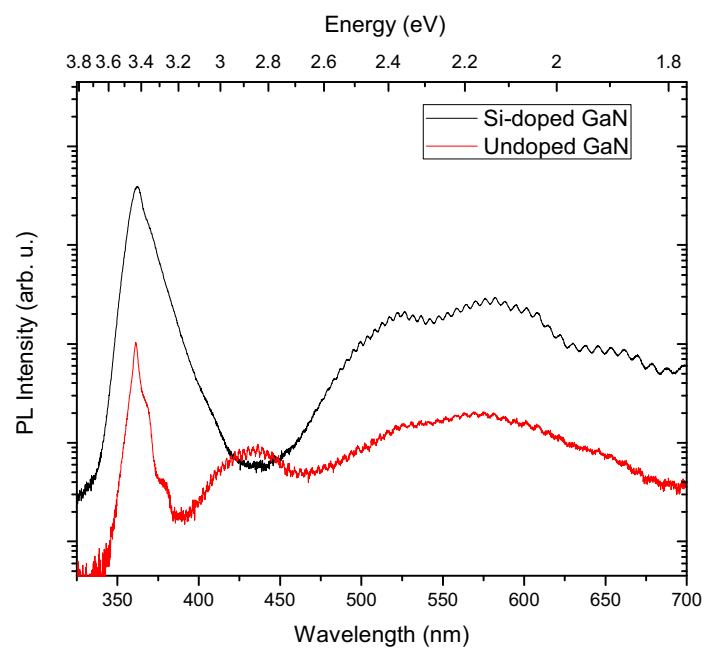

Figure 166. PL spectra of Si-doped and undoped GaN scintillators.

Figure 167 Figure 168 show the normalized front and back collected PL spectra for the undoped and Si-doped films. The back collected spectra have been normalized to have peak intensity equal to the intensity of the front collected spectra at the same wavelength. This is to highlight the effect of self-absorption and the nearly identical spectral shape for both orientations. Figure 168 is focused on the near-band-edge peaks of the two samples.
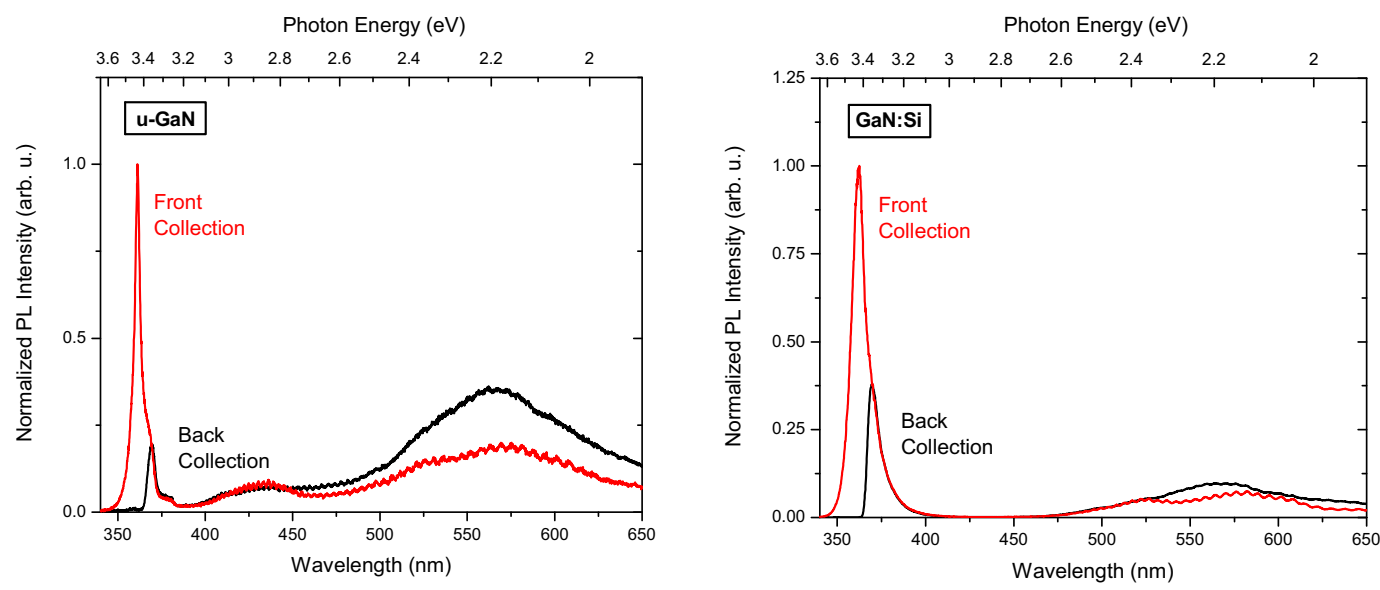

Figure 167. Front and back collected PL spectra from (a) undoped and (b) Si-doped GaN scintillators. 

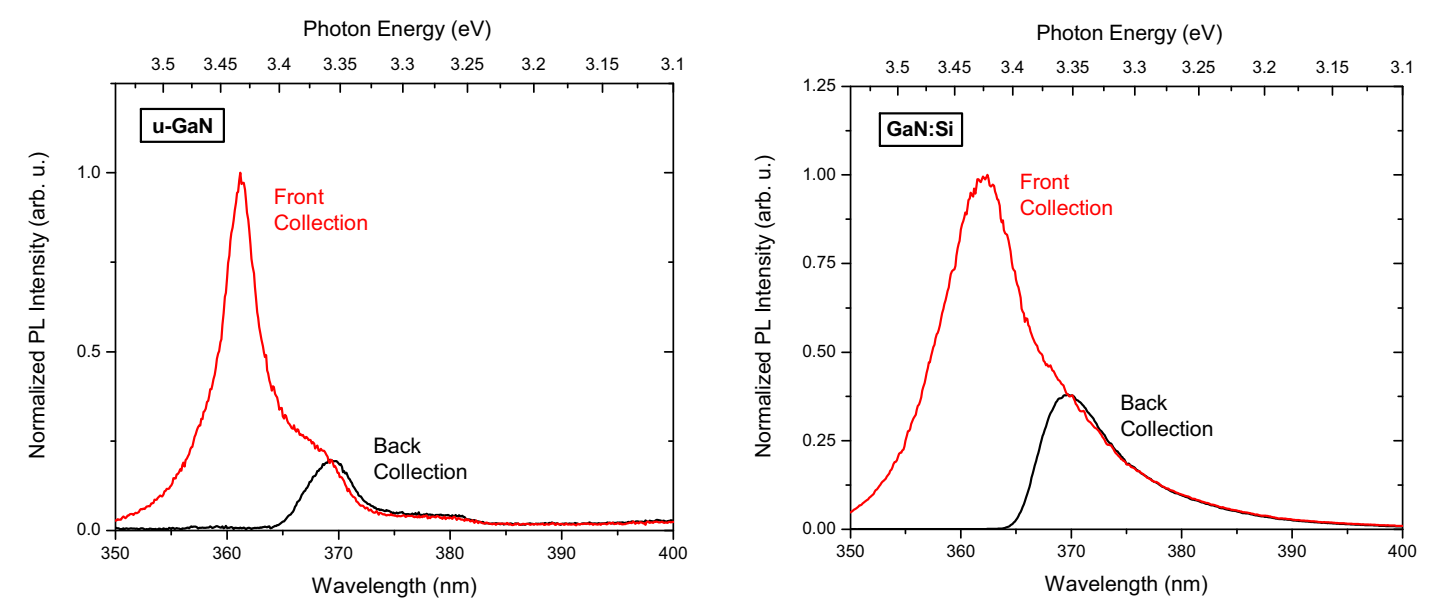

Figure 168. Front and back collected PL spectra from (a) undoped and (b) Si-doped GaN scintillators.

\section{Alpha Particle Response}

The $10 \mu \mathrm{m}$ thick undoped, Si-doped, and Gd-doped scintillators were tested tested for alpha-induced scintillation using an $241 \mathrm{Am}$ alpha source. This was intended to simulate the effect of using an alpha-emitting conversion layer such as $6 \mathrm{LiF}$. 241Am emits 5.48 $\mathrm{MeV}$ alpha particles, which have a range of $14.4 \mu \mathrm{m}$ in $\mathrm{GaN}$. Therefore only a portion of the total energy is deposited in the GaN. The scintillation spectra for the three films and a bare sapphire wafer are shown in Figure 169. The Si-doped GaN scintillator showed a distinct alpha-induced peak with very good gamma-discrimination, while the undoped and Gd-doped scintillators did not show a distinct alpha peak. This is due to the lower optical efficiency of these films relative to the Si-doped film, as seen in PL measurements. A material that produces fewer photons produced for a given alpha ionization trail will show these light pulses at lower channel numbers. In the undoped and Gd-doped films the alpha-induced events were compressed to the point that they occupy the same channel numbers as gamma-induced pulses, and thus gamma discrimination was not possible.
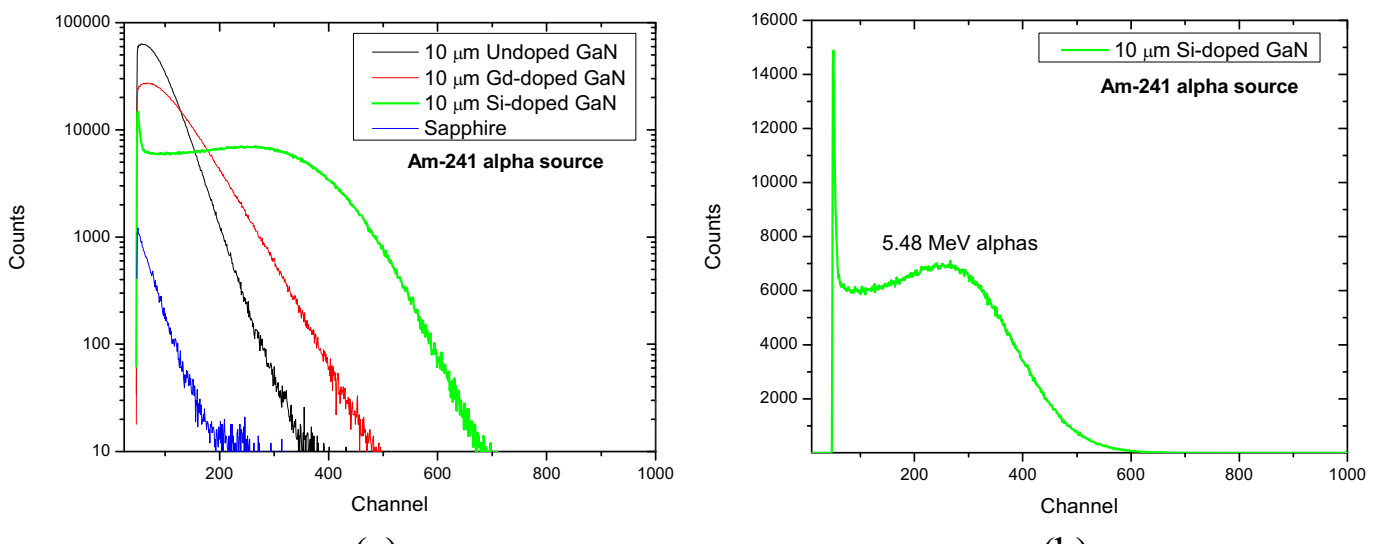

Figure 169. (a) Alpha-induced scintillation in GaN-based scintillators. (b) Scintillation from Sidoped scintillator plotted in linear scale to emphasize the alpha-induced peak. 


\section{Neutron Response}

The GaN scintillators were exposed to both a thermalized Am-Be neutron source and the thermal neutron column of a research reactor. Both of these sources produce gamma radiation, therefore reference spectra were taken with a Cd shield in place which blocks all thermal neutrons. These reference spectra were subtracted from the unshielded spectra to isolate the neutron-induced response.

Figure 170 shows the neutron induced luminescence in the Si-doped GaN scintillator. The scintillator was tested bare, with a $6 \mathrm{LiF}$ conversion layer, and a Gd metal conversion layer. All three configurations show a prominent peak which corresponds to the $584 \mathrm{keV}$ proton produced by the $14 \mathrm{~N}$ neutron reaction. This confirms that $\mathrm{GaN}$ is intrinsically neutron sensitive. An additional peak corresponding to the $2.05 \mathrm{MeV}$ alpha particle produced by the $6 \mathrm{Li}$ reaction is visible in the $6 \mathrm{LiF}$ conversion layer spectrum. This spectrum exhibits a reduction in the $14 \mathrm{~N}$ peak, which is due to the conversion of a portion of incident neutrons prior to entering the GaN scintillator. The spectrum for the Gd coated sample shows an even further reduced $14 \mathrm{~N}$ peak, which is due to the higher conversion efficiency of this layer compared to the $6 \mathrm{LiF}$. No discernible peak is visible in the $\mathrm{Gd}$ spectrum that can be attributed to the $157 \mathrm{Gd}$ reaction. Scintillation pulses caused by the energetic electrons produced in this reaction would be binned in similar channels as gamma-induced pulses. Thus it is difficult to separate them when subtracting the $\mathrm{Cd}$ shielded reference spectrum.

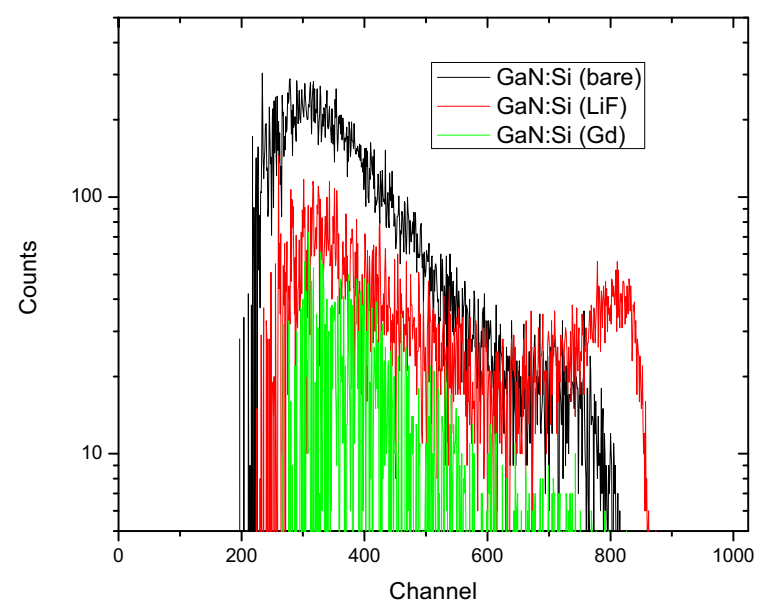

Figure 170. Neutron-induced luminescence in Si-doped GaN with varying conversion layers.

The thermalized Am-Be source has a limited, fixed flux of $\sim 103$ thermal neutrons per $\mathrm{cm} 2$ per second. For this reason a reactor neutron source was also used. The thermal column of the AGN-201 reactor has a power-dependent flux of $5.3 \times 106$ thermal neutrons per $\mathrm{cm} 2$ per second per Watt. This allowed more thorough measurements in much less time. Figure 171 shows the separated gamma and neutron responses of the bare Si-doped GaN scintillator. Again, a clear peak from the $584 \mathrm{keV} 14 \mathrm{~N}$ proton is visible, confirming the intrinsic neutron sensitivity of GaN. The gamma response of the scintillator is also plotted in Figure 171, illustrating the excellent pulse-height based gamma-discrimination in this detector. 


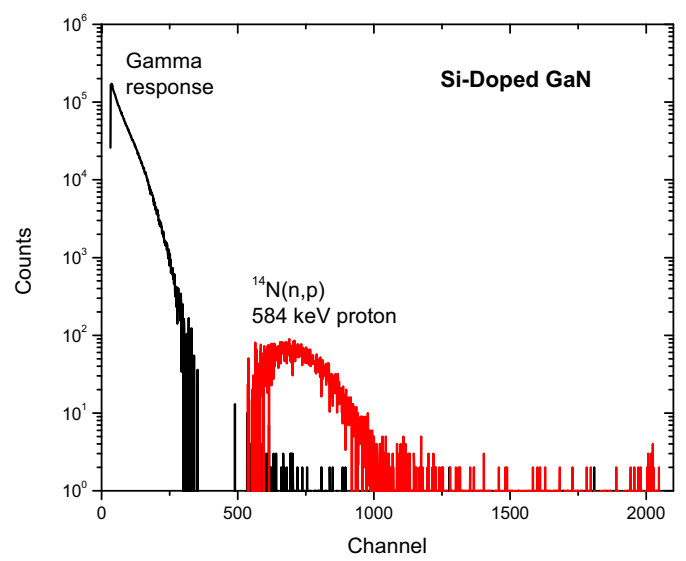

Figure 171. Gamma- and neutron-induced scintillation spectra of Si-doped GaN.

The Gd conversion layer also failed to show a discernible Gd-produced peak in reactor testing, however the $6 \mathrm{LiF}$ conversion layer continued to clearly discernible alpha peaks. Figure 172(a) shows the results of a reactor linearity test in which scintillation spectra were recorded from the Si-doped GaN sample with $6 \mathrm{LiF}$ conversion layer at a range of reactor power levels. Linear response to reactor power is a key characteristic for neutron detectors used in the nuclear energy industry. Figure 172 (b) shows the integrated neutron-induced counts from the detector plotted versus reactor power with two linear fits: a least squares regression and the line formed by the points at lowest and highest reactor power. Both fits have R2 values greater than 0.95 , indicating that the scintillator detector is highly linear over three orders of magnitude of reactor power.

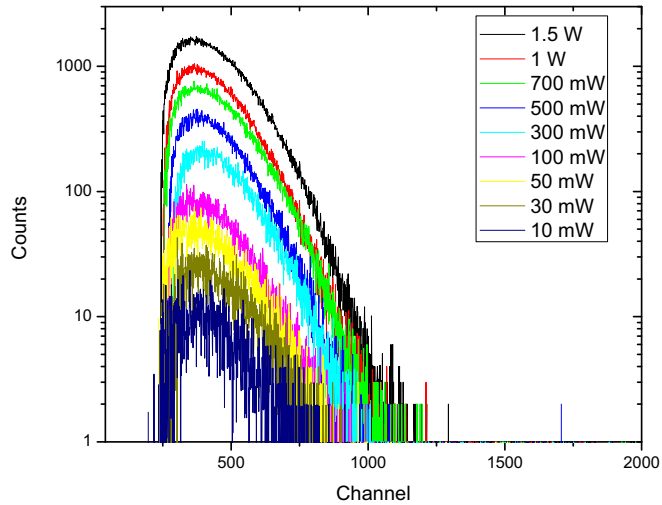

(a)

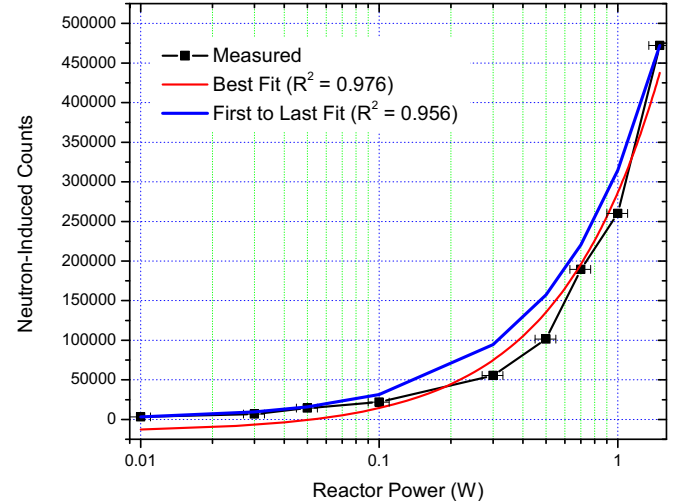

(b)

Figure 172(a) Neutron-induced scintillation spectra of Si-doped GaN with 6LiF conversion layer at different reactor power levels. (b) Linear fit of integrated neutron counts to reactor power.

\section{GaN P-I-N Characterization and Performance}

GaN p-i-n diodes were grown by MOCVD and fabricated into devices. Figure 173 (a) shows I-V curves for diodes with $200 \mathrm{~nm}, 2 \mu \mathrm{m}$, and $5 \mu \mathrm{m}$ thick i-regions. All three devices exhibited reverse bias dark current density of $\sim 10-6 \mathrm{~A} / \mathrm{cm} 2$. Figure 173 (b) shows the photoresponse of the $200 \mathrm{~nm}$ i-region diode illuminated by a broadband Xe lamp. 


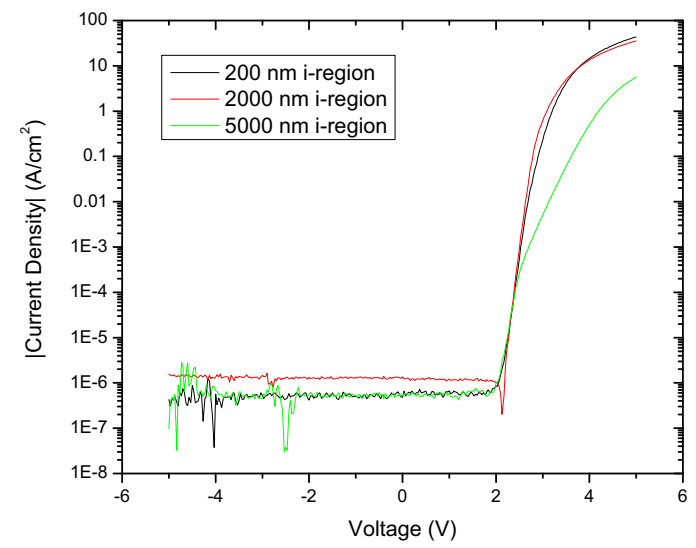

(a)

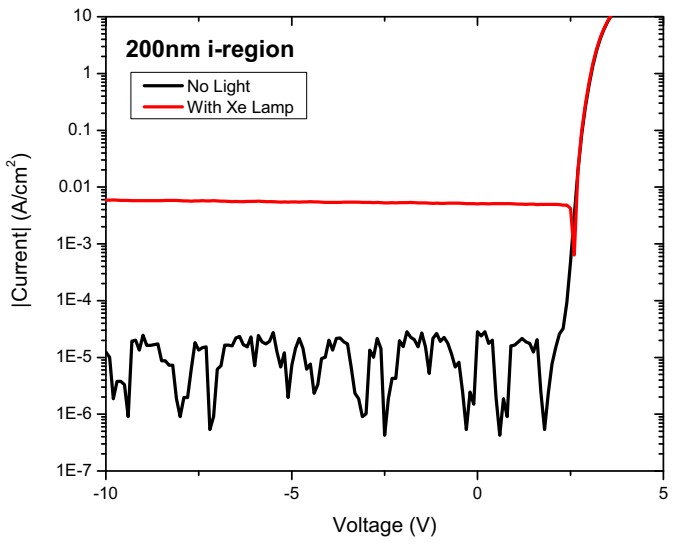

(b)

Figure 173 I-V curves for GaN p-i-n diodes (a) with varying i-region thickness and (b) with and without illumination.

$\mathrm{C}-\mathrm{V}$ profiling was used to determine the depletion width in the $200 \mathrm{~nm}$ and $2 \mu \mathrm{m}$ thick i-region diodes. Two individual devices from the same wafer were tested at $1 \mathrm{kHz}$ and $100 \mathrm{kHz}$. The results are shown in Figure 174. The $200 \mathrm{~nm}$ devices showed a fully depleted i-region, as predicted by PC1D simulation. The $2 \mu \mathrm{m}$ devices leveled off at different values, but each was below the i-region thickness. The depletion regions measured in this test gave allowed calculation of the electron concentration in the iregion. One of the $2 \mu \mathrm{m}$ devices had a concentration of $\sim 7.5 \times 1015 \mathrm{~cm}-3$, and the other had a concentration of $\sim 1.3 \times 1016 \mathrm{~cm}-3$. Both of these values are similar to the concentrations routinely observed in undoped GaN.

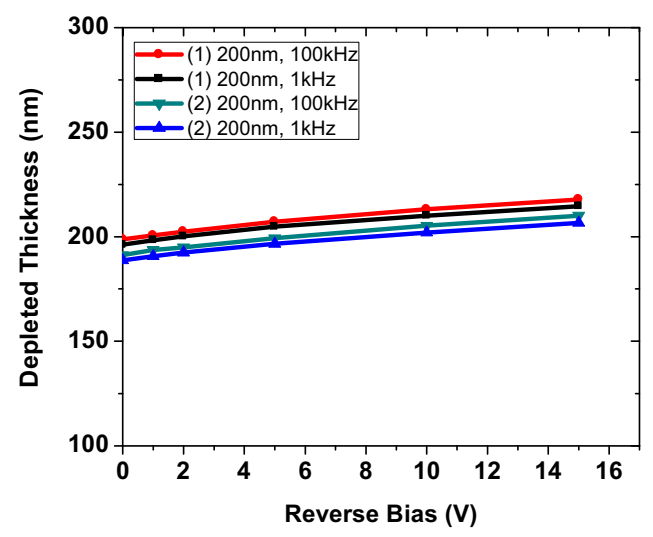

(a)

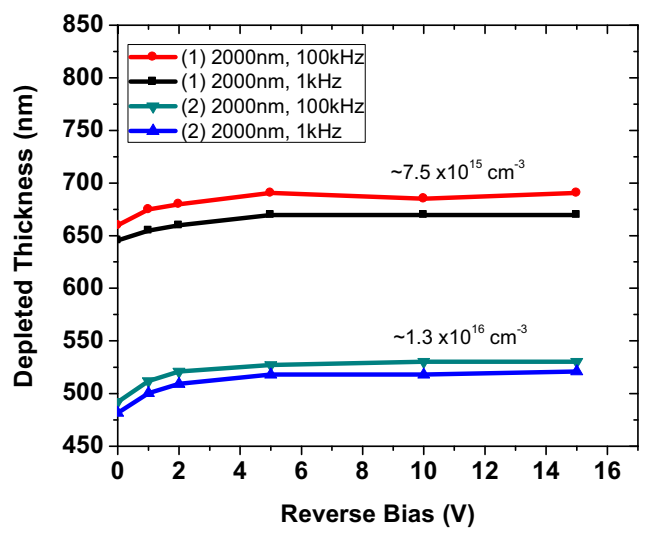

(b)

Figure 174. Calculated depletion thicknesses for GaN p-i-n diodes with (a) $200 \mathrm{~nm}$ i-regions and (b) $2000 \mathrm{~nm}$ i-regions.

These devices were exposed to the thermal neutron beam of the AGN reactor, but neither produced a discernible neutron response. This is likely due to the relatively high reverse bias dark current in the devices as well as the small area.

GaN-based scintillators and diodes were grown by MOCVD and characterized for material properties and neutron response. Self-absorption of near-band-edge emission was observed in both undoped and Si-doped films. Si-doped GaN was 
observed to be the best candidate for scintillator devices due to its superior optical gain. The low optical gain of undoped and Gd-doped scintillators caused any neutroninduced optical events to be indistinguishable from gamma events. The $14 N(n, p)$ reaction was observed in the Si-doped GaN scintillator with both the Am-Be and reactor sources, confirming the intrinsic neutron sensitivity of $\mathrm{GaN}$. This reaction was observed to offer excellent gamma discrimination as well. Conversion layers of $\mathrm{Gd}$ metal and $6 \mathrm{LiF}$ were also investigated. The neutron absorbing effects of both conversion layers were observed via the reduction in the $14 \mathrm{~N}(\mathrm{n}, \mathrm{p})$ peak, however only the $6 \mathrm{LiF}$ produced a distinguishable neutron-induced signal. Scintillation from the secondary ionizing radiation produced by the Gd layer was not discernible from the gamma-induced pulses. Scintillation spectra from Si-doped $\mathrm{GaN}$ with a $6 \mathrm{LiF}$ conversion layer were recorded at varying reactor power levels, and the detector was found to be highly linear over three orders of magnitude, indicating that GaN-based neutron detectors would be suitable for nuclear power application.

\section{Plasmonically Cloaked and Metamaterial Neutron Scintillators}

This research is a newly (FY12) funded NEUP contract to pursue advanced measurement techniques utilizing cutting edge Plasmonic cloaked and metamaterial neutron scintillators. The goal of the project is to measure the fission neutron spectrum, fission neutron yield, and time dependence of the neutron yield as a function of incident fission neutron energy. These innovative ideas bring game changing technology to the table, which is applicable to nuclear data, national security and the DOE. The project is centered around cutting edge advances in plasmonic cloaking, metamaterials and custom growth techniques to produce neutron scintillators who are intrinsically gamma blind. This new team will develop new measurement systems to address the nuclear data needs for the FCR\&D program and will work in conjunction with the nuclear data working group and other NEUP funded projects such as the elastic/inelastic measurement team and the fission Time Projection Chamber to develop the next generation of neutron detectors. This proposal addresses many of the pitfalls of other scintillator systems that had been proposed previously.

\section{Proposed Scope Description}

Significant work has been done over the last few years on the use of nanoparticle based scintillators. This work focused primarily on incorporating new scintillator material nanoparticles into a composite matrix to create large volume scintillators for gamma ray detection. These methods however suffer from the inability to collect the scintillation light. The light is either trapped internally in the nanoparticle, or is lost in scattering/absorption between the nanoparticle, host matrix interface. The idea behind plasmonic cloaking was originally published by Pendry et al. in 2006 . These cloaking devices are characterized by:

- Creating a closed space from which electromagnetic radiation is excluded, and the incident radiation is steered around it.

- The application of mathematical transformation on the original space creating a warped space, implemented by spatially varying permittivity and permeability. 
- [ [5

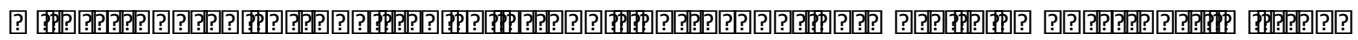

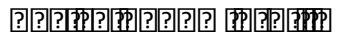

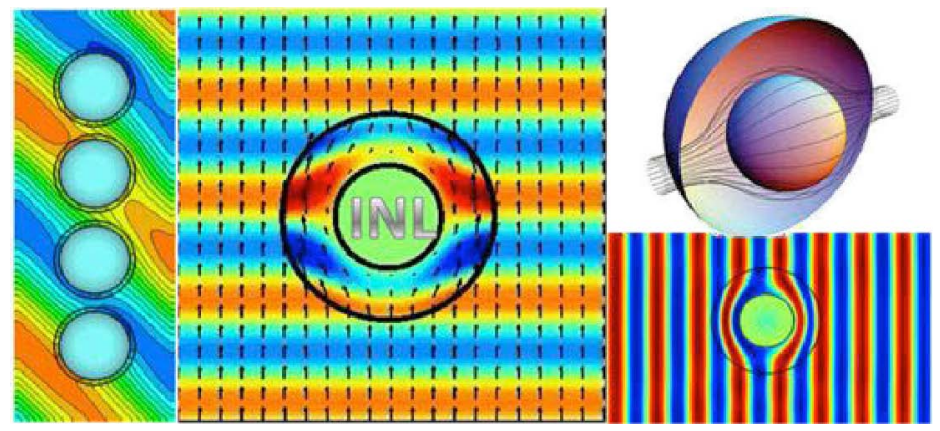

Figure 175 On the left are an array of cloaked spheres. The wave forms do not deform or get scattered by the spheres themselves which are absorbing. The center image is a close up of the simulation. Each particle was simulated as a GdO nanoparticle of $145 \mathrm{~nm}$ and a $15 \mathrm{~nm}$ plasmonic cloak of TiO. On the right are field lines of the light bending around the nanoparticle showing the arbitrary nature of the cloak.

Through the use of metamaterials, the Pls aim to develop planar neutron detectors and large volume neutron detectors. These detectors will have high efficiencyies for neutron detection and will have a high gamma discrimination capability. Each of these components will be described in subsequent sections.

\section{Plasmonic Cloak Theory}

Plasmonic cloaking is a method for creating a metamaterial in which light is bent around a highly absorbing material without interacting with the core. A metamaterial is a nanomaterial with an engineered structure of two or more materials which elicits specific phenomena including improved reflection, improved light yields, quantum wells, quantum confined materials, surface plasmon layers, and plasmonically cloaked materials. This bleeding edge technology is taken from the most recent advances in materials science and nanotechnology. In cloaking, the light is bent around the central core of arbitrary shape by designing a specially engineered structure to surround the core. This specially engineered structure is designed to have a low or negative permittivity. The intrinsic robustness of this system can be designed for a band of wavelengths. These methods have been demonstrated in several different applications including random orientations and clusters of various dielectric and conducting objects. This method has been successfully used to hide or cloak an object in a two dimensional array. The PIs have demonstrated this technology by producing this two dimensional structure.

\section{Neutron Detection Using Metamaterials}

Simulations and fabrication of a novel neutron detector material has begun by the PIs and shows significant promise as a large volume wide energy band neutron detector material. This new detector scheme has high sensitivities to fast neutrons from energies of $20 \mathrm{MeV}$ down to thermal energies. By using cutting edge nanotechnology 
research, a novel scintillator structure based on metamaterials is being proposed. The detector is designed around a plasmonically cloaked metamaterial. Here, nanoparticles of arbitrary shape are coated through a fluidized bed reactor that the Pls are currently developing. A thin plasmonic layer is used to bend scintillation light around the light absorbing nanoparticle core. Light is no longer scattered by the nanoparticle or trapped inside its unique facets. The nanoparticles can be created in bulk, kilogram size batches. ${ }^{6} \mathrm{LiF},{ }^{6} \mathrm{LiCO}_{3}$ or $\mathrm{GdO}$ would be used as the core material. $\mathrm{HfO}, \mathrm{TiO}, \mathrm{ZrO}$ or other high index material would be used in the plasmonic layer. The cloaking layer can be applied through an atomic layer deposition process in a fluidized bed reactor (FBR).

Several designs of scintillator will be considered. The first scintillator type is comprised of a plastic scintillator matrix that has suspended in the matrix a high density of plasmonically cloaked nanoparticles of neutron absorbing materials. This type is ideally suited for producing highly efficient neutron scintillators that do not suffer from light collection problems. The second scintillator design builds on the first scintillator design by producing a multilayered metamaterial who is cloaked with a plasmonic cloaking layer. This scintillator is cast in an inert ultra violet transmitting matrix.
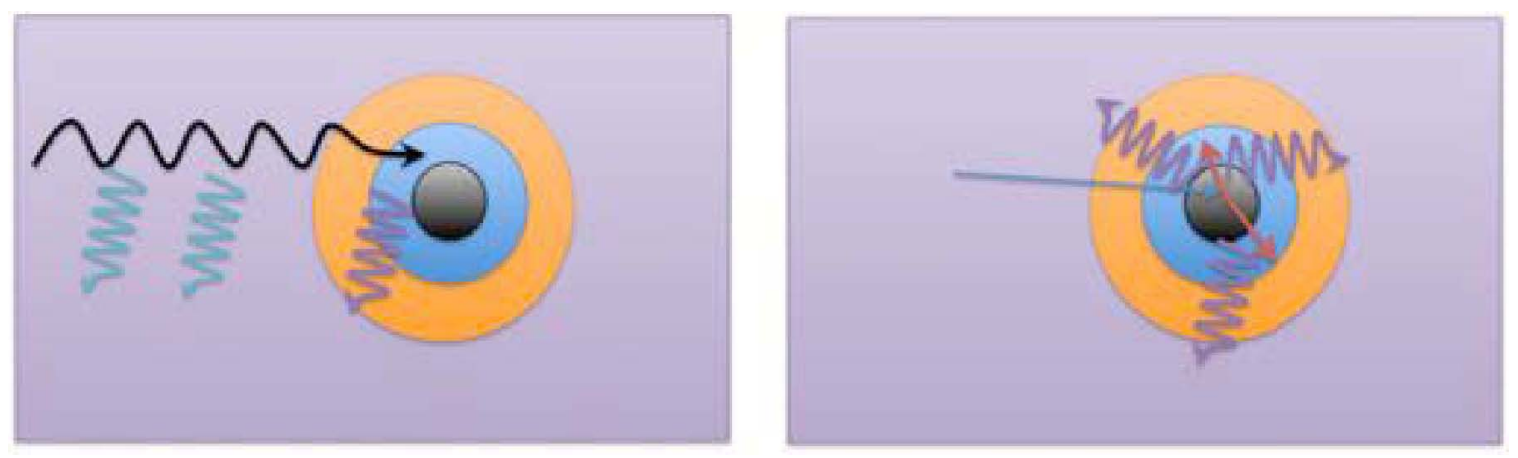

Figure 176 Design of neutron versus gamma discrimination for design one. The orange layer is the plasmonic cloak, the blue layer is the scintillator layer made from $\mathrm{ZnO}$, the kernel is made from ${ }^{6} \mathrm{Li}$ purple is a scintillator matrix. Gamma rays shown on the left, produce both scintillation wavelengths, while neutrons only produce one scintillation wavelength.

In the first method, the metamaterial is then cast into a plastic scintillator matrix such as $\mathrm{BC} 408$ or $\mathrm{BC} 400$. This metamaterial is a nanoparticle with a radius of $5-100 \mathrm{~nm}$ of ${ }^{6} \mathrm{LiF},{ }^{6} \mathrm{LiCO}_{3}$ or $\mathrm{GdO}$ which is coated with a plasmonic cloak. Through either the $(n, \alpha)$ reaction in ${ }^{6} \mathrm{Li}$ or neutron capture in gadolinium, the charged particle produced by the nanoparticle easily passes through the plasmonic cloak and deposits $98 \%$ of it's energy in the plastic scintillator. This design is shown in Figure 176. The light generated in the scintillator is produced isotropically. As opposed to striking another nanoparticle, the light wave interacts quantum mechanically with the plasmonic cloak. In doing so, the light bends around the opaque particle and continues on it's original path without loss or scattering. Due to the low absorption in the scintillator matrix, large volumes of scintillator are possible with no noticeable loss in light collection. 


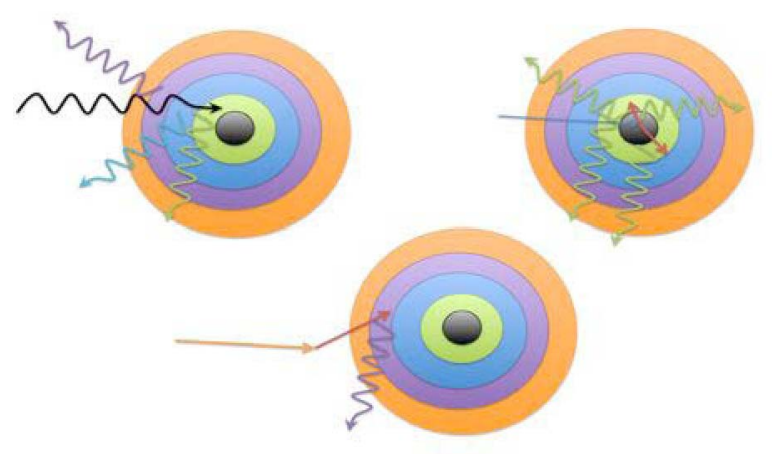

Figure 177. Design of neutron versus gamma discrimination for design three incorporating fast neutron response. The orange layer is the plasmonic cloak, the blue layer is the first scintillator layer made from $\mathrm{ZnO}$, the green layer is a bangap engineered layer scintillating in the green, the kernel is made from ${ }^{6} \mathrm{Li}$,. Gamma rays shown on the left, produce both scintillation wavelengths, while neutrons only produce one scintillation wavelength. Proton recoil events only interact in the outer region, shown on the bottom cartoon.
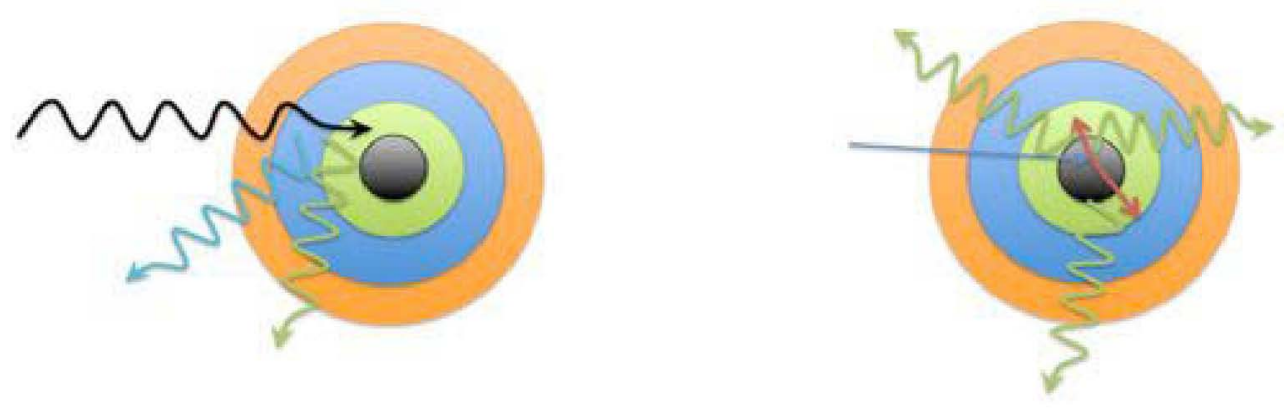

Figure 178. Design of neutron versus gamma discrimination for design two. The orange layer is the plasmonic cloak, the blue layer is the first scintillator layer made from $\mathrm{ZnO}$, the green layer is a bandgap engineered layer scintillating in the green, the kernel is made from ${ }^{6} \mathrm{Li}$,. Gamma rays shown on the left, produce both scintillation wavelengths, while neutrons only produce one scintillation wavelength.

In a second method, the metamaterial is cast into an intert ultraviolet transmitting plastic matrix. The matrix is no longer the scintillator. The nanoparticle kernel is still a nanoparticle with a radius of $5-100 \mathrm{~nm}$ of ${ }^{6} \mathrm{LiF}$ or ${ }^{6} \mathrm{LiCO}_{3}$ which is then coated with a 10 um scintillator material with a long wavelength in the blue, coated with a second scintillator of 10 um which scintillates in the near ultraviolet, and then coated with a plasmonic cloak layer. In this configuration, the resulting charged particles (the alpha and the triton) produced from the $(n, \alpha)$ reaction in ${ }^{6} \mathrm{Li}$ range out in the first scintillator. This scintillator produces a blue wavelength light pulse. The next scintillator layer is transparent to the blue wavelength and thus it passes out of the metamaterial nanoparticle and into the inert matrix. However, when gamma rays interact in the material, they interact by producing recoiling electrons or photoelectrons. These electrons range is significantly larger than the metamaterials. This will produce both 
blue and near ultraviolet light pulses. The plasmonic cloak will prevent the light from interacting with the other natnoparticles in the matrix. For a neutron only event, light pulses in the blue are the only scintillations to be produced. In gamma ray events, blue and ultraviolet light pulses are produced. A coincidence system can be used to provide the neutron versus gamma discrimination. By having two different sensitive PMTs or APDs one for blue, one for ultraviolet and blue, the two signals can be compared and run in anti-coincidence mode. This method is shown in Figure 179.

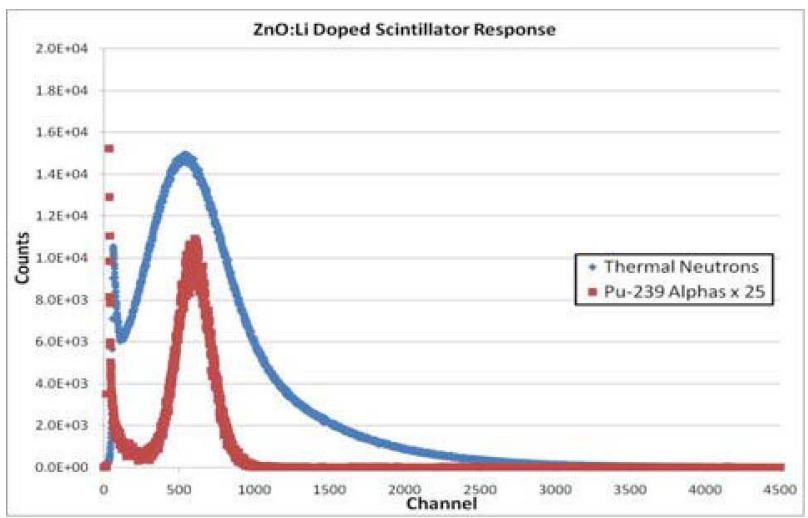

Figure 179. Pulse height distribution from lithium doped $\mathrm{ZnO}$ scintillator.
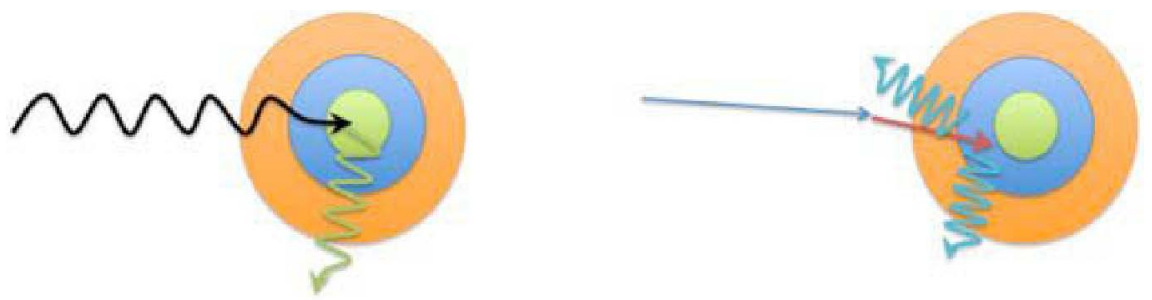

Figure 180. Design of neutron versus gamma discrimination for design three incorporating fast neutron response. The orange layer is the plasmonic cloak, the blue layer is the first scintillator layer made from $\mathrm{ZnO}$, the green layer is a bangap engineered layer scintillating in the green. In this design there is no kernel. Gamma rays shown on the left, produce both scintillation wavelengths, while neutrons only produce one scintillation wavelength. Proton recoil events only interact in the outer region, shown on the bottom cartoon.

This scintillator design is sensitive to both fast and thermal neutron fields. This unique solution can easily obtain $100 \%$ efficiency for thermal neutrons and absolute efficiencies in excess of $50 \%$ for fast neutrons. Fast neutron scintillators can run in two different modes. The first is a capture gated method. This is possible due to the hydrogen content of the plastic scintillator or the inert UVT matrix. The neutrons thermalize in the scintillator and are captured producing a large light pulse. This method is usually considered capture gated neutron spectroscopy and is shown in Figure 177. Design of neutron versus gamma discrimination for design three incorporating fast neutron response. The orange layer is the plasmonic cloak, the 
blue layer is the first scintillator layer made from $\mathrm{ZnO}$, the green layer is a bangap engineered layer scintillating in the green, the kernel is made from ${ }^{6} \mathrm{Li}$,. Gamma rays shown on the left, produce both scintillation wavelengths, while neutrons only produce one scintillation wavelength. Proton recoil events only interact in the outer region, shown on the bottom cartoon.. Neutron versus gamma discrimination can be achieved by utilizing a third configuration for the metamaterial including the plasmonic cloak. This configuration has three distinct scintillators, and a neutron absorbing kernel. Recoiling protons interact in only the outer layer scintillator, scintillating in the UV. The middle layer scintillates in the blue, and is out of the range of the charged particles produced from the central kernel or the recoiling protons. The inner kernel charged particles interact in the inner scintillator that scintillates in the green. By using a three PMT or APD light collection system, each one sensitive to a narrow band of scintillation wavelengths, and coincidencing the signals, neutron versus gamma discrimination can be achieved and even discriminate between fast and thermal neutrons. Thermal neutron only events would scintillate in green, Fast neutrons would scintillate in the UV, whereas the gamma events would scintillate in green, blue and UV. This three wavelength method can achieve theoretical neutron verses gamma discrimination levels of $10^{6}$.

The second fast neutron scintillator resembles the traditional fast neutron scintillator method. Here, only fast neutrons would interact with two different scintillators. The central kernel of neutron target nuclei has been removed, and replaced with a scintillator. The plasmonic cloak keeps the scintillators from reabsorbing their own scintillation. Neutrons would produce only UV scintillations while gammas produce both blue and UV. This method is shown in Figure 180.

To further improve the detector performance, an anti-coincidence system will be created by segmenting the detectors. With anticoincidence system, the detector becomes fairly insensitive to room return and scattered neutron contributions. This will further improve the detector response and the spectroscopic resolution of the detector system.

The low energy component of the detector array will be comprised of doped $\mathrm{ZnO}$ scintillators. The doped scintillators show excellent neutron versus gamma discrimination and can be grown in large planar arrays. These scintillators can use the shift in the $(n, \alpha)$ peak from ${ }^{6} \mathrm{Li}(n, \alpha)$ reactions to ascertain the incident neutron energy. This fills the neutron detection gap between $500 \mathrm{keV}$ and thermal neutron energies where many detection systems cannot perform. 


\section{Fluidized Bed Reactor Design}

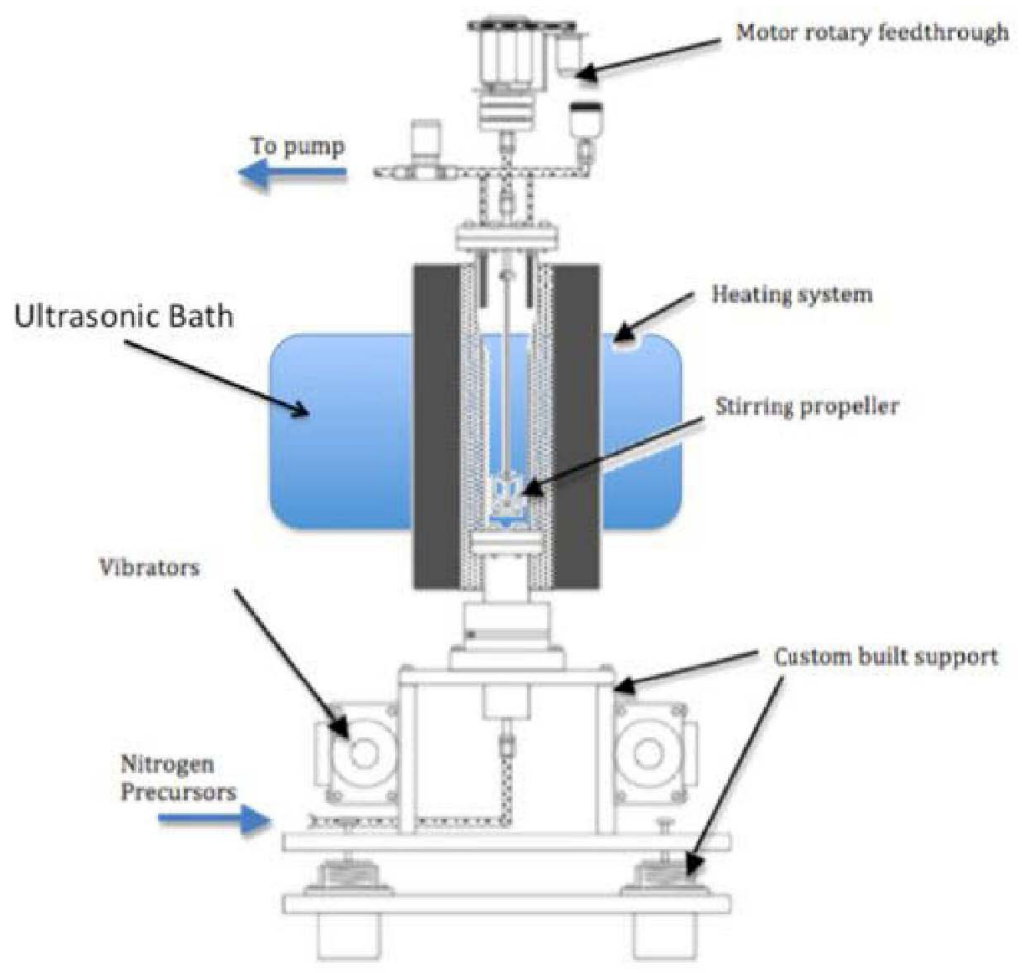

Figure 181. Proposed fluidized bed ALD reactor schematic. Vibration motors, a mixer impeller, and an ultrasonic bath are designed to keep the system fluidized.

The fluidized bed reactor design is not a novel concept. The adaption to be an atomic layer deposition system is however. The fluidized bed reactor takes and converts a bed of solid particles into a liquid like substance. This is accomplished through very specific gas flow rates through the nanoparticle media. The system is comprised of many mass flow controllers as well as reactant gas mass flow controllers. Like an ALD system, half cycle, self-limiting pulses of gas deposit one half of the two component material on the surface. The fluidized bed is needed to ensure uniform coating of the nanoparticles. A sample design of the fluidized bed reactor is shown in Figure 181. To prevent conglomeration of the nanoparticles, a combination of vibration, mixing and ultrasonic perturbation is used to keep the nanoparticles from clumping during growth.

The Pls have extensive experience in growth of these novel neutron scintillator materials and have a diverse background in materials science, nanotechnology and nuclear engineering with numerous years specializing in neutron detection. The Pls have grown novel plasmonic cloak materials before and have successfully demonstrated the technology. They currently have a preliminary design for the fluidized bed ALD reactor design for the system. 


\section{Design of Large Area Array}

The large area array will first be created out of planar devices. As the fluidized bed comes online and is able to produce plasmonically cloaked particles, large area devices will be fabricated. This will take on the shape and configuration similar to the Euroball detector array. The specific design will be finalized while working with the other data groups including the Elastic/Inelastic, Chi-Nu, and Fission TPC projects. Each detector design will be specific to the measurement program it is designed for. The detector array aims to help fill in the gap between traditional thermal neutron scintillators and fast neutron scintillators while maintaining excellent neutron versus gamma discrimination. No complicated electronics or pulse shape discrimination are needed, merely coincidence measurements for rejection of gamma ray backgrounds. The plasmonic cloaked scintillators engineer out the gamma ray response. This system has the potential to produce a highly efficient neutron detection system with cutting edge technology.

\section{Technical Relevance:}

This system aims to create a 4 pi geometry novel neutron scintillator array for measuring the fission neutron spectrum energy, angle, time, as a function of incident neutron energy. This will be done through the use of a small fission chamber, and the novel neutron scintillators proposed above. The goal of the project is to measure the fission neutron spectrum, fission neutron yield, and time dependence of the neutron yield as a function of incident fission neutron energy. These innovative ideas bring game changing technology to the table which is applicable to nuclear data, national security and the DOE. This new team will develop new measurement systems to address the nuclear data needs for the FCR\&D program and will work in conjunction with the nuclear data working group and other NEUP funded projects such as the elastic/inelastic measurement team.

\section{ZnO Photonic Crystal Neutron Detector Research}

As an offshoot of the plasmonic cloaking technology, another nanotechnology solution has been found. This is the $\mathrm{ZnO}$ neutron scintillator incorporating a photonic crystal structure. The $\mathrm{ZnO}$ scintillator was also investigated as a photonic crystal scintillator. The photonic crystal structure is an alternating array of different dielectric materials of controlled thickness. They are designed to control the optical flow of light. By varying an array of holes and/or rods, the wavelengths are confined to propagation in certain planes. A series of calculations was performed to calculate this very effect. These are shown below. One is trying to confine the Schrödinger wave equations to propagate in only one direction. This is achieved in certain patterns. Traditional research in square and triangular band structures has resulted in photonic crystal structures. To improve the performance, superlattice structures have been created by the PIs. This is a combination of square and triangular and can be in the form of orthorhombal and honeycomb hexagonal. A sample is shown below. The red and blue are the real and imaginary component to the propagating wave as it is created and traverses the photonic crystal structure. Tight confinement results in band gaps promoting single direction light propagation. 


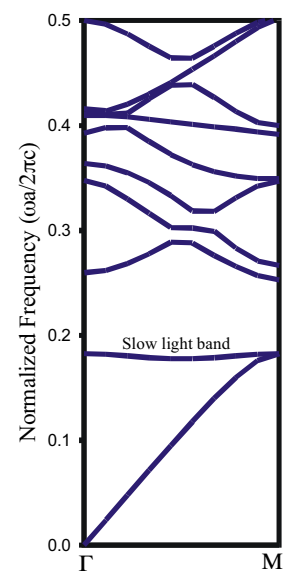

Superlattice PC

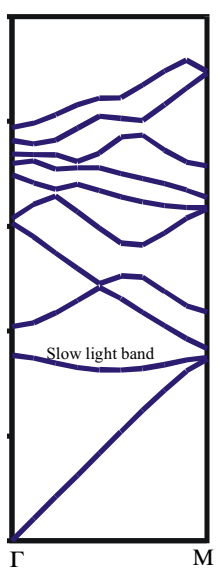

Rectangular PC
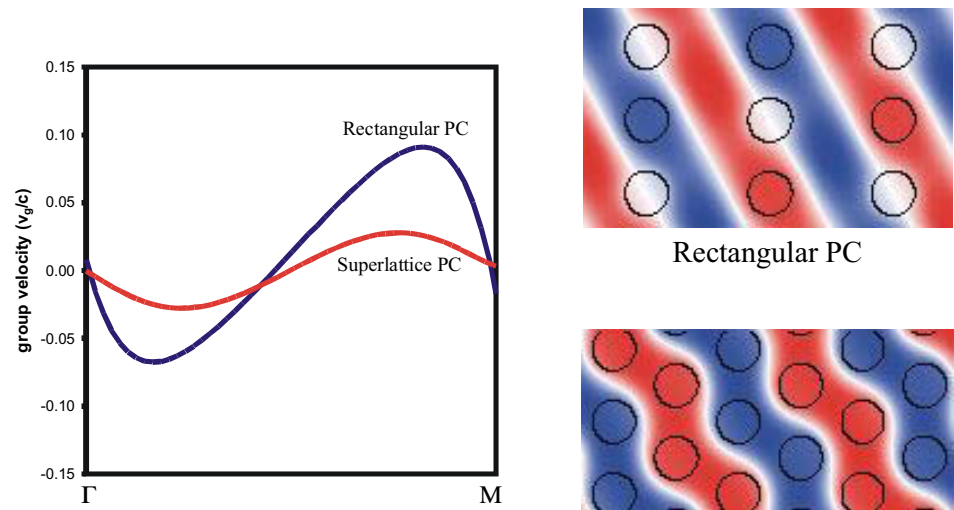

Rectangular PC

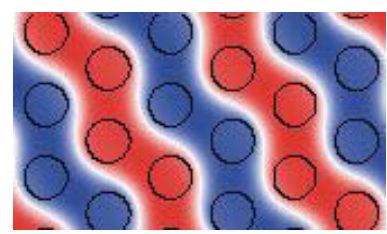

Superlattice PC

Figure 182 photonic crystal lattice calculations for rectangular PC and superlattice PC.

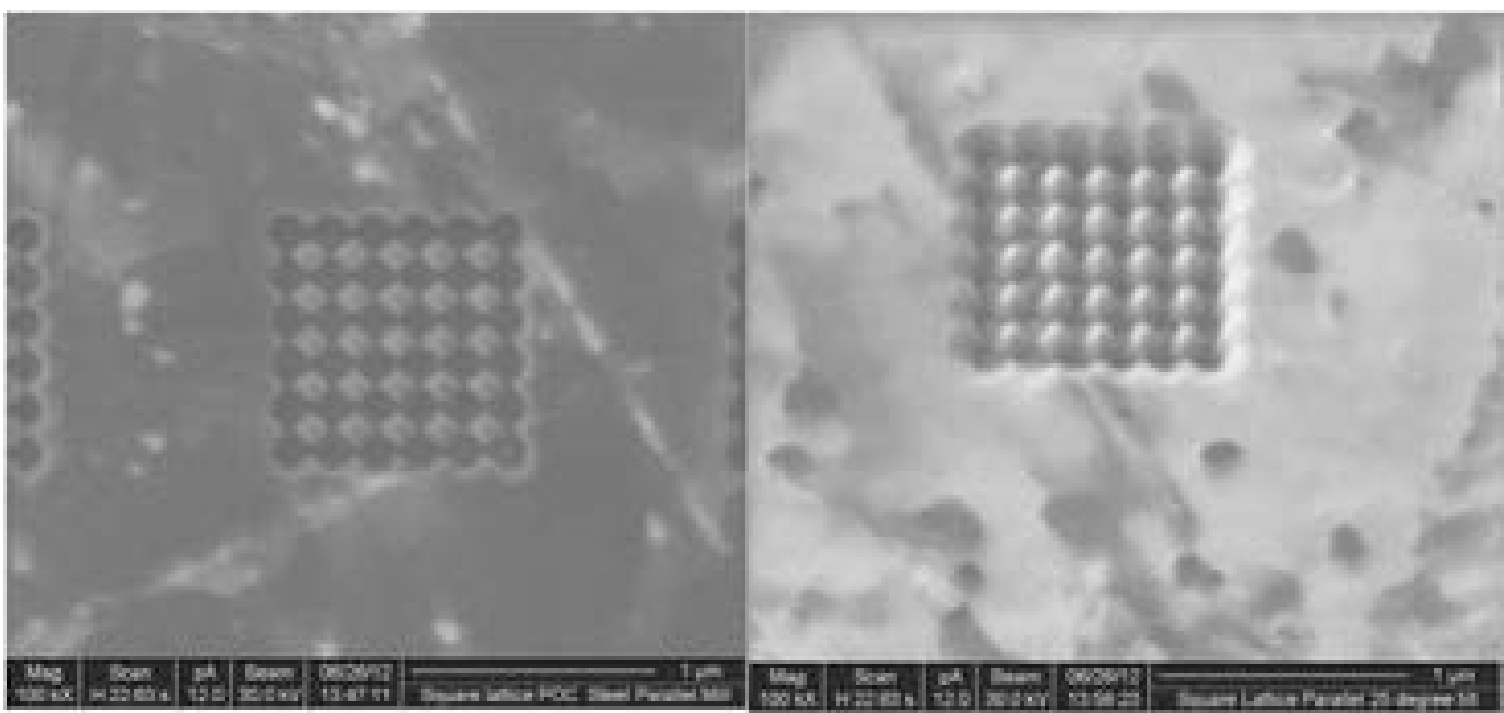

Figure 183 Photonic crystal superlattice structure cut into steel surrogate. On the left is the vertical view, on the right is the sample tilted at 25 degrees to show the depth of the milled structure.

These structures can lead to a high degree of pixilation. This is due to the quantum confinement of the scintillator light within a region of the photonic crystal structure. To improve imaging resolutions of imaging neutron detectors, this technology can be leveraged to produce exceptionally high resolution, high magnification images. Imaging pixilation can be as small as 10 lattice constants, which is can be as small as $500 \mathrm{~nm}$. With image magnification through neutron beam optics, unprecedented neutron imaging is possible with these systems. One such system is shown below. 


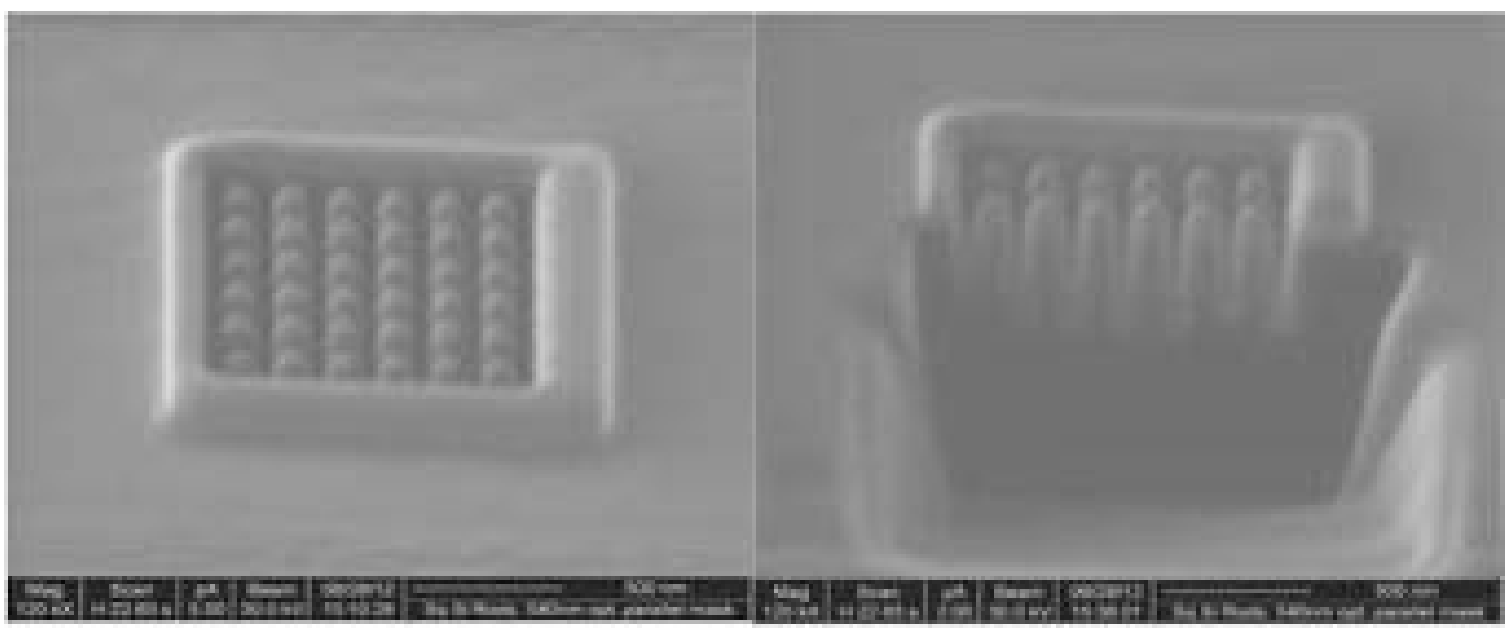

Figure 184. Pixilated detector design cut in surrogate material. The sample is tilted on the left and a cross sectional cutaway was performed on the right showing the crystalline structure.

These materials have been grown and have shown good results in the improvement of light outcoupling. A sapphire substrate was templated with a photonic crystal which was designed for the optical wavelength of $532 \mathrm{~nm}$. Zinc Oxide was grown with a green band emission wavelength to match the photonic crystal structure. This was achieved through carbon doping in the $\mathrm{ZnO}$ scintillator. In future work, dopants other than carbon will be elicited to achieve a more desired emission spectrum. The green band directly couples with the photonic crystal structure. The structure was directly grown on the sapphire template through the modified ALD technique resulting in $\mathrm{ZnO}$ being grown conformally into the sample. PL measurements were made of similar devices without the photonic crystal structure cut into them. These structures provided a baseline for comparison. The light outcoupling has improved by a factor of two. This can be seen below in Figure 186. This is a documented thin film scintillator with two times the light outcoupling.

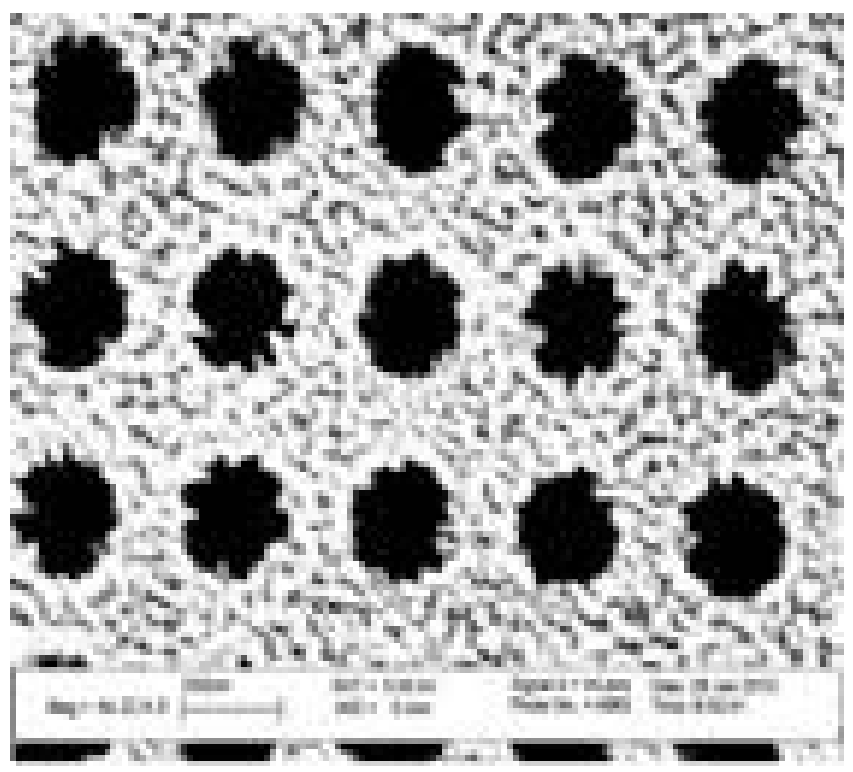

Figure 185. ZnO sample grown over a photonic crystal structure. 


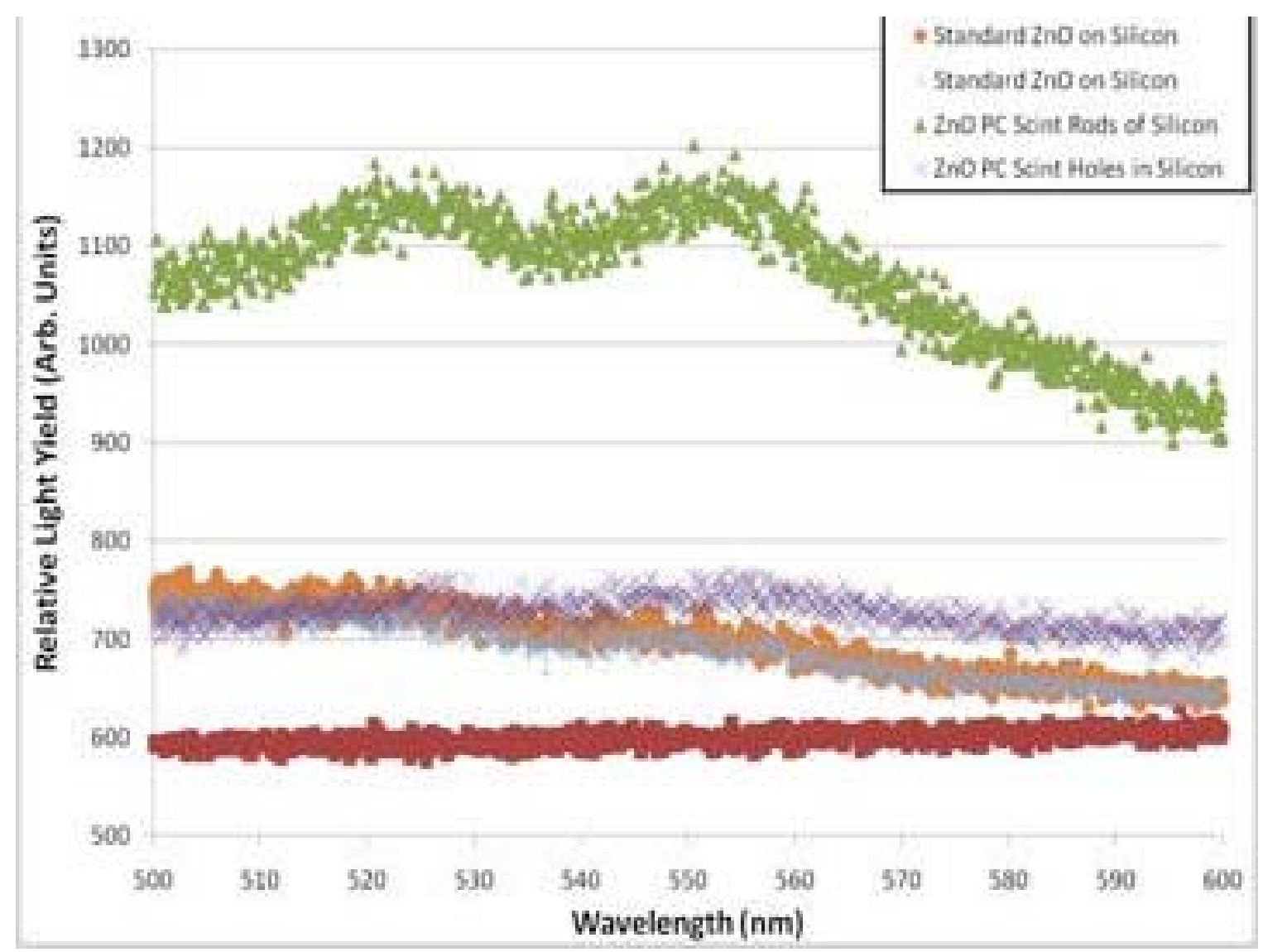

Figure 186 PL measurement of photonic crystal structured ZnO scintillator. A factor of 2 light increase was observed.

\section{Scintillator Materials Development (UML)}

The UML effort in the first year has been focused on evaluating the capabilities of emerging scintillation detectors for neutron-gamma detection and discrimination via digital signal processing techniques, as well as renovating and upgrading the capabilities of the 5.5-MV Van de Graaff at UML for thermal and fast neutron measurements with the new detectors. One undergraduate student (Thomas Harrington, NEUP scholar 2009-2011) and two graduate students (Emily Jackson and Nathan D'Olympia) are part the UML team on this project.

Leveraging last year's work in fabrication and characterizing of two emerging scintillation detectors, Cs2LiYCI6 (CLYC) and Cs2LiLaBr6 (CLLB), the focus for FY12 has been the use of Cs2LiYCI6:Ce (CLYC) as a fast neutron scintillator with excellent gamma ray pulse discrimination. The novel scintillator was tested and characterized with regard to energy and time resolutions, and, more importantly, the ability to distinguish between neutrons and gamma rays using pulse shape discrimination techniques. This is the first reported work on fast neutron detection since CLYC was first developed (Delft, 1999). 


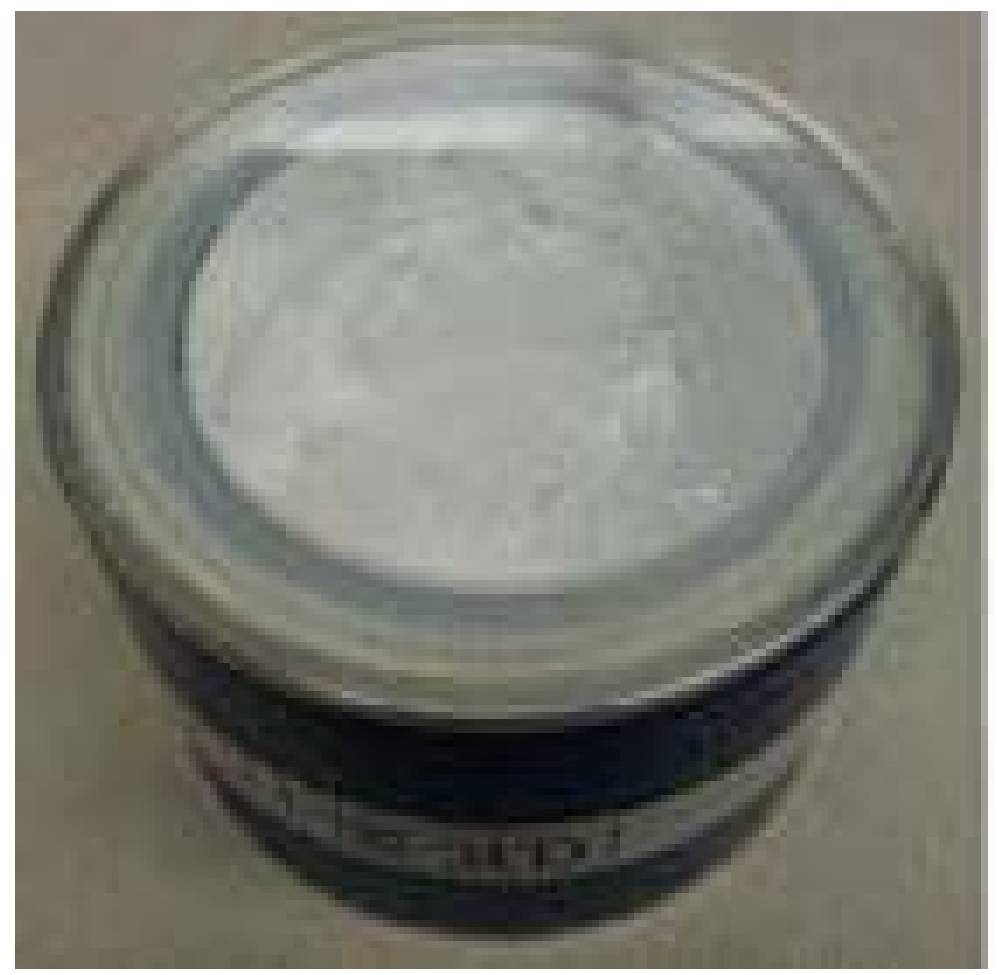

Fig. 187: The prototype 1"x1" CLYC scintillation detector being tested at UML The detector, approximately 1" diameter by 1 " thick and coupled to a Hamamatsu R6233-100 photomultiplier tube, was tested at UML with monoenergetic, fast neutrons $(0.81 \mathrm{MeV}$ to $2.6 \mathrm{MeV})$ from the $7 \mathrm{Li}(p, n)$ reaction. One of the most interesting and potentially useful discoveries was a large detection signal contribution from the $35 \mathrm{Cl}(n, p)$ reaction in the $C L Y C$ material. This was not expected to contribute to the fast neutron detection signal, as the signal is typically dominated by the thermal neutron capture in $\mathrm{Li}$ and the fast signal from the $6 \mathrm{Li}(\mathrm{n}$, alpha) reaction. A recent example of the $35 \mathrm{Cl}(\mathrm{n}, \mathrm{p})$ fast neutron signal is shown in Error! Reference source not found. 


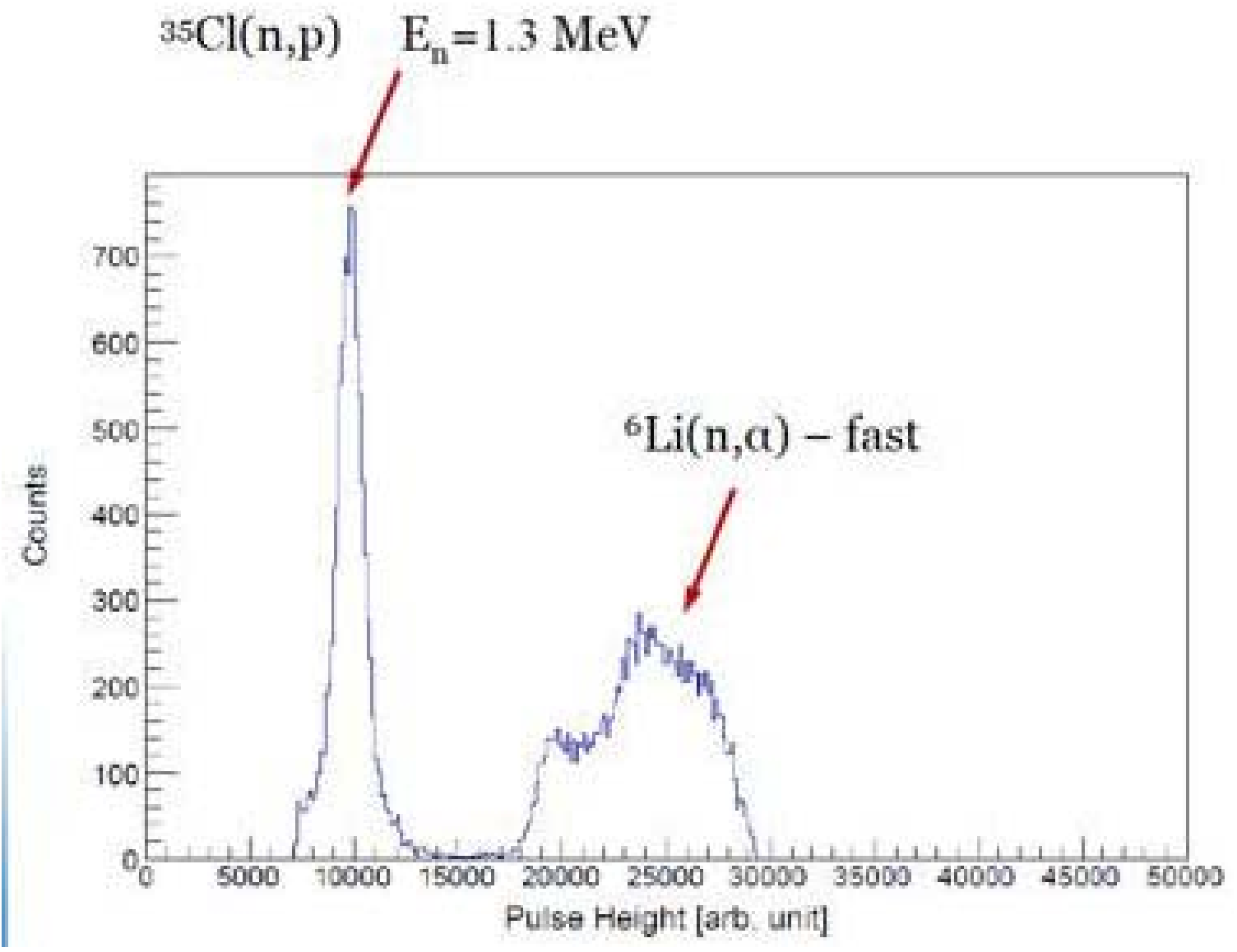

Error! Reference source not found. 


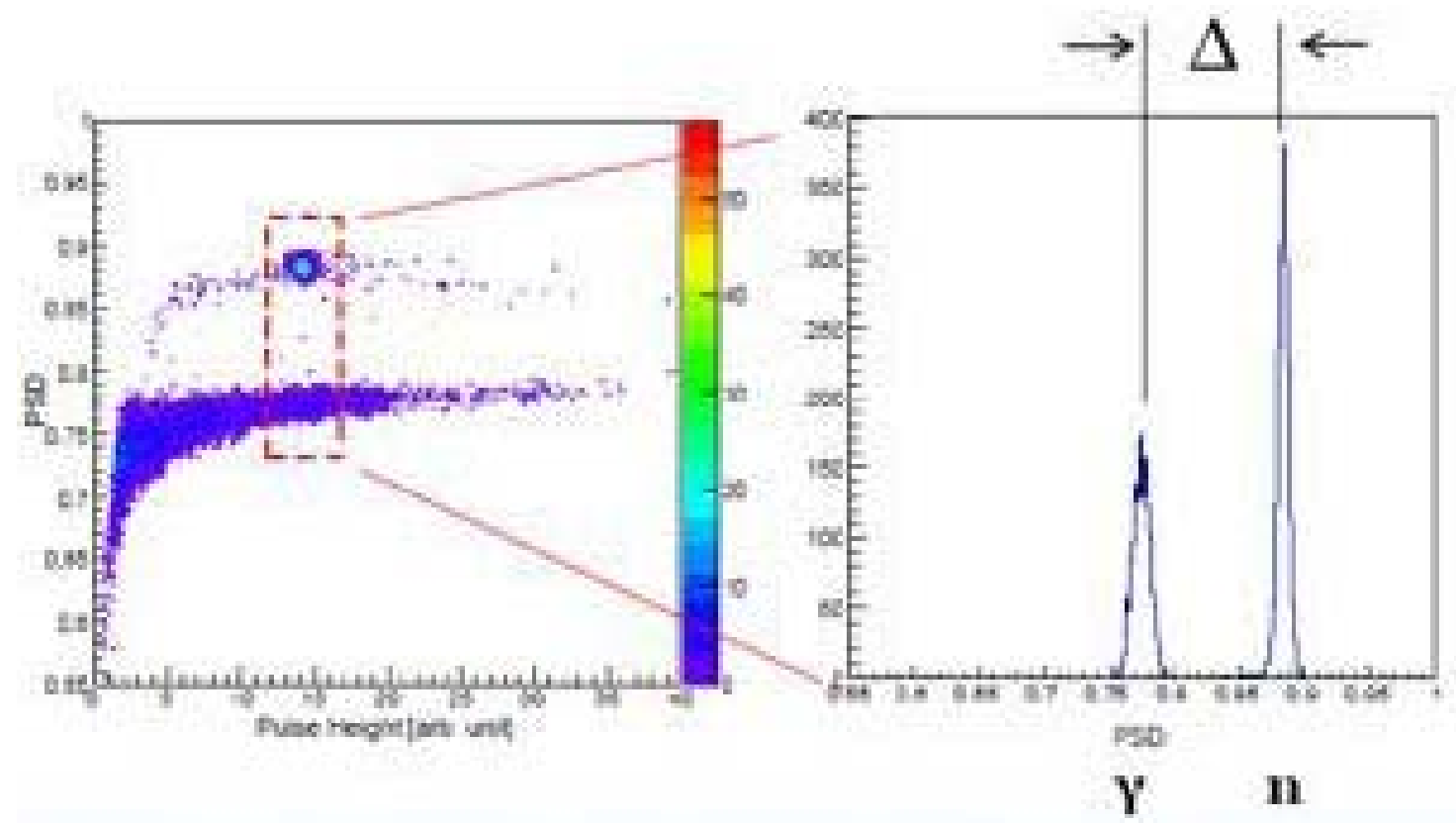

Figure 188. Preliminary neutron-gamma discrimination results for the CLYC prototype scintillator via digital pulse processing.

Captured signal waveforms were initially analyzed using LABVIEW. Two different areas under the signal waveform were integrated, and the ratios of the area were plotted as a function of the total area under the full pulse. Initial results for the neutron-gamma discrimination capabilities of the prototype CLYC scintillator are shown in Figure 188. The figure shows clear separation between gamma ray and neutron signals. The digital signal processing codes for neutron-gamma pulse-shape discrimination were initially written in LABVIEW and then converted to C++. Initially, the $\mathrm{C}++$ scripts were executed with the standard Linux compiler, and plots were generated using ORIGIN. Since the ROOT software from CERN has superior plotting and histogramming capabilities, the analysis software was then split into individual C++ scripts, which could then be executed in ROOT using simple user defined commands. All variables can now be changed without having to reload the data or restart the program. A user's manual was written for the n-gamma analysis.

Measurements with fast neutrons were performed with the 5.5 MV Van de Graaff accelerator at UML. Significant renovations have been ongoing for the experimental area of the UML 5.5-MV Van de Graaff accelerator facility, which can provide fast, pulsed neutrons. Floor asbestos was removed, the new concrete floor and walls painted, and the neutron measurement area platform upgraded. Renovations have been undertaken at the fast neutron beamline for neutron scattering and crosssection measurements. The goniometer drive assembly was repaired and a new controller was built for it. New collimators pre and post buncher were fabricated and tested with beam. In trying to obtain pulsed beam, the tank had to be removed and the source and all internal controls were tested individually. During tank gas removal, the compressor developed some problems, and some gas was lost. Some internal valves had to be sent off for repair, and 40 bottles of gas had to be added when the 
tank was reinstalled. At the present time, a 200-ns pulsed proton beam has been successfully generated. A test of the Mobley bunching system is the next item on the agenda.

A full description of the CLYC prototype as a fast neutron scintillator is reproduced here from a recent NIM-A publication obtained from the authors. 


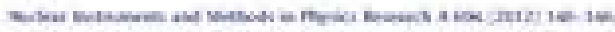

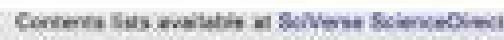 \\ Nuclear Instruments and Methods in Physics Research A

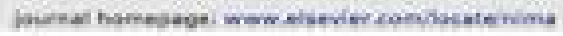

\section{Optimizing $\mathrm{Cs}_{2} \mathrm{LiYCl}_{6}$ for fast neutron spectroscopy}

N. Dolympia 2, , P. Chowdhury 2, CJ. Guess", T. Harrington ", EC. Jackson", S. Lakshmi"

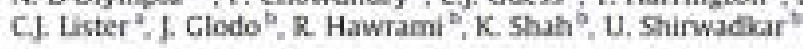

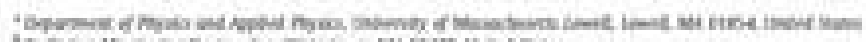

antete into

\section{sise $\operatorname{ling}$}

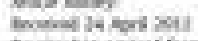

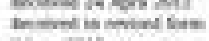

parasis?

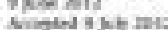

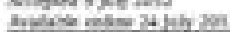

sowith

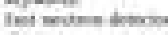

anc

P.tenrinimetis

icin

tuses:

raven

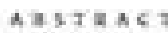

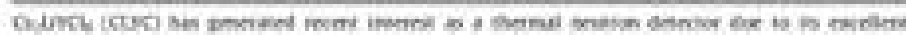

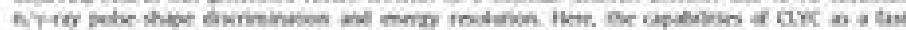

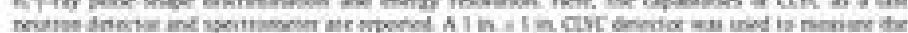

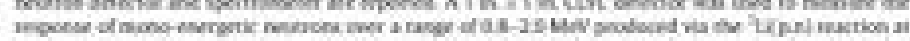

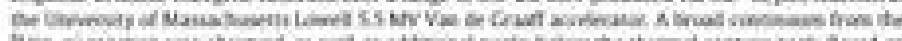

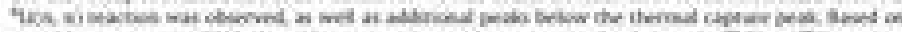

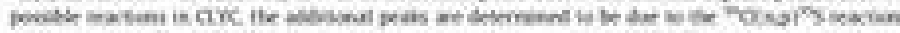

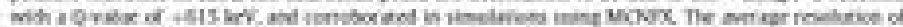

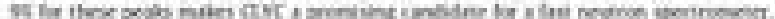

Nesihet by Bume E.

\section{1. thendurisen}

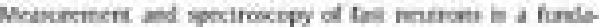

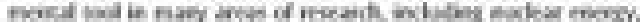

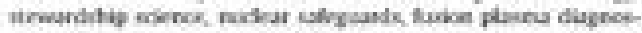

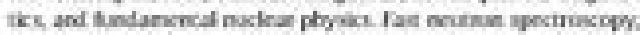

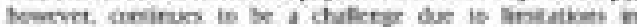

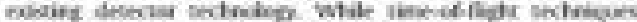

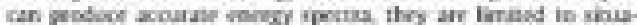

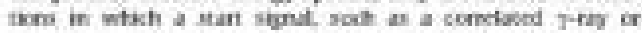

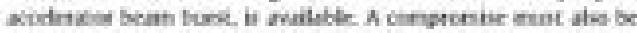

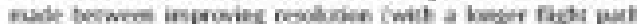

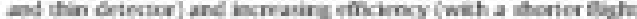

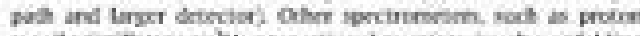

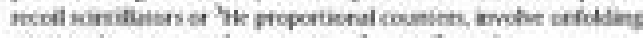

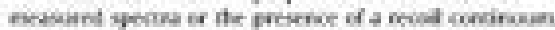

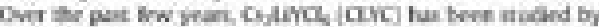

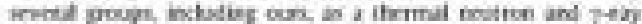

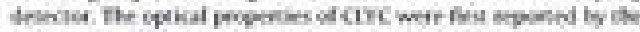

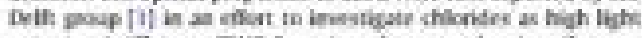
outpit scintalowes. Cre has sinop been und primerily an a

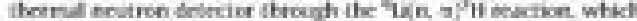

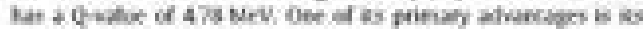

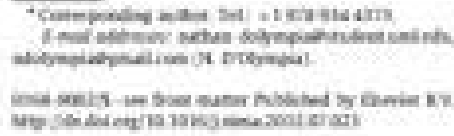

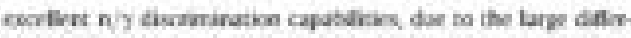

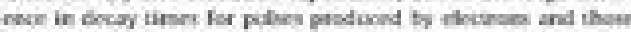

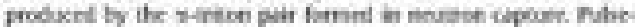

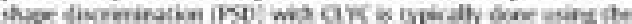

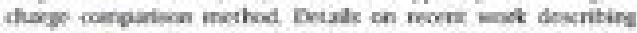
rosp and thermal notren dronction wh carc cap be found in Eris. $[23]$

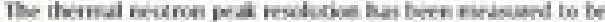

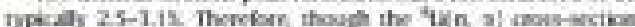

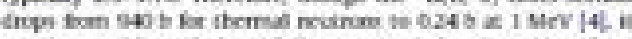

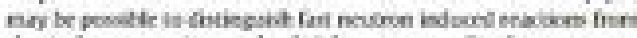

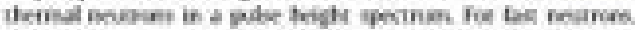

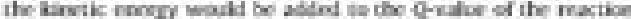
and shand betwosh the g-purikik and inson. etrponiking a boul

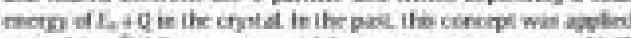

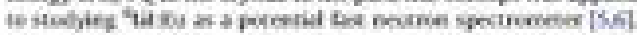

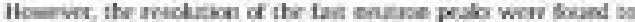

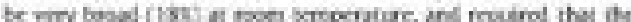

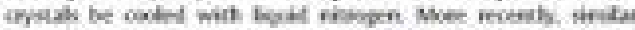

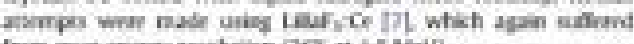

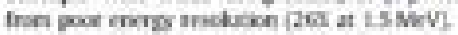

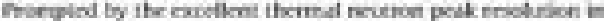

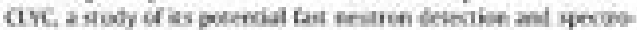

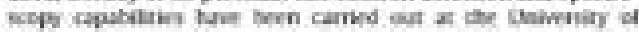

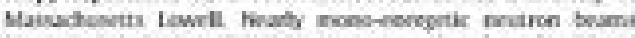
between oin MeV and 202 Merv wore prodered at the 5.5 NV

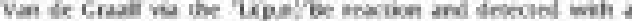

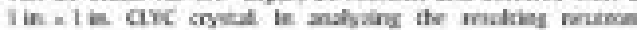




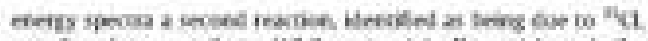

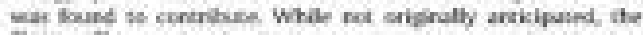

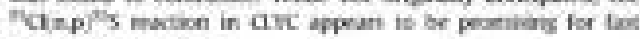
neirob spetimoliogy.

\section{Everimernal seap}

21 Nratou production

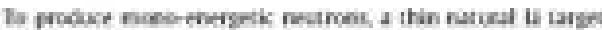

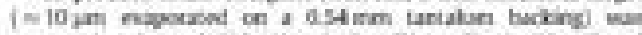

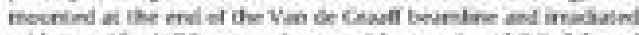

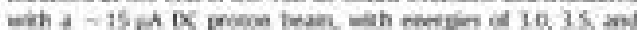

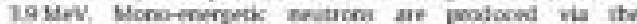

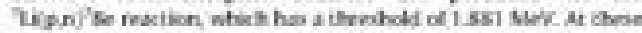

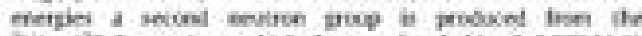

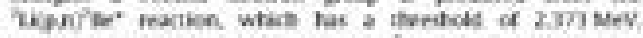

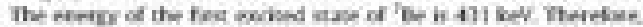

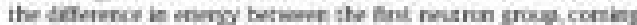

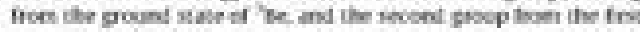

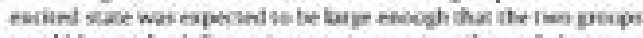

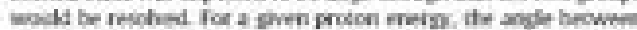
it be ben anertioe and deteolat was abs virind io oblai alleres furmos encryin.

\section{Dobeiner ietsy}

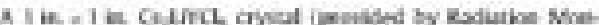

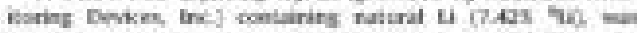

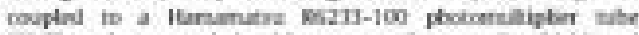

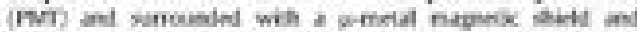

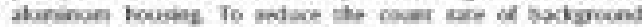

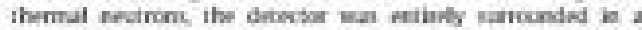

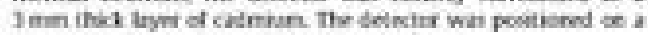

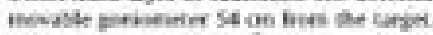

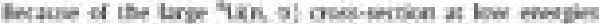

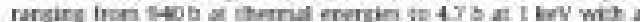

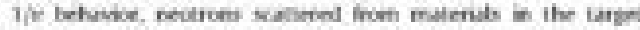

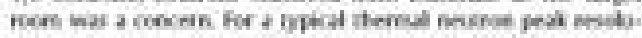

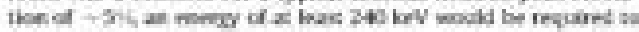

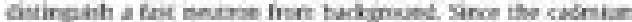

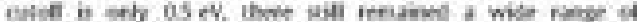

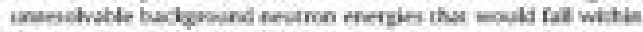
the so-called therrul prak.

Aliempes mere made to shich the dreciar wat a thich

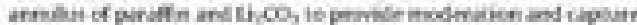

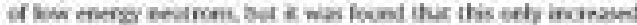

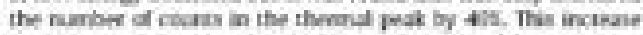

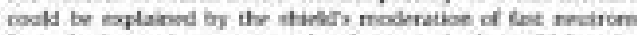

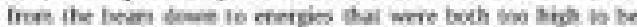

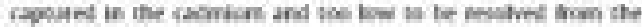
itermal poust

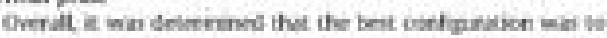

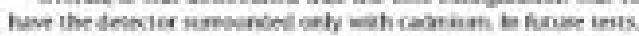

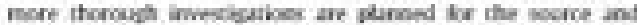

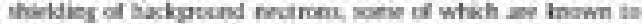

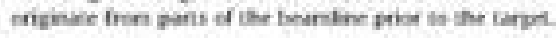

\section{Cata ondivas}

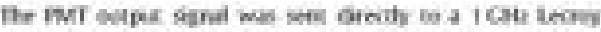

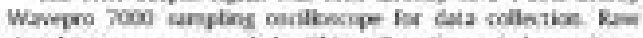

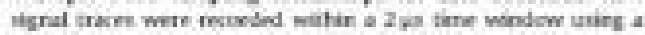

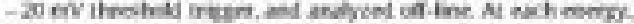

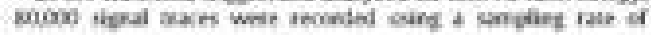

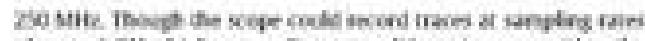

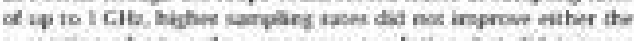
mparicion of $\mathrm{n} / \mathrm{z}$ pulen of mory tenchitise but ded increase

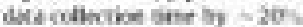

is itr afline arubsik, ny Gecresination wak ferfumed

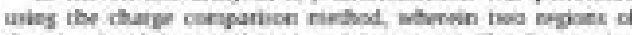
the signd pelbe are selncind and iniestalied the fine nopon

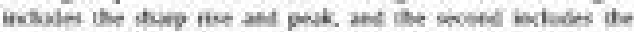

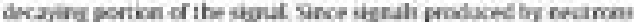

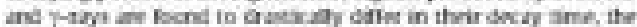
nave of areds for thre tho incegation windiows diflers drgond-

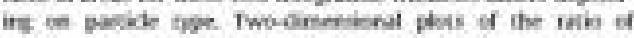

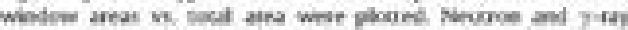

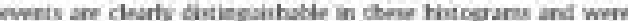

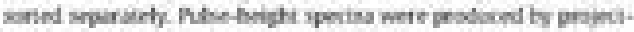

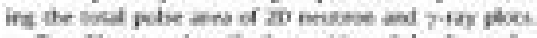

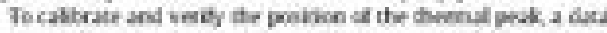

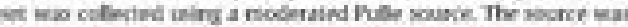

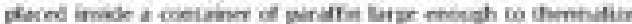

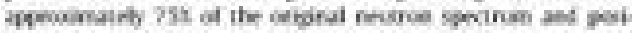
tored $5 \mathbf{4} \mathrm{cm}$ from the decester A 7-ray cabraibe war camied

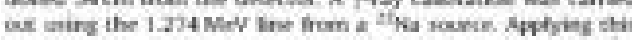

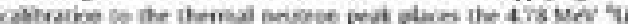

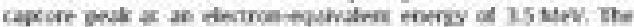

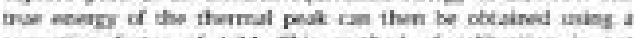

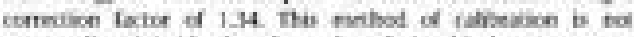

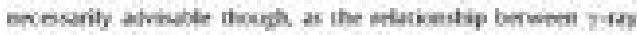

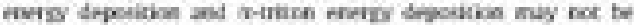

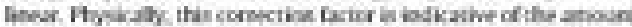

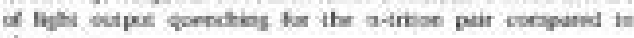
eleciroto.

\section{3. krows}

\section{1. $n / 7$ Dentrasotina}

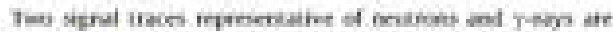

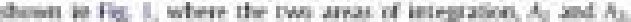

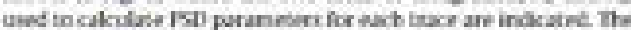

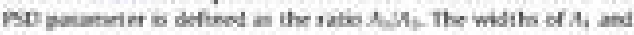

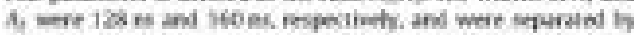

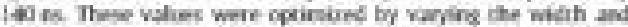

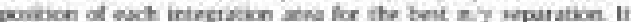

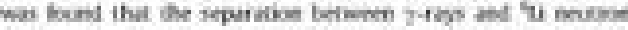

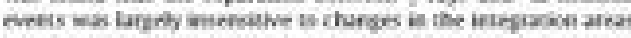
and alwas will equrated. The bade by which the gadaty a n/s

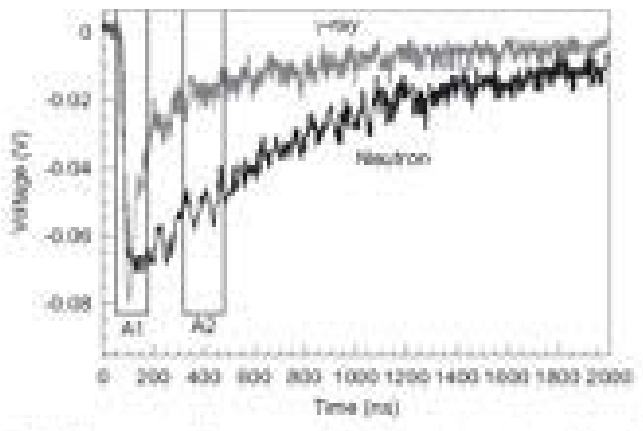

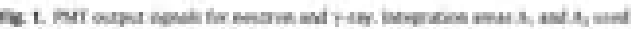

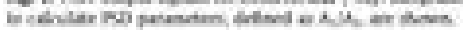




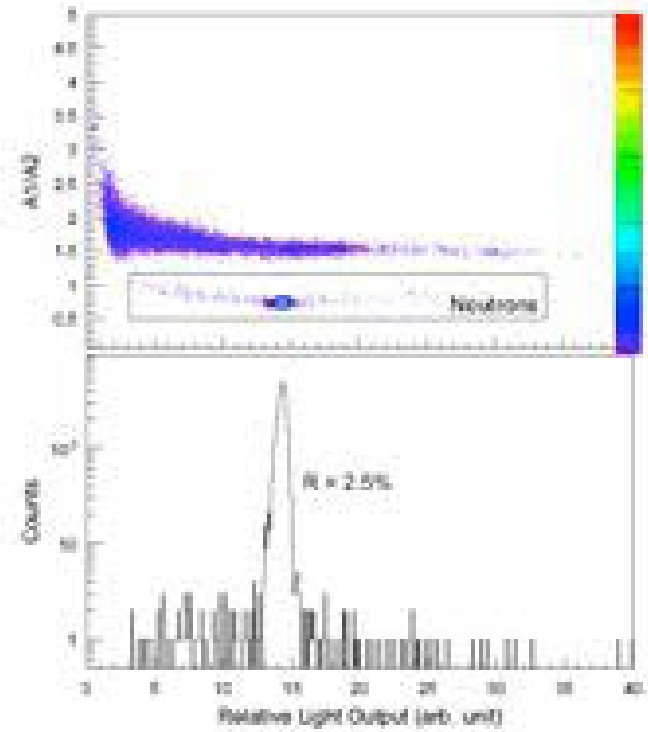

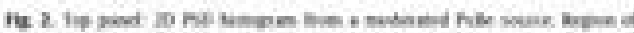

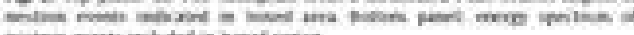

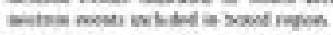

inpatation was jobled was therefoen haned os the spuration of

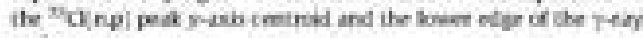
tegin.

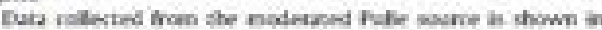
Is 2 . Insen the $2 \mathrm{D}$ hotogr am, crecan ine the clear beperition of

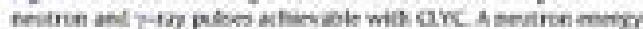

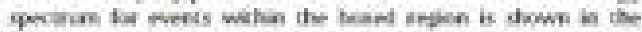

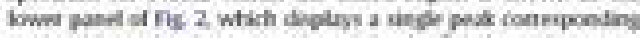

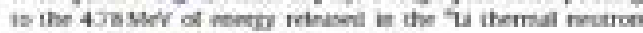
copouse. The resubsice of abs peis is 235

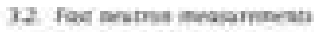

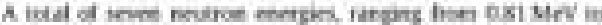

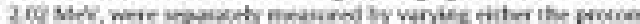

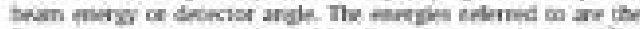

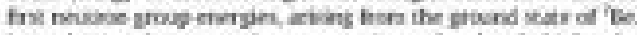
in eab hin. ite grouse bears was dowe de itrestedt is the

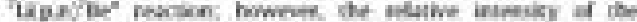

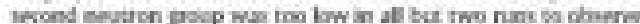

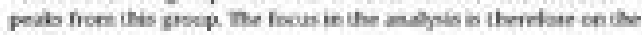

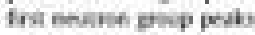

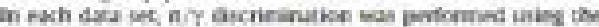

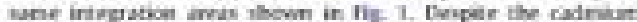

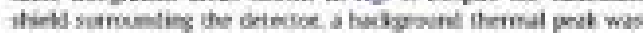

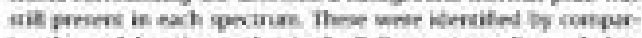
ing the peak locatioe so thut in the Puille apeciran, for each dana

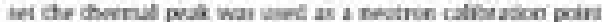

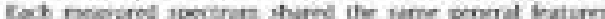

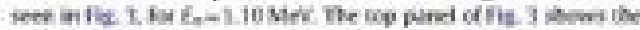

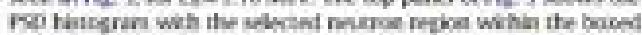

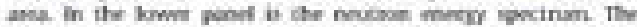

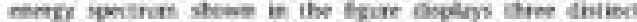

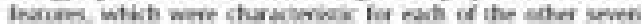

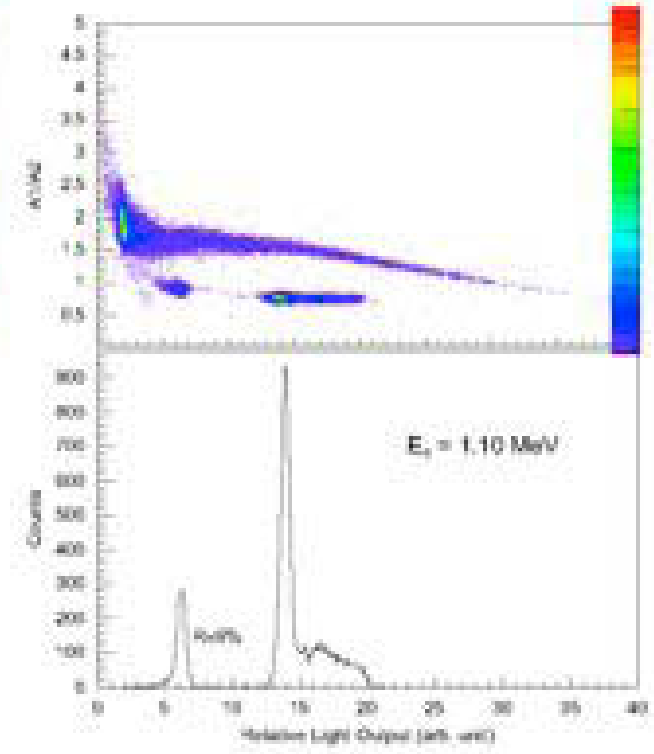

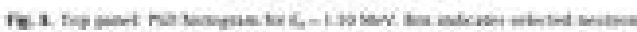

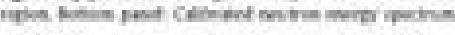

rums. The mot prominest prok were in the apectrom in the

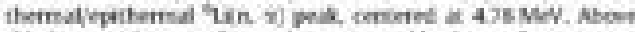

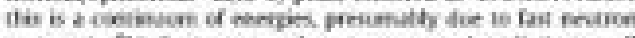

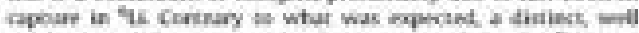

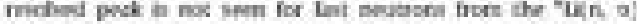
imbilin.

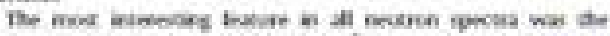

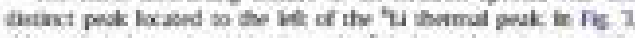

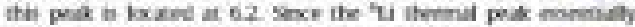

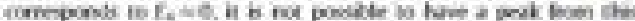

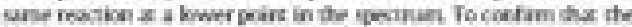

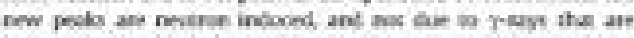

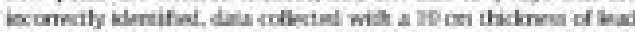

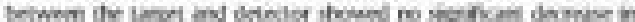

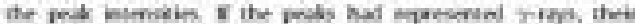

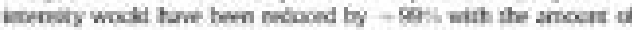

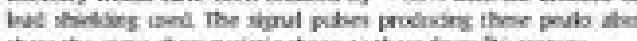

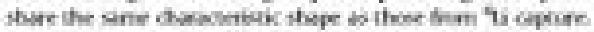

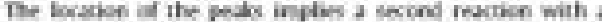

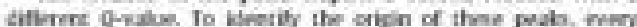

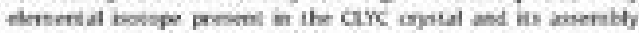

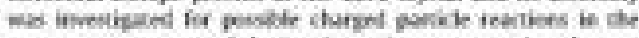

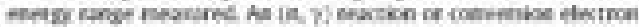

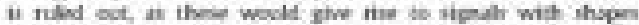

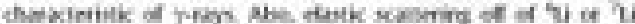

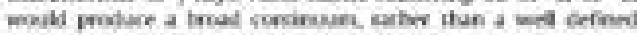

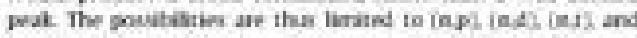

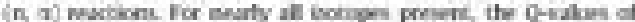

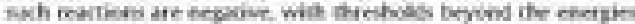

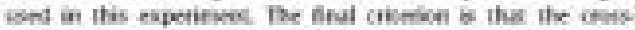

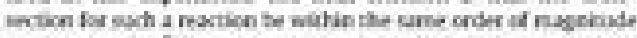
$a$ that for the $7 \mathrm{~K}, \mathrm{n}, \mathrm{z}$ ) maction, sine the ndatine namber af

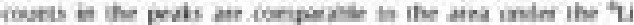
ceschunes. 


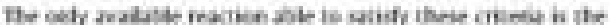

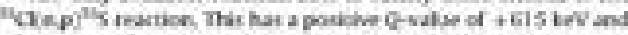

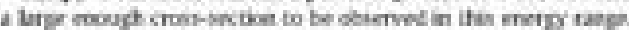

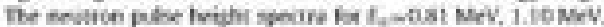

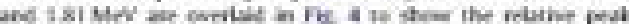

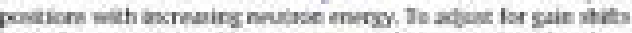

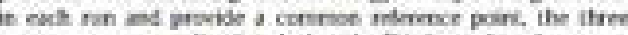

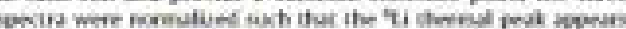

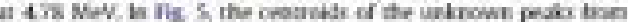

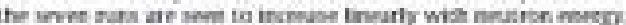

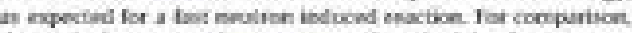

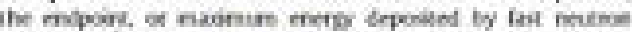

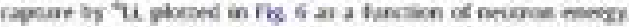

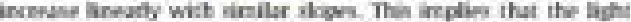

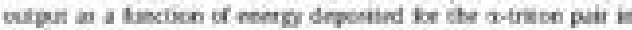

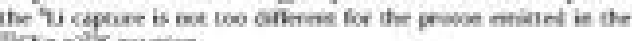
atrepils neacion

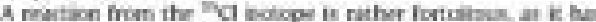

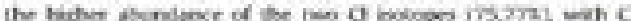

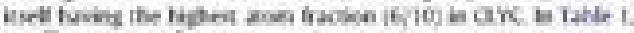

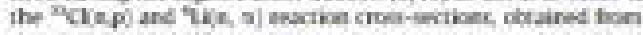

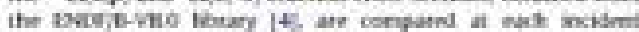

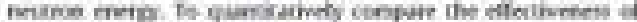

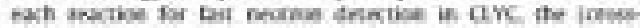

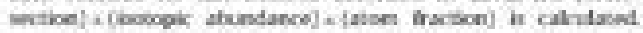

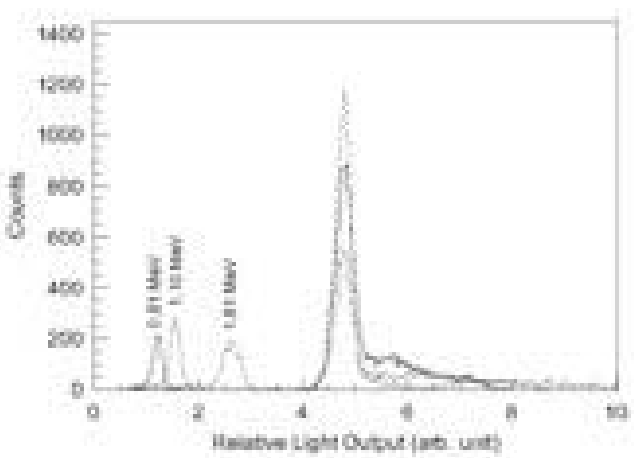

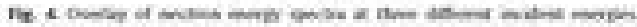

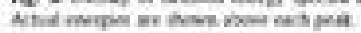

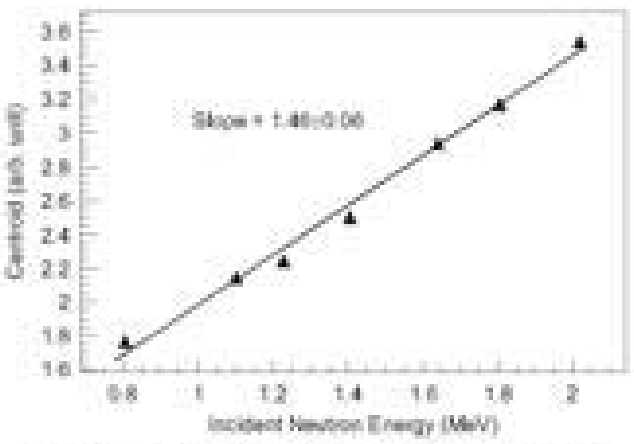

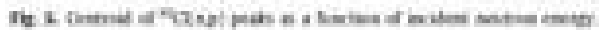

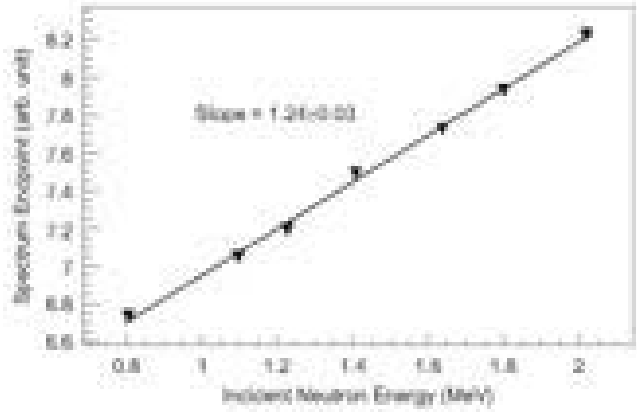

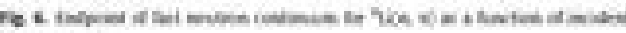
moin rown

1ate!

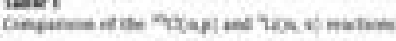

\begin{tabular}{|c|c|c|c|c|}
\hline $\begin{array}{l}\text { mans } \\
\text { mowp } \\
\text { then }\end{array}$ & 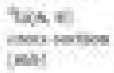 & 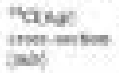 & 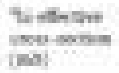 & 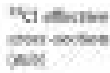 \\
\hline ex & 10 & a & $10 z$ & $20 \pi$ \\
\hline 118 & 31 & 4 & iाi & ฉที \\
\hline is & 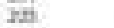 & 18 & 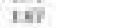 & in \\
\hline 14 & 21 & $\omega$ & 16 & sei \\
\hline is & $m$ & tis & iss & sid \\
\hline 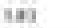 & 201 & (AII & ist & 4.16 \\
\hline$x_{00}$ & $7 \mathrm{x}$ & $w$ & is & 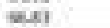 \\
\hline
\end{tabular}

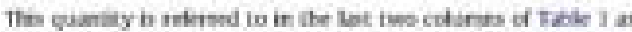

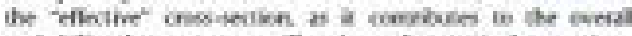

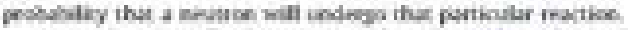

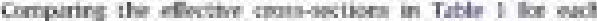

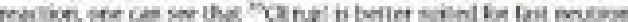

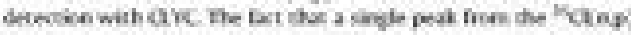
mastion it oherved in this mengr range denchatrates that CIr.

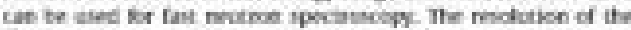

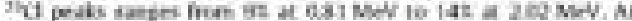

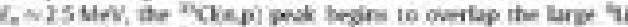

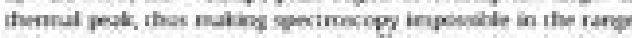

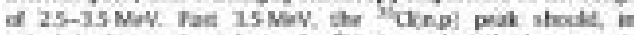

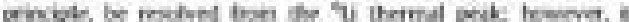

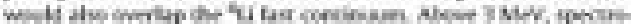

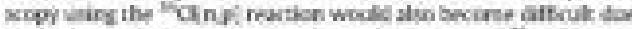

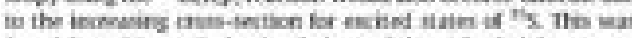

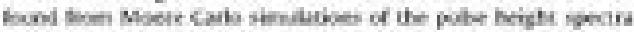

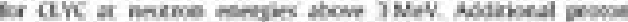

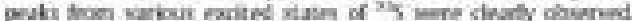

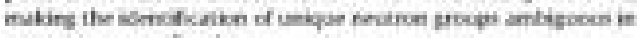
sectirecrey apdicaikes.

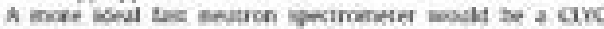

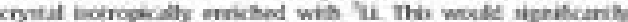

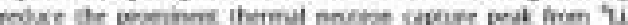

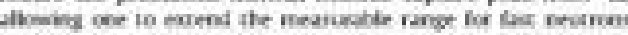

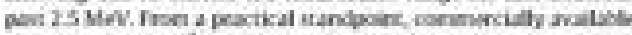

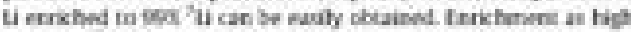

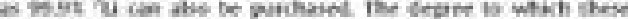

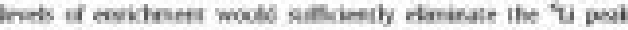

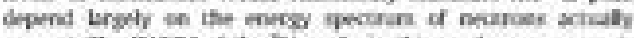

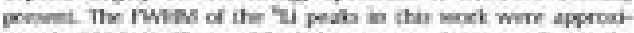

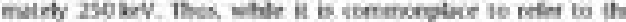

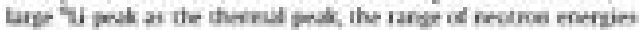




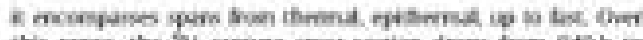

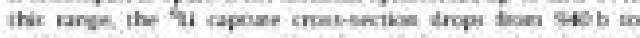
oess at is lownt potei. Nvidon, if the majokey of werts

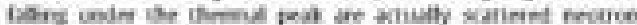

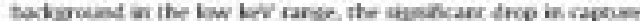

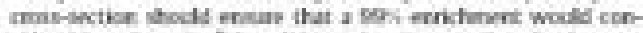

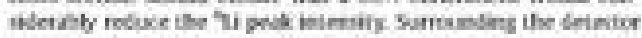

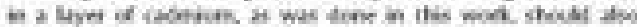

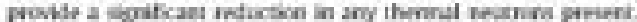

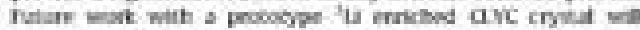
provide a mone quandiane secwest is its ethomerwis in lase thotren spocirodepy.

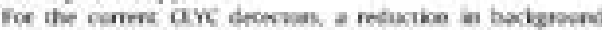

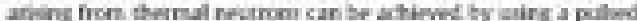

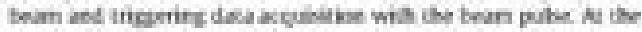

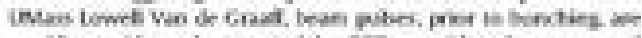

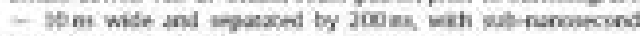
beam burtis obtairsble with a Moleby boncher.

\section{SACNOx nenatives}

To fariber werfy that the porvioully urbrown peds is ibe

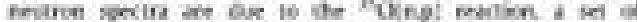

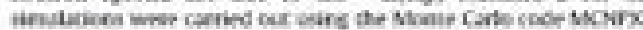

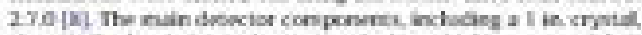

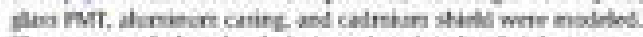

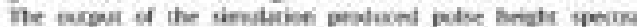

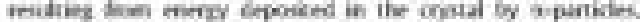

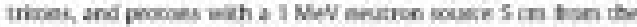

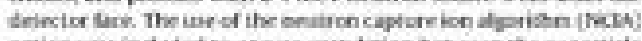

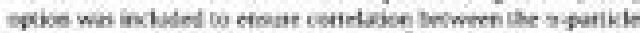

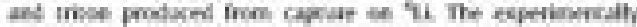

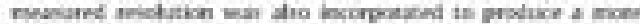
reshik sonthm.

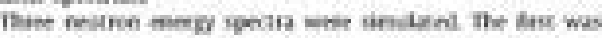

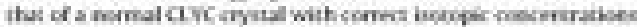

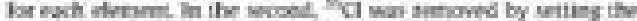

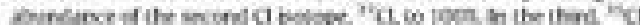

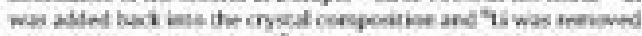

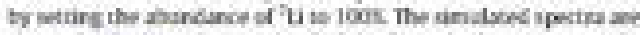

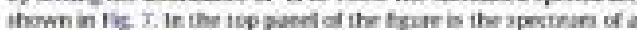

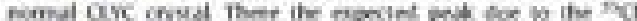

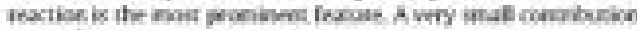

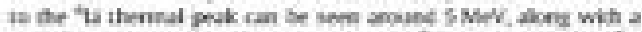

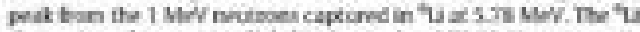

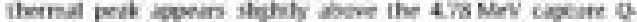

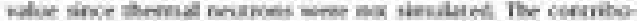
then to this pesk ane actiully from faet neuanoes whist bat a

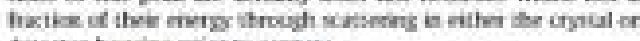
Aelecter hovies pris be cepoure.

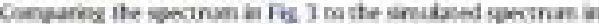

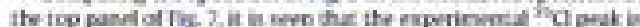

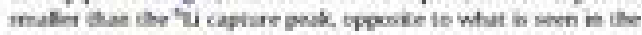

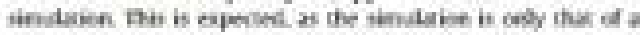

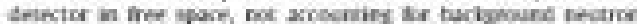

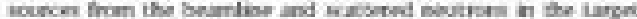

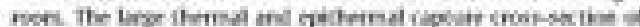

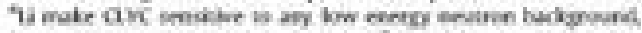

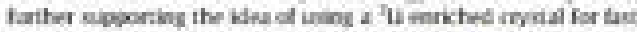

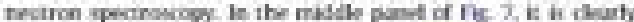

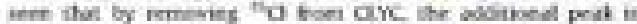

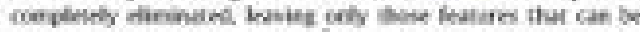

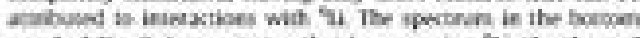

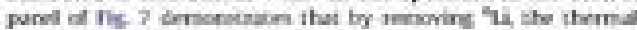

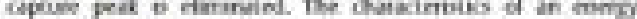

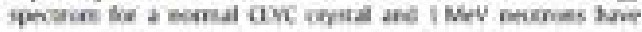

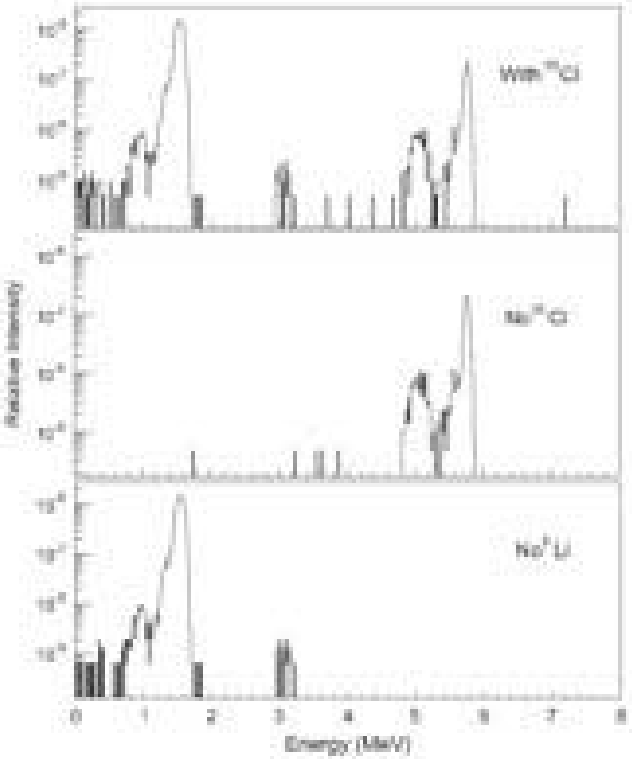

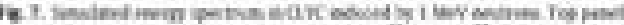

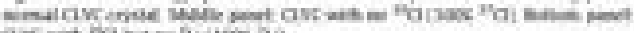

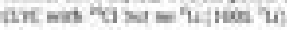

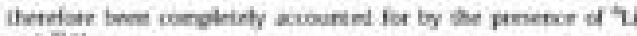
and Net

\section{I4. Nor kiomorio and calbasine}

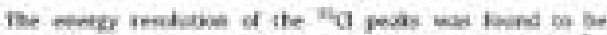

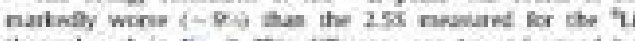

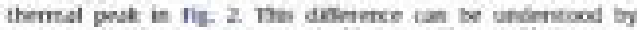

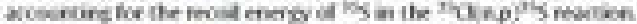

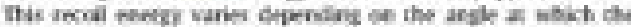

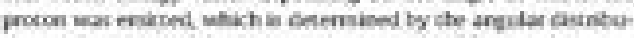

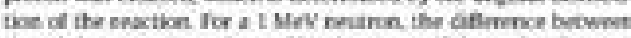

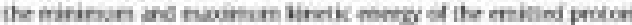

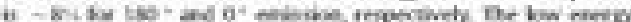

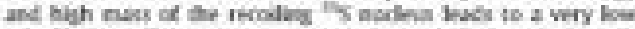

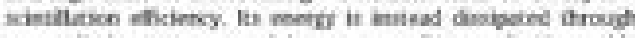

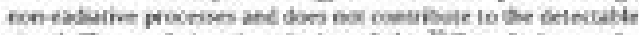

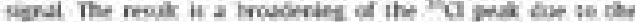

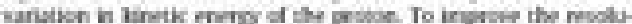

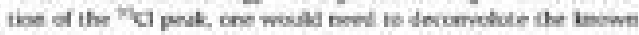

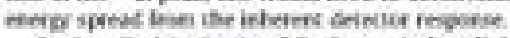

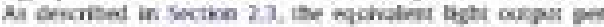
UrV in ook the curne for mass as it it for ' 4 saptum. The

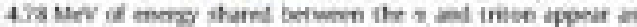

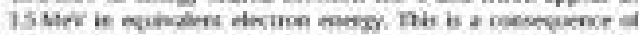
athers that kac to a sataration or qoenching of the Befe octpis:

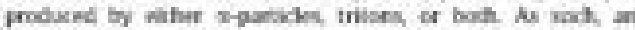

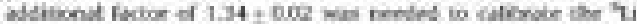

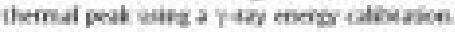

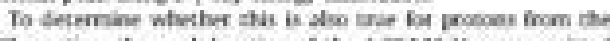

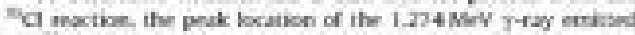

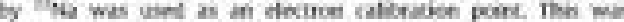

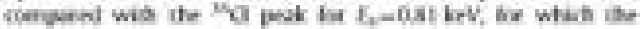




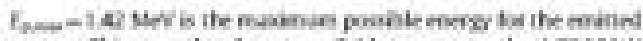

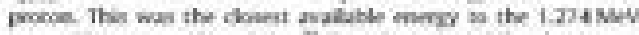

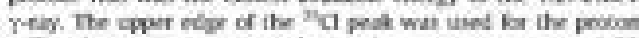

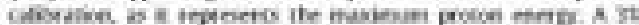

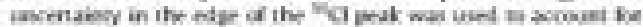
the eltwout arelation of the descice

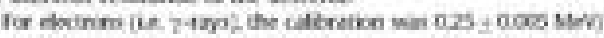

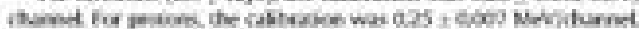

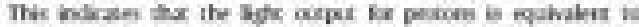

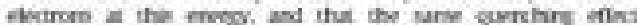

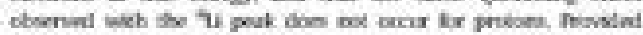

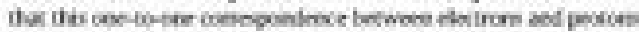

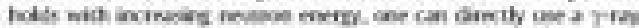

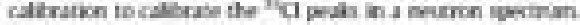

\section{4 ropese}

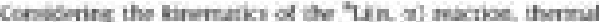

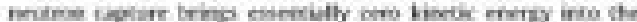

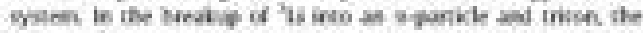

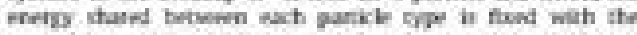

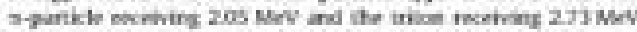

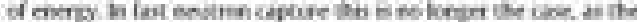

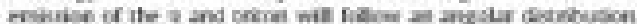

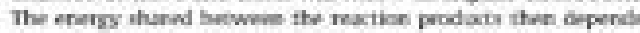

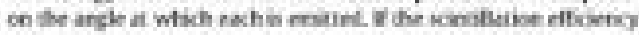

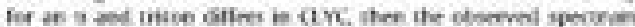

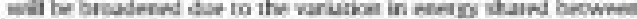

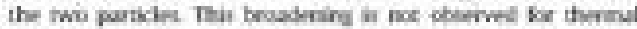

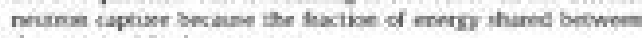
ite towe piriolos is weestare.

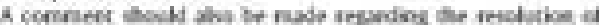

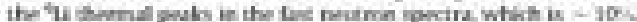

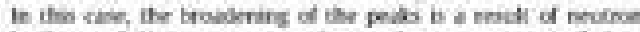

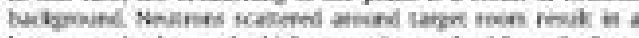

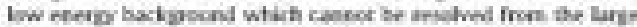

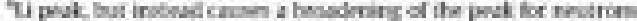
lefimen ones 160 ane 4 robev.

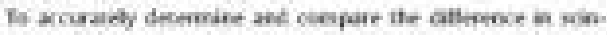
illation efficioy at a functione of mars for w-partides and

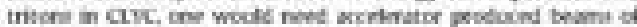

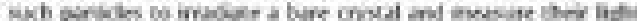

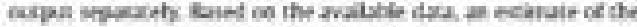
ansure of quenteks for r's and winces cas be made. To an

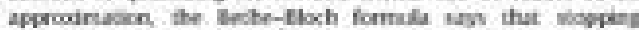

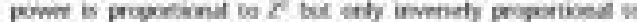

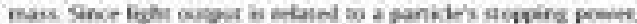

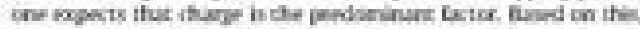
the mey anume that the bght outper protused by a tnich ti

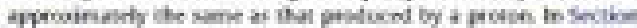

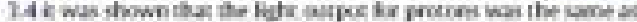
for electasors. Thas knwwieg the location of the 4 sternal peak and the energs of the a and inoen in thmnd enutrinc apture. the

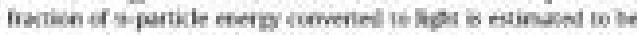

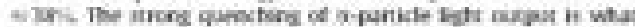

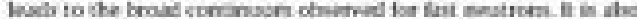

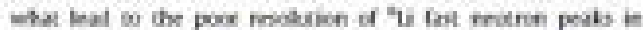

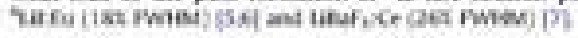

\section{6. hile ihape calporives}

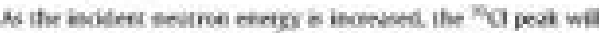

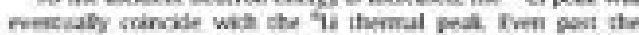
thermal pedk the mpone fanctice will becoene a coevidicien ef

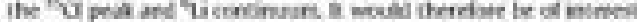

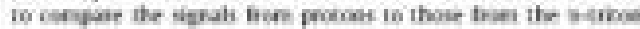

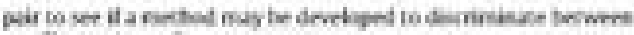

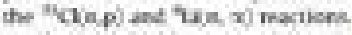

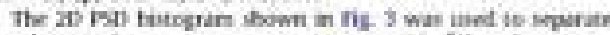

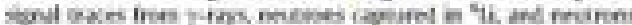

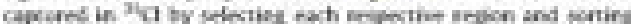

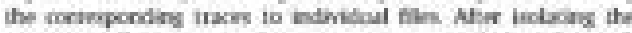
irace, stial ruts around a narrow energ wendow for that

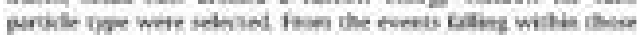

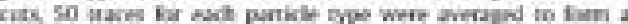

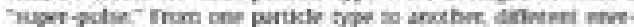

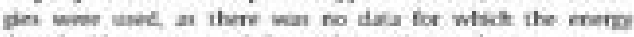

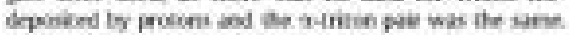

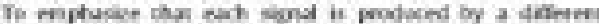

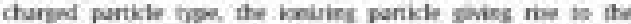

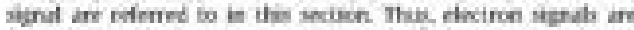

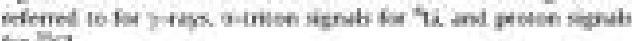
ir : Na

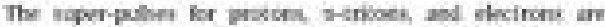

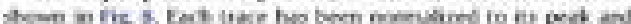

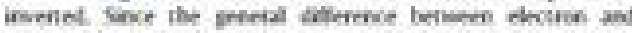

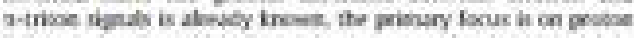

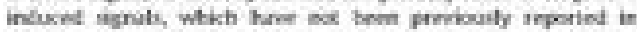

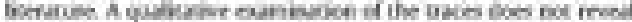

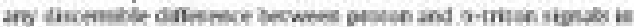
dither che rive of deray of the vigut.

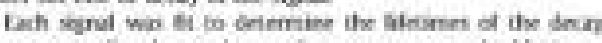

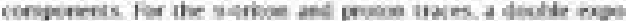

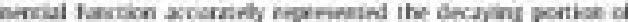

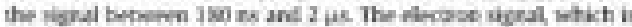

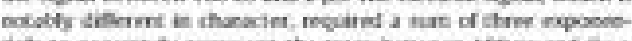

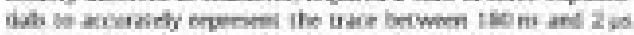

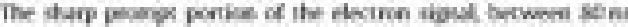

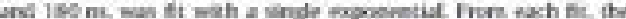

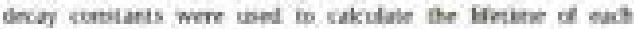

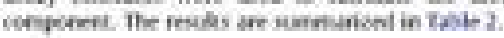

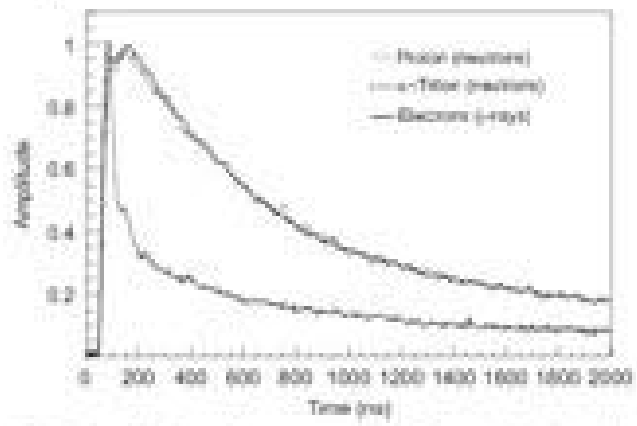

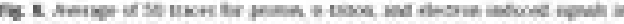
OKK livien

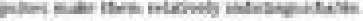

tane 2

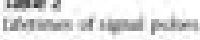

\begin{tabular}{|c|c|c|c|}
\hline Cesponeres & $\begin{array}{l}\text { Hetes } \\
\text { Inimes }\end{array}$ & $\begin{array}{l}\text { hitis } \\
\text { Onp? } \\
\text { ons }\end{array}$ & $\begin{array}{l}\text { tases } \\
\text { andice }\end{array}$ \\
\hline masel & $\operatorname{ten}$ & $=$ & * \\
\hline 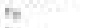 & 48 & - & $t$ \\
\hline 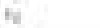 & $=16$ & $=64$ & 44 \\
\hline 6 & 13 & 203 & 320 \\
\hline
\end{tabular}




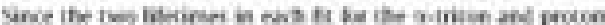
traces ane whin an coller of rusritude, theep in sisnifrank

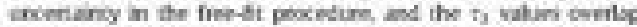

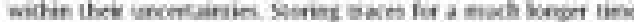

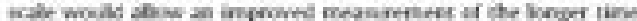

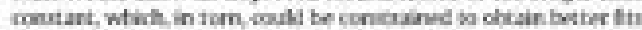
bre the shicher tiene comiant.

\section{Canclavios}

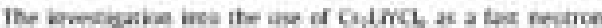

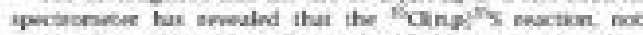

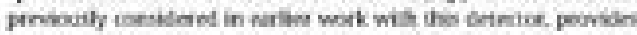

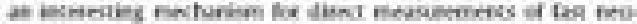

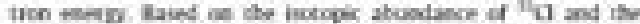

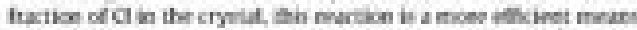

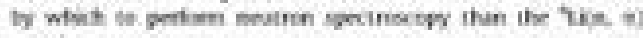
rearibe:

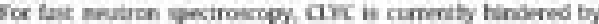

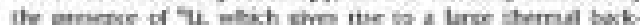

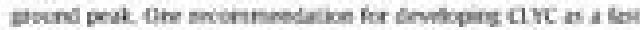

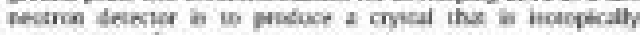

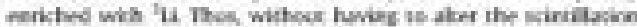

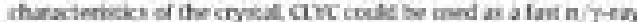

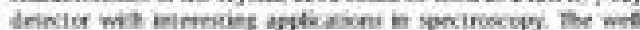

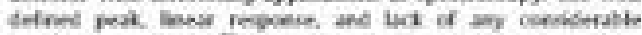

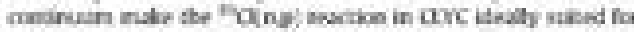

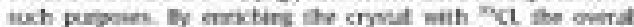

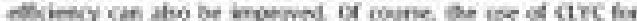

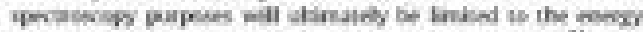

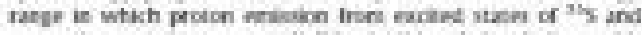

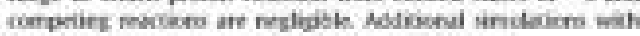

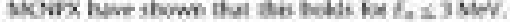

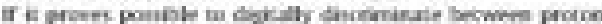

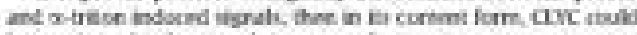

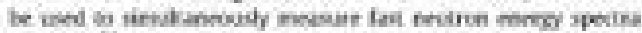

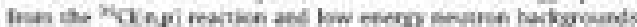

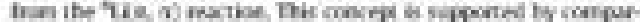

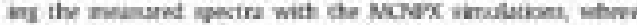

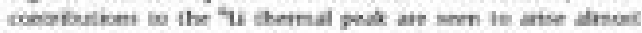

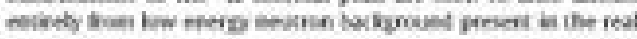

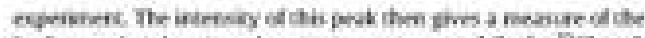

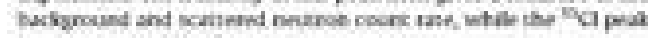
if a muanue of the fac mornse nocre.

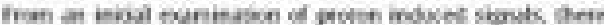

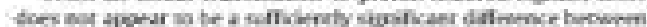

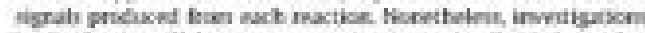
in the fatare odl focas on exirbetins moen detakd infornation

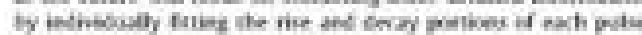
anc norgaing the neracted paramers.

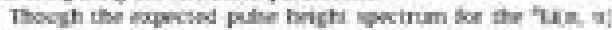

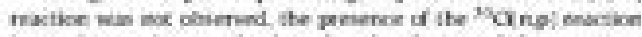

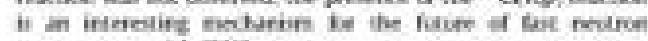
secusespy wh are

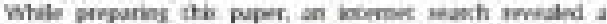

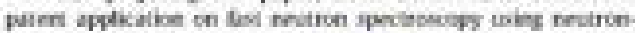

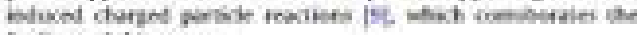
tindingi af the paper.

\section{Ackeowledyerest}

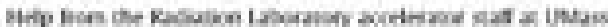
Lowel in patefidly actrowinded The werath is finded in pore

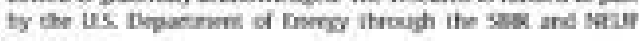
prosrims

\section{Beternen}

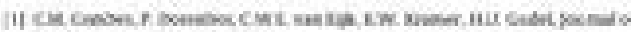

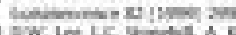

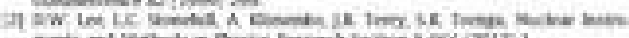

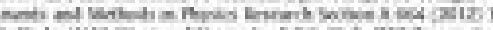

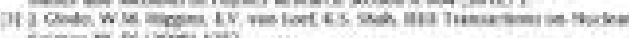

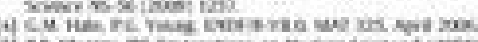

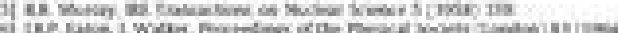
Ni

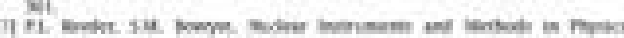

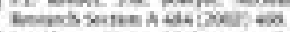

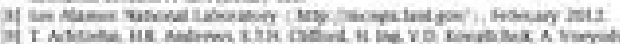

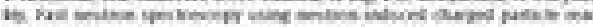

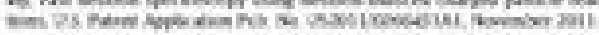




\section{Modeling Scintillator Performance (WSU)}

The initial scintillator selected is a composite that incorporate a number of unique technologies that, when integrated, provide an efficient means to measure neutrons. The scintillator composite uses LGB crystals that comprise of 10 percent by weight (3 percent by volume) of the detector head. When using a fast scintillating plastic, high energy neutrons can collide with hydrogen (proton recoil) and, to a lesser extent, carbon. Neutron capture is accomplished by the interaction of the neutrons with isotopic $\mathrm{Li} \mathrm{Gd}$, or B that process high cross-sections.

The structure of $\mathrm{Li}_{6} \mathrm{Gd}\left(\mathrm{BO}_{3}\right)_{3}$ complex consists of oxygen polyhedra of rare-earth and alkali ions connected by isolated boron-oxygen triangles into a three dimensional mixed frame work ${ }^{3}$. The unit cell for the single $\mathrm{Li}_{6} \mathrm{Gd}\left(\mathrm{BO}_{3}\right)_{3}$, is made up of both covalent and ionic bonds.

It is believed that the crystal structure and the bonding gives rise to amplification of the light that is emitted. Czochralski and Melt-Freeze techniques are used to manufacture the LGB crystals. In growing the LGB, $\mathrm{Ce}^{+3}$ is substituted for 4 percent of the $\mathrm{Gd}$ atoms in the crystal structure. It is the cerium, in the correct concentration and +3 oxidative state, which absorbs the energy of the emitted alpha produced by the neutron capture of $\mathrm{Li}, \mathrm{B}$, and $\mathrm{Gd}$ and translates it into visible light at $370-470 \mathrm{~nm}$. The LGB crystal operates as a transparent scintillator where the neutron absorption efficiency increases with increasing thickness. The reflective index is 1.66 , allowing it to be reasonably incorporated into plastics with similar reflective indexes. The detector head is composed of $0.1 \mathrm{~mm}$ shards LGB crystals uniformly dispersed in a commercially available scintillating plastic polyvinyl toluene (PVT, EJ-290).

\section{Signal Analysis and Diagnostics}

The composite scintillator is optically coupled to a 5 inch ADT Model: B133D01S Photo-Multiplier Tube. The signal from the PMT is directly linked to a Gage 14200 CompuScope Digitizer. The waveform digitizer operates at 14 bit and a sampling rate of $200 \mathrm{MS}$ (Mega-Sample)/second. The time between samples is $5 \mathrm{~ns} @ 200 \mathrm{MS} / \mathrm{s}$ with a PCl 32 Bit bus interface. The current digitizer is not capable of performing realtime acquisition, due to size of on-board memory and low data transfer rate to PC memory. Segment width for every trigger was $680 \mu \mathrm{s}$ with non-consecutive data segments acquired and saved to binary signal files (.sig) Appendix 3.2 describes the new advanced near real-time digitizers that will replace the current system.

The neutron identification (N-ID) software for this study was written to provide programmable signal analysis and diagnostic capabilities to characterize, identify, classify and associate (pair) pulse signals. Once paired, the software also estimates the background and adjusts the total counts to obtain those associated with neutrons. The software is integrated on the Lab View platform so it is capable of utilizing internal signal processing and analysis tools and also allows for programmable external mathematical functions, when necessary. A number of template parameters that are used to describe and classify the signal are discussed briefly. 
The classification process uses algorithms that correctly place each pulse in either a plastic (e.g., gamma and moderating) pulse or capture pulse category. Four distinct pulse characteristics were considered for pulse classification; namely, lambda ()ג, total area, pulse width at various percentages of pulse height, and length ratio.

Lambda is the exponential decay constant of the pulse decreasing back edge, based on the formula:

$$
\mathrm{N}=\mathrm{N}_{0} \times \mathrm{e}^{-\lambda t}
$$

Where $\mathrm{N}_{0}$ is the pulse height and $\mathrm{t}$ is the time difference between two adjacent data points on the digitized pulse decreasing edge. This equation is used to curve fit to the actual decreasing edge data points and lambda $(\lambda)$ is the constant for the exponential curve that best fits the data. When lambda is very small, e.g. $<0.02$ the pulse has a long decay and "large tail" classifying it as a capture pulse, while all other pulses having a lambda greater than 0.02 are classified as moderating pulses. The total area is simply the integrated area under the shape of the pulse. The other parameters are defined in Figure 189.

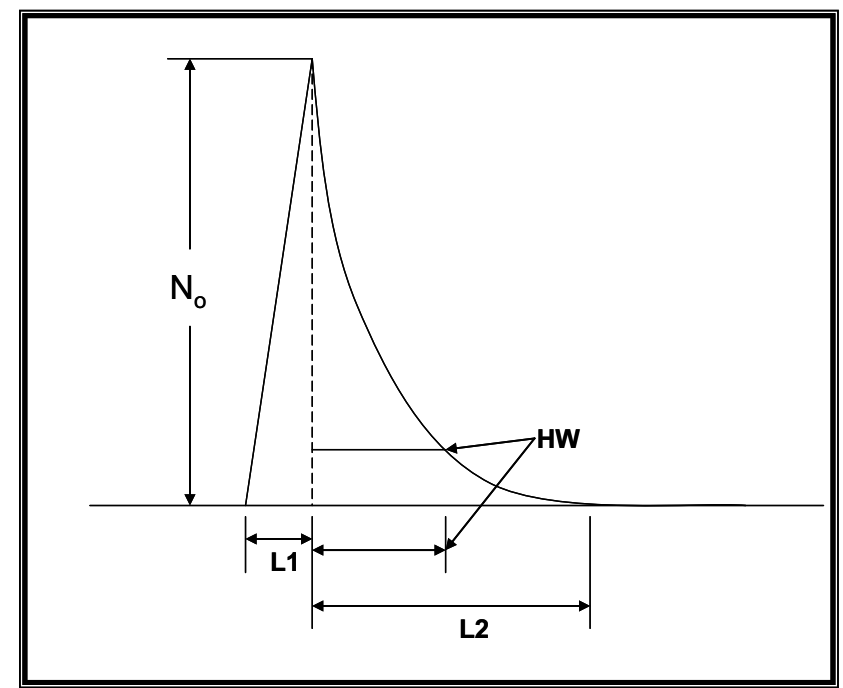

Figure 189. Pulse identification parameters.

Three types of signals may occur within the LGB scintillator. Of these, two are related to the interactions of neutrons and the third is due to gamma rays. The interaction of the fast neutrons with the plastic scintillator produces recoil protons that can be detected in the plastic by the emission of light. If the neutrons continue to slow down (moderate) in the detector volume, the probability that neutron capture will occur by $\mathrm{Li}, \mathrm{B}$, or $\mathrm{Gd}$. the moderating and the ultimate capture of the neutron will be reasonably close in time. The multi-scattering events are seen to be rapid thus resulting in a single short pulse (100 ns). The other potential event is the capture of the thermalized neutrons which results in a pulse width greater than $220 \mathrm{~ns}$. The capture of the neutron after being moderated is approximately 10 microseconds. If the recoil signal is followed by the capture signal within the prescribed time, then the identification of a neutron is likely. 
Of the paired signals, the moderating pulse contains the information to estimate the energy of the impinging neutron. Besides the sensitivity to neutrons, PVT (EJ290) also scintillates in the presence of gamma rays. The neutron capture events and gamma rays signal are quite distinctive and can be readily be identified by using PSD algorithms described to identity the salient features of the signal to a high degree of certainty to accurately classify them. In contrast, the signal from the gamma ray is very similar to the signal generated from the moderating neutron. Therefore, care must be exercised when identifying the signal produced from a gamma ray or moderating neutron.

When a plastic pulse is followed by a capture pulse, they are paired. The possible pairs are then grouped and evaluated based on time between the plastic (originating from moderating neutron or gamma ray) and the capture event. If the time difference exceeds what is expected for a legitimate moderating signal followed by capture, then it is considered a gamma ray and the pair is classified as an accidental pair. Since the gamma rays are random, it is assumed that the number of accidentals pairings for any a comparative time interval will be constant. Thus the number of accidentals (background) must be subtracted from the total pairs in the time difference of 10 microseconds as well. Elimination of the accidentals (background) from the total pairings yield the counts associated with neutrons not yet adjusted for detection efficiency.

\section{Advanced Systems Development}

\section{Spatialy Resolved Positron Analysis (SRPA)}

Advanced detector development is an integral part of the INSIGHTS program as innovative detector and measurement system designs are needed to characterize radioactive materials and material properties before, during and after irradiation. Primary detector systems under development include both direct radiation measurement systems suitable for use in high radiation fields after irradiation and materials examination systems such as the Spatially Resolved Positron Analysis (SRPA) system developed at the INL that can be used to characterize changes in strain and atomic structure before, during and after irradiation. The following sections discuss specialized radiation measurement systems and pre, during and post irradiation SRPA systems including an integrated scanning electron microscope/ SRPA system that can be used to assess materials behavior during high temperature transients and to optimize pre irradiation heat treatment effects.

\section{Pre-irradiation SRPA Materials Characterization}

SRPA is a recent INL developed measurement technology, which provides the capability to nondestructively characterize changes in atomic structure, dislocation density, and strain effects in reactor fuels and other materials. Figure 190 shows a Hot Isostatically Pressed (HIP) fuel plate and the SRPA response from this fuel plate. The SRPA data indicates significant strain effects and defect concentrations in the outer area of the plate with lower concentrations of defects in the surrogate fuel portion of the plate. (The center of the plate produces a SRPA response similar to virgin aluminum alloy.) The SRPA data suggest that fuel failures during irradiation 
may have likely resulted from differential strain effects from the outer section of fuel plates relative to the fueled central section of the plate and likely occurred at the interface between the high and low strain regions of the plate. The SRPA system allows direct characterization of changes in atomic structure including strain and dislocation density in the material with a precision of $0.1 \%$.

The two primary pre-irradiation SRPA systems that have been developed are a Doppler broadening fuel plate scanner and an integrated positron lifetime/coincidence Doppler broadening measurement system. Figure 191 a) shows the SRPA plate scanner and Figure $191 \mathrm{~b}$ ) shows the integrated system. The fuel plate scanner was developed for use on large $(15 \mathrm{~cm} \times 100 \mathrm{~cm})$ fuel plates prior to irradiation to assess fabrication-induced strain and defects in the fuel plates. The integrated positron life time/coincidence Doppler broadening measurement systems provide additional information on defect characteristics and the atomic structure of fuel material being examined beyond that provided by the plate scanner.

The SRPA pre-irradiation measurement systems allow direct evaluation of cladding and fuel plate characteristics prior to irradiation that can be used for comparison with SRPA measurement responses that will be performed post irradiation and possibly during pauses in irradiation.

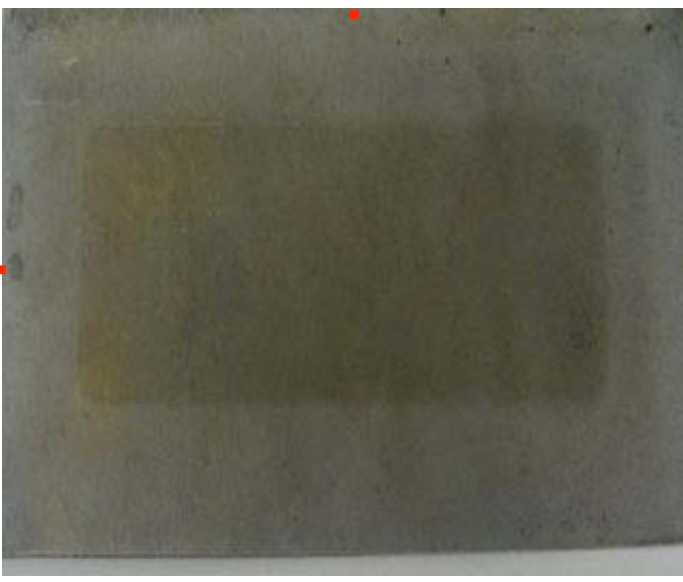

Figure 190. a) Sandwich Fuel Plate Surrogate

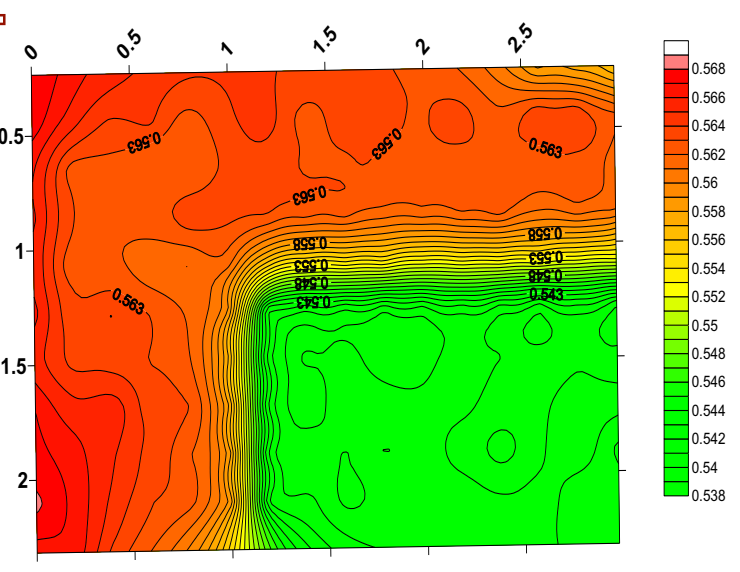

b) SRPA Response 


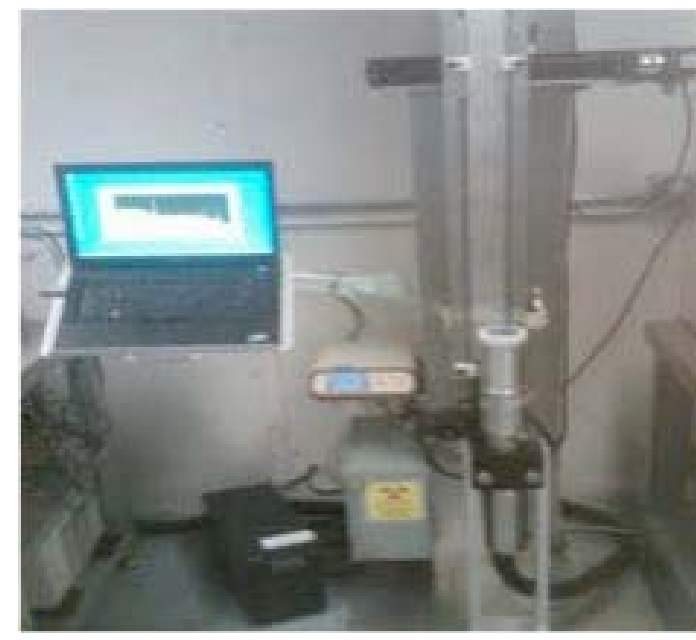

Figure 191. a) SRPA Plate Scanner

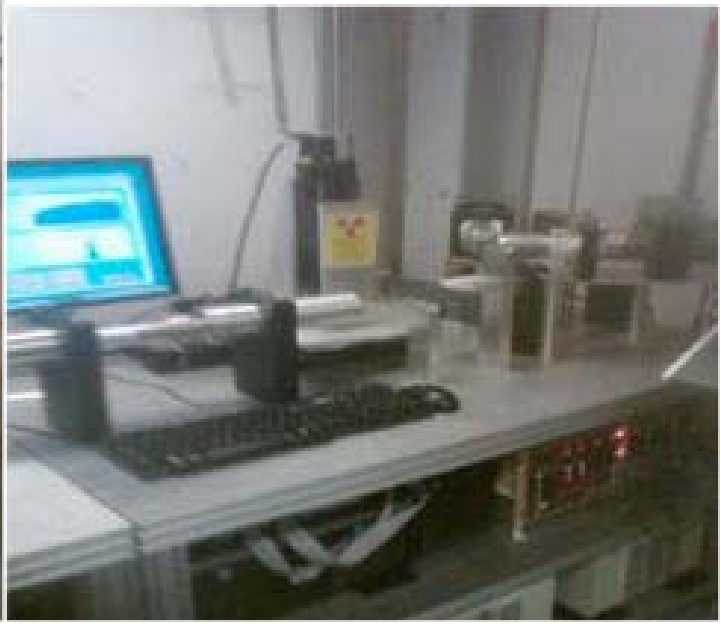

b) SRPA Coincidence Lifetime Scanner

\section{Irradiation and Post Irradiation SRPA Materials Characterization}

SRPA measurement systems are being developed to assess the properties of cladding and fuel materials following irradiation testing and possibly during pauses in the irradiation testing. These systems are primarily designed for use in a shielded cave or hotcell environment. Radiation hardened SRPA scanning systems have been developed that can be placed in a hotcell environment and used to perform measurements in cell. Figure 192 shows the conceptual design of a hotcell-based SRPA system with the detector mounted in the hotcell wall.

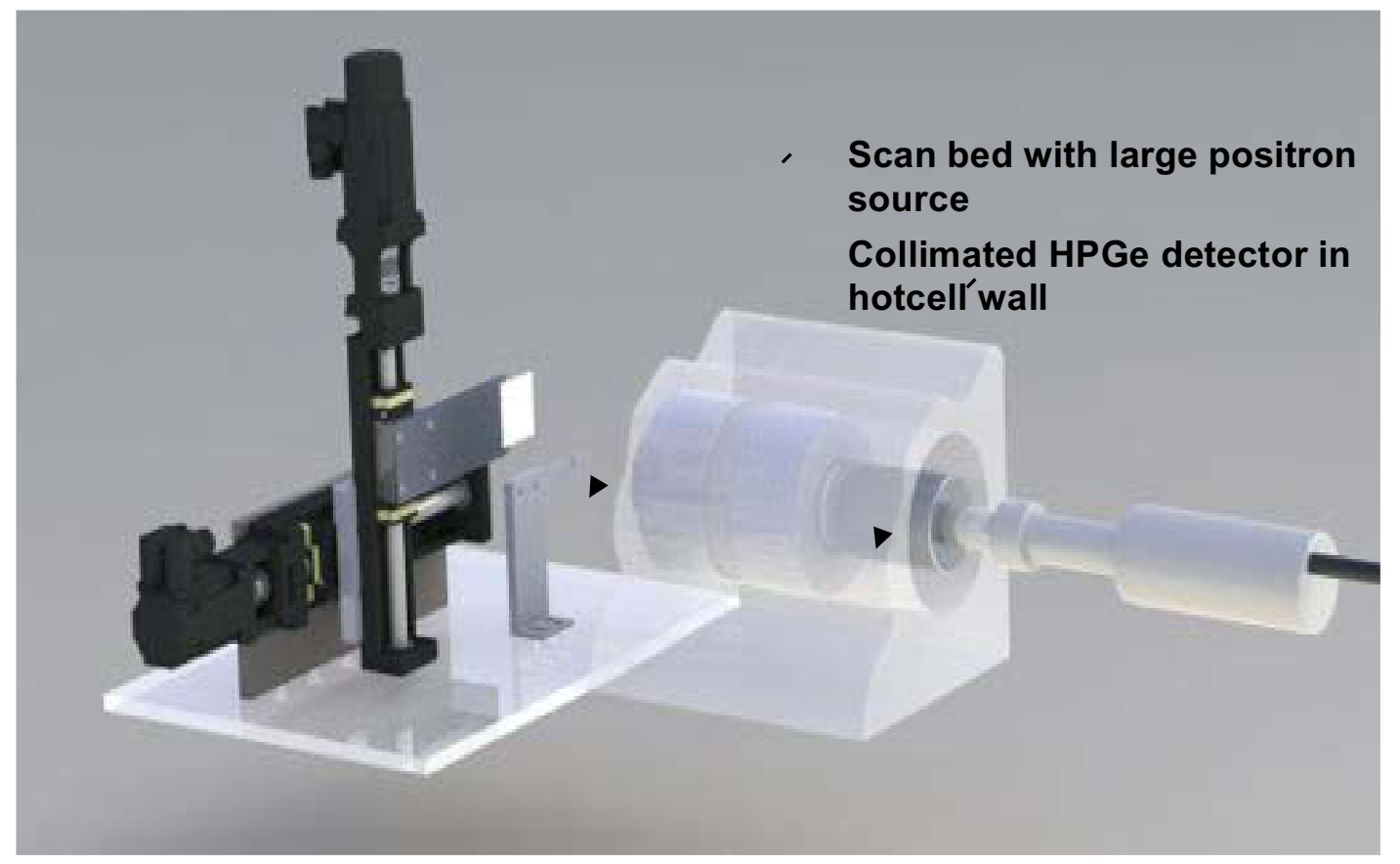

Figure 192. Hotcell SRPA System 
It is expected that similar detector system designs will be used to characterize fuels during pauses in the irradiation cycle using in-situ decay-based positron production or large positron sources similar to the hotcell-based system. The SRPA technology can provide a prognostic measurement capability for irradiated specimens to assess changes in microstructure during materials aging and degradation, and as part of radiation effects studies to better understand induced changes in reactor materials during irradiation.

In addition to the INSIGHTS efforts to develop high radiation field SRPA measurement systems, work is being performed as part of a collaboration with GE Global Nuclear Fuels to develop specialized SRPA systems that can be used to characterize irradiated fuel characteristics and cladding interactions such as hydride formation and particle cladding interactions. A specific goal of this program is to utilize the SRPA technology for ziracaloy cladding life extension issues related to hydride formation.

\section{Integrated SEM/SRPA High Temperature System}

An integrated SEM/SRPA measurement system is being developed to allow positron scanning measurements to be performed on reactor fuel materials during high temperature transients and to assess the effects of varying heat treatments on materials. A Semtech model 1645 SEM is being modified to incorporate a high temperature stage (up to $1200^{\circ} \mathrm{C}$ ) and a SRPA measurement system that can be used to characterize thermally induced changes in the atomic structure of materials during thermal transients. In addition, the more sensitive SRPA measurement data can be cross correlated with the SEM back scattered electron (BSE) and other SEM data to assess induced changes in fuel and cladding material properties as a function of temperature, which is not detectable using standard SEM capabilities. A critical advantage of the SRPA technology is that it can be used to monitor material property changes during the heat up to melting, through the liquid state and during cool down. The combined SEM/SRPA technology also has an added capability in that it can be used to directly monitor heat treatment effects in materials and can be used to optimize heat treatments by quantifying the strain and structural changes in the material during the heat treatment process.

\section{Management}

The NIFFTE university collaborators are funded directly by the Advanced Fuel Campaign of the FCT Program. The PI of this contract has reporting responsibilities directly to the Technical Point of Contact at INL. The laboratories are being funded directly by the FCT and ARC programs to not only participate but to provide guidance and project oversight, including reporting requirements within the DOE/NE management system. 


\section{References}

[1] "Observation of resonances in ${ }^{239} \mathrm{Pu}(\mathrm{n}, \alpha)$ reactions", Cole, J.D. and M.W. Drigert, Physical Review C, 79 (2009) (017603): p.3.

[2] M. Haupt, A. Ladenburger, R. Sauer, K. Thonke, R. Glass, W. Roos, J. P. Spatz, H. Rauscher, S. Riethmüller, M. Möller, "Ultraviolet-emitting ZnO Nanowhiskers Prepared by a Vapor Transport Process on Prestructured Surfaces with Selfassembled Polymers", J. Appl. Phys. 93, 6252 (2003).

[3] V. Avrutin, G. Cantwell, J. Zhang, J.J. Song, D.J. Silversmith, H. Morkoç, "Bulk ZnO: Current Status, Challenges, and Prospects", Proceedings of the IEEE 98, 1339 (2010).

[4] S. Wang, "Method and Apparatus for Zinc Oxide Single Boule Growth", U.S. Patent 7279040B1 United States of America, issued October 9, 2007

[5] C. R. Gorla, N. W. Emanetoglu, S. Liang, W. E. Mayo, and Y. Lu, "Structural, Optical, and Surface Acoustic Wave Properties of Epitaxial ZnO Films Grown on (012) Sapphire by Metalorganic Chemical Vapor Deposition", J. Appl. Phys. 85, 2595 (1999).

[6] J. Nause, "ZnO broadens the spectrum", III-Vs Review 12, 28 (1999).

[7] E. Ohshima, H. Ogino, I. Niikura, K. Maeda, M. Sato, M. Ito, T. Fukuda, "Growth of the 2-in-size Bulk ZnO Single Crystals by the Hydrothermal Method", J. Cryst. Growth 260, 166 (2004).

[8] D. F. Croxall, R. C. C. Ward, C. A. Wallace, R. C. Kell, "Hydrothermal Growth and Investigation of Li-doped Zinc Oxide Crystals of High Purity and Perfection", J. Cryst. Growth 22, 117 (1974).

[9] M.J. Suscavage, M. Harris, D. Bliss, P. Yip, S.-Q. Wang, D. Schwall, L. Bouthillette, J. Bailey, M. Callahan, D.C. Look, D.C. Reynolds, R.L. Jones, and C.W. Litton, "High Quality Hydrothermal ZnO Crystals", MRS Internet J. Nitride Semicond. Res. 4, G3.40 (2004)

[10] M. Razeghi, "The MOCVD Challenge: Volume 2: A Survey of GaInAsP-GaAs for Photonic and Electronic Device Applications", CRC Press, 1995.

[11] A.N. Jansen, M.E. Orazem, B.A. Fox, W.A. Jesser, "Numerical Study of the Influence of Reactor Design on MOCVD with a Comparison to Experimental Data", J. of Crystal Growth 112, 316 (1991).

[12] A. Gurary, P.T. Fabiano, D.R. Voorhees, S. Beherrell, "Induction Heated Chemical Vapor Deposition Reactor." U.S. Patent 6368404 B1, issued April 9, 2002.

[13] B. P. Zhang, N. T. Binh, K. Wakatsuki, Y. Segawa, Y. Yamada, N. Usami, M. Kawasaki, and H. Koinuma, "Pressure-Dependent ZnO Nanocrystal Growth in a Chemical Vapor Deposition Process", J. Phys. Chem. B 108, 10899 (2004).

[14] R. Tena-Zaera, J. Zuniga-Perez, C. Marti'nez-Tomas, V. Munoz-Sanjose, "Numerical Study of the ZnO Growth by MOCVD", J. Cryst. Growth 264, 237 (2004). 
[15] K.F. Jensen, D.I. Fotiadis and T.J. Mountziaris, "Detailed Models of the MOVPE Process", J. Cryst. Growth 107, 1 (1991)

[16] Y. Liu, C. R. Gorla, S. Liang et al., "Ultraviolet Detectors Based on Epitaxial ZnO Films Grown by MOCVD," Journal of Electronic Materials 29, 69 (2000)

[17] W. Xu, Z. Ye, T. Zhou, B. Zhao, L. Zhu and J. Huang, "Low-pressure MOCVD Growth of p-type ZnO Thin Films by using NO as the Dopant Source", J. Cryst. Growth 265, 133 (2004)

[18] S. Nakamura, "Analysis of Real-Time Monitoring Using Interference Effects", Jpn. Jour. Appl. Phys. 30, 1348 (1991)

[19] J.M. Olson, A. Kibbler, "In Situ Characterization of MOCVD Growth Processes by Light Scattering Techniques”, J. Cryst. Growth 77, 182 (1986).

[20] Harry Moffat, Klavs F. Jensen, "Complex Flow Phenomena in MOCVD Reactors: I. Horizontal Reactors", J. Cryst. Growth 77, 108 (1986)

[21] A. Ohtomo, R. Shiroki, I. Ohkubo, H. Koinuma, M. Kawasaki, "Thermal Stability of Supersaturated $\mathrm{Mg}_{\mathrm{x}} \mathrm{Zn}_{1-\mathrm{x}} \mathrm{O}$ Alloy Films and $\mathrm{Mg}_{\mathrm{x}} \mathrm{Zn}_{1-\mathrm{x}} \mathrm{O} / \mathrm{ZnO}$ Heterointerfaces", Appl. Phys. Lett. 75, 4088 (1999).

[22] S.J. Lim, Soonju Kwon, H. Kim, "ZnO Thin Films Prepared by Atomic Layer Deposition and RF Sputtering as an Active Layer for Thin Film Transistor", Thin Solid Films 516, 1523 (2008)

[23] S. M. George, A. W. Ott, and J. W. Klaus, "Surface Chemistry for Atomic Layer Growth", J. Phys. Chem. 100, 13121 (1996)

[24] T. Williams and C. Kelly, "GNUplot, An Interactive Plotting Program", (2010).

[25] S. Nakamura, The blue laser diode: GaN based light emitters and lasers. Berlin: Springer, 1997.

[26] R. Stevenson. (2011) GaN HEMTs: faster, more capable and better understood. Compound Semiconductor. 21-23.

[27] T. Chung, J. Limb, J.-H. Ryou, W. Lee, P. Li, D. Yoo, X.-B. Zhang, S.-C. Shen, R. D. Dupuis, D. Keogh, P. Asbeck, B. Chukung, M. Feng, D. Zakharov, and Z. LilienthalWeber, "Growth of InGaN HBTs by MOCVD," Journal of Electronic Materials, vol. 35, p. 695, 2006.

[28] B.-T. Liou, S.-H. Yen, and Y.-K. Kuo, "Vegard's law deviation in band gaps and bowing parameters of the wurtzite III-nitride ternary alloys," Proceedings of the SPIE, vol. 5628, p. 296, 2005.

[29] L. F. Eastman and U. K. Mishra. (2002) The Toughest Transistor Yet. IEEE Spectrum. 28.

[30] H. Y. Xiao, F. Gao, X. T. Zu, and W. J. Weber, "Threshold displacement energy in GaN: Ab initio molecular dynamics study," Journal of Applied Physics, vol. 105, p. 123527, 2009. 
[31] D. C. Look, D. C. Reynolds, J. W. Hemsky, J. R. Sizelove, R. L. Jones, and R. J. Molnar, "Defect Donor and Acceptor in GaN," Physical Review Letters, vol. 79, p. 2273, 1997.

[32] A. Ionascut-Nedelcescu, C. Carlone, A. Houdayer, H. J. von Bardeleben, J. L. Cantin, and S. Raymond, "Radiation hardness of gallium nitride," IEEE Transactions on Nuclear Science, vol. 49, pp. 2733-8, 2002.

[33] J. Nord, K. Nordlund, J. Keinonen, and K. Albe, "Molecular dynamics study of defect formation in GaN cascades," Nuclear Inst. and Methods in Physics Research, B, vol. 202, pp. 93-99, 2003.

[34] S. H. Park, T. W. Kang, and T. W. Kim, "p-Type conversion of Si-doped ntype GaN epilayers due to neutron transmutation doping and annealing," Journal of Materials Science, vol. 39, pp. 6353-5, 2004.

[35] K. Kuriyama, Y. Mizuki, H. Sano, A. Onoue, K. Kushida, M. Okada, M. Hasegawa, I. Sakamoto, and A. Kinomura, "Lattice disorder in neutron irradiated GaN: Nuclear reaction analysis and Rutherford backscattering studies," Nuclear Instruments \&amp; Methods in Physics Research, Section B (Beam Interactions with Materials and Atoms), vol. 249, pp. 132-5, 2006.

[36] J. G. Marques, K. Lorenz, N. Franco, and E. Alves, "Defect productin in neutron irradiated GaN," Nuclear Instruments and Methods in Physics Research Section B: Beam Interactions with Materials and Atoms, vol. 249, pp. 358-361, 2006.

[37] J. Grant, R. Bates, W. Cunningham, A. Blue, J. Melone, F. McEwan, J. Vaitkus, Gaubas, and V. O'Shea, "GaN as a radiation hard particle detector," Nuclear Instruments \&amp; Methods in Physics Research, Section A (Accelerators, Spectrometers, Detectors and Associated Equipment), vol. 576, pp. 60-5, 2007.

[38] A. Y. Polyakov, N. B. Smirnov, A. V. Govorkov, A. V. Markov, N. G. Kolin, D.Merkurisov, V. M. Boiko, K. D. Shcherbatchev, V. T. Bublik, M. I. Voronova, H. Lee, C. R. Lee, S. J. Pearton, A. Dabirian, and A. V. Osinsky, "Fermi level pinning in heavily neutron-irradiated GaN," Journal of Applied Physics, vol. 100, pp. 93715-1, 2006.

[39] A. Y. Polyakov, N. B. Smirnov, A. V. Govorkov, A. V. Markov, N. G. Kolin, D. Merkurisov, V. M. Boiko, K. D. Shcherbatchev, V. T. Bublik, M. I. Voronova, J. Pearton, A. Dabiran, and A. V. Osinsky, "Neutron irradiation effects in p-GaN," Journal of Vacuum Science \&amp; Technology B (Microelectronics and Nanometer Structures), vol. 24, pp. 2256-61, 2006.

[40] A. Y. Polyakov, N. B. Smirnov, A. V. Govorkov, A. V. Markov, S. J. Pearton, N. Kolin, D. I. Merkurisov, V. M. Boiko, L. Cheul-Ro, and L. In-Hwan, "Fast neutron irradiation effects in n-GaN," Journal of Vacuum Science \&amp; Technology B (Microelectronics and Nanometer Structures), vol. 25, pp. 436-42, 2007.

[41] T. Baghdadli, S. O. S. Hamady, S. Gautier, T. Moudakir, B. Benyoucef, and A. Ougazzaden, "Electrical and structural characterizations of BGaN thin films grown by metal-organic vapor-phase epitaxy," physica status solidi c, vol. 6, pp. S1029S1032, 2009. 
[42] S. Gupta, A. Melton, E. Malguth, W. E. Fenwick, T. Zaidi, H. Yu, and I. T. Ferguson, "Rare earth doping of GaN with gadolinium by MOCVD," MRS Proceedings, vol. 1111, p. 71, 2009.

[43] Y. K. Zhou, S. W. Choi, S. Emura, S. Hasegawa, and H. Asahi, "Large magnetization in high Gd concentration GaGdN and Si-doped GaGdN grown at low temperatures," Applied Physics Letters, vol. 92, p. 062505, 2008.

[44] B. Witek, A. Wysmolek, M. Kaminska, A. Twardowski, M. Potemski, M. Bockowski, and I. Grzegory, "Time-Resolved Studies of Gallium Nitride Doped with Gadolinium," Acta Physica Polonica A, vol. 114, p. 1425, 2008.

[45] R. P. Davies, B. P. Gila, C. R. Abernathy, S. J. Pearton, and C. J. Stanton, "Defect-enhanced ferromagnetism in Gd- and Si-coimplanted GaN," Applied Physics Letters, vol. 96, p. 212502, 2010.

[46] W. Utsumi, H. Saitoh, H. Kaneko, T. Watanuki, K. Aoki, and O. Shimomura, "Congruent melting of gallium nitride at $6 \mathrm{GPa}$ and its application to single-crystal growth," Nature Materials, vol. 2, p. 735, 2003.

[47] R. Stevenson. (2010) The World's Best Gallium Nitride. IEEE Spectrum. 40.

[48] V. Avutrin, D. J. Silversmith, Y. Mori, F. Kawamura, Y. Kitaoka, and H. Morkoc, "Growth of Bulk GaN and AlN: Progress and Challenges," Proceedings of the IEEE, vol. 98, p. 1302, 2010.

[49] R. Dwilinski, R. Doradzinski, J. Garczynski, L. Sierzputowski, R. Kucharski, M. Zajac, M. Rudzinski, R. Kudrawiec, J. Serafinczuk, and W. Strupinski, "Recent achievements in AMMONO-bulk method," Journal of Crystal Growth, vol. 312, 2499, 2010.

[50] I. Akasaki, H. Amano, Y. Koide, K. Hiramatsu, and N. Sawaki, "Effects of AlN Buffer Layer on Crystallographic Structure and on Electrical and Optical Properties of GaN and Ga1-xAlxN $(0<x<0.4)$ Films Grown on Sapphire Substrate by MOVPE," Journal of Crystal Growth, vol. 98, pp. 209-219, 1989.

[51] S. Nakamura, "GaN Growth Using GaN Buffer Layer," Japanese Journal of Applied Physics, vol. 30, pp. L1705-L1707, 1991.

[52] M. A. Moram, C. S. Ghedia, D. V. S. Rao, J. S. Barnard, Y. Zhang, M. J. Kappers, and C. J. Humphreys, "On the origin of threading dislocations in GaN films," Journal of Applied Physics, vol. 106, p. 0735513, 2009.

[53] S. D. Lester, F. A. Ponce, M. G. Craford, and D. A. Steigerwald, "High dislocation densities in high efficiency GaN-based light-emitting diodes," Applied Physics Letters, vol. 66, p. 1249, 1995.

[54] P. Kozodoy, H. Xing, S. P. DenBaars, U. K. Mishra, A. Saxler, R. Perrin, S. Elhamri, and W. C. Mitchel, "Heavy doping effects in Mg-doped GaN," Journal of Applied Physics, vol. 87, p. 1832, 2000. 
[55] C. Bayram, J. L. Pau, R. McClintock, and M. Razeghi, "Delta-doping optimization for high quality p-type GaN," Journal of Applied Physics, vol. 104, 083512, 2008.

[56] S. D. Burnham, G. Namkoong, D. C. Look, B. Clafin, and W. A. Doolittle, "Reproducible increased Mg incorporation and large hole concentration in GaN using metal modulated epitaxy," Journal of Applied Physics, vol. 104, p. 024902, 2008.

[57] F. Wang, J. Li, S.-S. Li, and J.-B. Xia, "Mg acceptor energy levels in AlxInyGa1-x-yN quaternary alloys: An approach to overcome the p-type doping bottleneck in nitrides," Physical Review B, vol. 77, p. 113202, 2008.

[58] I.-H. Lee, I.-H. Choi, C.-R. Lee, E.-J. Shin, D. Kim, S. K. Noh, S.-J. Son, K. Y. Lim, and H. J. Lee, "Stress relaxation in Si-doped GaN studied by Raman spectroscopy," Journal of Applied Physics, vol. 83, p. 5787, 1998.

[59] Y. Yan and S.-H. Wei, "Doping asymmetry in wide-bandgap semiconductors: Origins and solutions," physica status solidi b, vol. 245, pp. 641-652, 2008.

[60] B. N. Pantha, A. Sedhain, J. Li, J. Y. Lin, and H. X. Jiang, "Electrical and optical properties of p-type InGaN," Applied Physics Letters, vol. 95, p. 261904, 2009.

[61] M. Roever, D.-D. Mai, A. Bedoya-Pinto, J. Malindretos, and A. Rizzi, "Electron stabilized ferromagnetism in GaGdN," physica status solidi c, vol. 5, pp. 2352-2354, 2008.

[62] G. Martinez-Criado, O. Sancho-Juan, N. Garro, J. A. Sans, A. Cantarero, J. Susini, M. Roever, D.-D. Mai, A. Bedoya-Pinto, J. Malindretos, and A. Rizzi, "X-ray absorption in GaGdN: A study of local structure," Applied Physics Letters, vol. 93, p. 021916, 2008.

[63] J. Hejtmanek, K. Knizek, M. Marysko, Z. Jirak, D. Sedmidubsky, Z. Sofer, V. Perina, H. Hardtdegen, and C. Buchal, "On the magnetic properties of Gd implanted GaN," Journal of Applied Physics, vol. 103, p. 07D107, 2008.

[64] J. E. Sicre, J. T. Dubois, K. J. Eisentraut, and R. E. Sievers, "Volatile Lanthanide Chelates. II. Vapor Pressures, Heats of Vaporization, and Heats of Sublimation," Journal of the American Chemical Society, vol. 91, pp. 3476-3481, 1969.

[65] J. Niinisto, M. Putkonen, M. Ritala, P. Raisanen, A. Niskanen, and M. Lekela, "Method of Depositing Rare Earth Oxide Thing Films," United States Patent US20090035949A1, 2009.

[66] D. K. Schroder, Semiconductor Material and Device Characterization, Third ed. Hoboken, NJ: John Wiley \& Sons, 2006.

[67] X. H. Wu, P. Fini, E. J. Tarsa, B. Heying, S. Keller, U. K. Mishra, S. P. DenBaars, and J. S. Speck, "Dislocation generation in GaN heteroepitaxy," Journal of Crystal Growth, vol. 189-190, pp. 231-243, 1998. 
[68] M. A. Moram and M. E. Vickers, "X-ray diffraction of III-nitrides," Reports on Progress in Physics, vol. 72, p. 036502, 2009.

[69] L. J.v. d. Pauw, "A Method of Measuring Specific Resistivity and Hall Effect of Discs of Arbitrary Shape," Philips Research Reports, vol. 13, pp. 1-9, 1958.

[70] R. Chwang, B. J. Smith, and C. R. Crowell, "Contact Size Effects on the Van Der Pauw Method for Resistivity and Hall Coefficient Measurement," Solid-State Electronics, vol. 17, pp. 1217-1227, 1974.

[71] M. Nagamori, S. Ito, H. Saito, K. Shiojima, S. Yamada, N. Shibata, and M. Kuzuhara, "Optimum Rapid Thermal Activation of Mg-Doped p-Type GaN," Japanese Journal of Applied Physics, vol. 47, pp. 2865-2867, 2008.

[72] C. Y. Hu, Z. B. Ding, Z. X. Qin, Z. Z. Chen, K. Xu, Z. J. Yang, B. Shen, S. D. Yao, and G. Y. Zhang, "Ohmic contact mechanism of Ni/Au contact to p-type GaN studied by Rutherford backscattering spectrometry," Journal of Crystal Growth, vol. 298, pp. 808-810, 2007.

[73] S.-T. Park, "Neutron energy spectra of 252Cf, Am-Be source and of the D(d,n)3He reaction," Journal of Radioanalytical and Nuclear Chemistry vol. 256, pp. 163-166, 2003.

[74] E. Burgett, N. E. Hertel, and W. Lee, "Thermal Neutron Collimated Beam Line," Transactions of the American Nuclear Society, vol. 93, pp. 600-602, 2008.

[75] C. A. Klein, "Bandgap Dependence and Related Features of Radiation Ionization Energies in Semiconductors," Journal of Applied Physics, vol. 39, p. 2029, 1968.

[76] M. V. S. Chandrashekhar, C. I. Thomas, and M. G. Spencer, "Measurement of the mean electron-hole pair ionization energy in $4 \mathrm{H} \mathrm{SiC,"} \mathrm{Applied} \mathrm{Physics} \mathrm{Letters,} \mathrm{vol.}$ 89, p. 042113, 2006.

[77] J. Grant, W. Cunningham, A. Blue, V. O'Shea, J. Vaitkus, E. Gaubas, and M. Rahman, "Wide bandgap semiconductor detectors for harsh radiation environments," Nuclear Instruments \&amp; Methods in Physics Research, Section A (Accelerators, Spectrometers, Detectors and Associated Equipment), vol. 546, pp. 21317, 2005.

[78] M. A. Reschikov and H. Morkoc, "Luminescence properties of defects in GaN," Journal of Applied Physics, vol. 97, p. 061301, 2005.

[79] H. P. D. Schenk, S. I. Borenstain, A. Berezin, A. Schon, E. Cheifetz, A. Dadgar, and A. Krost, "Cathodoluminescence of epitaxial GaN and $\mathrm{ZnO}$ thin films for scintillator applications," Journal of Crystal Growth, vol. 311, pp. 3984-3988, 2009.

[80] A. A. Porporati and G. Pezzotti, "Absorption effect on the cathodoluminescence spectra of n-GaN," European Physical Journal of Applied Physics, vol. 48, p. 30901, 2009. 
[81] P. Pittet, G.-N. Lu, J.-M. Galvan, J.-M. Bluet, I. Anas, J.-Y. Giraud, and J. Balosso, "PL characterization of $\mathrm{GaN}$ scintillator for radioluminescence-based dosimetry," Optical Materials, vol. 31, p. 1421, 2009.

[82] P. Ramvall, Y. Aoyagi, A. Kuramata, P. Hacke, K. Domen, and K. Horino, "Doping dependent optical gain in GaN," Applied Physics Letters, vol. 76, p. 2994, 2000.

[83] H. P. D. Schenk, S. I. Borenstain, A. Berezin, A. Schon, E. Cheifetz, S. Khatsevich, and D. H. Rich, "Band gap narrowing and radiative efficiency of silicon doped GaN," Journal of Applied Physics, vol. 103, p. 103502, 2008.

[84] M. Godlewski, V. Y. Ivanov, E. Lusakowska, R. Bozek, S. Miasojedovas, S. Jursenas, K. Kazlauskas, E. M. Goldys, M. R. Phillips, T. Bottcher, S. Figge, andD. Hommel, "Influence of n-type doping on light emission properties of GaN layers and GaN-based quantum well structures," physica status solidi $c$, vol. 2, pp. 1056-1059, 2005.

[85] H. C. Yang, T. Y. Lin, M. Y. Huang, and Y. F. Chen, "Optical properties of Sidoped GaN films," Journal of Applied Physics, vol. 86, p. 6124, 1999.

[86] E. F. Schubert, Light-Emitting Diodes, Second ed. New York: Cambridge University Press, 2006.

[87] S. Gupta, H. Kang, M. Strassburg, A. Asghar, M. Kane, W. E. Fenwick, N. Dietz, and I. T. Ferguson, "A nucleation study of group III-nitride multifunctional nanostructures," Journal of Crystal Growth, vol. 287, pp. 596-600, 2006.

[88] N. Kuwano, M. Hijikuro, S. Hata, M. Takeuchi, and Y. Aoyagi, "TEM analysis of an interface layer formed by the anti-surfactant treatment on a GaN template," Journal of Crystal Growth, vol. 298, pp. 284-287, 2007.

[89] S. Tanaka, M. Takeuchi, and Y. Aoyagi, "Anti-Surfactant in III-Nitride Epitaxy-Quantum Dot Formation and Dislocation Termination-," Japanese Journal of Applied Physics, vol. 39, pp. L831-L834, 2000.

[90] Y. P. Hsu, S. J. Chang, Y. K. Su, S. C. Chen, J. M. Tsai, W. C. Lai, C. H. Kuo, and C. S. Chang, "InGaN-GaN MQW LEDs With Si Treatment," IEEE Photonics Technology Letters, vol. 17, p. 1620, 2005.

[91] S. Dhar, L. Perez, O. Brandt, A. Trampert, K. H. Ploog, J. Keller, and B. Beschoten, "Gd-doped GaN: a very dilute ferromagnetic semiconductor with a Curie temperature above 300 K," Physical Review B, vol. 72, pp. 245203-1, 2005.

[92] S. A. Wolf, A. Y. Chtchelkanova, and D. M. Treger, "Spintronics- a retrospective and perspective," IBM Journal of Research and Development, vol. 50, pp. 101-110, 2006.

[93] S. A. Wolf, D. D. Awschalom, R. A. Buhrman, J. M. Daughton, S. v. Molnar, M. L. Roukes, A. Y. Chtchelkanova, and D. M. Treger, "Spintronics: a spin-based electronics vision for the future," Science, vol. 294, pp. 1488-1495, 2001. 
[94] G. Schmidt, D. Ferrand, L. W. Molenkamp, A. T. Filip, and B. J. v. Wees, "Fundamental obstacle for electrical spin injection from a ferromagnetic metal into a diffusive semiconductor," Physical Review B, vol. 62, pp. R4790-R4793, 2000.

[95] Y. Ohno, I. Arata, F. Matsukura, H. Ohno, D. K. Young, B. Beschoten, and D. D. Awschalom, "Electrical spin injection in ferromagnetic/nonmagnetic semiconductor heterostructures," Physica E, vol. 10, pp. 489-492, 2001.

[96] S. Krishnamurthy, M. v. Schilfgaarde, and N. Newman, "Spin lifetimes of electrons injected into GaAs and GaN," Applied Physics Letters, vol. 83, pp. 17611763, 2003.

[97] M. H. Kane, M. Strassburg, A. Asghar, N. Li, W. Fenwick, and I. T. Ferguson, "Review of Recent Efforts on the Growth and Characterization of Nitride-based Diluted Magnetic Semiconductors," Proceedings of the SPIE, vol. 6121, pp. 61210L-1, 2006.

[98] A. Y. Polyakov, A. V. Govorkov, N. B. Smirnov, N. Y. Pashkova, G. T. Thaler, M. E. Overberg, R. Frazier, C. R. Abernathy, S. J. Pearton, K. Jihyun, and F. Ren, "Optical and electrical properties of GaMnN films grown by molecular-beam epitaxy," Journal of Applied Physics, vol. 92, pp. 4989-4993, 2002.

[99] N. Teraguchi, A. Suzuki, Y. Nanishi, Y.-K. Zhou, M. Hashimoto, and H. Asahi, "Room-temperature observation of ferromagnetism in diluted magnetic semiconductor GaGdN grown by RF-molecular beam epitaxy," Solid State Communications, vol. 122, pp. 651-653, 2002.

[100] S. Dhar, L. Perez, O. Brandt, A. Trampert, K. H. Ploog, J. Keller, and B. Beschoten, "Ferromagnetism and colossal magnetic moment in Gd-focused ionbeam-implanted GaN," Applied Physics Letters, vol. 89, pp. 62503-1, 2005.

[101] G. H. Zhong, J. L. Wang, and Z. Zeng, "Electronic and magnetic structures of 4f in Ga1-xGdxN," Journal of Physics: Condensed Matter, vol. 20, p. 2952221, 2008.

[102] G. M. Dalpian and S.-H. Wei, "Electron-induced stabilization of ferromagnetism in Ga1-xGdxN," Physical Review B, vol. 72, p. 115201, 2005.

[103] A. Ney, T. Kammermeier, V. Ney, S. Ye, K. Ollefs, E. Manuel, S. Dhar, K. H. Ploog, E. Arenholz, F. Wilhelm, and A. Rogalev, "Element specific magnetic properties of Gd-doped GaN: Very small polarization of Ga and paramagnetism of Gd," Physical Review B, vol. 77, p. 233308, 2008.

[104] A. Ney, T. Kammermeier, K. Ollefs, V. Ney, S. Ye, S. Dhar, K. H. Ploog, M. Rover, J. Malindretos, A. Rizzi, F. Wilhelm, and A. Rogalev, "Gd-doped GaN studied with element specificity: Very small polarization of Ga paramagnetism of Gd and the formation of magnetic clusters," Journal of Magnetism and Magnetic Materials, vol. 322, pp. 1162-1166, 2010.

[105] J. K. Hite, R. M. Frazier, R. Davies, G. T. Thaler, C. R. Abernathy, S. J. Pearton, and J. M. Zavada, "Effect of growth conditions on the magnetic characteristics of GaGdN," Applied Physics Letters, vol. 89, p. 092119, 2006. 
[106] C. Mitra and W. R. L. Lambrecht, "Interstitial-nitrogen and oxygen-induced magnetism in Gd-doped GaN," Physical Review B, vol. 80, p. 081202R, 2009.

[107] L. Liu, P. Y. Yu, M. Zhixun, and S. S. Mao, "Ferromagnetism in Gan:Gd: a density functional theory study," Physical Review Letters, vol. 100, p. 127203, 2008.

[108] P. Dev, Y. Xue, and P. Zhang, "Defect-Induced Intrinsic Magnetism in WideGap Nitrides," Physical Review Letters, vol. 100, p. 117204, 2008.

[109] M. Kohda, Y. Ohno, F. Matsukura, and H. Ohno, "Effect of $n+-G a A s$ thickness and doping density on spin injection of GaMnAs/n+-GaAs Esaki tunnel junction," Physica E, vol. 32, pp. 438-441, 2006.

[110] S. Gautier, G. Orsal, T. Moudakir, N. Maloufi, F. Jomard, M. Alnot, Z. Djebbour, A. Sirenko, M. Abid, K. Pantzas, I. T. Ferguson, P. L. Voss, and A. Ougazzaden, "Metal-organic vapour phase epitaxy of BInGaN quaternary alloys and characterization of boron content," Journal of Crystal Growth, vol. 312, pp. 641-644, 2010 . 